\title{
X-RAY ABSORPTION SPECTROSCOPIC STUDIES OF MONONUCLEAR NON-HEME IRON ENZYMES
}

Tami E. Westre

\section{RECEIVED}

AUG 121997

OSTI

SLAC-Report-499

January 1996
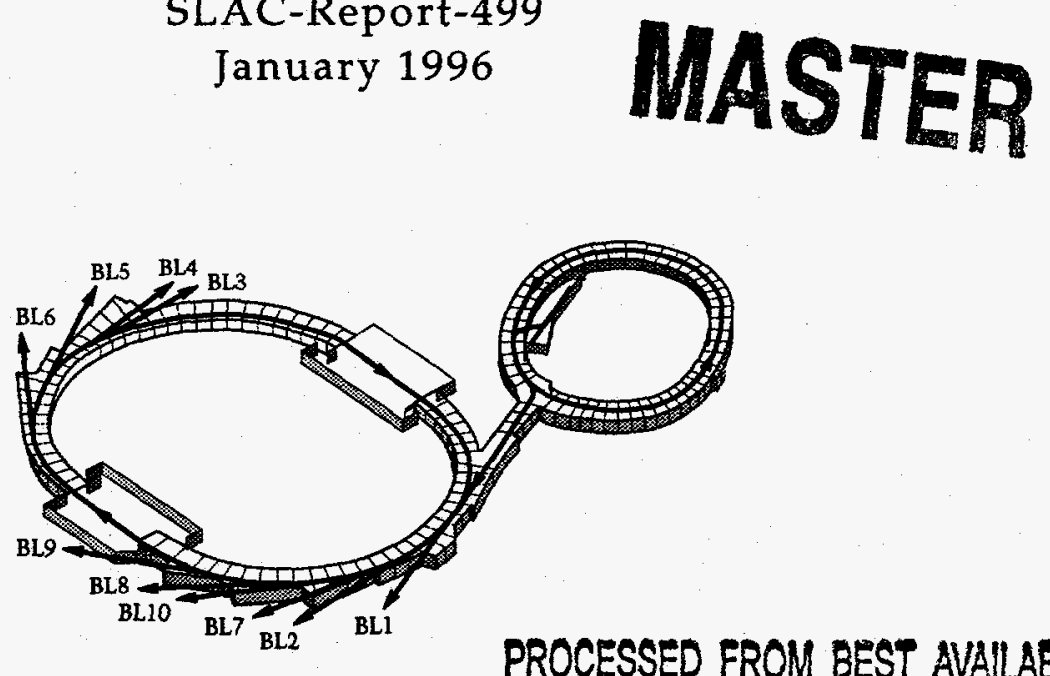

PROCESSED FRON BEST AVAILABLE COPY

Prepared for the Department of Energy

under contract number DE-AC03-76SF00515

STANFORD LINEAR ACCELERATOR CENTER

STANFORD SYNCHROTRON RADIATION LABORATORY

Stanford University - Stanford, California 


\section{DISCLAMMER}

Portions of this document may be illegible in electronic image products. Images are produced from the best available original document. 
This document and the material and data contained therein, was developed under sponsorship of the United States Government. Neither the United States nor the Department of Energy, nor the Leland Stanford Junior Unjversity, nor their employees, nor their respective contractors, subcontractors, or their employees, makes any warranty, express or implied, or assumes any liability or responsibility for accuracy, completeness or usefulness of any information, apparatus, product or process disclosed, or represents that its use will not infringe privately-owned rights. Mention of any product, its manufacturer, or suppliers shall not, nor is it intended to, imply approval, disapproval, or fitness for any particular use. A royalty-free, nonexclusive right to use and disseminate same for any purpose whatsoever, is expressly reserved to the United States and the University.

\title{
DISCLAIMER
}

\begin{abstract}
This report was prepared as an account of work sponsored by an agency of the United States Government. Neither the United States Government nor any agency thereof, nor any of their employees, makes any warranty, express or implied, or assumes any legal liability or responsibility for the accuracy, completeness, or usefulness of any information, apparatus, product, or process disclosed, or represents that its use would not infringe privately owned rights. Reference herein to any specific commercial product, process, or service by trade name, trademark, manufacturer, or otherwise does not necessarily constitute or imply its endorsement, recommendation, or favoring by the United States Government or any agency thereof. The views and opinions of authors expressed herein do not necessarily state or reflect those of the United States Government or any agency thereof.
\end{abstract}




\title{
X-RAY ABSORPTION SPECTROSCOPIC STUDIES OF MONONUCLEAR NON-HEME IRON ENZYMES
}

\author{
Tami E. Westre
}

Stanford Linear Accelerator Center

Stanford Synchrotron Radiation Laboratory

Stanford University, Stanford, California 94309

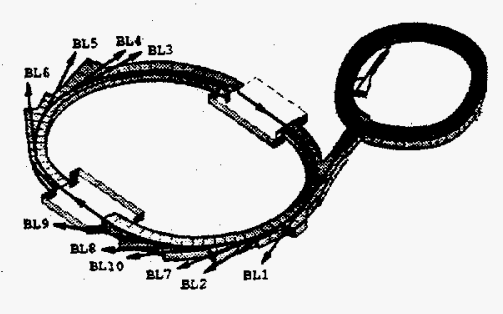

SLAC-Report-499

January 1996

Prepared for the Department of Energy under contract number DE-AC03-76SF00515 and the NIH, Biomedical Resource Technology Program,

Division of Research Resources

Printed in the United States of America. Available from the National Technical Information Service, U.S. Department of Commerce, 5285 Port Royal Road, Springfield, VA 22161

*Ph.D. Thesis Stanford University, Stanford, CA 94309 
X-RAY ABSORPTION SPECTROSCOPIC STUDIES OF MONONUCLEAR NON-HEME IRON ENZYMES

\author{
A DISSERTATION \\ SUBMITTED TO THE DEPARTMENT OF CHEMISTRY \\ AND THE COMMITTEE ON GRADUATE STUDIES \\ OF STANFORD UNIVERSITY \\ IN PARTIAL FULFILLMENT OF THE REQUIREMENTS \\ FOR THE DEGREE OF \\ DOCTOR OF PHILOSOPHY
}

By

Tami E. Westre

January 1996 


\begin{abstract}
Fe K-edge X-ray absorption spectroscopy (XAS) has been used to investigate the electronic and geometric structure of the iron active site in non-heme iron enzymes. A new theoretical extended X-ray absorption fine structure (EXAFS) analysis approach, called GNXAS, has been tested on data for iron model complexes to evaluate the utility and reliability of this new technique, especially with respect to the effects of multiplescattering. In addition, a detailed analysis of the $1 \mathrm{~s} \longrightarrow 3 \mathrm{~d}$ pre-edge feature has been developed as a tool for investigating the oxidation state, spin state, and geometry of iron sites. Edge and EXAFS analyses have then been applied to the study of non-heme iron enzyme active sites.
\end{abstract}

GNXAS and Its Application to Inorganic Iron Model Complexes. GNXAS, a recently developed integrated approach to the analysis of EXAFS data is presented in detail. Using the GNXAS approach, it is possible to calculate all the signals related to two-, three- and four-atom correlation functions with the proper treatment of correlated distances and Debye-Waller factors. The technique is particularly well-suited for the analysis of multiple-scattering effects and thus allows for accurate determination of bond distance and angular information of second and third neighbors. Herein the application of GNXAS to several chemical systems of known structure is reported. The reliability of GNXAS was evaluated on both a well-ordered inorganic complex, $\mathrm{Fe}$ (acac) 3 , as well as a lower-symmetry coordination complex with mixed ligation, $\mathrm{Na}\left[\mathrm{Fe}\left(\mathrm{OH}_{2}\right) \mathrm{EDTA}\right]$. The total EXAFS signal generated by GNXAS matches closely the experimental data for both complexes, especially when all the multiple-scattering contributions were included in the theoretical signal. First neighbor distances obtained from refinement using GNXAS, as well as distances and angles for further neighbors, compared very well with crystallographic values. The angle dependence of the Fe-C-N multiple-scattering contribution in $\mathrm{K}_{3} \mathrm{Fe}(\mathrm{CN})_{6}$ was also examined. The results indicate that GNXAS can be used to determine angles relatively accurately for Fe-C-N configurations with angles greater than about $150^{\circ}$. These results establish the utility and reliability of the GNXAS approach and provide a reliable means to determine additional structural information from EXAFS analysis of structures of chemical interest.

Angle Determination Using GNXAS. The Fe-N-O bond angle in a series of $\{\text { FeNO }\}^{7}$ complexes has been probed by EXAFS, utilizing the new theoretical data analysis package, GNXAS. Since it is possible with GNXAS to calculate all the signals related to two-, three-, and four-atom correlation functions with the proper treatment of correlated distances and Debye-Waller factors, the methodology is particularly 
well-suited for analysis of multiple-scattering effects and bond angle determination. EXAFS data were ob'ained on a series of crystallographically-characterized $\{\mathrm{FeNO}\}^{7}$ inorganic complexes with varying $\mathrm{Fe}-\mathrm{N}-\mathrm{O}$ angles to examine the sensitivity of the GNXAS fit to this angle. The compounds studied were $\mathrm{Fe}(\mathrm{TMC}) \mathrm{NO}$ which has an Fe-N$\mathrm{O}$ bond angle of $177.5(5)^{\circ}, \mathrm{Fe}(\mathrm{TACN})\left(\mathrm{N}_{3}\right)_{2} \mathrm{NO}$ which has an angle of $156(1)^{\circ}$ and $\mathrm{Fe}$ (salen)NO which has a bond angle of $127(6)^{\circ}$ at $-175^{\circ} \mathrm{C}$ and $147(5)^{\circ}$ at $23^{\circ} \mathrm{C}$. EXAFS data for FeEDTA-NO (whose crystal structure has not been determined and thus the angle is unknown) were also obtained and analyzed using GNXAS to determine the Fe$\mathrm{N}-\mathrm{O}$ bond angle. Results are presented which indicate that it is possible to determine whether the Fe-N-O unit is bent or linear, with the GNXAS analysis being extremely sensitive when the angle is between $150^{\circ}$ and $180^{\circ}$. Using this method the $\mathrm{Fe}-\mathrm{N}-\mathrm{O}$ angle in FeEDTA-NO is found to be $156(5)^{\circ}$. The results of this study establish that EXAFS analysis using GNXAS can provide reliable angular information for small molecules coordinated to transition metals with rather complex coordination environments. This study thus provides the basis for the determination of the coordination geometry of molecules like $\mathrm{NO}$ and $\mathrm{O}_{2}$ to metalloprotein active sites.

A Multiplet Analysis of the 1s $\longrightarrow$ 3d Pre-Edge Feature. In this study, XAS Fe $\mathrm{K}$-edge data on high spin and low spin ferrous and ferric model complexes with varying geometries, as well as binuclear complexes with varying oxidation state, geometry, and bridging ligation, were collected in order establish a detailed understanding of the $1 \mathrm{~s}-$ $>3 d$ pre-edge feature and its sensitivity to the electronic and geometric structure of the iron site. The energy splitting and intensity distribution of the pre-edge feature of these complexes varied with spin state, oxidation state, geometry, and bridging ligation (in the binuclear complexes). A methodology for interpreting the energy splitting and intensity distribution of the $1 \mathrm{~s} \rightarrow 3 \mathrm{~d}$ pre-edge features was developed for high spin ferrous and ferric complexes in octahedral, tetrahedral and square pyramidal environments and low spin ferrous and ferric complexes in octahedral environments. In each case, the allowable many-electron excited states were determined using ligand field theory. The energies of the excited states were calculated and compared to the energy splitting in the $1 \mathrm{~s} \rightarrow 3 \mathrm{~d}$ pre-edge features and the relative intensities of transitions into the many-electron excited states were obtained and compared to the intensity pattern of the pre-edge feature. The effect of distorting the iron site to tetrahedral and square-pyramidal geometries was analyzed. The contribution to the pre-edge intensity from both a quadrupole and a dipole (from $4 \mathrm{p}$-3d mixing) intensity mechanism was determined for these distorted cases where the amount of $4 \mathrm{p}$ mixing was experimentally determined and compared to a theoretical estimate of the amount of $4 p$ mixing determined from density functional calculations. 
The results presented should further aid in the interpretation of the $1 \mathrm{~s} \rightarrow 3 \mathrm{~d}$ pre-edge region for non-heme iron enzymes as the energy splitting and intensity pattern of the preedge features are directly related to the oxidation state, spin state and geometry of the iron site.

Characterization of the Active Sites in Non-Heme Iron Enzymes. Edge and EXAFS analyses have been used to characterize the active sites in several non-heme iron enzymes. A detailed analysis of the intensity and splitting of the $1 \mathrm{~s} \longrightarrow 3 \mathrm{~d}$ pre-edge feature allowed for determination of the oxidation state, spin state, and coordination geometry of the iron active site. In addition, an EXAFS analysis provided accurate first shell distances with information on the number and type of coordinating atoms. Not only were the resting enzymes studied, but stable substrate and oxygen bound intermediates were also investigated including the nitric oxide derivatives of the ferrous active sites which serve as reversible analogues of possible dioxygen intermediates.

As a probe of non-heme iron active sites, nitric oxide has been shown to react with the ferrous state of many mononuclear non-heme iron enzymes and model complexes to form an $[\mathrm{FeNO}\}^{7}$ complex which has a distinct $\mathrm{S}=3 / 2$ ground state. An edge analysis of the XAS data for three $\{\mathrm{FeNO}]^{7}$ model complexes was used to determine the oxidation state of the iron site in these iron-nitrosyl systems. The edge results were used in combination with results from other spectroscopies and theoretical methods to produce a new bonding description of the $[\mathrm{FeNO}]^{7}$ unit which involves high spin ferric iron ( $S=5 / 2)$ antiferromagnetically coupled to $\mathrm{NO}^{-}(S=1)$ to produce the $S=3 / 2$ ground state.

Bleomycin (BLM) is a glycopeptide antibiotic currently used in the treatment against a variety of carcinomas and lymphomas due to its ability to selectively cleave DNA. The geometric and electronic structure of high spin ferrous complexes of BLM and the structural analog PMAH have been investigated by XAS edge and EXAFS analyses. The XAS results have been used in combination with results from optical absorption (Abs), magnetic circular dichroism (MCD), and resonance Raman ( $\mathrm{rR}$ ) studies to define the electronic and geometric structure of the ferrous active site. The results indicate that there is a short $\mathrm{Fe}-\mathrm{N}$ bond which increases along the series solid [Fe(II)PMA] $]^{+}>$solution [Fe(II)PMA] ${ }^{+}>\mathrm{Fe}$ (II)BLM. The short bond is attributed to the pyrimidine ligand which is involved in pyrimidine $\pi$-backbonding. This pyrimidine $\pi$-backbonding mediates the electron density localized on the $\mathrm{Fe}^{2+}$ center which contributes to the unique chemistry of $\mathrm{Fe}$ (II)BLM relative to other non-heme iron sites.

Activated BLM is the first mononuclear non-heme iron oxygen intermediate stable enough for detailed spectroscopic study. It has been postulated that activated BLM 
is an oxoferryl intermediate on the basis of its reactivity and analogy with cytochrome P-450 chemistry. Altematively, spectroscopic and model studies have indicated activated BLM to have an Fe(III)-peroxide site. XAS has buen used to directly probe the oxidation and spin states of the iron in activated BLM and to determine if a short iron-oxo bond is present, which would be characteristic of the oxo-ferryl species of heme iron. Both the pre-edge and edge regions of the Fe K-edge spectra indicate that activated BLM is a low spin ferric complex. The pre-edge intensity of activated BLM is also similar to that of low spin ferric BLM and does not show the intensity enhancement which would be present if there were a short $\mathrm{Fe}-\mathrm{O}$ bond. Furthermore, bond distances obtained from EXAFS are similar to those in low spin Fe(III)BLM and show no evidence for a short iron-oxo bond. These data indicate that activated BLM is a peroxy-low spin ferric complex and suggest that such an intermediate may play an important role in activating $\mathrm{O}_{2}$ for further chemistry in the catalytic cycles of mononuclear non-heme iron enzymes.

Lipoxygenases (LOs) are non-heme iron enzymes which catalyze the reaction of dioxygen with cis, cis-1,4-pentadiene containing fatty acids to form hydroperoxide products, which in mammals are the precursors to the inflammation and immunity mediating compounds lipoxins and leukotrienes. Recent X-ray crystal structures of ferrous soybean lipoxygenase-1 (SLO-1) offer two different descriptions of the active site: one four-coordinate and one five- or six-coordinate. Near infrared (NIR) circular and magnetic circular dichroism (CD/MCD) and variable temperature, variable field (VTVH) MCD have been used to study SLO-1 in solution which is found to exist as a $40 / 60 \%$ mixture of five- and six-coordinate forms, respectively. An XAS edge and pre-edge analysis also shows that the mammalian 15-LOs and SLO-1 in glycerol are six-coordinate. This is consistent with the EXAFS results of SLO-1 in glycerol which show the iron active site to have $5 \pm 1$ N/O at $-2.16 \AA$. VTVH MCD data on the six-coordinate sites show that the mammalian and soybean enzymes have very different ground state splittings, indicative of differences in $\pi$ bonding interactions with the ligand set. These differences in ferrous site coordination in solution and ground state splittings are attributed to the substitution of a stronger histidine ligand in the mammalian 15-LOs for an asparagine in SLO-1.

Phenylalanine hydroxylase (PAH) is involved in the metabolism of phenylalanine and its dysfunction is responsible for the genetic disorder phenylketonuria which is characterized by irreversible, progressive brain damage. A transition between the resting low affinity state (the " $T$ " state) and the high affinity state (the " $R$ " state) is required for the enzyme to achieve catalytic competence where phenylalanine binds to an allosteric effector site (as distinguished from the active site phenylalanine binding site). XAS was 
used to define the geometric and electronic structure of the non-heme iron active site of $\mathrm{Fe}(\mathrm{I}) \mathrm{PAH}^{\mathrm{R}}, \mathrm{Fe}$ (II)PAH $\mathrm{PA}^{\mathrm{T}}, \mathrm{Fe}(\mathrm{III}) \mathrm{PAH}^{\mathrm{R}}$, and $\mathrm{Fe}$ (III)PAH ${ }^{\mathrm{T}}$. The edge and pre-edge features for the four forms of PAH studied indicate that the iron site is six-coordinate. The fits to the EXAFS data for all four forms of the protein give ligand distances typical of six-coordinate iron model complexes with oxygen and nitrogen ligation. There were subtle differences in the EXAFS fits to the data of Fe(II)PAHR $v$ s. Fe(II)PAH ${ }^{T}$. The differences seen in the EXAFS data of Fe(II)PAHR and Fe(II)PAH ${ }^{\mathrm{T}}$ can either be attributed to changes caused by phenylalanine in the active site or by the activation process (i.e. phenylalanine in the allosteric site). Further studies need to be done to sort out the effects of phenylalanine in the active site $v s$. the allosteric site.

Protocatechuate 3,4-dioxygenase (PCD), one of the most well-studied intradiol dioxygenases, catalyzes the intradiol cleavage of protocatechuic acid to produce $\beta$-carboxy-cis,cis-muconic acid. It is clear from previous studies that the native intradiol dioxygenases have a high spin ferric active site and that the enzyme mechanism involves initial substrate binding followed by $\mathrm{O}_{2}$ attack. Herein XAS studies on $\mathrm{Fe}$ (II)PCD, $\mathrm{Fe}$ (III)PCD, Fe(III)PCD + 3,4 dihydroxybenzoic acid (Fe(III)PCA), and FePCD-NO are reported. The XAS edge and pre-edge features of Fe(II)PCD indicate that the iron active site is six-coordinate with an EXAFS analysis showing those ligands to be oxygens and nitrogens. The iron active site of both Fe(III)PCD and Fe(III)PCA appear to be five-coordinate as the pre-edge and edge features are similar to those of five-coordinate ferric complexes. An analysis of the EXAFS data for $\mathrm{Fe}(\mathrm{III}) \mathrm{PCD}$ shows $\mathrm{O} / \mathrm{N}$ ligands at $1.92 \AA$ and $2.12 \AA$. The EXAFS of Fe(III)PCA could not be well simulated without a longer O/N contribution at $2.47 \AA$ in addition to contributions at 1.97 and $2.10 \AA$. The edge and pre-edge results for FePCD-NO indicate that the iron atom is in the ferric oxidation state with the rising edge of FePCD-NO being at lower energy than that of $\mathrm{Fe}$ (III)PCD due to the highly covalent nature of the $\mathrm{Fe}(\mathrm{III})-\mathrm{NO}^{-}$bond. An analysis of the pre-edge intensity for FePCD-NO predicts that the iron site is five-coordinate with a bent Fe-N-O unit. FePCD-NO having a bent Fe-N-O unit is also supported by a GNXAS analysis of the EXAFS data of FePCD-NO. In addition the EXAFS data of FePCD-NO were fit well with $1 \mathrm{O} / \mathrm{N}$ at $1.89 \AA, 4 \mathrm{O} / \mathrm{N}$ at $2.11 \AA$, and $1 \mathrm{O} / \mathrm{N}$ at $2.45 \AA$. The shorter distance is attributed to the $\mathrm{Fe}-\mathrm{N}(\mathrm{O})$ bond distance. An Fe-N(O) distance of $1.89 \AA$ is much longer than previously observed $\mathrm{Fe}-\mathrm{N}(\mathrm{O})$ distances for $\{\mathrm{FeNO}\}^{7}$ model complexes. The nature of the longer $\mathrm{Fe}-\mathrm{N}(\mathrm{O})$ bond in FePCD-NO needs to be further investigated keeping in mind that tyrosinate $\rightarrow \mathrm{Fe}$ (III) charge donation may limit the ability of $\mathrm{NO}^{-}$to donate electron density to the ferric site, thereby weakening the $\mathrm{Fe}-\mathrm{N}(\mathrm{O})$ interaction. 


\section{Acknowledgments}

I have been very fortunate in my graduate career to not only work on very interesting projects, but also to have worked as a joint student - reaping the benefits of two advisors and two research groups. My advisors Ed Solomon and Keith Hodgson have encouraged and guided me through my various projects over the past five and a half years. They have very different advising styles, but I feel as if I have benefited from working under both of them. Ed was always the motivator and Keith allowed for independent thinking, while the both provided unequaled insight into their respective fields of expertise. I appreciate their guidance and also the opportunities that they have given me to present the projects that I worked on at conferences locally and internationally.

Britt Hedman and Pat Frank are two remarkable people that have accurately been referred to as the "secret weapons" of the Hodgson group. Their many years of scientific experience have proven to be an invaluable resource and their many years of doing research in Keith's lab provided continuity to the group. I not only respect Pat and Britt as scientists, but they have both become friends and confidantes. I will miss working with them!

One of the benefits of working for two advisors is the opportunity to interact with so many different people. Numerous people have influenced my graduate career both directly and indirectly including all the past and present members of the Solomon and Hodgson groups: Heather Bufford, Jason Chen, Ling Ling Chen, Jane DeWittt, David Eliezer, Isaac Liu, Kent Nakagawa, Dan Segel, Susan Shadle (1/2), Chrisie Stanfel, Grace Tan, Trevor Tyson, Erik Wasinger (1/2), Kendra Williams (1/2), Holly Zhang, Mike Baldwin, Carl Brown, Cecelia Campochiaro, Pat Clark, Sabine (Pulver) Coates, Jim Cole, Mindy Davis, Daniel Gamelin, Jeff Guckert, Melissa Hanson, Brooke Hemming, Ted Holman, Paul Jones, Pierre Kennepohl, Marty Kirk, Lou LaCroix, Kelly Loeb, Mike Lowery, Tim Machonkin, Jennifer May, Jim McCormick, Amy Palmer, Elizabeth Pavel, Mark Pavlosky, Brad Reitz, Dave Root, Uma Sandaram, Susan Shadle (1/2), Woon Shin, Felix Tuczek, Erik Wasinger (1/2), Kendra Williams (1/2), Yi-Shan Yang, Mark Yeager, Jeff Zaleski, Yan Zhang, Jing Zhou. They are all terrific people and I grateful for the chance to work with them and get to know them. I have enjoyed interacting with my labmates both scientifically and socially (gambling for mooncakes, dining on authentic Swedish food, and the numerous barbecues and Christmas parties). I wish them all the best of luck in the future! 
Collecting data at SSRL is a very tiring and stressful process. The success of our experiments can in part be attributed to the staff at SSRL who work hard to ensure that all the equipment is working properly and that there is "beam". I have really enjoyed the camaraderie that ensues during a beamtime experiment. Typically there are twice as many samples as we have time for and so everyone must work together to get the experiments done as efficiently as possible. Most of the time there are other obstacles placed in the way - no beam, faulty power supplies, mis-labelled gas cylinders, etc. that make beamtime experiments challenging. Such an intense, stressful time could bring out the worst in people, but that has not been my experience. I am grateful to all the people involved in these experiments for making beamtime fun and challenging instead of dreadful and challenging. I should also thank the people that actually provided the samples for me to run. Yan Zhang, Mark Pavlosky, Kelly Loeb and Jeff Zaleski have spent a lot of time preparing and characterizing protein samples for me to run. I appreciate the time that they spent ensuring the quality of the samples.

I worked closely with Rino Natoli, Andrea Di Cicco, and Adriano Filipponi on the GNXAS approach to data analysis. Not only did they develop the theory behind the approach and the software, but their guidance was crucial in implementing this new software in our lab. I really enjoyed working with Rino, Andrea, and Adriano on their numerous visits here and on my trip to Frascati, Italy. They were great hosts!

Sabine (Pulver) Coates, Jane DeWitt, and Susan Shadle have played very special roles in my graduate career. Being a year, three years, and two years ahead of me, respectively, they taught me the ropes and served as role models. They indoctrinated me into the world of X-ray absorption spectroscopy, non-heme iron enzymes, and ligand field theory, encouraged me throughout my years here, and bolstered my confidence in the my ability to do science. Not only were they great lab-mates, but they are AWESOME friends. I am extremely thankful for the opportunity to work with them and for their friendship.

I have also had the pleasure of working with $1 / 2$ people, that is joint Hodgson/Solomon students. Susan Shadle, myself, Kendra Williams, and Erik Wasinger (the newest member) have had joint projects over the past few years. Susan, Kendra, and I have worked closely together building a vacuum chamber and running our first I-edge experiments, as well as discussing the intricacies of ligand field theory and how it relates to our X-ray absorption experiments. By being thrown in the same boat so to speak, we have developed close relationships. I wish Kendra and Erik success in their future experiments and speedy graduate careers! 
I want to give a special thanks to my family and friends for supporting and encouraging me over the past 5 (or so) years and for providing the entertainment! Geoff and Sabine Coates, Jane DeWitt, Carl (OhNO) Brown and Susan Shadle, Tom and Lendy Vail, and Erik and Kim Westre have provided breaks away from lab to have a nice dinner, a weekend get-away, a challenging game of ravine ball, and camping trips (you can throw in a few good wedding celebrations too!) - which have been imperative to my sanity. Long live Gila Monsters and Vodka Collins!! My family, Chris and Faye Gansberg, Todd and Julie Gansberg, Wendy and Anthony Reis, Barb and Doug Westre (plus my grandparents, aunts and uncles and cousins), has also provided their share of both relaxing (and rejuvenating) and fun-filled get-aways. In addition, I should acknowledge Star, Belle, Beau, and Cassie, our horses which have had stints at a stable in Portola Valley. They were probably the first to know when I had a frustrating day and I am sure that Sjon was grateful for the stress relief that they provided for me before I got home to him. Lastly, I want to thank Sjon for putting up with me as I experienced the ups and downs of research over the past few years. Having gone through the graduate experience himself, he could rejoice and empathize with me as the situation warranted and he was always there to put things into perspective and encourage me to move forward. I should also thank him for not having me committed during beamtime!

This dissertation is dedicated to all the teachers in my life: first and foremost, my parents, who taught me that anything worth doing is worth doing right; my brother and sister, who encouraged me to have fun (even during the most monotonous tasks); my elementary and high school teachers, who gave me an interest science (especially Charlie Condron and Barb Gallagher); my undergraduate advisor, Peter Kelly, who gave me my first taste of research; my advisors, Ed Solomon and Keith Hodgson, who guided me through my graduate career; Britt Hedman and Pat Frank, who never tired (at least they never told me so) of my endless questions about experimental techniques, data analysis, and metalloprotein chemistry; past and present members of the Hodgson and Solomon labs, who taught me about these things called enzymes and X-ray absorption spectroscopy; and lastly and most importantly my husband, Sjon, who taught me to take research one step at a time, one day at a time and things will get done! 


\section{Contents}

Abstract

iii

Acknowledgments viii

Contents $\quad x i$

List of Tables $\quad$ xvi

List of Figures xviii

List of Abbreviations $\quad$ xxiii

Chapter 1 Introduction to Mononuclear Non-Heme Iron Enzymes and X-ray Absorption Spectroscopy

1.1. Scope and Organization of this Dissertation 2

1.2. Mononuclear Non-Heme Iron Enzymes 3

1.3. X-ray Absorption Spectroscopy 6

1.3.1. General Background 6

1.3.2. Experimental Considerations 8

1.3.3. Edge Theory and Analysis $\quad 10$

1.3.4. EXAFS Theory and Analysis 11

1.3.4.1. Information Obtainable from EXAFS 11

1.3.4.2. Single-Scattering Process 12

1.3.4.3. Multiple-Scattering Process 16

$\begin{array}{ll}\text { 1.4. References } & 19\end{array}$

Chapter 2 GNXAS, a New Multiple-Scattering EXAFS Analysis Package, and Its Application to Iron Inorganic Model Complexes 23

2.1. Introduction 24

2.2. GNXAS Methodology 27

2.2.1. Background $\quad 27$

2.2.2. GNXAS Theoretical and Analytical Approach 29

2.2.3. The GNXAS Program Set 42

2.3. Applications to Iron Complexes 43

2.3.1. Sample Preparation and Data Collection 43

2.3.2. GNXAS Data Analysis $\quad 44$

2.3.3. Results and Discussion 44 
2.5. Acknowledgments 67

2.6. References and Notes . 67

Chapter 3 Determination of the Fe-N-O Angle in \{FeNO $\}^{7}$ Complexes Using Multiple-Scattering EXAFS Analysis by GNXAS

3.1. Introduction 72

3.2. Experimental Section 74

3.2.1. Sample Preparation and Data Collection 74

3.2.2. GNXAS Data Analysis 75

3.2.3. Empirical EXAFS Analysis 78

3.3. Results and Discussion 79

3.3.1. GNXAS Fits of $\{\mathrm{FeNO}\}^{7}$ Complexes with Known Fe-N-O Angles $\quad 79$

3.3.2. Fe-N-O Angle Determination of an $\{\mathrm{FeNO}\}^{7}$ Complex of Unknown Structure $\quad 92$

3.4. Summary

3.5. Acknowledgments 103

3.6. References and Notes 103

Chapter 4 A Multiplet Analysis of Fe K-Edge 1s $\rightarrow$ 3d Pre-Edge Features of Iron Complexes

4.1. Introduction

4.2. Experimental Section

4.2.1. Sample Preparation

4.2.2. XAS Data Collection and Reduction

4.2.3. Data Analysis

4.3. Results and Analysis

4.3.1. High Spin Ferrous Complexes 
4.3.1.3. $\mathrm{C}_{4 \mathrm{v}}$ Geometry

4.3.2. High Spin Ferric Complexes 128

4.3.2.1. $\mathrm{O}_{\mathrm{h}}$ Geometry 128

4.3.2.2. $T_{d}$ Geometry 131

4.3.2.3. $\mathrm{C}_{4 \mathrm{v}}$ Geometry $\quad 134$

4.3.3. Binuclear Complexes 138

4.3.4. Low Spin Iron Complexes 145

4.3.4.1. Ferrous Complexes 145

4.3.4.2. Ferric Complexes 148

4.4. Discussion 151

4.5. Acknowledgments 153

4.6. References and Notes 154

Chapter 5 Characterization of the Electronic and Geometric Structure of Non-Heme Iron Active Sites Using Fe K-Edge XAS 159

5.1. Mononuclear Non-Heme Iron Enzymes 160

5.1.1. Mechanistic and Structural Background 160

$\begin{array}{ll}\text { 5.1.2. References } & 168\end{array}$

5.2. $\{\mathrm{FeNO}\}^{7}$ Complexes 173

5.2.1. Introduction 173

5.2.2. Experimental Section 174

5.2.3. Results and Analysis $\quad \cdot 175$

5.2.4. Discussion 185

5.2.5. Acknowledgments 187

5.2.6. References and Notes 187

5.3. Iron(II) Bleomycin 190

5.3.1. Introduction 190

5.3.2. Experimental Section 192

5.3.3. Results and Analysis 195

5.3.3.1. Fe K-Edge XAS 195

5.3.3.2. EXAFS 197

5.3.4. Discussion 201

5.3.5. Acknowledgments 205

5.3.6. References and Notes 205

$\begin{array}{ll}\text { 5.4. Activated Bleomycin } & 210\end{array}$ 
5.4.1. Introduction 210

5.4.2. Experimental Section 211

5.4.3. Results and Analysis 214

5.4.3.1. Fe K-Edge XAS 214

5.4.3.2. EXAFS 218

5.4.4. Discussion . 218

5.4.5. Acknowledgments 222

5.4.6. References and Notes $\quad 222$

5.5. Lipoxygenases 225

5.5.1. Introduction 225

5.5.2. Experimental Section $\quad 226$

5.5.3. Results and Analysis 229

5.5.3.1. Fe K-Edge XAS 229

5.5.3.2. EXAFS 232

5.5.4. Discussion 236

5.5.5. Acknowledgments 239

5.5.6. References and Notes . 239

5.6. Phenylalanine Hydroxylase 243

5.6.1. Introduction 243

5.6.2. Experimental Section 245

5.6.3. Results and Analysis 248

5.6.3.1. Fe K-Edge XAS 248

5.6.3.2. EXAFS 254

5.6.4. Discussion 259

5.6.5. Acknowledgments 260

5.6.6. References and Notes 261

5.7. Protocatechuate 3,4-Dioxygenase 264

5.7.1. Introduction 264

5.7.2. Experimental Section 266

5.7.2.1. Sample Preparation 266

5.7.2.2. Data Collection and Reduction 267

5.7.2.3. Empirical EXAFS Analysis 268

5.7.2.4. GNXAS Data Analysis 269

5.7.2.5. $1 \mathrm{~s} \rightarrow 3 \mathrm{~d}$ Pre-Edge Analysis $\quad 270$

5.7.3. Results and Analysis 271

5.7.3.1. Fe K-Edge XAS 271 


\section{List of Tables}

\section{Chapter 1}

Table 1.1. Description of the Variables in the Single-Scattering

Expression for $\chi$

\section{Chapter 2}

Table 2.1. Comparison of $\mathrm{Fe}(\mathrm{acac})_{3}$ GNXAS Distance and Angle

Fitting Results to Crystallographic Values

Table 2.2 Comparison of the $\mathrm{Na}\left[\mathrm{Fe}\left(\mathrm{OH}_{2}\right)\right.$ EDTA] GNXAS Values to the

Crystallographic Values of $\mathrm{Li}\left[\mathrm{Fe}\left(\mathrm{OH}_{2}\right)\right.$ EDTA] $2 \mathrm{H}_{2} \mathrm{O}$

\section{Chapter 3}

Table 3.1. Crystallographic Bond Distances and Angles Compared to GNXAS Results for $\{\text { FeNO }\}^{7}$ Complexes with Known Fe-N-O Angles

Table 3.2. Results of First-Shell Empirical Fits of FeEDTA Complexes

Table 3.3. Comparision of the $\mathrm{Li}\left[\mathrm{Fe}\left(\mathrm{OH}_{2}\right) \mathrm{EDTA}\right] \cdot 2 \mathrm{H}_{2} \mathrm{O}$ Crystallographic Bond Distances and Angles to the GNXAS and Empirical Fitted Bond Distances and Angles for $\mathrm{Na}\left[\mathrm{Fe}\left(\mathrm{OH}_{2}\right)\right.$ EDTA] Solution and Powder and FeEDTA-NO

\section{Chapter 4}

Table 4.1. XAS Pre-Edge Energies and Areas for High Spin Iron Model Complexes

Table 4.2. XAS Pre-Edge Energies and Areas for Binuclear Model Complexes

Table 4.3. XAS Pre-Edge Energies and Areas for Low Spin Iron Model Complexes 


\section{Chapter 5}

Table 5.1. Mononuclear Non-Heme Iron Enzymes

Table 5.2. XAS Pre-Edge Energies and Areas for $\{\mathrm{FeNO}\}^{7}$ and Related Complexes

Table 5.3. Summary of EXAFS Curve-Fitting Results for Fe(T)BLM and Fe(II)PMA

Table 5.4. Summary of EXAFS Curve-Fitting Results for Fe(II)BLM, $\mathrm{Fe}$ (III)BLM and Activated BLM

Table 5.5. XAS Pre-Edge Energies and Areas for LO's and Model Complexes 231

Table 5.6. Summary of EXAFS Curve-Fitting Results for SLO-1 235

Table 5.7. XAS Pre-Edge Energies and Areas for PAH and Model Complexes

Table 5.8. Summary of EXAFS Curve-Fitting Results for $\mathrm{Fe}(\mathrm{I}) \mathrm{PAH}^{\mathrm{T}}$, $\mathrm{Fe}$ (II)PAH ${ }^{\mathrm{R}}, \mathrm{Fe}$ (III)PAH $\mathrm{PA}^{\mathrm{T}}$, and $\mathrm{Fe}$ (III)PAHR

Table 5.9. XAS Pre-Edge Energies and Areas for PCD and Model Complexes

Table 5.10. Summary of EXAFS Curve-Fitting Results for Fe(II)PCD, $\mathrm{Fe}$ (III)PCD, Fe(III)PCA, and FePCD-NO

Table 5.11. Crystallographic Bond Distances and Angles Compared to GNXAS Results for Fe(III)PCD

Table 5.12. GNXAS Results for FePCD-NO 


\section{List of Figures}

\section{Chapter 1}

Figure 1.1. Typical Fe K-Edge XAS Spectrum 7

Figure 1.2. Typical Hard X-ray Experimental Set-Up a 9

Figure 1.3. Diagram Depicting EXAFS Constructive and Destructive Interference 13

$\begin{array}{ll}\text { Figure 1.4. Example of a Multiple-Scattering Pathway } & 17\end{array}$

\section{Chapter 2}

Figure 2.1. Angular Dependence of the Expansion Parameter 33

Figure 2.2. Molecular Structure of $\mathrm{Fe}(\mathrm{acac})_{3} \quad 46$

Figure 2.3. GNXAS Fits to the EXAFS Data of $\mathrm{Fe}(\mathrm{acac})_{3}$

Figure 2.4. Comparison of the Fe- $\mathrm{C}_{1} \gamma(2)$ EXAFS Signal to the Fe-O-C $\mathrm{C}_{1} \gamma(3)$ Signal and the Total Fe-O-C $\mathrm{C}_{1}$ Signal

Figure 2.5. FT of the EXAFS Signals of $\mathrm{Fe}(\mathrm{acac})_{3}$ for the Individual Contributions Shown in Figure 2.3D

Figure 2.6. Molecular Structure of $\left[\mathrm{Fe}\left(\mathrm{OH}_{2}\right) \text { EDTA }\right]^{-}$

Figure 2.7. GNXAS Fit to the EXAFS Data of $\mathrm{Na}\left[\mathrm{Fe}\left(\mathrm{OH}_{2}\right)\right.$ EDTA]

Figure 2.8. FT of the EXAFS Signals of $\mathrm{Na}\left[\mathrm{Fe}\left(\mathrm{OH}_{2}\right) \mathrm{EDTA}\right]$ for the Individual Contributions Shown in Figure 2.7

Figure 2.9. GNXAS Fit to the EXAFS Data of $\mathrm{K}_{3} \mathrm{Fe}(\mathrm{CN})_{6}$

Figure 2.10. EXAFS Signals of the $\gamma(3) \mathrm{Fe}-\mathrm{C}-\mathrm{N}$ Contribution and the $\gamma(2) \mathrm{Fe}-\mathrm{N}$ Contribution with $\mathrm{Fe}-\mathrm{C}-\mathrm{N}$ Angles of $180^{\circ}, 150^{\circ}, 120^{\circ}$ and $90^{\circ}$

\section{Chapter 3}

Figure 3.1. Molecular Structures of $[\mathrm{Fe}(\mathrm{TMC}) \mathrm{NO}]\left(\mathrm{BF}_{4}\right)_{2}$, $\mathrm{Fe}(\mathrm{TACN})\left(\mathrm{N}_{3}\right)_{2} \mathrm{NO}$, and $\mathrm{Fe}$ (salen) NO at $23^{\circ} \mathrm{C}$

Figure 3.2. GNXAS Fit to the EXAFS Data of [Fe(TMC)NO] $\left(\mathrm{BF}_{4}\right)_{2}$

Figure 3.3. GNXAS Fit to the EXAFS Data of $\mathrm{Fe}(\mathrm{TACN})\left(\mathrm{N}_{3}\right)_{2} \mathrm{NO}$

Figure 3.4. GNXAS Fit to the EXAFS Data of Fe(salen)NO at $10 \mathrm{~K}$

Figure 3.5. GNXAS Fit to the EXAFS Data of Fe(salen)NO at $220 \mathrm{~K}$ 
Figure 3.6. Comparison of Theoretical and Experimental FTs of [Fe(TMC)NO] $\left(\mathrm{BF}_{4}\right)_{2}$ EXAFS Data with Differing

Fe-N-O Angles

Figure 3.7. Comparison of Theoretical and Experimental FTs of $\mathrm{Fe}(\mathrm{TACN})\left(\mathrm{N}_{3}\right)_{2} \mathrm{NO}$ EXAFS Data with Differing $\mathrm{Fe}-\mathrm{N}-\mathrm{O}$ Angles

Figure 3.8. Comparison of Theoretical and Experimental FTs of

[Fe(salen)NO at $10 \mathrm{~K}$ EXAFS Data with Differing

$\mathrm{Fe}-\mathrm{N}-\mathrm{O}$ Angles

Figure 3.9. Comparison of Theoretical and Experimental FTs of

$\mathrm{Fe}$ (salen)NO at $220 \mathrm{~K}$ EXAFS Data with Differing

$\mathrm{Fe}-\mathrm{N}-\mathrm{O}$ Angles

Figure 3.10. Plots of the $\log (R$ value $) v s$. Fe-N-O Angle for

$[\mathrm{Fe}(\mathrm{TMC}) \mathrm{NO}]\left(\mathrm{BF}_{4}\right)_{2}, \mathrm{Fe}(\mathrm{TACN})\left(\mathrm{N}_{3}\right)_{2} \mathrm{NO}$,

$\mathrm{Fe}$ (salen)NO at $220 \mathrm{~K}$, and $\mathrm{Fe}$ (salen)NO at $10 \mathrm{~K}$

Figure 3.11. Empirical First-Shell Fits of $\mathrm{Na}\left[\mathrm{Fe}\left(\mathrm{OH}_{2}\right) \mathrm{EDTA}\right]$ Powder, $\mathrm{Na} F \mathrm{Fe}\left(\mathrm{OH}_{2}\right)$ EDTA] Solution, and FeEDTA-NO EXAFS Data

Figure 3.12. GNXAS Fit to the EXAFS Data of $\mathrm{Na}\left[\mathrm{Fe}\left(\mathrm{OH}_{2}\right)\right.$ EDTA] in Solution

Figure 3.13. GNXAS Fit to the EXAFS Data of FeEDTA-NO

Figure 3.14. Comparison of Theoretical and Experimental FTs of FeEDTA-NO EXAFS Data with Differing Fe-N-O Angles

Figure 3.15. Plot of the $\log (R$ value) vs. Fe-N-O Angle for FeEDTA-NO

\section{Chapter 4}

Figure 4.1. XAS Fe K-Edge Spectra of $\mathrm{FeF}_{2}, \mathrm{FeCl}_{2}, \mathrm{FeBr}_{2}$, and $\mathrm{FeI}_{2}$

Figure 4.2. XAS Fe K-Edge Spectra of Rinneite, $\mathrm{FeSiF}_{6} 6^{\circ} 6 \mathrm{H}_{2} \mathrm{O}$, $\left(\mathrm{NH}_{4}\right)_{2} \mathrm{Fe}\left(\mathrm{SO}_{4}\right)_{2} \cdot 6 \mathrm{H}_{2} \mathrm{O}$, and [ $\mathrm{Fe}$ (imidazole $\left.)_{6}\right] \mathrm{Cl}_{2}$

Figure 4.3. Fit to the Fe K-Edge XAS Pre-Edge Region of $\mathrm{FeSiF}_{6} \cdot{ }^{\circ} \mathrm{H}_{2} \mathrm{O}$

Figure 4.4. XAS Fe K-Edge Spectra of $\left(\mathrm{Et}_{4} \mathrm{~N}\right)_{2}\left[\mathrm{FeCl}_{4}\right], \mathrm{Cs}_{3} \mathrm{FeCl}_{5}$, and $\mathrm{Fe}\left(\mathrm{HB}\left(3,5-i \mathrm{Pr}_{2} \mathrm{pz}\right)_{3}\right) \mathrm{Cl}$

Figure 4.5. Fit to the $\mathrm{Fe}$ K-Edge XAS Pre-Edge Region of $\left(\mathrm{Et}_{4} \mathrm{~N}\right)_{2}\left[\mathrm{FeCl}_{4}\right]$ 
Figure 4.6. XAS Fe K-Edge Spectra of $\left(\mathrm{BF}_{4}\right)[\mathrm{Fe}(\mathrm{TMC}) \mathrm{Cl}]$, $\left(\mathrm{BF}_{4}\right)[\mathrm{Fe}(\mathrm{TMC}) \mathrm{Br}]$, $\left(\mathrm{BF}_{4}\right)\left[\mathrm{Fe}(\mathrm{TMC}) \mathrm{CH}_{3} \mathrm{CN}\right]$ and $\left(\mathrm{BF}_{4}\right)\left[\mathrm{Fe}(\mathrm{TMC}) \mathrm{N}_{3}\right]$

Figure 4.7. Fit to the Fe K-Edge XAS Pre-Edge Region of $\left(\mathrm{BF}_{4}\right)[\mathrm{Fe}(\mathrm{TMC}) \mathrm{Cl}]$

Figure 4.8. XAS Fe K-Edge Spectra of $\mathrm{FeF}_{3}, \mathrm{FeCl}_{3}, \mathrm{FeBr}_{3}$, and $\left[\mathrm{FeCl}_{6}\right]\left[\mathrm{Co}\left(\mathrm{NH}_{4}\right)_{6}\right]$

Figure 4.9. XAS Fe K-Edge Spectra of $\mathrm{Fe}(\mathrm{acac})_{3}$, $\left(\mathrm{NH}_{4}\right)_{3} \mathrm{Fe}$ (malonate) $\left(\mathrm{NH}_{4}\right) \mathrm{Fe}\left(\mathrm{SO}_{4}\right)_{2} \cdot 12 \mathrm{H}_{2} \mathrm{O}$, and $\mathrm{Fe}(\text { urea })_{6}\left(\mathrm{ClO}_{4}\right)_{3}$

Figure 4.10. Fit to the Fe K-Edge XAS Pre-Edge Region of Fe(acac) 3

Figure 4.11. XAS Fe K-Edge Spectra of $\left(\mathrm{Et}_{4} \mathrm{~N}\right)\left[\mathrm{FeCl}_{4}\right]$ and $\mathrm{Fe}($ salen $) \mathrm{Cl}$

Figure 4.12. Fit to the Fe K-Edge XAS Pre-Edge Region of $\left(\mathrm{Et}_{4} \mathrm{~N}\right)\left[\mathrm{FeCl}_{4}\right]$

Figure 4.13. Fit to the Fe K-Edge XAS Pre-Edge Region of $\mathrm{Fe}($ salen)Cl

Figure 4.14. XAS Fe K-Edge Spectra of $\left.\left(\mathrm{Et}_{4} \mathrm{~N}\right)_{2}\left[\mathrm{Fe}_{2} \text { (salmp }\right)_{2}\right] \cdot 2 \mathrm{DMF}$, $\left.\mathrm{FeSiF}_{6} \cdot 6 \mathrm{H}_{2} \mathrm{O}, \mathrm{Fe}_{2}(\mathrm{OBz})(\mathrm{et}-\mathrm{HPTB})\right]\left(\mathrm{BF}_{4}\right)_{2}$, and $\left(\mathrm{BF}_{4}\right)[\mathrm{Fe}(\mathrm{TMC}) \mathrm{Cl}]$

Figure 4.15. XAS Fe K-Edge Spectra of $\left[\mathrm{Fe}_{2} \mathrm{OH}(\mathrm{OAc})_{2}\left(\mathrm{HB}(\mathrm{pz})_{3}\right)_{2}\right]\left(\mathrm{ClO}_{4}\right)$, $\mathrm{Fe}(\mathrm{acac})_{3},\left(\mathrm{BzPHMe} \mathrm{N}_{2}\right)_{2}\left[\mathrm{Fe}_{2} \mathrm{OCl}_{6}\right]$, and $\left(\mathrm{Et}_{4} \mathrm{~N}\right)\left[\mathrm{FeCl}_{4}\right]$

Figure 4.16. XAS Fe K-Edge XAS Spectra of

$\left[\mathrm{Fe}_{2} \mathrm{OH}(\mathrm{OAC})_{2}\left(\mathrm{HB}(\mathrm{pz})_{3}\right)_{2}\right]\left(\mathrm{ClO}_{4}\right),\left[\mathrm{Fe}_{2} \mathrm{O}(\mathrm{OAc})_{2}\left(\mathrm{HB}(\mathrm{pz})_{3}\right)_{2}\right]$,

$\left[\mathrm{Fe}_{2}(\mathrm{TPA})_{2} \mathrm{O}(\mathrm{OAc})\right]\left(\mathrm{ClO}_{4}\right)_{2}$, and $\left(\mathrm{enH}_{2}\right)\left[\mathrm{Fe}_{2} \mathrm{O}(\mathrm{HEDTA})_{2}\right] \cdot 6 \mathrm{H}_{2} \mathrm{O}$

Figure 4.17. XAS Fe K-Edge Spectra of $\mathrm{Fe}\left(\mathrm{HB}(\mathrm{pz})_{3}\right)_{2}, \mathrm{Fe}$ (prpep) $)_{2}$, and $\mathrm{K}_{4} \mathrm{Fe}(\mathrm{CN})_{6}$

Figure 4.18. XAS Fe K-Edge Spectra of $\left[\mathrm{Fe}\left(\mathrm{HB}(\mathrm{pz})_{3}\right)_{2}\right]\left(\mathrm{ClO}_{4}\right)$,

[Fe(prpep) $\left.)_{2}\right]\left(\mathrm{ClO}_{4}\right)$, and $\mathrm{K}_{3} \mathrm{Fe}(\mathrm{CN})_{6}$

Figure 4.19. Fit to the Fe K-Edge XAS Pre-Edge Region of $\mathrm{K}_{3} \mathrm{Fe}(\mathrm{CN})_{6}$

\section{Chapter 5}

Figure 5.1. XAS Fe K-Edge Spectra of $\left[\mathrm{Fe}\left(\mathrm{OH}_{2}\right) \mathrm{EDTA}\right]^{2-}$,

[Fe( $\left(\mathrm{OH}_{2}\right)$ EDTA] ${ }^{-}$, and FeEDTA-NO

Figure 5.2. XAS Fe K-Edge Spectra of $\left[\mathrm{Fe}(\mathrm{TMC}) \mathrm{N}_{3}\right]\left(\mathrm{BF}_{4}\right)$ and [Fe(TMC) NO] $\left(\mathrm{BF}_{4}\right)_{2}$

Figure 5.3. XAS Fe K-Edge Spectra of $\mathrm{Fe}$ (salen) $\mathrm{Cl}$ and $\mathrm{Fe}($ salen)NO

Figure 5.4. Schematic of Bleomycin and the PMAH Ligand

Figure 5.5. XAS Fe K-Edge Spectra of Fe(II)BLM, Fe(II)PMA Solid, and $\mathrm{Fe}$ (II)PMA Solution 
Figure 5.6. EXAFS Data and FTs of that Data for Fe(I)BLM, $\mathrm{Fe}$ (II)PMA Solid, and Fe(II)PMA Solution

Figure 5.7. Empirical First-Shell Fits to the Fourier-Filtered EXAFS Data of Fe(II)BLM, Fe(II)PMA Solid, and Fe(II)PMA Solution

Figure 5.8. XAS Fe K-Edge Spectra of Fe(II)BLM, Fe(III)BLM, and Activated BLM

Figure 5.9. The $1 \mathrm{~s} \rightarrow 3 \mathrm{~d}$ Pre-Edge Feature of Representative Iron Model Complexes

Figure 5.10. EXAFS Data and FTs of that Data for Fe(II)BLM, Fe(III)BLM, and Activated BLM

Figure 5.11. Empirical First-Shell Fits to the Fourier-Filtered EXAFS Data of $\mathrm{Fe}$ (II)BLM, Fe(III)BLM, and Activated BLM

Figure 5.12. XAS Fe K-Edge Spectra of SLO-1, 15-RLO, and 15-HLO and Related Model Complexes

Figure 5.13. EXAFS Data, an Empirical First-Shell Fit to the EXAFS Data, and FT of the EXAFS Data for SLO-1

Figure 5.14. XAS Fe K-Edge Spectra of Fe(II)PAH ${ }^{\mathrm{T}}$, Fe(II)PAHR, $\mathrm{Fe}$ (III) $\mathrm{PAH}^{\mathrm{T}}$, and $\mathrm{Fe}$ (III) $\mathrm{PAH}^{\mathrm{R}}$

Figure 5.15. XAS Fe K-Edge Spectra of Four-, Five-, and Six-Coordinate Ferrous and Ferric Model Complexes 250

Figure 5.16. EXAFS Data and FTs of that Data for Fe(II)PAH ${ }^{\mathrm{T}}$ and $\mathrm{Fe}$ (II)PAHR

Figure 5.17. EXAFS Data and FTs of that Data for $\mathrm{Fe}(\mathrm{III}) \mathrm{PAH}^{\mathrm{T}}$ and $\mathrm{Fe}$ (III)PAHR

Figure 5.18. Empirical First-Shell Fits to the Fourier-Filtered EXAFS Data of $\mathrm{Fe}$ (II)PAH ${ }^{\mathrm{T}}, \mathrm{Fe}$ (II)PAHR $\mathrm{Pe}^{\mathrm{R}}$ (III)PAH ${ }^{\mathrm{T}}$, and $\mathrm{Fe}$ (III)PAHR

Figure 5.19. XAS Fe K-Edge Spectra of Fe(II)PCD, Fe(III)PCD, $\mathrm{Fe}$ (III)PCA, and FePCD-NO

Figure 5.20. XAS Fe K-Edge Spectra of Four-, Five-, and Six-Coordinate Ferrous and Ferric Model Complexes

Figure 5.21. EXAFS Data for Fe(II)PCD, Fe(III)PCD, Fe(III)PCA, and FePCD-NO

Figure 5.22. FTs of the EXAFS Data for Fe(II)PCD, Fe(III)PCD, $\mathrm{Fe}$ (III)PCA, and FePCD-NO 
Figure 5.23. Empirical First-Shell Fits to the Fourier-Filtered EXAFS

Data of $\mathrm{Fe}$ (II)PCD, Fe(III)PCD, Fe(III)PCA, and FePCD-NO

Figure 5.24. GNXAS Fit to the EXAFS Data of Fe(III)PCD

Figure 5.25. Comparison of Theoretical and Experimental FT of the EXAFS Signals of Fe(III)PCD with the FTs of the Individual Contributions Shown in Figure 5.25C

Figure 5.26. GNXAS Fit to the EXAFS Data of FePCD-NO 291

Figure 5.27. Plots of the $\log (R$ value) $v s$. Fe-N-O Angle for FePCD-NO

Figure 5.28. Comparison of Theoretical and Experimental FTs of FePCD-NO EXAFS Data with Differing Fe-N-O Angles for Calculated Spectra with Only First-Shell Contributions

Figure 5.29. Comparison of Theoretical and Experimental FTs of FePCD-NO EXAFS Data with Differing Fe-N-O Angles for Calculated Spectra that Included Second-Shell Signals with Fixed Parameters

Figure 5.30. Comparison of Theoretical and Experimental FTs of FePCD-NO EXAFS Data with Differing Fe-N-O Angles for Calculated Spectra that Included Second-Shell Signals with Varied Parameters

Figure 5.31. Comparison of the Individual EXAFS Signals for Calculated Spectra that Included Second-Shell Signals with Fixed and Varied Parameters with an Fe-N-O Angle of $165^{\circ}$ 


\section{List of Abbreviations}

1,2-DBD

2,3-CTD

13-HPOD

$\alpha-\mathrm{KG}$

Abs

Asn

acac

$\mathrm{BIPhMe}_{2}$

BLM

$\mathrm{BzPhMe}_{2} \mathrm{~N}$

CD

Chel

CS

CT

CVPAH

Dipic

DNA

DOE

DW

EPR

EDTA

ENDOR

ESEEM

EXAFS

FeSOD

FT

FWHM

GNXAS

$\mathrm{H}_{4} \mathrm{BP}$

$\mathrm{HB}(\mathrm{pz})_{3}$

$\mathrm{HB}\left(3,5-i \operatorname{Pr}_{2} \mathrm{Pz}\right)_{3}$ 2,3-dihydroxybiphenyl 1,2-dioxygenase catechol 2,3-dioxygenase

13(S)-hydroperoxy-9,11-(E,Z)-octadecadienoic acid $\alpha$-ketoglutarate

optical absorption

asparagine

acetylacetonate (2,4-pentanedionate)

2,2'-bis(1-methylimidazolyl)phenylmethoxymethane bleomycin

benzyldimethylphenylammonium

circular dichroism

4-hydroxo-2,6-pyridinedicarboxylate

clavaminate synthase

charge transfer

phenylalanine hydroxylase from Chromobacterium

violaceum

2,6-pyridinedicarboxylate

deoxyribonucleic acid

Department of Energy

Debye-Waller

electron paramagnetic resonance

ethylenediaminetetraacetic acid

electron-nuclear double resonance

electron spin echo envelope modulation

extended X-ray absorption fine structure

iron superoxide dismutase

Fourier transform

full-width-at-half-maximum

EXAFS data analysis package where $g_{n}$ stands for $n$-atom distribution function and XAS stands for

$\mathrm{X}$-ray absorption spectroscopy

tetrahydrobiopterin

hydrotris-1-pyrazolylborate

hydrotris(3,5-diisopropyl-1-pyrazolyl)borate 
His

$\mathrm{HL}$

$\mathrm{HLO}$

HPTB

Dle

IPNS

LF

LLD-ACV

LO

$\mathrm{MCD}$

MLCT

MOPS

MS

$\mathrm{NADH}$

$\mathrm{NIH}$

NIR

NMR

NSF

NSLS

$\mathrm{PAH}$

PCD

PDO

PDR

PrpepH

RLO

$\mathrm{rR}$

salen

salmp

SCF-X $\alpha-S W$

SLO

SS

SSRL

TACN

TH histidine

Hedin-Lundqvist

human lipoxygenase

$\left[N, N, N^{\prime}, N^{\prime}\right.$-tetrakis(2-benzimidazoylmethyl)-2-

hydroxy-1,3-diaminopropane] $]^{-}$

isoleucine

isopenicillin $\mathrm{N}$ synthase

ligand field

$\delta$-(L- $\alpha$-aminoadipoyl)-L-cysteinyl-D-valine

lipoxygenase

magnetic circular dichroism

metal to ligand charge transfer

3-( $N$-morpholino)propanesulfonic acid

multiple-scattering

reduced nicotinamide-adenine dinucleotide

National Institutes of Health

near infrared

nuclear magnetic resonance

National Science Foundation

National Synchrotron Light Source

phenylalanine hydroxylase

protocatechuate 3,4-dioxygenase

phthalate dioxygenase

phthalate dioxygenase reductase

$N$-(2-(4-imidazole)ethyl)pyrimidine-4-carboxamide

rabbit lipoxygenase

resonance Raman

$N, N$-ethylenebis(salicylideneiminato)

[2-bis(salicylideneamino)methylphenolate] ${ }^{3-}$

self-consistent field-X $\alpha$-scattered wave

soybean lipoxygenase

single-scattering

Stanford Synchrotron Radiation Laboratory

$N, N^{\prime}, N^{\prime \prime}$-trimethyl-1,4,7-triazacyclononane

tyrosine hydroxylase 
TMC

TPA

TPH

Tyr

VTVH

$\omega \mathrm{H}$

XAS 1,4,8,11-tetramethyl-1,4,8,11-

tetraazacyclotetradecane

tris(2-pyric ylmethyl)amine

tryptophan hydroxylase

tyrosine

variable temperature, variable field

$\omega$-Hydroxylase

$X$-ray absorption spectroscopy 


\section{Chapter 1}

Introduction to Mononuclear Non-Heme Iron Enzymes and X-ray Absorption Spectroscopy 


\subsection{Scope and Organization of this Dissertation}

This dissertation focuses on the use of Fe K-edge X-ray absorption spectroscupy (XAS) as a tool in defining the geometric and electronic structure of the iron active sites in mononuclear non-heme iron enzymes. Chapter 1 provides a general introduction to mononuclear non-heme iron enzymes, in particular putting these types of enzymes into functional context with classes of iron proteins. In addition, Chapter 1 contains a brief overview of X-ray absorption spectroscopy, including the information content of both the edge and extended X-ray absorption fine structure (EXAFS) regions. In the course of the last five years, several studies on inorganic iron model complexes have been completed in order to redefine the information content available in the edge and EXAFS regions. These studies are presented in Chapters 2, 3, and 4. Chapter 2 describes the methodology of a new multiple-scattering approach to EXAFS analysis, called GNXAS, and the application of this approach to iron model complexes. GNXAS was also used to obtain angular information for $\{\mathrm{FeNO}\}^{7}$ model complexes (presented in Chapter 3). In Chapter 4, a multiplet analysis of $\mathrm{Fe} \mathrm{K}$-edge pre-edge features for iron model complexes of varying oxidation states, spin states, and geometries is presented.

Chapter 5 describes XAS edge and EXAFS studies of the active sites of mononuclear non-heme iron enzymes and $\{\mathrm{FeNO}\}^{7}$ complexes. An overview of the reactions catalyzed by these enzymes, as well as a review of previous structural and mechanistic studies, is given in the first section. The following sections contain the results from individual studies of $\{\mathrm{FeNO}\}^{7}$ complexes, ferrous bleomycin, activated bleomycin, lipoxygenase, phenylalanine hydroxylase, and protocatechuate 3,4-dioxygenase. Each of these sections are divided into parts with an introduction to the enzyme studied, results and analysis of the XAS edge and EXAFS, and a discussion relating the XAS results to those of previous studies. Complementary electron paramagnetic resonance, magnetic circular dichroism, resonance Raman, and optical absorption studies were frequently performed on these enzymes in conjunction with the XAS studies by members of Prof. Ed Solomon's research group. In such cases, the results of the other spectroscopies are discussed for each enzyme in order obtain a more detailed description of the iron active site. 


\subsection{Mononuclear Non-Heme Iron Enzymes}

Metals are commonly found as natural constituents of proteins and nature has learned to use the special properties of metal ions to perform a wide variety of specific functions associated with life processes. Iron is the most abundant transition metal in the Earth's crust and is essential for all plants, animals, and bacteria (with the exception of Lactobacillus and some strains of Bacillus). ${ }^{1}$ Iron can be found in several oxidation states with $\mathrm{Fe}^{2+}$ and $\mathrm{Fe}^{3+}$ being the most common. The iron can be high spin or low spin in each of these oxidation states depending on the ligand environment. Iron is usually complexed to four, five, or six ligands. Different iron coordination environments alter the reactivity of the iron allowing for a large diversity in protein function. Due to its abundance and versatility, iron is distributed into a variety of proteins with varying biological functions: iron transport, electron transfer, oxygen binding, oxygen activation, and multi-electron reduction. Iron-containing proteins can be classified, based upon the coordination of the iron active site, into heme, iron-sulfur, and non-heme sub-groups.

The most studied class of iron-containing enzymes are the heme proteins. These systems are responsible for oxygen binding, oxygen activation, and multi-electron reduction and include such examples as: hemoglobin, cytochrome P-450, prostaglandin synthase, cytochrome oxidase, and catalase. ${ }^{2}$ Hemoglobin is involved in respiration by reversibly binding oxygen in the lungs and transporting it to cells throughout the body. Prostaglandin synthase is a dioxygenase that catalyzes the cyclooxygenase reaction where two dioxygen molecules are inserted into arachidonic acid. ${ }^{3}$ Cytochrome $P-450$ activates dioxygen for monooxygenase chemistry and is thought to involve an oxo-ferryl intermediate, which is believed to be responsible for the oxygen transfer chemistry. ${ }^{4}$ Cytochrome oxidase acts as a proton pump across cell membranes requiring four electron reduction from cytochrome $c$ to reduce molecular oxygen to water. ${ }^{5,6}$ Catalase catalyzes the dismutation of the toxic peroxide byproduct to oxygen and water in the cells of nearly all aerobic organisms. Interestingly enough, all these heme proteins involve the same kind of cofactor in their active site, an iron porphyrin, and a common iron axial imidazole from a histidine residue (except for cytochrome P-450). However, because of a very different structure of the protein and distal environment of the beme, they have clearly different roles and/or catalyze different reactions.

There is another class of proteins which contains iron-sulfur clusters that are involved in electron transfer and storage. ${ }^{7}$ Rubredoxin is an electron transfer protein which contains one iron tetrahedrally coordinated to four cysteine sulfurs. Electron storage is accomplished by [2Fe-2S] plant ferredoxins, in which the two iron atoms are 
tetrahedrally coordinated to four sulfur atoms (two bridging sulfides and two cysteine sulfurs). Rieske centers also perform electron storage, however, they differ from the plant ferredoxins in that one of the two tetrahedrally coordinated irons is ligated to two histidine nitrogens. [4Fe-4S] ferredoxins are low molecular weight proteins which are also involved in oxidation/reduction reactions in substrate metabolism and have a distorted cubane geometry. Iron-sulfur clusters are also found in complex iron-sulfur proteins, ${ }^{8}$ such as nitrogenases which are the enzymes responsible for the biological fixation of atmospheric dinitrogen to ammonia. ${ }^{9}$ Whereas most iron-sulfur clusters, even when they are found in association with enzyme activities, play essentially the role of electron transport, at least one case is known where an iron-sulfur protein catalyzes a chemical reaction. This is aconitase, which transforms citrate to isocitrate in the Kreb's cycle, catalyzing successive reactions of dehydration and rehydration. When purified aerobically from beef heart mitochondria, aconitase is obtained in an inactive [3Fe-4S] form which can be activated with $\mathrm{Fe}^{2+}$ under reducing conditions to give a [4Fe-4S] cluster. $^{8}$

It is important to realize that there are also large number of non-heme iron enzymes which perform reactions similar to those of the heme enzymes involving oxygen binding, oxygen activation, four-electron reduction, and disproportionation. The non-heme iron enzymes can be subdivided into binuclear and mononuclear classes. For the binuclear proteins, the nature of the oxo or hydroxy bridge appears to play a key role in the catalytic mechanisms. ${ }^{10,11}$ Hemerythrin is an oxygen carrier protein analogous to hemoglobin, methane monooxygenase catalyzes the conversion of methane to methanol, and ribonucleotide reductase reduces ribonucleotides to deoxyribonucleotides in the first committed step in DNA synthesis. Finally, the mononuclear non-heme iron enzymes, which are the focus of this dissertation, are an extensive class of iron proteins which do not have the dominant structural features of the above proteins (the heme ligand, ironsulfur bonds, or oxo bridges) and are thus the least well understood. Several recent reviews describe in detail the current understanding of the structure and mechanistic function of these non-heme iron enzymes. ${ }^{12-15}$

Mononuclear iron enzymes are involved in a variety of important biological functions requiring dioxygen. These enzymes are classified according to the types of reactions catalyzed: dismutation, oxidation, monooxygenation, dioxygenation, hydroperoxidation, and DNA cleavage. The dioxygenases may be further subdivided into extra- and intradiol dioxygenases, cis-dihydroxylases, and pterin- and $\alpha$-ketoglutarate-dependent hydroxylases. The latter two systems incorporate one oxygen atom from dioxygen into substrate and one into the organic cofactor and are thus formally 
dioxygenases. Specific enzyme reactions from each class are given in Table 5.1 in Chapter 5. Briefly, iron superoxide dismutase is one of three superoxide dismutases that catalyze the dismutation of superoxide ions to oxygen and hydrogen peroxide. ${ }^{16}$ Isopenicillin $\mathrm{N}$ synthase is an oxidase which is unusual in that it catalyzes the four-electron oxidative double ring closure of its substrate which is the key step in the biosynthesis of penicillins. ${ }^{17}$ The hydroxylation of fatty acids and alkanes and the epoxidation of alkenes using molecular oxygen are catalyzed by whydroxylase. ${ }^{18}$ Several different types of dioxygenases are involved in the bacterial degradation of aromatic rings. The final ring cleavage in the degradation of aromatic rings (breakdown of a catechol) is catalyzed by the extra- and intradiol dioxygenases (e.g., catechol 2,3-dioxygenase and protocatechuate 3,4-dioxygenase, respectively) which exhibit fundamental differences in structure and reactivity. ${ }^{13,19}$ Prior to this reaction, conversion of an unactivated aromatic to the cis-dihydrodiol is required and is catalyzed by the cisdihydroxylase phthalate dioxygenase and related enzymes. ${ }^{20}$ Phenylalanine hydroxylase, one of the three pterin-dependent hydroxylases, catalyzes the hydroxylation of phenylalanine to tyrosine. ${ }^{21} \mathrm{~A}$ deficiency in this enzyme is responsible for the genetic disorder phenylketonuria that is associated with severe mental retardation. Clavaminate synthase is an $\alpha$-ketoglutarate-dependent hydroxylase which catalyzes the key biosynthetic ring closure step in the formation of clavulanic acid, a potent $\beta$-lactamase inhibitor. ${ }^{22}$ This type of inhibitor is important since bacterial resistance to penicillin antibiotics is largely due to the hydrolytic activity of the $\beta$-lactamase enzymes. The lipoxygenases catalyze the hydroperoxidation of cis,cis-1,4-pentadiene-containing fatty acids. Mammalian lipoxygenases catalyze the conversion of arachidonic acid to leukotrienes, which mediate hypersensitiviy and inflammation, and lipoxins, which inhibit cellular immunity. ${ }^{23}$ Bleomycin is a non-heme iron glycopeptide that reversibly binds and activates oxygen for hydrogen atom abstraction which is similar to heme chemistry (cytochrome $\mathrm{P}-450)^{24,25}$ but involves a different oxygen intermediate. Bleomycin is used as an anti-cancer agent due to its ability to selectively cleave DNA. More detailed mechanistic and structural information for these enzymes is presented in Chapter 5.

Both ferrous and ferric oxidation states have been determined to be involved in catalysis for the different mononuclear non-heme iron enzymes, and substrate- and oxygen-bound intermediates have been observed for several of these enzymes. Much less is known about the active sites in these enzymes relative to heme systems as the non-heme iron centers are less spectroscopically accessible particularly in the ferrous oxidation state. This is due to the fact that non-heme ferrous active sites are generally 
high spin $S=2$ non-Kramers systems and, therefore, do not have an EPR signal. Also, these non-heme ferrous active sites do not have ligand-to-metal charge transfer transitions in a spectroscopically accessible region. For high spin ferric systems the $\mathrm{d} \longrightarrow \mathrm{d}$ transitions, which are powerful probes of active site geometric and electronic structure, are spin forbidden. Only ligand $\rightarrow$ metal charge transfer transitions are observed. Fortunately, X-ray absorption spectroscopy is ideally suited for studying dilute metal proteins and the information obtainable from the iron active site is not dependent on the oxidation state or spin state of the iron as in other spectroscopies.

\subsection{X-ray Absorption Spectroscopy}

\subsubsection{General Background}

X-ray absorption spectroscopy (XAS) involves the measurement of the absorption coefficient, $\mu$, as a function of energy. ${ }^{26}$ A typical X-ray absorption spectrum (Figure 1.1) exhibits a decreasing absorption as the photon energy is increased with a sharp discontinuity, called an absorption edge, superimposed on the smooth background. An absorption edge occurs when the incident photon has sufficient energy to promote a core electron to unoccupied valence orbitals or to the continuum. Thus, the edge occurs at a characteristic threshold energy which is specific to the absorbing atom. Edges are named according to the Bohr atomic level from which the photoionized electron originates. Hence, a K-edge refers to the ionization of a $1 \mathrm{~s}$ electron, a $L_{1}$ edge to the ionization of a $2 \mathrm{~s}$ electron, etc. The data in this dissertation were measured at the Fe $\mathrm{K}$-edge where the ionization of a $1 \mathrm{~s}$ electron requires $-7130 \mathrm{eV}$.

XAS spectra can be divided into several regions (Figure 1.1). In the pre-edge and edge region the incident energy is below the ionization threshold. This region contains transitions from core levels to unoccupied or partially occupied atomic and molecular orbitals localized on the absorbing atom, as well as to localized and delocalized continuum levels. ${ }^{27}$ These features occur below or are superimposed on the rising edge. Throughout this dissertation, features which are at energies well-separated from the onset of the edge will be referred to as pre-edge features, while those transitions which actually overlap the rising edge intensity will be called edge or rising-edge features. At X-ray energies above the threshold for ionization, electrons are promoted into the continuum. The oscillations in this region are known as extended X-ray absorption fine structure (EXAFS) and result from interference between the photoelectron wave propagating from the absorbing atom and the wave back scattered by neighboring atoms. ${ }^{28}$ There is an 


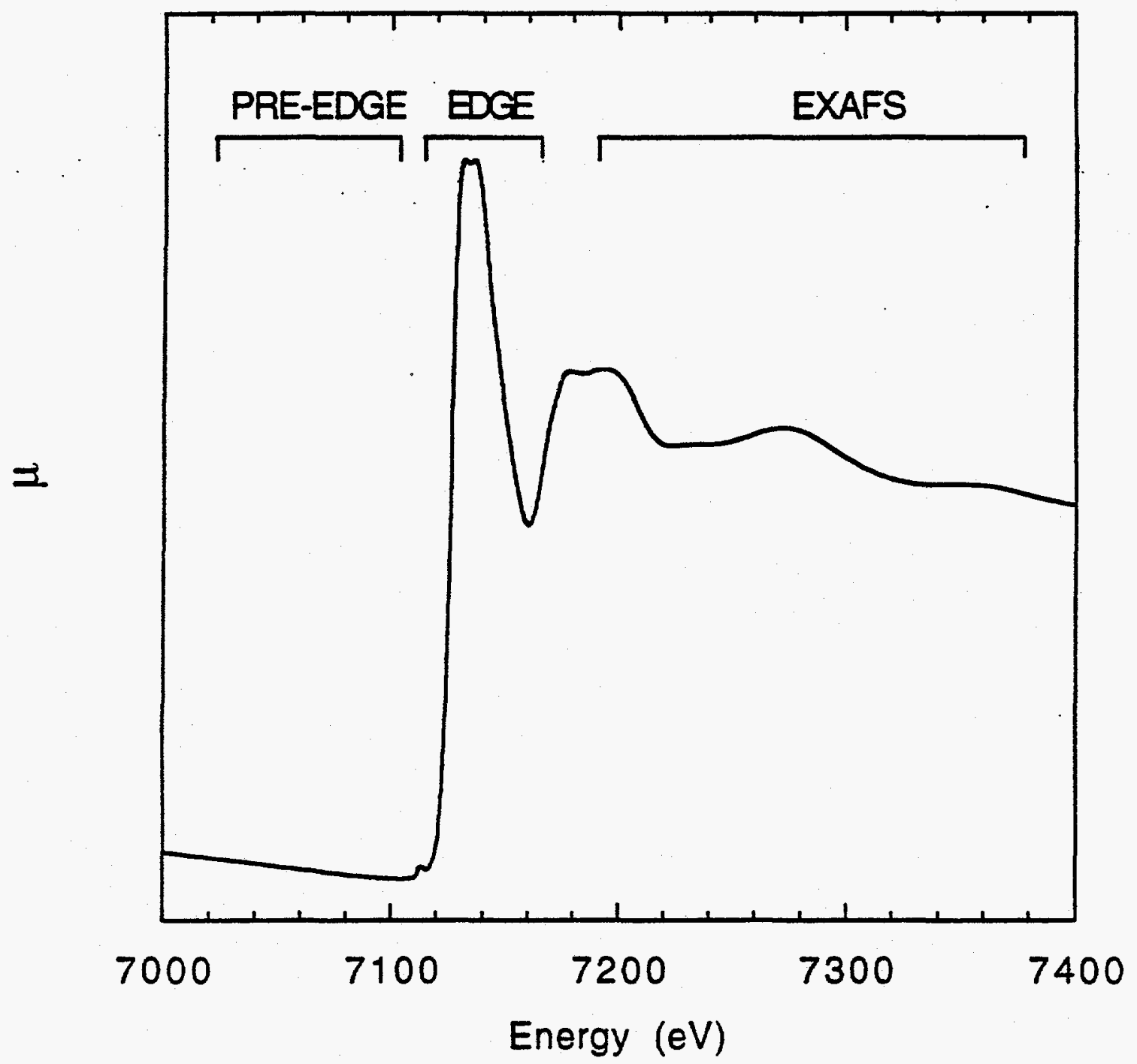

Figure 1.1. A typical Fe K-edge XAS spectrum of an inorganic iron model complex. 
increase in the absorption if the scattered wave is in phase with the outgoing photoelectron wave, or a decrease in the absorption if the scattered wave is out of phase with the outgoing photoelectron wave.

Although edge structure and EXAFS have different physical origins, they contain complementary information about the absorbing atom and its environment. Absorption features in the pre-edge and edge regions are sensitive to the local electronic and geometric environment of the absorbing atom. Analysis of the position and relative intensities of the absorption edge features can reveal details about the absorbing atom's site symmetry, oxidation state, and the nature of the surrounding ligands. Interpretation of the phase, amplitude, and frequency of the EXAFS oscillations can provide information about the type, number, and distances of the atoms in the vicinity of the absorber. XAS is ideally suited for studying the local electronic and geometric structure of metal active sites in metalloproteins. With the advent of synchrotron radiation sources, which provide $\mathrm{X}$-ray fluxes many orders of magnitude higher than those previously obtainable with conventional X-ray tubes, XAS data can be collected on very dilute metalloproteins in a reasonable amount of time. Since XAS does not depend on long-range order, samples in any physical state can be studied. Even without long-range order, EXAFS provides very detailed metrical information about the local environment of the absorber (within $\sim 4 \AA$ of the absorber). Interatomic distances can be determined with an accuracy typically of $\pm 0.02 \AA$ or better with the accuracy in coordination numbers being about $25 \%$. $^{29}$

\subsubsection{Experimental Considerations}

The design of a basic X-ray absorption experiment is presented in Figure 1.2. Synchrotron radiation provides a polychromatic source of X-ray energies. The X-ray beam, which is highly vertically collimated, is further defined vertically and horizontally by a pair of slits and then energy resolved with a double-crystal monochromator. After passing through a set of tantalum slits that minimize scatter, the incident intensity is measured with a gas-filled ionization chamber (nitrogen is used at the Fe K-edge ). There are two basic configurations for a standard XAS experiment: transmission and fluorescence. Transmission mode is used for concentrated samples, such as solid model complexes, where the absorption of the sample is determined by measuring the $\mathrm{X}$-ray intensity before and after the sample using ionization chambers $\left(I_{0}\right.$ and $I_{1}$ in Figure 1.2). Fluorescence mode is used for dilute samples, where the fluorescence signal emitted as the excited nucleus relaxes after photoionization is measured at $90^{\circ}$ from the incident 


\section{SIDE·VIEW}

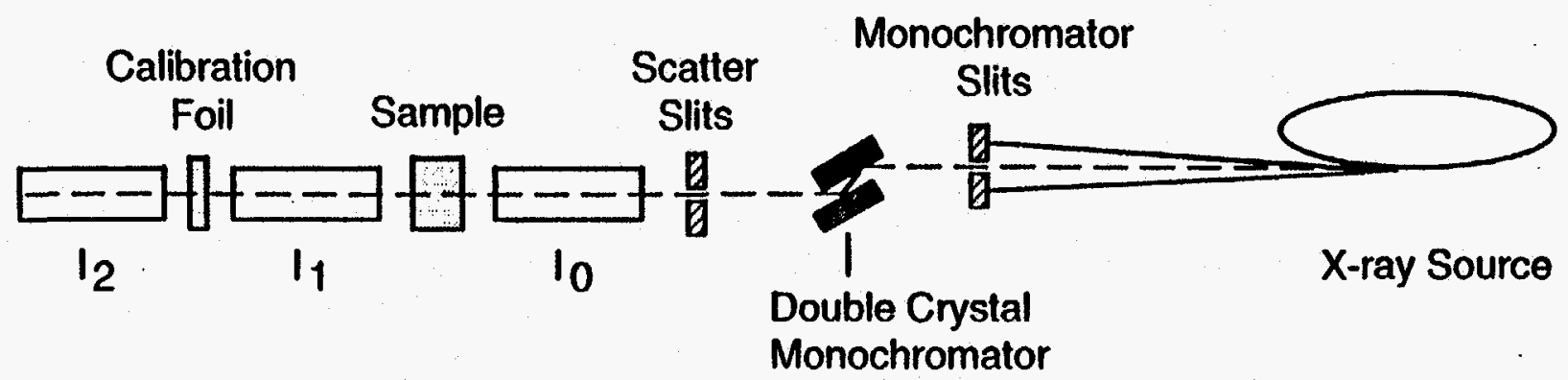

TOP VIEW

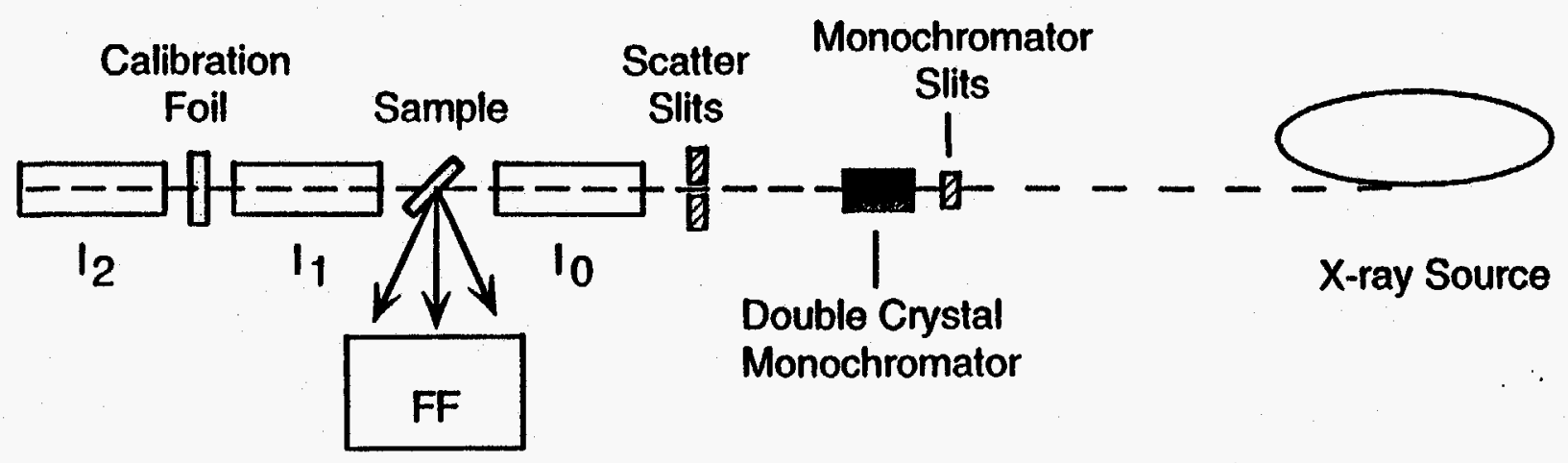

Figure 1.2. A typical hard X-ray experimental set-up (side and top views) for transmission and fluorescence measurements. 
$\mathrm{X}$-ray beam (FF in Figure 1.2). In both configurations, the transmitted or fluorescence intensity is ratioed by the incident intensity to correct for any X-ray beam instabilities and for the continuous decay of intensity with decreasing ring current. Internal energy calibration for each scan is achieved by placing an Fe foil after $I_{1}$ followed by a third ionization chamber, $I_{2}$. Thus, the transmitted intensity of the $F e$ foil is measured simultaneously with that of the sample.

A primary consideration in XAS data collection is the energy resolution of the experiment. For a given monochromator, the resolution is controlled through the use of the defining slits placed before the monochromator and the choice of monochromator crystals. For the experiments in this dissertation, the slits were set to optimize the spectral resolution at a vertical height of $1 \mathrm{~mm}$ (unless otherwise stated). By defining the vertical height of the beam to be $1 \mathrm{~mm}$, the experimental resolution is determined by the intrinsic resolution of the monochromator and the core-hole lifetime of the absorber. ${ }^{30}$ The resolution is also, in principle, affected by mirrors in the optical path of the beam. The experiments described herein were performed on beam lines where no mirrors were present or were used in such a way that the degradation of resolution did not occur. At the Fe K-edge, using $1 \mathrm{~mm}$ pre-monochromator defining slits and a $\mathrm{Si}(220)$ double-crystal monochromator, the resolution is about $1.4 \mathrm{eV} \cdot{ }^{30}$ A more detailed discussion of all the factors to be considered in XAS data collection can be found in references 30-33.

\subsubsection{Edge Theory and Analysis}

Edge structure consists of absorption bands superimposed on the steeply rising continuum absorption caused by transitions of core electrons to discrete bound valence levels. The bound state transitions in the pre-edge and edge region can be interpreted using the $\mathrm{X}$-ray absorption cross-section, $\sigma$, given in equation $1.1,{ }^{34}$

$$
\sigma=c\left|\left\langle\phi_{f}|\hat{O}| \phi_{i}\right\rangle\right|^{2}
$$

where $c$ is a constant, $\hat{O}$ is the transition moment operator, and $\phi_{f}$ and $\phi_{i}$ are the final and initial state wavefunctions, respectively. Pre-edge and edge features are governed primarily by electric dipole selection rules $(\Delta \ell= \pm 1)$. The intensity of these features, then, is related to the density of the final states of the appropriate symmetry which have measurable overlap with the initial state wavefunction. Based on a dipole-coupling mechanism, the features in a K-edge spectrum reflect transitions from a core $1 \mathrm{~s}$ orbital to p-type final states. 
XAS spectra of first row transition metal complexes typically have a weak pre-edge feature $-10 \mathrm{eV}$ below the rising edge. ${ }^{35}$ This was attributed to the $1 \mathrm{~s} \rightarrow 3 \mathrm{~d}$ transition, as spectra of $\mathrm{Zn}^{2+}$ (a $3 \mathrm{~d}^{10}$ system) do not have this feature. ${ }^{36}$ The $1 \mathrm{~s} \longrightarrow 3 \mathrm{~d}$ transition is electric dipole forbidden, however, it gains intensity through an allowed quadrupole mechanism and by $4 p$ mixing into the $3 d$ orbitals due to a noncentrosymmetric iron site. An Fe K-edge spectrum typically has a very weak pre-edge feature at $\sim 7112 \mathrm{eV}$ due to the $1 \mathrm{~s} \rightarrow 3 \mathrm{~d}$ transition (Figure 1.1). It has been observed that the intensity of this feature increases with decreasing coordination number. ${ }^{37,38}$ Decreasing the coordination number distorts the iron site, allowing for $4 p$ mixing into the $3 \mathrm{~d}$ orbitals, which increases the intensity of the pre-edge feature. A detailed analysis of the energy splittings and intensity pattern of the $1 \mathrm{~s} \rightarrow 3 \mathrm{~d}$ pre-edge feature can give information on the spin state, oxidation state, and geometry of the iron site (see Chapter 4).

The abrupt increase in the absorption coefficient at $\sim 7125 \mathrm{eV}$ in an Fe K-edge spectrum is attributed to the electric dipole allowed $1 \mathrm{~s} \rightarrow 4 \mathrm{p}$ transition (Figure 1.1). The energy of the rising edge is dependent on the effective nuclear charge of the iron. ${ }^{35-37,39-41}$ An atom with a higher effective nuclear charge has a deeper core level and, thus, a higher photon energy is need to ionize the core electron. On this basis, the position of the edge can be related to the oxidation state of the iron, to a first approximation. Further, for complexes of the same oxidation state, variations in edge energies can be related to differences in the covalency of the ligands. However, one must keep in mind that there are numerous factors that influence the effective nuclear charge including the formal oxidation state of the metal and the number and type of coordinating ligands.

\subsubsection{EXAFS Theory and Analysis}

1.3.4.1. Information Obtainable from EXAFS. EXAFS spectroscopy is an invaluable technique for investigating the local coordination environment of specific atomic species in systems ranging from metalloproteins ${ }^{31,42}$ to catalysts. ${ }^{43,44}$ The method is sensitive to short-range order (distances typically within about 4-5 $\AA$ of the absorber) and provides information on: 1) the distances to a neighboring atom, 2) the numbers of neighboring atoms, and 3 ) the types of neighboring atoms. EXAFS has the advantages of focusing on particular atoms and of being applicable to any physical state, including liquid or frozen solutions and amorphous solids. However, the analysis of EXAFS data requires accurately known experimental or theoretical pairwise phase and 
amplitude functions. Experimental standards have been widely used to extract reliable amplitude and phase functions and used with success to obtain structural information from EXAFS data, particularly for nearest neighbors. ${ }^{29,31,32}$ Alternatively, reliable theoretical phases and amplitudes have recently become available that enable more information, including angular distribution, to be obtained from EXAFS analysis. ${ }^{45-50}$

The empirical data-analysis technique $29,31,32$ involves the use of phase and amplitude parameters which bave been extracted from the EXAFS data of a suitable model complex. The empirical technique allows for the determination of first neighbor distances with high accuracy (typically $\pm 0.02 \AA$ ) but determines with less accuracy the coordination number (one atom in 4 or 5) and the identity of the ligating atoms (not differentiating \pm 1 or 2 in $Z$ ). The empirical approach is of questionable utility for atoms beyond $-3 \AA$ because of phase and amplitude transferability problems. A break down of the phase and amplitude transferability occurs because of intervening atoms that give rise to multiple-scattering (MS) signals. These MS signals can contribute significantly to the total EXAFS signal and very often interfere with the single-scattering (SS) signal. Moreover, it can be difficult to obtain suitable models for extraction of reliable empirical amplitudes and phases. As a result of these limitations, determination of distances beyond the first coordination shell and of bond angles has been difficult using empirically-derived phase and amplitude parameters.

The alternative to the empirical data-analysis technique is the theoretical technique, ${ }^{45-50}$ where the phase and amplitude functions are calculated theoretically. Thus, the reliability of the result is determined by the accuracy of the theory. In this approach, an expected theoretical signal is calculated assuming a structural model for the system under study. The resulting signal is then fit to the experimental data, varying structural and non-structural parameters until a minimum of a selected reliability function is reached. The quality of the fit is determined by visual inspection of EXAFS and Fourier transform (FT) residuals. The theoretical approach is advantageous to the empirical approach in that MS contributions can be modeled and, therefore, bond distance and bond angle information from distant shells of atoms can be determined. Also a theoretical approach is not dependent upon obtaining suitable model compounds to extract phase and amplitude parameters.

1.3.4.2. Single-Scattering Process. This section includes a brief theoretical description of the single-scattering process along with the type of information that can be obtained from a single-scattering analysis. EXAFS results from the interference between the outgoing photoelectron wave from the photoabsorber with the backscattered waves from surrounding atoms (Figure 1.3). The interference generated by each surrounding 


\section{Constructive Interference}

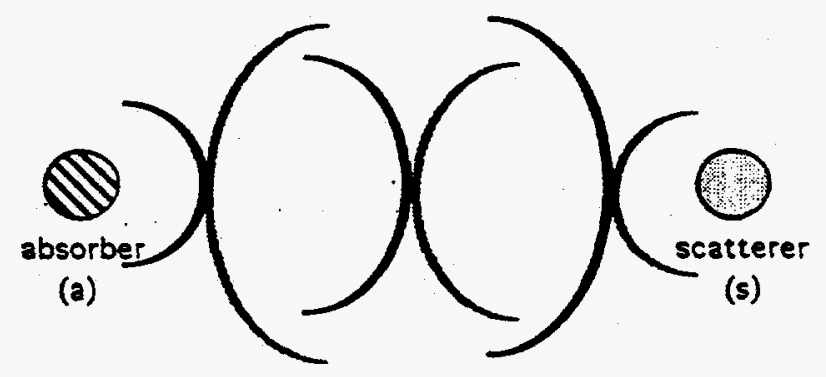

Destructive Interference
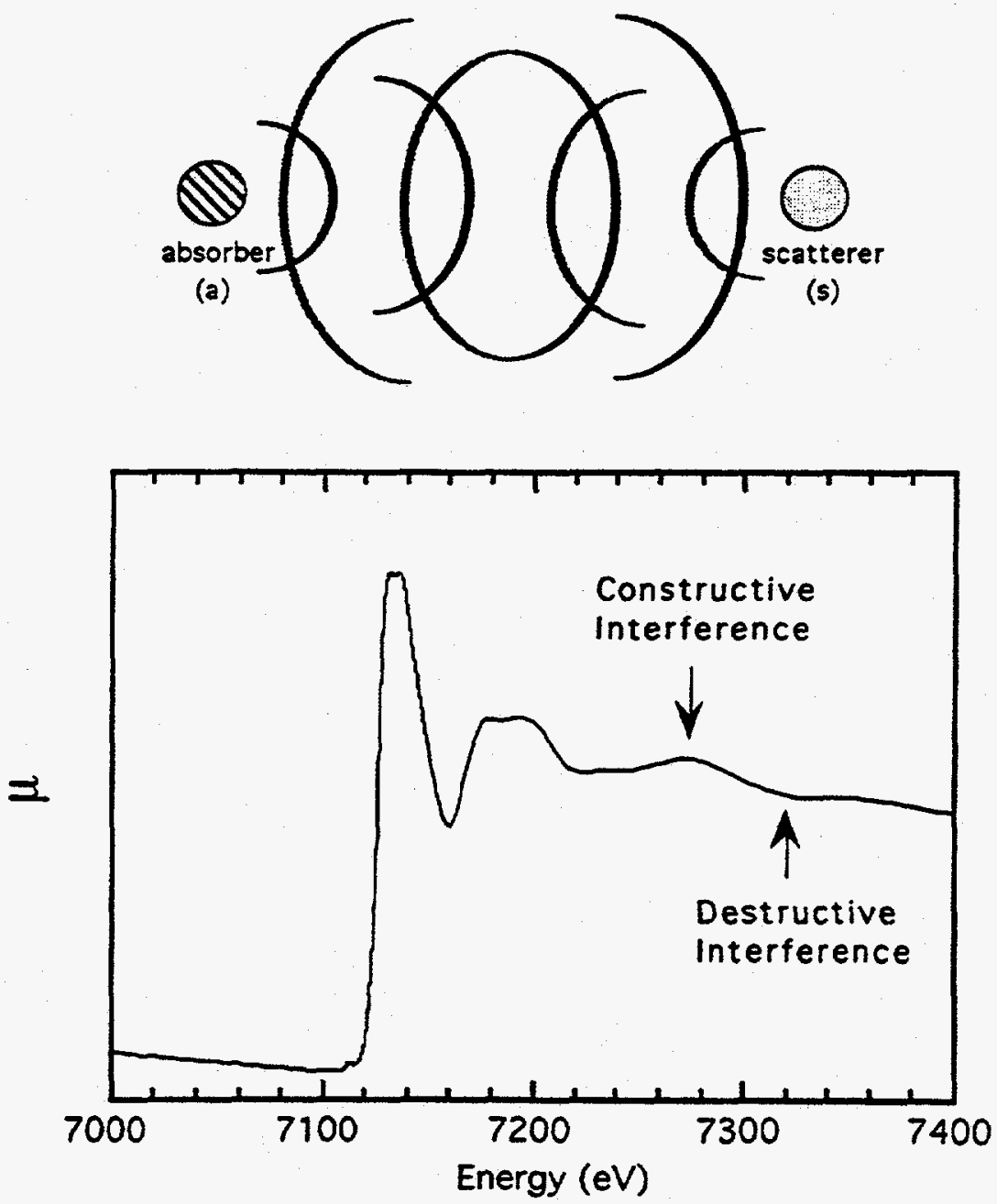

Figure 1.3. Diagram of photoelectron waves generated by $X$-ray absorption of the absorber. In the top case, the outgoing photoelectron wave constructively interferes with the backscattered wave resulting in a maximum in the absorption coefficient. At slightly higher energy, the outgoing photoelectron wave destructively interferes with the backscattered wave resulting in a minimum in the absorption coefficient. 
(scattering) atom contributes a damped sine wave to the overall EXAFS spectrum, where each sine wave can be described by three measurable quantities: frequency, amplitude, and phase. These three cbservables contain structural information about the nature and location of the scattering atom. The frequency of the sine wave is a measure of the distance between the absorbing atom and the scattering atom, the amplitude of the sine wave is a measure of the number of scattering atoms, and the phase of the sine wave is indicative of the identity of the scattering atom.

EXAFS, denoted here by $\chi$, is the relative modulation of the absorption coefficient, $\mu$, of a particular atom compared to the smooth background absorption coefficient, $\mu_{s}$, normalized by the absorption coefficient $\mu_{0}$ that would be observed for the free atom. Thus, as defined in reference 51 ,

$$
\chi=\frac{\mu-\mu_{s}}{\mu_{0}}
$$

Since $\mu_{s} \cong \mu_{o}$, the EXAFS may alternatively be defined by $\chi=\left(\mu-\mu_{o}\right) / \mu_{0}$ or $\left(\mu-\mu_{s)} / \mu_{s}\right.$. It is now conventional to plot $\chi$ versus the photoelectron wave vector, $k$,

$$
k=\left[\left(\frac{2 m}{\hbar^{2}}\right)\left(E-E_{0}\right)\right]^{1 / 2}
$$

where $E_{0}$ is the threshold energy for liberation of a photoelectron wave.

The complete mathematical derivation of the single-scattering EXAFS equation is presented in references ${ }^{51}$ and ${ }^{52}$. The resultant theoretical single-scattering expression for $\chi$ is given by:

$$
\chi(k)=\sum_{s} \frac{N_{s}\left|f_{s}(\pi, k)\right|}{k R_{a s}^{2}} \exp \left(-R_{a s} / \lambda_{f}\right) \exp \left(-2 \sigma_{a s}^{2} k^{2}\right) \sin \left[2 k R_{a s}+\alpha_{a s}(k)\right]
$$

\begin{tabular}{|c|c|c|}
\hline symbol & units & definition \\
\hline$N_{S}$ & --- & the number of atoms in a shell \\
\hline$\left|f_{S}(\pi, k)\right|$ & -- & $\begin{array}{l}\text { the inherent backscattering amplitude for this type of } \\
\text { scattering atom }\end{array}$ \\
\hline$R_{a s}$ & $\AA$ & $\begin{array}{l}\text { the distance between the absorbing atom and the } \\
\text { scattering atom }\end{array}$ \\
\hline$\lambda_{f}$ & $\AA$ & $\begin{array}{l}\text { the mean free path for inelastic scattering of the } \\
\text { photoelectron }\end{array}$ \\
\hline$\sigma_{a s}{ }^{2}$ & $\AA^{2}$ & $\begin{array}{l}\text { the rms deviation of } R_{a s}\left(\exp \left(-2 \sigma_{a s}{ }^{2} k^{2}\right) \text { is referred to as }\right. \\
\text { the Debye-Waller factor) }\end{array}$ \\
\hline$\alpha_{a s}(k)$ & -- & $\begin{array}{l}\text { the inherent backscattering phase shift for this absorbing } \\
\text { atom/scattering atom combination }\end{array}$ \\
\hline
\end{tabular}

where a description of the variables is given in Table 1.1.

Table 1.1. Description of the Variables in the Single-Scattering Expression for $\chi$. 
Equation 1.4 expresses the EXAFS, $\chi(k)$, as a sum of damped sine waves with each term within the summation consisting of an amplitude term, an exponential damping term (Debye-Waller factor) and a sine function to describe the (quasi-periodic) behavior of the EXAFS. The EXAFS for any absorbing atom/scattering atom pair can be represented a damped sine wave with the amplitude $\left(\left[N_{s} \mid f_{s}(\pi, k) \exp \left(-2 \sigma_{a s}^{2} k^{2}\right) \exp \left(-R_{a s} / \lambda_{f}\right)\right] /\left[k\left(R_{a s}^{2}\right)\right]\right)$, frequency $\left(2 R_{a s}\right)$, and phase shift $\left(\alpha_{a s}(k)\right)$ characteristic of the atoms involved. The $R_{a s}{ }^{-2}$ dependence makes the EXAFS of long-distance shells much weaker than that from nearby atoms. Thus, only atoms within a radius of $-4-5 \AA$ of the absorbing atom contribute significant scattering to the EXAFS. This fall-off of EXAFS amplitude at high $R_{a s}$ also has a contribution from inelastic losses of the photoelectron which are more serious for longer distances. This is usually treated by defining a mean free path for the photoelectron, $\lambda_{f}$, and incorporating the $\exp \left(-R_{a s} / \lambda_{f}\right)$ term in the equation. Within a shell of scattering atoms, there is some variation in $R_{a s}$, which may be static (a spread in the a-s distances from structural distortion or site heterogeneity) or dynamic (due to a stretching vibration in the a-s bond). This variation leads to a damping of the EXAFS oscillations which is physically described by $\sigma_{a s}{ }^{2}$, a root-mean-square (rms) deviation in the distance $R_{a s}$. The vibrational portion of $\sigma_{a s}{ }^{2}$ has a characteristic temperature dependence. It should be noted that the derivation of this expression for $x^{51,52}$ involves a number of approximations that break down at low $k$ values, i.e. close to the absorption edge. Therefore, most plane wave single-scattering EXAFS analyses only use the data for $k>4 \AA^{-1}$.

EXAFS data analysis requires accurately known experimental or theoretical absorber/scatterer pair phase and amplitude parameters, $\left|f_{s}(\pi, k)\right|$ and $\alpha_{a s}(k)$, respectively. To obtain distances from EXAFS data it is necessary to know the phase shift $\alpha_{a s}(k)$. Empirical methods for obtaining phase shifts involve the fitting the EXAFS of a known structure with $\sin \left[2 k R_{a s}+\alpha_{a s}(k)\right]$. Typically, $\alpha_{a s}(k)$ is parameterized as a quadratic function, $a_{0}+a_{1} k+a_{2} k^{2}$, where each of these parameters are optimized in the fitting of the EXAFS of a structure with known $R_{\text {as. }}{ }^{29,31,32}$ The phase shift parameters are then fixed, and the EXAFS of an unknown structure is fit by varying $R_{a s}$. In a large number of cases this procedure has been shown to yield distances with an accuracy better than $0.03 \AA$. Altematively, $\alpha_{a s}(k)$ can be calculated from first principles. ${ }^{53}$ As can be seen in equation 1.4, the EXAFS amplitude depends on the number of scatterers, $N_{s}$, as well as $R_{a s}, k,\left|f_{s}(\pi, k)\right|$, and $\sigma_{a s}{ }^{2}$. Since $R_{a s}$ is obtained from the frequency of the EXAFS and $k$ is known, calculation of the number of scatterers from EXAFS is possible if $\left|f_{\mathcal{S}}(\pi, k)\right|$ and $\sigma_{a s}{ }^{2}$ are known. The two approaches towards EXAFS amplitude have been: 1) to 
parameterize $\left|f_{s}(\pi, k)\right|$ and $\exp \left(-2 \sigma_{a s}{ }^{2} k^{2}\right)$ together and neglect variations in thermal motion in the fits $29,31,32$ or 2$)$ to use theoretical values for $\left|f_{\mathcal{S}}(\pi, k)\right|$ and to vary both the Debye-Waller factor and the number of scatterers. ${ }^{54}$

1.3.4.3. Multiple-Scattering Process. Thus far only the plane wave single-scattering theory of EXAFS has been discussed. This treatment ignores the possibility that the photoelectron might encounter two (or more) scattering atoms in its "round trip" back to the photoabsorber, as seen in Figure 1.4. A proper analysis of such multiple-scattering processes would enable metrical information to be obtained on second and third shell neighbors. Of particular interest would be the ability to obtain angular information. Multiple-scattering processes cannot by studied with the traditional empirical data analysis approach since the MS effects are incorporated within the empirical parameter approach in such a way that the phase and amplitude parameters reflect the exact geometry of the model compound and cannot be transferred to an unknown of different geometry. Thus, there has been concentrated effort in the last fifteen years to obtain an accurate theoretical approach, so that the multiple-scattering processes can be analyzed properly and exploited.

Multiple-scattering effects in EXAFS can become especially important when atoms are arranged in an approximately collinear array (A-B-C angle $>150^{\circ}$ ). In such cases, the outgoing photoelectron is strongly forward scattered by the intervening atom, resulting in significant amplitude enhancement. This effect was first observed when theoretical calculations of EXAFS were compared with measurements on copper metal. ${ }^{52,55}$ The observed amplitude of the scattered wave for the fourth copper shell was larger than the amplitude calculated from single-scattering theory, and the observed phase shift was off by approximately $\pi$ from the calculated phase shift. These discrepancies were explained as an effect of first-shell atoms that intervene directly in the absorber-toscatterer path to the fourth-shell atoms in the face-centered cubic lattice. Rather than occluding the EXAFS from the fourth-shell atoms as might have been expected, the intervening atoms actually accentuate the EXAFS of the shadowed atoms by enhanced forward scattering of both the outgoing and backscattered photoelectron waves. These MS effects also cause additional phase shifts. Multiple-scattering effects have also been observed in many inorganic $\pi$-acceptor complexes, where di- or triatomic ligands (e.g. $\left.\mathrm{CO}, \mathrm{CN}^{-}, \mathrm{NCS}^{-}\right)$are linearly bound to the absorbing transition metal..$^{31,56-59}$ For example, in $\left[\mathrm{Mo}(\mathrm{NCS})_{6}\right]^{3-}$ the amplitude of the carbons and sulfurs of the isothiocyanate ligands are distinctly enhanced in the Fourier transform spectrum. ${ }^{29}$ In the case of $\mathrm{Mo}(\mathrm{CO})_{6}$ the amplitude of the oxygen shell is even larger than that of the carbon shell in dramatic contrast to the "normal" $1 / R_{a s}{ }^{2}$ falloff in the EXAFS amplitude. ${ }^{29}$ 


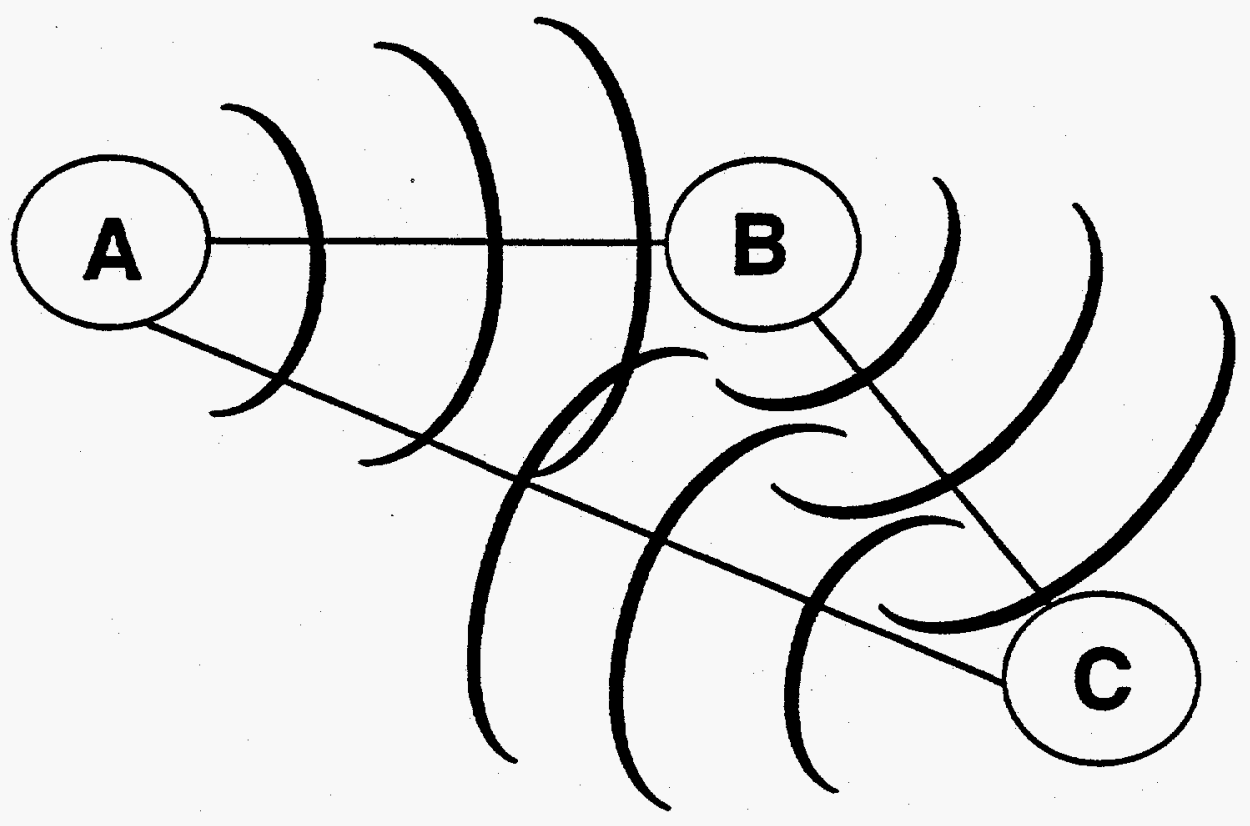

Figure 1.4. A multiple-scattering pathway for a photoelectron generated by $\mathrm{X}$-ray absorption by atom $A$ in the presence of two scattering atoms, $B$ and $C$. The scattering pathway indicated is A-B-C-A. The multiple-scattering contribution to the EXAFS depends on the distances involved and the A-B-C angle. 
Once the effects of multiple-scattering were observed, new EXAFS analysis methods were formulated to take into account multiple-scattering effects. Inclusion of MS contributions could in principle improve the accuracy of the EXAFS analysis and make it possible to exploit the strong angular dependence of multiple-scattering terms. Teo theoretically calculated scattering amplitude and phase functions at various A-B-C scattering angles and assessed the relative importance of various MS pathways as the scattering angle varied. ${ }^{59}$ There are three scattering pathways for a three atom A-B-C system (Figure 1.4) where each pathway originates and terminates at the absorbing atom A. Pathway $I$ is the direct backscattering from atom $A$ to $C$ and back. Pathway II is the multiple-scattering via atom $B$ and around the triangle in either direction and pathway III is the multiple-scattering via atom $B$ in both outgoing and incoming trips. When the A-B-C bridging angle is small $\left(-100^{\circ}\right)$ the three pathways are resolvable in a FT of the EXAFS data and can be analyzed separately. When the bridging angle is large $\left(>150^{\circ}\right)$, pathway III is dominant and signified by an amplitude enhancement in the EXAFS. Co and coworkers exploited these effects and studied a series of oxygen-bridged iron complexes. ${ }^{60}$ The analysis showed that it was possible to estimate the bridging angle to $\pm 8^{\circ}$ and calculate the metal-metal distances to within $\pm 0.05 \AA$. However, both of these studies indicated that angle determination by EXAFS was only possible when outer-shell peaks are well-resolved in the FT and can be correctly identified. Such cases are infrequent, especially for unknown systems. Thus, a more generally applicable multiple-scattering EXAFS analysis method was needed.

Recently, the proper theoretical formulation of the photoabsorption process has allowed for such a theoretical MS data analysis approach. Currently, there are three widely used theoretical data analysis packages that are capable of computing multiple-scattering processes as well as single-scattering processes. The GNXAS approach (where $g_{n}$ stands for the $n$-body distribution function and XAS stands for X-ray absorption spectroscopy) was developed as an integrated theoretical approach to the analysis of EXAFS data. ${ }^{45}$ The program EXCURVE was developed at Daresbury. ${ }^{46}$ The program FEFF, ${ }^{47,50}$ developed at the University of Washington, Seattle, initially was only capable of calculating single-scattering processes, however, later versions incorporated multiple-scattering (FEFF5). ${ }^{48,49}$ Chapter 2 of this dissertation presents a detailed description of the GNXAS methodology and then reports the application of GNXAS to iron model complexes. An analysis of the multiple-scattering pathways in $\{\mathrm{FeNO}\}^{7}$ complexes using GNXAS is given in Chapter 3. 


\subsection{References}

(1) Bioinorganic Catalysis; Reedijk, J., Ed.; Marcel Dekker, Inc.: New York, 1993.

(2) Heme Proteins; Eichorn, G. L.; Marzilli, L. G., Ed.; Elsevier Science Publishing Co., Inc.: New York, 1988; Vol. 7.

(3) Mansuy, D.; Battioni, P. In Bioinorganic Catalyis; Reedijk, J., Ed.; Marcel Dekker Inc.: New York, 1993; pp 395.

(4) Cytochrome P-450: Structure, Mechanism, and Biochemistry; Ortiz de Montellano, P. R., Ed.; Plenum Press: New York, 1986.

(5) Babcock, G. T.; Wikström, M. Nature 1992, 356, 301.

(6) Malmström, B. G. Chem. Rev. 1990, 90, 1247.

(7) Iron-Sulfur Proteins; Spiro, T. G., Ed.; John Wiley \& Sons: New York, 1982; Vol. 4.

(8) Inorganic Chemistry of Iron Metabolism; Crichton, R. R., Ed.; Ellis Horwood Limited: Chichester, 1991.

(9) Evans, D. J.; Henderson, R. A.; Smith, B. E. In Bioinorganic Catalysis; Reedijk, J., Ed.; Marcel Dekker, Inc.: New York, 1993; p 89.

(10) Sanders-Loehr, J. In Iron Carriers and Iron Proteins; Loehr, T. M., Ed.; VCH Publishers, Inc.: New York, 1989; Vol. 5; p 373.

(11) Brown, C. B.; Remar, G. J.; Musselman, R. L.; Solomon, E. I. Inorg. Chem. 1995, 34,688 .

(12) Solomon, E. I.; Zhang, Y. Acc. Chem. Res. 1992, 25, 343.

(13) Que, L., Jr. In Bioinorganic Catalysis; Reedijk, J., Ed.; Marcel Dekker, Inc.: New York, 1993; p 467.

(14) Feig, A. L.; Lippard, S. J. Chem. Rev. 1994, 94, 759.

(15) Solomon, E. I.; Pavel, E. G.; Loeb, K. E.; Campochiaro, C. Coord. Chem. Rev. 1995 , in press.

(16) Stoddard, B. L.; Howell, P. L.; Ringe, D.; Petsko, G. A. Biochemistry 1990, 29, 8885.

(17) Baldwin, J. E.; Bradley, M. Chem. Rev. 1990, 90, 1079.

(18) Katopodis, A. G.; Wimalasena, K.; Lee, J.; May, S. W. J. Am. Chem. Soc. 1984, 106, 7928.

(19) Lipscomb, J. D.; Orville, A. M. In Metals in Biological Systems; Sigel, H.; Sigel, A., Eds.; Marcel Dekker Inc.: New York, 1992; Vol. 28; 243.

(20) Batie, C. J.; Lahaie, E.; Ballou, D. P. J. Biol. Chem 1987, 262, 1510. 
(21) Shiman, R. In Folates and Pterins: Chemistry and Biochemistry of Pterins; Blakley, R. L.; Benkovic, S. J., Ed.; John Wiley and Sons: New York, 1985; Vol. 2; $\mathrm{p} 179$.

(22) Salowe, S. P.; Marsh, E. N.; Townsend, C. A. Biochemistry 1990, 29, 6499.

(23) Samuelsson, B.; Dahlén, S.-E.; Lindgren, J. Å.; Rouzer, C. A.; Serhan, C. N. Science 1987, 237, 1171.

(24) Stubbe, J.; Kozarich, J. W. Chem. Rev. 1987, 87, 1107.

(25) Petering, D. H.; Bymes, R. W.; Antholine, W. E. Chem.-Biol. Interactions 1990 , 73, 133.

(26) X-ray Absorption: Principles, Applications, Techniques of EXAFS, SEXAFS and $X A N E S$; Koningsberger, D. C.; Prins, R., Eds.; John Wiley and Sons Inc.: New York, 1988.

(27) Bianconi, A. In X-ray Absorption: Principles, Applications, Techniques of EXAFS, SEXAFS and XANES; Koningsberger, D. C.; Prins, R., Eds.; John Wiley and Sons Inc.: New York, 1988; 573.

(28) Stern, E. A. In X-ray Absorption: Principles, Applications, Techniques of EXAFS, SEXAFS and XANES; Koningsberger, D. C.; Prins, R., Eds.; John Wiley and Sons Inc.: New York, 1988; $\mathrm{p} 1$.

(29) Cramer, S. P.; Hodgson, K. O.; Stiefel, E. I.; Newton, W. E. J. Am. Chem. Soc. 1978, 100, 2748.

(30) Lytle, F. W. In Applications of Synchrotron Radiation; Winick, H.; Xiam, D.; Ye, M.-h.; Huang, T., Eds.; Gordon and Breach Science Publishers: New York, 1989; p 135.

(31) Cramer, S. P.; Hodgson, K. O. Prog. Inorg. Chem. 1979, $25,1$.

(32) Scott, R. A. Meth. Enzymol. 1985, 117, 414.

(33) Heald, S. M. In X-ray Absorption: Principles, Applications, Techniques of EXAFS, SEXAFS and XANES; Koningsberger, D. C.; Prins, R., Eds.; John Wiley and Sons Inc.: New York, 1988; $\mathrm{p} 87$.

(34) Agarwal, B. K. X-ray Spectroscopy; Springer-Verlag: New York, 1979.

(35) Stivastava, U. C.; Nigam, H. L. Coord. Chem. Rev. 1973, 9, 275.

(36) Shulman, R. G.; Yafet, Y.; Eisenberger, P.; Blumberg, W. E. Proc. Natl. Acad. Sci. USA 1976, 73, 1384.

(37) Roe, A. L.; Schneider, D. J.; Mayer, R. J.; Pyrz, J. W.; Widom, J.; Que, L., Jr. J. Am. Chem. Soc. 1984, 106, 1676.

(38) Randall, C. R.; Shu, L.; Chiou, Y.-M.; Hagen, K. S.; Ito, M.; Kitajima, N.; Lachicotte, R. J.; Zang, Y.; Que, L., Jr. Inorg. Chem. 1995, 34, 1036. 
(39) Cramer, S. P.; Eccles, T. K.; Kutzler, F. W.; Hodgson, K. O. J. Am. Chem. Soc. 1976, 98, 1287.

(40) Wong, J.; Lytle, F. W.; Messmer, R. P.; Maylotte, D. H. Phys. Rev. B. 1984, 30 , 5596.

(41) Kau, L.-S.; Spira-Solomon, D. J.; Penner-Hahn, J. E.; Hodgson, K. O.; Solomon, E: I. J. Am. Chem. Soc. 1987, 109, 6433.

(42) Cramer, S. P. In X-ray Absorption: Principles, Applications, Techniques of EXAFS, SEXAFS and XANES; Koningsberger, D. C.; Prins, R., Eds.; John Wiley and Sons Inc.: New York, 1988; $\mathrm{p} 573$.

(43) Lytle, F. W.; Via, G. H.; Sinfelt, J. H. In Synchrotron Radiation Research; Winick, H.; Doniach, S., Eds.; Plenum Press: New York, 1980; Chp. 12.

(44) Iwasawa, Y. Tailored Metal Catalyst; D. Reidel Publishing Co.: Dordrecht, 1986.

(45) Filipponi, A.; Di Cicco, A.; Tyson, T. A.; Natoli, C. R. Solid State Commun. 1991, 78, 265.

(46) Binsted, N.; Campbell, J. W.; Gurman, S. J.; Stephenson, P. C. SERC Daresbury Laboratory EXCURVE 92 program, 1991.

(47) Rehr, J. J.; Mustre de Leon, J.; Zabinsky, S. I.; Albers, R. C. J. Am. Chem. Soc. $1991,113,5135$.

(48) Rehr, J. J.; Albers, R. C.; Zabinsky, S. I. Phys. Rev. Lett. 1992, 69, 3397.

(49) Rehr, J. J. Jpn. J. Appl. Phys. 1993, 32, 8.

(50) Mustre de Leon, J.; Rehr, J. J.; Zabinsky, S. I.; Albers, R. C. Phys. Rev. B 1991, 44, 4146.

(51) Stern, E. A. Phys. Rev. B 1974, 10, 3027.

(52) Ashley, C. A.; Doniach, S. Phys. Rev. B 1975, 11, 1279.

(53) Lee, P. A.; Teo, B.-K.; Simons, A. L. J. Am. Chem. Soc. 1977, 99, 3856.

(54) Shulman, R. G.; Eisenberger, P.; Teo, B.-K.; Kincaid, B. M.; Brown, G. S. J. Mol. Biol. 1978, 124, 305.

(55) Lee, P. A.; Pendry, J. B. Phys. Rev. B 1975, 11, 2795.

(56) Filipponi, A.; Di Cicco, A.; Zanoni, R.; Bellatreccia, M.; Sessa, V.; Dossi, C.; Psaro, R. Chem. Phys. Lett. 1991, 184, 485.

(57) Binsted, N.; Cook, S. L.; Evans, J.; Greaves, G. N.; Price, R. J. J. Am. Chem. Soc. 1987, 103, 3669.

(58) · Binsted, N.; Evans, J.; Greaves, G. N.; Price, R. J. J. Chem. Soc., Chem. Comm. 1987, 1130.

(59) Teo, B.-K. J. Am. Chem. Soc. 1981, 109, 3990. 
(60) Co, M. S.; Hendrickson, W. A.; Hodgson, K. O.; Doniach, S. J. Am. Chem. Soc. 1983, $105,1144$. 


\section{Chapter 2}

GNXAS,

a New Multiple-Scattering EXAFS Analysis Package, and Its Application to Iron Inorganic Model Complexes 


\subsection{Introduction}

Extended X-ray absorption fine structure (EXAFS) spectroscopy is a valuable technique for investigating the local coordination environment of specific atomic species in systems ranging from metalloproteins ${ }^{1,2}$ to catalysts. ${ }^{3-5}$ The method is sensitive to short-range order (distances typically within about 3-5 $\AA$ of the absorber) and provides information on the distances to, numbers of, and types of neighboring atoms. EXAFS has the advantages of being able to focus on a selected type of atom and of being applicable to any physical state, including liquid or frozen solutions and amorphous solids. However, the analysis of EXAFS data requires accurately known experimental or theoretical pairwise phase and amplitude functions. Experimental standards have been widely used to extract reliable empirical amplitude and phase functions and these have been used with success to obtain structural information from EXAFS data, particularly for nearest neighbors. Alternatively, reliable theoretical phases and amplitudes have recently become available that enable more information, including angular distributions, to be obtained from EXAFS analysis.

The empirical data analysis technique ${ }^{1,2,6,7}$ involves the use of pairwise phase and amplitude functions which have been extracted from the EXAFS data of suitable model complexes. The empirical technique allows for the determination of first neighbor distances with high accuracy (typically $\pm 0.02 \AA$ ) but determines with less accuracy the coordination number (one atom in four or five) and the identity of the ligating atoms (not differentiating \pm 2 in $Z$ ). The empirical approach is of questionable utility for atoms beyond $\sim 3 \AA$ because of phase and amplitude transferability problems. A breakdown of the phase and amplitude transferability occurs because of intervening atoms that give rise to multiple-scattering (MS) signals. These MS signals can contribute significantly to the total EXAFS signal and very often interfere with the single-scattering (SS) signal. The MS effects are particularly evident when an intervening atom lies in a close-to-linear relationship with the absorber and a more distant scatterer, as occurs, for example, in Fe-oxo dimers ${ }^{8}$ and metal carbonyls. ${ }^{2,9-12}$ Multiple-scattering effects can also be quite prominent for certain rigid ligands such as imidazoles and porphyrins ${ }^{13,14}$ and can be of such magnitude that they dominate over SS signals even in structures that are not collinear (vide infra). Moreover, it can be difficult to obtain suitable models for extraction of reliable pairwise empirical amplitudes and phases because of the requirement for single well-ordered coordination shells that are separated from other EXAFS contributions. As a result of these limitations, determination of distances beyond 
the first coordination shell and of bond angles has been difficult using empirically-derived phase and amplitude functions.

An alternative to the empirically-based EXAFS data analysis is to calculate the phase and amplitude functions theoretically. In this approach, an expected theoretical signal is calculated assuming a structural model for the system under study. The resulting signal is then fit to the experimental data, varying the input parameters until a minimum of a selected reliability function is reached. The quality of the fit is further determined by inspection of EXAFS and Fourier transform (FT) residuals. The theoretical approach is advantageous relative to the empirical approach in that MS contributions can be modeled and therefore bond distance and bond angle information from distant shells of atoms can in principle be determined. Also a theoretical approach is not dependent upon obtaining suitable model compounds to extract pairwise phase and amplitude functions. While the reliability of the results are limited by the accuracy of the theory, it is becoming clear that accuracy comparable to that available with the empirical technique is now possible as illustrated by this work and that published in some of the references cited below.

The GNXAS approach (where $g_{n}$ stands for the $n$-atom distribution function and XAS stands for X-ray absorption spectroscopy) has recently been developed as an integrated theoretical approach to the analysis of EXAFS data. ${ }^{15-17}$ Three distinctive features of the integrated GNXAS approach in comparison with other existing analysis packages (FEFF5 ${ }^{18,19}$ and EXCURVE ${ }^{20}$ ) are: (1) an improved solution for the one-particle Green's function equation with complex optical potential of the Hedin-Lundquist type in the muffin-tin approximation (from which the total photoabsorption cross section is calculated), (2) SS and MS signals are classified according to the appropriate $n$-atom distribution function with proper treatment of the configurational average of MS terms, and (3) the fit to the experimental spectrum is performed by comparing directly in energy space the raw data with a global model absorption coefficient that includes the structural signal, the edge jump normalization, the post-edge background, and if present, shake-up/shake-off edges, so that the structural signal is optimized together with other components of the absorption spectrum. Since GNXAS is able to calculate all the signals relating to two-, three-, and four-atom correlation functions with the proper treatment of correlated distances and Debye-Waller factors, it is particularly well-suited for the analysis of MS effects and for bond angle determination. GNXAS has been initially used on several simpler systems (including $\mathrm{SiX}_{4}, \mathrm{X}=\mathrm{F}, \mathrm{Cl}$, and $\mathrm{CH}_{3},{ }^{21} \mathrm{Os}_{3}(\mathrm{CO})_{12},{ }^{9} \mathrm{Br}_{2}$ and $\mathrm{HBr},{ }^{22}$ and brominated hydrocarbons ${ }^{23}$ ) and more recently on a complex polynuclear metal cluster. ${ }^{24}$ 
In this chapter, the background and brief theoretical description of the GNXAS methodology is presented, along with a description of the GNXAS programs and their use for analysis of molecular systems. The GNXAS methodology is followed by its specific application to three iron coordination complexes. ${ }^{25}$ These complexes were chosen to investigate the characteristics, advantages, and limitations of the method, in particular in the study of MS effects in chemical systems. Further, this detailed analysis enables an accurate error assessment by examining the variance between crystallographically known and EXAFS-determined metrical details.

The GNXAS method was applied to $\mathrm{Fe} \mathrm{K}$-edge EXAFS data for $\mathrm{Fe}(\mathrm{acac})_{3}$, $\mathrm{Na}\left[\mathrm{Fe}\left(\mathrm{OH}_{2}\right) \mathrm{EDTA}\right]$, and $\mathrm{K}_{3} \mathrm{Fe}(\mathrm{CN})_{6}$ (where acac = acetylacetonate and EDTA = ethylenediaminetetraacetic acid). The applicability and utility of the GNXAS method was determined by studying the MS effects in the EXAFS data and evaluating the reliability of structural parameters (bond distances and angles) obtained from GNXAS. The study of the magnitude and complexity of MS contributions in the EXAFS data of $\mathrm{Fe}$ (acac) 3 was of particular interest since $\mathrm{Fe}(\mathrm{acac})_{3}$, due to the regularity of its structure, has been widely used to extract both $\mathrm{Fe}-\mathrm{O}$ and second shell $\mathrm{Fe}-\mathrm{C}$ phase and amplitude backscattering parameters for empirical EXAFS analysis. The empirical $\mathrm{Fe}-\mathrm{O}$ backscattering parameters have been used quite successfully to model first-shell iron-oxygen distances and coordination numbers in many iron-containing models and enzymes, ${ }^{26-29}$ while the use of the $\mathrm{Fe}-\mathrm{C}$ second shell backscattering parameters has met with much more limited success ${ }^{26,29}$ due to MS contributions. MS effects are incorporated within the empirical approach in such a way that the phase and amplitude parameters reflect the exact geometry of the model compound and cannot be transferred to an unknown of different geometry. The GNXAS technique was also applied to $\mathrm{Na}\left[\mathrm{Fe}\left(\mathrm{OH}_{2}\right)\right.$ EDTA] to test the ability of GNXAS to interpret the EXAFS data for a lower-symmetry compound with mixed ligation, such complexes being a better approximation to the situation typically found in metalloenzymes, where the GNXAS approach can prove especially valuable in EXAFS data analysis. Finally, the EXAFS data of $\mathrm{K}_{3} \mathrm{Fe}(\mathrm{CN})_{6}$ was analyzed in detail with GNXAS to study the MS behavior of the linear $\mathrm{Fe}-\mathrm{C}-\mathrm{N}$ unit and to evaluate the use of this analytical approach for angle determination of small molecules liganded to transition metals. The results of these three applications together establish the validity and reliability of GNXAS as an approach for EXAFS data of chemical systems. Given this, the technique may be used to analyze unknown systems, as further elaborated in Chapter 3.

It should be noted here that other groups have developed analysis packages similar in concept to GNXAS. The program EXCURVE, developed at Daresbury, is 
probably the oldest. ${ }^{20}$ The program FEFF, ${ }^{30,31}$ developed at the University of Washington, Seattle, came later, roughly at the same time as GNXAS, initially with the possibility of calculating single scattering only, then including multiple scattering in later versions (FEFF5). ${ }^{18,19}$ The relationship of these programs to GNXAS shall be briefly discussed in the course of the methodology presentation which follows.

\subsection{GNXAS Methodology}

\subsubsection{Background}

Until relatively recently, the lack of a proper theoretical formulation of the photoabsorption process has limited the use of theoretical functions for reliable analysis of EXAFS data. It was necessary to obtain a correct mathematical description of the spherical wave propagation of the photoelectron through the system (in an inner core photoabsorption process the photoelectron is created in an eigenstate of the angular momentum operator or a definite mixture of them) and to use an appropriate optical potential in describing this propagation. Early plane-wave SS theories ${ }^{32}$ failed even in the high energy limit ${ }^{33}$ and had to be replaced with MS theories with spherical wave propagation. ${ }^{34-39}$ It was also realized that the "universal" atomic potentials used to calculate standard theoretical amplitudes and phases were not sufficiently reliable because the electrostatics was not modeled correctly. Therefore, it was necessary to construct a realistic charge density on and around the photoabsorber, as is done in band theory calculations. The Mattheiss ${ }^{40}$ prescription of overlapping neutral atom charge densities provided charge densities that are acceptably close to those obtained by self-consistent procedures. The Coulomb, exchange and correlation potentials could then be calculated from this cluster charge density.

Additionally, in the statistical interpretation, the local density approximation of the Hedin-Lundqvist ${ }^{41,42}$ (HL) exchange-correlation potential proved to be a good starting point for the photoelectron optical potential. The $\mathrm{HL}$ exchange-correlation potential takes into account the energy dependence of the exchange and correlation (Coulomb) hole around the propagating electron in the dispersive (real) part and has an imaginary part capable of reproducing the observed electron mean-free path in metals and semiconductors. ${ }^{43}$ The optical potential could be approximated by the self-energy of a uniform interacting electron gas with a density given by the local density of the system. In the spirit of this statistical approximation, Lee and Beni ${ }^{44}$ extended the $\mathrm{HL}$ potential, which was initially devised to describe exchange and correlation corrections to the 
Coulomb potential due to the valence charge only, to the atomic core. When put in context with other components of MS theory correctly formulated in presence of a complex effective potential, this statistical potential proved to be a good starting point for the photoelectron optical potential. ${ }^{30,45-47}$ The effect of the intrinsic inelastic channels has not been included in the theory. However, a reasonable estimate of the size of this effect on the amplitude of the EXAFS signal is less than 10\% of the total signal, which is acceptable.

Another important aspect for a correct EXAFS analysis is the proper description of structural correlations in a system and the possibility of doing configurational averages. EXAFS has an almost unique advantage over other structural techniques in that it can probe atomic correlation functions of order greater than two, i.e. position correlations of more than two atoms at a time. In fact, diffraction techniques only probe the pair correlation function, since the technique is based on the weak coupling between the probe (X-rays, neutrons) and the system under study. The double scattering events of the probe which would allow access to higher order correlations are generally negligible. This is not the case with EXAFS, in that the primary probe (the photon) couples weakly enough with matter so that the simple "golden rule" is sufficient to describe the photoabsorption cross section. However, the secondary probe, i.e. the emitted photoelectron, can couple strongly with the atoms of the system so that in addition to SS, MS becomes quite detectable and exploitable in many cases. This feature is shared by other techniques that use electrons either as a secondary probe (as in photoelectron diffraction) or as a primary probe (as in low energy electron diffraction). A good description of the dynamical strong coupling of the electron and matter is not easy to obtain, but once this is achieved, the next step is to have a general method for describing geometric structural correlations. Since the MS series is known to converge slowly, it can be resummed in such a way that the interrelation between the dynamic and the structural parts of the theory is transparent (while at the same time improving the convergence rate). In GNXAS this has been accomplished by summing together all the terms in the series referring to the same set of atoms in all their equivalent configurations with respect to the photoabsorber. This sum is done so as to treat all the MS signals relating to definite structural configurations together to give the various $n$-atom correlation functions. ${ }^{15-17,45-49}$ This involves a topological structural analysis that is done on the chemical structure under study. Moreover, an efficient way to perform proper thermal or structural configurational averages, using probability distributions that are either chosen a priori or conveniently parameterized, has been devised in this integrated approach to EXAFS analysis. ${ }^{50}$ 


\subsubsection{GNXAS Theoretical and Analytical Approach}

Before discussing how the GNXAS package functions, it is useful to present a brief derivation of the photoabsorption cross section for a cluster of atoms in physical terms. It will serve to define concepts, to give an intuitive feeling of what is actually going on in the process, and provides the background for understanding the angle-dependent aspects of MS and how they can be used to obtain geometric information.

In an absorption measurement, the emitted photoelectron is not detected, rather the total number of created holes (the total cross section) is measured. This is equivalent to integrating over all the photoemitted electrons. The integration process suppresses all the electron paths that do not come back to the photoabsorber so that the observed modulations of the absorption coefficient are due to the interference (constructive or destructive according to the photoelectron energy) between the outgoing and returning photoelectronic waves. Only electrons in the completely relaxed (elastic) channel with the maximum available kinetic energy $E=\omega-I_{c}$ contribute to the effect. Therefore, in studying the modulations in the absorption coefficient, the propagation of the coherent electrons can be described through the introduction of an effective optical potential. In this way a truly many-body problem can be reduced to an effective and tractable one-electron problem. The total many-body absorption cross section can be written as 45

$$
\sigma_{m b}(E)=S_{i n}(E) \sigma_{e}(E)
$$

where $\sigma_{e}(E)$ is the one-electron absorption cross section in the elastic channel, calculated with the optical potential, and $S_{i n}(E)$ describes the inelastic channels. Examples of inelastic channels are the shake-up or shake-off double-electron excitations. ${ }^{51}$ These may need to be taken into account since they can distort the EXAFS signal. Notice that in this approach $\sigma_{e}(E)$ includes the many-body amplitude reduction factor $S_{o}{ }^{2}{ }^{30,45}$

As mentioned earlier, the construction of the one-electron optical potential, although in principle feasible, is very difficult. The one-electron optical potential should take into account both the extrinsic and intrinsic effects and their interference. Moreover, the potential needs to be simple and versatile enough to describe the many varied situations encountered in practical applications. On the basis of statistical considerations, it has been found that the $\mathrm{HL}$ potential ${ }^{41,42}$ is a good starting point for approximating the optical potential. ${ }^{30,45-47}$ In this approximation, the optical potential is complex. Its imaginary part $\Gamma(E)$ gives rise to a finite lifetime that describes the attenuation of the 
photoelectron wave in the elastic coherent channel due to the possibility of inelastic excitations of the system. Therefore, the optical potential acts as a medium that diffracts the coherent electron wave with its real part and attenuates it via the imaginary part.

The attenuation process has the consequence that the actual size of the system probed by a photoelectron with wave vector $k$ and kinetic energy $E=k^{2}$ reduces to a sphere around the photoabsorber with a radius roughly equal to the mean-free path $\lambda(E)$ of the electron probe at that energy. The mean free path is linked to the imaginary part of the optical potential $\Gamma(E)$ through the relation ${ }^{52,53}$

$$
\lambda(E)(a u)=\frac{k(a u)^{-1}}{\Gamma(E)(R y d)} \quad \text { or equivalently } \quad \lambda(E)(\AA)=\frac{E}{\Gamma(E)} \frac{1}{k(\AA)^{-1}}
$$

In a one-electron picture it is necessary to start from the description of the potential associated with a cluster of atoms. Since in the statistical approximation the HL potential depends on the local density of the system under study, as does the Coulomb potential, a rapid and efficient way of generating such a density has to be devised. As mentioned before, the Mattheiss ${ }^{40}$ prescription of overlapping neutral atom charge densities present in the molecular cluster is able to generate charge densities that are acceptably close to those obtained by self-consistent procedures. At this point, a further approximation is made to the charge density to simplify the solution of the one-electron Schrödinger equation. After partitioning the cluster space into touching spheres around the atoms, an outer sphere encircling all the cluster and an interstitial region in between, one spherically averages the charge density inside the atomic spheres and calculates an averaged charge density in the interstitial region. The potential is set to a constant in this latter region. This approximation is likely to distort the calculated signal within $-30 \mathrm{eV}$ of the absorption edge, but its effects diminish quite rapidly with increasing energy.

Having constructed the potential, the derivation of the one-electron photoabsorption cross section $\sigma_{e}(E)$ in eq 1 follows from the application of MS theory. The main results relevant to the present discussion are summarized here. The reader is referred to the Appendix in reference 25 for a more detailed derivation. The equation for $\sigma_{e}(E)$ can be written as

$$
\sigma_{e}(E)=\sigma_{0}^{l}(E)\left[1+\chi^{l}(E)\right]
$$

where $\sigma_{0}^{\prime}(E)$ is the final state $l$, dipole-allowed, atomic absorption cross section for the photoabsorbing atom and $\chi^{l}(E)$ represents the contribution due to the other atoms in the 
cluster. GNXAS uses an improved solution for the one-particle Green's function equation with complex potential in the muffin-tin approximation, from which the photoabsorption cross section is calculated. In this scheme the total cross section can be written as the sum of two contributions, the first one having the form of an "atomic" cross section relative to the muffin-tin sphere of the photoabsorber, the second one being the contribution coming from the neighboring scatterers. However the latter does not factorize into an "atomic" cross section times a dimensionless structure signal, as is the case with a real potential, therefore the structural signal which appears in eq 3 has to be defined as the ratio of the two contributions. This is different from that used in other codes. In most cases, this difference is negligible over almost the entire EXAFS spectrum but it may affect the amplitude of the structural signal in the low-energy part of the spectrum. For more details on this point the reader is referred to reference 45 , eq 3.8 3.12. In the region of convergence of the MS series the structural term $\chi^{l}(E)$ can be expressed as $^{45}$

$$
\chi^{l}(E)=\sum_{n=2}^{\infty} \chi_{n}^{l}(E)=\sum_{n=2}^{\infty} \sum_{p_{n}} \Im A_{p_{n}}^{l}\left(E ; R_{i j}^{p_{n}}\right)
$$

where each $\chi_{n}^{l}(E)$ term represents the contribution originating from processes in which the excited photoelectron is scattered $\mathrm{n}-1$ times by the surrounding atoms before returning to the photoabsorber. Each $\chi_{n}^{l}(E)$ term is obtained by taking the imaginary part ( $(\mathfrak{I})$ of the scattering amplitudes $A_{p_{n}}^{l}\left(E ; R_{i j}^{p_{n}}\right)$ relative to all the individual paths $p_{n}$ of order $n$ that involve at most $n$ atoms, including the photoabsorber. The functional form of the contribution of any path $p_{n}$ is of the type (see Appendix in reference 25)

$$
A_{n}^{l}\left(\kappa ; R_{i j}^{P_{n}}\right) \sin \left[\kappa R_{p_{n}}^{\text {lot }}+\phi\left(\kappa ; R_{i j}^{P_{n}}\right)\right]
$$

where $R_{p_{n}}^{\text {rot }}$ is the total length of the path and $A_{n}^{l}\left(\kappa ; R_{i j}^{p_{n}}\right)$ and $\phi\left(\kappa ; R_{i j}^{p_{n}}\right)$ are, respectively, the amplitude and the phase of the signal associated with it. Due to the use of the optical complex potential $V(r)$ the amplitude of the path contains a damping factor which (in the Wentzel-Kramers-Brillouin (WKB) approximation for the potential phase shifts) can be written as

$$
\exp \left[-3 \oint_{p_{n}} d r \sqrt{k^{2}-V(r)}\right]
$$


where the integral is taken along the closed path of the photoelectron. Therefore the longer the path, the more heavily its contribution is damped. Notice that the complex nature of the central atom phase shift has also been taken into account. In contrast, Lee and $\mathrm{Beni}^{44}$ and $\mathrm{TeO}^{12}$ only take into account the complex nature of the phase shifts for the backscattering atoms. These authors also only use the plane-wave approximation for the spherical wave propagators. On the basis of eqs 3-5 the absorption cross section consists of the superposition of various oscillating signals of different amplitudes and periods onto a more or less smooth background given by the atomic absorption. The most important contributions coming from the various paths should be summed, and the resulting signal should be compared to the observed spectrum. However, the criterion of assessing the importance of the various contributions according to the number of scattering events, as suggested by the expansion in eq 4 , is not of general validity since in some cases paths running along the same atoms with a different number of scattering events might contribute signals of similar strength. As presented in more detail in the Appendix in reference 25, this might occur because the expansion parameter for the MS series, which is given by $|f(\theta) /(x R)|$ where $R$ is the typical nearest neighbor interatomic distance in the system, has a peculiar behavior as a function of the scattering angle $\theta$. In fact, even at moderately high energies $(\geq 200 \mathrm{eV}$ ) this quantity falls off quite rapidly from values on the order of unity in a forward cone of aperture $\sim 20^{\circ}$ to values typically on the order of less than 0.1. This behavior leads to the so-called "focusing effect", whereby forward scattering events enhance rather than depress the corresponding signal. In the case of a collinear path involving three sites $0, i$ and $j$ at distance $R$ from each other, the ratio of the triple-scattering signal to the double scattering one is $2|f(0) /(\kappa R)|$. Therefore the amplitude of the fourth order path is nearly twice that of the third order which in turn is twice that of second order SS. At lower energies, the scattering becomes more isotropic and $|f(\theta) /(\kappa R)|$ may attain sizable values $(\sim 0.2-0.4)$ for $\sim 30^{\circ}<\theta<180^{\circ}$, as illustrated in Figure 2.1, so that the rate of convergence of the MS series is slower.

From these considerations, it is evident that the rate of convergence of the MS series is controlled by an interplay between (a) the strength of the scattering, which depends on the energy, (b) the number of scattering events and the angles at which the scattering events occur (c) the electron damping, which in turn depends on the energy, the length of the path, and the types of atoms along the path and d) the degeneracy of the various paths. As mentioned in the Background section above, an efficient way to cope with this situation and one that improves the rate of convergence of the MS series is to sum together up to infinite order (or to the necessary order to get convergence) all the terms which refer to the same set of atoms in all their equivalent configurations with 


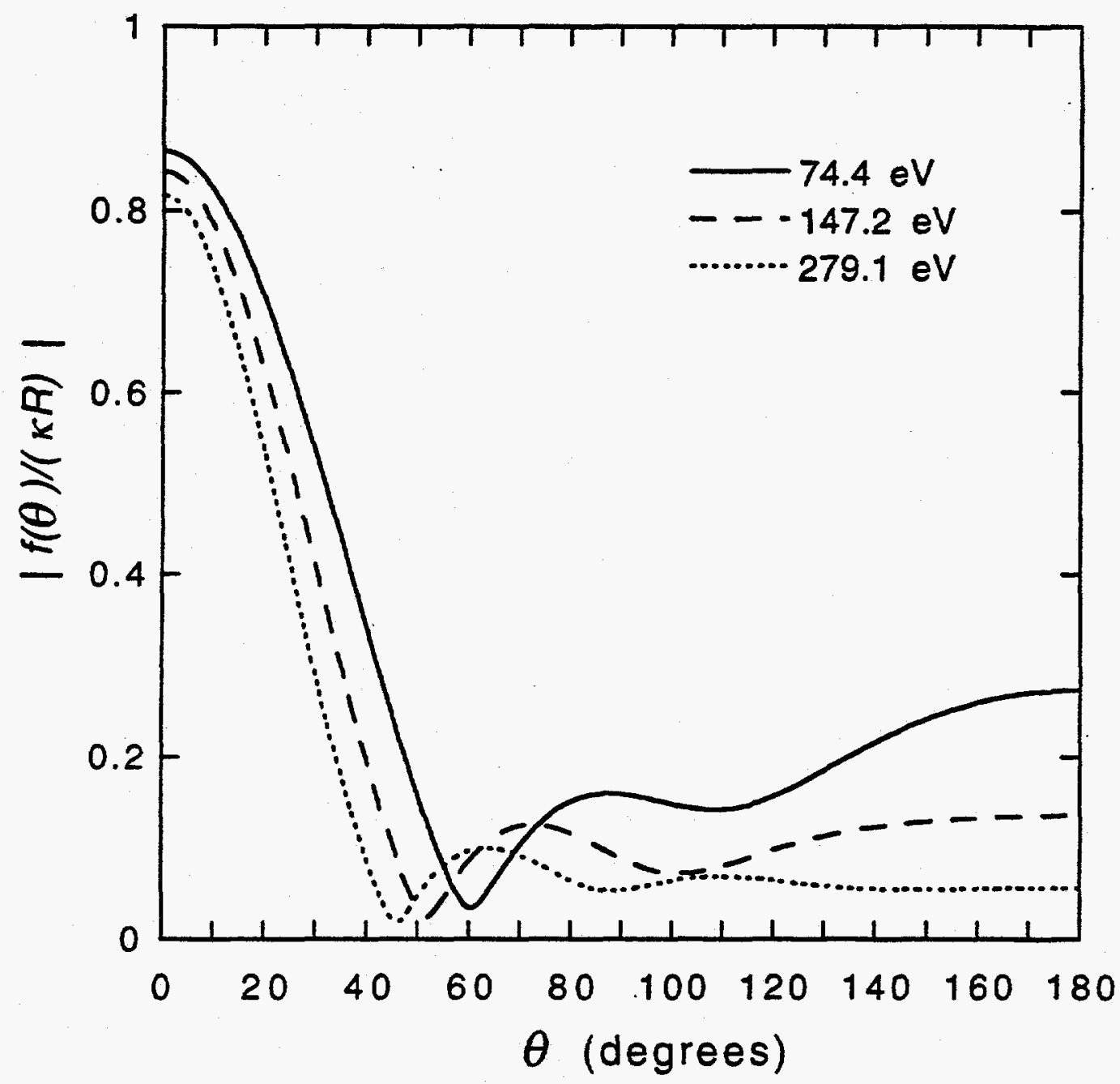

Figure 2.1. Plot of the expansion parameter $|f(\theta) /(\kappa R)|$ as a function of the scattering angle $\theta$ at different energies using the oxygen phase shifts and the Fe-O distance $R=$ $1.99 \AA$ for an Fe-O-C scattering pathway. The quantity $|f(\theta) / \kappa|^{2}$ is the effective scattering cross section for the excited photoelectron impinging onto the $O$ atom at an angle $\theta$ away from the incoming Fe-O direction. From inspection of the behavior of this function, it is clear that forward scattering directions are enhanced by factors of 3 - 4 . Notice that with this definition of the scattering angle $\theta$ the Fe-O-C angle is $180^{\circ}-\theta$. This latter angle $\left(180^{\circ}-\theta\right)$ is the one used throughout this chapter for defining the bond angle in a triangle, besides the two short sides. 
respect to the photoabsorber, starting with pairs of atoms, then triplets, quadruplets, and so on. ${ }^{15-17,48,49}$ The cut-off distance which limits the size of the model cluster, and therefore the number of structural configurations to be taken into account, can be deduced by inspecting the FT of the experimental absorption spectrum under consideration. A topological structural analysis of the cluster will then provide all the relevant configurations whose path lengths are less than the chosen cut-off distance.

On the basis of these considerations, the structural term, in eq 3, related to a cluster of $N$ atoms with the photoabsorber in site $o$ can be rewritten as ${ }^{15-17,48,49}$

$$
\chi^{\prime}(E)=\sum_{i=1}^{N-1} \gamma_{(0, i)}^{(2)}+\sum_{i=1}^{N-1} \sum_{j>1}^{N-1} \gamma_{(o, i, j)}^{(3)}+\sum_{i=1}^{N-1} \sum_{j>i}^{N-1} \sum_{k>j}^{N-1} \gamma_{(o, i, j, k)}^{(4)}+\ldots=\gamma^{(2)}+\gamma^{(3)}+\gamma^{(4)}+\ldots
$$

where $\gamma^{(2)_{(0, i)},} \gamma^{(3)_{(0, i, j)}}$, and $\left.\gamma^{(4)}\right)_{(0, i, j, k)}$ are the proper two-atom, three-atom, and four-atom signals associated with configurations of two $(o, i)$, three $(o, i, j)$, and four $(o, i, j, k)$ atoms, respectively. The idea here is to sum all the MS signals that refer to the same subclusters of atoms.

In general, the $\gamma^{(n)}$ signals can be defined through the terms of the MS series. For example, in the case of a two-atom signal involving atoms $o$ (photoabsorber) and $i$

$$
\gamma^{(2)}=\chi_{2}^{0 i 0}+\chi_{4}^{\text {oioio }}+\chi_{6}^{\text {oicioio }}+\chi_{8}^{\text {oioioioio }}+\ldots . O\left(\chi_{10}\right)
$$

where the leading term is the SS process with obvious meaning of the superscripts. Similarly for a $\gamma^{(3)}$ signal involving sites $0, i$, and $j$ one has

$$
\gamma^{(3)}=2 \chi_{3}^{0 i j o}+2 \chi_{4}^{0 i j o}+\chi_{4}^{o i j i o}+\chi_{4}^{o i j i o}+\ldots O\left(\chi_{5}\right)
$$

where the coefficients count the time reversal degeneracy of the paths. Usually $\gamma(2)$ and $\chi_{2}$ differ very little since the higher order MS contributions are very small, thus the $\gamma(2)$ signal often is referred to as the SS contribution. However $\gamma(3)$ and $2 \chi_{3}$ can be quite different due to the sizable contributions from the higher order terms.

For higher-order $\gamma^{(n)}$ signals, only the terms $\chi_{m}(E)$, with $m \geq n$, appear in the infinite summation. Higher-order terms are meaningful only when the MS series converges (see Appendix in reference 25). Nevertheless, the $n$-body $\gamma(n)$ signals can be defined independently. In a system with only two atoms, the $\gamma(2)$ signal coincides with the total structural term $\chi(E)$. For such a system, one can carry out the matrix inversion of eq $A 12$ of the Appendix in reference 25. 
For a triplet of atoms $(o, i, j)$, the proper three-atom signal is defined by subtracting the lower-order terms

$$
\gamma_{(0, i, j)}^{(3)}=\chi^{(0, i, j)}-\gamma_{(0, i)}^{(2)}-\gamma_{(0, j)}^{(2)}
$$

This procedure can be used to define the higher-order $\gamma(n)$ signals. In general, the evaluation of the $n$-atom terms $\gamma(n)$ is obtained by calculating the total signal for $n$ atoms and subtracting all the lower-order $m<n$ terms. The exact calculation of the $\gamma(n)$ signals is obtained by performing matrix inversions for defined sets of two, three, or four atoms. A very fast algorithm based on the continued fraction expansion has been developed to calculate the total $n$-atom signals, ${ }^{49}$ since it is difficult to perform such inversions at high energies, due to the high number of angular momenta needed. It is assumed that the rearranged MS series in eq 7 always converges, especially after proper configurational averaging of the individual terms.

Since the MS series can now be written in terms of $n$-atom signals, an average over all the configurations, whether thermal or structural, in the system can be written as $^{16,17,48}$

$$
\begin{aligned}
& \langle\chi(E)\rangle=\rho_{0} \int_{0}^{\infty} 4 \pi r^{2} d r g_{2}(r) \gamma^{(2)}(r ; E)+ \\
& \rho_{0}^{2} \int 8 \pi^{2} r_{1}^{2} r_{2}^{2} \sin \theta d r_{1} d r_{2} d \theta g_{3}\left(r_{1}, r_{2}, \theta\right) \gamma^{(3)}\left(r_{1}, r_{2}, \theta ; E\right)+ \\
& \rho_{0}^{3} \int 8 \pi^{2} r_{1}^{2} r_{2}^{2} r_{3}^{2} \sin \theta d r_{1} d r_{2} d r_{3} d \theta d \omega g_{4}\left(r_{1}, r_{2}, \theta, r_{3}, \omega\right) \gamma^{(4)}\left(r_{1}, r_{2}, \theta, r_{3}, \omega ; E\right)+\ldots
\end{aligned}
$$

where the various $g_{n}$ are the $n$-atom correlation functions which give the probability of the occurrence of a given configuration as seen from the absorbing site. The distances $r_{i}$ and angles $\theta$ and $\omega$ are the structural variables, which parameterize the relative position of $n$ atoms at a time, and $\rho_{0}$ is the average density of the system. Since the various $g_{n}$ are not known a priori, unless a definite model to describe thermal or structural disorder is known, a decomposition can be made of the distribution functions into sums of well defined peaks associated with particular $n$-atom configurations. To each peak there corresponds a $\gamma^{(n)}$ signal which is dependent on peak shape, where the peak shape is defined by a certain number of parameters that can be varied during the fitting procedure. An initial background structural model must exist to establish such a decomposition. For molecules of biological interest, the various bond lengths and the angle between the 
bonds are the natural variables to describe thermal disorder and the various peaks can be described in terms of correlated Gaussian distributions. In this case, correlation variances and average distances and angles describing the various configurations can be fitted directly to the experimental signal.

Other ways of path selection and configuration averaging are obviously possible and each code uses different criteria. FEFF5, ${ }^{18,19}$ for example, retains only the most significant MS paths in order to avoid unnecessary computations. The default presorting criterion for retaining a path is that the amplitude of the contribution of a given path, estimated in the plane wave approximation, is above $2.5 \%$ of the first-shell amplitude. Configurational averages are made via the method of cumulant expansion, ${ }^{18,19}$ where the cumulants of various order enter among the fitting parameters. For instance, the first cumulant is the linear phase shift, the second is the Debye-Waller factor, the third is the cubic phase shift, etc. As is apparent from the previous discussion GNXAS classifies the MS paths according to a physical criterion that improves the convergence of the MS series and at the same time is suitable for configurational averaging. This approach has three advantages: (1) the number of structural parameters to be fit can be minimized (e.g. two bond lengths and an angle can serve to parameterize two SS scattering contributions and a MS contribution), (2) bond lengths and angles can be chosen as variables in the configuration space or given fixed values, and (3) correlations between the variables can be taken into account. In this respect, the cumulant expansion method is one of the possible choices in the GNXAS package for performing configurational averages.

The GNXAS program set incorporates all the advances described above on $a b$-initio calculations of the $X$-ray absorption cross section and configurational averages and directly fits the theoretical results with the experimental EXAFS data. Raw data are compared directly in $E$ space with a model absorption coefficient $\alpha_{\bmod }(E)$

$$
\alpha_{\bmod }(E)=J \alpha_{0}(E)[1+\chi(E)]+\beta(E)
$$

composed of an atomic absorption of hydrogenic type $\alpha_{0}(E)$, a structural $\chi(E)$ term and an appropriate function $\beta(E)$. The function $\beta(E)$ accounts for remaining background effects and can include many-body features like double-electron excitation channels (the $S_{\text {in }}(E)$ factor in eq 1). $J$ is the absorption coefficient jump which takes into account thickness and density of the photoabsorbing centers of the particular sample. The comparison of the experiment with the theoretical cross section also requires the inclusion of a few parameters which do not have direct structural meaning. The XAS experiment is not a measure of the pure $\mathrm{K}$-edge or L-edge absorption as there is always a 
background present mainly due to lower energy excitations and to instrumental effects. A smooth background is taken into account as a sum of polynomial functions. Also there are often spikes, steps, or small edges arising from instrumental effects or from intrinsic photoabsorption phenomena which are necessary to identify and to remove in the definition of the structural signal. It is possible to exclude particular energy regions affected by glitches, spikes, etc., and contributions coming from multi-electron excitation channels can be included with arc-tangent, step-like or Lorentzian line shapes.

The procedure of fitting a global model absorption coefficient directly to the raw data is unique to the GNXAS package. In the usual approach, a structural signal $\chi(E)$ is separated from the measured absorption cross section $\alpha(E)$ according to the formula

$$
\chi(E)=\left\{\alpha(E)-\alpha_{0}(E)\right\} / \alpha_{0}(E)
$$

where $\alpha_{0}(E)$ is the absorption of an isolated embedded atom. This separation is achieved in three steps: (a) a pre-edge background removal that eliminates the energy dependence of the absorption other than the one under investigation; (b) a normalization to an edge jump that takes into account the thickness and density of the photoabsorbing atoms; and (c) a post-edge background removal that eliminates the energy dependence due to the absorption from an isolated atom. This last step is the most crucial one as it can affect the final form of the structural signal. Up until recently, the practice followed was to perform the three steps without optimization in a partially subjective way. Recently a method has been suggested ${ }^{54}$ that for the third step subtracts a spline that best eliminates the nonstructural, low- $R$ portion of $\chi(R)$, the Fourier transform of $\chi(E)$, through an iterative procedure. Instead, GNXAS optimizes all three steps in $E$ space, since the three contributions cannot be separately defined in an unambiguous way, neither theoretically nor experimentally. The atomic cross section of the photoabsorber, for example, is a concept that can be defined theoretically in the framework of multiple scattering theory only in the muffin-tin approximation for the cluster potential. However, the cross section so calculated contains unphysical oscillations due to the truncation of the atomic potential. In a non-muffin-tin approach of MS theory, there is no way to define unambiguously the central atom absorption, since this latter depends on the scattering amplitude of the region of space surrounding the photoabsorber, which is not well defined. The ideal situation would be to have a reliable theory that calculates altogether the pre-edge, edge, and post-edge absorptions, including the structural signal and shake-up/shake-off processes, to be fitted to the experiment. Unfortunately, this is too complicated and the present status of the theory is not yet sufficiently developed. 
However, this concept is retained in GNXAS by constructing a global model signal to fit to the whole absorption. It is true that the $\beta$ term in eq $11 \mathrm{a}$, which contains the pre-edge contribution plus shake-up/shake-off :dges, couples this background to the structural model, but this is unavoidable and physical. In fact the method suggested in reference 54 has the drawback that it misrepresents the intensity and the shape of the double-excitations channels. Indeed these spectral features peak in the low- $R$ region of configuration space, since they contain high frequency components. By trying to minimize this low- $R$ nonstructural portion of $\chi(R)$ in order to define an optimal atomic background absorption, one is bound to misrepresent this contribution, since the intensity and the shape of the double excitations channels are determined by the physics of the process.

The nonlinear fitting procedure is applied to the unfiltered data by a residual function

$$
R_{N-n}\left(x_{1}, x_{2} \ldots x_{n}\right)=\frac{N}{N-n} \frac{\sum_{i=1}^{N}\left[\alpha\left(k_{i}\right)-\alpha_{\text {mod }}\left(k_{i} ; x_{1}, x_{2} \ldots x_{n}\right)\right]^{2} k_{i}^{p}}{\sum_{i=1}^{N}\left[\alpha\left(k_{i}\right)\right]^{2} k_{i}^{p}}
$$

which is a $\chi^{2}$-like statistical function dependent on the structural and background parameters $\left(x_{1}, x_{2}, \ldots x_{n}\right)$ and on the noise level. This function is not a true statistical $\chi^{2}$ function since a true $\chi^{2}$ function weights the data inversely according to the variance of each data point. However, the two functions can be roughly proportional in a situation in which the collection times are such that all data points, at low and high $k$, have roughly the same variance, the latter being determined by calculating the standard deviation during averaging of the experimental spectra. This requires a careful selection of the experimental count times to ensure that high- and low- $k$ data contribute significantly to the spectrum. In any case, in the GNXAS package, there is also the capability for generating error bars for each data point and constructing a true $\chi^{2}$ function. In eq 12 $k=\sqrt{E}, N$ is the number of experimental points, and $n$ is the number of fitting parameters. Structural parameters, such as equilibrium distances, angles, and Debye-Waller factors, can be refined around model values by using a Taylor expansion of phases and amplitudes up to sufficient-order to calculate the theoretical signals relative to each new configuration in the refinement procedure. Signals need to be recalculated only when the structural parameters vary significantly (typically $10 \%$ or more) from the 
starting values. For details on applied nonlinear multiparametric fitting procedures, see reference 55 .

It is useful to consider the number of i dependent data points present in a spectrum for comparison with the number of fit variables. At first sight, it would seem from eq 12 that this number is the total number of points; however, this is not so. In fact, doubling the number of points in a set of sinusoidal signals defined in $k$ space does not lead to a doubling of the information content in the spectrum, especially if one has already enough points to determine the phases and amplitudes. More quantitatively, if $\delta k$ is the interval in $k$ space where the spectrum is defined and if this latter is analyzed only on a finite interval $\delta R$ of the conjugate variable $R$, then it has recently been shown ${ }^{56}$ that the number of truly independent points $N_{I}$ in a spectrum is given by $N_{I}=(2 \delta k \delta R / \pi)+2$. This conclusion does not contradict the procedure of nonlinear least-squares minimization in $k$ space, since this latter is in principle able to lead to the determination not only of the number but also of the type of parameters relevant to the fit. In fact, trying to fit more parameters than the number allowed by the above formula will result in some of them being determined with very large errors, indicating which parameters are relevant. A parameter which is not relevant will not lead to a decrease of the squared residual function of the type shown in eq 12. Of course, it is very useful to have an $a$ priori estimate of the number of parameters one can reasonably fit to a spectrum as a guide, but in principle, this is not essential. In the data analysis section, the independent data-to-parameter ratio, which is an indication of the degree of determinacy of the fit, is presented for each compound.

The inclusion of three-atom signals provides for determination of quantities such as bond angles, angle variances, and bond-bond and bond-angle correlations. The structural parameters associated with a pair of atoms are the distance $R$ and the variance $\sigma_{R}^{2}$ (i.e. the mean square variation of the distance $R$ ) if a Gaussian distribution of distances is used. ${ }^{50}$ By considering the explicit contributions associated with triplets of atoms, one has to include three average quantities to define the triangle (e.g., the two short sides $R_{1}$ and $R_{2}$ and $\theta$, the angle between them). Thermal and configurational Gaussian disorder is taken into account through six parameters

$$
M=\left(\begin{array}{ccc}
\sigma_{R_{1}}^{2} & \sigma_{R_{1} R_{2}}^{2} & \sigma_{R_{1} \theta}^{2} \\
\sigma_{R_{1} R_{2}}^{2} & \sigma_{R_{2}}^{2} & \sigma_{R_{2} \theta}^{2} \\
\sigma_{R_{1} \theta}^{2} & \sigma_{R_{2} \theta}^{2} & \sigma_{\theta}^{2}
\end{array}\right)
$$


which belong to the symmetric covariance matrix. In the case of a pair of atoms, the symmetric covariance matrix is represented by the variance $\sigma_{R}^{2}$. In a simple vibrational model for the two atoms, the DW factor in the EXAFS formula is given by $\exp \left(-2 \sigma_{R}^{2} k^{2}\right)$. For a more complete treatment of configurational averages of a general EXAFS signal, the reader is referred to reference 50.

Besides the above structural parameters, other nonstructural parameters are to be refined in the fit (although their variation is limited by theoretical considerations). One of these nonstructural parameters is $E_{0}$, which aligns the experimental energy spectrum to the theoretical one. Physically $E_{0}$ is the origin of the photoelectron kinetic energy and should be defined as the core ionization threshold (vacuum level) so that $E=\omega-E_{0}$. Even though in the theoretical treatment an internal photoelectron wavenumber $K$ is defined relative to a muffin-tin origin $\bar{V}_{0}$, this origin is energy dependent (since the $\mathrm{HL}$ potential is energy dependent) and the only reason for its existence is that the true molecular potential has been approximated by its muffin-tin counterpart. Since non-self-consistent molecular charge densities are used, $E_{0}$ can be estimated only within an uncertainty of $2-3 \mathrm{eV}$. Self-consistent calculations might provide a more accurate determination of this quantity. However, the ionization threshold $I_{c}$ is very seldom experimentally determined in current measurements of absorption spectra. Therefore, in practice, it is convenient to leave $E_{0}$ as a parameter in the fit. Another nonstructural parameter that can be varied in the fit is the many-body amplitude reduction factor $S_{0}^{2}$. The presence of $S_{0}^{2}$ is justified since intrinsic processes are not incorporated in the optical potential as described by the HL potential. The magnitude of $S_{0}^{2}$ should be related to the weight of the intrinsic processes in the absorption spectrum, which should be typically less than -0.1 . An additional source of broadening of the experimental spectra comes from the core hole width $\Gamma_{c}$ that adds to the imaginary part of the potential. The value of $\Gamma_{c}$ in the fit is usually kept fixed to some experimentally-determined value or good theoretical estimate. ${ }^{57}$ Finally, the calculated signal should be convoluted with the experimental resolution function as determined by the specific optics of the experimental system used to measure the data. ${ }^{58}$ In practice this function is modeled as a Gaussian with standard deviation $E_{r}$, which is allowed to vary in a range of $1-2 \mathrm{eV}$ around the expected value.

Standard statistical concepts can be used to estimate the error affecting the fitted values of the parameters since the data analysis is performed using raw absorption spectra. ${ }^{59,60}$ By neglecting systematic errors in the experimental data and in the theoretical calculations, the definition of the residual function given by eq 12 allows one to estimate parameter values, statistical standard deviations, and the quality of the fit. 
The expected value of this expression can be calculated in terms of the variance of the experimental and model signals. In particular, for $p=0$, the expected value is the variance of the experimental points which is usually on the order of $10^{-6}-10^{-8}$. This is the lower limit of the residual. Therefore, the quality of the fit is measured by the value of the residual. In the limit of a "perfect" simulation, the quality of the fit is on the order of the variance of the experimental data. Once the residual is near the variance of the experimental data, the statistical standard deviation of a specific structural parameter can be estimated by the increase of the residual as the parameter is varied. This kind of procedure is commonly used in multi-parametric nonlinear fitting procedures. The statistical significance of the inclusion of particular fitting parameters can be tested by using the well-known $F$-test, valid for $\chi^{2}$ distributions.

These considerations do not take into account correlations between different fitting parameters. Correlation effects can increase the standard deviation of the measured parameters. A rigorous way to account for these effects is by estimating correlation through contour plots in parameter space. ${ }^{61}$ However, the size of correlation effects can be greatly reduced by extending the number of independent points in the fitting procedure. Calculation of correlation among all the parameters is time consuming for standard data analysis. For EXAFS spectra recorded over a wide energy range and composed of a reasonable number of points, one can reasonably assume that correlations are within $3 \sigma$ of the estimated standard deviation $(\sigma)$. Error bars are estimated as three times the statistical standard deviation, an assumption that tends to overestimate the error. Usually the statistical errors determined are quite small. Systematic errors in the experimental data collection and the intrinsic limitation of the theory (arising mainly from the approximations) give rise to errors that can be much larger than the statistical ones. When GNXAS is applied to a particular class of unknown systems, the best indication would be the variance between GNXAS results on a number of similar structures for which crystallographic results are known. A thorough study of the effects of the approximations on the derived structural data is currently under way. According to comparisons in fits to known, less complex structures, theoretical cross sections are quite accurate for the determination of distances and angles (on the order of $0.01 \AA$ for bond distances, around $1^{\circ}$ for bond angles) and are less accurate in the determination of covariance matrices (errors up to $10-20 \%$ for bond variances $\sigma_{R}^{2}$ have been observed). These limits are explored further in the applications described below for much more complex multishell transition metal complexes. 


\subsubsection{The GNXAS Program Set}

The GNXAS package consists of five independent subprograms, each performing. a specific task in the general layout of the method described above. Briefly, in the order of application, the CRYMOL subprogram (a) generates a cluster of sufficient size to count all the two-, three-, and four-atom configurations associated with any nonequivalent photoabsorber up to a given cutoff with the correct degeneracy, so that one can define all the SS and MS paths involving up to four atoms and (b) selects the various types of atoms differing in atomic number, types of neighbors, and distances within a given tolerance to build appropriate miniclusters to be used in the construction of the overlapped charge density to obtain the potential.

The PHAGEN subprogram takes the minicluster generated by CRYMOL, defines muffin-tin radii according to Norman's criterion, ${ }^{62}$ and uses the Mattheiss prescription to overlap self-consistent atomic charge densities to construct the cluster charge density. In order to model the charge relaxation around the core hole and to mimic the screening of the excited photoelectron, the self-consistent charge density of the photoabsorbing atom with one core hole and one electron added to the first nonoccupied valence state is used. On the basis of the cluster charge density obtained, the Coulomb and the HL exchange and correlation potential are generated, the latter being recalculated at each new energy point. Finally, the radial Schrödinger equation is solved with the complex potential and the $t$ atomic matrix elements calculated on the basis of eq A13 in the Appendix in reference 25 for any nonequivalent atom in the cluster.

The GNPEAK subprogram accepts as input a file generated by CRYMOL specifying the type, position, and neighbors of all the atoms in the cluster and searches for all two-, three-, and four-atom local configurations around each nonequivalent photoabsorber which are associated with SS and MS contributions to the absorption coefficient. These atomic configurations are referred to as peaks of the two-atom $\left(g_{2}\right)$, three-atom $\left(g_{3}\right)$, and four-atom $\left(g_{4}\right)$ distribution functions. This information is passed to the GNXAS subprogram. The GNXAS subprogram also reads the atomic $t$ matrix file generated by PHAGEN and calculates all the $\gamma(n)$ signals relative to all the configurations calculated by GNPEAK.

Finally, the subprogram FTTHEO builds up a model absorption signal (see eq 11) composed of an appropriate background plus the oscillatory structural contribution $\chi(E)$ already calculated by GNXAS. The parameters contained in the model absorption signal are then refined during a fitting procedure that tries to minimize the difference between the calculated and experimental signals. The function minimized is given by eq 12 . Fits 
are done directly in $E$ space. A standard statistical procedure commonly used in multiparametric nonlinear fitting is implemented here to perform $\chi^{2}$ and $F$-tests in order to answer typical questions arising in model refinements.

A comment is relevant on the relationship of GNXAS to other theoretical-parameter based EXAFS analysis programs. EXCURVE, FEFF5, and GNXAS all have conceptual similarities regarding the calculation of SS and MS signals. All three programs at present use a theoretical scheme consisting of the reduction of the photoabsorbtion many-body problem to a one-particle problem with a complex Hedin-Lundqvist effective potential based on a charge distribution obtained by overlapping atomic charge densities following the Mattheiss prescription ${ }^{40}$ in a muffin-tin approximation. The calculated signals are therefore in general very similar, with the differences arising from the definition of the muffin-tin parameters and the different way of defining the central atom absorption cross section mentioned above.

\subsection{Applications to Iron Complexes}

\subsubsection{Sample Preparation and Data Collection}

$\mathrm{Fe}$ (acac) ${ }_{3}$ was purchased from Aldrich, $\mathrm{K}_{3} \mathrm{Fe}(\mathrm{CN})_{6}$ was purchased from J. T. Baker, and $\mathrm{Na}\left[\mathrm{Fe}\left(\mathrm{OH}_{2}\right)\right.$ EDTA] was prepared according to the published procedures. ${ }^{63}$ The crystalline samples were ground into a fine powder and diluted with BN. The BN powder mixture was pressed into a $1 \mathrm{~mm}$ thick slotted $\mathrm{Al}$ spacer and sealed with Mylar tape windows. The X-ray absorption spectra were recorded at the Stanford Synchrotron Radiation Laboratory on unfocused beamlines 7-3 and 4-3 during dedicated conditions ( 3 $\mathrm{GeV}, 25-90 \mathrm{~mA}$ ). The radiation was monochromatized using a $\mathrm{Si}(220)$ double-crystal monochromator detuned to $50 \%$ at $7998 \mathrm{eV}$ to minimize harmonic contamination. The $\mathrm{X}$-ray beam was defined to be $1 \mathrm{~mm}$ vertically by pre-monochromator slits. An Oxford Instruments continuous-flow liquid helium CF1208 cryostat was used to maintain a constant temperature of $10 \mathrm{~K}$. Data were measured in transmission mode with three nitrogen-filled ionization chambers, using an Fe foil between the second and third ionization chambers for internal energy calibration. The spectra were calibrated by assigning the first inflection point of the Fe foil spectrum to $7111.2 \mathrm{eV}$. The data represent an average of two to four scans. The effects of a quartet monochromator glitch were removed from the averaged data by four single point replacements at around $k=$ $11.8,12: 1,12.3$, and $12.6 \AA^{-1}$. 


\subsubsection{GNXAS Data Analysis}

The following approach was used for the GNXAS analysis of the three iron complexes. The atomic coordinates were input into CRYMOL, and the appropriate cluster was determined. Phase shifts were calculated in PHAGEN using the standard muffin-tin approximation with the entire cluster and up to an energy limit of 70 Ry $(950 \mathrm{eV})$ above the $\mathrm{Fe} \mathrm{K}$ edge. The muffin-tin radii were chosen by scaling Norman radii of the cluster atoms by a factor of about 0.8 in such a way as to match the nearest neighbor distance. The GNPEAK program was then run to identify and select the relevant peaks in the $g_{n}$ distribution functions and associate each atom with the appropriate phase shifts. GNXAS calculated the various signals from each $g_{n}$ contribution. Least-square fits were performed in the subprogram FITHEO on the averaged, energy-calibrated, raw absorption data without prior background subtraction or Fourier filtering. The minimization program uses the MINUIT subroutine of the CERN Library. The residual function is minimized by refining parameters for which specified intervals can be input. The nonstructural parameters, $E_{0}, S_{0}{ }^{2}, \Gamma_{c}$, and $E_{\mathrm{r}}$ were, as usual, calibrated on model compounds and allowed to vary within narrow intervals, observing if they refined to one of the hard limits. ${ }^{57,58}$ The structural parameters varied in the refinements were the distance and the associated bond variance $\sigma_{R}^{2}$ for each two-atom configuration and the distances, the angle, and the covariance matrix elements for the three-atom configurations (unless stated otherwise). Distances and angles were allowed to vary within a preset range, typically $\pm 0.05 \AA$ and $\pm 5^{\circ}$, respectively. Bond and angle variances and the off-diagonal covariance elements were also allowed to vary in restricted ranges: $\pm 0.005 \AA^{2}, \pm 50$ (degrees) ${ }^{2}$ and \pm 0.5 , respectively. The results were carefully monitored to ensure that all parameters refined inside the allowed range. The coordination numbers were kept fixed to known values.

\subsubsection{Results and Discussion}

2.3.3.1. $\mathrm{Fe}(\mathrm{acac})_{3}$. The GNXAS method was applied to $\mathrm{Fe}(\mathrm{acac})_{3} \mathrm{Fe} \mathrm{K}$-edge EXAFS data to determine the feasibility of studying the metrical details of inorganic compounds. The ability of GNXAS to provide an accurate description of the MS contributions in the EXAFS data of a compound with a non-collinear arrangement of atoms and the reliability of the structural parameters obtained from GNXAS were evaluated. The GNXAS set of programs were used to generate theoretical EXAFS signals corresponding to both two-atom- and three-atom scattering processes. The 
structure of $\mathrm{Fe}(\mathrm{acac})_{3}$ has been determined by $\mathrm{X}$-ray diffraction. ${ }^{64}$ The iron atom is in an octahedral arrangement (Figure 2.2) surrounded by six oxygen atoms at $1.99 \AA$, six carbons $\left(C_{1}\right)$ at $2.95 \AA$, three carbons $\left(C_{2}\right)$ at $3.34 \AA$, and six carbons $\left(C_{3}\right)$ at $4.32 \AA$ (where the ranges of the distances are given in Table 2.1). The atomic coordinates of $\mathrm{Fe}(\mathrm{acac})_{3}$ were entered into $\mathrm{CRYMOL}$ and the appropriate cluster (neglecting the hydrogens) was determined (shown in Figure 2.2). In this case, the cut-off distance was $4.4 \AA$ since the longest Fe- $C$ distance is $4.32 \AA$ and the FT showed no significant features beyond this value. The reduced Norman sphere radii used to calculate the phase shifts were $1.13 \AA$ for $\mathrm{Fe}, 0.873 \AA$ for $\mathrm{O}$, and $0.899 \AA$ for $\mathrm{C}$. The prototypical two-atom and three-atom configurations ( $g_{2}$ and $g_{3}$ peaks) were identified in the cluster up to $4.4 \AA$ and averaged with a frequency tolerance of $0.1 \AA$. The resultant coordinates of the atomic configurations were used to calculate the various signals associated with two-atom and three-atom contributions. The signal associated with four two-atom configurations were generated: $\mathrm{Fe}-\mathrm{O}, \mathrm{Fe}-\mathrm{C}_{1}, \mathrm{Fe}-\mathrm{C}_{2}$, and $\mathrm{Fe}-\mathrm{C}_{3}$. Five signals associated with three-atom configurations were calculated: $\mathrm{Fe}-\mathrm{O}-\mathrm{C}_{1}, \mathrm{O}-\mathrm{Fe}-\mathrm{O}\left(90^{\circ}\right), \mathrm{O}-\mathrm{Fe}-\mathrm{O}\left(180^{\circ}\right), \mathrm{Fe}-\mathrm{O}-\mathrm{C}_{2}$, and $\mathrm{Fe}-\mathrm{O}-\mathrm{C}_{3}$ (where the three-atom configuration is defined by the two short distances and the intervening angle). The appropriate crystallographic distances and angles for the above mentioned two- and three-atom configurations are listed in Table 2.1. The fitting program used at the final step of the data analysis built the theoretical absorption spectrum by summing all the two-atom and three-atom contributions. The final spline was in three segments of order $4,4,4$ with defining energy points of $7147,7269,7577$, and $7999 \mathrm{eV}$. The least-squares fits were done with $k^{3}$ weighting over the $k$ range of 2.4 $15.1 \AA^{-1}$.

To analyze the MS effects in $\mathrm{Fe}(\mathrm{acac})_{3}$, signals from two-atom configurations were systematically replaced with the appropriate three-atom contributions, while monitoring the residual in the EXAFS and the components in the FT. For these fits all the distances and angles were fixed to the crystallographic values while permitting the associated variances and nonstructural parameters to vary. Fit $\mathrm{A}$ (Figure 2.3A) contains only two-atom contributions from $\mathrm{Fe}-\mathrm{O}, \mathrm{Fe}-\mathrm{C}_{1}, \mathrm{Fe}-\mathrm{C}_{2}$, and $\mathrm{Fe}-\mathrm{C}_{3}$. The $R$ value for Fit $\mathrm{A}$ is $0.181 \times 10^{-4}$, and the EXAFS residual clearly contains high frequency components. In Fit A, the first peak of the FT of the data is fit fairly well by the FT of the theoretical signal, but the intensity of the theoretical signal does not match the experimental intensity above $2 \AA$. The second fit, Fit $B$, includes three-atom signals from $\mathrm{Fe}-\mathrm{O}-\mathrm{C}_{1}, \mathrm{O}-\mathrm{Fe}-\mathrm{O}$ $\left(90^{\circ}\right)$, and $\mathrm{O}-\mathrm{Fe}-\mathrm{O}\left(180^{\circ}\right)$ while the second and third shells of carbon are still treated as two-atom configurations (Figure 2.3B). The $R$ value of Fit $\mathrm{B}$ is $0.635 \times 10^{-5}$, almost a factor of three better than the $R$ value in Fit $\mathrm{A}$, indicating the importance of treating 


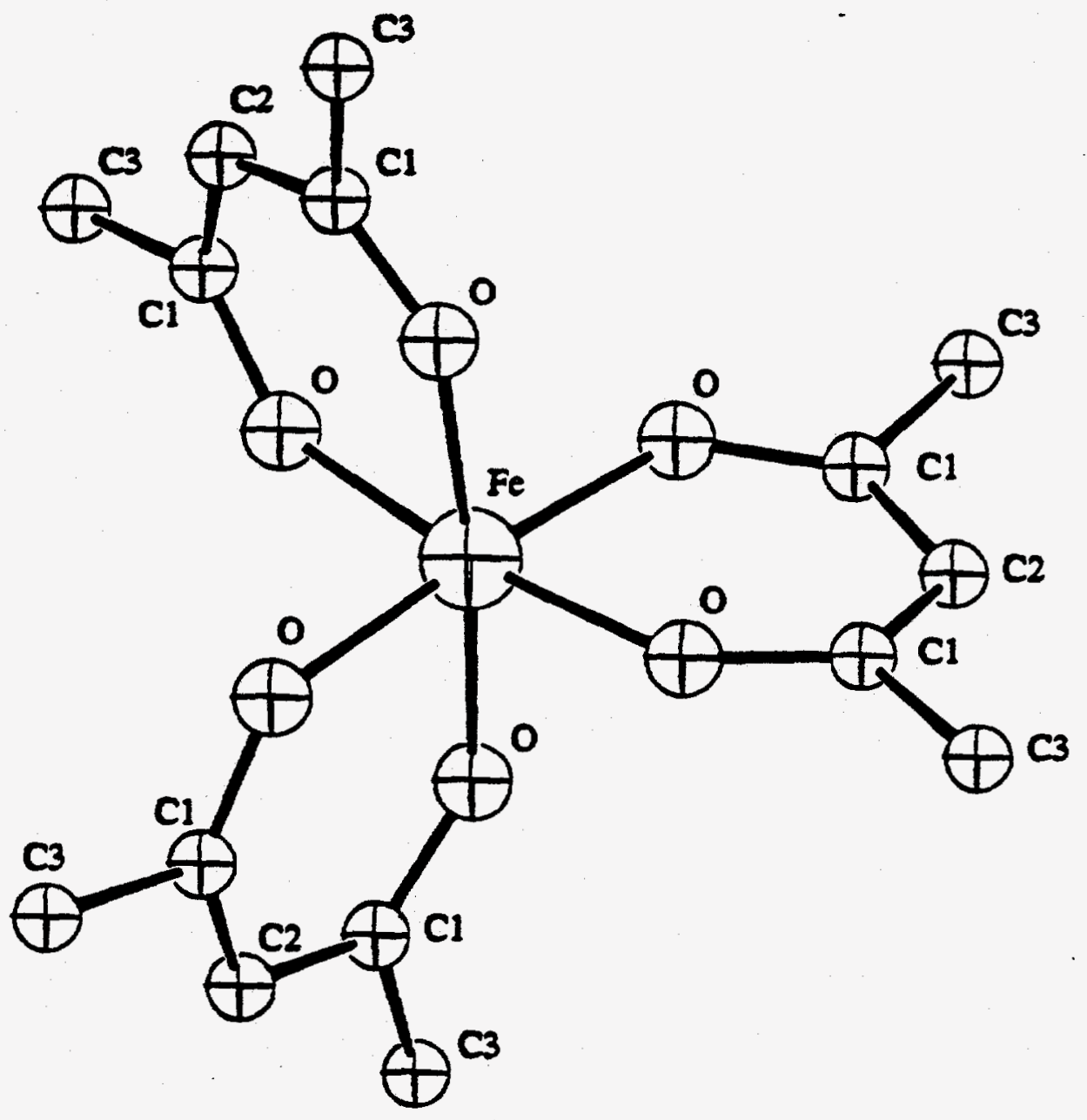

Figure 2.2. Molecular structure of $\mathrm{Fe}(\mathrm{acac})_{3}$ with atom designations as used in the text. 
Table 2.1. Comparison of $\mathrm{Fe}(\mathrm{acac})_{3}$ GNXAS Distance and Angle Fitting Results to Crystallographic Values.

\begin{tabular}{|c|c|c|c|}
\hline $\begin{array}{l}\text { structural feature } \\
\text { (\# of configur- } \\
\text { ations in complex) }\end{array}$ & $\begin{array}{l}\text { GNXAS } \\
\text { distances/angles }\end{array}$ & $\begin{array}{l}\text { GNXAS } \\
\text { bond variance }\left(\sigma_{R}^{2}\right) / \\
\text { angle variance }\left(\sigma_{\theta}^{2}\right)^{a}\end{array}$ & $\begin{array}{l}\text { crystallographic } \\
\text { distances/angles } \\
\text { average [range] }\end{array}$ \\
\hline $\mathrm{Fe}-\mathrm{O}(6)$ & $1.99 \AA$ & 0.002 & $1.99 \AA[1.99-2.00]$ \\
\hline $\mathrm{Fe}-\mathrm{C}_{1}(6)$ & $(2.98 \AA)^{*}$ & -- & $2.95 \AA[2.93-2.97]$ \\
\hline $\mathrm{Fe}-\mathrm{C}_{2}(3)$ & $(3.37 \AA)^{*}$ & $\ldots$ & $3.34 \AA[3.29-3.43]$ \\
\hline $\mathrm{Fe}-\mathrm{C}_{3}(6)$ & $(4.34 \AA)^{*}$ & - & $4.32 \AA[4.30-4.33]$ \\
\hline $\mathrm{O}-\mathrm{C}_{1}(6)$ & $1.22 \AA$ & 0.001 & $1.26 \AA[1: 24-1.28]$ \\
\hline $\mathrm{O}-\mathrm{C}_{2}(6)$ & $2.38 \AA$ & 0.006 & $2.34 \AA[2.31-2.39]$ \\
\hline $\mathrm{O}-\mathrm{C}_{3}(6)$ & $2.38 \AA$ & 0.008 & $2.36 \AA[2.34-2.38]$ \\
\hline $\mathrm{Fe}-\mathrm{O}-\mathrm{C}_{1}(6)$ & $134^{\circ}$ & $1 \times 10^{1}$ & $128^{\circ}[128-130]$ \\
\hline $\mathrm{Fe}-\mathrm{O}-\mathrm{C}_{2}(6)$ & $101^{\circ}$ & $4 \times 10^{1}$ & $101^{\circ}[99-103]$ \\
\hline $\mathrm{Fe}-\mathrm{O}-\mathrm{C}_{3}(6)$ & $165^{\circ}$ & $3 \times 10^{1}$ & $165^{\circ}[164-166]$ \\
\hline $\mathrm{O}-\mathrm{Fe}-\mathrm{O}(6)$ & $89^{\circ}$ & $6 \times 10^{1}$ & $91^{\circ}[87-94]$ \\
\hline $\mathrm{O}-\mathrm{Fe}-\mathrm{O}(3)$ & $175^{\circ}$ & $3 \times 10^{1}$ & $175^{\circ}[174-176]$ \\
\hline
\end{tabular}


Figure 2.3. Comparison of the theoretical and experimental signals of the $k^{3}$-weighted EXAFS data and the FT of Fit A, Fit B, Fit C, and Fit D of Fe(acac)3. The top portion of the figu e contains the non-phase-shift-corrected FT of the $k^{3}$-weighted EXAFS data of the experimental data $(-$ ) and that of the total theoretical signal (--). Also shown is the FT of the residual (...). The lower portion of the figure presents the EXAFS signals for the individual contributions. The total theoretical signal is also shown $(-)$ and compared with the experimental data (...) with the residual being the difference between the experimental and the theoretical EXAFS. (The ordinate scale is 10 between two consecutive tick marks.) Fit A contains only $\gamma(2)$ contributions. The residual in Fit A contains many high frequency components, and the fit does not match the data between 2 $\AA$ and $4 \AA$ in the FT. Fit B includes contributions from Fe-O- $C_{1}$ and $90^{\circ}$ and $180^{\circ} \mathrm{O}-\mathrm{Fe}-$ $O$ configurations. Notice the reduction of the residual in the low $k$-region of the EXAFS and the improvement of the fit to the FT between 2 and $3 \AA$. Fit $C$ includes contributions from $\mathrm{Fe}-\mathrm{O}-\mathrm{C}_{2}$. There is no noticeable improvement in the fit to the data since the Fe-O$\mathrm{C}_{2}$ signal is weak. Fit $\mathrm{D}$ includes contributions from $\mathrm{Fe}-\mathrm{O}-\mathrm{C}_{3}$. Note the considerable improvement in the fit to the data between 3.5 and $4.1 \AA$ in the FT. 


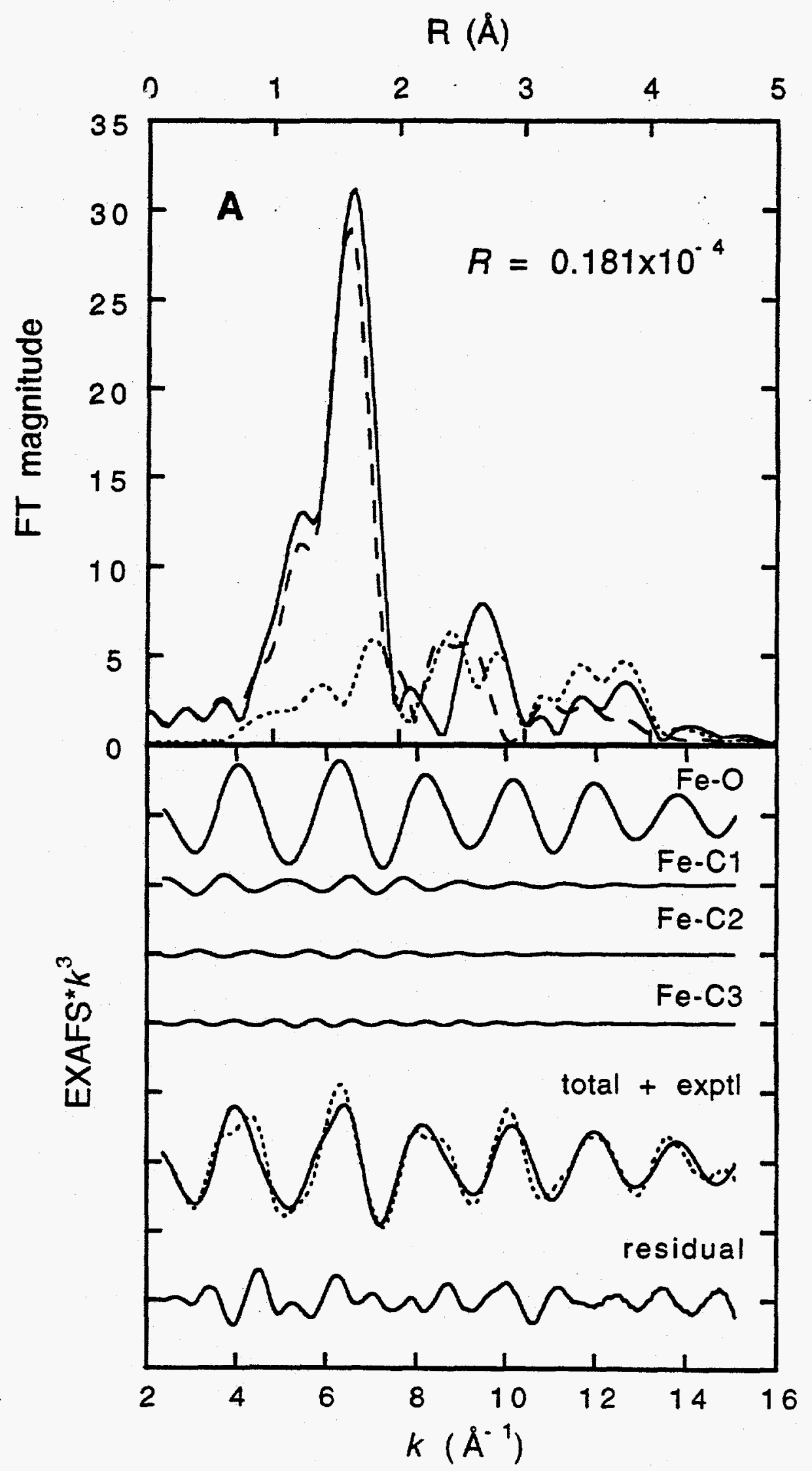




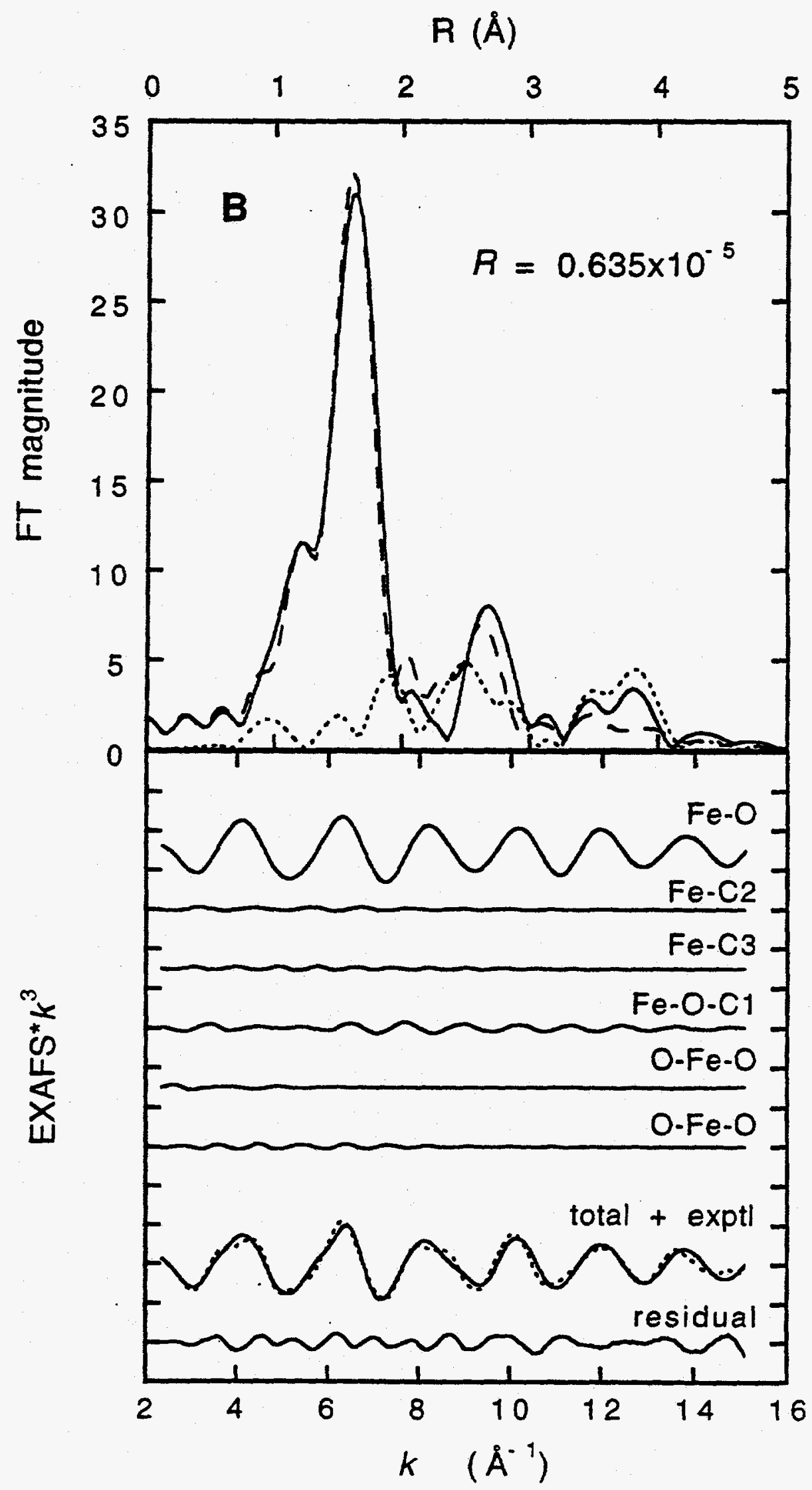




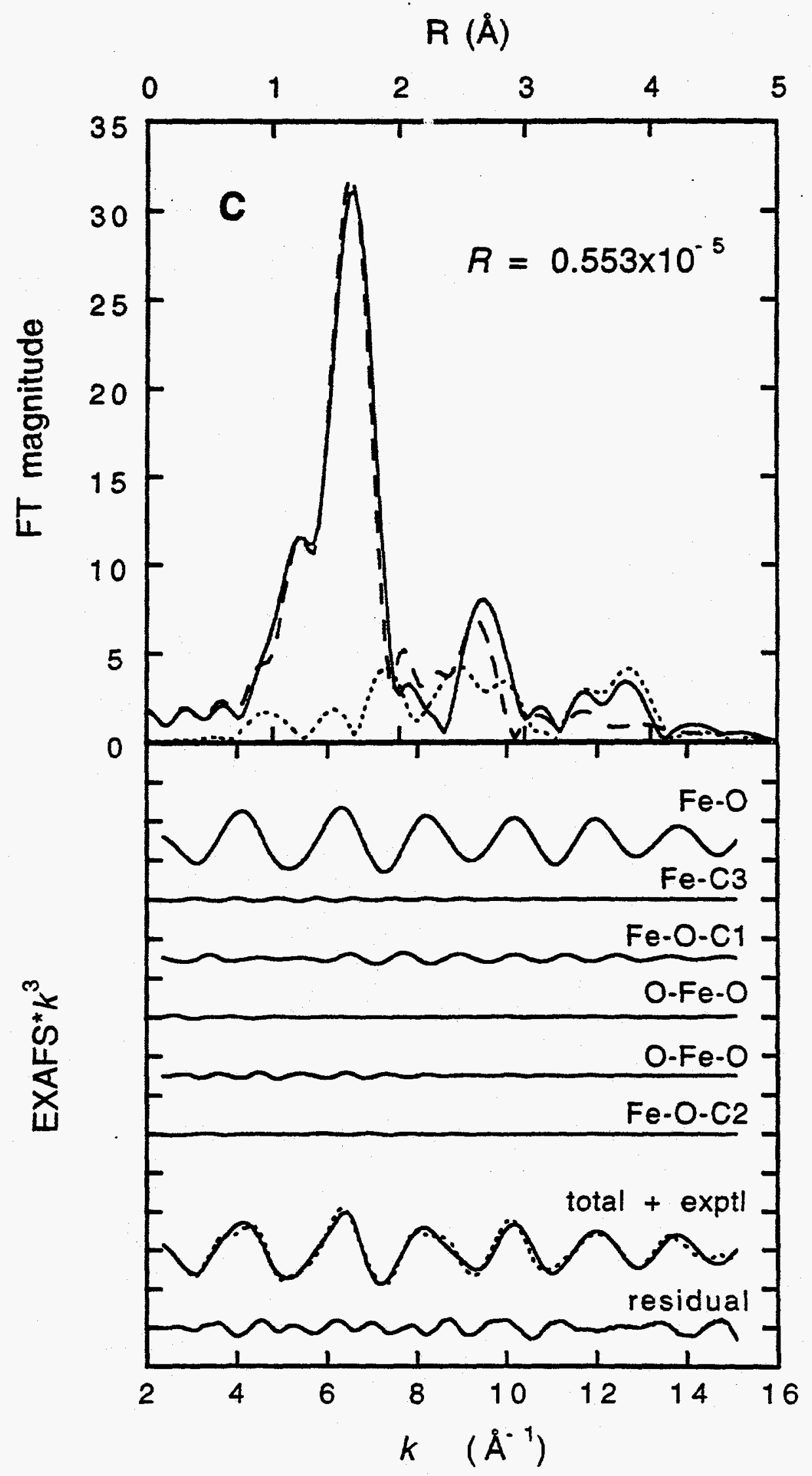




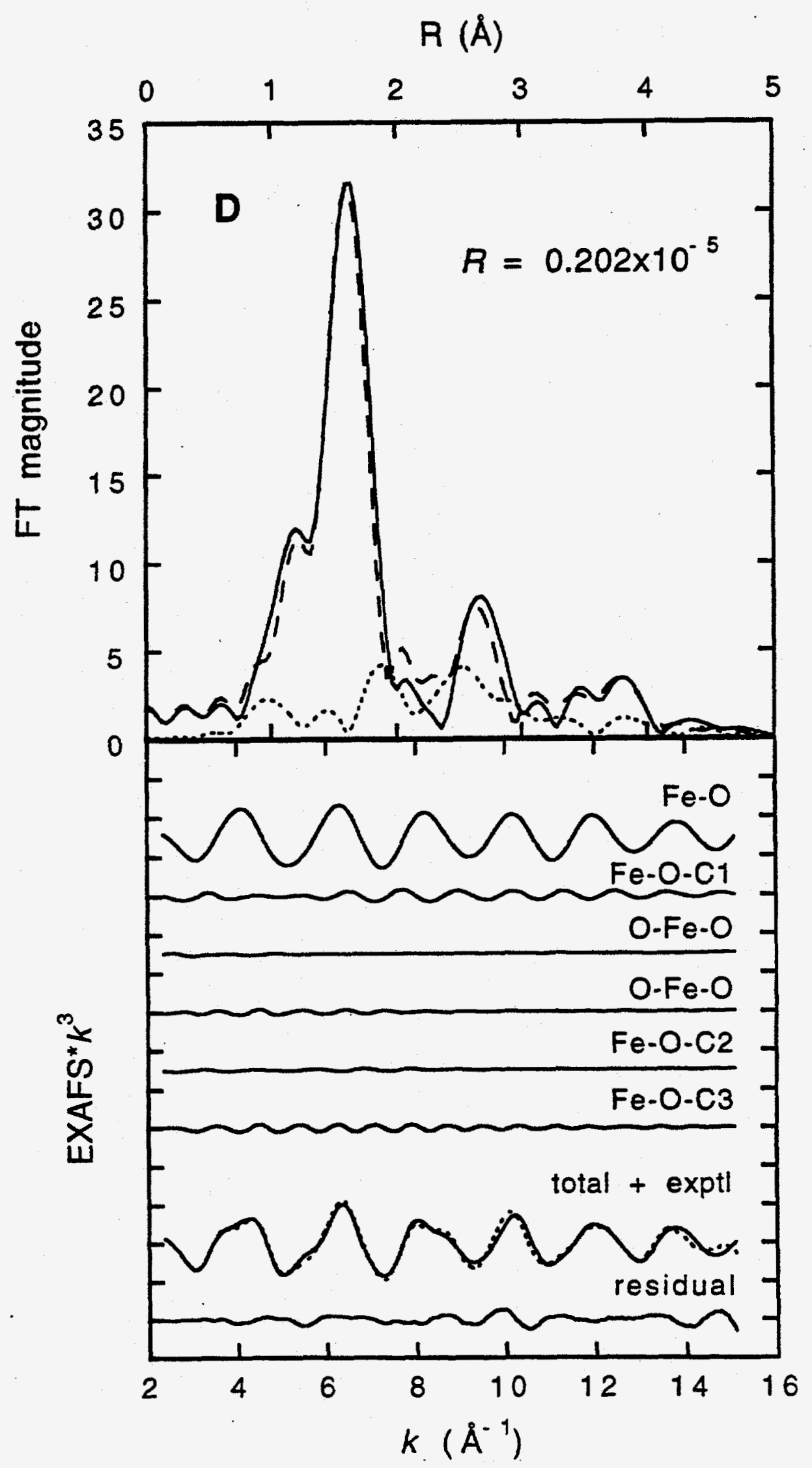


Fe-O- $\mathrm{C}_{1}$ as a three-atom configuration. Also notice the significant improvement in the fit to the low- $k$ region of the EXAFS, where the $\mathrm{Fe}-\mathrm{C}_{1} \gamma(2)$ signal and the $\mathrm{Fe}-\mathrm{O}-\mathrm{C}_{1} \gamma^{(3)}$ signals differ the most (Figure 2.4). The contributions from the $90^{\circ}$ and $180^{\circ} \mathrm{O}-\mathrm{Fe}-\mathrm{O}$ configurations are relatively small as seen by comparison of their amplitudes with the total Fe-O- $\mathrm{C}_{1}$ signal. The EXAFS residual in Fit B still contains some high frequency components, but the FT of the theoretical signal of Fit B begins to match the second peak in the FT of the experimental data at $\sim 2.6 \AA$. In Fit $C$ the second shell of carbons is treated with a three-atom signal. The $R$ value of Fit $C$ is $0.553 \times 10^{-5}$. Fit $C$ is not a significant improvement over Fit $B$ because the signal generated by the Fe-O- $\mathrm{C}_{2}$ configuration is negligible (Figure 2.3C). Both the EXAFS data and the FT look very similar to those in Fit B. In Fit D a signal from $\mathrm{Fe}-\mathrm{O}-\mathrm{C}_{3}$ is included. The signal from Fe-O- $C_{3}$ is fairly strong, and the $R$ value of Fit $D$ decreased to $0.202 \times 10^{-5}$. All of the distinguishable regular high frequency components have been removed in the Fit $D$ residual, and the FT of theoretical signals is in very good agreement with the experimental FT up to $\sim 4 \AA$ (Figure 2.3D). Notice that even though the Fe- $\mathrm{C}_{3}$ distance is longer than $4 \AA$ the $\mathrm{Fe}-\mathrm{O}-\mathrm{C}_{3}$ signal is significant. This enhancement is in part due to a focusing effect since the $\mathrm{Fe}-\mathrm{O}-\mathrm{C}_{3}$ angle is relatively large $\left(165^{\circ}\right)$. Figure 2.5 displays the individual contributions of each signal in the FT. The dominant feature in the FT is the $\mathrm{Fe}-\mathrm{O}$ signal with the $\mathrm{Fe}-\mathrm{O}-\mathrm{C}_{1}$ and $\mathrm{O}-\mathrm{Fe}-\mathrm{O}\left(180^{\circ}\right)$ and $\mathrm{Fe}-\mathrm{O}-\mathrm{C}_{3}$ signals contributing at higher $R$ values.

The ability of the GNXAS method to accurately determine bond distances and angles was also evaluated. Fits were done by varying the distances and angles and applying constraints to keep them within $5 \%$ of the crystallographic values. The initial covariance matrix elements were obtained from Fit $D$ and were allowed to vary within $10 \%$ of those values. A comparison of the crystallographic values with the distances and angles obtained from the best fit to the data is presented in Table 2.1. The $R$ value of this fit was $0.142 \times 10^{-5}$ (slightly better than Fit $D$ ) and the bond distances and angles were quite close to the crystallographic values. The fit to the experimental data looks very similar to Fit $D$ with a slight improvement of the fit in the Fourier transformed data between 2.5 and $3.0 \AA$. The bond distances and angles obtained from the GNXAS fit to the experimental data are within the range of the crystallographic values as given in Table 2.1 with a few exceptions. The values obtained from the $\mathrm{Fe}-\mathrm{O}-\mathrm{C}_{1}$ signal deviate from the range of crystallographic values by $0.02 \AA$ for the $\mathrm{O}-\mathrm{C}_{1}$ distance and $4^{\circ}$ for the $\mathrm{Fe}-\mathrm{O}-\mathrm{C}_{1}$ angle, causing the $\mathrm{Fe}-\mathrm{C}_{1}$ distance to deviate from the crystallographic value by $0.03 \AA$.

The level of accuracy in this fit indicates that the theory is quite reliable in reproducing the phase of the experimental signal, as has been confirmed by previous 


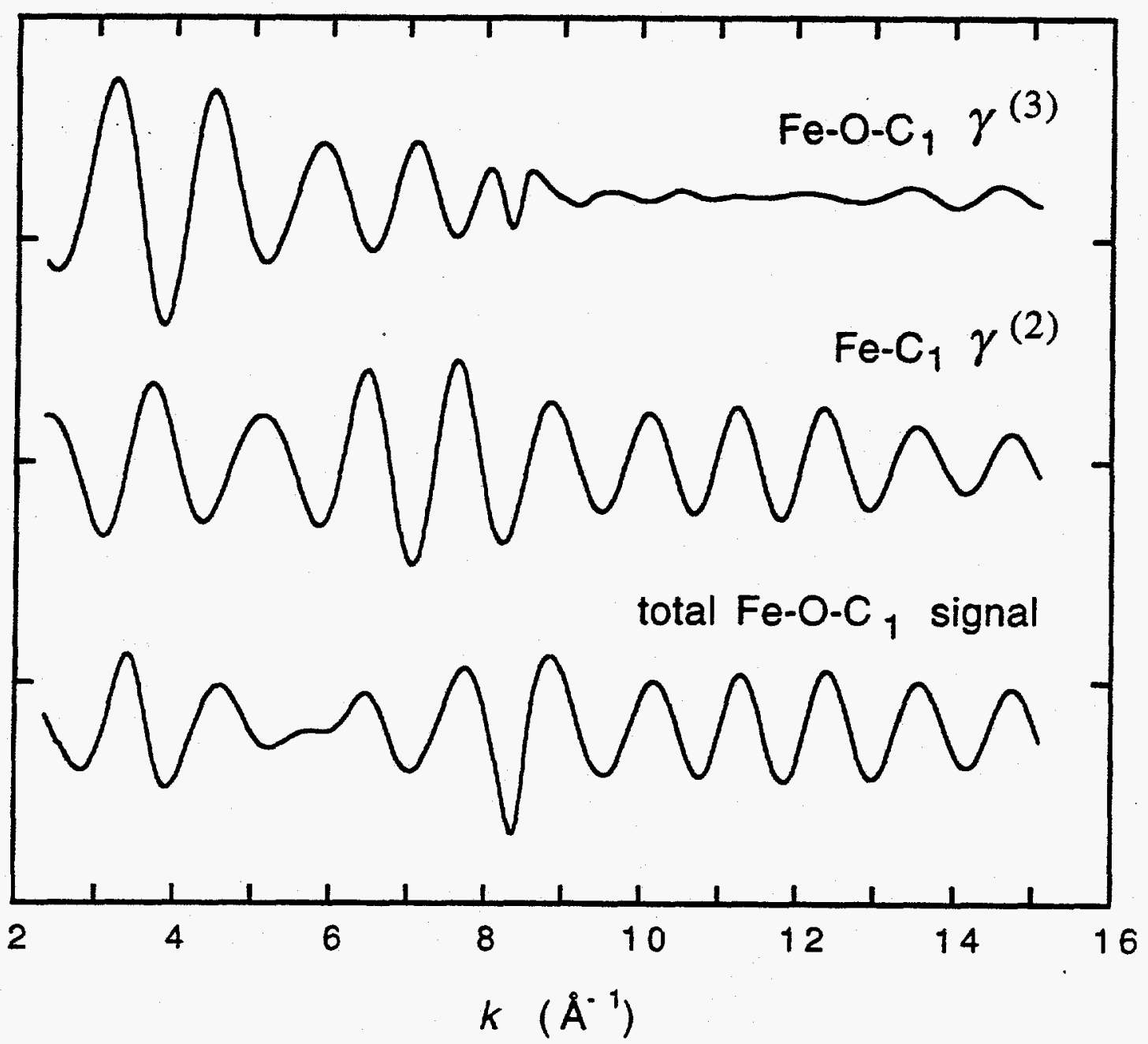

Figure 2.4. Comparison of the $\mathrm{Fe}-\mathrm{C}_{1} \gamma^{(2)}$ EXAFS signal to the Fe-O-C $\mathrm{C}_{1} \gamma^{(3)}$ signal and the total Fe-O- $C_{1}$ signal. The anomalous behavior of the Fe-O-C $C_{1} \gamma(3)$ signal near $8 \AA^{-1}$ is due to the existence of a deep minimum in the amplitude function of the three-atom signal. Note that the Fe-O- $\mathrm{C}_{1} \gamma(3)$ signal is out of phase with the $\mathrm{Fe}-\mathrm{C}_{1} \gamma(2)$ signal between 2.5 and $7.5 \AA^{-1}$. The low-k EXAFS can only be properly accounted for when the first shell of carbons $\left(C_{1}\right)$ is treated in a three-atom configuration (Fe-O- $C_{1}$ ), including both the SS and MS contributions. (The ordinate scale is 5 between two consecutive tick marks.) 


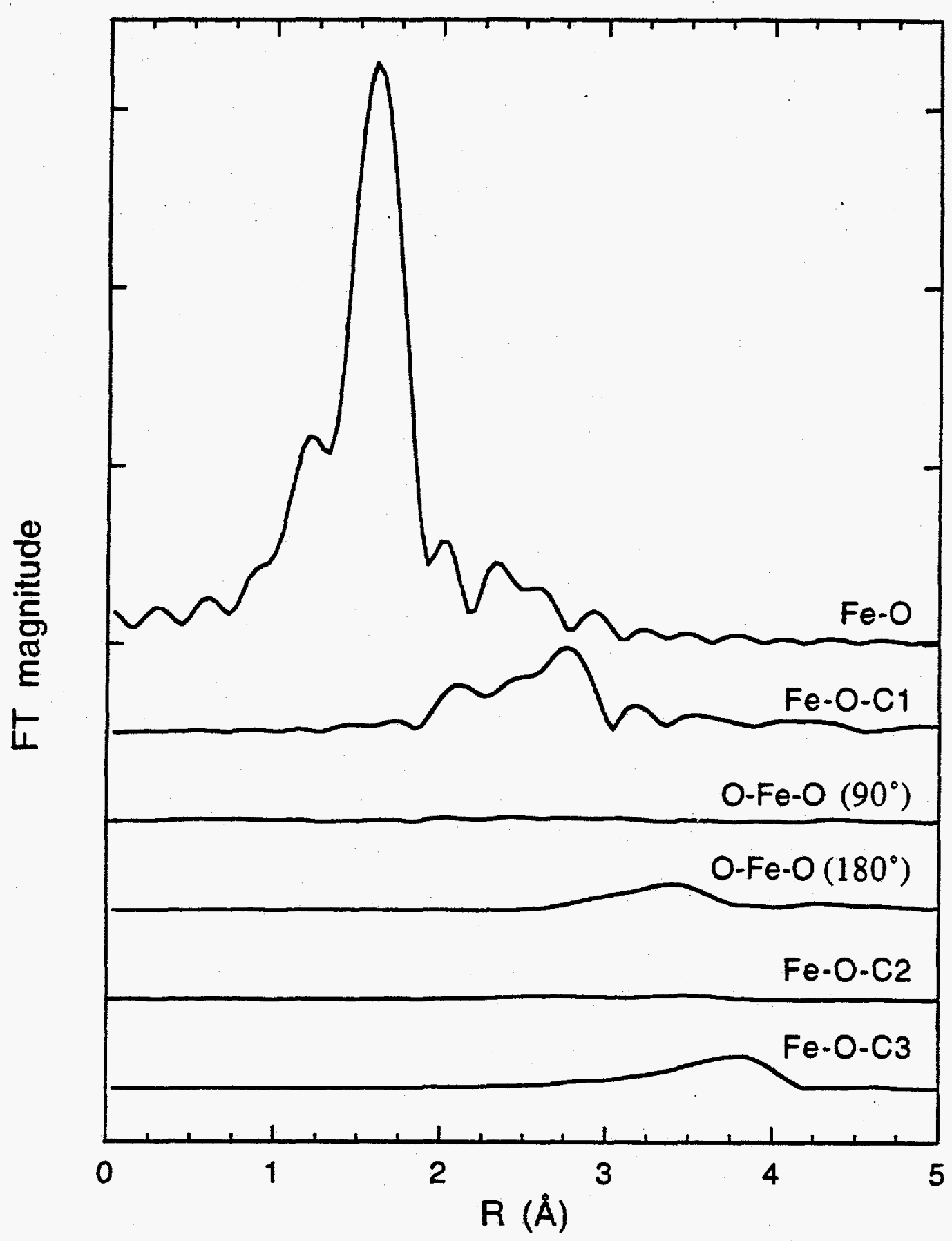

Figure 2.5. FT of the EXAFS signals of $\mathrm{Fe}(\mathrm{acac})_{3}$ for the individual contributions shown in Figure 2.3D. This display is a useful way to determine which signals contribute in which regions and shows clearly the significant and complex contributions from $\mathrm{Fe}-\mathrm{O}-\mathrm{C}_{1}$ and $\mathrm{Fe}-\mathrm{O}-\mathrm{C}_{3}$. (The ordinate scale is 10 between two consecutive tick marks.) 
experiments. $^{21-25}$ Over a large number of fits varying the nonstructural parameters and spline and differing the number of contributions, the Fe-O distance varied by less than $0.01 \AA$ and the Fe-O-C angles by less than $1^{\circ}$, while the $\mathrm{O}-\mathrm{C}_{1}$ distance varied up to $0.04 \AA$, the $\mathrm{O}-\mathrm{C}_{2}$ distance $0.1 \AA$, and the $\mathrm{O}-\mathrm{C}_{3}$ distance $0.02 \AA$. The stronger the signal the smaller the variation in the distance/angle between fits. The amplitude of the signal is determined with slightly less accuracy because amplitudes are affected in the fits by several variables which can be strongly correlated $\left(S_{0}^{2}, E_{I}, \Gamma_{\mathrm{c}}\right.$, bond variances, and the covariance matrix elements). However, the variations of the above-mentioned parameters were confined in narrow ranges determined by physical constraints. In the $\mathrm{Fe}(\mathrm{acac})_{3}$ case, values for the bond variances are not well known since a theoretical approximation of the molecular vibrations is not available. However, fitted values followed reasonable trends with the $\mathrm{Fe}-\mathrm{O}$ distance having the lowest variance of all the $\gamma^{(2)}$ contributions in Fit $A$. In a comparison of the variances for the $\mathrm{O}-\mathrm{C}$ distances, $\mathrm{O}-\mathrm{C}_{1}$ had the lowest variance, $\mathrm{O}-\mathrm{C}_{2}$ had a much higher variance with the static disorder in the $\mathrm{O}-\mathrm{C}_{2}$ distances being over twice that of the $O-C_{1}$ distances, and $O-C_{3}$ (with $C_{3}$ being the carbon in the methyl groups) had the highest mean square deviation.

The EXAFS signal generated by GNXAS matches closely that of the experimental signal of $\mathrm{Fe}(\mathrm{acac})_{3}$ when all the MS contributions were added into the theoretical signal. A comparison of the FT in Figure 2.3A, where only the two-atom signals were taken into account, with the FT in Figure 2.3D, where the three-atom signals were also used, shows the importance of including MS contributions from the three shells of carbon atoms. Not only does the GNXAS theoretical fit match the experimental data but the bond distances and angles in the final fit were within $4 \%$ of the crystallographic average values with the majority of the bond distances and angles being within the range of the crystallographic values (see.Table 2.1). The number of parameters used in the fit is 18 (two parameters for each bond, the length and its variance, and two for each angle, the angle and its variance, since in this application the off-diagonal elements of the correlation matrix have been fixed to zero) plus $3\left(S_{0}{ }^{2}, E_{\mathrm{T}}\right.$, and $\left.\Gamma_{c}\right)$, for a total of 21 . This number can be compared with the number of independent data points $N_{l}=(2 \delta k \delta R \pi)+2$ $=36$, for $\delta k=12 \AA^{-1}$ and $\delta R=4.5 \AA$. Notice that the the number of neighbors has been fixed and that the two-body parameters are also associated with three-body signals. In this manner, the same parameters can be associated with both a strong and a weak signal. The independent data-to-parameter ratio determined above shows that the fit is overdetermined by nearly a factor of two, pointing to the reliability of the fit. A point worth mentioning is that the three-atom MS signal from $\mathrm{Fe}-\mathrm{O}-\mathrm{C}_{1}$ is out of phase with the Fe- $C_{1}$ signal between 2.5 and $7.5 \AA^{-1}$ (the Fe-O- $C_{1}$ signal dies off after $7.5 \AA^{-1}$ ) (this can 
be seen in Figure 2.4). This has implications when using SS Fe-C second-shell parameters in empirical fits. One can only assume phase and amplitude transferability in the second shell if the two distances and the angle of the model are very close to the comparable distances and angle in the unknown. Therefore, the GNXAS method is advantageous in that it can account for the strength and the complexity of MS contributions in an inorganic compound with a noncollinear arrangement of atoms. Once the MS signals are modeled correctly, reliable bond distances and angles can be obtained not only from the first-shell but also from second- and third- shell neighbors without dependence on obtaining suitable models from which to extract such empirical parameters.

2.3.3.2. $\mathrm{Na}\left[\mathrm{Fe}\left(\mathrm{OH}_{2}\right) \mathrm{EDTA}\right]$. Similar methodology was applied to $\mathrm{Na}\left[\mathrm{Fe}\left(\mathrm{OH}_{2}\right)\right.$ EDTA] Fe K-edge EXAFS data to evaluate the ability of GNXAS to theoretically analyze the EXAFS data of a lower symmetry coordination complex with mixed ligation. The structure of $\left[\mathrm{Fe}\left(\mathrm{H}_{2} \mathrm{O}\right) \text { EDTA }\right]^{-}$(shown in Figure 2.6) is not as well-ordered as that of $\mathrm{Fe}(\mathrm{acac})_{3}$ and more like the structures of metalloenzymes for which it is expected that the GNXAS methodology will be particularly useful. The crystal structure of $\mathrm{Li}\left[\mathrm{Fe}\left(\mathrm{OH}_{2}\right)\right.$ EDTA] $\cdot 2 \mathrm{H}_{2} \mathrm{O}$ was previously reported. ${ }^{63}$ The iron atom is surrounded by five oxygens and two nitrogens in the first shell with two $\mathrm{O}_{1}$ 's at $1.97 \AA$, two $\mathrm{O}_{2}$ 's at $2.11 \AA$, a water at $2.11 \AA$, and two N's at $2.32 \AA$. Each oxygen (except for the water) is bound to a carbon which is bound to another oxygen. Each nitrogen is bound to three carbons that link the hexadentate ligand. The crystallographic values of $\mathrm{Li}\left[\mathrm{Fe}\left(\mathrm{OH}_{2}\right)\right.$ EDTA] $2 \mathrm{H}_{2} \mathrm{O}$ were used to generate the two-atom and three-atom configurations up to $4.5 \AA$ with a frequency tolerance of $0.1 \AA$. The reduced Norman sphere radii used in the phase shift calculation were $1.17 \AA$ for $\mathrm{Fe}, 0.730 \AA$ for $\mathrm{O}$, $0.751 \AA$ for $N$, and $0.772 \AA$ for $C$. The peaks in the two-atom distribution include two short $\mathrm{Fe}-\mathrm{O}_{1}$ distances, two long $\mathrm{Fe}-\mathrm{O}_{2}$ distances, one $\mathrm{Fe}-\mathrm{OH}_{2}$ distance, two $\mathrm{Fe}-\mathrm{N}$ distances, ten $\mathrm{Fe}-\mathrm{C}$ distances between 2.83 and $3.16 \AA$, and four $\mathrm{Fe}-\mathrm{O}_{3}$ distances between 3.91 and $4.22 \AA$. There were approximately 30 unique three-atom contributions which ranged in distance from $3.04 \AA$ to $4.5 \AA$. The signals attributed to each of the two- and three-atom configurations were calculated. Due to the complexity of the structure, contributions to the fits were systematically introduced. The spline was in three segments of order $3,4,4$ with defining energy points of $7155,7250,7600$, and $7999 \mathrm{eV}$. Least-squares fits were done with $k^{3}$ weighting over the $k$ range $2.8-15.1 \AA^{-1}$.

The first-shell fit contained waves from the following two-atom configurations: $\mathrm{Fe}^{-\mathrm{O}_{1}}$ [2] at $1.97 \AA, \mathrm{Fe}-\mathrm{O}_{2}[3]$ at $2.11 \AA$, and $\mathrm{Fe}-\mathrm{N}$ [2] at $2.32 \AA$, where the number in the brackets indicates the coordination number (see Table 2.2 for the range of 


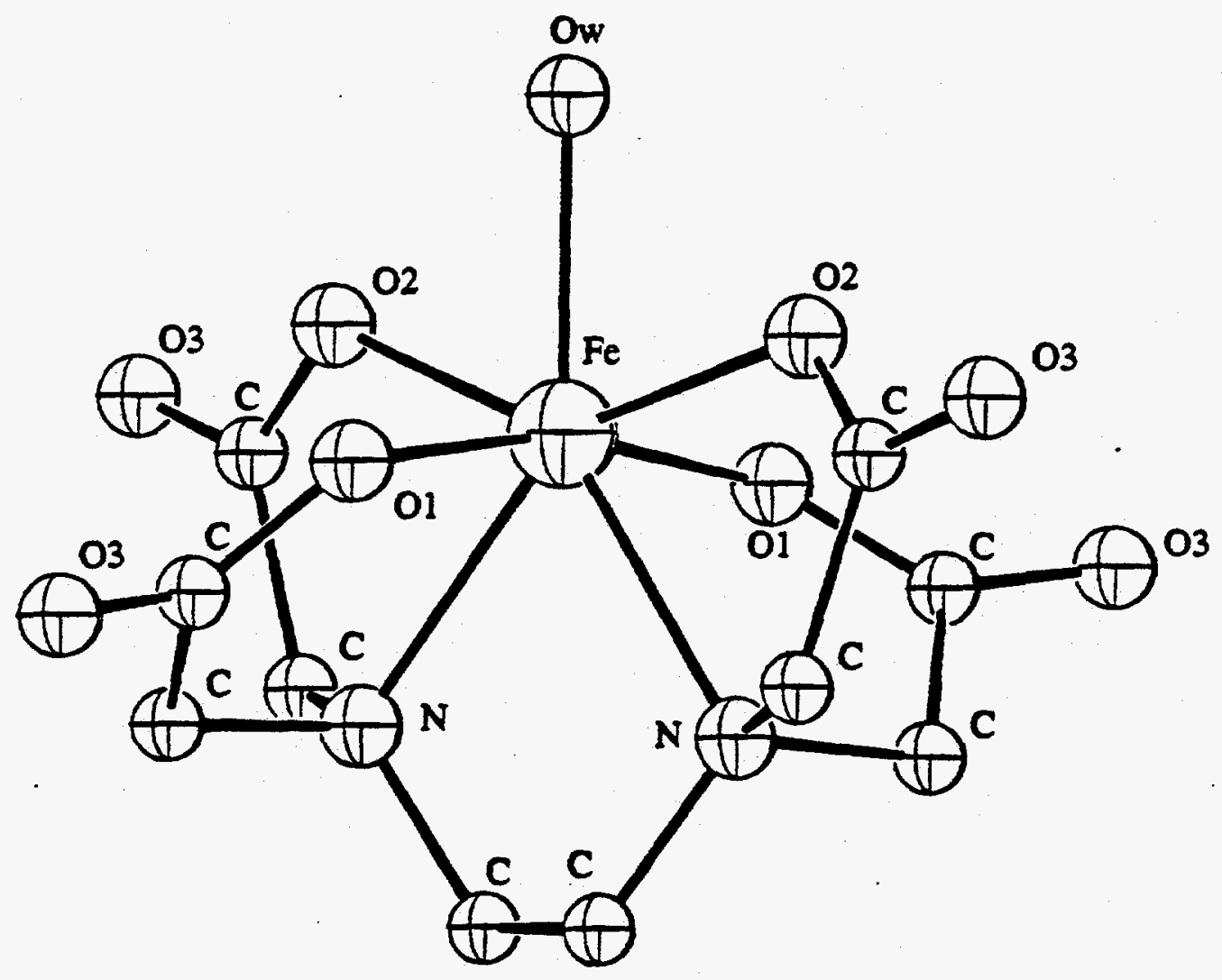

Figure 2.6. Molecular structure of $\left[\mathrm{Fe}\left(\mathrm{OH}_{2}\right) \mathrm{EDTA}\right]^{-}$with atom designations as used in the text. 
Table 2.2. Comparison of the $\mathrm{Na}\left[\mathrm{Fe}\left(\mathrm{OH}_{2}\right) \mathrm{EDTA}\right] \mathrm{GNXAS}$ Values to the Crystallographic Values of $\mathrm{Li}\left[\mathrm{Fe}\left(\mathrm{OH}_{2}\right)\right.$ EDTA] $\cdot 2 \mathrm{H}_{2} \mathrm{O}$.

$\mathrm{Li}\left[\mathrm{Fe}\left(\mathrm{OH}_{2}\right) \mathrm{EDTA}\right]$.

$\mathrm{Na}\left[\mathrm{Fe}\left(\mathrm{OH}_{2}\right) \mathrm{EDTA}\right] \quad 2 \mathrm{H}_{2} \mathrm{O}$

\begin{tabular}{|c|c|c|c|}
\hline $\begin{array}{l}\text { structural feature } \\
\text { (\# of configur- } \\
\text { ations in complex) }\end{array}$ & $\begin{array}{l}\mathrm{Na}\left[\mathrm{Fe}\left(\mathrm{OH}_{2}\right) \mathrm{EDTA}\right] \\
\mathrm{GNXAS} \\
\text { distances/angles }\end{array}$ & $\begin{array}{l}\text { GNXAS } \\
\text { bond variance }\left(\sigma_{R}^{2}\right) / \\
\text { angle variance }\left(\sigma_{\theta}^{2}\right)^{a}\end{array}$ & $\begin{array}{l}\text { crystallographic } \\
\text { distances/angles } \\
\text { average [range] }\end{array}$ \\
\hline $\mathrm{Fe}-\mathrm{O}_{1}(2)$ & $1.97 \AA$ & 0.003 & $1.97 \AA[1.94-2.00]$ \\
\hline $\mathrm{Fe}-\mathrm{O}_{2}(3)$ & $2.10 \AA$ & 0.004 & $2.11 \AA$ [2.11-2.13] \\
\hline $\mathrm{Fe}-\mathrm{N}(2)$ & $2.33 \AA$ & 0.003 & $2.32 \AA[2.30-2.35]$ \\
\hline $\mathrm{O}_{1}-\mathrm{C}(2)$ & $1.33 \AA$ & 0.005 & $1.28 \AA[1.27-1.29]$ \\
\hline $\mathrm{O}_{2}-\mathrm{C}(2)$ & $1.30 \AA$ & 0.004 & $1.26 \AA[1.26-1.27]$ \\
\hline $\mathrm{N}-\mathrm{C}(6)$ & $1.48 \AA$ & 0.002 & $1.47 \AA[1.47-1.48]$ \\
\hline $\mathrm{O}_{1,2}-\mathrm{O}_{3}(4)$ & $2.30 \AA$ & 0.006 & $2.23 \AA[2.20-2.25]$ \\
\hline $\mathrm{C}-\mathrm{O}_{3}(4)$ & $1.27 \AA$ & 0.006 & $1.23 \AA[1.21-1.25]$ \\
\hline $\mathrm{Fe}-\mathrm{C}(4)$ & $2.91 \AA$ & 0.008 & $2.91 \AA[2.79-2.99]$ \\
\hline $\mathrm{Fe}-\mathrm{O}_{1}-\mathrm{C}(2)$ & $121^{\circ}$ & $3 \times 10^{1}$ & $120^{\circ}[119-121]$ \\
\hline $\mathrm{Fe}-\mathrm{O}_{2}-\mathrm{C}(2)$ & $119^{\circ}$ & $6 \times 10^{1}$ & $122^{\circ}[121-123]$ \\
\hline $\mathrm{Fe}-\mathrm{N}-\mathrm{C}(6)$ & $106^{\circ}$ & $1 \times 10^{1}$ & $108^{\circ}[103-112]$ \\
\hline $\mathrm{O}_{1}-\mathrm{Fe}-\mathrm{O}_{1}(1)$ & $170^{\circ}$ & $1 \times 10^{1}$ & $166^{\circ}$ \\
\hline $\mathrm{O}_{2}-\mathrm{Fe}-\mathrm{O}_{2}(1)$ & $150^{\circ}$ & $2 \times 10^{0}$ & $145^{\circ}$ \\
\hline $\mathrm{Fe}-\mathrm{O}_{1}-\mathrm{O}_{3}(2)$ & $150^{\circ}$ & $5 \times 10^{1}$ & $145^{\circ}[142-148]$ \\
\hline $\mathrm{Fe}-\mathrm{O}_{2}-\mathrm{O}_{3}(2)$ & $155^{\circ}$ & $5 \times 10^{1}$ & $149^{\circ}[148-150]$ \\
\hline $\mathrm{Fe}-\mathrm{C}-\mathrm{O}_{3}(4)$ & $158^{\circ}$ & $1 \times 10^{1}$ & $158^{\circ}[153-161]$ \\
\hline
\end{tabular}

a Bond and angle variances are reported in $\AA^{2}$ and degrees ${ }^{2}$, respectively. 
crystallographic distances). The $\mathrm{Fe}-\mathrm{OH}_{2}$ and long $\mathrm{Fe}-\mathrm{O}_{2}$ distances were treated together since they both have a distance of $2.11 \AA$. All five oxygens could not be averaged and treated as a single shell because the EXAFS signals from the short $\mathrm{Fe}-\mathrm{O}_{1}$ and longer $\mathrm{Fe}-\mathrm{O}_{2}$ strongly interfere at higher $k$ (Figure 2.7). This first-shell fit (not shown) gave an $R$ value of $0.118 \times 10^{-4}$ with good agreement between the FT of the experimental data and the fit signal up to $2.0 \AA$ (corresponding to $-2.4 \AA$ in the cluster when the phase shift is taken into account). The major contributions in the EXAFS signal were accounted for using the three first-shell distances, with especially good agreement at higher $k$. The next fit included signals from three-atom configurations: $\mathrm{Fe}-\mathrm{O}_{1}-\mathrm{C}, \mathrm{Fe}-\mathrm{O}_{2}-\mathrm{C}$, and $\mathrm{Fe}-\mathrm{N}-\mathrm{C}$. The $R$ value decreased to $0.437 \times 10^{-5}$. The total theoretical EXAFS signal fits extremely well to the experimental EXAFS above $k=8 \AA^{-1}$, and there were several peaks between 2.0 and $3.0 \AA$ in the FT. However, peaks above $3.0 \AA$ in the FT were not being fit well and high frequency components could be seen in the EXAFS residual, especially at lower $k$. Therefore other three-atom components were examined for signals that were relatively strong and of the same frequency as those in the residual.

All the $\sim 90^{\circ}$ signals associated with O-Fe-O, O-Fe-N, and N-Fe-N were extremely weak. Both the $\mathrm{O}_{1}-\mathrm{Fe}-\mathrm{O}_{1}$ and $\mathrm{O}_{2}-\mathrm{Fe}-\mathrm{O}_{2} \mathrm{MS}$ signals contributed only a small amount at low $k$. The $\mathrm{Fe}-\mathrm{O}_{1}-\mathrm{O}_{3}$ and $\mathrm{Fe}-\mathrm{O}_{2}-\mathrm{O}_{3}$ signals were significant. However, the $\mathrm{Fe}-\mathrm{C}-\mathrm{O}_{3}$ contribution was found to be extremely strong and largely responsible for the peak in the FT at $\sim 3.5 \AA$. The best fit was obtained when the last five mentioned contributions were included. The results of this fit are shown in Figure 2.7, and a comparison of the distances and angles to the $\mathrm{Li}\left[\mathrm{Fe}\left(\mathrm{OH}_{2}\right) \mathrm{EDTA}\right] \cdot 2 \mathrm{H}_{2} \mathrm{O}$ crystallographic values are given in Table 2.2. The individual contributions to the FT are shown in Figure 2.8. With an $R$ value of $0.735 \times 10^{-6}$, this fit was a factor of 6 better than the fit that included the first neighbors and $\mathrm{Fe}-\mathrm{O}_{1}-\mathrm{C}, \mathrm{Fe}-\mathrm{O}_{2}-\mathrm{C}$, and $\mathrm{Fe}-\mathrm{N}-\mathrm{C}$ signals. The fit compares extremely well to the experimental EXAFS with the exception of high frequency components between 7.5 and $12 \AA^{-1}$ (see results in Figure 2.7). These higher frequency components can possibly be attributed to intermolecular signals that were not accounted for because the cluster was only generated up to $4.5 \AA$. The FT of the theoretical fit is in close agreement with the FT of the experimental data up to $4.0 \AA$. The low-frequency EXAFS is dominated by three waves from the $g_{2}$ contributions: $\mathrm{Fe}-\mathrm{O}_{1}, \mathrm{Fe}-\mathrm{O}_{2}$, and $\mathrm{Fe}-\mathrm{N}$. The EXAFS distances for these three shells show excellent agreement with the $\mathrm{Li}\left[\mathrm{Fe}\left(\mathrm{OH}_{2}\right)\right.$ EDTA $] \cdot 2 \mathrm{H}_{2} \mathrm{O}$ crystallographic values, deviating by $<0.01 \AA$. The $\mathrm{Fe}-\mathrm{O}_{1}-\mathrm{C}$, $\mathrm{Fe}-\mathrm{O}_{2}-\mathrm{C}$, and $\mathrm{Fe}-\mathrm{N}-\mathrm{C}$ waves have significant contributions in the FT region between 2.0 and $3.0 \AA$, with $\mathrm{Fe}-\mathrm{N}-\mathrm{C}$ having the largest signal because of the 6 -fold degeneracy. The 


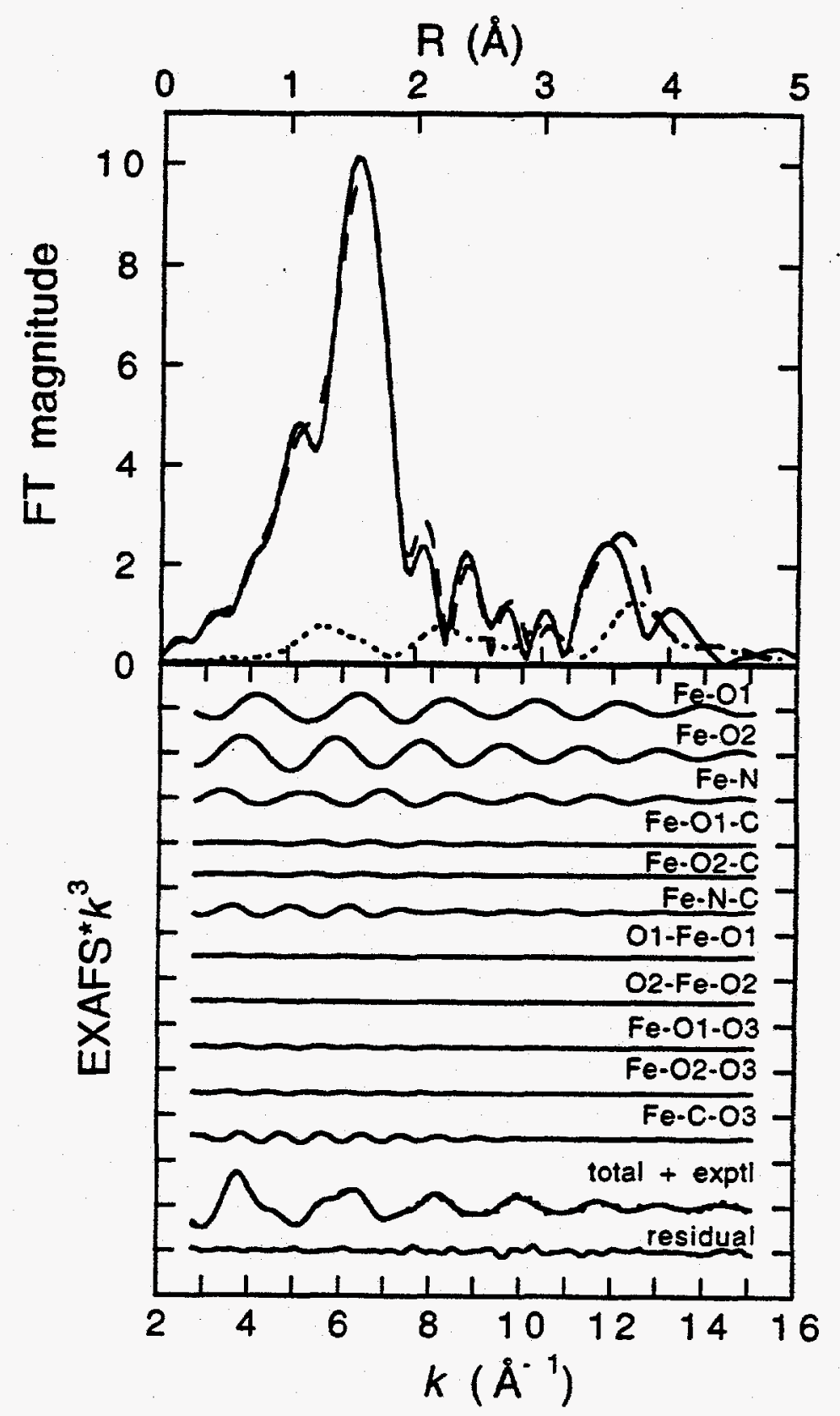

Figure 2.7. Comparison of the GNXAS theoretical signal with experimental data of $\mathrm{Fe}$ K-edge $k^{3}$-weighted EXAFS data between 7155 and $7999 \mathrm{eV}$ of $\mathrm{Na}\left[\mathrm{Fe}\left(\mathrm{OH}_{2}\right)\right.$ EDTA]. The top portion of the figure contains the non-phase shift corrected FT of the $k^{3}$-weighted experimental EXAFS data ( $\longrightarrow$ ) and that of the total theoretical signal (---). Also shown is the FT of the residual (…). The lower portion of the figure presents the EXAFS signals for the individual contributions. The total theoretical signal is also shown (-) and compared with the experimental data (...) with the residual being the difference between the experimental and the theoretical EXAFS. (The ordinate scale is 10 between two consecutive tick marks.) 


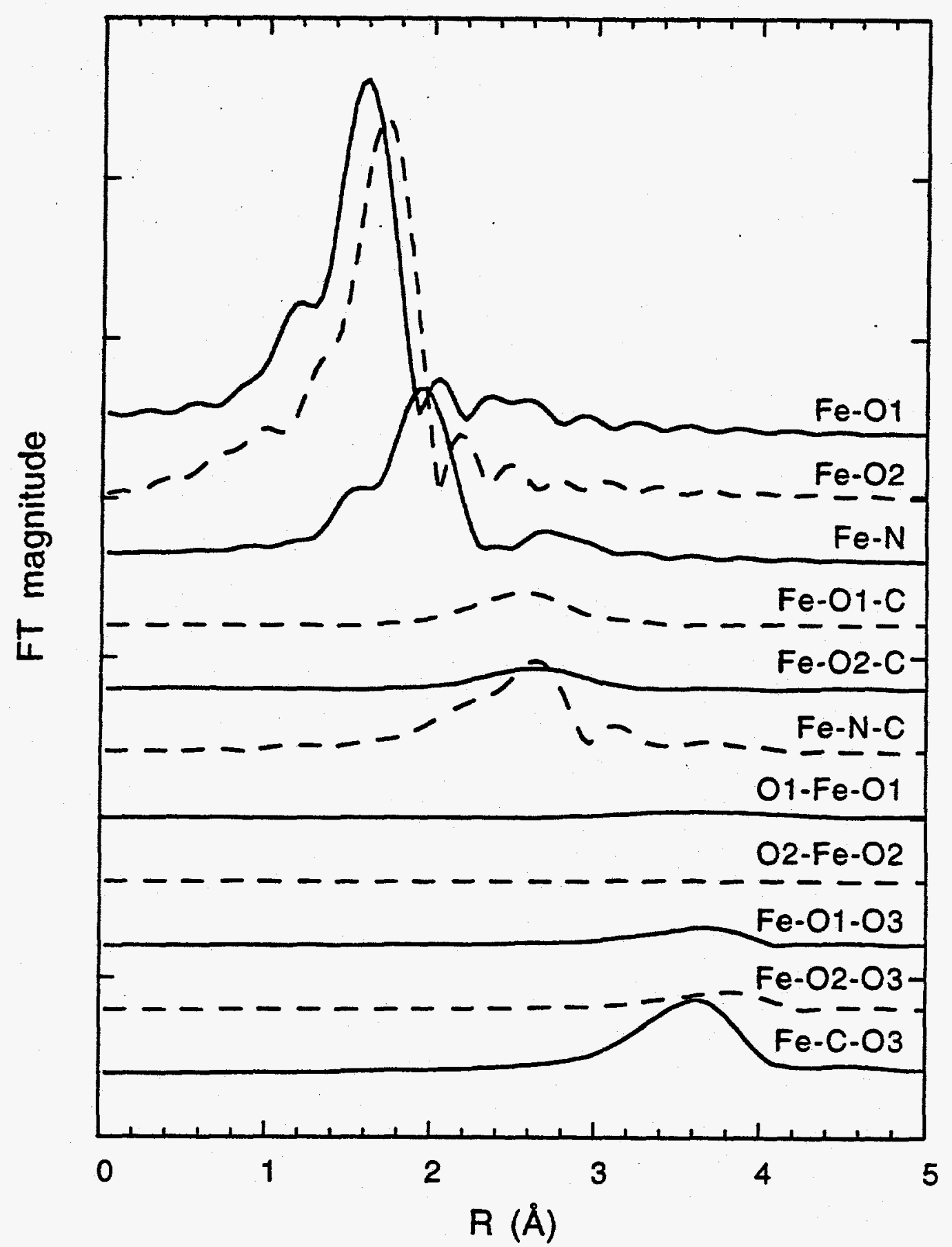

Figure 2.8. FT of the EXAFS signals of $\mathrm{Na}\left[\mathrm{Fe}\left(\mathrm{OH}_{2}\right) \mathrm{EDTA}\right]$ for the individual contributions shown in Figure 2.7. The first-shell signals contribute significantly below $2.5 \AA$ with the $\mathrm{Fe}-\mathrm{O}_{1}-\mathrm{C}, \mathrm{Fe}-\mathrm{O}_{2}-\mathrm{C}$, and $\mathrm{Fe}-\mathrm{N}-\mathrm{C}$ signals contributing between 2.5 and 3.2 $\AA$. The main contribution above $3.0 \AA$ comes from $\mathrm{Fe}-\mathrm{C}-\mathrm{O}_{3}$. (The ordinate is 5 between two consecutive tick marks.) 
higher frequency region is dominated by the $\mathrm{Fe}-\mathrm{C}-\mathrm{O}_{3}$ signal. A focusing effect occurs because of the large $\mathrm{Fe}-\mathrm{C}-\mathrm{O}_{3}$ angle $\left(161^{\circ}\right)$.

Not only are the structural values obtained from the GNXAS fit consistent with the crystallographic values but the GNXAS total EXAFS signal compared to the data and the respective FT of the fit and the data agree remarkably well for a low-symmetry coordination complex with mixed ligation. The fitted parameters are in substantially good agreement with crystallographic data, even though the fit is slightly under-determined ( 37 fitting parameters compared to 36 independent points). The bond distances obtained from GNXAS for the three first neighbors distance are all within 0.01 $\AA$ of the crystallographic values (see Table 2.2). The GNXAS bond distances and angles that make up the 83 contributions are within $4 \%$ of the average crystallographic values, with the strength of the signal influencing the goodness of the match. The configurations with stronger signals have distances and angles that are closer to the crystallographic values than the configurations with weaker signals. For example, the first shell has the strongest contributions and the calculated distances are within the range of the $\mathrm{Li}\left[\mathrm{Fe}\left(\mathrm{OH}_{2}\right) \mathrm{EDTA}\right] \cdot 2 \mathrm{H}_{2} \mathrm{O}$ crystallographic values. The Fe-N-C signal is much stronger than the Fe-O-C signals. The difference between the crystallographic and calculated N-C distance is $0.01 \AA$ while the difference between the crystallographic and calculated O-C distance is $0.05 \AA$. Since the $\mathrm{Fe}-\mathrm{C}-\mathrm{O}_{3}$ signal is strong, accurate distances and angles are obtainable, even though the $\mathrm{O}_{3}$ atoms are over $4 \AA$ away from the $\mathrm{Fe}$ atom. GNXAS also proved to be internally consistent, in that the first-shell distances varied $<0.01 \AA$, the low- $Z$ bond distances (i.e., O-C and N-C) varied $\pm 0.04 \AA$, and the bond angles varied $\pm 3^{\circ}$ over a large number of fits with varying contributions, splines, and nonstructural parameters.

2.3.3.3. $\mathrm{K} 3 \mathrm{Fe}(\mathrm{CN})_{6}$. The $\mathrm{GNXAS}$ programs were applied to $\mathrm{K}_{3} \mathrm{Fe}(\mathrm{CN})_{6} \mathrm{EXAFS}$ data to investigate the MS of the linear Fe-C-N unit and to test the feasibility of using GNXAS for angle determination studies for low- $Z$ diatomics coordinated to transition metal centers. The iron atom in $\mathrm{K}_{3} \mathrm{Fe}(\mathrm{CN})_{6}$ is in an octahedral environment ${ }^{65}$ with an average $\mathrm{Fe}-\mathrm{C}$ bond distance of $1.94 \AA$ and a range of $1.93-1.94 \AA$. The Fe-C-N angle ranges from 177 to $179^{\circ}$ with a C-N distance of $1.15 \AA$. The crystallographic values of $\mathrm{K}_{3} \mathrm{Fe}(\mathrm{CN})_{6}$ were used to characterize the two-atom and three-atom configurations up to $4.5 \AA$ with a frequency tolerance of $0.1 \AA$. The reduced Norman sphere radii used in the phase shift calculation were $0.946 \AA$ for $\mathrm{Fe}, 0.654 \AA$ for $\mathrm{C}$, and $0.668 \AA$ for $\mathrm{N}$. The two-atom configurations included $\mathrm{Fe}-\mathrm{C}$ while the three-atom configurations included $\mathrm{Fe}-\mathrm{C}-\mathrm{N}, \mathrm{C}-\mathrm{Fe}-\mathrm{C}\left(90^{\circ}\right)$, and C-Fe-C $\left(180^{\circ}\right)$. The two-region spline had orders of 3,4 with defining energy points of 7160,7300 , and $7999 \mathrm{eV}$. The coordination numbers were 
fixed to the known values, and the $\mathrm{Fe}-\mathrm{C}$ and $\mathrm{C}-\mathrm{N}$ distances were allowed to vary along with the respective variances. The independent points to parameters ratio is 36 to 19 , indicating that the fit to the data will be overdetermined by almost a factor of 2 . A comment has to be made for collinear configurations. In the GNXAS programs, a Taylor expansion of amplitudes and phases is used during the fitting procedure with first order derivatives. For a collinear structure $\left(\theta=180^{\circ}\right)$, the first order derivative is zero and therefore the program uses the second-derivative. Thus, the thermal and configurational averages of the Fe-C-N contributions were performed using a second-order Taylor expansion for the amplitude and phase around $\theta=180^{\circ}$, as described elsewhere. ${ }^{9}$ The agreement with the experimental data was found to be much worse with fits having angles $\theta<178^{\circ}$, thus indicating a strong sensitivity of the signal to the geometry of the collinear configuration. In addition, the angles around the iron were constrained to be octahedral. Least-squares fits were done with $k^{3}$ weighting over the $k$ range of 2.9-15.1 $\AA^{-1}$.

The best fit gave an Fe-C distance of $1.92 \AA$ and a $C-N$ distance of $1.18 \AA$. The EXAFS contributions and the FT of the best fit are presented in Figure 2.9 and show good agreement to the experimental data. The Fe-C SS signal and the Fe-C-N MS signal dominate the EXAFS spectrum. The angular sensitivity of the Fe- $\mathrm{C}-\mathrm{N}$ signal was investigated by fixing all the distances and variances and generating the MS signal from Fe-C-N and the SS signal from Fe-N as a function of the Fe-C-N angle (Figure 2.10). The MS signal from the Fe-C-N unit shows considerable amplitude enhancement for angles greater than about $150^{\circ}$, as reported in earlier papers for $\mathrm{Fe}-\mathrm{O}$-Fe and metal carbonyl systems. ${ }^{8-12}$ This indicates that GNXAS can be used to analyze MS effects as a function of angle, and where the angular dependent amplitude/phase effects become significant (above about $150^{\circ}$ ), angles can be fairly accurately determined for Fe-C-N configurations. This should also be the case for similar systems such as nitrosyl and dioxygen complexes even when contributions from other outer shell scatterers may be present.

\subsection{Summary}

In this chapter an $a b$ initio, integrated approach to EXAFS data analysis, called GNXAS, has been described. The characteristics and advantages of this approach were investigated by applying the method to Fe K-edge EXAFS data of three iron coordination complexes of known structure. Accurate structural results were obtained by using a fitting procedure which takes into account two-atom and three-atom MS signals. The raw: 


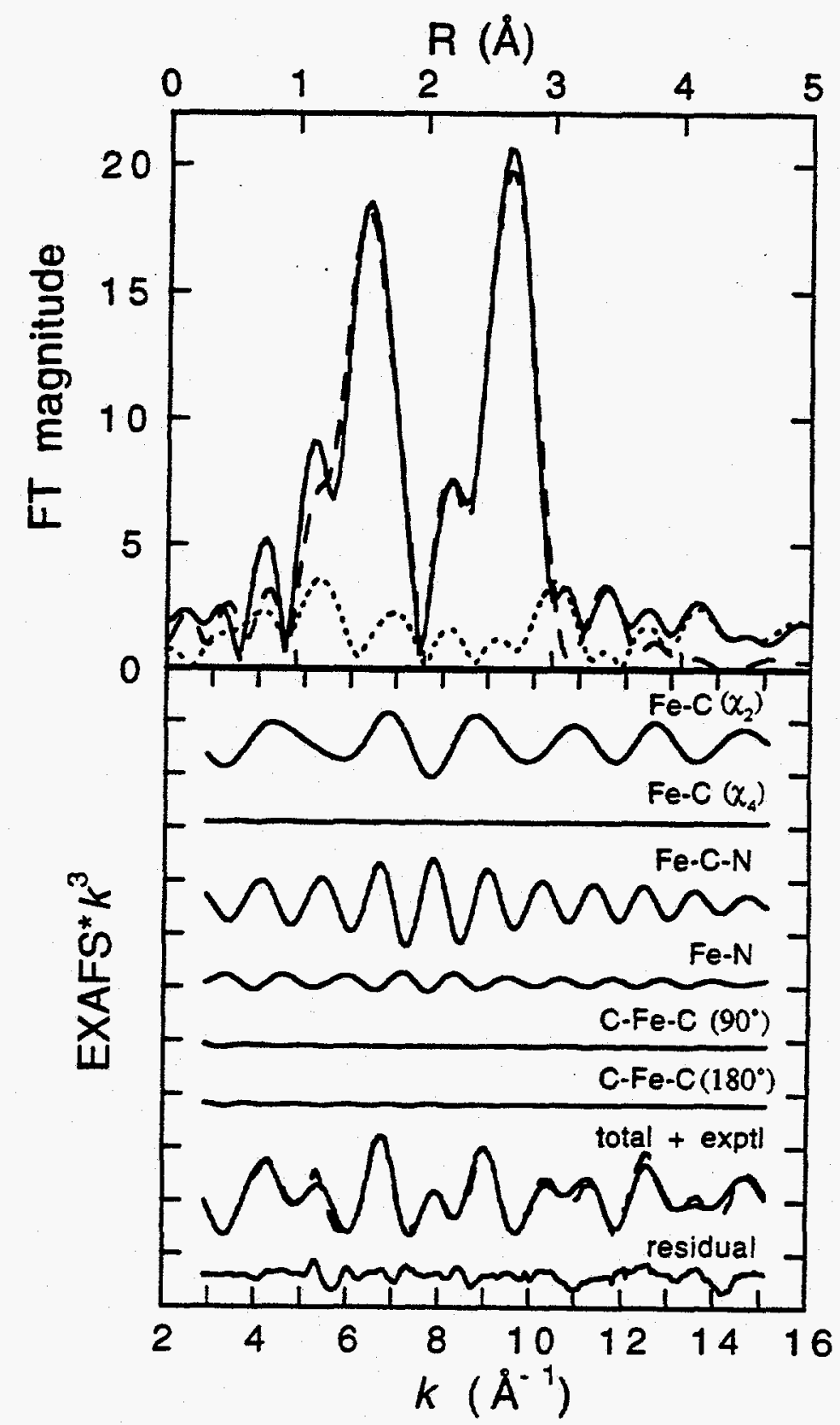

Figure 2.9. Comparison of the GNXAS theoretical signal with experimental data of $\mathrm{Fe}$ $\mathrm{K}$-edge $k^{3}$-weighted EXAFS data between 7160 and $7999 \mathrm{eV}$ of $\mathrm{K}_{3} \mathrm{Fe}(\mathrm{CN})_{6}$. The top portion of the figure contains the non-phase shift corrected FT of the $k^{3}$-weighted experimental EXAFS data (-) and that of the total theoretical signal (---). Also shown is the FT of the residual (...). The lower portion of the figure presents the EXAFS signals for the individual contributions. The total theoretical signal is also shown (-) and compared with the experimental data (...) with the residual being the difference between the experimental and the theoretical EXAFS. (The ordinate scale is 10 between two consecutive tick marks. 


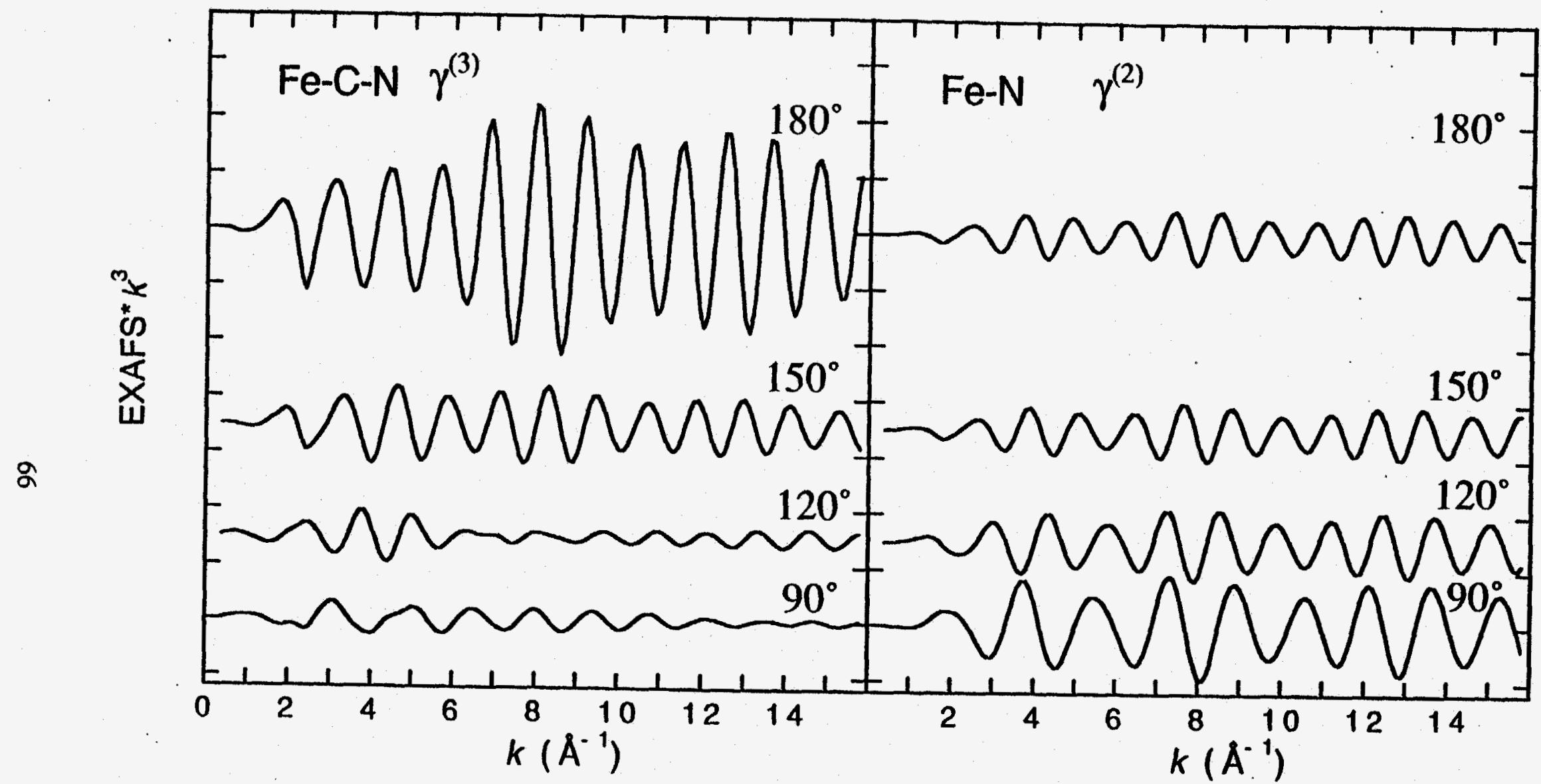

Figure 2.10. Comparison of the EXAFS signals of the $\gamma(3) \mathrm{Fe}-\mathrm{C}-\mathrm{N}$ contribution to the $\gamma(2) \mathrm{Fe}-\mathrm{N}$ contribution when the $\mathrm{Fe}-\mathrm{C}-\mathrm{N}$ angle equals $180^{\circ}, 150^{\circ}, 120^{\circ}$, and $90^{\circ}$. Notice the increased amplitude enhancement of the $\gamma^{(3)} \mathrm{Fe}-\mathrm{C}-\mathrm{N}$ signal above $150^{\circ}$. This indicates that GNXAS can be used to determine angles fairly accurately above $150^{\circ}$ for $\mathrm{Fe}-\mathrm{C}-\mathrm{N}$ configurations and other similar systems where diatomics may be coordinated to a transition metal center. (The ordinate scale is 10 between two consecutive tick marks.) 
data were fit in a way that reduces the tedious standard preanalysis of manual spline-background removal and without dependence on obtaining suitable models from which to extract empirical phase and amplitude parameters. First neighbor distances deviated less than $0.01 \AA$ from the crystallographic values, which is comparable or better than that which can be obtained by empirical-based methods. Bond distances and angles of second (and in some cases third) neighbors were also obtained due to the accurate modeling of MS contributions. The second and third neighbor distances and angles were found to be in good agreement with crystallographic values, typically within the crystallographic range and varying only $4 \%$ in distance and angle from the average. These findings are of general importance for structural studies of chemical systems, including inorganic complexes and metalloproteins. Further, they demonstrate that a proper treatment of the MS components in the EXAFS signal is necessary to get reliable structural information on distant neighbors. Moreover, accurate bond angle determination for angles over about $150^{\circ}$ is feasible for Fe-C-N and other similar systems using the GNXAS approach to accurately analyze MS effects.

\subsection{Acknowledgments}

This research was supported by grants from the NIH (RR01209, K.O.H.; GM40392, E.I.S.) and NSF (CHE91-21576, K.O.H.). Stanford Synchrotron Radiation Laboratory is supported by the Department of Energy, Office of Basic Energy Science, Divisions of Chemical Science and Material Science, and in part by the National Institutes of Health, National Center of Research Resources, Biomedical Research Technology Program (RR-01209) and DOE's Office of Health and Environmental Research. Rino Natoli, Andrea Di Cicco and Adriano Filipponi (who are supported by the Italian INFN and CNR research institutions) are gratefully acknowledged for providing the GNXAS program set and for their help in introducing me to the intricacies of the this data analysis package.

\subsection{References and Notes}

(1) Cramer, S. P. In X-ray Absorption: Principles, Applications, Techniques of EXAFS, SEXAFS and XANES; Koningsberger, D. C, Prins, R., Eds.; John Wiley and Sons Inc.: New York, 1988; p 573.

(2) Cramer, S. P.; Hodgson, K. O. Prog. Inorg. Chem. 1979, $25,1$.

(3) Lytle, F. W.; Via, G. H.; Sinfelt, J. H. In Synchrotron Radiation Research; Winick, H., Doniach, S. Eds.; Plenum Press: New York, 1980; Chapter 12. 
(4) Lagarde, P.; Dexpert, H. Adv. Phys. 1984, 33, 567.

(5) Iwasawa, Y. Tailored Metal Catalysts; D. Reidel Publishing Co.: Dordrecht, The Netherlands, 1986.

(6) Cramer, S. P.; Hodgson, K. O.; Stiefel, E. I.; Newton, W. E. J. Am. Chem. Soc. 1978, 100, 2748.

(7) a) Scott, R. A. Methods Enzymol. 1985, 117, 414.

b) Shulman, R.G.; Eisenberger, P.; Kincaid, B.M. Ann. Rev. Biophys. Bioeng. 1978, 7, 559.

(8) Co, M. S.; Hendrickson, W. A.; Hodgson, K. O.; Doniach, S. J. Am. Chem. Soc. 1983, $105,1144$.

(9) Filipponi, A.; Di Cicco, A.; Zanoni, R.; Bellatreccia, M.; Sessa, V.; Dossi, C.; Psaro, R. Chem. Phys. Lett. 1991, 184, 485.

(10) Binsted, N.; Cook, S. L.; Evans, J.; Greaves, G. N.; Price, R. J. J. Am. Chem. Soc. 1987, 109, 3669.

(11) Binsted, N.; Evans, J.; Greaves, G. N.; Price, R. J. J. Chem. Soc., Chem. Commun. 1987, 1130.

(12) Teo, B. -K. J. Am. Chem. Soc. 1981, 103, 3990.

(13) Co, M. S.; Scott, R. A.; Hodgson, K. O. J. Am. Chem. Soc. 1981, 103, 986.

(14) Hasnain, S. S.; Strange, R. W. In Synchrotron Radiation and Biophysics; Hasnain, S. S., Ed.; Ellis Horwood: Chichester, U.K., 1990; Chapter 4 and references therein.

(15) Filipponi, A.; Di Cicco, A.; Tyson, T. A.; Natoli, C. R. Solid State Commun. 1991, 78, 265.

(16) Filipponi, A.; Di Cicco, A. Synchrotron Radiation News 1993, 6, 13.

(17) Filipponi, A.; Di Cicco, A. Unpublished data.

(18) a) Rehr, J. J.; Albers, R. C.; Zabinsky, S. I. Phys. Rev. Lett. 1992, 69, 3397.

b) Rehr, J. J. Jpn. J. Appl. Phys. 1993, 32, 8.

(19) a) Frenkel, A. I.; Stern, E. A.; Qian, M.; Newville, M. Phys. Rev. B 1993, 48, 12449.

b) Frenkel, A. I.; Stern, E. A.; Voronel, A.; Qian, M.; Newville, M. Phys. Rev. Letr. 1993, 71, 3485.

(20) Binsted, N.; Campbell, J. W.; Gurman, S. J.; Stephenson, P. C. "SERC Daresbury Laboratory EXCURVE92 program" 1991.

(21) Di Cicco, A.; Stizza, S.; Filipponi, A.; Boscherini, F.; Mobilio, S. J. Phys. B: At. Mol. Opt. Phys. 1992, 25, 2309. 
(22) D'Angelo, P.; Di Cicco, A.; Filipponi, A.; Pavel, N. V. Phys. Rev. A 1993, 47, 2055.

(23) Burattini, E.; D'Angelo, P.; Di Cicco, A.; Filipponi, A.; Pavel, N. V. J. Phys. Chem. 1993, 97, 5486.

(24) Nordlander, E.; Lee, S. C.; Cen, W.; Wu, Z. Y.; Natoli, C. R.; Di Cicco, A.; Filipponi, A.; Hedman, B.; Hodgson, K. O.; Holm, R. H. J. Am. Chem. Soc. 1993, $115,5549$.

(25) Westre, T. E.; Di Cicco, A.; Filipponi, A.; Natoli, C. R.; Hedman, B.; Solomon, E. I.; Hodgson, K. O. J. Am. Chem. Soc. 1995, 117, 1566.

(26) DeWitt, J. G.; Bentsen, J. G.; Rosenzweig, A. C.; Hedman, B.; Green, J.; Pilkington, S.; Papaefthymiou, G. C.; Dalton, H.; Hodgson, K. O.; Lippard, S. J. J. Am. Chem. Soc. 1991, 113, 9219.

(27) Hedman, B.; Co, M. S.; Armstrong, W. H.; Hodgson, K. O.; Lippard, S. J. Inorg. Chem. 1986, 25, 3708.

(28) Tsang, H. -T.; Batie, C. J.; Ballou, D. P.; Penner-Hahn, J. E. Biochemistry 1989, $28,7233$.

(29) Scarrow, R. C.; Maroney, M. J.; Palmer, S. M.; Que, L., Jr.; Roe, A. L.; Salowe, S. P.; Stubbe, J. J. Am. Chem. Soc. 1987, 109, 7857.

(30) Mustre de Leon, J.; Rehr, J. J.; Zabinsky, S. I.; Albers, R. C. Phys. Rev. B 1991, $44,4146$.

(31) Rehr, J. J.; Mustre de Leon, J.; Zabinsky, S. I.; Albers, R. C. J. Am. Chem. Soc. $1991,113,5135$.

(32) Lee, P. A.; Citrin, P. H.; Eisenberger, P.; Kincaid, B. M. Rev. Mod. Phys. 1981, 53,769 .

(33) Rehr, J. J.; Albers, R. C.; Natoli, C. R.; Stern, E. A. Phys. Rev. B 1986, 34, 4350.

(34) Müller, J. E.; Schaich, W. L. Phys. Rev. B 1983, 27, 6489.

(35) Schaich, W. L. Phys. Rev. B 1984, 29, 6513.

(36) Lee, P. A.; Pendry, J. B. Phys. Rev. B 1975, 11, 2795.

(37) Natoli, C. R.; Benfatto, M. J. Phys. 1986, 47, C8.

(38) Vvedensky, D. D.; Saldin, D. K.; Pendry, J. B. Comput. Phys. Commun. 1986, 40, 421.

(39) Durham, P. J.; Pendry, J. B.; Hodges, C. H. Solid State Commun. 1981, 38, 159.

(40) Mattheiss, L. F. Phys. Rev. 1964, 134, A970.

(41) Hedin, L.; Lundqvist, S. Solid State Phys. 1969, 23, 1.

(42) Lundqvist, B. I. Phys. Kondens. Materie 1967, 6, 193.

(43) Penn, D. R. Phys. Rev. B 1987, 35, 482. 
(44) Lee, P. A.; Beni, G. Phys. Rev. B 1977, 15, 2862.

(45) Tyson, T. A.; Hodgson, K. O.; Natoli, C. R.; Benfatto, M. Phys. Rev. B 1992, 46, 5997.

(46) Natoli, C. R. In X-ray Absorption Fine Structure; Hasnain, S. S., Ed.; Ellis Horwood: Chichester, U.K., 1991; p 6.

(47) Tyson, T. A.; Benfatto, M.; Natoli, C. R.; Hedman, B.; Hodgson, K. O. Physica B $1989,158,425$.

(48) Filipponi, A.; Di Cicco, A.; Benfatto, M.; Natoli, C. R. Europhys. Lett. 1990, 13, 319.

(49) Filipponi, A. J. Phys.: Condens. Matter 1991, 3, 6489.

(50) Benfatto, M.; Natoli, C. R.; Filipponi, A. Phys. Rev. B 1989, 40, 9626.

(51) Stern, E. A.; Bunker, B. A.; Heald, S. M. Phys. Rev. B 1980, $21,5521$.

(52) Müller, J. E.; Jepsen, O.; Wilkins, J. W. Solid State Commun. 1982, 42, 365.

(53) Müller, J. E.; Wilkins, J. W. Phys. Rev. B 1984, $29,4331$.

(54) Newville, M.; Livins, P.; Yacoby, Y.; Rehr, J. J.; Stern, E. A. Phys. Rev. B 1993, 47, 14126.

(55) Bevington, P. R.; Robinson, D. K. Data Reduction and Error Analysis for the Physical Sciences; McGraw-Hill: New York, 1992.

(56) Stern, E. A. Phys. Rev. B 1993, 48, 9825.

(57) Krause, M. O.; Oliver, J. H. J. Phys. Chem. Ref. Data 1979, 8, 329.

(58) The principal determining factor is the monochromator and associated vertical slit opening, with the resolution determined by the relationship $\Delta E / E=\cot (\Theta) \Delta \Theta$, where $\Theta$ is a function of the Darwin width and the vertical angular acceptance of the monochromator. The value at the Fe $\mathrm{K}$-edge for the experimental conditions used for these experiments were $\sim 1.4 \mathrm{eV}$. Lytle, F. W. In Applications of Synchrotron Radiation; Winick, H.; Xiam, D.; Ye, M.-h; Huang,T., Eds.; Gordon and Breach Science Publishers: New York, 1989; p. 135.

(59) Di Cicco, A. Ph.D. Thesis, University of Rome "La Sapienza", 1991.

(60) Di Cicco, A.; Filipponi, A. Unpublished.

(61) Joyner, R. W.; Martin, K. J.; Meehan, P. J. Phys. C 1987, 20, 4005.

(62) Norman, J. G., Jr. Mol. Phys. 1976, 31, 1191.

(63) Lind, M. D.; Hamor, M. J.; Hamor, T. A.; Hoard, J. L. Inorg. Chem. 1964, 3, 34.

(64) Iball, J.; Morgan, C. H. Acta Crystallogr. 1967, 23, 239.

(65) Figgis, B. N.; Skelton, B. W.; White, A. H. Aust. J. Chem. 1978, 31, 1195. 
Chapter 3

Determination of the Fe-N-O Angle in $\{\mathrm{FeNO}\}^{7}$ Complexes Using Multiple-Scattering EXAFS Analysis by GNXAS 


\subsection{Introduction}

Mononuclear high spin non-heme ferrous centers are present in the catalytic active sites of a large number of enzymes involved in the binding and activation of molecular dioxygen. ${ }^{1}$ An understanding of the reactivity of these enzymes requires knowledge of the geometric and electronic structures of the active sites as well as their interactions with substrate, dioxygen, and other molecules of relevance to catalysis. Understanding the oxygen intermediates involved in catalysis is key to obtaining molecular insight into the mechanism of the reaction. Unfortunately, these intermediates are often too unstable to allow detailed spectroscopic study. Nitric oxide reversibly binds to the ferrous active site of the native form of many of these non-heme iron enzymes to generate stable nitrosyl complexes. ${ }^{2}$ These enzyme-NO complexes can serve as analogs of the possible dioxygen intermediates involved in catalysis and can be readily studied spectroscopically to determine geometric and electronic structure differences which could provide insight into differences in oxygen activation by the enzymes.

However, in order to use the NO derivative of these non-heme iron enzymes as a probe of electron distribution related to dioxygen reactivity, a detailed understanding of the electronic and geometric structure of the $\{\mathrm{FeNO}\}^{7}$ unit $^{3}$ is required. $\{\mathrm{FeNO}\}^{7}$ complexes have been described in the literature as having different electronic structures for different geometric structures, linear $\mathrm{Fe}-\mathrm{N}-\mathrm{O}$ being viewed as $\mathrm{Fe}^{+}$and $\mathrm{NO}^{+}$and bent $\mathrm{Fe}-\mathrm{N}-\mathrm{O}$ as $\mathrm{Fe}^{3+}$ and $\mathrm{NO}$. Enzyme-NO complexes as well as several $\{\mathrm{FeNO}\}^{7}$ model compounds (in particular FeEDTA-NO, vide infra) exhibit an unusual $S=3 / 2$ EPR signal. ${ }^{2}$ A wide range of bonding descriptions have appeared ${ }^{4}$ to describe this $S=3 / 2$ sigrial including $\left[\mathrm{Fe}^{+} \mathrm{d}^{7}(\mathrm{~S}=3 / 2)-\mathrm{NO}^{+}(\mathrm{S}=0)\right],\left[\mathrm{Fe}^{2+} \mathrm{d}^{6}(\mathrm{~S}=2)-\mathrm{NO}^{0}(\mathrm{~S}=1 / 2)\right]$ antiferromagnetically coupled, $\left[\mathrm{Fe}^{3+} \mathrm{d}^{5}(\mathrm{~S}=1 / 2)-\mathrm{NO}^{-}(\mathrm{S}=1)\right]$ ferromagnetically coupled, and $\left[\mathrm{Fe}^{3+} \mathrm{d}^{5}(\mathrm{~S}=3 / 2)-\mathrm{NO}^{-}(\mathrm{S}=0)\right]$. Recently a combination of spectroscopic and theoretical methods was used to determine that the appropriate description of the $S=3 / 2$ $\{\mathrm{FeNO}\}^{7}$ unit is high spin $\mathrm{Fe}^{3+}(\mathrm{S}=5 / 2)$ antiferromagnetically coupled to an $\mathrm{NO}^{-}(\mathrm{S}=1)$ to produce the $S=3 / 2$ ground state. ${ }^{5}$

In the present study, extended X-ray absorption fine structure (EXAFS) data on a series of $\{\mathrm{FeNO}\}^{7}$ model compounds were measured and analyzed to characterize the geometric structure of the Fe-N-O unit. Multiple-scattering effects from distant shells can contribute significantly to the EXAFS of inorganic molecules, ${ }^{6}$ and these effects have been used in a few favorable cases to obtain angular information. ${ }^{7}$ The effects are particularly evident when an intervening atom nears a linear relationship with an absorber and a distant scatterer, as occurs in Fe-oxo dimers ${ }^{7 b}$ and metal carbonyls. ${ }^{8}$ An empirical 
data analysis approach was utilized to determine the $\mathrm{Fe}-\mathrm{O}-\mathrm{Fe}$ angle in oxygen-bridged iron complexes. ${ }^{7 b}$ The analysis demonstrated that it was possible to estimate the $\mathrm{Fe}-\mathrm{O}-\mathrm{Fe}$. bridging angle to within $\pm 8^{\circ}$ and calculate the Fe-Fe distance to $\pm 0.05 \AA$. In the present study, the same traditional empirical EXAFS technique was initially applied to the \{FeNO $\}^{7}$ systems to determine the Fe-N-O angle. However, determination of the Fe-N-O angle using the empirical technique was not found to be possible because the oxygen of the Fe-N-O is not a heavy back-scatterer, the quality of empirical Fe-N and $\mathrm{Fe}-\mathrm{O}$ (second shell) phases and amplitudes is poor, and other low $Z$ atoms are at approximately the same distance as the $\mathrm{Fe}-\mathrm{O}$ (second shell). ${ }^{9}$ Since angle determination by empirical methods did not prove to be feasible, a new theoretical EXAFS data analysis package, GNXAS, 10 was utilized to probe the $\mathrm{Fe}-\mathrm{N}-\mathrm{O}$ bond angle using a multiple-scattering analysis and establish the generality of the approach for angle determination of low- $Z$ small molecules liganded to transition metal complexes.

The GNXAS package provides a new integrated approach to the analysis of EXAFS data based on full curved-wave, multiple-scattering theoretical analysis. It incorporates direct fitting of theoretical spectra (calculated by utilizing the Hedin-Lundqvist complex exchange and correlations potential ${ }^{11}$ ) to the experimental data and utilizes single- and multiple-scattering signals with the proper treatment of correlated distances and Debye-Waller factors. GNXAS has been evaluated on simpler systems (including $\mathrm{SiX}_{4}\left(\mathrm{X}=\mathrm{F}, \mathrm{Cl}, \mathrm{CH}_{3}\right),{ }^{12} \mathrm{Os}_{3}(\mathrm{CO})_{12},{ }^{8 \mathrm{~d}} \mathrm{Br}_{2}$ and $\mathrm{HBr},{ }^{13}$ and brominated hydrocarbons ${ }^{14}$ ) and a more complex heterometal cluster. ${ }^{15}$ It has been demonstrated in the previous chapter that the GNXAS method can provide accurate bond distances and angles for second and third neighbors for Fe complexes. 16

In this study, the EXAFS data of a series of crystallographically-characterized $\{\mathrm{FeNO}\}^{7}$ compounds with varying $\mathrm{Fe}-\mathrm{N}-\mathrm{O}$ angles were analyzed using the GNXAS method to examine the sensitivity of this method to $\mathrm{Fe}-\mathrm{N}-\mathrm{O}$ angle determination. The compounds studied were $[\mathrm{Fe}(\mathrm{TMC}) \mathrm{NO}]\left(\mathrm{BF}_{4}\right)_{2}{ }^{17}$ (where $\mathrm{TMC}=$ 1,4,8,11-tetramethyl-1,4,8,11-tetraazacyclotetradecane), which has an Fe-N-O bond angle of $177.5(5)^{\circ}, \mathrm{Fe}(\mathrm{TACN})\left(\mathrm{N}_{3}\right)_{2} \mathrm{NO}^{4 e}$ (where TACN $=N, N^{\prime}, N^{\prime \prime}$-trimethyl-1,4,7triazacyclononane) which has an angle of $156(1)^{\circ}$, and $\mathrm{Fe}$ (salen)NO18 (where salen $=$ $N, N$-ethylenebis(salicylideneiminato)), which has a bond angle of $127(6)^{\circ}$ at $-175^{\circ} \mathrm{C}$ and $147(5)^{\circ}$ at $23^{\circ} \mathrm{C}$.

EXAFS data for FeEDTA-NO (whose crystal structure is not known due to lack of suitable crystals) were obtained and analyzed to determine the unknown $\mathrm{Fe}-\mathrm{N}-\mathrm{O}$ bond angle. In order to use the GNXAS method to calculate the theoretical EXAFS spectrum, an initial structural model is needed. Such a model for this unknown structure was 
obtained by comparing first-shell empirical fits of the EXAFS data of $\left[\mathrm{Fe}\left(\mathrm{H}_{2} \mathrm{O}\right)\right.$ EDTA]-, [Fe( $\left.\mathrm{H}_{2} \mathrm{O}\right)$ EDTA] ${ }^{2-}$, and FeEDTA-NO. The Fe-O and $\mathrm{Fe}-\mathrm{N}$ distances of the EDTA ligand in FeEDTA-NO were much closer to the distances in [ $\mathrm{Fe}\left(\mathrm{H}_{2} \mathrm{O}\right)$ EDTA] than the respective distances in $\left[\mathrm{Fe}\left(\mathrm{H}_{2} \mathrm{O}\right) \mathrm{EDTA}\right]^{2-}$, consistent with our description of the FeEDTA-NO complex as having a ferric center. 5 Thus bond distances and angles from the crystallographically-characterized [Fe( $\left.\mathrm{H}_{2} \mathrm{O}\right)$ EDTA]- were used as an initial structural model in the GNXAS analysis with NO substituted for the bound water. ${ }^{19}$ Since the EXAFS data for FeEDTA-NO were collected as a frozen solution, EXAFS data were also collected for $\mathrm{Na}\left[\mathrm{Fe}\left(\mathrm{OH}_{2}\right)\right.$ EDTA] as a solution as well as a powder to determine if the metrical details differed in the two states. The results of this study establish that EXAFS analysis by GNXAS can provide reliable angular information and serve as the basis for its application to NO complexes of non-heme iron protein active sites.

\subsection{Experimental Section}

\subsubsection{Sample Preparation and Data Collection}

X-ray absorption (XAS) spectra were recorded at the Stanford Synchrotron Radiation Laboratory on unfocused beamlines 7-3 and 4-3 during dedicated conditions ( $3 \mathrm{GeV}, 25-75 \mathrm{~mA}$ ). The radiation was monochromatized using a Si $(220)$ double-crystal monochromator detuned $50 \%$ at $7998 \mathrm{eV}$ to minimize harmonic contamination. An Oxford Instruments continuous-flow liquid helium CF 1208 cryostat was used to maintain a constant temperature. The XAS spectra were calibrated using an internal $\mathrm{Fe}$ foil standard, 20 assigning the first inflection point to $7111.2 \mathrm{eV}$.

[Fe(TMC)NO] $\left(\mathrm{BF}_{4}\right)_{2},{ }^{17} \mathrm{Fe}(\mathrm{TACN})\left(\mathrm{N}_{3}\right)_{2} \mathrm{NO}, 4 \mathrm{e} \quad \mathrm{Fe}$ (salen)NO,18 a n d $\mathrm{Na}\left[\mathrm{Fe}\left(\mathrm{OH}_{2}\right) \mathrm{EDTA}\right]^{19}$ were prepared as described in the literature. [Fe(TMC)NO] $\left(\mathrm{BF}_{4}\right)_{2}$, $\mathrm{Fe}(\mathrm{TACN})\left(\mathrm{N}_{3}\right)_{2} \mathrm{NO}$, and $\mathrm{Fe}$ (salen)NO are air-sensitive and were handled in a nitrogen-filled inert atmosphere dry glove box during the following sample preparation. The crystalline samples were mixed with $\mathrm{BN}$ and ground into a fine powder. The $\mathrm{BN} / \mathrm{sample}$ mixture was pressed into a $1 \mathrm{~mm}$ thick $\mathrm{Al}$ spacer that was sealed with $63.5 \mu \mathrm{m}$ Mylar tape windows. Immediately after preparation, the samples were frozen in liquid nitrogen. Data were measured in transmission mode at $10 \mathrm{~K}$ with nitrogen-filled ionization chambers. Since Fe(salen)NO undergoes a spin and structural transition at $180 \mathrm{~K}$, EXAFS data were also collected at $220 \mathrm{~K}$. 
The $\left[\mathrm{Fe}\left(\mathrm{OH}_{2}\right) \mathrm{EDTA}\right]^{2-}$ solution, $50 \mathrm{mM}$ in $\mathrm{Fe}$, was prepared by anaerobically adding ferrous ammonium sulfate to a $50 \mathrm{mM}$ solution of $\mathrm{Na}_{2} \mathrm{EDTA}$ in $\mathrm{pH}=6.5,0.1 \mathrm{M}$ deoxygenated phosphate buffer. Oxidizing this solution produced $\left[\mathrm{Fe}\left(\mathrm{OH}_{2}\right) \mathrm{EDTA}\right]:$ An FeEDTA-NO solution was prepared by purging an $\left[\mathrm{Fe}\left(\mathrm{OH}_{2}\right) \mathrm{EDTA}\right]^{2-}$ solution with NO gas under anaerobic conditions. To form an ice-free glass, the XAS solution samples were prepared by adding $50 \%$ (by volume) glycerol to the previously prepared solutions resulting in solutions $25 \mathrm{mM}$ in Fe. These samples were loaded into $140 \mu \mathrm{L}$ Lucite EXAFS cells ( $23 \times 2 \times 3 \mathrm{~mm}$ ) with $37 \mu \mathrm{m}$ Kapton windows in an anaerobic wet box under nitrogen. The samples were frozen in liquid nitrogen and were subsequently stored in a liquid nitrogen refrigerator until use. Data were collected in fluorescence mode at $10 \mathrm{~K}$. The fluorescence signal was collected by an argon-filled ionization chamber, 21 equipped with Soller slits and a $\mathrm{Mn}$ filter.

Data were also collected for $\mathrm{Fe}$ (acetylacetonate) 3 and [Fe(1,10-phenanthroline $\left.)_{3}\right]\left(\mathrm{ClO}_{4}\right)_{3}$ at $10 \mathrm{~K}$ to extract $\mathrm{Fe}-\mathrm{O}$ and $\mathrm{Fe}-\mathrm{N}$ backscattering parameters for empirical analysis. Fe(acetylacetonate) 3 was purchased from Aldrich and $\left[\mathrm{Fe}(1,10 \text {-phenanthroline })_{3}\right]\left(\mathrm{ClO}_{4}\right)_{3}$ was prepared according to the published procedure. ${ }^{22}$ The samples were prepared in air in an identical manner to the solids mentioned above. Data were measured in transmission mode with nitrogen-filled ionization chambers.

The EXAFS data were measured to $k=15 \AA^{-1}$ with $2 \mathrm{~mm}$ high pre-monochromator beam-defining slits for the $\mathrm{Na}\left[\mathrm{Fe}\left(\mathrm{OH}_{2}\right) \mathrm{EDTA}\right.$, $\mathrm{Na}_{2}\left[\mathrm{Fe}\left(\mathrm{OH}_{2}\right)\right.$ EDTA], and FeEDTA-NO solutions and $1 \mathrm{~mm}$ high pre-monochromator slits for all the powder samples. Two to seven scans were averaged for each transmission sample, while eight to twenty scans were averaged for the fluorescence samples. The effects of a quartet monochromator glitch were removed from the averaged data by four single point replacements at around $k=11.8,12.1,12.3$ and $12.6 \AA^{-1}$.

\subsubsection{GNXAS Data Analysis}

As described in detail in Chapter 2 and elsewhere, ${ }^{10,16}$ the GNXAS programs generate model EXAFS signals for each shell around the photoabsorber based on an initial structural model. Both single-scattering and multiple-scattering contributions are summed to generate a theoretical spectrum for the model which is then fit to the nonFourier-filtered experimental data. 10,16

The crystallographic coordinates were used as input for $\left[\mathrm{Fe}(\mathrm{TMC}) \mathrm{NO}\left(\mathrm{BF}_{4}\right)_{2},{ }^{17}\right.$ $\mathrm{Fe}(\mathrm{TACN})\left(\mathrm{N}_{3}\right)_{2} \mathrm{NO},{ }^{4 e}$ and $\mathrm{Fe}\left(\right.$ salen) $\mathrm{NO}^{18}$ at high and low temperatures (Figure 3.1 shows the structure of each compound). Phase shifts were calculated using the standard 


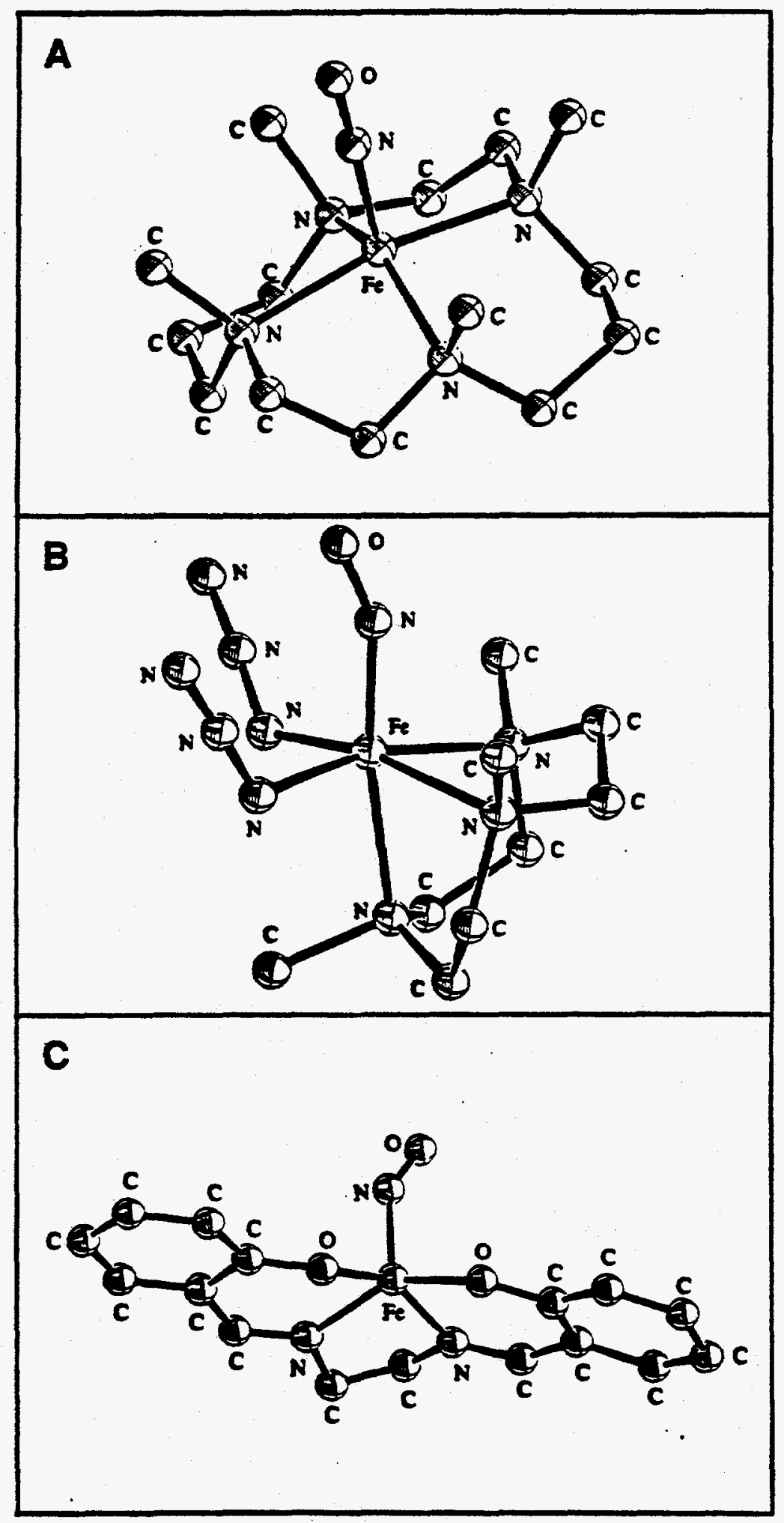

Figure 3.1. Molecular structures of (A) $\left[\mathrm{Fe}(\mathrm{TMC}) \mathrm{NO}\left(\mathrm{BF}_{4}\right)_{2}\right.$, (B) $\mathrm{Fe}(\mathrm{TACN})\left(\mathrm{N}_{3}\right)_{2} \mathrm{NO}$ and (C) $\mathrm{Fe}\left(\right.$ salen)NO at $23^{\circ} \mathrm{C}$. 
muffin-tin approximation with all the atoms associated with each compound and up to an energy limit of $70 \mathrm{Ry}(950 \mathrm{eV})$ above the $\mathrm{Fe} \mathrm{K}$ edge. The Mattheiss prescription ${ }^{23}$ of overlapping, self-consistent atomic charge densities of the atoms of the cluster was used to construct the Coulomb portion of the effective one-electron potential. Proper account of the charge relaxation around the core hole was taken. The. Hedin-Lundqvist plasmon-pole approximation was used to model its exchange and correlation part. ${ }^{11}$. The imaginary part of the latter takes into account inelastic scattering processes of the photoelectron propagating out of the system and models a priori its mean-free path. The muffin-tin radii were chosen by scaling Norman radii of the cluster atoms by a factor of about 0.8 as to match the nearest neighbor distance.

The theoretical EXAFS spectrum was calculated to include contributions from two-atom and three-atom configurations. Within each $n$-atom configuration, all the MS contributions were taken into account. ${ }^{10,16}$ The two-atom and three-atom configurations were identified in each cluster up to $4.4 \AA$ and averaged with a frequency tolerance of $0.1 \AA$. The resultant information was used to calculate the various EXAFS $\gamma^{(2)}$ and $\gamma^{(3)}$ signals associated with each two-atom and three-atom contribution using the crystallographic bond lengths and distances.

The GNXAS fitting program constructs the theoretical absorption spectrum by summing all the $\gamma^{(2)}$ and $\gamma^{(3)}$ signals and compares this theoretical spectrum with the experimental absorption spectrum with the residual function $R$ being a measure of the quality of the fit. ${ }^{16}$ Least-square fits are performed on the averaged, energy-calibrated, raw absorption data without prior background subtraction or Fourier filtering. Raw data are compared directly with a model absorption coefficient composed of an appropriate background plus the oscillatory structural contribution from the theoretically calculated EXAFS. ${ }^{16}$ A spline of orders $3,4,4$ with defining energy points of $7155,7250,7600$, and $7998 \mathrm{eV}$ was used for most cases. If there was low-frequency noise in the Fourier transform (FT) the first defining energy point was adjusted by a maximum of $5 \mathrm{eV}$ until the noise was minimized. Least-squares fits were done with $k^{3}$ weighting where the first and the last spline points determined the range of the fit.

The structural parameters varied in the refinements were the distance $(R)$ and the bond variance $\left(\sigma_{R}^{2}\right)$, the mean square variation in the bond distance, for each two-atom configuration and the distances, the angle and the covariance matrix elements 10,16 for the three-atom configurations. Distances and angles were allowed to vary within a preset range, typically $\pm 0.05 \AA$ and $\pm 5^{\circ}$. Bond and angle variances and the off-diagonal covariance matrix elements were also allowed to vary in restricted ranges: $\pm 0.005 \AA^{2}$, \pm 50 (degrees) ${ }^{2}$ and \pm 0.5 , respectively. The coordination numbers were kept fixed to 
known crystallographic values. The nonstructural parameters in the fits were $E_{\mathrm{o}}$ (a parameter that aligns the experimental energy scale to the theoretical energy scale), $S_{0}{ }^{2}$ (many-body amplitude reduction factor), $\Gamma_{\mathrm{c}}$ (core-hole lifetime), and $E_{\mathrm{r}}$ (experimental resolution). These parameters were refined within narrow limits around expected values. 24,25

\subsubsection{Empirical EXAFS Analysis}

Data reduction and analysis using empirical phase and amplitude parameters was performed to obtain first-shell fits of the $\mathrm{Na}\left[\mathrm{Fe}\left(\mathrm{OH}_{2}\right) \mathrm{EDTA}\right]$ powder and solution, $\mathrm{Na}_{2}\left[\mathrm{Fe}\left(\mathrm{OH}_{2}\right)\right.$ EDTA] solution, and FeEDTA-NO solution according to the methods described previously $8 \mathrm{a}, 26$ and briefly summarized here. A pre-edge subtraction was performed by fitting the EXAFS region with a smooth second order polynomial function which was extrapolated into the pre-edge region and subtracted. A three segment spline approximately even in $k$-space with orders of two, three, and three was fit to the EXAFS region and subtracted and the data normalized to an edge jump of one at $7130 \mathrm{eV}$. The polynomial spline was chosen so that it minimized residual low-frequency noise but did not reduce the amplitude of the EXAFS, as judged by monitoring the FT of the EXAFS as a function of the spline fitting process. The normalized data were converted to $k$ space. The photoelectron wave vector, $k$, is defined by $\left[2 \mathrm{me}_{\mathrm{e}}\left(\mathrm{E}-\mathrm{E}_{0}\right) \pi^{2}\right]^{1 / 2}$ where $\mathrm{m}_{\mathrm{e}}$ is the electron mass, $E$ is the photon energy, $h$ is Planck's constant divided by $2 \pi$, and $E_{0}$ is the threshold energy of the absorption edge, which was defined to be $7130 \mathrm{eV}$ for the $\mathrm{Fe}$ $\mathrm{K}$ absorption edge. The empirical EXAFS data analyses were performed with nonlinear least-square curve-fitting $8 \mathrm{a}, 20,26$ techniques using empirical phase and amplitude parameters. The following models were used to obtain the empirical Fe-X backscattering parameters of interest: $\mathrm{Fe}-\mathrm{O}$ from $[\mathrm{Fe} \text { (acetylacetonate) }]^{27}$ and $\mathrm{Fe}-\mathrm{N}$ from $\left[\mathrm{Fe}(1,10 \text {-phenanthroline })_{3}\right]\left(\mathrm{ClO}_{4}\right)_{3} \cdot{ }^{22}$

Fourier transforms (from $k$ to $\mathrm{R}$ space) were performed for the data range 3.5$14.5 \AA^{-1}$ with a Gaussian window of $0.1 \AA^{-1}$. The window widths used in the backtransforms (from $\mathrm{R}$ to $k$ space) are presented in the Results and Discussion section. The window widths were kept as similar as possible to the windows used to extract amplitude and phase parameters from the model compounds to minimize artifacts introduced by the Fourier filtering technique. All curve-fitting was based on $k^{3}$-weighted data and applied to the individual filtered shell of interest. Only the structure-dependent parameters, the distance and coordination number, were varied. A "goodness of fit" parameter, $F$, was calculated as $F=\left\{\left[k^{6} \text { (data - fit }\right)^{2}\right] /($ no. of points $\left.)\right\}^{1 / 2}$ for each fit. 


\subsection{Results and Discussion}

\subsubsection{GNXAS Fits of $\{\mathrm{FeNO}\}^{7}$ Complexes with Known Fe-N-O Angles}

The GNXAS approach was used to fit the experimental EXAFS data of [Fe(TMC)NO] $\left(\mathrm{BF}_{4}\right)_{2}, \mathrm{Fe}(\mathrm{TACN})\left(\mathrm{N}_{3}\right)_{2} \mathrm{NO}$, and $\mathrm{Fe}(\mathrm{salen}) \mathrm{NO}$ at 10 and $220 \mathrm{~K}$. EXAFS contributions for each two-atom and three-atom configuration were calculated using crystallographic distances and bond angles. The individual contributions were then summed to generate a theoretical EXAFS spectrum which was then fit to the non-Fourier filtered experimental EXAFS data without prior background subtraction. In the fits, the crystallographic bond distances and angles were allowed to vary to fit the experimental EXAFS data. A comparison of the theoretical EXAFS spectrum to the experimental data (along with the individual EXAFS signal from each contribution) for each compound is presented in Figures 3.2 - 3.5. A comparison of the bond distances and angles obtained from the GNXAS fits to the crystallographic values is given in Table 3.1.

The best fit to the EXAFS data of [Fe(TMC)NO] $\left(\mathrm{BF}_{4}\right)_{2}$ is presented in Figure 3.2, with the corresponding FT presented in Figure 3.6A. The total EXAFS spectrum was accounted for by four contributions: $\mathrm{Fe}-\mathrm{N}(\mathrm{O}), \mathrm{Fe}-\mathrm{N}(\mathrm{TMC})$, Fe-N-O, and $\mathrm{Fe}-\mathrm{N}-\mathrm{C}$ [throughout this chapter, signals from three-atom configurations contain contributions from the three-atom multiple-scattering pathways $(\gamma(3)$ signal) and a two-atom contribution ( $\gamma^{(2)}$ signal) from the distant atom $\left.{ }^{16}\right]$. The GNXAS bond distances and angles match extremely well with the crystallographic values, deviating less than $0.01 \AA$ and $1^{\circ}$, respectively (Table 3.1). The linear Fe-N-O multiple-scattering signal is very strong due to the intervening atom focusing effect, ${ }^{16}$ allowing for very accurate bond angle determination. The crystallographic $\mathrm{Fe}-\mathrm{N}-\mathrm{O}$ bond angle is $177.5(5)^{\circ}$ and the $\mathrm{Fe}-\mathrm{N}-\mathrm{O}$ bond angle obtained from the GNXAS fit is $178^{\circ}$. In the numerous fits that were calculated the bond distances varied by $<0.02 \AA$ and the bond angles varied by $<1^{\circ}$.

The EXAFS data of $\mathrm{Fe}(\mathrm{TACN})\left(\mathrm{N}_{3}\right)_{2} \mathrm{NO}$ and the best fit to the data are presented in Figure 3.3 and the FT is shown in Figure 3.7B. The EXAFS spectrum is dominated by three two-atom signals: $\mathrm{Fe}-\mathrm{N}(\mathrm{O}), \mathrm{Fe}-\mathrm{N}\left(\mathrm{N}_{2}\right)$ and $\mathrm{Fe}-\mathrm{N}(\mathrm{TACN})$. The significant three-atom signals come from $\mathrm{Fe}-\mathrm{N}-\mathrm{O}, \mathrm{Fe}-\mathrm{N}-\mathrm{N}$ and $\mathrm{Fe}-\mathrm{N}-\mathrm{C}$ groups. The resultant bond distances and angles are all within $5 \%$ of the crystallographic values (see Table 3.1) with the $\mathrm{Fe}-\mathrm{N}-\mathrm{O}$ angle equal to $157^{\circ}$ (as compared to the crystallographic value of $156(1)^{\circ}$ ).

The fit to the Fe(salen)NO EXAFS data at $10 \mathrm{~K}$ with an Fe-N-O angle of $131^{\circ}$ is presented in Figure 3.4, with the FT of this fit to the data shown in Figure 3.8C. The two-atom and three-atom contributions included in the fit to the data were $\mathrm{Fe}-\mathrm{N}(\mathrm{O})$, 


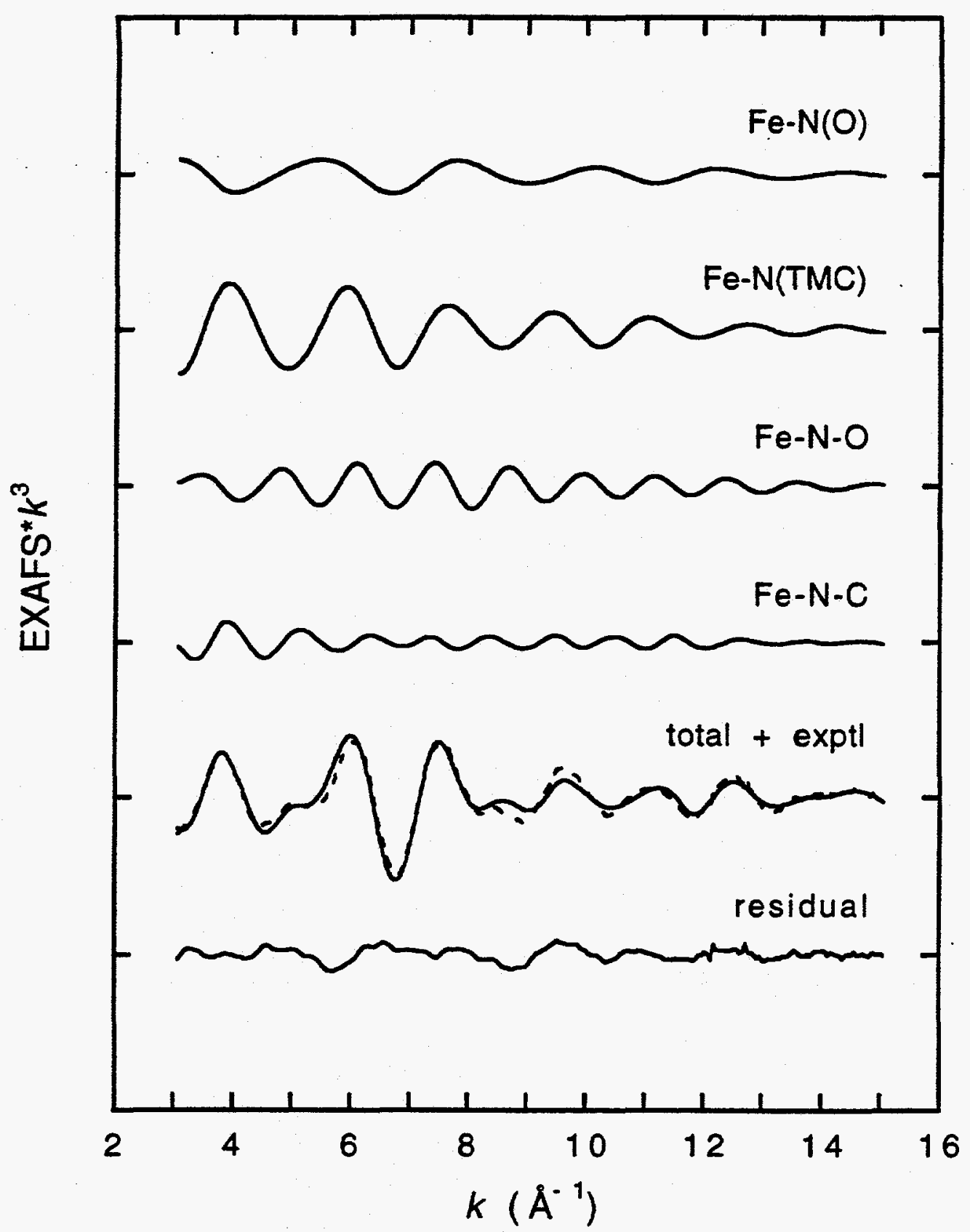

Figure 3.2. EXAFS signals for individual contributions in the best fit for the [Fe(TMC)NO] $\left(\mathrm{BF}_{4}\right)_{2}$ data. The total signal (-) is also shown and compared with the experimental data (---) with the residual being the difference between the experimental EXAFS and the theoretical EXAFS. (The ordinate scale is 10 between two consecutive tick marks.) Note the strength of the Fe-N-O contribution. 


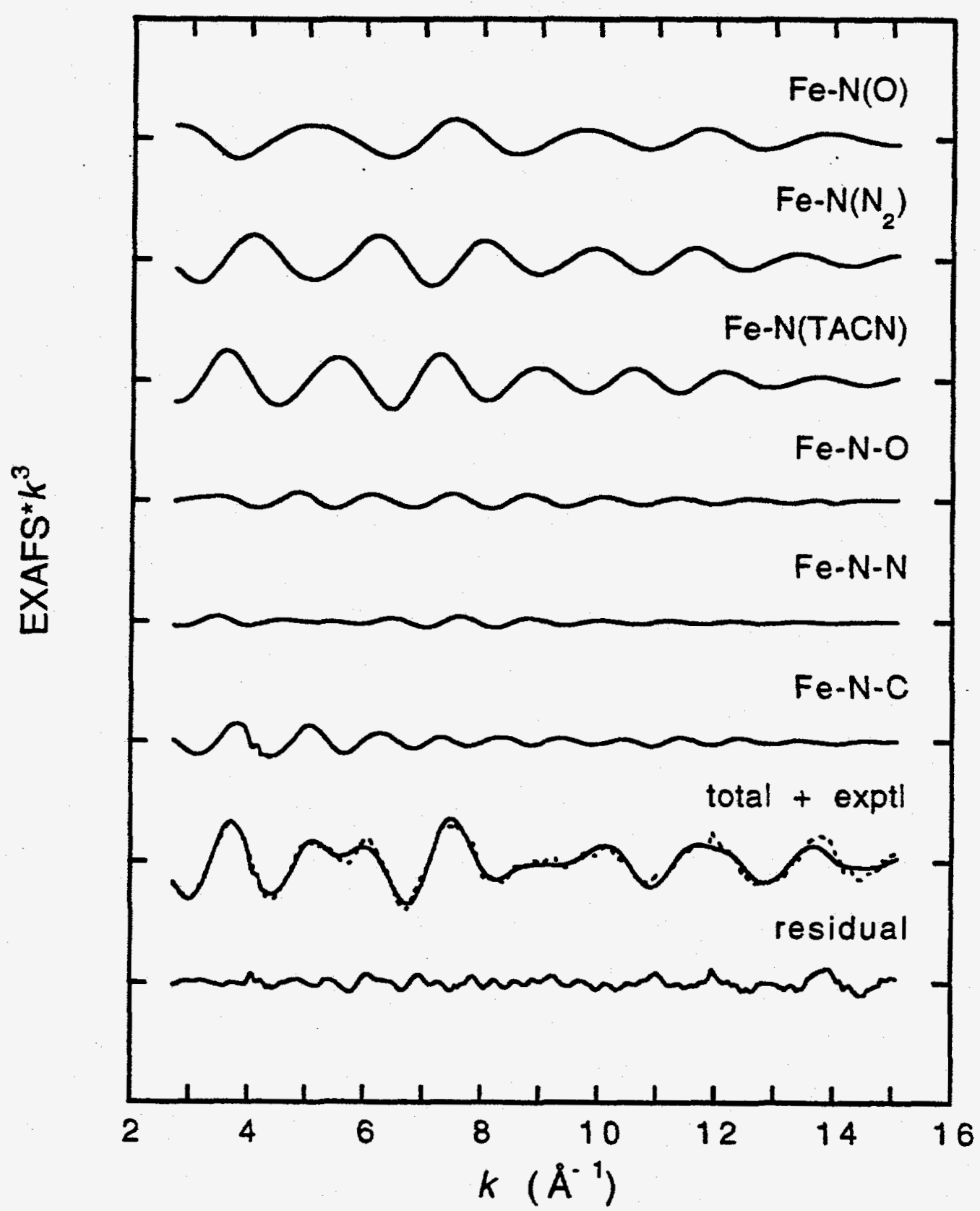

Figure 3.3. EXAFS signals for individual contributions in the best fit for the $\mathrm{Fe}(\mathrm{TACN})\left(\mathrm{N}_{3}\right)_{2} \mathrm{NO}$ data. The total signal (-) is also shown and compared with the experimental data (---) with the residual being the difference between the experimental EXAFS and the theoretical EXAFS. (The ordinate scale is 10 between two consecutive tick'marks.) 


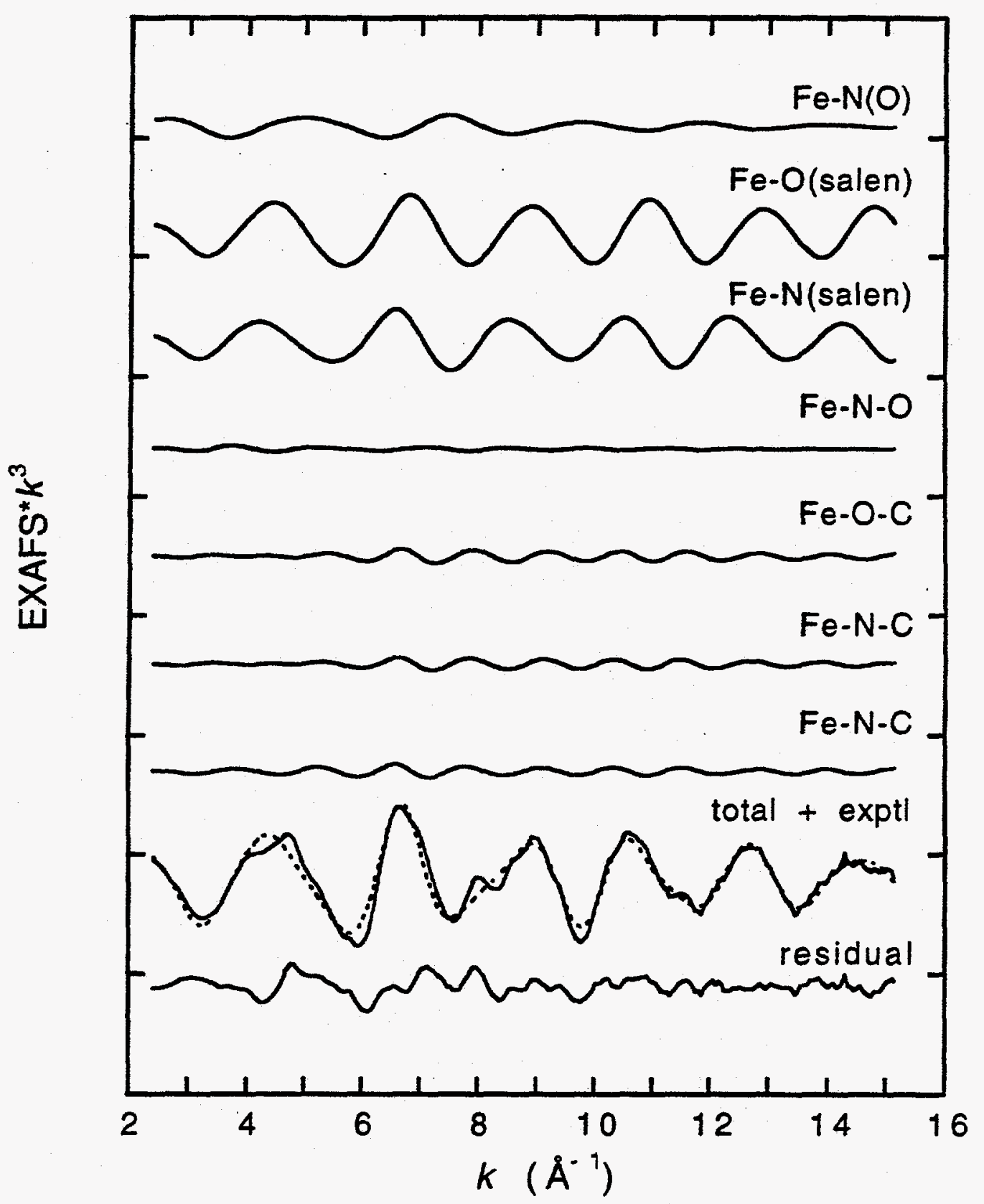

Figure 3.4. EXAFS signals for individual contributions in the best fit for the $\mathrm{Fe}$ (salen)NO at $10 \mathrm{~K}$ data. The total signal (-) is also shown and compared with the experimental data (--) with the residual being the difference between the experimental EXAFS and the theoretical EXAFS. (The ordinate scale is 10 between two consecutive tick marks.) Note that the $\mathrm{Fe}-\mathrm{N}$ and $\mathrm{Fe}-\mathrm{N}-\mathrm{O}$ signals are extremely weak. 
Table 3.1. Crystallographic Bond Distances and Angles Compared to GNXAS Results for $\{\mathrm{FeNO}\}^{7}$ Complexes with Known Fe-N-O Angles.

\section{GNXAS}

bond variance

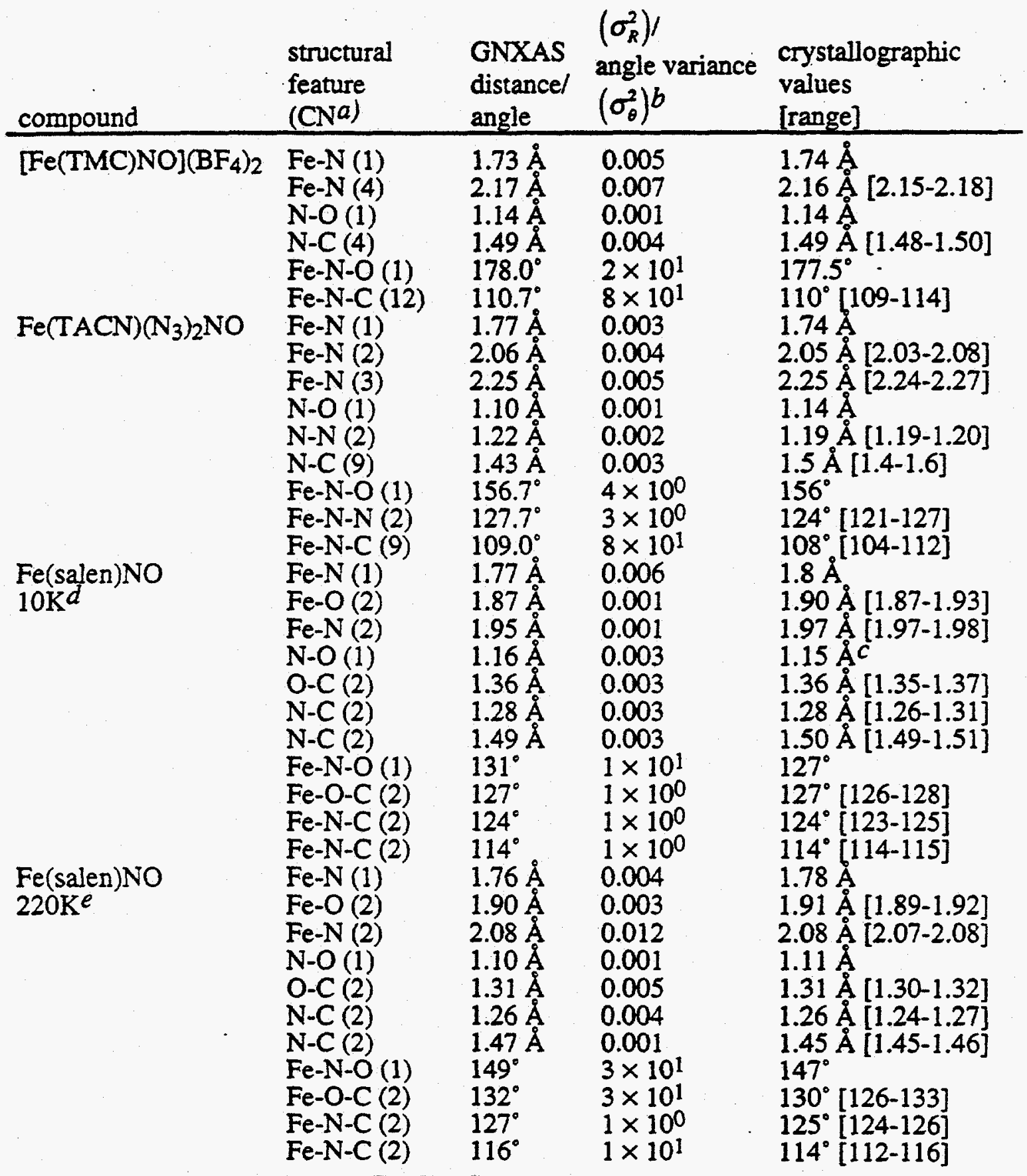

$a \mathrm{CN}=$ number of configurations in the complex. $b$ Bond and angle variances are reported in $\AA^{2}$ and degrees ${ }^{2}$, respectively. ${ }^{c}$ value was fixed in the crystal structure. $d_{\text {The }}$ crystal structure was determined at $-175^{\circ} \mathrm{C}$ and the EXAFS was measured at $10 \mathrm{~K}$. ${ }^{e}$ The crystal structure was determined at $23^{\circ} \mathrm{C}$ and the EXAFS was measured at $220 \mathrm{~K}$. 
Fe-O(salen), Fe-N(salen), Fe-N-O, Fe-O-C, and two Fe-N-C signals. The distances and angles obtained from the GNXAS fits were all within $1 \%$ of the crystallographic values, Table 3.1. In the Fe(salen)NO EXAFS data at $10 \mathrm{~K}$ the two-atom signals from the $O$ and $N$ of the salen ligand were very strong relative to the signal from the $N$ of the nitrosyl ligand. The bond variances $\left(\sigma_{R}^{2}\right)$ are $0.001 \AA^{2}$ for the Fe-O(salen) and Fe-N(salen) contributions and $0.006 \AA^{2}$ for the Fe-N(O) signal. The high bond variance and the associated weak signal for the $\mathrm{Fe}-\mathrm{N}(\mathrm{O})$ contribution could be due to the fact that the nitrosyl group is disordered. 18 The crystal structure of $\mathrm{Fe}$ (salen)NO at $-175^{\circ} \mathrm{C}$ shows a strongly disordered nitrosyl group with the standard deviation of the $\mathrm{Fe}-\mathrm{N}(\mathrm{O})$ distance being $0.1 \AA$ and a $1 \sigma$ variation of the Fe-N-O angle ranging from $115^{\circ}$ to $137^{\circ}$. Not only is the nitrosyl group disordered, but the Fe-N-O angle is below 150.' Significant enhancement of the multiple-scattering signal results when the atoms are arranged in approximately a collinear array, in which case the outgoing photoelectron is strongly forward scattered by the intervening atom. This effect drops off very rapidly for bond angles below $-150^{\circ} .6,7,16$ Since the $\mathrm{Fe}-\mathrm{N}(\mathrm{O})$ contribution has a high bond variance and the Fe-N-O angle is low $\left(-130^{\circ}\right)$, the Fe-N-O signal is extremely weak.

The best fit to the Fe(salen)NO EXAFS data at $220 \mathrm{~K}$ is presented in Figure 3.5, with the FT of the best fit to the data shown in Figure 3.9B. The two-atom and three-atom contributions included in the fit to the data were $\mathrm{Fe}-\mathrm{N}(\mathrm{O}), \mathrm{Fe}-\mathrm{O}$ (salen), $\mathrm{Fe}-\mathrm{N}$ (salen), Fe-N-O, Fe-O-C and two Fe-N-C signals. The distances and angles obtained from the GNXAS fits were all within $1 \%$ of the crystallographic values, Table 3.1. The crystal structure of $\mathrm{Fe}$ (salen)NO taken at $23^{\circ} \mathrm{C}$ was more accurately determined than the structure at $-175^{\circ} \mathrm{C}$, although the oxygen of the nitrosyl group showed some disorder. Two oxygens (OA and $O B$ ) were introduced into the crystallographic model with fixed occupancies of 0.5 ; the Fe-N-OA angle is $144(5)^{\circ}$ and the Fe-N-OB angle is $150(4)^{\circ}$. The Fe-N-O angle obtained from the GNXAS fit to the EXAFS data in Figure 3.5 was $149^{\circ}$ with an angle variance of 31 (degrees) $^{2}$, see Table 3.1. Predictably, the bond variances were higher for the $\mathrm{Fe}$ (salen)NO data collected at $220 \mathrm{~K}$, which is also seen in the lower magnitude at high $k$ in the EXAFS data (Figures 3.4 and 3.5).

Once best fits were obtained for each $\{\mathrm{FeNO}\}^{7}$ complex, the sensitivity of the fit to the Fe-N-O angle was tested by fixing all the distances, angles, and nonstructural parameters and calculating a theoretical EXAFS spectrum with $\mathrm{Fe}-\mathrm{N}-\mathrm{O}$ angles ranging from $90^{\circ}$ to $180^{\circ}$. The FTs of relevant calculated spectra for each compound are presented in Figures 3.6 - 3.9. Plots of $\log$ ( $R$ values) vs Fe-N-O angle for each complex are shown in Figure 3.10 (the log function allows the plots to be scaled for comparison). A minimum in these plots is indicative of a better fit to the experimental EXAFS data. 


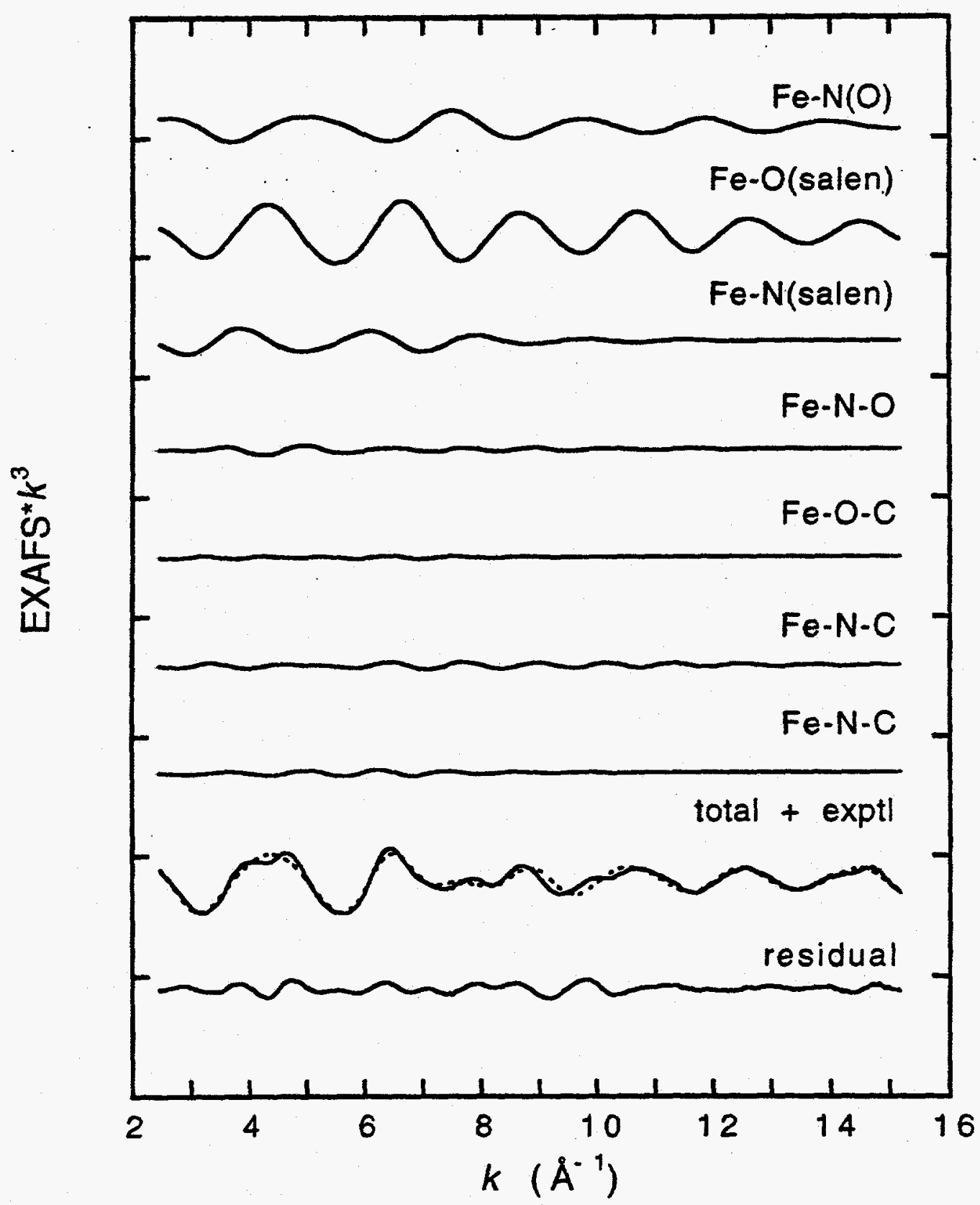

Figure 3.5. EXAFS signals for individual contributions in the best fit for the $\mathrm{Fe}$ (salen)NO at $220 \mathrm{~K}$ data. The total signal (-) is also shown and compared with the experimental data (---) with the residual being the difference between the experimental EXAFS and the theoretical EXAFS. (The ordinate scale is 10 between two consecutive tick marks.) 
The FTs of the calculated GNXAS spectra with the Fe-N-O angles of $178^{\circ}$ (best fit), $150^{\circ}$, and $120^{\circ}$ for the [Fe(TMC)NO] $\left(\mathrm{BF}_{4}\right)_{2}$ data are presented in Figure 3.6. The second peak in the FT at $2.5 \AA$, which is due to the Fe-N-O multiple-scattering signal, cannot be accounted for without an Fe-N-O angle that is close to linear. The $R$ value dramatically increases in calculated spectra where the $\mathrm{Fe}-\mathrm{N}-\mathrm{O}$ angle is below $170^{\circ}$ (Figure 3.10A). Due to the strength of the multiple-scattering signal from an approximately linear $\mathrm{Fe}-\mathrm{N}-\mathrm{O}$ unit, the calculated spectrum is extremely sensitive to the Fe-N-O angle.

Figure 3.7 shows the FTs of calculated spectra for $\mathrm{Fe}(\mathrm{TACN})\left(\mathrm{N}_{3}\right)_{2} \mathrm{NO}$ with an Fe-N-O angle of $180^{\circ}, 157^{\circ}$ (best fit), and $120^{\circ}$. The FT of the calculated spectrum with Fe-N-O equal to $180^{\circ}$ does not match the FT of the experimental EXAFS data. When the $\log (R$ value $)$ is plotted $v s$ the Fe-N-O angle there is a minimum between $155^{\circ}$ and $160^{\circ}$ (Figure 3.10B) with the crystallographic Fe-N-O angle for $\mathrm{Fe}(\mathrm{TACN})\left(\mathrm{N}_{3}\right)_{2} \mathrm{NO}$ being $156^{\circ}$. There is a second shallower minimum in the $R$ value at $110^{\circ}$. Upon inspection of the $\mathrm{Fe}-\mathrm{N}-\mathrm{O}$ multiple-scattering signal and the $\mathrm{Fe}-\mathrm{O}$ (of the $\mathrm{Fe}-\mathrm{N}-\mathrm{O}$ ) single-scattering signal, it was observed that in the low- $k$ region the $\mathrm{Fe}-\mathrm{O}$ signal with an $\mathrm{Fe}-\mathrm{N}-\mathrm{O}$ angle of $110^{\circ}$ was in-phase and of the same order of magnitude as the Fe-N-O multiple-scattering signal with an Fe-N-O angle equal to $156^{\circ}$. Therefore the single-scattering $\mathrm{Fe}-\mathrm{O}$ signal with a Fe-N-O angle of $110^{\circ}$ was able to mimic the multiple-scattering $\mathrm{Fe}-\mathrm{N}-\mathrm{O}$ signal with a Fe-N-O angle equal to $156^{\circ}$ for $k$ less than $6 \AA^{-1}$, giving a false minimum in the $\log (R$ value $)$ vs Fe-N-O angle plot. The multiple-scattering contribution for a three-atom configuration dominates for angles above $150^{\circ}$, while the single-scattering signal is important for values below $150^{\circ}, 6,7,16$ Thus, due to the sinusoidal nature of EXAFS, a double minimum occurs when the $\log (R$ value $)$ is plotted $v s$ the Fe-N-O angle, where in one case the single-scattering signal $(\mathrm{Fe}-\mathrm{O})$ has a phase and amplitude that matches the experimental data and in the other case the multiple-scattering signal ( $\mathrm{Fe}-\mathrm{N}-\mathrm{O}$ ) has a phase and amplitude that matches the data.

The FTs for calculated spectra of $\mathrm{Fe}($ salen)NO at $10 \mathrm{~K}$ with $\mathrm{Fe}-\mathrm{N}-\mathrm{O}$ values of $180^{\circ}, 150^{\circ}$, and $131^{\circ}$ are shown in Figure 3.8. Since the theoretical spectra were calculated using the bond distances, angles, and the covariance matrix elements of the fit in Figure 3.4, the bond variance associated with the $\mathrm{Fe}-\mathrm{N}(\mathrm{O})$ signal was very high, $0.006 \AA^{2}$. The high $\mathrm{Fe}-\mathrm{N}(\mathrm{O})$ bond variance made the $\mathrm{Fe}-\mathrm{N}-\mathrm{O}$ signal extremely weak at all angles, even at $180^{\circ}$. Due to the weak Fe-N-O signal, the $R$ values of these fits are all very similar and insensitive to the Fe-N-O angle (Figure 3.10D). Thus, a signal from a three-atom configuration must be a significant component in the total EXAFS signal in order for GNXAS to provide angular information. 


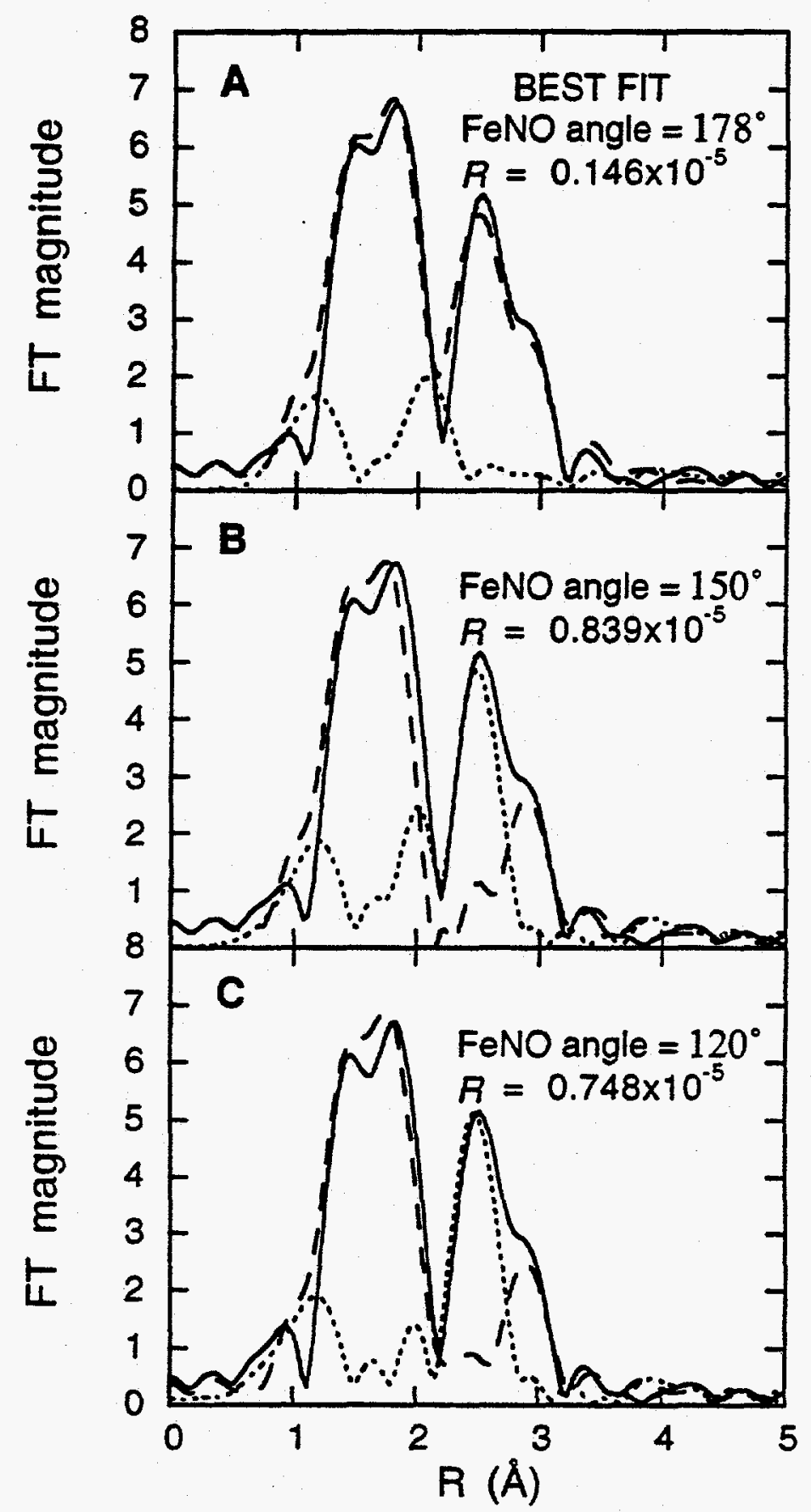

Figure 3.6. A comparison of the theoretical (--) and experimental (-) non-phase shift corrected FT of [Fe(TMC)NO] $\left(\mathrm{BF}_{4}\right)_{2}$ EXAFS data, along with the FT of the EXAFS residual (....). The $R$ value is an indication of the goodness of the fit. Calculated spectra for several different Fe-N-O bond angles are shown: (A)178 (best fit), (B) $150^{\circ}$, and (C) $120^{\circ}$. This $\{\mathrm{FeNO}\}^{7}$ complex has a crystallographic Fe-N-O bond angle of $177.5(5)^{\circ}$. 


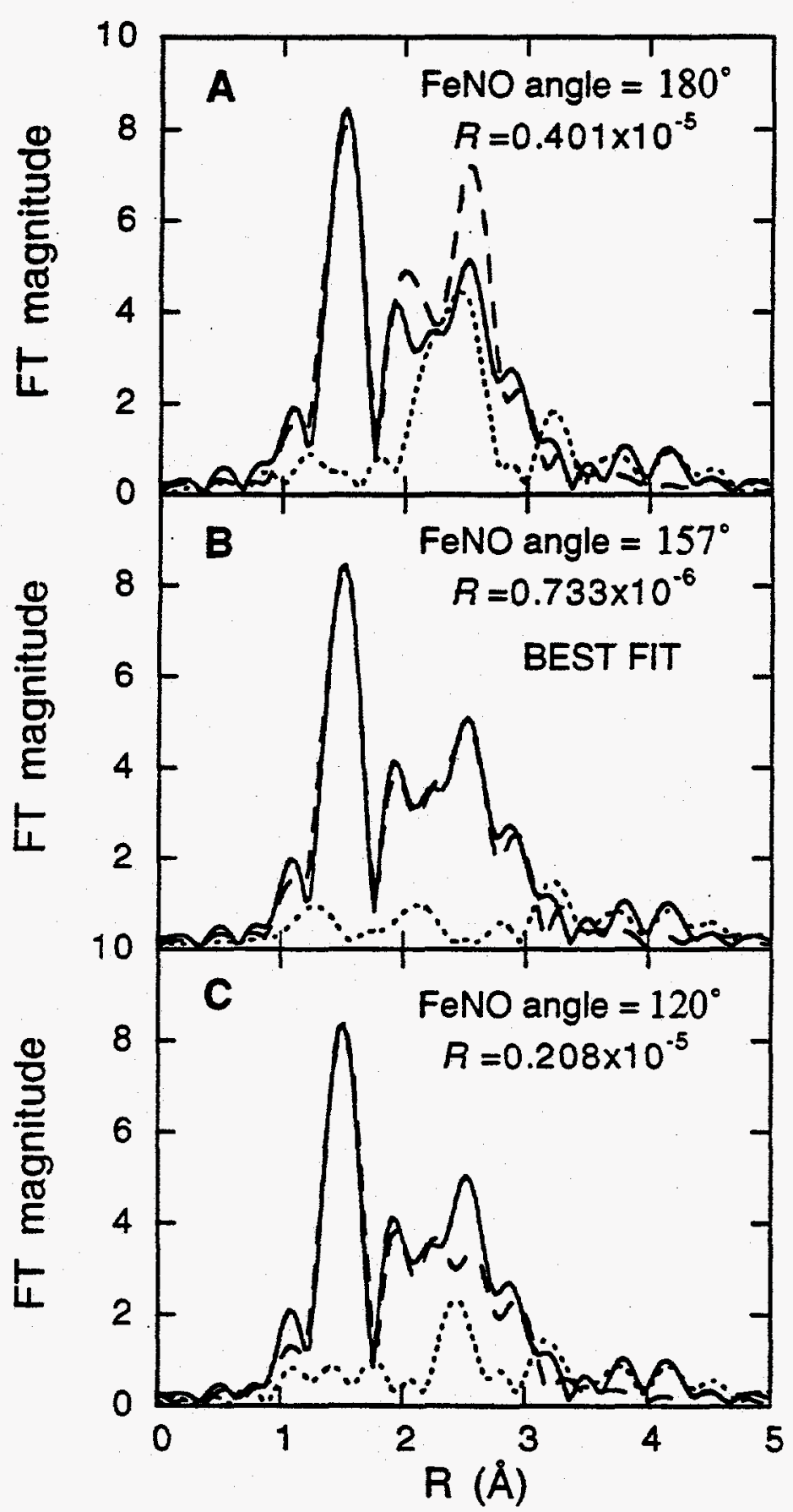

Figure 3.7. A comparison of the theoretical (--) and experimental $(-)$ non-phase shift corrected FT of $\mathrm{Fe}(\mathrm{TACN})\left(\mathrm{N}_{3}\right)_{2} \mathrm{NO}$ EXAFS data, along with the FT of the EXAFS residual (...). The $R$ value is an indication of the goodness of the fit. Calculated spectra for several different Fe-N-O bond angles are shown: (A) $180^{\circ}$, (B) $157^{\circ}$ (best fit), and (C) $120^{\circ}$. This $\{\mathrm{FeNO}\}^{7}$ complex has a crystallographic $\mathrm{Fe}-\mathrm{N}-\mathrm{O}$ bond angle of $156(1)^{\circ}$. 


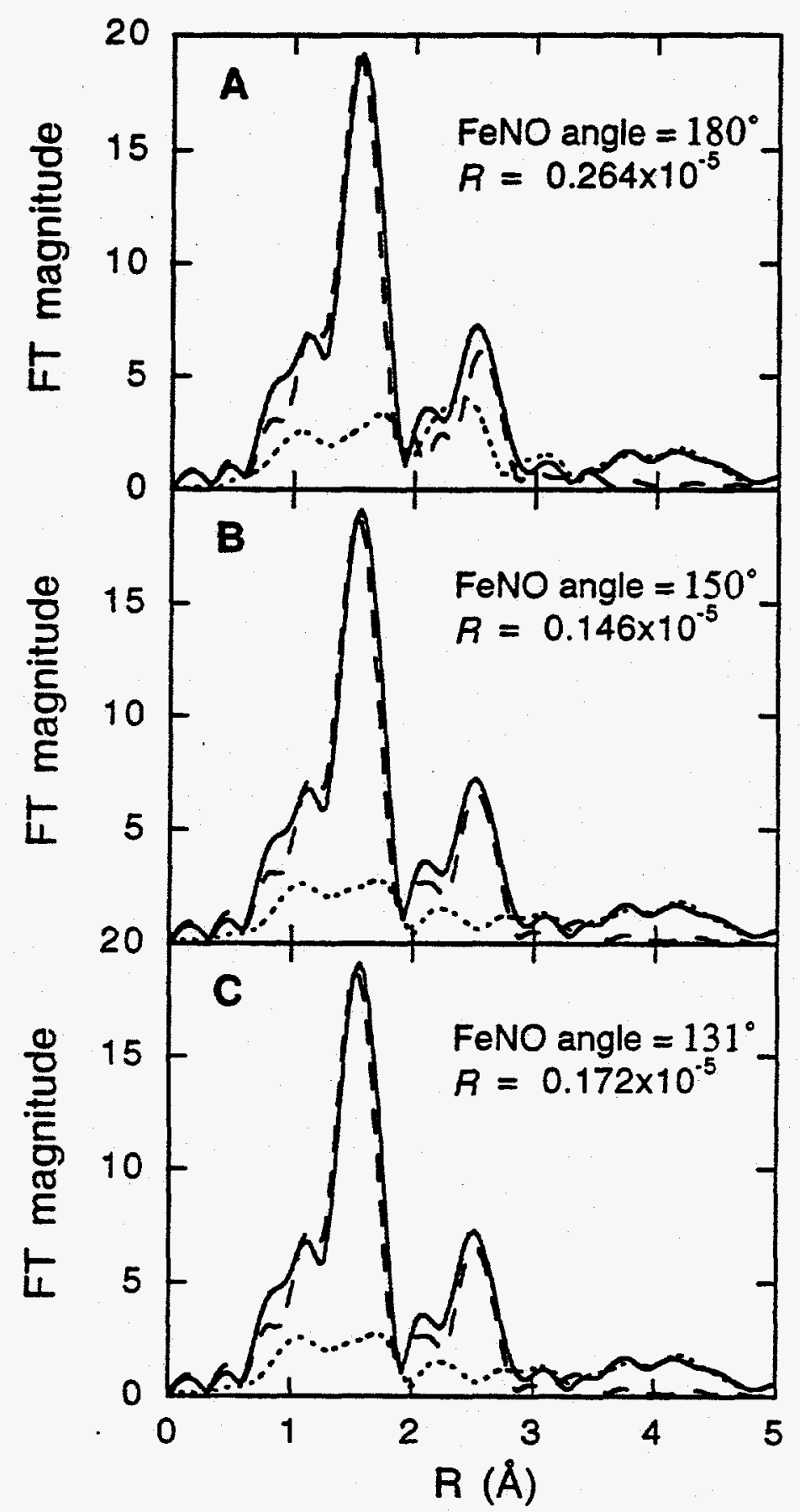

Figure 3.8. A comparison of the theoretical (--) and experimental (-) non-phase shift corrected FT of Fe(salen)NO at $10 \mathrm{~K}$ EXAFS data, along with the FT of the EXAFS residual $(\cdots . \cdots)$. The $R$ value is an indication of the goodness of the fit. Calculated spectra for several different $\mathrm{Fe}-\mathrm{N}-\mathrm{O}$ bond angles are shown: (A) $180^{\circ}$, (B) $150^{\circ}$, and (C) $131^{\circ}$. This $\{\mathrm{FeNO}\}^{7}$ complex has a crystallographic Fe-N-O bond angle of $127(6)^{\circ}$. 


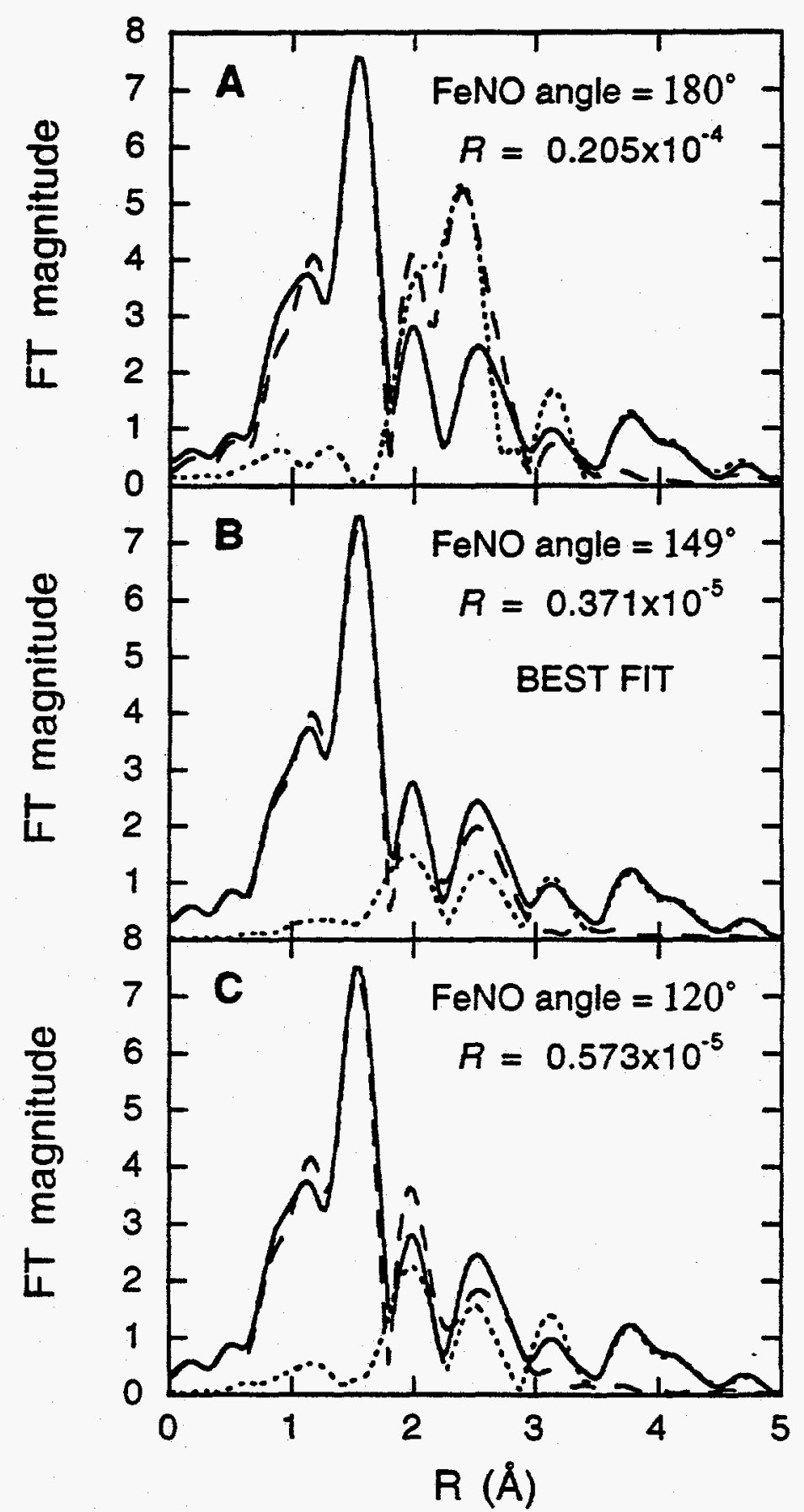

Figure 3.9. A comparison of the theoretical $(--)$ and experimental $(-)$ non-phase shift corrected FT of $\mathrm{Fe}$ (salen)NO at $220 \mathrm{~K}$ EXAFS data, along with the FT of the EXAFS residual (....). The $R$ value is an indication of the goodness of the fit. Calculated spectra for several different Fe-N-O bond angles are shown: (A) $180^{\circ}$, (B) $149^{\circ}$ (best fit), and (C) $120^{\circ}$. This model compound has a crystallographic bond angle of $147(5)^{\circ}$. 


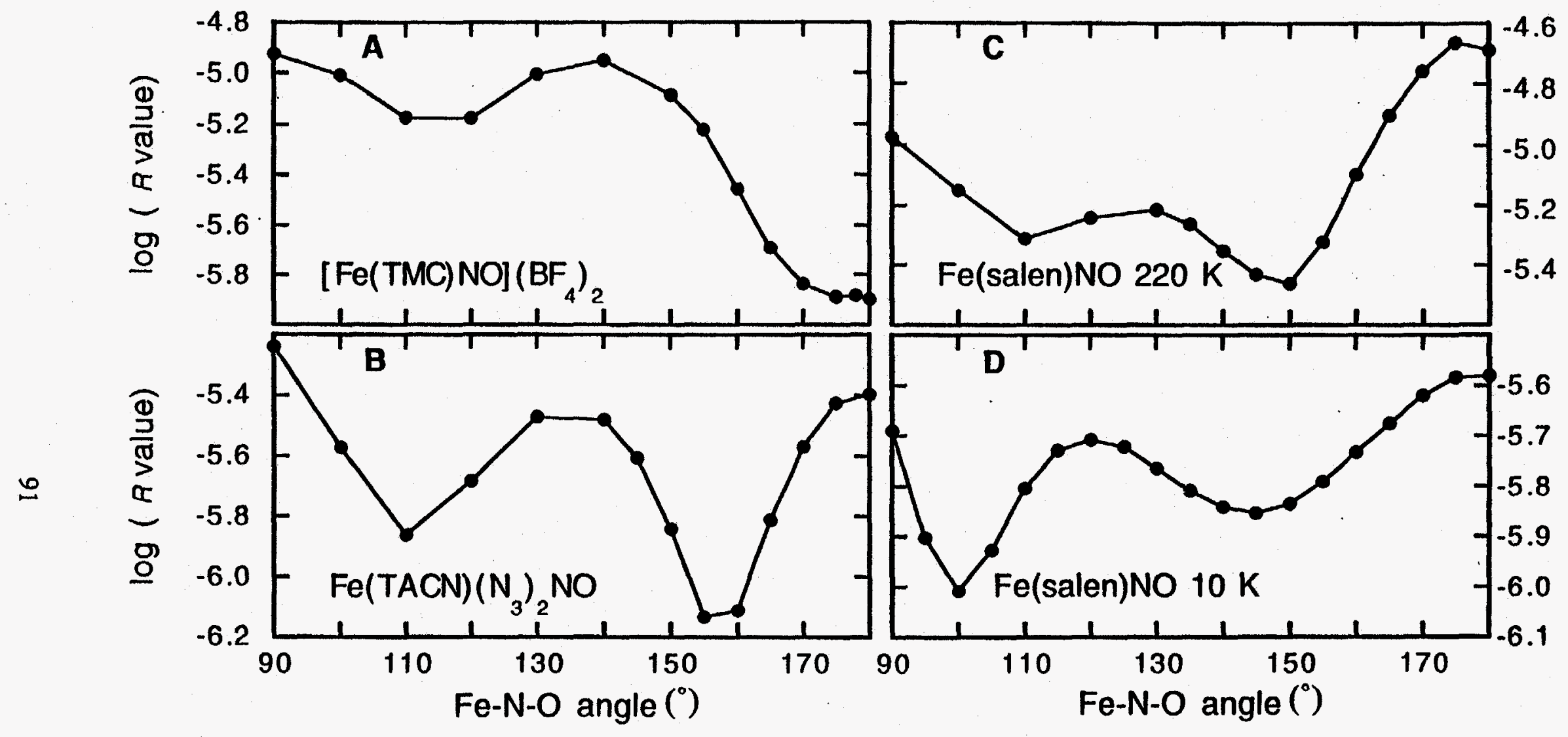

Figure 3.10. Plots of the $\log \left(R\right.$ value) vs Fc-N-O angle for (A) $[\mathrm{Fe}(\mathrm{TMC}) \mathrm{NO}]\left(\mathrm{BF}_{4}\right)_{2}$, (B) $\mathrm{Fe}(\mathrm{TACN})\left(\mathrm{N}_{3}\right)_{2} \mathrm{NO}$ and (C) $\mathrm{Fe}(\mathrm{salen}) \mathrm{NO}$ at $220 \mathrm{~K}$ and (D) $10 \mathrm{~K}$ where each point represents a match of the calculated EXAFS spectrum to the data. The lower the $R$ value the better the fit. The deep minimum in the $[\mathrm{Fe}(\mathrm{TMC}) \mathrm{NO}]\left(\mathrm{BF}_{4}\right)_{2}$ data at high angles indicates the Fe- $\mathrm{N}-\mathrm{O}$ angle must be over $170^{\circ}$. The plot for the $\mathrm{Fe}(\mathrm{TACN})\left(\mathrm{N}_{3}\right)_{2} \mathrm{NO}$ data has a double minimum with the dominant minimum being around $155^{\circ}$. The minimum in the $\mathrm{Fe}$ (salen) $220 \mathrm{~K}$ data is at $\sim 150^{\circ}$, while $R$ values for the $\mathrm{Fe}$ (salen)NO at $10 \mathrm{~K}$ were nearly insensitive to variations of the $\mathrm{Fe}-\mathrm{N}-\mathrm{O}$ angle due to the weak Fe-N-O signal. 
The FTs for calculated spectra of Fe(salen)NO at $220 \mathrm{~K}$ with Fe-N-O angles of $180^{\circ}, 149^{\circ}$ (best fit), and $120^{\circ}$ are shown in Figure 3.9. Fits above $155^{\circ}$ have relatively high $R$ values, however the $R$ values for all the fits below $155^{\circ}$ are very similar (Figure $3.10 \mathrm{C}$ ). In this case, application of GNXAS to the data only allows an upper limit of $155^{\circ}$ to be set for the Fe-N-O angle.

\subsubsection{Fe-N-O Angle Determination of an $\{\mathrm{FeNO}\}^{7}$ Complex of Unknown Structure}

GNXAS analysis was used to investigate the Fe-N-O angle of FeEDTA-NO, a complex whose structure is unknown. GNXAS requires an initial structural model. Therefore, EXAFS data of several FeEDTA complexes were obtained and compared to FeEDTA-NO to determine a suitable initial structural model. Using the empirical EXAFS data analysis method, first-shell distances were obtained for $\mathrm{Na}\left[\mathrm{Fe}\left(\mathrm{OH}_{2}\right) \mathrm{EDTA}\right]$ powder, $\mathrm{Na}\left[\mathrm{Fe}\left(\mathrm{OH}_{2}\right)\right.$ EDTA] solution, $\mathrm{Na} 2\left[\mathrm{Fe}\left(\mathrm{OH}_{2}\right)\right.$ EDTA] solution, and FeEDTA-NO solution. The EXAFS of both the powder and solution forms of $\mathrm{Na}\left[\mathrm{Fe}\left(\mathrm{OH}_{2}\right) \mathrm{EDTA}\right]$ were studied to determine if there is any significant structural change between solid and solution forms. The results of the first-shell empirical fits are given in Table 3.2 and Figure 3.11 .

The first-shell distances obtained from the best fit, Fit \#1, to the $\mathrm{Na}\left[\mathrm{Fe}\left(\mathrm{OH}_{2}\right)\right.$ EDTA] powder data match extremely well to the crystallographic values of $\mathrm{Li}\left[\mathrm{Fe}\left(\mathrm{OH}_{2}\right)\right.$ EDTA $] \cdot 2 \mathrm{H}_{2} \mathrm{O}^{19}$ (Table 3.3) with two shells of $\mathrm{O}$ at $\sim 2.0$ and $\sim 2.1 \AA$ and $2 \mathrm{~N}$ at $\sim 2.3 \AA$. The first-shell distances obtained from the best fit, Fit \#2, to the $\mathrm{Na}\left[\mathrm{Fe}\left(\mathrm{OH}_{2}\right) \mathrm{EDTA}\right]$ solution data are very similar to those of the powder sample. The shorter Fe-O distance was elongated by $0.02 \AA$ in the solution sample and the coordination numbers were slightly different. It appears that there are no major changes in the first shell of the $\mathrm{Na}\left[\mathrm{Fe}\left(\mathrm{OH}_{2}\right) \mathrm{EDTA}\right]$ structure between the solution and the powder since both the difference in the short Fe-O distance and the differences in the coordination numbers are within the uncertainty of the technique. The first-shell distances obtained from the best fit, Fit \#3, to the $\mathrm{Na}_{2}\left[\mathrm{Fe}\left(\mathrm{OH}_{2}\right)\right.$ EDTA] EXAFS data are given in Table 3.2. While the presence of two shells of $O$ could not be resolved in the reduced form, an average distance of $2.17 \AA$ was obtained which is $0.12 \AA$ longer than the average $\mathrm{Fe}-\mathrm{O}$ distance in $\mathrm{Na}\left[\mathrm{Fe}\left(\mathrm{OH}_{2}\right)\right.$ EDTA].

Two fits (Fits \#4 and \#5) are shown (Figure 3.11) for the FeEDTA-NO solution, one fit with and one without a short Fe-N bond from the Fe-N-O unit. Fit \#4, which includes the short Fe- $\mathrm{N}$ bond, is substantially better than Fit \#5, without the short $\mathrm{Fe}-\mathrm{N}$ bond, with the $F$ value being over a factor of 2 lower for Fit \#4. The best fit to the 


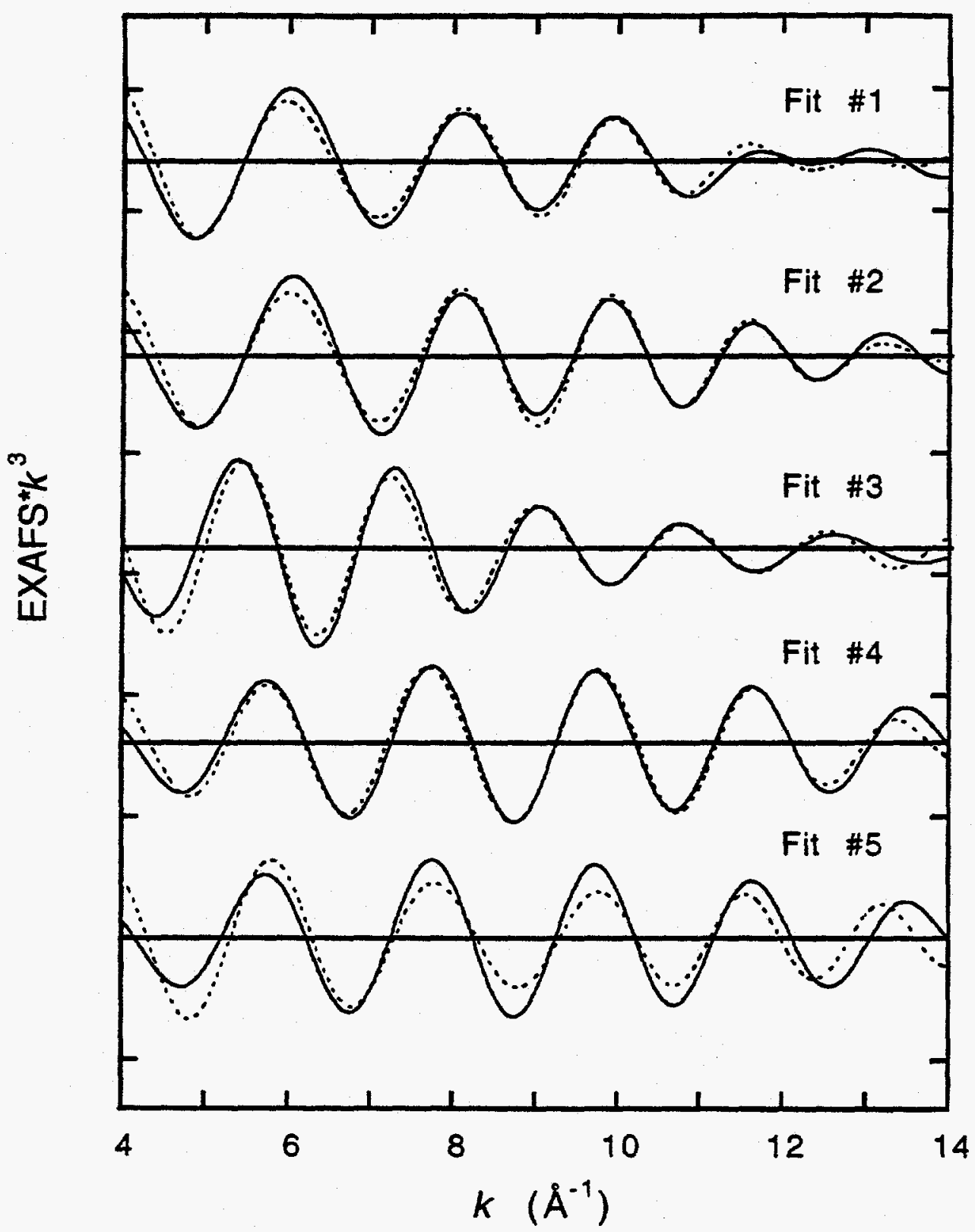

Figure 3.11. Empirical first-shell fits to the Fourier-filtered EXAFS data with the solid line representing the experimental data and the dashed line representing the fit to the data. Fits $\# 1, \# 2$, and $\# 3$ are the best empirical fits to the $\mathrm{Na}\left[\mathrm{Fe}\left(\mathrm{OH}_{2}\right) \mathrm{EDTA}\right]$ powder data (Table 3.2), to the $\mathrm{Na}\left[\mathrm{Fe}\left(\mathrm{OH}_{2}\right) \mathrm{EDTA}\right]$ solution data, and to the $\mathrm{Na}_{2}\left[\mathrm{Fe}\left(\mathrm{OH}_{2}\right) \mathrm{EDTA}\right]$ solution data, respectively. Fits \#4 and \#5 are fits to the FeEDTA-NO data with Fit \#4 containing a short $\mathrm{Fe}-\mathrm{N}$ distance. (The ordinate scale is 5 between consecutive tick marks with solid horizontal lines going through the zero point of each plot.) 
Table 3.2. First Shell Empirical Fits of FeEDTA Complexes.

\begin{tabular}{|c|c|c|c|c|c|c|}
\hline sample & Fit \# & $\begin{array}{l}\text { FT window } \\
\text { width }(\AA)\end{array}$ & element & $\mathrm{CN}^{a}$ & $\begin{array}{c}\text { bond length } \\
(\AA)\end{array}$ & $F^{b}$ \\
\hline $\begin{array}{c}\mathrm{Na}\left[\mathrm{Fe}\left(\mathrm{OH}_{2}\right) \mathrm{EDTA}\right] \\
\text { (fowder) }\end{array}$ & $\# 1$ & {$[0.8-2.2]$} & $\begin{array}{l}\mathrm{O} \\
\mathrm{O} \\
\mathrm{N}\end{array}$ & $\begin{array}{l}2.5 \\
2.5 \\
1.8\end{array}$ & $\begin{array}{l}1.98 \\
2.12 \\
2.34\end{array}$ & 0.40 \\
\hline$\underset{\text { (solution) }}{\mathrm{Na}\left[\mathrm{Fe}\left(\mathrm{OH}_{2}\right) \mathrm{EDT}\right]}$ & \#2 & {$[0.8-2.2]$} & $\begin{array}{l}\mathrm{O} \\
\mathrm{O} \\
\mathrm{N}\end{array}$ & $\begin{array}{l}2.7 \\
1.6 \\
1.6\end{array}$ & $\begin{array}{l}2.00 \\
2.12 \\
2.34\end{array}$ & 0.40 \\
\hline$\underset{\text { (solution) }}{\mathrm{Na}_{2}\left[\mathrm{Fe}\left(\mathrm{OH}_{2}\right) \mathrm{EDTA}\right]}$ & $\# 3$ & {$[1.0-2.2]$} & $\stackrel{\mathrm{O}}{\mathrm{N}}$ & $\begin{array}{l}3.4 \\
1.9\end{array}$ & $\begin{array}{l}2.17 \\
2.34\end{array}$ & 0.46 \\
\hline \multirow[t]{2}{*}{$\begin{array}{l}\text { FeEDTA-NO } \\
\text { (solution) }\end{array}$} & \#4 & {$[1.1-2.0]$} & $\begin{array}{l}\mathbf{N} \\
\mathbf{O} \\
\mathbf{N}\end{array}$ & $\begin{array}{l}1.1 \\
3.2 \\
1.5\end{array}$ & $\begin{array}{l}1.76 \\
2.05 \\
2.27\end{array}$ & 0.39 \\
\hline & \#5 & & $\stackrel{\mathrm{O}}{\mathrm{N}}$ & $\begin{array}{l}3.5 \\
1.9\end{array}$ & $\begin{array}{l}2.06 \\
2.28\end{array}$ & 0.85 \\
\hline
\end{tabular}


Table 3.3. Comparision of the Li[Fe( $\left.\mathrm{OH}_{2}\right)$ EDTA] $2 \mathrm{H}_{2} \mathrm{O}$ Crystallographic Bond Distances and Angles to the GNXAS and Empirical Fitted Bond Distances and Angles for $\mathrm{Na}$ [Fe( $\left.\mathrm{OH}_{2}\right)$ EDTA] Solution and Powder and FeEDTA-NO.

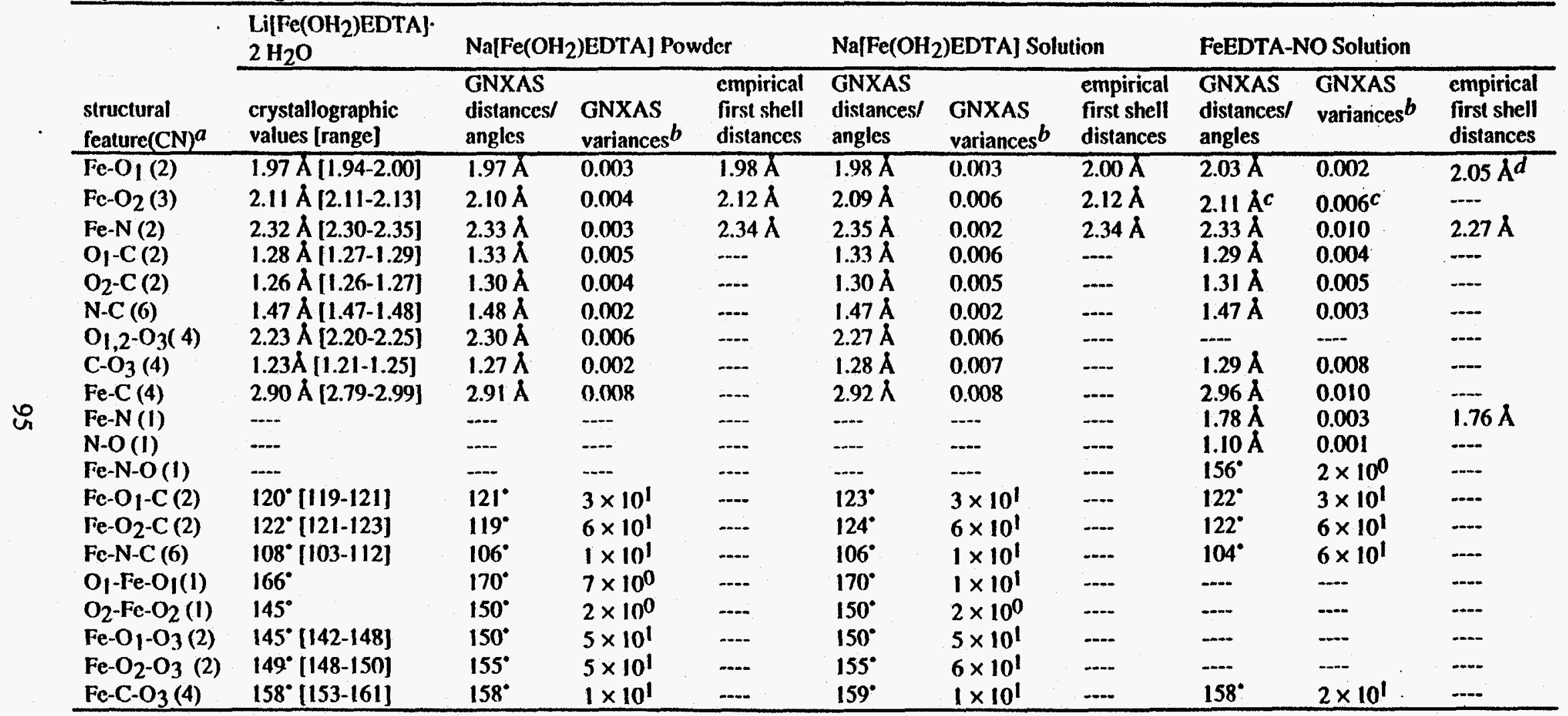

$a_{\mathrm{CN}}=$ number of configurations in the complex. ${ }^{b}$ Bond variances $\left(\sigma_{R}^{2}\right)$ and angle variances $\left(\sigma_{\theta}^{2}\right)$ are reported in $\AA^{2}$ and degrecs ${ }^{2}$, respectively.

$c$ coordination number was fixed at $2 . d$ average of both Fe-O shells 
FeEDTA-NO data has $1.1 \mathrm{~N}$ at $1.76 \AA, 3.2 \mathrm{O}$ at $2.05 \AA$, and $1.5 \mathrm{~N}$ at $2.27 \AA$. The short $1.76 \AA$ Fe-N distance is typical for the $\{\mathrm{FeNO}\}^{7}$ systems. ${ }^{4}$ The $\mathrm{Fe}-\mathrm{O}$ distance appears to be an average of two Fe-O shells, which could not be resolved given the range of available data. A fit with four contributions was attempted (Fe-N at $\sim 1.8 \AA, \mathrm{Fe}-\mathrm{O}$ at $2.0 \AA, \mathrm{Fe}-\mathrm{O}$ at $\sim 2.1 \AA$ and $\mathrm{Fe}-\mathrm{N}$ at -2.3 ) but both $\mathrm{Fe}-\mathrm{O}$ distances coalesced at $2.05 \AA$ with an $F$ value identical to that for Fit \#4.

The $\mathrm{Fe}-\mathrm{O}$ and $\mathrm{Fe}-\mathrm{N}$ distances of the EDTA ligand in FeEDTA-NO are more similar to the respective distances in $\mathrm{Na}\left[\mathrm{Fe}\left(\mathrm{OH}_{2}\right) \mathrm{EDTA}\right]$ than those in $\mathrm{Na}_{2}\left[\mathrm{Fe}\left(\mathrm{OH}_{2}\right)\right.$ EDTA]. In addition, the XAS edge of FeEDTA-NO is more similar to the edge of $\mathrm{Na}\left[\mathrm{Fe}\left(\mathrm{OH}_{2}\right) \mathrm{EDTA}\right]$ than to that of $\mathrm{Na} 2\left[\mathrm{Fe}\left(\mathrm{OH}_{2}\right) \mathrm{EDTA}\right] .^{5}$ The coordination number of the oxygens varies in a chemically reasonable way for the solid $\mathrm{Na}\left[\mathrm{Fe}\left(\mathrm{OH}_{2}\right) \mathrm{EDTA}\right]$, solution $\mathrm{Na}\left[\mathrm{Fe}\left(\mathrm{OH}_{2}\right) \mathrm{EDTA}\right]$, and FeEDTA-NO. The crystallographically-characterized [Fe( $\left(\mathrm{OH}_{2}\right)$ EDTA] $]^{-}$has 5 oxygens in the first shell and the best fits to the $\mathrm{Na}\left[\mathrm{Fe}\left(\mathrm{OH}_{2}\right) \mathrm{EDTA}\right]$ powder and solution data give an oxygen coordination number of 5.0 and 4.3 , respectively. The somewhat lower coordination number in solution could be related to an increased disorder in the solution. The NO seems to take the place of the $\mathrm{H}_{2} \mathrm{O}$ at $2.11 \AA$, since the oxygen coordination number has decreased to 3.2 in the best FeEDTA-NO fit.

The GNXAS approach was used to analyze EXAFS data of $\mathrm{Na}\left[\mathrm{Fe}\left(\mathrm{OH}_{2}\right)\right.$ EDTA] powder and solution to ensure that the MS contributions could be properly accounted for and that reliable second and third shell bond distances and bond angles could be obtained. The results of the fits to the $\mathrm{Na}\left[\mathrm{Fe}\left(\mathrm{OH}_{2}\right) \mathrm{EDTA}\right]$ solution data also provided values for bond and angle variances and the off-diagonal covariance matrix elements for the fits to the FeEDTA-NO data.

The best fit to the $\mathrm{Na}\left[\mathrm{Fe}\left(\mathrm{OH}_{2}\right)\right.$ EDTA] powder data is presented and discussed in Chapter 2. The low-frequency EXAFS is dominated by three waves from two-atom contributions: $\mathrm{Fe}-\mathrm{O}_{1}, \mathrm{Fe}-\mathrm{O}_{2}$ and $\mathrm{Fe}-\mathrm{N}$, where $\mathrm{O}_{1}$ refers to the oxygen at $1.97 \AA$ and $\mathrm{O}_{2}$ refers to the oxygen at $2.11 \AA$. The EXAFS distances for these three shells show excellent agreement with the crystallographic values of the $\mathrm{Li}\left[\mathrm{Fe}\left(\mathrm{OH}_{2}\right) \mathrm{EDTA}\right] \cdot 2 \mathrm{H}_{2} \mathrm{O}, 19$ deviating by $<0.01 \AA$ (Table 3.3). There were approximately 30 unique three-atom configurations which ranged in distance from 3.0 to $4.5 \AA$. The eight main contributions were from $\mathrm{Fe}-\mathrm{O}_{1}-\mathrm{C}, \mathrm{Fe}-\mathrm{O}_{2}-\mathrm{C}, \mathrm{Fe}-\mathrm{N}-\mathrm{C}, \mathrm{O}_{1}-\mathrm{Fe}-\mathrm{O}_{1}, \mathrm{O}_{2}-\mathrm{Fe}-\mathrm{O}_{2}, \mathrm{Fe}-\mathrm{O}_{1}-\mathrm{O}_{3}, \mathrm{Fe}-\mathrm{O}_{2}-\mathrm{O}_{3}$, and Fe-C-O $\mathrm{O}_{3}$, where $\mathrm{O}_{3}$ refers to the oxygen outside the first shell. The GNXAS determined bond distances and angles that make up the three-atom contributions are within $5 \%$ of the crystallographic values, with the strength of the signal influencing the goodness of the match. ${ }^{16}$ Contributions with stronger signals have distances and angles that match closer 
to the crystallographic values than do contributions with weaker signals. Over a large number of fits with varying contributions, splines, and nonstructural parameters the firstshell distances varied by $<0.01 \AA$, the low- $Z$ bonu distances (i.e. $\mathrm{O}-\mathrm{C}$ and $\mathrm{N}-\mathrm{C}$ ) varied by $\pm 0.04 \AA$, and the bond angle varied by $\pm 3^{\circ}$. The GNXAS first-shell distances are within $0.02 \AA$ of the empirical first-shell distances, with the GNXAS distances being slightly closer to the crystallographic values.

The EXAFS data and the FT of the best fit to the $\mathrm{Na} F \mathrm{Fe}\left(\mathrm{OH}_{2}\right)$ EDTA solution data are presented in Figure 3.12 and the bond distances and angles from that fit are given in Table 3.3. The main contributions to the EXAFS are the same as for the $\mathrm{Na}\left[\mathrm{Fe}\left(\mathrm{OH}_{2}\right) \mathrm{EDTA}\right]$ powder. The best fit shows excellent agreement with the experimental EXAFS as does the FT of the experimental data and the fit. The bond distances obtained from the best fit to the $\mathrm{Na}\left[\mathrm{Fe}\left(\mathrm{OH}_{2}\right) \mathrm{EDTA}\right]$ solution data are within $0.02 \AA$ of the $\mathrm{Na}\left[\mathrm{Fe}\left(\mathrm{OH}_{2}\right)\right.$ EDTA] powder values and the bond angles are all within $2^{\circ}$ with two exceptions. There is a $0.03 \AA$ difference in the $\mathrm{O}_{1,2}-\mathrm{O}_{3}$ distance and a $5^{\circ}$ difference in the $\mathrm{Fe}-\mathrm{O}_{2}-\mathrm{C}$ angle. However, both the $\mathrm{O}_{1,2}-\mathrm{O}_{3}$ distance and the $\mathrm{Fe}-\mathrm{O}_{2}-\mathrm{C}$ angle show large disorder, with a bond variance of $0.006 \AA^{2}$ and an angle variance of 60 (degrees) ${ }^{2}$ (Table 3.3). The bond and angle variances are very similar, but slightly larger than the powder values, which is expected since there should be more disorder in solution. The increase in the solution variances is also consistent with the fact that in the empirical analysis (where the Debye-Waller factors are fixed) the coordination numbers for the solution were lower than those of the powder. The similarities in bond lengths, bond angles, and the respective variances indicate that the $\left[\mathrm{Fe}\left(\mathrm{OH}_{2}\right) \mathrm{EDTA}\right]^{-}$unit is structurally the same in the powder and the solution form. Therefore it is a reasonable approximation to initially model FeEDTA-NO in the solution form using the crystallographic coordinates of $\mathrm{Li}\left[\mathrm{Fe}\left(\mathrm{OH}_{2}\right)\right.$ EDTA] $\cdot 2 \mathrm{H}_{2} \mathrm{O}$ with NO replacing the $\mathrm{H}_{2} \mathrm{O}$.

The best fit to the EXAFS of the FeEDTA-NO solution data is shown in Figure 3.13 and the bond distances and angles are presented in Table 3.3. The FT of the EXAFS data of the best fit is shown in Figure 3.14B. The initial [Fe( $\left(\mathrm{OH}_{2}\right)$ EDTA] structural model was modified by including a short Fe-N distance $(-1.8 \AA)$ and fixing the coordination number for the $2.1 \AA \mathrm{Fe}-\mathrm{O}$ distance at two. Fits were done using $\gamma^{(2)}$ signals exclusively to determine first-shell distances. Once a reasonable fit was obtained, the first-shell distances were fixed and a $\mathrm{Fe}-\mathrm{N}-\mathrm{O}$ signal was added to the fit. $\mathrm{Fe}-\mathrm{N}-\mathrm{O}$ signals were calculated every $10^{\circ}$ between $90^{\circ}$ and $180^{\circ}$, fixing the Fe-N distance at $1.8 \AA$ and the $\mathrm{N}-\mathrm{O}$ distance at $1.1 \AA$. Fits were then performed including the Fe-N-O signal at each angle. Reasonable fits were obtained for $\mathrm{Fe}-\mathrm{N}-\mathrm{O}$ angles between $150^{\circ}$ and $160^{\circ}$. At this point, other three-atom signals were included in the fits allowing the distances, 


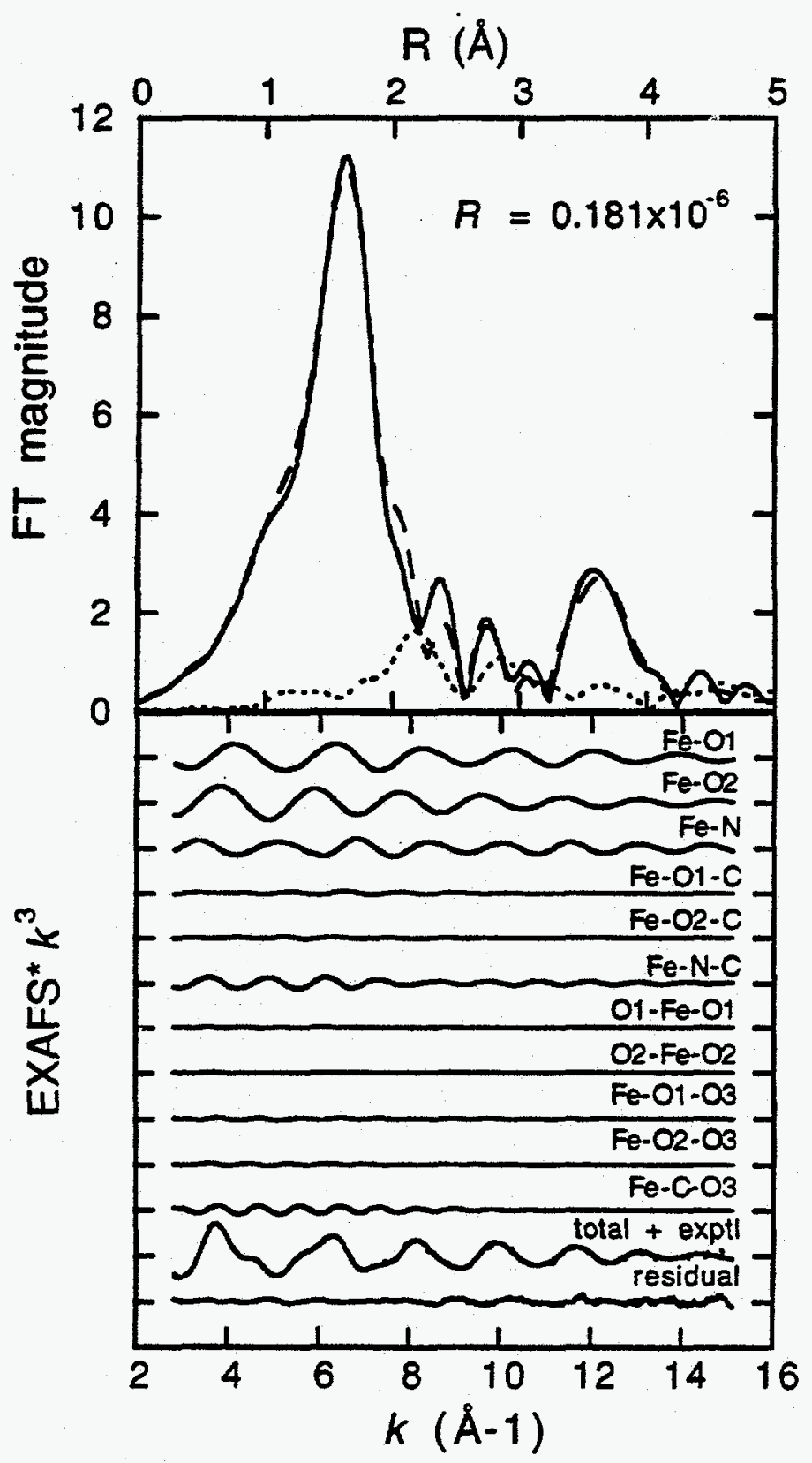

Figure 3.12. Comparison of the GNXAS theoretical signal with the experimental data of Fe K-edge $k^{3}$-weighted EXAFS of $\mathrm{Na}\left[\mathrm{Fe}\left(\mathrm{OH}_{2}\right)\right.$ EDTA] solution data. The top portion of the figure contains the non-phase shift corrected FT of the $k^{3}$-weighted EXAFS data of the experimental data $(-)$ and that of the total theoretical signal (---). Also shown is the FT of the residual (…). The lower portion of the figure presents the EXAFS signals for the individual contributions. The total theoretical signal is also shown (-) and compared with the experimental data (....) with the residual being the difference between the experimental EXAFS and the theoretical EXAFS. (The ordinate scale is 10 between two consecutive tick marks.) 


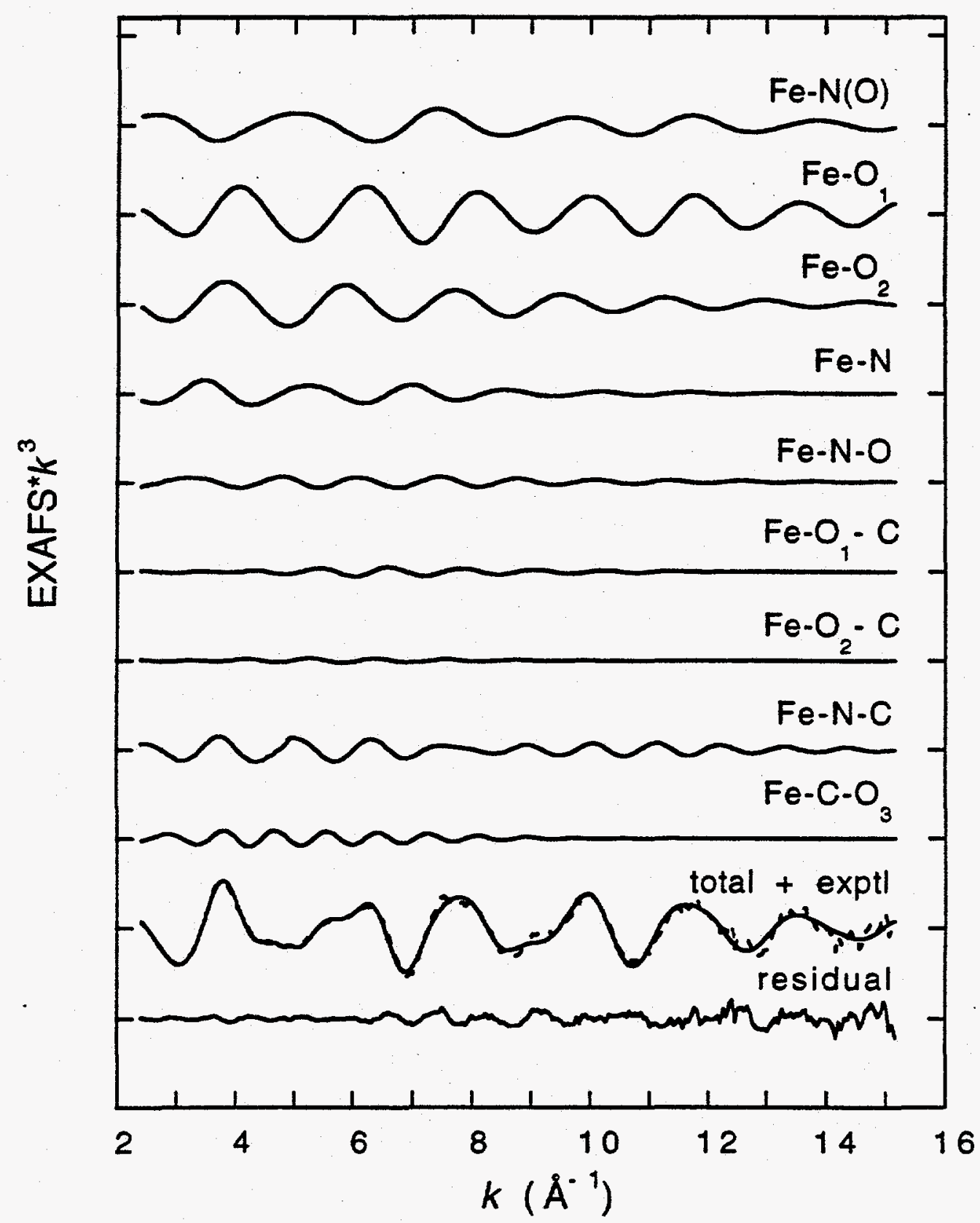

Figure 3.13. EXAFS signals for individual contributions in the best fit for the FeEDTANO data. The total signal (-) is also shown and compared with the experimental data (--) with the residual being the difference between the experimental EXAFS and the theoretical EXAFS. (The ordinate scale is 10 between two consecutive tick marks.) 
angles, and elements of the covariance matrix to vary within a restricted range. The Fe-N-O angle was allowed to vary between $145^{\circ}$ and $165^{\circ}$. Several of the three-atom contributions included in the fit to the $\mathrm{Na}\left[\mathrm{Fe}\left(\mathrm{OH}_{2}\right)\right.$ EDTA] solution data (Figure 3.12) were left out of the FeEDTA-NO fits $\left(\mathrm{O}_{1}-\mathrm{Fe}-\mathrm{O}_{1}, \mathrm{O}_{2}-\mathrm{Fe}-\mathrm{O}_{2}, \mathrm{Fe}-\mathrm{O}_{1}-\mathrm{O}_{3}\right.$, and $\left.\mathrm{Fe}-\mathrm{O}_{2}-\mathrm{O}_{3}\right)$, since the signals were relatively weak and only increased the number of variables in the fit. The distances and bond angles obtained from the GNXAS final fit are very similar to the distances and bond angles from the GNXAS fits of the Na[Fe $\left(\mathrm{OH}_{2}\right)$ EDTA] powder and solution data as can be seen in Table 3.3. The complicated EXAFS spectrum in Figure 3.13 is dominated by four waves of lower frequency: $\mathrm{Fe}-\mathrm{N}(\mathrm{O}), \mathrm{Fe}-\mathrm{O}_{1}, \mathrm{Fe}-\mathrm{O}_{2}$ and $\mathrm{Fe}-\mathrm{N}$. However, the longer $\mathrm{Fe}-\mathrm{N}$ contribution is much weaker than in the $\mathrm{Na}\left[\mathrm{Fe}\left(\mathrm{OH}_{2}\right) \mathrm{EDTA}\right]$ powder and solution EXAFS with a bond variance that is three to four times higher indicating that the bond between the Fe-N at $\sim 2.3 \AA$ may be weakened when the NO binds. The Fe-N-O signal is fairly strong compared to the other three-atom signals. The $\mathrm{Fe}-\mathrm{N}(\mathrm{O})$ and $\mathrm{N}-\mathrm{O}$ bond distances obtained from the GNXAS fit were 1.78 and $1.10 \AA$, respectively. These distances are consistent with other $\mathrm{Fe}-\mathrm{N}(\mathrm{O})$ and $\mathrm{N}-\mathrm{O}$ bond distances in $\{\mathrm{FeNO}\}^{7}$ systems. ${ }^{4}$ A fit with an $\mathrm{Fe}-\mathrm{N}-\mathrm{O}$ angle of $156^{\circ}$ shows excellent agreement with the experimental EXAFS data, Figure 3.13, and with the Fourier transformed data, Figure $3.14 \mathrm{~B}$, up to $3.5 \AA$. The discrepancy between the theoretical and experimental signal in the FT beyond $3.5 \AA$ can be attributed to the fact that several three-atom contributions associated with weaker signals in that region were not included in the fit.

As was done with the crystallographically-characterized $\{\mathrm{FeNO}\}^{7}$ complexes, the sensitivity of the calculated spectrum to the EXAFS data for FeEDTA-NO was tested as a function of Fe-N-O angle. The FTs for FeEDTA-NO with Fe-N-O values of $180^{\circ}, 156^{\circ}$ (best fit), and $120^{\circ}$ are shown in Figure 3.14. A plot of $\log (R$ value) vs Fe-N-O angle (Figure 3.15) of the FeEDTA-NO data displays a minimum at $156^{\circ}$. This looks extremely similar to the plot of the $\mathrm{Fe}(\mathrm{TACN})\left(\mathrm{N}_{3}\right)_{2} \mathrm{NO}$ data, where $\mathrm{Fe}(\mathrm{TACN})\left(\mathrm{N}_{3}\right)_{2} \mathrm{NO}$ has an Fe-N-O angle of $156^{\circ}$. It is not surprising that the geometric structures of the Fe-N-O unit in $\mathrm{Fe}(\mathrm{TACN})\left(\mathrm{N}_{3}\right)_{2} \mathrm{NO}$ and FeEDTA-NO are similar since both compounds exhibit very similar optical spectroscopy. 5

\subsection{Summary}

Multiple-scattering signals from three-atom configurations are accurately modeled by GNXAS to obtain angular information on the Fe-N-O unit of $\{\mathrm{FeNO}\}^{7}$ complexes. The GNXAS fits to the $\{\mathrm{FeNO}\}^{7}$ model compounds are sensitive to the $\mathrm{Fe}-\mathrm{N}-\mathrm{O}$ angle 


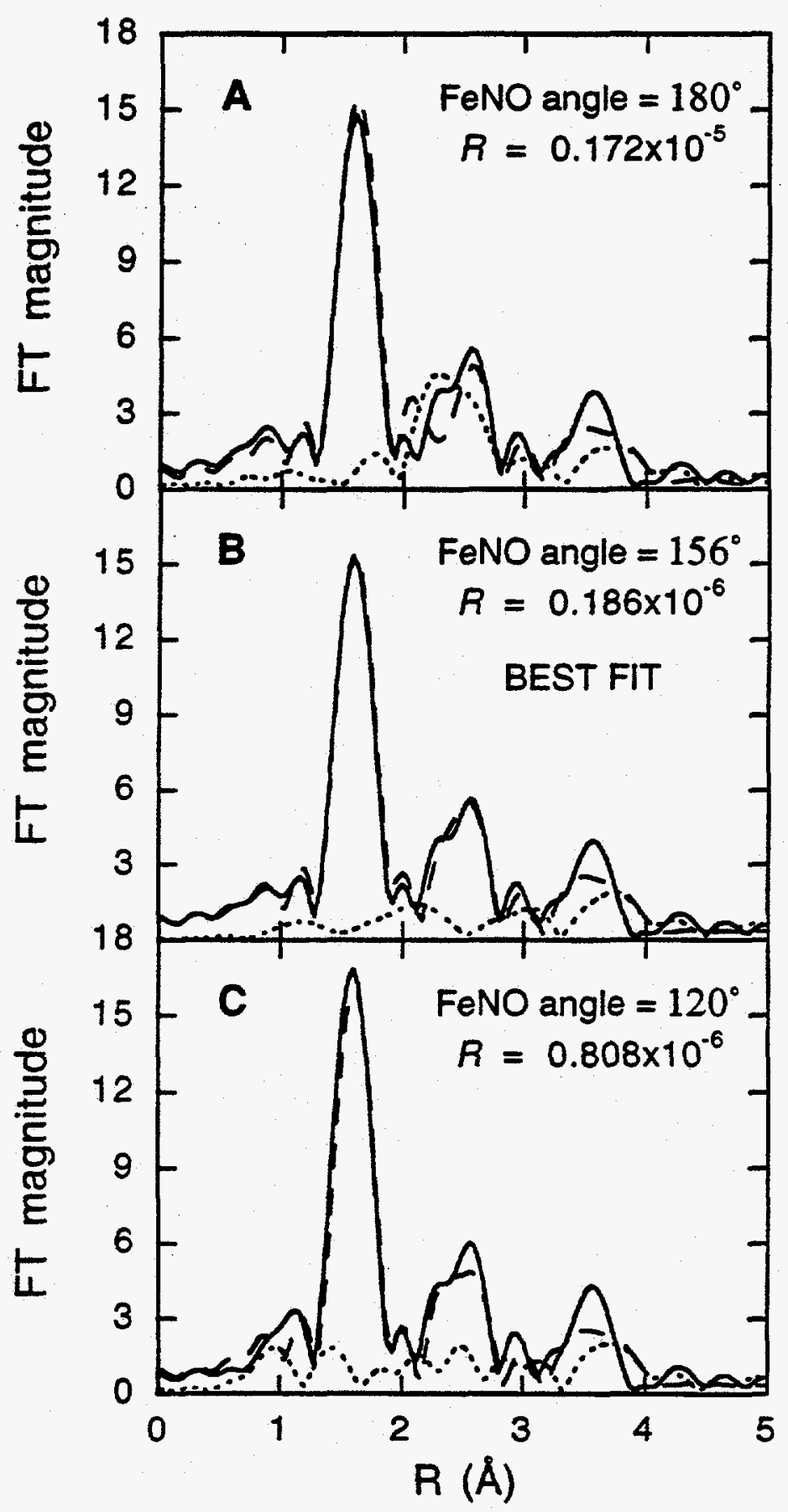

Figure 3.14. A comparison of the theoretical (---) and experimental $(-)$ non-phase shift corrected FT of FeEDTA-NO EXAFS data, along with the FT of the EXAFS residual (…). The $R$ value is an indication of the goodness of the fit. Calculated spectra are for Fe-N-O bond angles of: (A) $180^{\circ}$, (B) $156^{\circ}$ (best fit), and (C) $120^{\circ}$. 


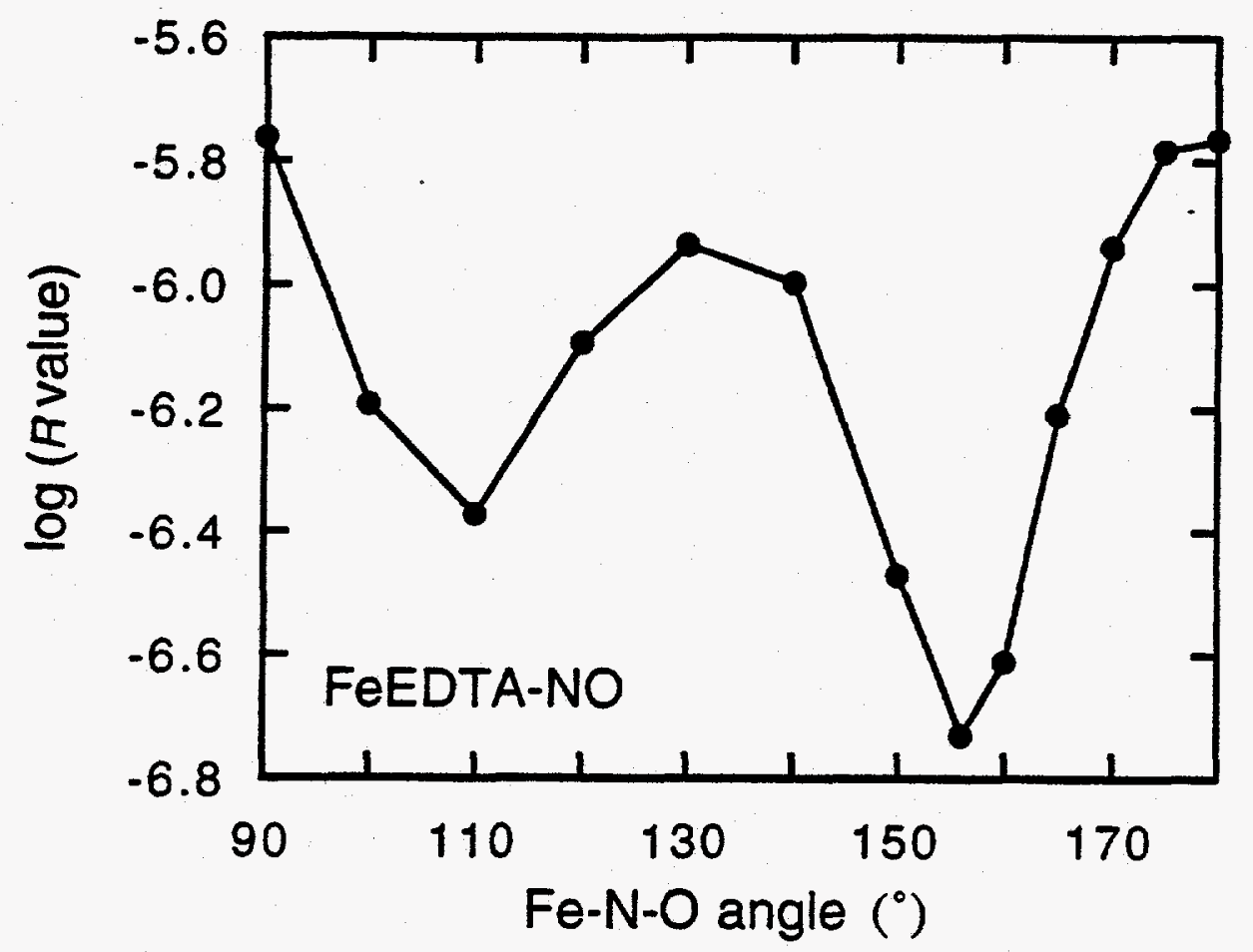

Figure 3.15. Plot of the $\log (R$ value $)$ vs Fe-N-O angle for FeEDTA-NO where each point represents a match of the calculated EXAFS spectrum to the data. The lower the $R$ value the better the fit. This plot of FeEDTA-NO calculated spectra exhibits similar behavior to the plot of the $\mathrm{Fe}(\mathrm{TACN})\left(\mathrm{N}_{3}\right)_{2} \mathrm{NO}$ calculated spectra (Figure 3.10B) with the dominant minimum being around $155^{\circ}$. 
when the Fe-N-O signal is significant in comparison with the total EXAFS signal. It is possible to determine whether the Fe-N-O unit is linear or bent and estimate the Fe-N-O angle, with the GNXAS fits being very sensitive when the angle is between $150^{\circ}$ and $180^{\circ}$. The Fe-N-O angle of a crystallographically-uncharacterized $\{\mathrm{FeNO}\}^{7}$ model complex was determined. Using this method the Fe-N-O angle of FeEDTA-NO is determined to be bent, and close to $156^{\circ}$. The results of this study establish that EXAFS analysis using GNXAS can provide reliable angular information on low- $Z$ small molecules liganded to transition metal complexes. This work provides the basis for studying NO complexes with transition metal active sites in metalloproteins. It is also straightforward to extend this methodology to study other diatomics such as $\mathrm{O}_{2}-$ or $\mathrm{O}_{2}{ }^{2-}$ liganded to transition metal sites.

\subsection{Acknowledgments}

The $\mathrm{Na}\left[\mathrm{Fe}\left(\mathrm{OH}_{2}\right) \mathrm{EDTA}\right], \mathrm{Na} 2\left[\mathrm{Fe}\left(\mathrm{OH}_{2}\right)\right.$ EDTA], and FeEDTA-NO solutions were prepared and provided by Yan Zhang and Mark Pavlosky. This research was supported by grants from the NIH (RR01209, K.O.H., GM40392, E.I.S) and NSF (CHE91-21576, K.O.H.). The Stanford Synchrotron Radiation Laboratory is supported by the Department of Energy, Office of Basic Energy Sciences, Divisions of Chemical Science and Material Science, and in part by the National Institutes of Health, National Center of Research Resources, Biomedical Research Technology Program and DOE's Office of Health and Environmental Research. Rino Natoli, Andrea Di Cicco, and Adriano Fillipponi (who are funded by the Italian INFN and CNR research institutions) should be thanked for providing the GNXAS data analyis package, as well as for their help in installing the package and providing insight into the intricacies of GNXAS fitting.

\subsection{References and Notes}

(1) (a) Solomon, E. I.; Zhang, Y. Acc. Chem. Res. 1992, 25, 343. (b) Smith, W. L.; Lands, W. E. M. J. Biol. Chem. 1972, 247, 1038. (c) Kohlmiller, N. A.; Howard, J. B. J. Biol. Chem. 1979, 254, 7302. (d) Nozaki, M.; Kagamiyama, H.; Hayaishi, O. Biochem. Z. 1963, 338, 582. (e) Burger, R. M.; Peisach, J.; Blumberg, W. E.; Wittenberg, J. B.; Sausville, E. A.; Horwitz, S. B. Electron Transport and Oxygen Utilization; Elsevier: New York, 1982; p 319. (f) Shiman, R. Folates and Pterins; Wiley: New York, 1984; Vol. 2, p 179. (g) Lindstedt, S.; Odelhög, B.; Rundgren, M. Biochemistr; 1977, 16, 3369. (h) Ruettinger, R. T.; Griffith, G. R.; 
Coon, M. J. Arch. Biochem. Biophys. 1977, 183, 528. (i) Batie, C. J.; LaHaie, E.; Ballou, D. P. J. Biol. Chem. 1987, 262, 1510. (j) Pang, C. -P.; Chakravarti, B.; Adlington, R. M.; Ting, H. -H.; White, R. L.; Jayatilake, G. S.; Baldwin, J. E.; Abraham, E. P. Biochem. J. 1984, 222, 789. (k) Slykhouse, T. O.; Fee, J. A. J. Biol. Chem. 1976, 251, 5472.

(2) (a) Nelson, M. J. J. Biol. Chem. 1987, 262, 12137. (b) Rich, P. R.; Salemo, J. C.; Leigh, J. S.; Bonner, W. D. FEBS Lett. 1978, 93, 323. (c) Arciero, D. M.; Lipscomb, J. D. J. Biol. Chem. 1986, 261, 2170. (d) Arciero, D. M.; Orville, A. M.; Lipscomb, J. D. J. Biol. Chem. 1985, 260, 14035. (e) Twilfer, H.; Bernhardt, F.-H.; Gersonde, K. Eur. J. Biochem. 1985, 147, 171. (f) Chen, V.J .; Orville, A. M.; Harpel, M. R.; Frolik, C. A.; Surerus, K. K.; Münck, E.; Lipscomb, J. D. J. Biol. Chem. 1989, 264, 21677.

(3) The Feltham-Enemark formalism for $\{\text { MNO }\}^{x}$ complexes is used here in which $x$ is the number of $\mathrm{d}$ type electrons in the system when the nitrosyl ligand is formally considered as NO+ (Enemark, J. H.; Feltham, R. D. Coord. Chem. Rev. 1974, 13, 339).

(4) (a) Wells, F. V.; McCann, S. W.; Wickman, H. H.; Kessel, S. L.; Hendrickson, D. N.; Feltham, R. D. Inorg. Chem. 1982, 21, 2306. (b) Bill, E.; Bernhardt, F. -H.; Trautwein, A. X.; Winkler, H. Eur. J. Biochem. 1985, 147, 177. (c) Eamshaw, A.; King, E. A.; Larkworthy, L. F. J. Chem. Soc. (A) Inorg. Phys. Theor. 1969, 2459. (d) Salemo, J. C.; Siedow, J. N. Biochim. Biophys. Acta 1979, 579, 246. (e) Pohl, K.; Wieghardt, K.; Nuber, B.; Weiss, J. J. Chem. Soc., Dalton Trans. $1987,187$.

(5) (a) Zhang, Y.; Pavlosky, M. A.; Brown, C. A.; Westre, T. E.; Hedman, B.; Hodgson, K. O.; Solomon, E. I. J. Am. Chem. Soc. 1992, 114, 9189. (b) Brown, C. A.; Pavlosky, M. A.; Westre, T. E.; Zhang, Y.; Hedman, B.; Hodgson, K. O.; Solomon, E. I. J. Am. Chem. Soc. 1995, 117, 715.

(6) (a) Ashley, C. A.; Doniach, S. Phys. Rev. B 1975, 11, 1279. (b) Lee, P. A.; Pendry, J. B. Phys. Rev. B 1975, 11, 2795. (c) Cramer, S. P. Ph.D. Thesis, Stanford University, 1978.

(7) (a) Teo, B. -K. J. Am. Chem Soc. 1981, 103, 3990. (b) Co, M. S.; Hendrickson, W. A.; Hodgson, K. O.; Doniach, S. J. Am Chem. Soc. 1983, 105, 1144.

(8) (a) Cramer, S. P.; Hodgson, K. O.; Stiefel, E. I.; Newton, W. E. J. Am. Chem Soc. 1978, 100, 2748. (b) Binsted, N.; Evans, J.; Greaves, G. N.; Price, R.J . J. Chem. Soc., Chem. Commun. 1987, 1330. (c) Binsted, N.; Cook, S. L.; Evans, J.; Greaves, G. N.; Price, R. J. J. Am. Chem. Soc., 1987, 109, 3669. (d) Filipponi, A.; 
Di Cicco, A.; Zanoni, R.; Bellatreccia, M.; Sessa, V.; Dossi, C.; Psaro, R. Chem. Phys. Lett. 1991, 184, 485.

(9) Westre, T. E.; Hedman, B.; Solomon, E. I.; Hodgson, K. O. Unpublished data.

(10) (a) Filipponi, A.; Di Cicco, A.; Tyson, T. A.; Natoli, C. R. Solid State Commun. 1991, 78, 265. (b) Filipponi, A.; Di Cicco, A. Synchrotron Radiation News 1992, $6,13$.

(11) Hedin, L.; Lundqvist, S. Solid State Phys. 1969, $23,1$.

(12) Di Cicco, A.; Stizza, S.; Filipponi, A.; Boscherini, F.; Mobilio, S. J. Phys. B: At. Mol. Opt. Phys. 1992, 25, 2309.

(13) D'Angelo, P.; Di Cicco, A.; Filipponi, A.; Pavel, N. V. Phys. Rev. A 1993; 47, 2055.

(14) Burattini, E.; D'Angelo, P.; Di Cicco, A.; Filipponi, A.; Pavel, N. V. J. Phys. Chem. 1993, 97, 5486.

(15) Nordlander, E.; Lee, S. C.; Cen, W.; Wu, Z. Y.; Natoli, C. R.; Di Cicco, A.; Filipponi, A.; Hedman, B.; Hodgson, K. O.; Holm, R. H. J. Am. Chem. Soc. 1993, 115, 5549.

(16) Westre, T. E.; Di Cicco, A.; Filipponi, A.; Natoli, C. R.; Hedman, B.; Solomon, E. I.; Hodgson, K. O. J. Am. Chem. Soc. 1995, 117, 1566.

(17) Hodges, K. D.; Wollman, R. G.; Kessel, S. L.; Hendrickson, D. N.; Van Derveer, D. G.; Barefield, E. K. J . Am. Chem. Soc. 1979, 101, 906.

(18) Haller, K. J.; Johnson, P. L.; Feltham, R. D.; Enemark, J. H. Inorg. Chim. Acta $1979,33,119$.

(19) Lind, M. D.; Hamor, M. J.; Hamor, T. A.; Hoard, J. L. Inorg. Chem. 1963, 3, 34.

(20) Scott, R. A.; Hahn, J. E.; Doniach, S.; Freeman, H. C.; Hodgson, K. O. J. Am. Chem. Soc. 1982, 104, 5364.

(21) (a) Stern, E. A.; Heald, S. M. Rev. Sci. Instrum. 1979, 50, 1579. (b) Lytle, F. W.; Greegor, R. B.; Sandstrom, D. R.; Marques, E. C.; Wong, J.; Spiro, C. L.; Huffman, G. P.; Huggins, F. E. Nucl. Instrum. Meth. Phys. Res. 1984, 226, 542.

(22) Johansson, L. Chem. Scr. 1976, 9, 30. The crystal structure of the perchlorate salt has not been determined, but the $[\mathrm{Fe} \text { (phenanthroline) }]^{2+}$ complex structure can be assumed to be identical with that of the corresponding iodide salt (Johansson, L.; Molund, M.; Oskarsson, A. Inorg. Chim. Acta 1978, 31, 117).

(23) Mattheiss, L. F. Phys. Rev. 1964, 134, A970.

(24) Krause, M. O.; Oliver, J. H. J. Phys. Chem. Ref. Data 1979, 8, 329.

(25) The principal determining factor for $E_{\mathrm{r}}$ is the monochromator and associated vertical slit opening, with the resolution determined by the relationship $\triangle E / E=$ 
$\cot (\Theta) \Delta \Theta$, where $\Theta$ is a function of the Darwin width and the vertical angular acceptance of the monochromator. The value at the $\mathrm{Fe} \mathrm{K}$-edge for the experimental conditions used for these experiments were $\sim 1.4 \mathrm{eV}$. Lytle, F. W. In Applications of Synchrotron Radiation; Winick, H.; Xiam, D.; Ye, M.-h; Huang,T., Eds.; Gordon and Breach Science Publishers: New York, 1989; p. 135.

(26) (a) Cramer, S. P.; Hodgson, K. O. Prog. Inorg. Chem. 1979, 25, 1.' (b) Scott, R. A. Methods Enzymol. 1985, 117, 414.
(a) Iball, J.; Morgan,
C. H. Acto
Crystallogr. 1967, 23, 239.
(b) Roof, R. B., Jr. Acta Crystallogr. 1956, 9, 781. 
Chapter 4

A Multiplet Analysis of Fe K-Edge 1s $\rightarrow 3 d$

Pre-Edge Features of Iron Complexes 


\subsection{Introduction}

X-ray absorption spectroscopic (XAS) studies have been used xtensively to characterize the iron active sites in both mononuclear and binuclear non-heme iron enzymes. ${ }^{1-18}$ While extended X-ray absorption fine structure (EXAFS) analysis provides. information on the types of ligating atoms and very accurate first-shell iron-ligand distances, it determines with less accuracy the coordination number and in general gives little or no information on the active site geometry. However, complementary information can be obtained from the edge region of the XAS spectra. In particular, the features in region of the $1 \mathrm{~s} \rightarrow 3 \mathrm{~d}$ transition have been shown to be sensitive to the oxidation state and geometry of the iron atom. ${ }^{19}$ Additionally, the total intensity of this transition has been shown to increase with decreasing coordination number for iron model complexes due to the loss of inversion symmetry at the iron site. ${ }^{1,20}$ Analysis of the $1 \mathrm{~s} \rightarrow 3 \mathrm{~d}$ pre-edge feature has already proven useful in the determination of the coordination number of the non-heme iron active sites in ovotransferrin, ${ }^{1}$ catechol 1,2-dioxygenase, ${ }^{1}$ protocatechuate 3,4-dioxygenase, ${ }^{1,8}$ uteroferrin, ${ }^{11}$ soybean lipoxygenase, ${ }^{13,16}$ rabbit lipoxygenase, ${ }^{16}$ human lipoxygenase, ${ }^{16}$ and bleomycin. ${ }^{15}$ Previously, the splitting of the pre-edge feature was used to characterize the oxidation state and spin state of the iron site in activated bleomycin. ${ }^{17}$ The feature from the $1 \mathrm{~s}->3 \mathrm{~d}$ transition has also been shown to change with differing bridging ligation in binuclear model complexes, with $\mu$-oxo bridged complexes having a fairly intense pre-edge feature with a distinctive shape. ${ }^{21}$

XAS edge spectra of first-row transition metals have a weak pre-edge feature $\sim 10 \mathrm{eV}$ below the absorption edge. This feature was unequivocally assigned as originating from the $1 \mathrm{~s} \rightarrow 3 \mathrm{~d}$ transition by Shulman $e t$. al. when they observed that $\mathrm{Zn}(\mathrm{II})$, a $\mathrm{d}^{10}$ system, did not have this feature. ${ }^{19}$ A dipole-coupled $1 \mathrm{~s} \rightarrow 3 \mathrm{~d}$ transition is forbidden by parity considerations for complexes in a centrosymmetric environment. Yet, experimentally, a very weak pre-edge feature is still observed for complexes in a centrosymmetric environment. In such molecules, the most likely $1 \mathrm{~s} \rightarrow 3 \mathrm{~d}$ transition intensity mechanism is electric quadrupole coupling which is theoretically calculated to be two orders of magnitude weaker than electric dipole coupling. ${ }^{22,23}$ Experimentally, the quadrupole nature of the $1 \mathrm{~s} \longrightarrow 3 \mathrm{~d}$ pre-edge feature in $\mathrm{D}_{4 \mathrm{~h}} \mathrm{CuCl}_{4}{ }^{2-}$ was determined by Hahn $e t$. al. by analysis of the angular dependence of the $1 \mathrm{~s} \rightarrow 3 \mathrm{~d}$ transition using polarized radiation and oriented single crystals. ${ }^{24}$ Complexes in noncentrosymmetric environments have more intense pre-edge features. ${ }^{1,20,24-28}$ The increase in intensity has been attributed to metal $4 p$ mixing into the $3 d$ orbitals which provides some $1 \mathrm{~s} \rightarrow 4 \mathrm{p}$ 
character to the transition which is electric dipole allowed. Since the quadrupole-coupled mechanism is two orders of magnitude weaker than the dipole-coupled mechanism, only a few percent of $4 \mathrm{p}$ mixing into the $3 \mathrm{~d}$ orbitals can have a dramatic effect on the intensity of the $1 \mathrm{~s} \rightarrow 3 \mathrm{~d}$ pre-edge feature.

In this study, XAS Fe K-edge data on high and low spin ferrous and ferric inorganic model complexes with varying geometries, as well as binuclear complexes with varying oxidation states, geometries, and bridging ligation, have been measured in order to establish a detailed understanding of the $1 \mathrm{~s} \rightarrow 3 \mathrm{~d}$ pre-edge feature and its sensitivity to the electronic and geometric structure of the iron site. The energy splitting and intensity distribution of the pre-edge features of these complexes vary with spin state, oxidation state, geometry, and bridging ligation (in the binuclear complexes). A methodology for interpreting this energy splitting and intensity distribution of the $1 \mathrm{~s} \rightarrow 3 \mathrm{~d}$ pre-edge features is developed for high spin ferrous and ferric complexes in octahedral, tetrahedral and square pyramidal environments and low spin ferrous and ferric complexes in octahedral environments. In each case, the allowable many-electron excited states are determined using ligand field theory. The energies of the excited states are calculated and compared to the measured energy splitting in the $1 \mathrm{~s} \rightarrow 3 \mathrm{~d}$ pre-edge features. The relative intensities of transitions into the many-electron excited states are obtained and also compared to the observed intensity pattern of the pre-edge feature. The effect of distorting the iron site to tetrahedral and square-pyramidal geometries is analyzed. The contribution to the pre-edge intensity from both an electric quadrupole and an electric dipole (from 4p-3d mixing) intensity mechanism is determined for these distorted cases where the amount of $4 \mathrm{p}$ mixing is experimentally obtained and compared to a theoretical estimate of the amount of $4 \mathrm{p}$ mixing determined from density functional calculations.

\subsection{Experimental Section}

\subsubsection{Sample Preparation}

$\mathrm{FeF}_{2}, \mathrm{FeCl}_{2}, \mathrm{FeBr}_{2}, \mathrm{FeI}_{2}, \mathrm{FeSiF}_{6} \cdot 6 \mathrm{H}_{2} \mathrm{O},\left(\mathrm{NH}_{4}\right)_{2} \mathrm{Fe}\left(\mathrm{SO}_{4}\right)_{2} \bullet^{\circ} \mathrm{H}_{2} \mathrm{O}, \mathrm{FeF}_{3}, \mathrm{FeCl}_{3}$, $\mathrm{FeBr}_{3}, \mathrm{Fe}(\mathrm{acac})_{3},\left(\mathrm{NH}_{4}\right) \mathrm{Fe}\left(\mathrm{SO}_{4}\right)_{2} \cdot 12 \mathrm{H}_{2} \mathrm{O}, \mathrm{K}_{3} \mathrm{Fe}(\mathrm{CN})_{6}$, and $\mathrm{K}_{4} \mathrm{Fe}(\mathrm{CN})_{6}$ were purchased from Aldrich in $>98 \%$ purity and used without further purification. $\mathrm{FeF}_{2}, \mathrm{FeCl}_{2}, \mathrm{FeBr}_{2}$, $\mathrm{FeI}_{2}, \mathrm{FeSiF}_{6} \cdot 6 \mathrm{H}_{2} \mathrm{O}$, and $\left(\mathrm{NH}_{4}\right)_{2} \mathrm{Fe}\left(\mathrm{SO}_{4}\right)_{2}{ }^{\bullet} 6 \mathrm{H}_{2} \mathrm{O}$ are air-sensitive and, therefore, these complexes were shipped in Ar-filled bottles and immediately placed in an inert atmosphere glove box upon delivery. All other model complexes were prepared as previously described (see references in Tables $4.1,4.2$, and 4.3). The crystalline samples 
were each mixed with boron nitride (BN) and ground into a fine powder. The BN/sample mixture was pressed into a $1 \mathrm{~mm}$ thick Al spacer that was sealed with $63.5 \mu \mathrm{m}$ Mylar tape windows. All air-sensitive complexes were prepared in an inert atmosphere nitrogen-filled glove box and immediately frozen in liquid nitrogen.

\subsubsection{XAS Data Collection and Reduction}

X-ray absorption spectra were recorded at the Stanford Synchrotron Radiation Laboratory (SSRL) and the National Synchrotron Light Source (NSLS) on beamlines 2-3, $4-2,4-3,7-3$, and X19A, during dedicated conditions $(3 \mathrm{GeV}, 30-100 \mathrm{~mA} / 2.5 \mathrm{GeV}$, 100-200 mA, respectively). Tables 4.1, 4.2, and 4.3 give the respective beamlines and temperatures at which the data were collected for each sample. ${ }^{29}$ In all cases, the radiation was monochromatized using a $\mathrm{Si}(220)$ double-crystal monochromator. For the majority of the data, the vertical aperature of the pre-monochromator slits was $1 \mathrm{~mm}$. The only exception was for the $\left(\mathrm{BF}_{4}\right)[\mathrm{Fe}(\mathrm{TMC}) \mathrm{X}]$ series (where $\mathrm{X}=\mathrm{Cl}^{-}, \mathrm{Br}^{-}, \mathrm{CH}_{3} \mathrm{CN}^{-}$, and $\mathrm{N}_{3}{ }^{-}$) in which case the pre-monochromator slit opening was $1.5 \mathrm{~mm}$ vertically. Data were measured in transmission mode with $\mathrm{N}_{2}$ filled ionization chambers to $k=9.5 \AA^{-1}$, detuning the monochromator $50 \%$ at $7474 \mathrm{eV}$ to minimize harmonic contamination (in some cases the data were measured to $k=15 \AA^{-1}$ in order to obtain EXAFS data as well). In general, two to five scans were measured for each sample. A smooth pre-edge background was removed from each averaged spectrum by fitting a first order polynomial to the pre-edge region and subtracting this polynomial from the entire spectrum. A twosegment spline of order two was fit to the EXAFS region and the data normalized to an edge jump of one at $7130 \mathrm{eV}$. Energies were calibrated using an internal $\mathrm{Fe}$ foil standard, assigning the first inflection point to $7111.2 \mathrm{eV} .^{30}$ The spectrometer energy resolution was approximately $1.4 \mathrm{eV}^{31}$ with reproducibility in edge position determination of $<0.2 \mathrm{eV}$.

\subsubsection{Data Analysis}

The intensities and energies of pre-edge features of the model complexes were quantitated by least-squares fits to the data. The fitting program EDG_FIT, which utilizes the double precision version of the public domain MINPAK fitting library ${ }^{32}$ was used. EDG_FIT was written by Dr. Graham N. George of the SSRI. Pre-edge features were modeled by pseudo-Voigt line shapes (simple sums of Lorentzian and Gaussian functions). ${ }^{31,33-35}$ A fixed 50:50 ratio of Lorentzian to Gaussian contribution for the 
pre-edge feature successfully reproduced these spectral features. Functions modeling the background underneath the pre-edge features were chosen empirically to give the best fit and included a pseudo-Voigt feature that mimicked the white line and in some cases pseudo-Voigt features that mimicked shoulders on the rising edge. Furthermore, the second derivative of the data was compared to the second derivative of the fit and only fits where a good overall match was obtained were accepted. In all cases, a number of acceptable fits, typically eight, were obtained which equally well reproduced the data and the second derivative varying the energy range over which the data was. fit and the background functions used. All spectra were fit over three energy ranges: 7108 $7116 \mathrm{eV}, 7108-7117 \mathrm{eV}$, and $7108-7118 \mathrm{eV}$. Typically, only functions modeling the pre-edge peaks and one function modeling the background were needed to obtain a good match to the data over the range $7108-7116 \mathrm{eV}$, while functions modeling shoulders on the rising edge were needed to obtain a good match to the data over the range 7108 $7118 \mathrm{eV}$. The value reported for the area of a fitted feature (where the peak area was approximated by the height $x$ full width at half-maximum (FWHM)) is the average of all the pseudo-Voigt functions that fit the pre-edge features from all the successful fits. To quantitate the error, the standard deviations for the peak energies and areas were calculated from all the pseudo-Voigt functions that fit the pre-edge features from all the successful fits for each sample.

\subsection{Results and Analysis}

Fe K-edge XAS data were measured for approximately 50 monomeric and dimeric iron model complexes. The energies and areas of the $1 \mathrm{~s} \rightarrow 3 \mathrm{~d}$ pre-edge features were determined by fits to the data and are presented in Tables 4.1 (high spin iron complexes), 4.2 (binuclear complexes), and 4.3 (low spin iron complexes). A multiplet analysis was used to explain the energy splitting and intensity patterns of the pre-edge features for the various cases: high spin ferrous complexes $\left(O_{h}, T_{d}\right.$ and $C_{4 v}$ geometries), high spin ferric complexes ( $\mathrm{O}_{\mathrm{h}}, \mathrm{T}_{\mathrm{d}}$ and $\mathrm{C}_{4 \mathrm{v}}$ geometries), binuclear complexes, and $\mathrm{O}_{\mathrm{h}}$ low spin ferrous and ferric complexes. In each case, the strong field many-electron states were determined for the $d^{(n+1)}$ excited state ${ }^{36}$ where the only effect of the core $1 \mathrm{~s}$ hole should be an increase in the potential since it is spherically symmetric. ${ }^{37}$ The energies of the many-electron states were determined by using reasonable values for $10 \mathrm{Dq}, \mathrm{B}$, and $\mathrm{C}$ (vide infra) and solving the Tanabe-Sugano matrices. ${ }^{38}$ The intensity of the pre-edge features due to the transition into each many-electron state was analyzed in terms of both a quadrupole intensity and a dipole intensity (i.e. $4 p$ mixing into the $3 d$ orbitals) 
mechanism. In the cases where the iron atom was in a noncentrosymmetric environment, the amount of $4 \mathrm{p}$ mixing into the $3 \mathrm{~d}$ orbitals was determined and compared to the theoretical estimate from density functional calculations.

\subsubsection{High Spin Ferrous Complexes}

4.3.1.1. $\mathrm{O}_{\mathrm{h}}$ Geometry. Fe K-edge XAS data were collected for eight high spin ferrous octahedral model complexes with varying ligation: $\mathrm{F}^{-}, \mathrm{Cl}^{-}, \mathrm{Br}^{-}, \mathrm{I}^{-}, \mathrm{H}_{2} \mathrm{O}$, and imidazole. All of these complexes have an iron atom in an approximately octahedral site. FeF 2 has the largest distortion away from $\mathrm{O}_{\mathrm{h}}$ with the axial ligands being $0.1 \AA$ closer to the iron than the equatorial ligands. ${ }^{39}$ The other seven complexes have iron sites that are nearly $O_{h}$ with ligand distances that differ by less than $0.05 \AA$. The XAS edge spectra for $\mathrm{FeF}_{2}, \mathrm{FeCl}_{2}, \mathrm{FeBr}_{2}$, and $\mathrm{FeI}_{2}$ are shown in Figure 4.1 and the spectra for rinneite, $\mathrm{FeSiF}_{6} \cdot 6 \mathrm{H}_{2} \mathrm{O},\left(\mathrm{NH}_{4}\right)_{2} \mathrm{Fe}\left(\mathrm{SO}_{4}\right)_{2} \cdot 6 \mathrm{H}_{2} \mathrm{O}$, and $\left.[\mathrm{Fe} \text { (imidazole) })_{6}\right]_{\mathrm{Cl}}$ are shown in Figure 4.2. The lowest energy transitions are the weak $1 \mathrm{~s} \rightarrow 3 \mathrm{~d}$ pre-edge peaks at approximately $7112 \mathrm{eV}$ followed by the $1 \mathrm{~s} \longrightarrow 4 \mathrm{p}$ transition at approximately $7125 \mathrm{eV}$. An expanded view of the $1 \mathrm{~s} \rightarrow 3 \mathrm{~d}$ pre-edge region is shown as insets in Figures 4.1 and 4.2. The energies and areas of the pre-edge features are presented in Table 4.1. All eight high spin ferrous octahedral model complexes have two very weak pre-edge features that are split by $\sim 2 \mathrm{eV}$ at $\sim 7111.5$ and $7113.5 \mathrm{eV}$.

The $1 \mathrm{~s} \rightarrow 3 \mathrm{~d}$ transition is formally electric dipole forbidden, but can gain intensity through an allowed quadrupole transition and by $4 p$ mixing into the $3 d$ states as a result of a noncentrosymmetric environment of the metal site. Since all eight of these complexes have a nearly centrosymmetric octahedral iron site, the only intensity mechanism available is the allowed quadrupole transition. The splitting of the high spin ferrous pre-edge feature has been observed before, ${ }^{19}$ and has been attributed to the free ion splitting of the ${ }^{4} \mathrm{~F}$ and ${ }^{4} \mathrm{P}$ terms (which is $-2 \mathrm{eV}$ ). However, the iron atom in these complexes is affected by an octahedral ligand field which causes the $3 \mathrm{~d}$ orbitals to split into $a t_{2}$ and an $e$ set. Removing the degeneracy of the $3 \mathrm{~d}$ orbitals causes the free ion terms to split into four many-electron states: ${ }^{4} \mathrm{~T}_{1},{ }^{4} \mathrm{~T}_{2},{ }^{4} \mathrm{~T}_{1}$, and ${ }^{4} \mathrm{~A}_{2} \cdot{ }^{36}$ The ground state of a high spin ferrous atom in an $\mathrm{O}_{\mathrm{h}}$ ligand field has an electronic (hole) configuration of $t_{2}{ }^{2} e^{2}$. Promoting an electron from a $1 s$ orbital into the $3 d$ manifold gives the $t_{2} e^{2}$ and $t_{2}{ }^{2} e$ configurations (Scheme 1). 
Table 4.1. XAS Pre-Edge Energies and Areas for High Spin Iron Model Complexes.

\begin{tabular}{|c|c|c|c|c|c|c|c|c|c|}
\hline compound & $\begin{array}{l}\text { oxidation } \\
\text { state }\end{array}$ & ligation & $\begin{array}{l}\text { beamline, } \\
\text { tempera- } \\
\text { ture }\end{array}$ & $\begin{array}{l}\text { pre-edge peak } \\
\text { energy }^{a}\end{array}$ & $\begin{array}{l}\text { pre-edge } \\
\text { peak } \\
\text { area } a, b\end{array}$ & $\begin{array}{l}\text { total pre- } \\
\text { edge peak } \\
\text { area } b\end{array}$ & $\begin{array}{l}\text { peak } \\
\text { energy } \\
\text { differ- } \\
\text { ences } c\end{array}$ & $\begin{array}{l}\text { peak } \\
\text { area } \\
\text { ratiod }\end{array}$ & $\begin{array}{l}\text { refer- } \\
\text { ence }\end{array}$ \\
\hline$\left(\mathrm{Et}_{4} \mathrm{~N}\right)_{2}\left[\mathrm{FeCl}_{4}\right]$ & ferrous & $4 \mathrm{Cl}$ & $\begin{array}{l}\text { SSRL 7-3, } \\
10 \mathrm{~K}\end{array}$ & $\begin{array}{l}7111.60(0.02) \\
7113.12(0.02)\end{array}$ & $\begin{array}{l}8.6(0.4) \\
4.3(0.7)\end{array}$ & $12.9(0.6)$ & 1.5 & 0.67 & 53 \\
\hline $\mathrm{Cs}_{3} \mathrm{FeCl}_{5}$ & ferrous & $4 \mathrm{Cl}$ & $\begin{array}{l}\text { SSRL 2-3, } \\
10 \mathrm{~K}\end{array}$ & $\begin{array}{l}7111.60(0.01) \\
7113.11(0.01)\end{array}$ & $\begin{array}{l}9.8(0.3) \\
3.3(0.3)\end{array}$ & $13.1(0.4)$ & 1.5 & 0.75 & 54 \\
\hline $\mathrm{Fe}\left(\mathrm{HB}\left(3.5-\mathrm{iPr} \mathrm{prz}_{3}\right) \mathrm{Cl}\right.$ & ferrous & $3 \mathrm{~N}, 1 \mathrm{Cl}$ & $\begin{array}{l}\text { SSRL 2-3, } \\
10 \mathrm{~K}\end{array}$ & $\begin{array}{l}7111.64(0.02) \\
7113.17(0.02)\end{array}$ & $\begin{array}{l}14.2(0.5) \\
5.6(0.9)\end{array}$ & $19.8(0.9)$ & 1.5 & 0.72 & 55 \\
\hline gillespite $\left(\mathrm{BaFeSi}_{4} \mathrm{O}_{10}\right)$ & ferrous & 40 & $\begin{array}{l}\text { SSRL 4-3, } \\
100 \mathrm{~K}\end{array}$ & $\begin{array}{l}7111.74(0.02) \\
7113.25(0.03)\end{array}$ & $\begin{array}{l}4.1(0.3) \\
1.2(0.2)\end{array}$ & $5.3(0.3)$ & 1.5 & 0.78 & 56,57 \\
\hline$\left(\mathrm{BF}_{4}\right)\{\mathrm{Fe}(\mathrm{TMC}) \mathrm{Cl}\}$ & ferrous & $4 \mathrm{~N}, 1 \mathrm{Cl}$ & $\begin{array}{l}\text { SSRL 7-3, } \\
300 \mathrm{~K}\end{array}$ & $\begin{array}{l}7111.41(0.01) \\
7113.43(0.02)\end{array}$ & $\begin{array}{l}10.9(0.1) \\
2.0(0.3)\end{array}$ & $12.9(0.2)$ & 2.0 & 0.84 & 58 \\
\hline$\left(\mathrm{BF}_{4}\right)[\mathrm{Fe}(\mathrm{TMC}) \mathrm{Br}]$ & ferrous & $4 \mathrm{~N}, 1 \mathrm{Br}$ & $\begin{array}{l}\text { SSRL 7-3, } \\
300 \mathrm{~K}\end{array}$ & $\begin{array}{l}7111.35(0.01) \\
7113.24(0.04)\end{array}$ & $\begin{array}{l}9.0(0.3) \\
2.1(0.4)\end{array}$ & $11.1(0.3)$ & 1.9 & 0.81 & 58 \\
\hline$\left(\mathrm{BF}_{4}\right)\left[\mathrm{Fe}(\mathrm{TMC}) \mathrm{CH}_{3} \mathrm{CN}\right]$ & ferrous & $\begin{array}{l}4 \mathrm{~N}, 1 \mathrm{C} \\
\left(\mathrm{CH}_{3} \mathrm{CN}\right)\end{array}$ & $\begin{array}{l}\text { SSRL 7-3, } \\
300 \mathrm{~K}\end{array}$ & $\begin{array}{l}7111.52(0.04) \\
7113.30(0.12)\end{array}$ & $\begin{array}{l}10.5(0.8) \\
2.2(1.2)\end{array}$ & $12.7(0.5)$ & 1.8 & 0.83 & 58 \\
\hline$\left(\mathrm{BF}_{4}\right)\left[\mathrm{Fe}(\mathrm{TMC}) \mathrm{N}_{3}\right]$ & ferrous & $\begin{array}{l}4 \mathrm{~N}, 1 \mathrm{~N} \\
\left(\mathrm{~N}_{3}\right)\end{array}$ & $\begin{array}{l}\text { SSRL 7-3, } \\
300 \mathrm{~K}\end{array}$ & $\begin{array}{l}7111.47(0.01) \\
7113.30(0.05)\end{array}$ & $\begin{array}{l}12.4(0.5) \\
1.0(0.2)\end{array}$ & $13.4(0.3)$ & 1.8 & 0.92 & 58 \\
\hline $\mathrm{FeF}_{2}$ & ferrous & $6 \mathrm{~F}$ & $\begin{array}{l}\text { SSRL 7-3, } \\
10 \mathrm{~K}\end{array}$ & $\begin{array}{l}7111.38(0.03) \\
7112.28(0.09) \\
7113.48(0.06)\end{array}$ & $\begin{array}{l}2.2(0.2) \\
1.0(0.1) \\
1.2(0.2)\end{array}$ & $4.4(0.2)$ & 2.1 & 0.73 & 39 \\
\hline $\mathrm{FeCl}_{2}$ & ferrous & $6 \mathrm{Cl}$ & $\begin{array}{l}\text { SSRL 7-3, } \\
10 \mathrm{~K}\end{array}$ & $\begin{array}{l}7111.32(0.05) \\
7112.05(0.11) \\
7113.42(0.05)\end{array}$ & $\begin{array}{l}1.9(0.4) \\
1.4(0.6) \\
0.9(0.2)\end{array}$ & $4.3(0.2)$ & 2.1 & 0.78 & 59 \\
\hline $\mathrm{FeBr}_{2}$ & ferrous & $6 \mathrm{Br}$ & $\begin{array}{l}\text { SSRL 7-3, } \\
10 \mathrm{~K}\end{array}$ & $\begin{array}{l}7111.32(0.03) \\
7111.98(0.25) \\
7113.15(0.04)\end{array}$ & $\begin{array}{l}2.5(0.6) \\
0.7(0.8) \\
0.6(0.1)\end{array}$ & $3.8(0.3)$ & 1.8 & 0.84 & 60 \\
\hline $\mathrm{Fel}_{2}$ & ferrous & $6 I$ & $\begin{array}{l}\text { SSRL 4-3, } \\
30 \mathrm{~K}\end{array}$ & $\begin{array}{l}7111.35(0.05) \\
7112.70(0.1)\end{array}$ & $\begin{array}{l}2.2(0.2) \\
0.8(0.2)\end{array}$ & $3.1(0.2)$ & 1.3 & 0.73 & 60 \\
\hline $\begin{array}{l}\text { rinneite } \\
\left(\mathrm{K}_{3} \mathrm{NaFeCl}_{6}\right)\end{array}$ & ferrous & $6 \mathrm{Cl}$ & $\begin{array}{l}\text { SSRL 7-3, } \\
10 \mathrm{~K}\end{array}$ & $\begin{array}{l}7111.32(0.06) \\
7111.81(0.19) \\
7113.38(0.04)\end{array}$ & $\begin{array}{l}1.2(0.9) \\
1.8(1.2) \\
0.6(0.1)\end{array}$ & $3.6(0.2)$ & 2.1 & 0.84 & 61 \\
\hline
\end{tabular}




\begin{tabular}{|c|c|c|c|c|c|c|c|c|c|c|}
\hline & $\mathrm{FeSiF}_{6} \cdot 6 \mathrm{H}_{2} \mathrm{O}$ & ferrous & 60 & SSRL 2-3, & $7111.34(0.07)$ & $2.2(0.5)$ & $4.0(0.5)$ & 2.3 & 0.73 & 62,63 \\
\hline & & & & $10 \mathrm{~K}$ & $7112.26(0.08)$ & $0.7(0.3)$ & & & & \\
\hline & & & & & $7113.60(0.04)$ & $1.1(0.3)$ & & & & \\
\hline & $\left(\mathrm{NH}_{4}\right)_{2} \mathrm{Fe}\left(\mathrm{SO}_{4}\right)_{2} \cdot 6 \mathrm{H}_{2} \mathrm{O}$ & ferrous & 60 & SSRL 2-3, & $7111.18(0.07)$ & $1.5(0.6)$ & $3.9(0.3)$ & 2.4 & 0.77 & 64 \\
\hline & & & & $10 \mathrm{~K}$ & $7112.03(0.15)$ & $1.6(0.7)$ & & & & \\
\hline & & & & & $7113.58(0.03)$ & $0.9(0.2)$ & & & & \\
\hline & [Fe(imidazole) $\left.{ }_{6}\right] \mathrm{Cl}_{2}$ & ferrous & $6 N$ & SSRL 2-3, & $7111.24(0.03)$ & $1.6(0.5)$ & $3.8(0.3)$ & 2.4 & 0.85 & 65,66 \\
\hline & & & & $10 \mathrm{~K}$ & $7112.35(0.16)$ & $1.6(0.8)$ & & & & \\
\hline & & & & & $7113.66(0.02)$ & $0.6(0.2)$ & & & & \\
\hline & $\left(\mathrm{Et}_{4} \mathrm{~N}\right)\left[\mathrm{FeCl}_{4}\right]$ & ferric & $4 \mathrm{Cl}$ & $\begin{array}{l}\text { SSRL 7-3, } \\
10 \mathrm{~K}\end{array}$ & $7113.16(0.00)$ & $20.7(0.8)$ & $20.7(0.8)$ & -- & -- & 67 \\
\hline & $\mathrm{Fe}($ salen)Cl & ferric & $20,2 \mathrm{~N}$ & SSRL 7-3, & $7112.91(0.01)$ & $12.9(0.3)$ & $14.4(0.6)$ & 1.3 & 0.89 & 68 \\
\hline & & & $1 \mathrm{Cl}$ & $10 \mathrm{~K}$ & $7114.25(0.07)$ & $1.5(0.4)$ & & & & \\
\hline & $\mathrm{FeF}_{3}$ & ferric & $6 \mathrm{~F}$ & SSRL 4-3, & $7113.38(0.02)$ & $3.3(0.2)$ & $5.3(0.3)$ & 1.4 & 0.63 & 69 \\
\hline & & & & $55 \mathrm{~K}$ & $7114.79(0.02)$ & $1.9(0.2)$ & & & & \\
\hline & $\mathrm{FeCl}_{3}$ & ferric & $6 \mathrm{Cl}$ & SSRL 4-3, & $7112.60(0.02)$ & $2.2(0.2)$ & $4.0(0.2)$ & 1.2 & 0.54 & 70 \\
\hline & & & & $30 \mathrm{~K}$ & $7113.77(0.03)$ & $1.8(0.2)$ & & & & \\
\hline & FcBr3 & ferric & $6 \mathrm{Br}$ & SSRL 4-3, & $7112.37(0.02)$ & $2.6(0.2)$ & $4.4(0.2)$ & 1.1 & 0.59 & 70 \\
\hline & & & & $30 \mathrm{~K}$ & $7113.52(0.03)$ & $1.8(0.2)$ & & & & \\
\hline & {$\left[\mathrm{FeCl}_{6}\right]\left[\mathrm{Co}\left(\mathrm{NH}_{4}\right)_{6}\right]$} & ferric & $6 \mathrm{Cl}$ & SSRL 7-3, & $7112.78(0.03)$ & $2.6(0.3)$ & $4.0(0.2)$ & 1.2 & 0.65 & 61,62 \\
\hline & & & & $10 \mathrm{~K}$ & $7114.02(0.03)$ & $1.4(0.1)$ & & & & \\
\hline & $\mathrm{Fe}(\mathrm{acac}) 3$ & ferric & 60 & SSRL 4-3, & $7112.79(0.02)$ & $2.7(0.3)$ & $4.6(0.3)$ & 1.5 & 0.59 & 63 \\
\hline & & & & $55 \mathrm{~K}$ & $7114.31(0.04)$ & $1.9(0.1)$ & & & & \\
\hline & {$\left[\mathrm{Fe}(\text { urea })_{6}\right]\left(\mathrm{ClO}_{4}\right)_{3}$} & ferric & $6 \mathrm{~N}$ & SSRL 7-3, & $7112.97(0.02)$ & $2.6(0.2)$ & $4.5(0.3)$ & 1.5 & 0.58 & 64 \\
\hline & & & & $10 \mathrm{~K}$ & $7114.44(0.02)$ & $1.9(0.1)$ & & & & \\
\hline & $\left.\left(\mathrm{NH}_{4}\right)_{3}[\mathrm{Fe} \text { (malonate) })_{3}\right]$ & ferric & 60 & SSRL 2-3, & $7112.92(0.03)$ & $3.1(0.3)$ & $5.5(0.1)$ & 1.5 & 0.57 & 65 \\
\hline & & & & $10 \mathrm{~K}$ & $7114.44(0.03)$ & $2.4(0.3)$ & & & & \\
\hline & $\mathrm{K}_{3}\left[\mathrm{Fe}(\text { oxalatc) })_{3}\right]$ & ferric & 60 & SSRL 2-3, & $7112.93(0.00)$ & $4.9(0.1)$ & $7.3(0.2)$ & 1.5 & 0.67 & 66 \\
\hline & & & & $10 \mathrm{~K}$ & $7114.41(0.01)$ & $2.4(0.1)$ & & & & \\
\hline & $\left(\mathrm{NH}_{4}\right) \mathrm{Fe}\left(\mathrm{SO}_{4}\right)_{2} \cdot 12 \mathrm{H}_{2} \mathrm{O}$ & ferric & 60 & SSRL 2-3, & $7113.14(0.02)$ & $2.7(0.1)$ & $4.9(0.2)$ & 1.4 & 0.56 & 67 \\
\hline & & & & $10 \mathrm{~K}$ & $7114.57(0.01)$ & $2.2(0.2)$ & & & & \\
\hline
\end{tabular}

a Pre-edge energies and intensities were determined by fits to the data (see the Experimental Section for details on the fitting procedure). $b$ The values reported for the pre-edge areas were multiplied by 100 . $c$ Peak energy differences are the energy differences between the lowest cnergy and the highest encrgy pre-edge feature. $d$ Peak area ratios are the area of the first pre-edge feature divided by the total precuge area. 


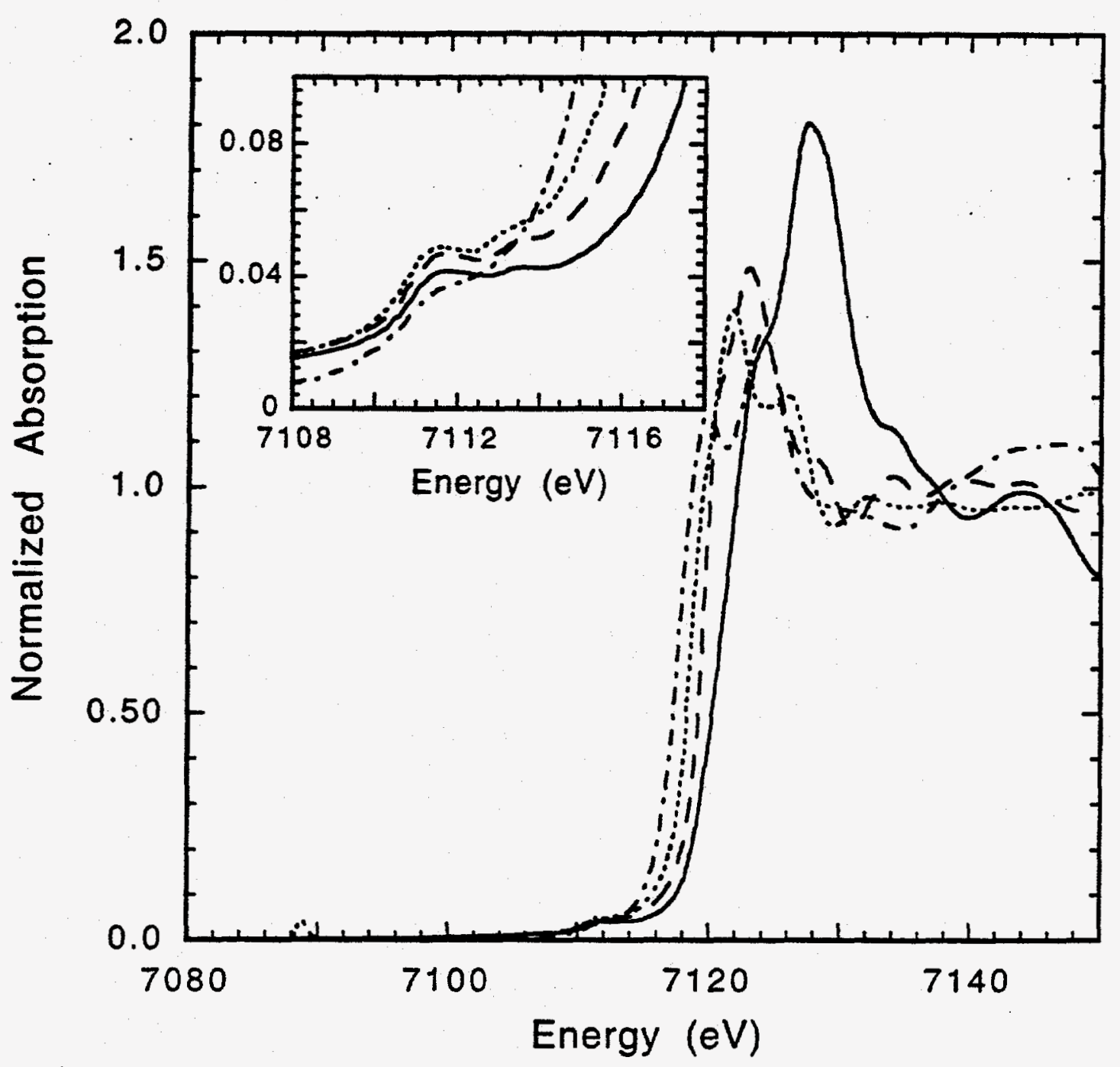

Figure 4.1. Fe K-edge XAS spectra of $\mathrm{FeF}_{2}(-), \mathrm{FeCl}_{2}(\cdots), \mathrm{FeBr}_{2}(\cdots \cdots)$, and $\mathrm{FeI}_{2}$ $(--)$, where the inset is an expansion of the $1 \mathrm{~s} \rightarrow 3 \mathrm{~d}$ pre-edge region. 


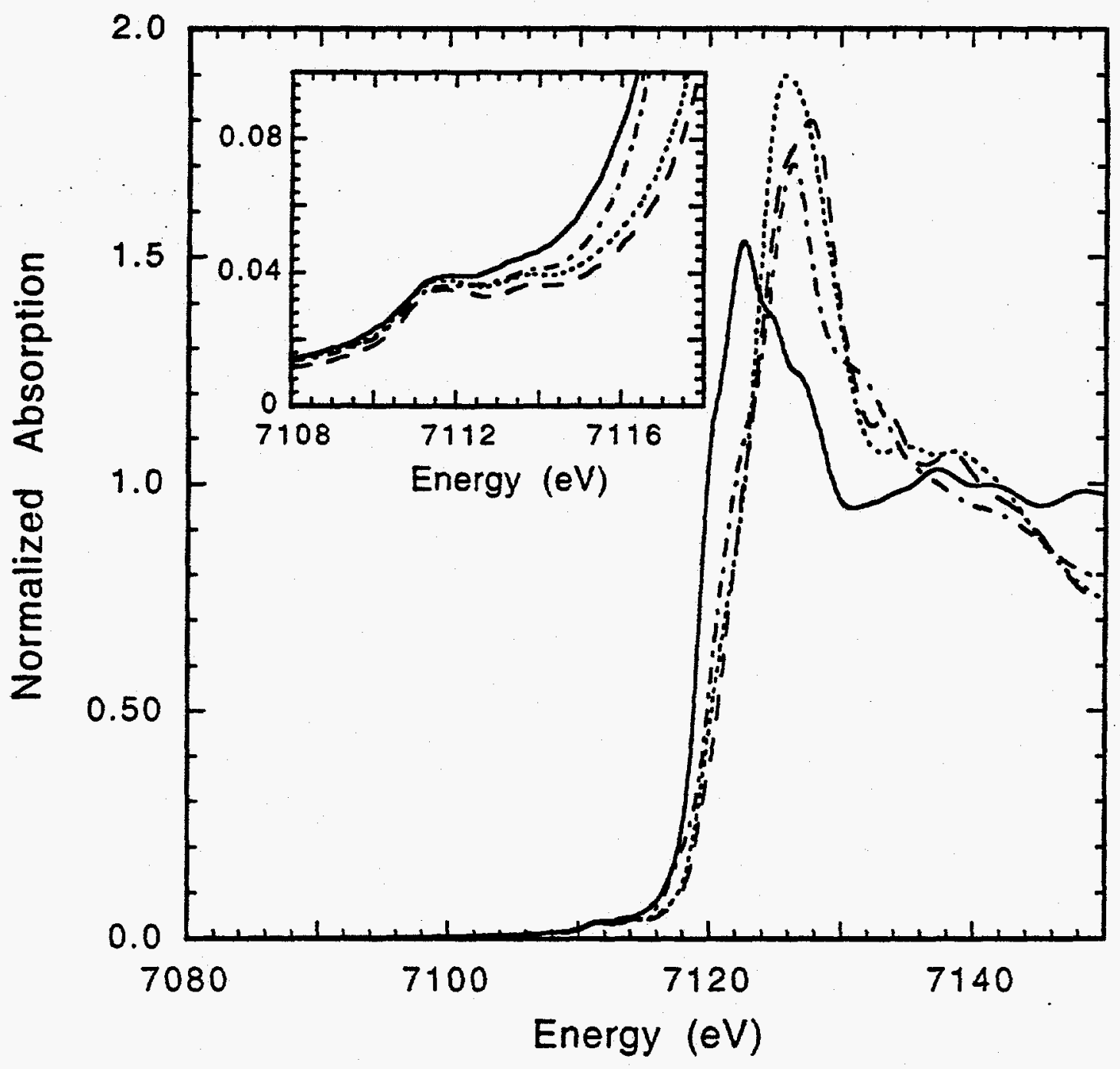

Figure 4.2. Fe K-edge XAS spectra of rinneite (-), $\mathrm{FeSiF}_{6} \cdot 6 \mathrm{H}_{2} \mathrm{O}(\cdots)$, $\left(\mathrm{NH}_{4}\right)_{2} \mathrm{Fe}\left(\mathrm{SO}_{4}\right)_{2} \cdot 6 \mathrm{H}_{2} \mathrm{O}(\cdots \cdot)$, and $\left.[\mathrm{Fe} \text { (imidazole })_{6}\right] \mathrm{Cl}_{2}(--)$; where the inset is an expansion of the $1 \mathrm{~s} \rightarrow 3 \mathrm{~d}$ pre-edge region. 
ground state configuration
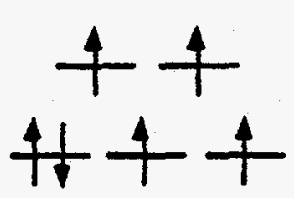

$t_{2} e^{2}$ ? excited state configurations

To obtain the strong-field many-electron excited states the holes in each of these configurations must be coupled. The $t_{2} e^{2}$ configuration gives rise to a ${ }^{4} \mathrm{~T}_{1}$ many-electron state, while the $t_{2}{ }^{2} e$ configuration gives a ${ }^{4} \mathrm{~T}_{1}$ and a ${ }^{4} \mathrm{~T}_{2}$ state. The ${ }^{4} \mathrm{~A}_{2}$ state originates from a $t_{2}{ }^{3}$ configuration and thus is forbidden from a $t_{2}{ }^{2} e^{2}$ ground state as it would involve a two-electron transition and it cannot mix with any other quartet state due to symmetry constraints. (Note that coupling of the 1 s hole to the these $d^{(n+1)}$ final states gives a set of ${ }^{5} \mathrm{~T}_{1},{ }^{5} \mathrm{~T}_{2}$, and ${ }^{5} \mathrm{~T}_{1}$ states and a set of ${ }^{3} \mathrm{~T}_{1},{ }^{3} \mathrm{~T}_{2}$, and ${ }^{3} \mathrm{~T}_{1}$ states. Only the quintet states are spin allowed and these will have the same relative energy splittings as the parent $d^{(n+1)}$ states. Thus, only the $d^{(n+1)}$ state nomenclature is used further.)

The energies of the ${ }^{4} \mathrm{~T}_{1},{ }^{4} \mathrm{~T}_{2}$, and ${ }^{4} \mathrm{~T}_{1}$ states can be determined by solving the Tanabe-Sugano matrices ${ }^{38}$ with appropriate values for the ligand field parameters, $10 \mathrm{Dq}$, $B$, and C. Ground state $10 \mathrm{Dq}$ values can be obtained from optical spectroscopy, however, excited state $10 \mathrm{Dq}$ values will be reduced in this case because of the addition of an electron into the $\mathrm{d}$-manifold. It has been found that $10 \mathrm{Dq}$ values obtained from charge transfer transition energies are $63 \%$ of the values obtained from ligand field transitions. ${ }^{40}$ In this case, however, the excited state $10 \mathrm{Dq}$ should not be reduced as much due to the increase in the effective nuclear charge from the $1 \mathrm{~s}$ hole. The energies of the manyelectron states were calculated with a $10 \mathrm{Dq}$ that is $80 \%$ of the ground state value as was experimentally determined from fits to the high spin ferric octahedral model complex data (vide infra). The $B$ value used was a $\mathrm{d}^{(\mathrm{n}+1)}$ free ion $\mathrm{B}$ value reduced by $10 \%$ to include covalency effects. The $C / B$ ratio was kept fixed at 4.0. High spin ferrous complexes typically have ground state $10 \mathrm{Dq}$ values of $-10,000 \mathrm{~cm}^{-1} .^{41}$ Using a $10 \mathrm{Dq}$ value of $8000 \mathrm{~cm}^{-1}$, a B value of $780 \mathrm{~cm}^{-1}$, and a $C$ value of $3120 \mathrm{~cm}^{-1}$, the energies of the ${ }^{4} \mathrm{~T}_{1},{ }^{4} \mathrm{~T}_{2}$, and ${ }^{4} \mathrm{~T}_{1}$ states were determined. The lowest energy excited state is the ${ }^{4} \mathrm{~T}_{1}\left(0.081 \mathrm{t}_{2}{ }^{2} \mathrm{e}>+0.921 \mathrm{t}_{2} \mathrm{e}^{2}>\right)$ state, $0.9 \mathrm{eV}$ higher in energy is the ${ }^{4} \mathrm{~T}_{2}\left(\mathrm{It}_{2}{ }^{2} \mathrm{e}>\right)$ state, and $1.3 \mathrm{eV}$ higher in energy than the ${ }^{4} \mathrm{~T}_{2}$ state is the ${ }^{4} \mathrm{~T}_{1}\left(0.92 / \mathrm{t}_{2}{ }^{2} \mathrm{e}>+0.081 \mathrm{t}_{2} \mathrm{e}^{2}>\right)$ state. Therefore, from ligand field theory one would expect that a high spin ferrous complex in an octahedral ligand field would have three pre-edge features of equal intensity split by 0.9 and $1.3 \mathrm{eV}$ (see Figure 4.3B). However, the first two features would be barely experimentally resolvable as the energy resolution at the $\mathrm{Fe} \mathrm{K}$-edge is $\sim 1.4 . \mathrm{eV}^{31}$ 


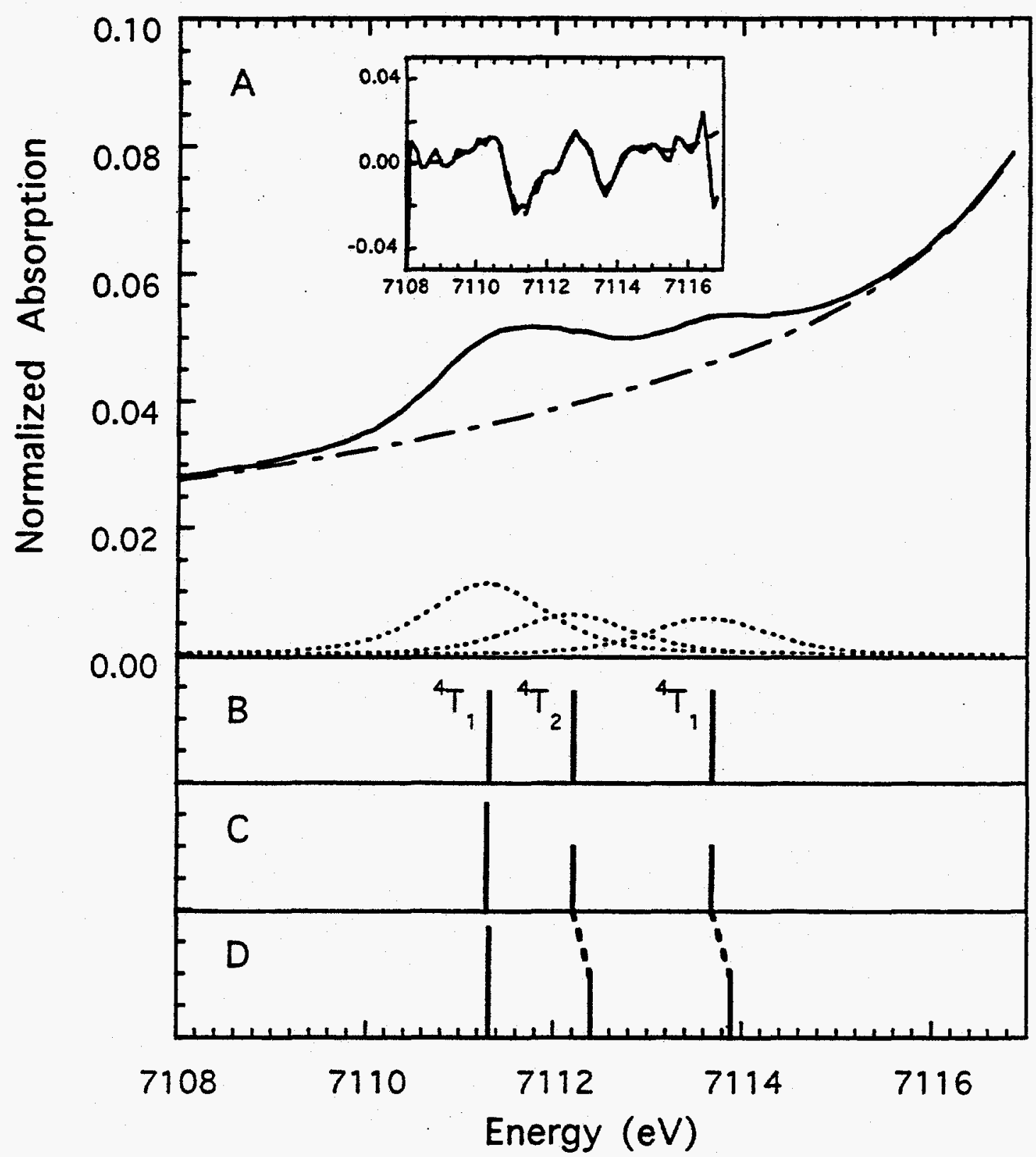

Figure 4.3. A) Fit to the Fe K-edge XAS pre-edge region of $\mathrm{FeSiF}_{6}{ }^{\circ} 6 \mathrm{H}_{2} \mathrm{O}$ where the solid line is the data, the dashed line is the fit to the data, the dashed-dot line is the background function, and the dotted lines are the individual pre-edge peaks. The inset is the second derivative of the data $(-)$ and the second derivative of the fit to the data (--). B) The energy splitting and the quadrupole intensity distribution of the ${ }^{4} \mathrm{~T}_{1},{ }^{4} \mathrm{~T}_{2}$ and ${ }^{4} \mathrm{~T}_{1}$ states. C) The energy splitting and the quadrupole intensity distribution of the ${ }^{4} \mathrm{~T}_{1},{ }^{4} \mathrm{~T}_{2}$ and ${ }^{4} \mathrm{~T}_{1}$ states when covalency effects are included. D) The effect of increased $10 \mathrm{Dq}$ on the energy splitting of the ${ }^{4} \mathrm{~T}_{1},{ }^{4} \mathrm{~T}_{2}$ and ${ }^{4} \mathrm{~T}_{1}$ states. 
Furthermore, the intensity of the three features should also reflect covalency effects. The e set of $3 \mathrm{~d}$ orbitals is more covalent than the $t_{2}$ set due to $\sigma$ bonding interactions with the ligands and, thus, states containing a $t_{2} \mathrm{e}^{2}$ component should be more intense than states containing a $t_{2}{ }^{2} \mathrm{e}$ component as there is more $d$-character in the $t_{2}$ set of orbitals. In the high spin ferrous case, only the lowest energy ${ }^{4} T_{1}$ state contains a $t_{2} e^{2}$ component. Thus, the lowest energy feature (arising from the transition to the lowest energy ${ }^{4} \mathrm{~T}_{1}$ state) should be more intense than the two features higher in energy (see Figure 4.3C). All of the ferrous high spin octahedral model complex pre-edge features needed to be fit with three peaks (with the exception of $\mathrm{Fel}_{2}$ which was adequately fit with two features) with the lower energy feature being more intense than the other two features (see Table 4.1 and Figure 4.3).

The total normalized pre-edge intensities of the octahedral high spin ferrous complexes range from 3.1 - 4.4 with an average intensity of 3.9. This is consistent with the quadrupole intensity observed for $\mathrm{D}_{4 \mathrm{~h}} \mathrm{CuCl}_{4}{ }^{2-} \cdot{ }^{24,42,43}$ The pre-edge feature of an isotropic sample of (creatininium) ${ }_{2} \mathrm{CuCl}_{4}$ (which has been shown to be quadrupole in character ${ }^{24}$ was fit using the same criteria as for the iron complexes. The results of the fit give a pre-edge feature at $8978.8(0.1) \mathrm{eV}$ with an intensity of $1.5(0.3) . \mathrm{Cu}$ (II) complexes are $3 d^{9}$ systems that have only one hole in the $3 d$ manifold. Therefore, to compare the total intensity of the $\mathrm{Cu}$ (II) complex to that of the $\mathrm{Fe}$ (II) complexes that are $3 d^{6}$ systems, the total pre-edge intensity has to be scaled by 4 and by $\left(Z_{\text {eff }}{ }^{2}\right.$ for $\mathrm{Fe}(\mathrm{II})) /\left(\mathrm{Z}_{\text {eff }}{ }^{2}\right.$ for $\left.\mathrm{Cu}(\mathrm{II})\right)$ as the quadrupole transition increases as $\mathrm{Z}_{\text {eff }}{ }^{2}$. $Z_{\text {eff }}$, estimated from Slater's rules, ${ }^{44}$ is 7.85 for $\mathrm{Cu}(\mathrm{II})$ and 5.90 for $\mathrm{Fe}(\mathrm{II})$. Thus, the predicted quadrupole intensity for a ferrous complex is $3.4(0.7)$ which is close to the experimental average intensity of 3.9. One would expect that the experimental value would be higher than the predicted quadrupole intensity as some of the complexes are slightly distorted from $\mathrm{O}_{\mathrm{h}}$ symmetry.

Trends in the pre-edge splittings of the three features in these complexes can be related to ligand field strength: $\mathrm{Fe}(\mathrm{II})\left(\right.$ imidazole) $6>\mathrm{Fe}(\mathrm{II})\left(\mathrm{H}_{2} \mathrm{O}\right)_{6}>\mathrm{FeF}_{2} \sim \mathrm{FeCl}_{2}>$ $\mathrm{FeBr}_{2}>\mathrm{FeI}_{2}$. This is the same trend that is observed for $10 \mathrm{Dq}$ values obtained from optical spectroscopy along the spectrochemical series. ${ }^{41}$ It is worth noting that the splittings of the pre-edge features observed for $\mathrm{FeSiF}_{6} \cdot 6 \mathrm{H}_{2} \mathrm{O}$ are identical to the calculated splittings of the states when using a $10 \mathrm{Dq}$ value that is $80 \%$ of the ground state 10Dq value of $\mathrm{FeSiF}_{6} \cdot 6 \mathrm{H}_{2} \mathrm{O}$. Increasing- $10 \mathrm{Dq}$ from 7500 to $13,000 \mathrm{~cm}^{-1}$, increases the splitting between the ${ }^{4} \mathrm{~T}_{1}$ and ${ }^{4} \mathrm{~T}_{2}$ states by $0.5 \mathrm{eV}$ and the splitting between the lowest and highest ${ }^{4} T_{1}$ states by $0.4 \mathrm{eV}$. 
4.3.1.2. $\mathbf{T}_{\mathbf{d}}$ Geometry. Fe K-edge XAS data were collected for three tetrahedral ferrous complexes: $\left(\mathrm{Et}_{4} \mathrm{~N}\right)_{2}\left[\mathrm{FeCl}_{4}\right], \mathrm{Cs}_{3} \mathrm{FeCl}_{5}$, and $\mathrm{Fe}\left(\mathrm{HB}\left(3,5-2 \mathrm{Pr}_{2} \mathrm{Pz}\right)_{3}\right) \mathrm{Cl}$. The iron environment in $\left(\mathrm{Et}_{4} \mathrm{~N}\right)_{2}\left[\mathrm{FeCl}_{4}\right]$ is very close to $\mathrm{T}_{\mathrm{d}}$ in geometry, $\mathrm{Cs}_{3} \mathrm{FeCl}_{5}$ is $\mathrm{D}_{2 \mathrm{~d}}$ distorted, and $\mathrm{Fe}\left(\mathrm{HB}\left(3,5-i \mathrm{Pr}_{2} \mathrm{Pz}\right)_{3}\right) \mathrm{Cl}$ is $\mathrm{C}_{3 \mathrm{v}}$ distorted. The XAS edge spectra for these three complexes are shown in Figure 4.4 with an expanded view of the $1 \mathrm{~s} \longrightarrow 3 \mathrm{~d}$ pre-edge region presented in the inset. All three complexes have a relatively intense pre-edge feature that is split by $1.5 \mathrm{eV}$ with the lower energy feature being more intense than the higher energy feature (Table 4.1). The $\mathrm{Fe}\left(\mathrm{HB}\left(3,5-i \mathrm{Pr}_{2} \mathrm{pz}\right)_{3}\right) \mathrm{Cl}$ pre-edge peaks are more intense than those of $\left(\mathrm{Et}_{4} \mathrm{~N}\right)_{2}\left[\mathrm{FeCl}_{4}\right]$ and $\mathrm{Cs}_{3} \mathrm{FeCl}_{5}$ (Figure 4.4 and Table 4.1).

The $1 \mathrm{~s} \longrightarrow 3 \mathrm{~d}$ pre-edge features of $\left(\mathrm{Et}_{4} \mathrm{~N}\right)_{2}\left[\mathrm{FeCl}_{4}\right], \mathrm{Cs}_{3} \mathrm{FeCl}_{5}$, and $\mathrm{Fe}\left(\mathrm{HB}(3,5-i \mathrm{Pr} 2 \mathrm{pz})_{3}\right) \mathrm{Cl}$ can gain intensity both through an allowed quadrupole transition and by $4 p$ mixing into the $3 \mathrm{~d}$ orbitals, since these complexes have a noncentrosymmetric environment around the iron site. In changing the symmetry from $\mathrm{O}_{h}$ to $T_{d}$, the e set of the $3 d$ orbitals is lower in energy than the $t_{2}$ set with $10 \mathrm{Dq}$ of the $T_{d}$ systems being $4 / 9$ that of the $\mathrm{O}_{\mathrm{h}} 10 \mathrm{Dq}$. In the $\mathrm{T}_{\mathrm{d}}$ case, the ground state has a hole configuration of $\mathrm{t}_{2}{ }^{3} \mathrm{e}$ and, therefore, the only allowed excited states are those containing the $t_{2}{ }^{3}$ or $t_{2}{ }^{2} \mathrm{e}$ configurations (Scheme 2).

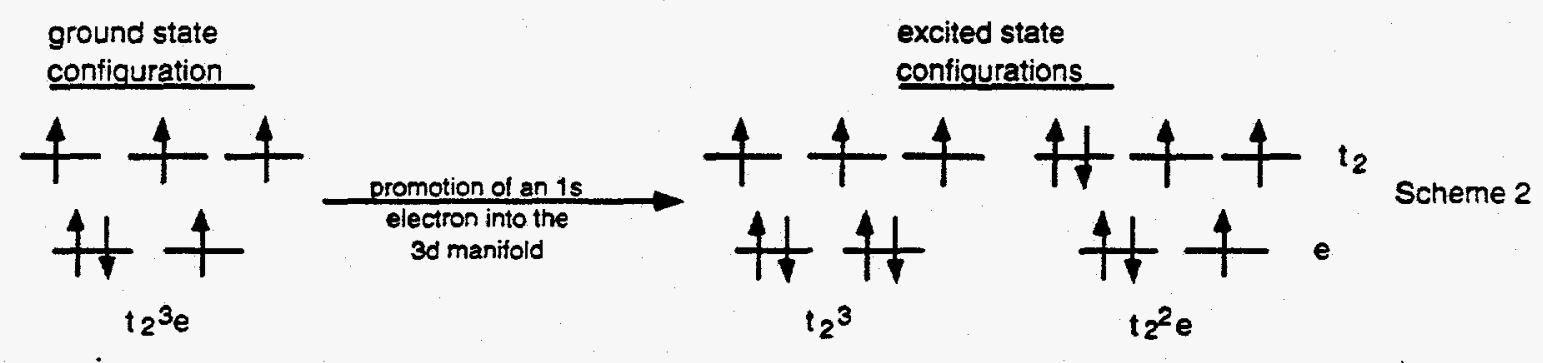

A $t_{2} e^{2}$ configuration would involve a forbidden two-electron transition. Coupling the three $\mathrm{t}_{2}$ holes gives a ${ }^{4} \mathrm{~A}_{2}$ many-electron state, while coupling the holes in the $\mathrm{t}_{2}{ }^{2} \mathrm{e}$ configuration give ${ }^{4} \mathrm{~T}_{1}$ and ${ }^{4} \mathrm{~T}_{2}$ states. The $\mathrm{t}_{2} \mathrm{e}^{2}$ configuration (arising from a twoelectron transition) also gives rise to a ${ }^{4} \mathrm{~T}_{1}$ state. Since the two ${ }^{4} \mathrm{~T}_{1}$ states are allowed to mix by symmetry, both ${ }^{4} \mathrm{~T}_{1}$ states will have an allowed $t_{2}{ }^{2} e$ component. The ${ }^{4} \mathrm{~A}_{2}$ and the ${ }^{4} \mathrm{~T}_{2}$ states only contain $\mathrm{t}_{2}{ }^{3}$ or $\mathrm{t}_{2}{ }^{2} \mathrm{e}$ components, respectively, and transitions into these states are fully allowed.

Energies of the four many-electron states were calculated using a 10Dq value of $3280 \mathrm{~cm}^{-1}\left(80 \%\right.$ of the ground state $10 \mathrm{Dq}$ of $\left.\left(\mathrm{Et}_{4} \mathrm{~N}\right)_{2}\left[\mathrm{FeCl}_{4}\right]\right),{ }^{45}$ a B value of $780 \mathrm{~cm}^{-1}$ $\left(90 \%\right.$ of the $\left.\mathrm{d}^{(\mathrm{n}+1)} \mathrm{B}\right)$, and a $\mathrm{C}$ value of $3120 \mathrm{~cm}^{-1}$. The ${ }^{4} \mathrm{~A}_{2}\left(\mathrm{lt}_{2}{ }^{3}>\right.$ ) state is the lowest energy excited state; the ${ }^{4} \mathrm{~T}_{2}\left(\mathrm{lt}_{2}{ }^{2} \mathrm{e}>\right)$ state is $0.4 \mathrm{eV}$ higher in energy; a ${ }^{4} \mathrm{~T}_{1}\left(0.31 \mathrm{lt}_{2}{ }^{2} \mathrm{e}>+\right.$ 


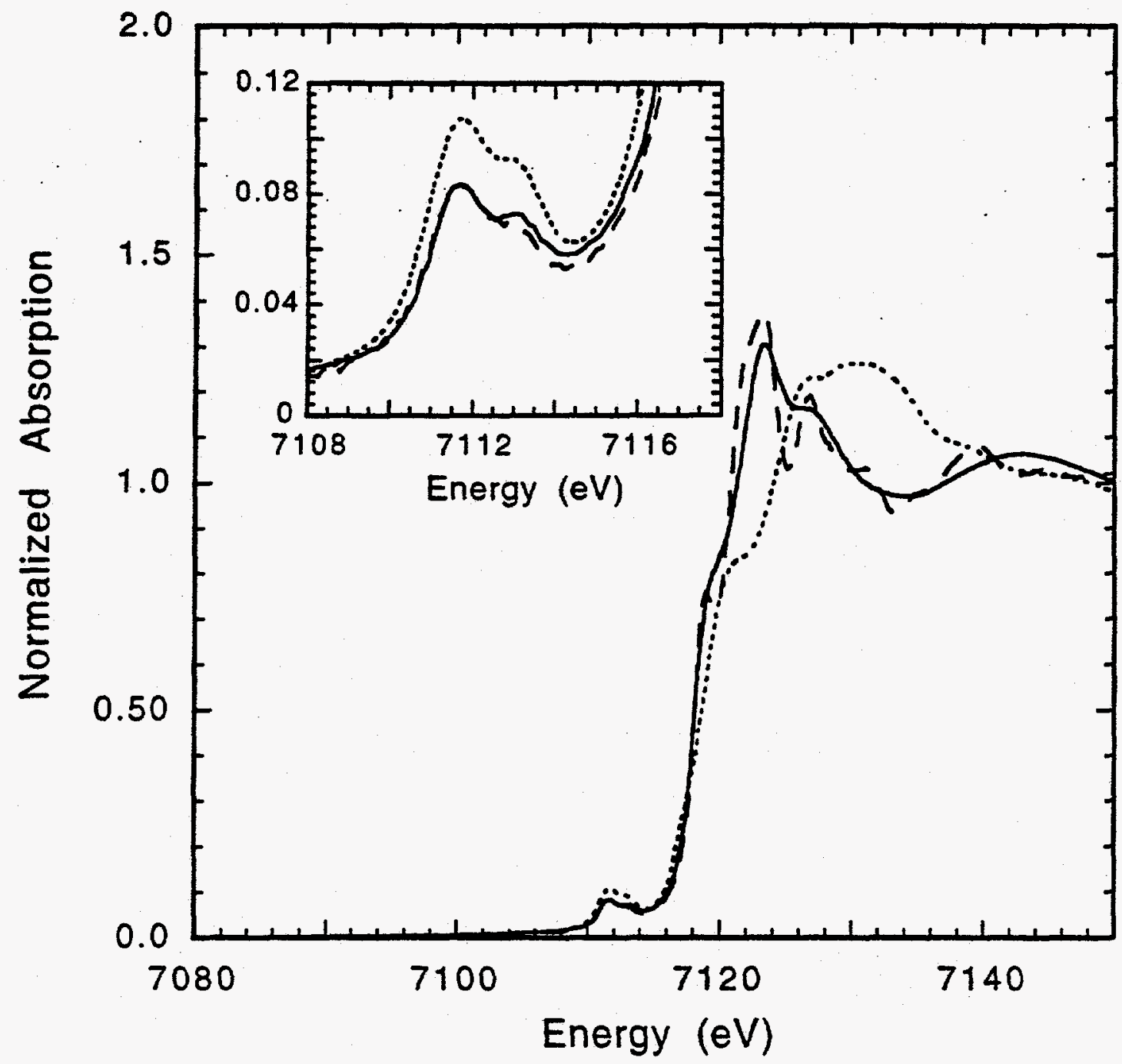

Figure 4.4. Fe K-edge XAS spectra of $\left(\mathrm{Et}_{4} \mathrm{~N}\right)_{2}\left[\mathrm{FeCl}_{4}\right](-), \mathrm{Cs}_{3} \mathrm{FeCl}_{5}(--)$, and $\mathrm{Fe}\left(\mathrm{HB}\left(3,5-i \mathrm{Pr}_{2} \mathrm{pz}\right)_{3}\right) \mathrm{Cl}(\cdots \cdot \cdot)$, where the inset is an expansion of the $1 \mathrm{~s} \rightarrow 3 \mathrm{~d}$ pre-edge region. 
$0.691 \mathrm{t}_{2} \mathrm{e}^{2}>$ ) state is $0.3 \mathrm{eV}$ higher in energy (than the ${ }^{4} \mathrm{~T}_{2}$ state); and a ${ }^{4} \mathrm{~T}_{1}\left(0.691 \mathrm{t}_{2}{ }^{2} \mathrm{e}>+\right.$ $0.31 t_{2} \mathrm{e}^{2}>$ ) state is $1.3 \mathrm{eV}$ higher in energy than the other ${ }^{4} \mathrm{~T}_{1}$ state. Since the $\mathrm{t}_{2} \mathrm{e}^{2}$ configuration requires a two-electron transition, only the $t_{2}{ }^{2} e$ components of the ${ }^{4} \mathrm{~T}_{1}$ states are allowed. The three lower energy states $\left({ }^{4} \mathrm{~A}_{2},{ }^{4} \mathrm{~T}_{2}\right.$, and $\left.{ }^{4} \mathrm{~T}_{1}\right)$ are all within $0.7 \mathrm{eV}$ of one another and, thus, will not be resolvable at the $\mathrm{Fe} \mathrm{K}$-edge, while the higher energy ${ }^{4} \mathrm{~T}_{1}$ state is positioned $2 \mathrm{eV}$ higher than the ${ }^{4} \mathrm{~A}_{2}$ state and should be resolvable.

The quadrupole intensity is divided between these states according to the degeneracy of the state and the percentage of the one-electron allowed strong field component (in the case of the ${ }^{4} \mathrm{~T}_{1}$ states), so that the quadrupole intensity ratio of the ${ }^{4} \mathrm{~A}_{2}:{ }^{4} \mathrm{~T}_{2}:{ }^{4} \mathrm{~T}_{1}:{ }^{4} \mathrm{~T}_{1}$ is 1:3:0.31 $3: 0.69 \times 3$ (see Figure 4.5B). The pre-edge features of these tetrahedral complexes will also gain intensity from $4 \mathrm{p}$ mixing into the $3 \mathrm{~d}$ orbitals. However, the $4 \mathrm{p}$ states transform as $\mathrm{t}_{2}{ }^{46}$ so there will only be mixing into the $3 \mathrm{~d} \mathrm{t}_{2}$ set and, thus, only the $d^{(n+1)}$ states that have $t_{2}{ }^{2} e$ components will have electric dipole intensity from $4 \mathrm{p}$ mixing into the $3 \mathrm{~d}$ orbitals. The ${ }^{4} \mathrm{~T}_{2},{ }^{4} \mathrm{~T}_{1}$, and ${ }^{4} \mathrm{~T}_{1}$ states have a $\mathrm{t}_{2}{ }^{2} \mathrm{e}$ component and will have an electric dipole intensity ratio of 3:0.31 $\times 3: 0.69 \times 3$, respectively (see Figure $4.5 \mathrm{C}$ ). Consequently, two pre-edge features are expected, with the first feature containing transitions into the ${ }^{4} \mathrm{~A}_{2},{ }^{4} \mathrm{~T}_{2}$, and ${ }^{4} \mathrm{~T}_{1}$ states and the second feature containing the transition into the higher energy ${ }^{4} \mathrm{~T}_{1}$ state. The intensity pattern of the data can be explained when both the quadrupole intensity and the dipole intensity from $4 \mathrm{p}$ character mixing into the $3 \mathrm{~d}_{2}$ orbitals are taken into account (see Figure 4.5). Using a total quadrupole intensity of $3.4(0.7)$ (the value calculated for the quadrupole contribution), the dipole intensity of $\left(\mathrm{Et}_{4} \mathrm{~N}\right)_{2}\left[\mathrm{FeCl}_{4}\right]$, which has a total intensity of 12.9(0.6) (Table 4.1), is 9.5(0.9). Taking the proper intensity ratio for each state (given above), the intensity of the first feature divided by the total intensity is 0.66 which is very close to the intensity ratio of 0.67 experimentally obtained for the pre-edge features of $\left(\mathrm{Et}_{4} \mathrm{~N}\right)_{2}\left[\mathrm{FeCl}_{4}\right]$ (Table 4.1).

The percentage of $4 \mathrm{p}$ mixing into the $3 \mathrm{~d}$ orbitals was also obtained. The dipole intensity (allowed by $4 p$ mixing into the $3 d t_{2}$ set of orbitals) is $9.5(0.9)$ for $\left(\mathrm{Et}_{4} \mathrm{~N}\right)_{2}\left[\mathrm{FeCl}_{4}\right]$. The quadrupole intensity is $-1 \%$ of the dipole intensity as has been calculated by Blair and Goddard ${ }^{22}$ and experimentally observed for $\mathrm{CuCl}_{4}{ }^{2-.42,43} \mathrm{~A}$ more rigorous figure can be obtained from an analysis of the pre-edge features of (creatininium) ${ }_{2} \mathrm{CuCl}_{4}, \mathrm{a} \mathrm{D}_{4 \mathrm{~h}}$ complex, and $\mathrm{Cs}_{2} \mathrm{CuCl}_{4}, \mathrm{a} \mathrm{D}_{2 \mathrm{~d}}$ complex. The fitted $1 \mathrm{~s}->3 \mathrm{~d}$ pre-edge areas of these two complexes are 1.5(0.3) and 7.1(0.5), respectively. The pre-edge feature of (creatininium) ${ }_{2} \mathrm{CuCl}_{4}$ only has quadrupole intensity from a transition into $3 d_{x^{2}}-y^{2}$ as the copper is in a centrosymmetric environment, while the pre-edge feature of $\mathrm{Cs}_{2} \mathrm{CuCl}_{4}$ has both quadrupole intensity from a transition into $3 \mathrm{~d}_{x} 2-\mathrm{y}^{2}$ and 


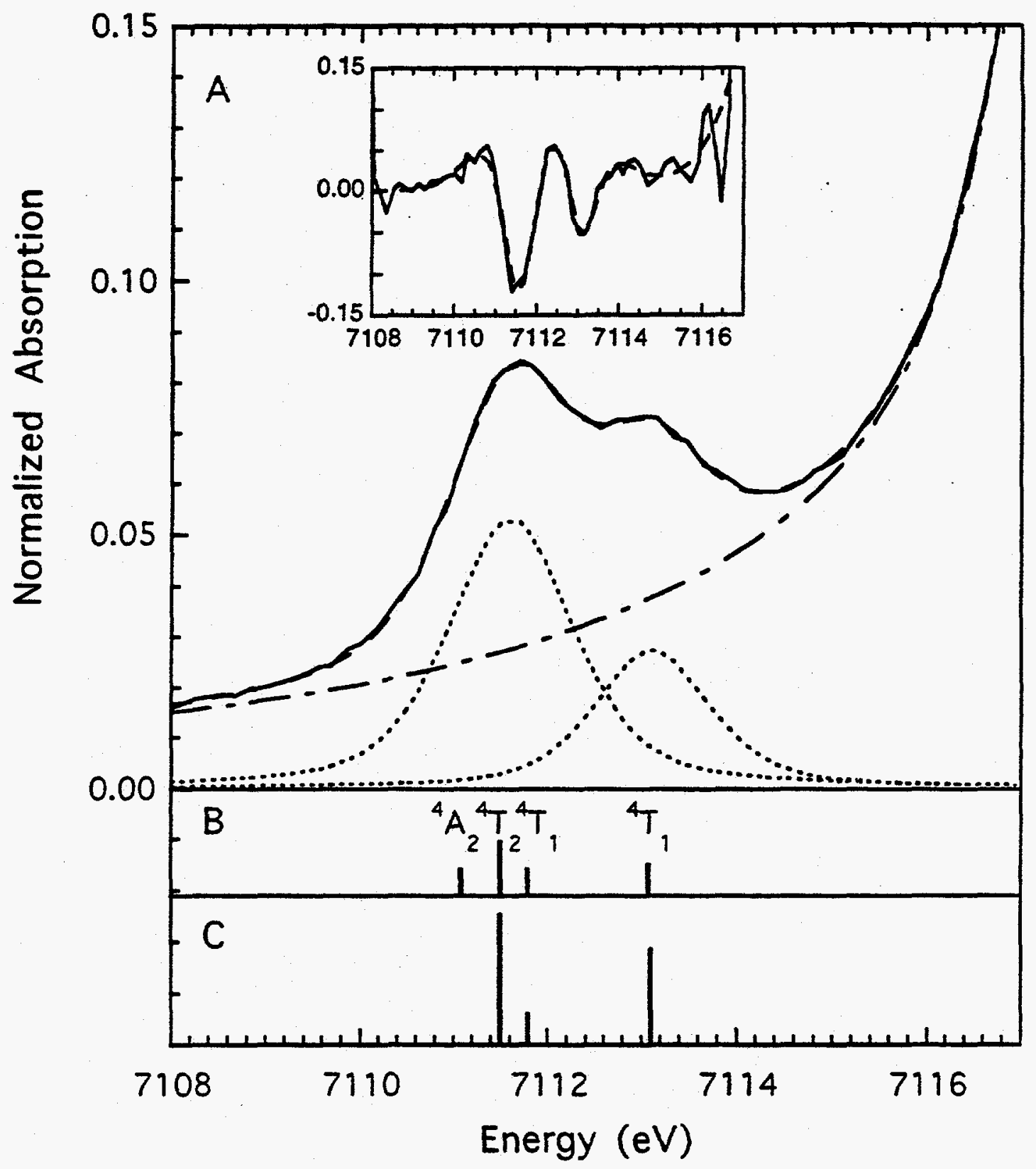

Figure 4.5. A) Fit to the $\mathrm{Fe}$ K-edge XAS pre-edge region of $\left(\mathrm{Et}_{4} \mathrm{~N}\right)_{2}\left[\mathrm{FeCl}_{4}\right]$, where the solid line is the data, the dashed line is the fit to the data, the dashed-dot line is the background function, and the dotted lines are the individual pre-edge peaks. The inset is the second derivative of the data $(-)$ and the second derivative of the fit to the data (--). B) The energy splitting and the quadrupole intensity distribution of the ${ }^{4} \mathrm{~A}_{2},{ }^{4} \mathrm{~T}_{2},{ }^{4} \mathrm{~T}_{1}$, and ${ }^{4} \mathrm{~T}_{1}$ states. $C$ ) The energy splitting and the dipole intensity distribution (allowed by $4 \mathrm{p}$ mixing into the $3 \mathrm{~d}$ states) of the ${ }^{4} \mathrm{~A}_{2},{ }^{4} \mathrm{~T}_{2},{ }^{4} \mathrm{~T}_{1}$, and ${ }^{4} \mathrm{~T}_{1}$ states. 
dipole intensity from $4 \mathrm{p}_{z}$ mixing into the $3 \mathrm{~d}_{\mathrm{x}} 2-\mathrm{y}^{2}$ orbital. The pre-edge feature of $\mathrm{Cs}_{2} \mathrm{CuCl}_{4}$ should also have $-1.5(0.3)$ units of quadrupole intensity from a transition into $3 d_{x} 2-y 2$, however, self-consistent field-X $\alpha$-scattered wave calculations indicate that the $3 d_{x^{2}}-y^{2}$ component of the lowest unoccupied molecular orbital is $61 \%$ for (creatininium) ${ }_{2} \mathrm{CuCl}_{4}$ and $67 \%$ for $\mathrm{Cs}_{2} \mathrm{CuCl}_{4}{ }^{42,43}$ Taking into account the differences in d character in the lowest unoccupied molecular orbital, the amount of quadrupole intensity in the pre-edge feature of $\mathrm{Cs}_{2} \mathrm{CuCl}_{4}$ should be 1.65 (0.3). Thus, the amount of dipole intensity in the pre-edge feature of $\mathrm{Cs}_{2} \mathrm{CuCl}_{4}$ should be 5.45(0.6), 7.1(0.5) minus 1.65(0.3). The amount of $4 \mathrm{p}_{z}$ mixing into the lowest unoccupied molecular orbital is known to be $3.8(1.5) \%$ from an analysis of the $\mathrm{Cu} 1 \mathrm{~s} \rightarrow 4 \mathrm{p}+$ ligand-to-metal charge-transfer shakedown transition. ${ }^{42,43}$ Hence, $3.8(1.5) \% 4 \mathrm{p}_{z}$ mixing into the lowest unoccupied molecular orbital provides for $5.45(0.6)$ units of pre-edge intensity. In other words, $1 \% 4 \mathrm{p}$ mixing yields $1.43(0.6)$ units of $1 \mathrm{~s} \longrightarrow 3 \mathrm{~d}$ pre-edge intensity. Using this ratio, the percentage of $4 \mathrm{p}$ mixing into the $3 \mathrm{~d}$ orbitals was obtained for $\left(\mathrm{Et}_{4} \mathrm{~N}\right)_{2}\left[\mathrm{FeCl}_{4}\right]$. Since the dipole intensity of the pre-edge feature for $\left(\mathrm{Et}_{4} \mathrm{~N}\right)_{2}\left[\mathrm{FeCl}_{4}\right]$ is $9.5(0.9)$ units, the amount of $4 \mathrm{p}$ mixing into the $3 \mathrm{~d}$ orbitals is $9.5(0.9) / 1.43(0.6)$, or $6.6(2.8) \%$. A density functional calculation on $\left(\mathrm{Et}_{4} \mathrm{~N}\right)_{2}\left[\mathrm{FeCl}_{4}\right]$ predicts $2-3 \% 4 \mathrm{p}$ mixing into each of the $3 \mathrm{~d}$ orbitals with $t_{2}$ symmetry, indicating that the total amount of $4 p$ mixing into the $3 \mathrm{~d}$ manifold is $6-9 \% .^{47}$

4.3.1.3. $\mathrm{C}_{4 \mathrm{v}}$ Geometry. Fe K-edge XAS data were collected for four square pyramidal high spin ferrous model complexes: $\left(\mathrm{BF}_{4}\right)[\mathrm{Fe}(\mathrm{TMC}) \mathrm{Cl}],\left(\mathrm{BF}_{4}\right)[\mathrm{Fe}(\mathrm{TMC}) \mathrm{Br}]$, $\left(\mathrm{BF}_{4}\right)\left[\mathrm{Fe}(\mathrm{TMC}) \mathrm{CH}_{3} \mathrm{CN}\right]$, and $\left(\mathrm{BF}_{4}\right)\left[\mathrm{Fe}(\mathrm{TMC}) \mathrm{N}_{3}\right]$. The spectra of these four complexes are shown in Figure 4.6 with the inset showing an expanded view of the $1 \mathrm{~s} \longrightarrow 3 \mathrm{~d}$. pre-edge region. All four of these square pyramidal complexes have an intense feature at $-7111.5 \mathrm{eV}$, with a second much weaker feature at $\sim 7113.3 \mathrm{eV}$ where the second feature is visible in the second derivative. $\left(\mathrm{BF}_{4}\right)\left[\mathrm{Fe}(\mathrm{TMC}) \mathrm{N}_{3}\right]$ has the most intense pre-edge feature while that of $\left(\mathrm{BF}_{4}\right)[\mathrm{Fe}(\mathrm{TMC}) \mathrm{Br}]$ is the least intense (Table 4.1).

An iron atom in a $\mathrm{C}_{4 \mathrm{v}}$ site has a noncentrosymmetric environment and thus the $1 \mathrm{~s} \rightarrow 3 \mathrm{~d}$ pre-edge transition gains intensity both from the allowed quadrupole and from dipole (associated with $4 \mathrm{p}$ mixing into the $3 \mathrm{~d}$ orbitals) mechanisms. $\mathrm{A} \mathrm{C}_{4 \mathrm{v}}$ site can be treated as a distorted $\mathrm{O}_{\mathrm{h}}$ site, in which the ${ }^{4} \mathrm{~T}_{2} \mathrm{~d}^{(\mathrm{n}+1)}$ state (vide supra) splits into a ${ }^{4} \mathrm{~B}_{2}$ and $a{ }^{4} \mathrm{E}$ state and the two ${ }^{4} \mathrm{~T}_{1}$ states split into $\mathrm{a}^{4} \mathrm{~A}_{2}$ and $\mathrm{a}^{4} \mathrm{E}$ state. If the ground state orbital splitting is as shown in Scheme 3, then promotion of a 1s electron into the 3d manifold produces three $d^{(n+1)}$ allowed excited state configurations: $e a_{1} b_{1}, e^{2} b_{1}$, and $e^{2} a_{1}$ (Scheme 3). 


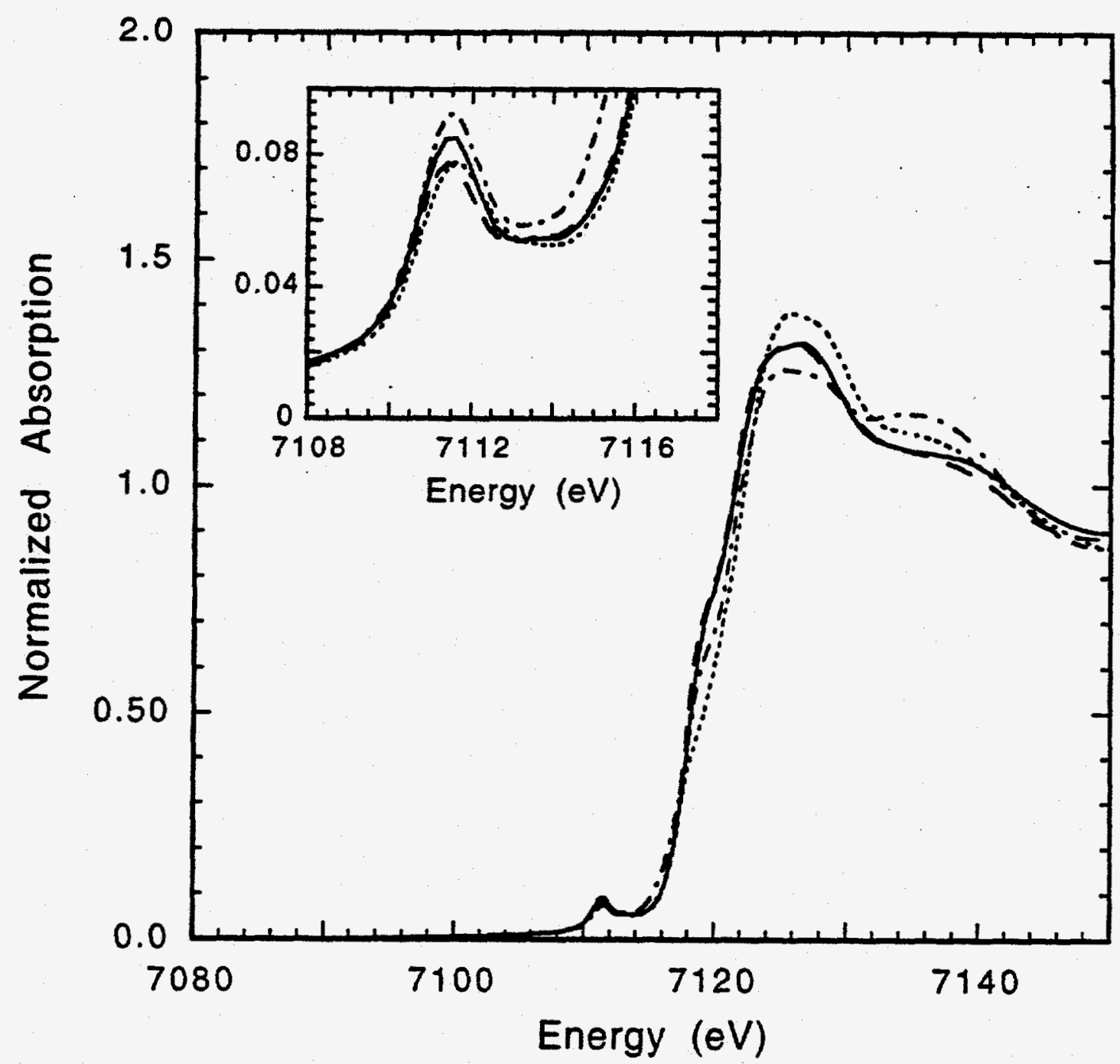

Figure 4.6. Fe K-edge XAS spectra of $\left(\mathrm{BF}_{4}\right)[\mathrm{Fe}(\mathrm{TMC}) \mathrm{Cl}](-),(\mathrm{BF} 4)[\mathrm{Fe}(\mathrm{TMC}) \mathrm{Br}](--)$, $\left(\mathrm{BF}_{4}\right)\left[\mathrm{Fe}(\mathrm{TMC}) \mathrm{CH}_{3} \mathrm{CN}\right](\cdots \cdot)$, and $\left(\mathrm{BF}_{4}\right)\left[\mathrm{Fe}\left(\mathrm{TMC}_{3} \mathrm{~N}_{3}\right](--)\right.$, where the inset is an expansion of the $1 \mathrm{~s} \rightarrow 3 \mathrm{~d}$ pre-edge region. 
ground state configuration

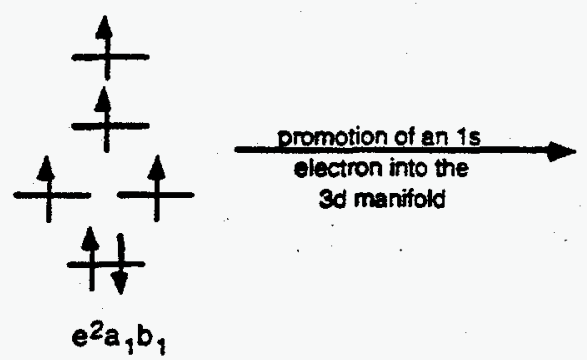

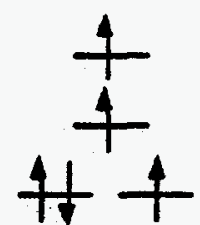

41

$e_{1} b_{1}$ excited state

configurations
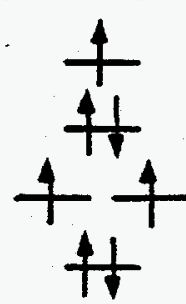

$e^{2} b_{1}$

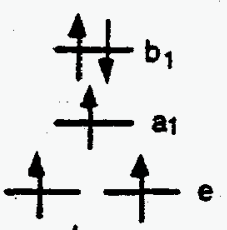

Scheme 3

The relative energies of these configurations have been determined from density functional calculations ${ }^{47}$ and are consistent with values obtained from optical absorption experiments for square pyramidal ferrous complexes. ${ }^{41}$ From these calculations, the $e^{2} b_{1}$ configuration (which contributes to the ${ }^{4} \mathrm{~B}_{2}$ state from the $\mathrm{O}_{\mathrm{h}}{ }^{4} \mathrm{~T}_{2}$ state) is the lowest in energy, with the $e a_{1} b_{1}$ configuration (which contributes to the ${ }^{4} E$ state from the lower energy $\mathrm{O}_{\mathrm{h}}{ }^{4} \mathrm{~T}_{1}$ state) being $0.4 \mathrm{eV}$ higher in energy, and the $\mathrm{e}^{2} \mathrm{a}_{1}$ configuration (which contributes to the ${ }^{4} \mathrm{~A}_{2}$ state from the higher energy $\mathrm{O}_{\mathrm{b}}{ }^{4} \mathrm{~T}_{1}$ state) being $1.6 \mathrm{eV}$ higher than the ea $\mathrm{b}_{1}$ configuration. ${ }^{47}$ Thus, the many-electron states that involve one-electron transitions are the ${ }^{4} B_{2},{ }^{4} \mathrm{E}$, and ${ }^{4} \mathrm{~A}_{2}$ states. The quadrupole intensity of these states will have an intensity ratio of 1:2:1, respectively (see Figure 4.7B).

The pre-edge features of these square pyramidal complexes can gain additional intensity from $4 \mathrm{p}$ mixing into the $3 \mathrm{~d}$ orbitals. In the $\mathrm{C}_{4 \mathrm{v}}$ case, the $4 \mathrm{p}$ orbitals transform as $e\left(4 p_{x, y}\right)$ and $a_{1}\left(4 p_{z}\right)$. As the main distortion is along the $z$ axis, one would predict that the $4 p_{z}$ orbital mixing into the $d_{z}^{2}$ orbital of $a_{1}$ symmetry will be the dominant effect. Thus, the transition to the ${ }^{4} \mathrm{~B}_{2}$ state that contains the $\mathrm{e}^{2} \mathrm{~b}_{1}$ component should be the most intense. This is consistent with the data where the lower energy feature is much more intense than the higher energy feature (see Figure 4.7). The density functional calculation for $\left(\mathrm{BF}_{4}\right)[\mathrm{Fe}(\mathrm{TMC}) \mathrm{Cl}]$ also shows that the $\mathrm{d}_{2}{ }^{2}$ orbital contains $4 \mathrm{pz}$ character, while the $d_{x z}$ and $d_{y z}$ e orbitals contained no significant $4 p_{x y}$ character. ${ }^{47}$ The amount of $4 p_{z}$ mixing into the $\mathrm{d}_{\mathrm{z}}{ }^{2}$ orbital can be estimated from the experimental data. The total pre-edge intensity for $\left(\mathrm{BF}_{4}\right)[\mathrm{Fe}(\mathrm{TMC}) \mathrm{Cl}]$ is $12.9(0.2)$ (Table 4.1). If 3.4(0.7) units are attributed to the quadrupole transition, then $9.5(0.7)$ units originate from $4 \mathrm{p}_{z}$ (dipole allowed) character in the $d_{z}{ }^{2}$ orbital. If $1 \% 4 p$ mixing account for $1.43(0.6)$ units of area (vide supra), the amount of $4 \mathrm{pz}_{\mathrm{z}}$ mixing into the $3 \mathrm{~d}_{\mathrm{z}}{ }^{2}$ orbital is $9.5(0.7) / 1.43(0.6)$, or $6.6(2.8) \%$. The density functional calculation for $\left(\mathrm{BF}_{4}\right)[\mathrm{Fe}(\mathrm{TMC}) \mathrm{Cl}]$ shows $9 \% 4 \mathrm{p}_{\mathrm{z}}$ mixing into the $\mathrm{d}_{\mathrm{z}}{ }^{2}$ orbital. ${ }^{47}$

Considering that there is only one state that gains intensity from the $4 p_{z}$ mixing into the $\mathrm{d}_{\mathrm{z}}{ }^{2}$ orbital, the axial interaction will be reflected in the intensity and position of 


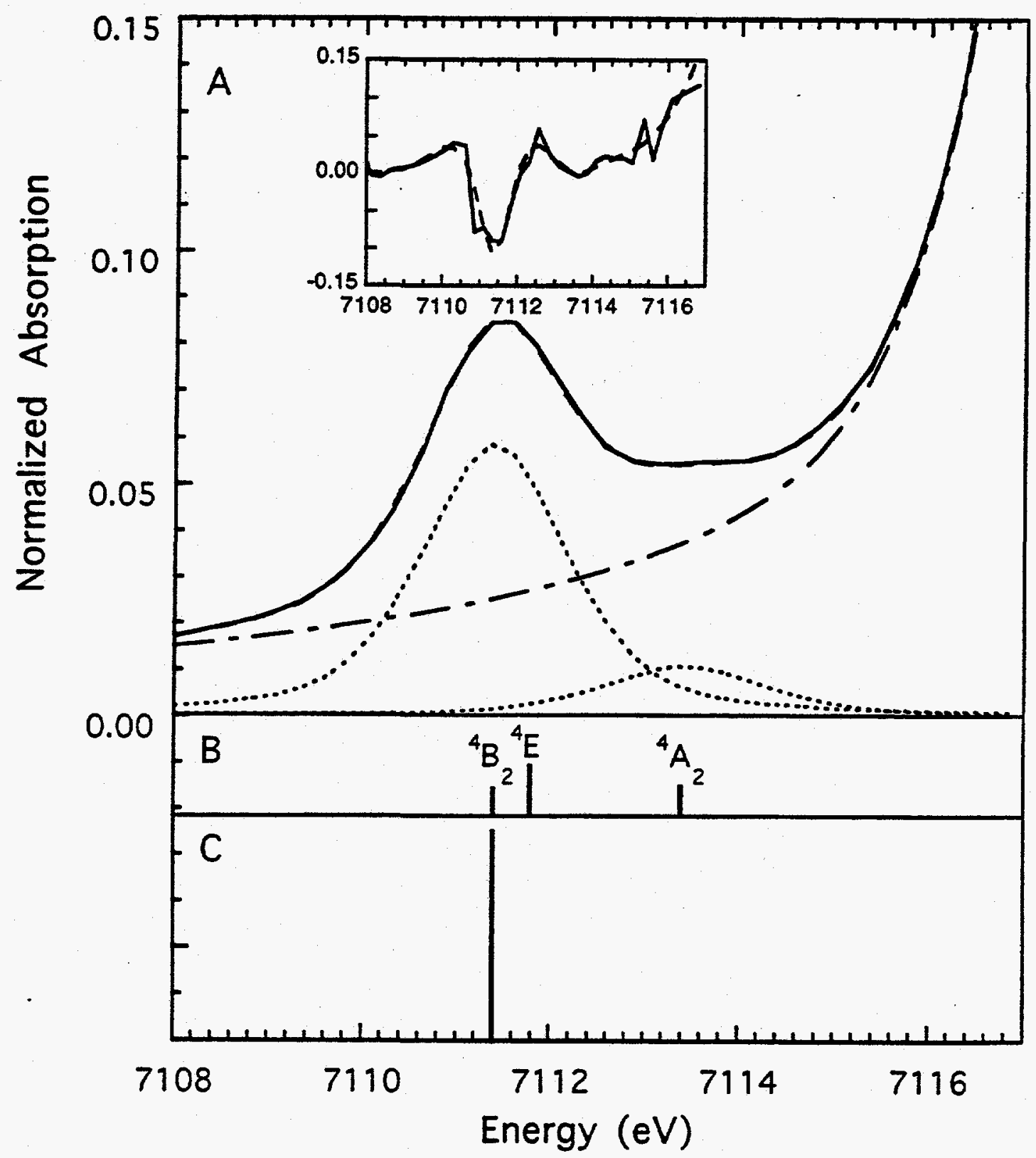

Figure 4.7. A) Fit to the $\mathrm{Fe} \mathrm{K}$-edge XAS pre-edge region of $\left(\mathrm{BF}_{4}\right)[\mathrm{Fe}(\mathrm{TMC}) \mathrm{Cl}]$, where the solid line is the data, the dashed line is the fit to the data, the dashed-dot line is the background function, and the dotted lines are the individual pre-edge peaks. The inset is the second derivative of the data (-) and the second derivative of the fit to the data (---). B) The energy splitting and the quadrupole intensity distribution of the ${ }^{4} \mathrm{~B}_{2},{ }^{4} \mathrm{E}$, and ${ }^{4} \mathrm{~A}_{2}$ states. C) The energy splitting and the dipole intensity distribution (allowed by $4 p$ mixing into the $3 \mathrm{~d}$ states) of the ${ }^{4} \mathrm{~B}_{2},{ }^{4} \mathrm{E}$, and ${ }^{4} \mathrm{~A}_{2}$ states. 
the most intense pre-edge feature. For example, the intensity of this pre-edge feature of the high spin ferrous complexes in this study decreases as: $\left(\mathrm{BF}_{4}\right)\left[\mathrm{Fe}(\mathrm{TMC}) \mathrm{N}_{3}\right]>$ $\left(\mathrm{BF}_{4}\right)[\mathrm{Fe}(\mathrm{TM} / \mathrm{C}) \mathrm{Cl}]>\left(\mathrm{BF}_{4}\right)\left[\mathrm{Fe}(\mathrm{TMC}) \mathrm{CH}_{3} \mathrm{CN}\right]>\left(\mathrm{BF}_{4}\right)[\mathrm{Fe}(\mathrm{TMC}) \mathrm{Br}]$. This trend matches the strength of the axial ligand with the azide having the strongest interaction and the bromide having the weakest.

It is important to note that both $\mathrm{T}_{\mathrm{d}}\left(\mathrm{Et}_{4} \mathrm{~N}_{2}\left[\mathrm{FeCl}_{4}\right]\right.$ and $\mathrm{C}_{4 \mathrm{v}}\left(\mathrm{BF}_{4}\right)[\mathrm{Fe}(\mathrm{TMC}) \mathrm{Cl}]$ have the same pre-edge intensity. Thus, one would not be able to determine the site symmetry of the iron solely on the basis of total pre-edge intensity. ${ }^{1,20}$ However, the distribution of intensity over the multiplet features of $T_{d}$ and $C_{4 v}$ complexes is very different. In the $T_{d}$ case, the dipole intensity (from $4 p$ mixing into the $t_{2} d$ orbitals) is distributed over both pre-edge features (Figure 4.5), while in the $\mathrm{C}_{4 \mathrm{v}}$ case (where one axial ligand is removed), the dipole intensity from $4 \mathrm{p}_{z}$ mixing into the $\mathrm{d}_{z}{ }^{2}$ orbital is located only in the lowest energy pre-edge features since removing an axial ligand lowers the energy of $d_{z} 2$ (Figure 4.7).

\subsubsection{High Spin Ferric Complexes}

4.3.2.1. $\mathrm{O}_{\mathrm{h}}$ Geometry. Fe K-edge XAS data were collected for nine high spin ferric octahedral model complexes with varying ligation: $\mathrm{F}^{-}, \mathrm{Cl}^{-}, \mathrm{Br}$, and $\mathrm{O}$ and $\mathrm{N}$ from acac, malonate, oxalate, urea, and $\mathrm{H}_{2} \mathrm{O}$. The XAS edge spectra for $\mathrm{FeF}_{3}, \mathrm{FeCl}_{3}, \mathrm{FeBr}_{3}$, and $\left[\mathrm{FeCl}_{6}\right]\left[\mathrm{Co}\left(\mathrm{NH}_{4}\right)_{6}\right]$ are shown in Figure 4.8 and the spectra for $\mathrm{Fe}(\mathrm{acac})_{3}$, $\left(\mathrm{NH}_{4}\right)_{3} \mathrm{Fe}$ (malonate) $)_{3}$, $\left(\mathrm{NH}_{4}\right) \mathrm{Fe}\left(\mathrm{SO}_{4}\right)_{2} \bullet 12 \mathrm{H}_{2} \mathrm{O}$, and $\mathrm{Fe}(\text { urea })_{6}\left(\mathrm{ClO}_{4}\right)_{3}$ are shown in Figure 4.9. The lowest energy transitions are the weak $1 \mathrm{~s} \rightarrow 3 \mathrm{~d}$ pre-edge peaks, which are expanded and shown as insets in Figures 4.8 and 4.9. All nine complexes have a low intensity split pre-edge feature (Table 4.1).

Since all nine of these complexes have a centrosymmetric octahedral iron site, the only intensity mechanism available for the $1 \mathrm{~s} \rightarrow 3 \mathrm{~d}$ feature is the allowed quadrupole transition. In the high spin ferric case, there are five $3 d$ electrons in the ground state with a $t_{2}{ }^{3} \mathrm{e}^{2}$ configuration. Promotion of a $1 \mathrm{~s}$ electron into the $3 \mathrm{~d}$ manifold produces two excited state configurations, $\mathrm{at}_{2} \mathrm{e}^{2}$ and $\mathrm{t}_{2}{ }^{3} \mathrm{e}$ (Scheme 4 ).
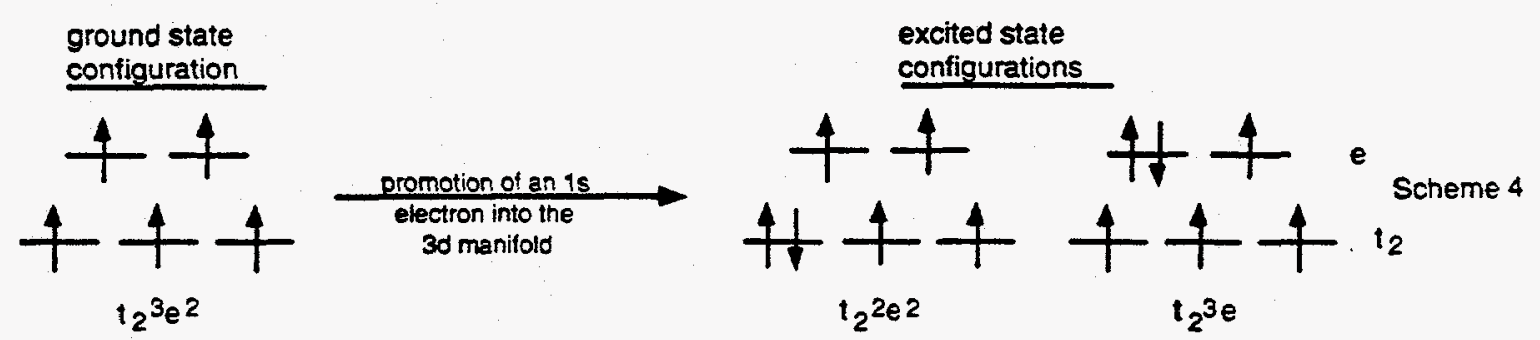


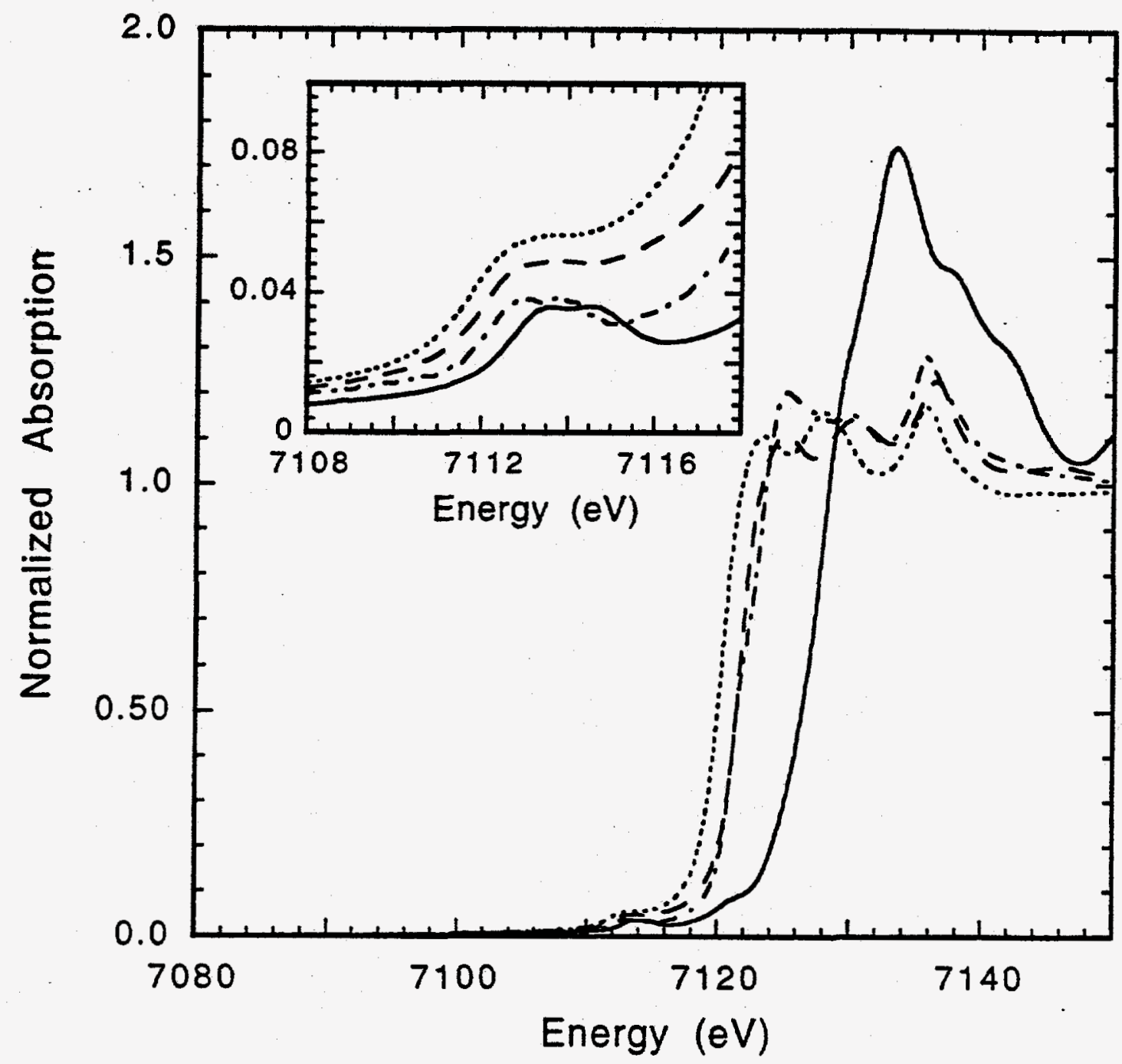

Figure 4.8. $\mathrm{Fe} \mathrm{K}$-edge XAS spectra of $\mathrm{FeF}_{3}(-), \mathrm{FeCl}_{3}(\cdots), \mathrm{FeBr}_{3}(\cdots \cdots)$, and $\left[\mathrm{FeCl}_{6}\right]\left[\mathrm{Co}\left(\mathrm{NH}_{4}\right)_{6}\right](--)$, where the inset is an expansion of the $1 \mathrm{~s} \rightarrow 3 \mathrm{~d}$ pre-edge region. 


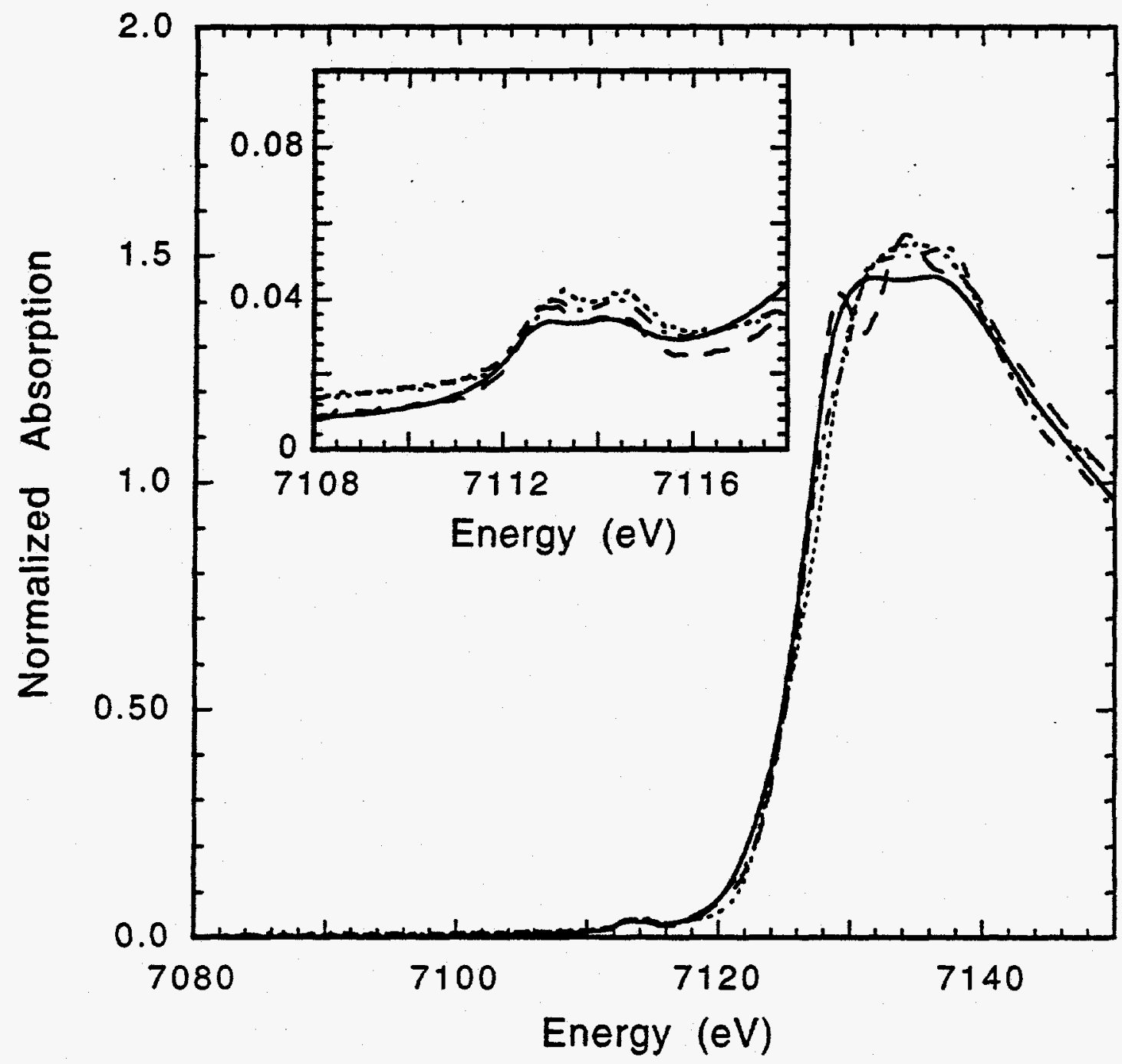

Figure 4.9. $\mathrm{Fe} \mathrm{K}$-edge XAS spectra of $\mathrm{Fe}(\mathrm{acac})_{3}(-),\left(\mathrm{NH}_{4}\right)_{3} \mathrm{Fe}$ (malonate) $)_{3}(--)$, $\left(\mathrm{NH}_{4}\right) \mathrm{Fe}\left(\mathrm{SO}_{4}\right)_{2} \cdot 12 \mathrm{H}_{2} \mathrm{O}(\cdots \cdot)$, and $\mathrm{Fe}(\text { urea })_{6}\left(\mathrm{ClO}_{4}\right)_{3}(--)$, where the inset is an expansion of the $1 \mathrm{~s} \rightarrow 3 \mathrm{~d}$ pre-edge region. 
Coupling of the holes in the $t_{2}{ }^{2} e^{2}$ and $t_{2}{ }^{3} e$ configurations gives a ${ }^{5} T_{2}$ and a ${ }^{5} \mathrm{E}$ state, respectively. ${ }^{36}$ Therefore, one predicts an octahedral high spin ferric complex to have two pre-edge features split by an excited state $10 \mathrm{Dq}$ with a quadrupole intensity ratio of $3: 2$. Fits to the data show that there are indeed pre-edge two features with an intensity ratio of $\sim 3: 2$ and splittings that range from 1.1 to $1.5 \mathrm{eV}$ (Figure 4.10 and Table 4.1). The energy splitting between the two pre-edge features is a direct measurement of the $10 \mathrm{Dq}$ value of the $d^{(n+1)}$ final state. In comparing these energy splitting with ground state $10 \mathrm{Dq}$ values from optical data, ${ }^{41}$ the excited state $10 \mathrm{Dq}$ values are $80 \%$ of the ground state values. The splitting of the two pre-edge features in these complexes can be related to ligand field strength with the splittings following the trend $\mathrm{Fe}-\mathrm{O} / \mathrm{Fe}-\mathrm{N}>\mathrm{FeF}_{3}>\mathrm{FeCl} 3$ $>$ FeBr3.

The total pre-edge intensities of the octahedral high spin ferric complexes range from 4.0 to 7.3 with the average intensity being 4.9. As was done for the high spin ferrous complexes in an $\mathrm{O}_{\mathrm{h}}$ field, an estimate of the quadrupole intensity was calculated using the area of the pre-edge feature for $\mathrm{D}_{4 h} \mathrm{CuCl}_{4}{ }^{2-}$ of $1.5(0.3)$. That value was scaled by 5 for the number of $3 d$ electrons and multiplied by $\left(Z_{\text {eff }}{ }^{2}\right.$ for $\left.\mathrm{Fe}(\mathrm{III})\right) /\left(\mathrm{Z}_{\mathrm{eff}}{ }^{2}\right.$ for $\mathrm{Cu}(\mathrm{II})$ ) where $Z_{\text {eff }}$ is 7.85 for $\mathrm{Cu}(\mathrm{II})$ and 6.25 for $\mathrm{Fe}(\mathrm{III})$ giving a predicted quadrupole intensity for a ferric complex of $4.7(0.9)$ which is very similar to the experimental averaged intensity of 4.9. One would expect that the experimental value would be higher than the predicted quadrupole intensity as some of the complexes are distorted from $\mathrm{O}_{\mathrm{h}}$ symmetry.

4.3.2.2. $T_{\mathbf{d}}$ Geometry. Fe K-edge XAS data were collected for one high spin ferric tetrahedral complex, (Et4N) $\left[\mathrm{FeCl}_{4}\right]$. The XAS spectrum is shown in Figure 4.11 (solid line) with the $1 \mathrm{~s} \longrightarrow 3 \mathrm{~d}$ pre-edge region expanded and shown in the inset.. $\left(\mathrm{Et}_{4} \mathrm{~N}\right)\left[\mathrm{FeCl}_{4}\right]$ has a very intense single pre-edge feature (Table 4.1).

The $1 \mathrm{~s} \rightarrow 3 \mathrm{~d}$ pre-edge feature of $\left(\mathrm{EL}_{4} \mathrm{~N}\right)\left[\mathrm{FeCl}_{4}\right]$ gains intensity both through an allowed quadrupole transition and dipole transition from $4 \mathrm{p}$ mixing into the $3 \mathrm{~d}$ orbitals, since this complex has a noncentrosymmetric environment around the iron site. In changing symmetry from $O_{h}$ to $T_{d}$, the e set of the $3 d$ orbitals is lower in energy than the $t_{2}$ set with $10 D q$ of the $T_{d}$ systems being $4 / 9$ that of the $O_{h} 10 D q$. As in the high spin ferric octahedral case, there are two one-electron allowed excited state configurations, $\mathrm{t}_{2}{ }^{2} \mathrm{e}^{2}$ and $\mathrm{t}_{2}{ }^{3} \mathrm{e}$ (Scheme 5), that produce two many-electron states, ${ }^{5} \mathrm{~T}_{2}$ and ${ }^{5} \mathrm{E}$, respectively. 


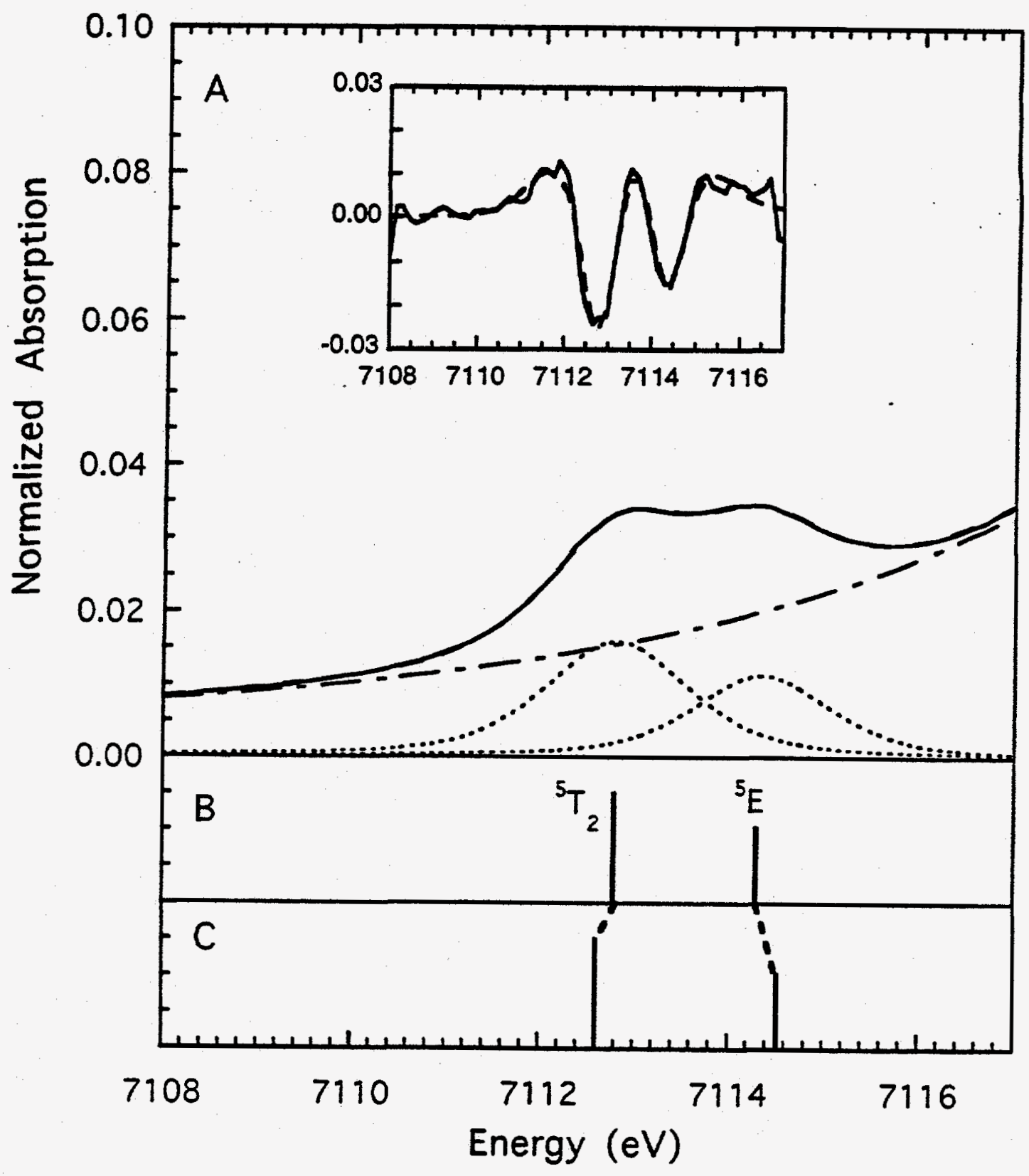

Figure 4.10. A) Fit to the Fe K-edge XAS pre-edge region of $\mathrm{Fe}(\mathrm{acac})_{3}$, where the solid line is the data, the dashed line is the fit to the data, the dashed-dot line is the background function, and the dotted lines are the individual pre-edge peaks. The inset is the second derivative of the data $(-)$ and the second derivative of the fit to the data (--). B) The energy splitting and the quadrupole intensity distribution of the ${ }^{5} \mathrm{~T}_{2}$ and ${ }^{5} \mathrm{E}$ states. C) The effect of increased $10 \mathrm{Dq}$ on the energy splitting of the ${ }^{5} \mathrm{~T}_{2}$ and ${ }^{5} \mathrm{E}$ states. 


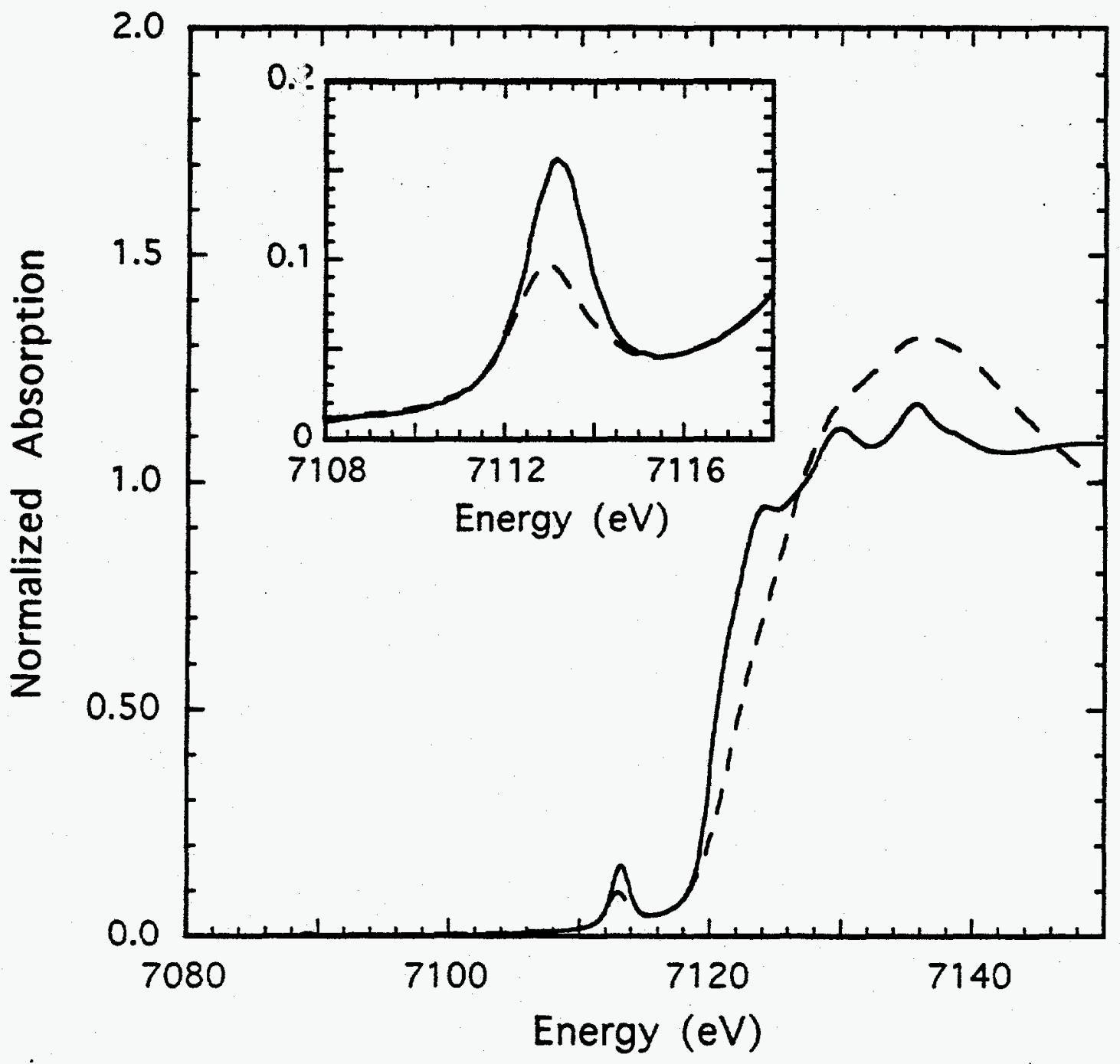

Figure 4.11. Fe K-edge XAS spectra of $\left(\mathrm{Et}_{4} \mathrm{~N}\right)\left[\mathrm{FeCl}_{4}\right](-)$ and $\mathrm{Fe}$ (salen) $\mathrm{Cl}(--)$, where the inset is an expansion of the $1 \mathrm{~s} \rightarrow 3 \mathrm{~d}$ pre-edge region. 


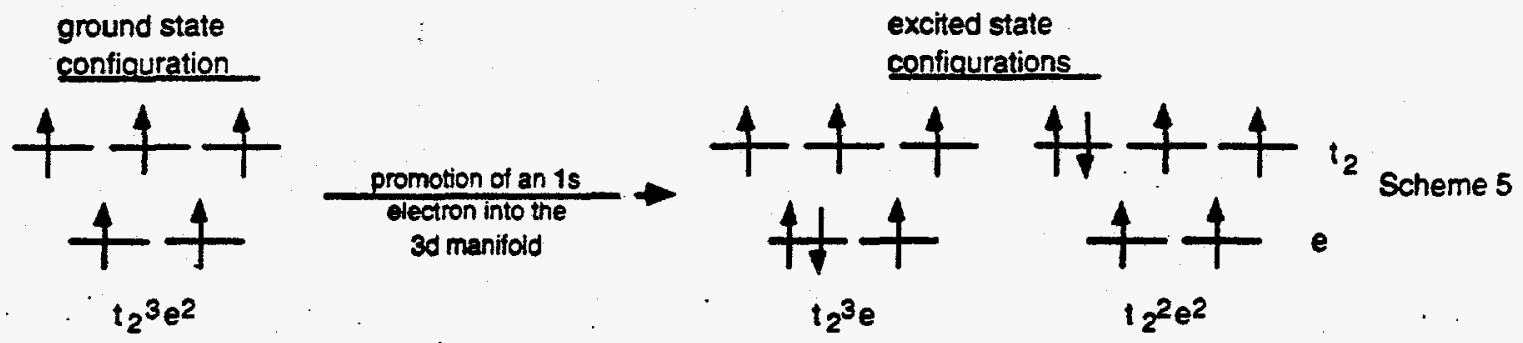

Consequently, a tetrahedral high spin ferric complex can have a transition to the doubly degenerate ${ }^{5} \mathrm{E}$ state and also to a triply degenerate ${ }^{5} \mathrm{~T}_{2}$ state at $10 \mathrm{Dq}$ higher in energy. Energies of the many-electron states were calculated using a $10 \mathrm{Dq}$ value of $5200 \mathrm{~cm}^{-1}$ $\left(80 \% \text { of the ground state } 10 \mathrm{Dq} \text { of }\left(\mathrm{E}_{4} \mathrm{~N}\right)\left[\mathrm{FeCl}_{4}\right]\right)^{48}$, a B value of $950 \mathrm{~cm}^{-1}(90 \%$ of the $\mathrm{d}^{(\mathrm{n}+1)} \mathrm{B}$ ), and a $C$ value of $3800 \mathrm{~cm}^{-1}$. Using these values the energy splitting between the ${ }^{5} \mathrm{E}$ and ${ }^{5} \mathrm{~T}_{2}$ states is $0.6 \mathrm{eV}$ (Figure 4.12) which is not resolvable at the Fe K-edge.

The pre-edge features of tetrahedral complexes also gain intensity from $4 p$ mixing into the $3 \mathrm{~d}$ orbitals. The $4 \mathrm{p}$ orbitals transform as $\mathrm{t}_{2}$ so there is only mixing into the $3 \mathrm{~d} \mathrm{t}_{2}$ set which contributes to the intensity of the transition into the ${ }^{5} \mathrm{~T}_{2}$ state (see Figure 4.12). If the total quadrupole intensity is $4.7(0.9)$ (the value calculated above for ferric complexes) then for ( $\left.\mathrm{Et}_{4} \mathrm{~N}\right)\left[\mathrm{FeCl}_{4}\right]$ which has a total intensity of 20.7(0.8) (Table 4.1) the dipole intensity is $16.0(1.2)$. Thus, the total amount of $4 p$ mixing into the $t_{2}$ set of orbitals observed experimentally is $11.2(4.7) \%$ (i.e. $16.0(1.2) / 1.43(0.6)$ ). A density functional calculation on ( $\left.\mathrm{Et}_{4} \mathrm{~N}\right)\left[\mathrm{FeCl}_{4}\right]$ predicts $2-3 \% 4 \mathrm{p}$ mixing into each of the $3 \mathrm{~d}$ orbitals of $t_{2}$ symmetry, giving a total of 6-9\%. ${ }^{47}$

4.3.2.3. $\mathrm{C}_{4 \mathrm{v}}$ Geometry. Fe K-edge data were collected for one square pyramidal high spin ferric complex, $\mathrm{Fe}($ salen)Cl. The XAS spectrum of $\mathrm{Fe}($ salen) $\mathrm{Cl}$ is shown in Figure 4.11 with the pre-edge region expanded and shown in the inset. The pre-edge feature of $\mathrm{Fe}$ (salen)Cl appears to be a relatively intense single feature (Table 4.1).

When the iron atom is in a $\mathrm{C}_{4 \mathrm{v}}$ site it is in a noncentrosymmetric environment and thus the $1 \mathrm{~s} \rightarrow 3 \mathrm{~d}$ pre-edge transition can gain intensity both from the allowed quadrupole transition and also from $4 \mathrm{p}$ mixing into the $3 \mathrm{~d}$ orbitals. $\mathrm{A} \mathrm{C}_{4 \mathrm{v}}$ site can be treated as a distorted $\mathrm{O}_{\mathrm{h}}$ site, in which the $\mathrm{d}^{(\mathrm{n}+1)}{ }^{5} \mathrm{~T}_{2}$ state splits into a ${ }^{5} \mathrm{~B}_{2}$ and a ${ }^{5} \mathrm{E}$ state and the ${ }^{5} \mathrm{E}$ state splits into a $5 \mathrm{~A}_{1}$ and $\mathrm{a} \mathrm{5}_{1}$ state. Due to the reduced ligand repulsion along the $z$-axis (from removing an axial ligand), the $d_{x z}, d_{y z}$ and the $d_{z} 2$ orbitals will be lower in energy with respect to the $d_{x y}$ and $d_{x}{ }^{2}-y^{2}$ orbitals. There are four one-electron allowed excited state configurations: $e b_{2} a_{1} b_{1}, e^{2} a_{1} b_{1}, e^{2} b_{2} b_{1}$, and $e^{2} b_{2} a_{1}$. 


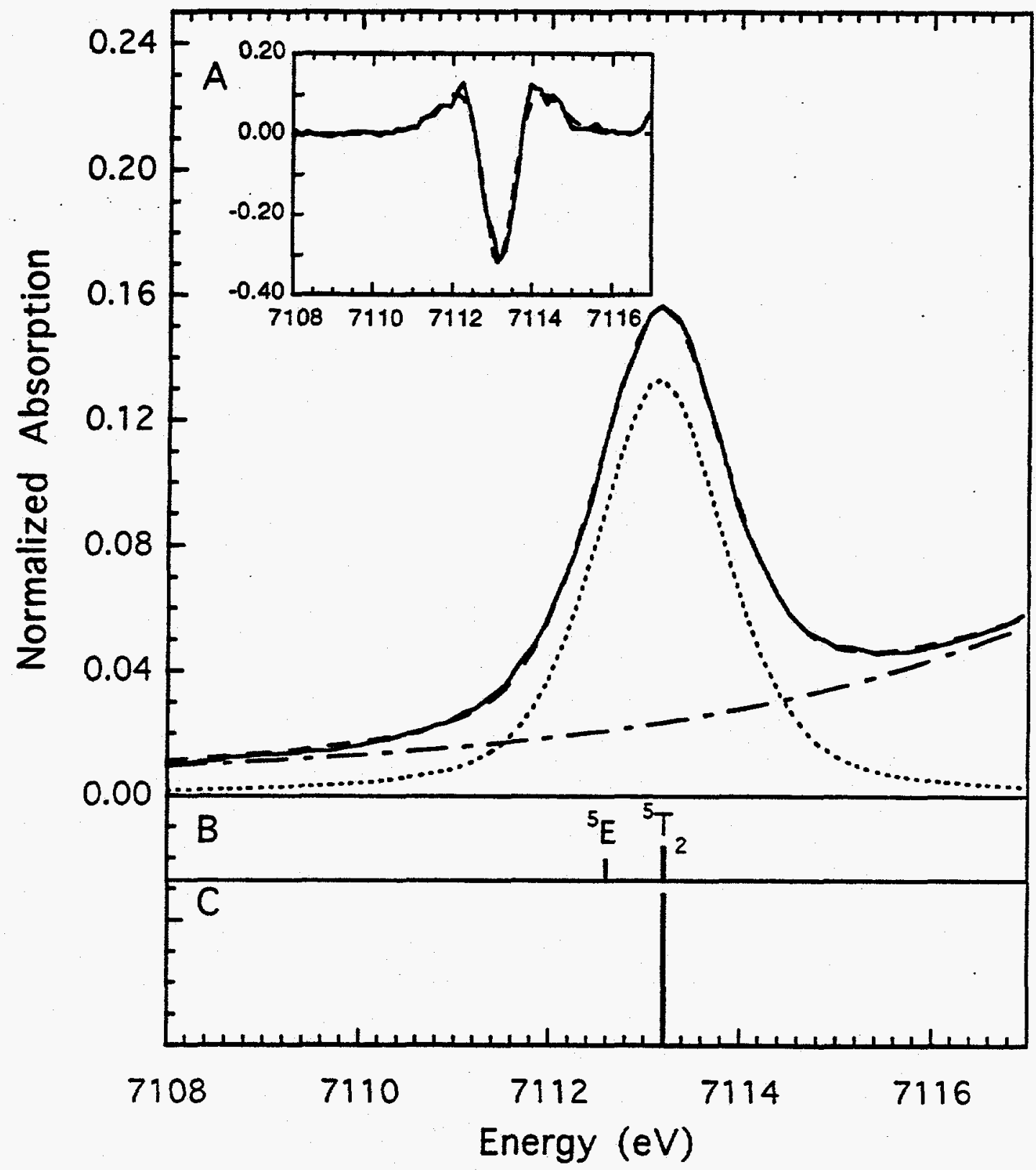

Figure 4.12. A) Fit to the $\mathrm{Fe} \mathrm{K}$-edge XAS pre-edge region of $\left(\mathrm{Et}_{4} \mathrm{~N}\right)\left[\mathrm{FeCl}_{4}\right]$, where the solid line is the data, the dashed line is the fit to the data, the dashed-dot line is the background function, and the dotted lines are the individual pre-edge peaks. The inset is the second derivative of the data (-) and the second derivative of the fit to the data (---). B) The energy splitting and the quadrupole intensity distribution of the ${ }^{5} \mathrm{E}$ and ${ }^{5} \mathrm{~T}_{2}$ states. C) The energy splitting and the dipole intensity distribution (allowed by $4 p$ mixing into the $3 \mathrm{~d}$ states) of the ${ }^{5} \mathrm{E}$ and ${ }^{5} \mathrm{~T}_{2}$ states. 

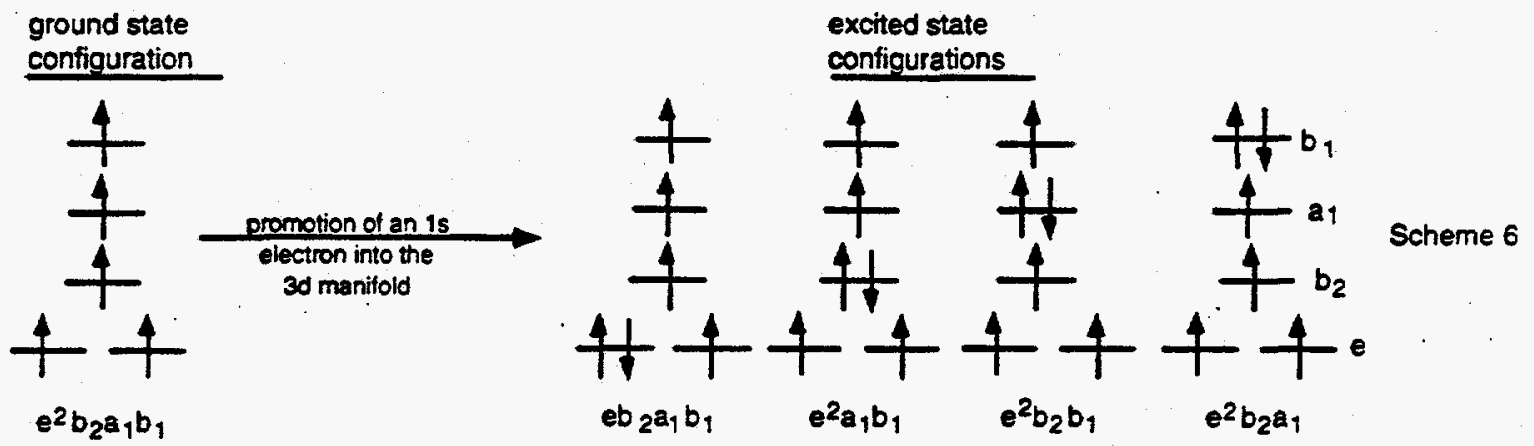

The relative energies of these configurations can be determined from density functional calculations and from experimental observations of the $3 \mathrm{~d}$ orbital energies. ${ }^{41}$ From these calculations, the $e^{2} a_{1} b_{1}$ configuration (which comprises the ${ }^{5} B_{2}$ state) is the lowest in energy, with the eb ${ }_{2} a_{1} b_{1}$ configuration (which comprises the ${ }^{5} E$ state) being $0.2 \mathrm{eV}$ higher in energy, the $e^{2} b_{2} b_{1}$ configuration (which comprises the ${ }^{5} A_{1}$ state) being $0.45 \mathrm{eV}$ above the e $b_{2} a_{1} b_{1}$ configuration, and the $e^{2} b_{2} a_{1}$ (which comprises the ${ }^{5} B_{1}$ state) being $1.05 \mathrm{eV}$ above the $\mathrm{e}^{2} \mathrm{~b}_{2} \mathrm{~b}_{1}$ configuration. ${ }^{47}$ The quadrupole intensity into these states will have an intensity ratio of 1:2:1:1, respectively (see Figure 4.13B).

The pre-edge features of square pyramidal complexes gains additional intensity from $4 p$ mixing into the $3 d$ orbitals. In the $C_{4 v}$ case, the $4 p$ orbitals transform as $e$ and $a_{1}$. However, the main distortion is along the $z$ axis, and one would thus predict that the $4 p_{z}$ orbital (of $a_{1}$ symmetry) will mix into the $d_{z}{ }^{2}$ orbital of $a_{1}$ symmetry. In this scenario the transition to the ${ }^{5} \mathrm{~A}_{1}$ state that contains the $\mathrm{e}^{2} \mathrm{~b}_{2} \mathrm{~b}_{1}$ component should be the most intense. This is consistent with the pre-edge data where the lower energy feature is much more intense than the higher energy feature (see Figure 4.13). The density functional calculation on $\mathrm{Fe}($ salen $) \mathrm{Cl}$ also shows that the $\mathrm{d}_{\mathrm{z}}{ }^{2}$ orbital contains $4 \mathrm{p}_{z}$ character, while the $d_{x z}$ and $d_{y z}$ orbitals do not contain significant $4 p_{x y}$ character. ${ }^{47}$ The amount of $4 p_{z}$ mixing into the $d_{z}{ }^{2}$ orbital can be estimated experimentally as follows. The total preedge intensity for $\mathrm{Fe}($ salen $) \mathrm{Cl}$ is $14.4(0.6)$; if $4.7(0.9)$ units are attributed to quadrupole intensity, then $9.7(1.1)$ units come from $4 p_{z}$ (dipole allowed) character in the $d_{z}{ }^{2}$ orbital. The amount of $4 \mathrm{p}_{z}$ mixing into the $\mathrm{d}_{z}{ }^{2}$ orbital is $9.7(1.1) / 1.43(0.6)$, or $6.8(2.9) \%$. The density functional calculation of $\left(\mathrm{BF}_{4}\right)[\mathrm{Fe}(\mathrm{TMC}) \mathrm{Cl}]$ shows $9 \% 4 \mathrm{p}_{z}$ mixing into the $\mathrm{d}_{\mathrm{z}}{ }^{2}$ orbital. As in the high spin ferrous square pyramidal case, there is only one state that gains intensity from the $4 p_{z}$ mixing into the $d_{z}^{2}$ orbital; thus, the axial interaction will be reflected in the intensity and position of the most intense pre-edge feature. 


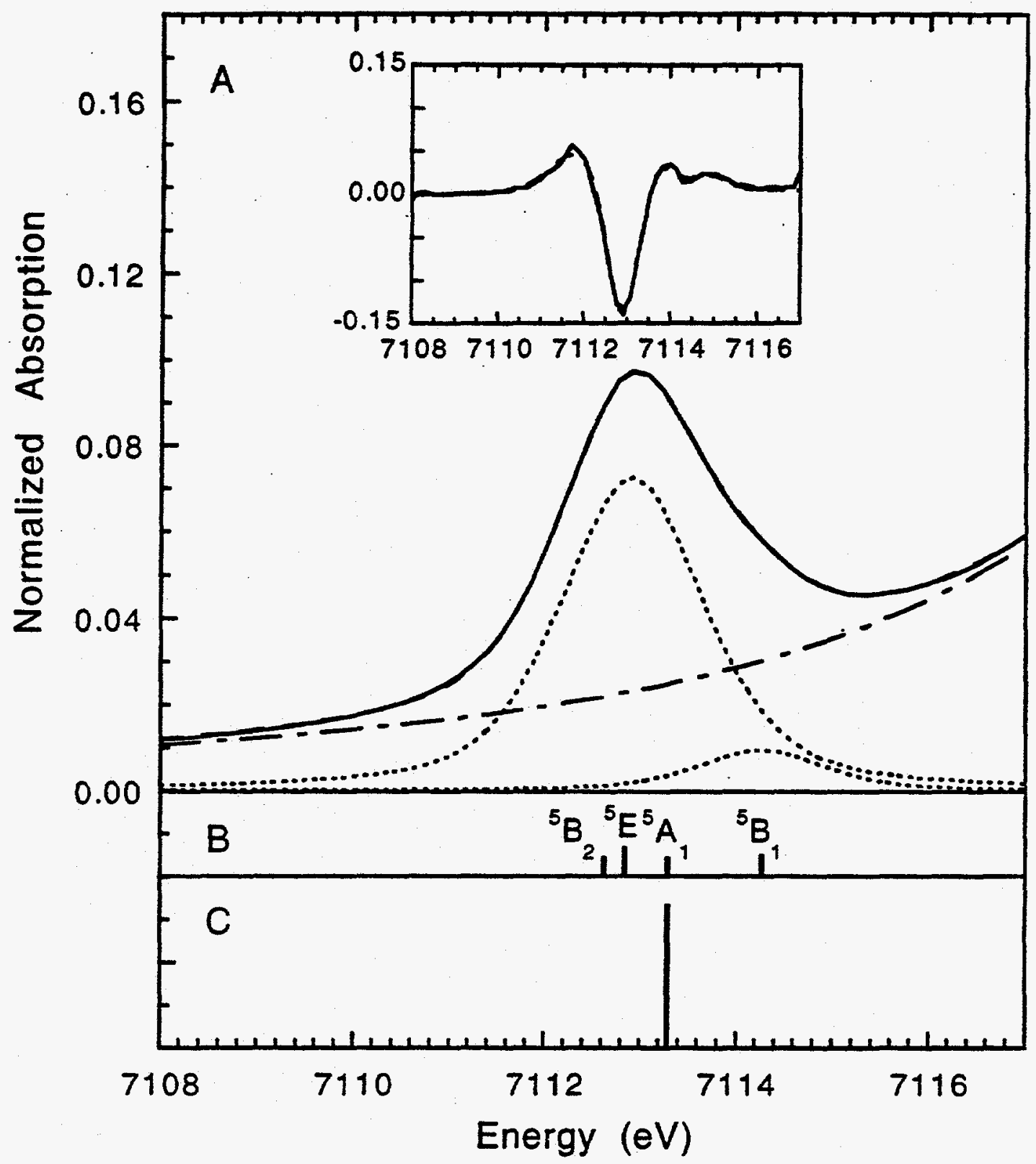

Figure 4.13. A) Fit to the $\mathrm{Fe} \mathrm{K}$-edge XAS pre-edge region of $\mathrm{Fe}(\mathrm{salen}) \mathrm{Cl}$, where the solid line is the data, the dashed line is the fit to the data, the dashed-dot line is the background function, and the dotted lines are the individual pre-edge peaks. The inset is the second derivative of the data (-) and the second derivative of the fit to the data (--). B) The energy splitting and the quadrupole intensity distribution of the ${ }^{5} \mathrm{~B}_{2},{ }^{5} \mathrm{E},{ }^{5} \mathrm{~A}_{1}$, and $5_{B_{1}}$ states. C) The energy splitting and the dipole intensity distribution (allowed by $4 p$ mixing into the $3 \mathrm{~d}$ states) of the ${ }^{5} \mathrm{~B}_{2},{ }^{5} \mathrm{E},{ }^{5} \mathrm{~A}_{1}$, and ${ }^{5} \mathrm{~B}_{1}$ states. 


\subsubsection{Binuclear Complexes}

Fe K-edge data were also collected for a series of binuclear iron model complexts with varying oxidation states, geometries, and bridging ligation. The complexes studied are listed in Table 4.2. Figures 4.14, 4.15, and 4.16 display XAS spectra of representative complexes with the insets containing an expanded view of the $1 \mathrm{~s} \rightarrow 3 \mathrm{~d}$ pre-edge region. Figure 4.14 shows a comparison of spectra of six- and five-coordinate diferrous complexes, $\left(\mathrm{Et}_{4} \mathrm{~N}_{2}\left[\mathrm{Fe}_{2} \text { (salmp) }\right)_{2}\right] \cdot 2 \mathrm{DMF}$ and $\left[\mathrm{Fe}_{2}(\mathrm{OBz})(\mathrm{et}-\mathrm{HPTB})\right]\left(\mathrm{BF}_{4}\right)_{2}$ to those of six- and five-coordinate monomeric complexes. ( $\left(\mathrm{Et}_{4} \mathrm{~N}\right)_{2}\left[\mathrm{Fe}_{2}(\mathrm{salmp})_{2}\right] \cdot 2 \mathrm{DMF}$ has a low intensity pre-edge feature that is split by $\sim 2 \mathrm{eV}$, very similar to the monomeric six-coordinate complex. [ $\left.\mathrm{Fe}_{2}(\mathrm{OBz})(\mathrm{et}-\mathrm{HPTB})\right]\left(\mathrm{BF}_{4}\right)_{2}$ also has a split feature; however, the lower energy feature is much more intense, similar to the monomeric five-coordinate complex. In Figure 4.15 is shown an analogous comparison of six- and four-coordinate diferric complexes, $\left[\mathrm{Fe}_{2} \mathrm{OH}(\mathrm{OAc})_{2}\left(\mathrm{HB}(\mathrm{pz})_{3}\right)_{2}\right]\left(\mathrm{ClO}_{4}\right)$ and $\left(\mathrm{BzPHMe}_{2} \mathrm{~N}\right)_{2}\left[\mathrm{Fe}_{2} \mathrm{OCl}_{6}\right]$, with those of the corresponding six- and four-coordinate monomeric complexes. $\left[\mathrm{Fe}_{2} \mathrm{OH}(\mathrm{OAc})_{2}\left(\mathrm{HB}(\mathrm{pz})_{3}\right)_{2}\right]\left(\mathrm{ClO}_{4}\right)$ has a low intensity pre-edge feature that is split, similar to the monomeric ferric complex. (BzPHMe $\left.2{ }_{2}\right)_{2}\left[\mathrm{Fe}_{2} \mathrm{OCl}_{6}\right]$ has a single very intense pre-edge feature, similar to the monomeric tetrahedral complex.

Spectra of $\left[\mathrm{Fe}_{2} \mathrm{OH}(\mathrm{OAc})_{2}\left(\mathrm{HB}(\mathrm{pz})_{3}\right)_{2}\right]\left(\mathrm{ClO}_{4}\right),\left[\mathrm{Fe}_{2} \mathrm{O}(\mathrm{OAc})_{2}\left(\mathrm{HB}(\mathrm{pz})_{3}\right)_{2}\right]$, $\left[\mathrm{Fe}_{2}(\mathrm{TPA})_{2} \mathrm{O}(\mathrm{OAC})\right]\left(\mathrm{ClO}_{4}\right)_{2}$, and $\left(\mathrm{enH}_{2}\right)\left[\mathrm{Fe}_{2} \mathrm{O}(\mathrm{HEDTA})_{2}\right] \cdot 6 \mathrm{H}_{2} \mathrm{O}$ are shown in Figure 4.16. All four of these complexes are diferric with various bridging ligands. $\left[\mathrm{Fe}_{2} \mathrm{OH}(\mathrm{OAC})_{2}\left(\mathrm{HB}(\mathrm{pz})_{3}\right)_{2}\right]\left(\mathrm{ClO}_{4}\right)$ and $\left[\mathrm{Fe}_{2} \mathrm{O}(\mathrm{OAc})_{2}\left(\mathrm{HB}(\mathrm{pz})_{3}\right)_{2}\right]$ have the same ligand set with the exception of the former having a hydroxide bridge, while the latter has a $\mu$-oxo bridge. $\left[\mathrm{Fe}_{2} \mathrm{OH}(\mathrm{OAc})_{2}\left(\mathrm{HB}(\mathrm{pz})_{3}\right)_{2}\right]\left(\mathrm{ClO}_{4}\right)$ and $\left[\mathrm{Fe}_{2} \mathrm{O}(\mathrm{OAc})_{2}\left(\mathrm{HB}(\mathrm{pz})_{3}\right)_{2}\right]$ display very different pre-edge features with the hydroxide bridged complex having a weak, split pre-edge feature and the $\mu$-oxo bridged complex having a much more intense higher energy feature with a lower energy shoulder. $\left[\mathrm{Fe}_{2}(\mathrm{TPA})_{2} \mathrm{O}(\mathrm{OAc})\right]\left(\mathrm{ClO}_{4}\right)_{2}$ and $\left(\mathrm{enH}_{2}\right)\left[\mathrm{Fe}_{2} \mathrm{O}(\mathrm{HEDTA})_{2}\right] \cdot 6 \mathrm{H}_{2} \mathrm{O}$ also contain a $\mu$-oxo bridge with $\left[\mathrm{Fe}_{2}(\mathrm{TPA})_{2} \mathrm{O}(\mathrm{OAc})\right]\left(\mathrm{ClO}_{4}\right)_{2}$ having an additional acetate bridge. Both of these complexes show a pre-edge feature similar to $\left[\mathrm{Fe}_{2} \mathrm{O}(\mathrm{OAc})_{2}\left(\mathrm{HB}(\mathrm{pz})_{3}\right)_{2}\right]$. In fact, all the dimer complexes studied that contained a $\mu$-oxo bridge had a distinctive pre-edge feature in their spectra with this higher energy intense feature and a lower energy shoulder (Table 4.2).

The first part to be addressed is the applicability of using the atomic multiplet analysis that was developed above for monomeric systems on dimeric iron systems. To resolve this question the pre-edge features of diferrous and diferric complexes were 
Table 4.2. XAS Pre-Edge Energies and Areas for Binuclcar Iron Model Complexes.

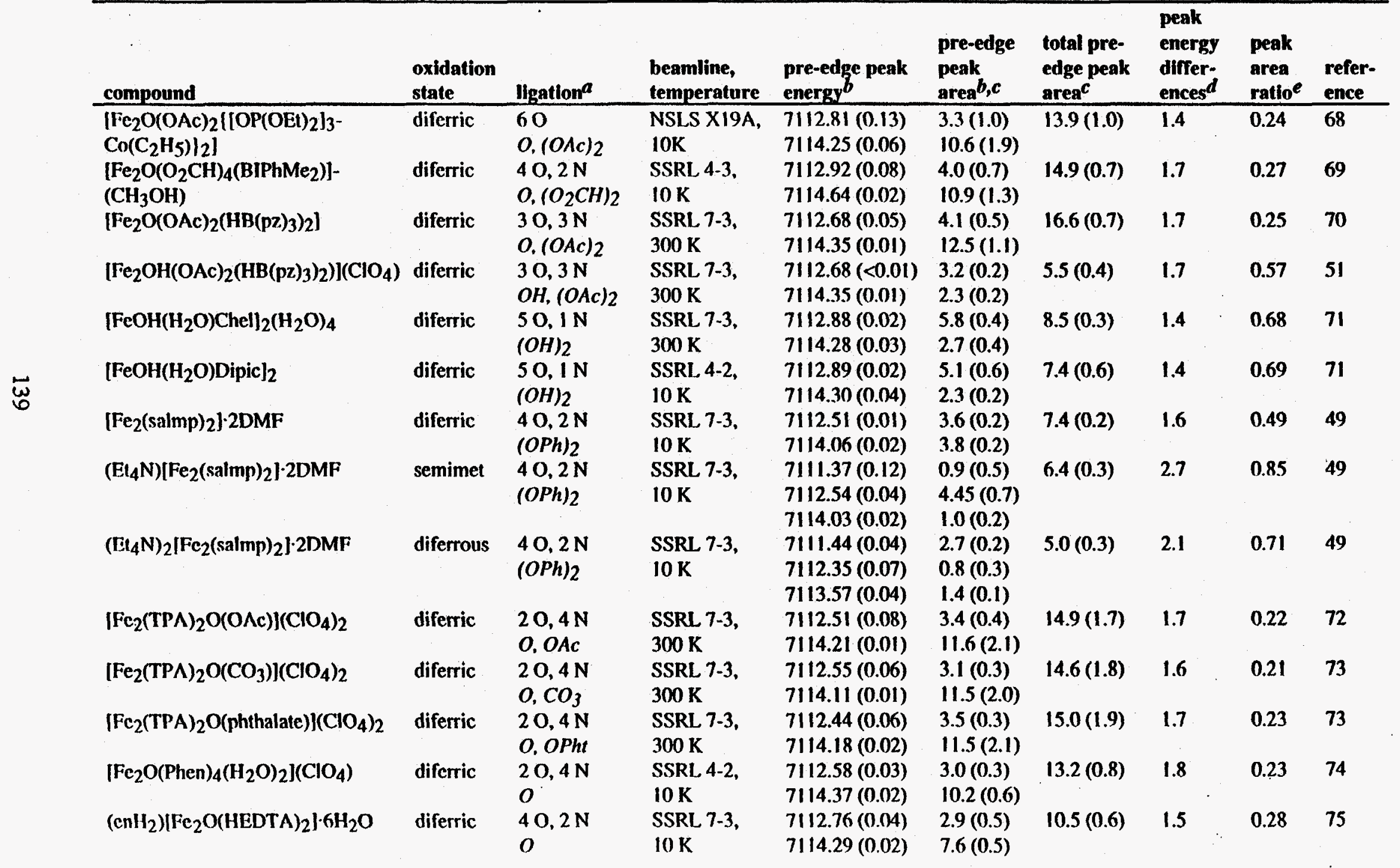




\begin{tabular}{|c|c|c|c|c|c|c|c|c|c|}
\hline$\left[\mathrm{Fe}_{2}(\mathrm{OBz})(\mathrm{et}-\mathrm{HPTB})\right\}\left(\mathrm{BF}_{4}\right)_{2}$ & diferrous & $\begin{array}{l}2 \mathrm{O}, 3 \mathrm{~N} \\
\text { alkoxo, } \mathrm{OBz}\end{array}$ & $\begin{array}{l}\text { SSRL 7-3, } \\
10 \mathrm{~K}\end{array}$ & $\begin{array}{l}7111.68(0.01) \\
7113.41(0.01)\end{array}$ & $\begin{array}{l}10.9(0.2) \\
2.2(0.1)\end{array}$ & $13.0(0.3)$ & 1.7 & 0.83 & 50 \\
\hline$\left(\mathrm{Bz} \mathrm{PhMe}{ }_{2} \mathrm{~N}\right)_{2}\left[\mathrm{Fe}_{2} \mathrm{OCl}_{6}\right]$ & diferric & $\begin{array}{l}10,3 \mathrm{Cl} \\
o\end{array}$ & $\begin{array}{l}\text { SSRL 2-3, } \\
10 \mathrm{~K}\end{array}$ & $7113.37(0.01)$ & $27.1(1.2)$ & $27.1(1.2)$ & --- & -- & 52 \\
\hline
\end{tabular}

$a$ Bridging ligands are denoted by italics. $b$ Pre-edge energies and intensities were determined by fits to the data (see the Experimental Section for details on the fitting procedure). $c$ The values reported for the pre-edge areas were multiplied by $100 . d$ Peak energy differences are the energy differences between the lowest encrgy and the highest energy pre-edge feature. $e$ Peak area ratios are the area of the first pre-edge feature divided by the total pre-edge area. 


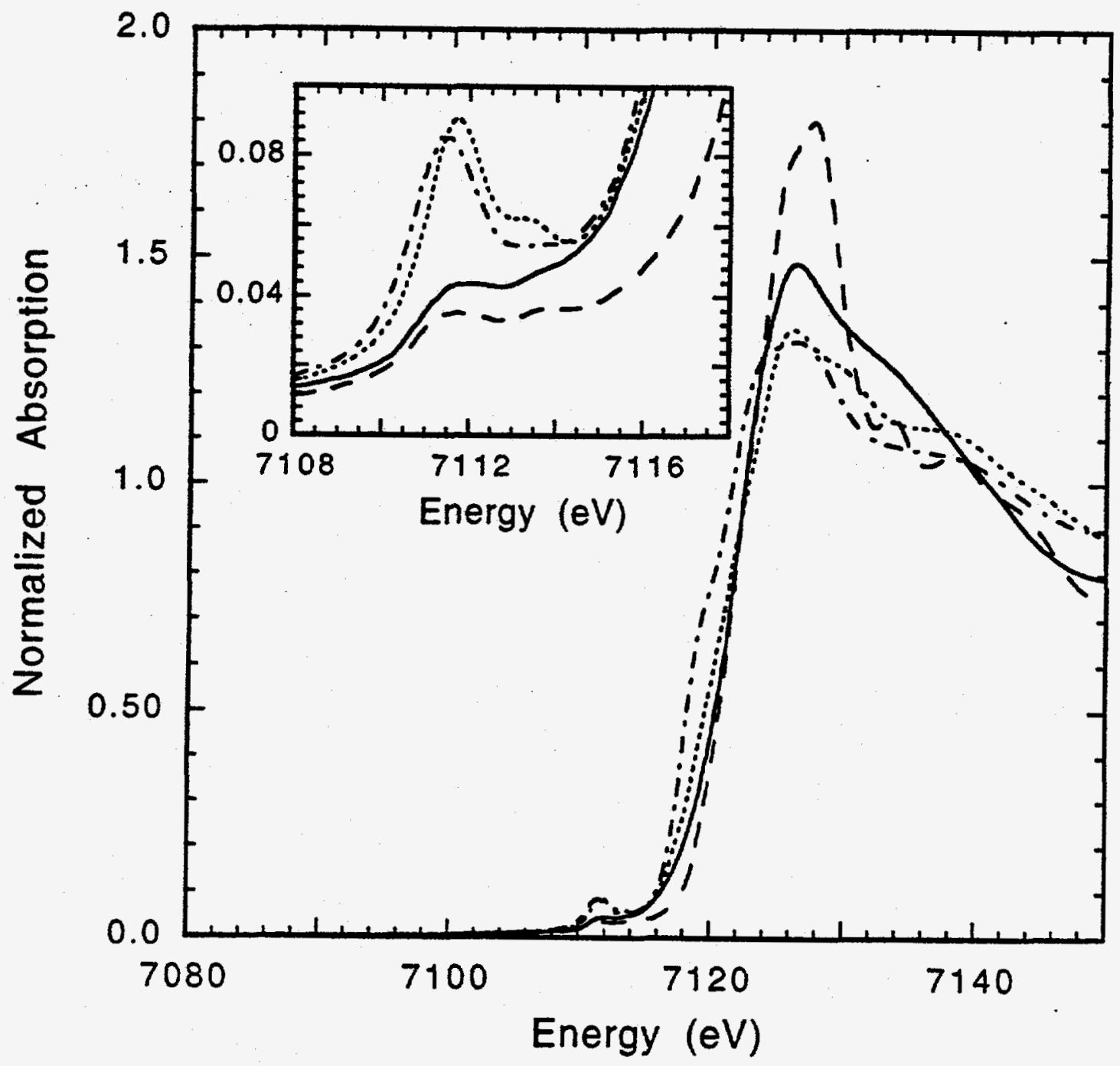

Figure 4.14. Fe K-edge XAS spectra of $\left(\mathrm{Et}_{4} \mathrm{~N}\right)_{2}\left[\mathrm{Fe}_{2}(\mathrm{salmp})_{2}\right] \cdot 2 \mathrm{DMF}(-)$, $\left.\mathrm{FeSiF}_{6}{ }^{\circ} 6 \mathrm{H}_{2} \mathrm{O}(--), \mathrm{Fe}_{2}(\mathrm{OBz})(\mathrm{et}-\mathrm{HPTB})\right]\left(\mathrm{BF}_{4}\right)_{2}(\cdots \cdot \cdot)$, and $\left(\mathrm{BF}_{4}\right)[\mathrm{Fe}(\mathrm{TMC}) \mathrm{Cl}](--)$, where the inset is an expansion of the $1 \mathrm{~s} \rightarrow 3 \mathrm{~d}$ pre-edge region. 


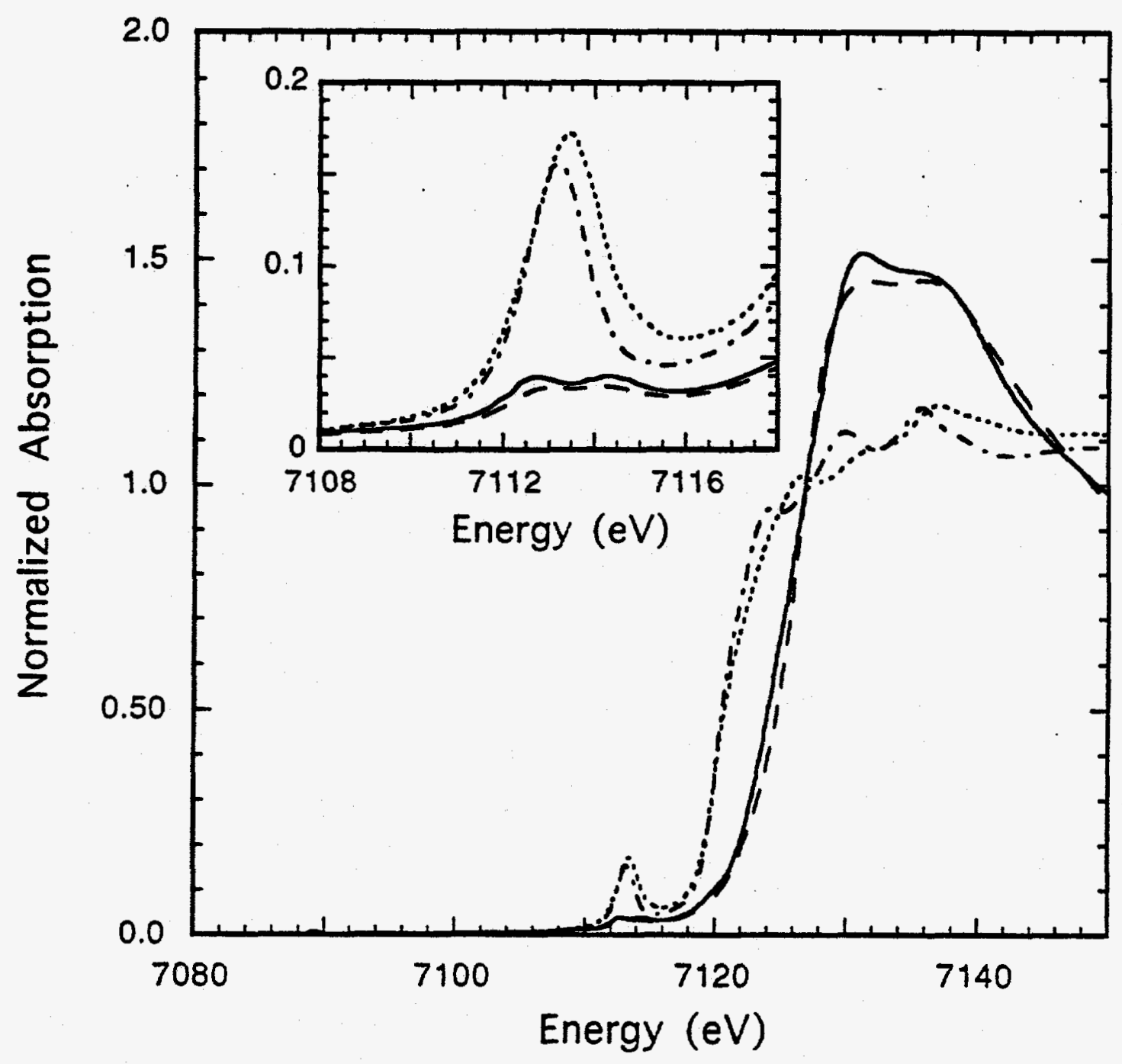

Figure 4.15. Fe K-edge XAS spectra of $\left[\mathrm{Fe}_{2} \mathrm{OH}(\mathrm{OAc})_{2}\left(\mathrm{HB}(\mathrm{pz})_{3}\right)_{2}\right]\left(\mathrm{ClO}_{4}\right)$ (-), $\mathrm{Fe}(\mathrm{acac})_{3}(--),\left(\mathrm{BzPHMe}_{2} \mathrm{~N}_{2}\left[\mathrm{Fe}_{2} \mathrm{OCl}_{6}\right](\cdots \cdot)\right.$, and $\left(\mathrm{Et}_{4} \mathrm{~N}\right)\left[\mathrm{FeCl}_{4}\right](--)$, where the inset is an expansion of the $1 \mathrm{~s} \rightarrow 3 \mathrm{~d}$ pre-edge region. 


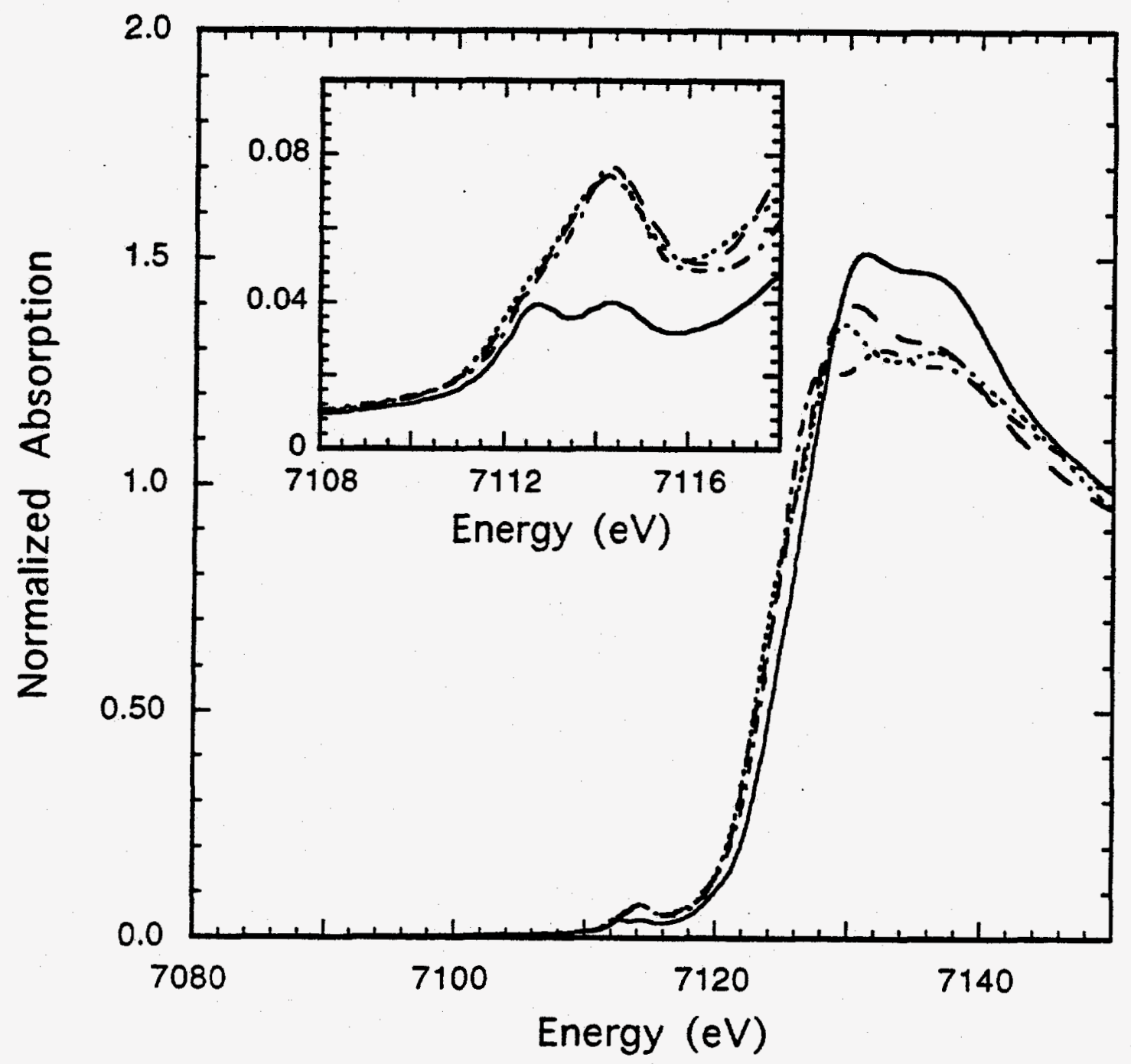

Figure 4.16. Fe K-edge XAS spectra of $\left[\mathrm{Fe}_{2} \mathrm{OH}(\mathrm{OAC})_{2}\left(\mathrm{HB}(\mathrm{pz})_{3}\right)_{2}\right]\left(\mathrm{ClO}_{4}\right)(-)$, $\left[\mathrm{Fe}_{2} \mathrm{O}(\mathrm{OAc})_{2}\left(\mathrm{HB}(\mathrm{pz})_{3}\right)_{2}\right] \quad(---),\left[\mathrm{Fe}_{2}(\mathrm{TPA})_{2} \mathrm{O}(\mathrm{OAc})\right]\left(\mathrm{ClO}_{4}\right)_{2}(\cdots)$, and $\left(\mathrm{enH}_{2}\right)\left[\mathrm{Fe}_{2} \mathrm{O}(\mathrm{HEDTA})_{2}\right] \cdot 6 \mathrm{H}_{2} \mathrm{O}(--)$, where the inset is an expansion of the $1 \mathrm{~s} \rightarrow 3 \mathrm{~d}$ pre-edge region. 
compared to those of monomer complexes with similar ligation and geometry. $\left(\mathrm{Et}_{4} \mathrm{~N}_{2}\left[\mathrm{Fe}_{2}\right.\right.$ (salmp) $\left.{ }_{2}\right] \cdot 2 \mathrm{DMF}$ has two six-coordinate iron centers with $\mathrm{O}$ and $\mathrm{N}$ ligation. ${ }^{49}$ The pre-edge of this complex (Figure 4.14) is very similar to the pre-edges of monomeric octahedral complexes with $\mathrm{O}$ and $\mathrm{N}$ ligation (Figure 4.2). The fits to the $\left(\mathrm{Et}_{4} \mathrm{~N}\right)_{2}\left[\mathrm{Fe}_{2}(\mathrm{salmp})_{2}\right] \cdot 2 \mathrm{DMF}$ data give pre-edge peak energies that are within $0.1 \mathrm{eV}$. of the monomeric octahedral complexes with $\mathrm{O}$ and $\mathrm{N}$ ligation, with very similar areas of the three fitted peaks (Table 4.1 and 4.2). $\left.\mathrm{Fe}_{2}(\mathrm{OBz})(\mathrm{et}-\mathrm{HPTB}) \mathrm{BBF}_{4}\right)_{2}$ has two five-coordinate iron sites ${ }^{50}$ and has a pre-edge feature that is very similar to the square pyramidal high spin ferrous monomers (Figure 4.14; Tables 4.1 and 4.2). $\left[\mathrm{Fe}_{2} \mathrm{OH}(\mathrm{OAc})_{2}\left(\mathrm{HB}(\mathrm{pz})_{3}\right)_{2}\right]\left(\mathrm{ClO}_{4}\right)$ has two six-coordinate iron sites that are very nearly octahedral. ${ }^{51}$ The pre-edge feature of this complex is very similar to the pre-edge features of octahedral high spin ferric monomeric complexes with $\mathrm{O}$ and $\mathrm{N}$ ligation with a low intensity split pre-edge feature (Figures 4.15; Tables 4.1 and 4.2). $\left(\mathrm{BzPHMe}_{2} \mathrm{~N}\right)_{2}\left[\mathrm{Fe}_{2} \mathrm{OCl}_{6}\right]$ contains iron sites with $3 \mathrm{Cl}^{-}$ligands and a $\mu$-oxo bridge in a $-T_{d}$ geometry ${ }^{52}$ and has a very intense single pre-edge feature that is very similar to that of $\left(\mathrm{Et}_{4} \mathrm{~N}\right)\left[\mathrm{FeCl}_{4}\right]$ (Figure 4.15; Tables 4.1 and 4.2). The pre-edge feature of $\left(\mathrm{BzPHMe}{ }_{2} \mathrm{~N}_{2}\left[\mathrm{Fe}_{2} \mathrm{OCl}_{6}\right]\right.$ is more intense since the iron site is more distorted due to the shorter $\mu$-oxo bridge.

From the above similarities, in pre-edge features between monomeric and dimeric complexes of similar oxidation state, ligation and geometry, we can conclude that the pre-edge splittings and intensities predicted from atomic multiplet theory can be used to explain dimeric as well as monomeric iron complexes.

Dimeric iron complexes that contain a $\mu$-oxo bridge all have a very similar and very distinctive pre-edge feature (Figure 4.16 ). The $\mu$-oxo bridge seems to have a dominant effect on the pre-edge features, as mono-, di-, and tri-bridged complexes that contain a $\mu$-oxo bridge all have very similar pre-edge features (Figure 4.16 and Table 4.2). There is also a striking difference in the pre-edge feature of hydroxide $v s$. $\mu$-oxo bridged complexes, as can be seen in the pre-edge features of $\left[\mathrm{Fe}_{2} \mathrm{OH}(\mathrm{OAc})_{2}\left(\mathrm{HB}(\mathrm{pz})_{3}\right)_{2}\right]\left(\mathrm{ClO}_{4}\right)$ and $\left[\mathrm{Fe}_{2} \mathrm{O}(\mathrm{OAc})_{2}\left(\mathrm{HB}(\mathrm{pz})_{3}\right)_{2}\right]$ (Figure 4.16). The six-coordinate $\mu$-oxo bridged dimers can be viewed as having an iron site with $\mathrm{C}_{4} \mathrm{v}$ symmetry in which an axial ligand (the $O$ of the $\mu$-oxo bridge) has moved closer to the iron site. This shortening of the axial bond strongly perturbs the iron site allowing for $4 p$ mixing into the $3 \mathrm{~d}$ orbitals. As discussed previously, in the high spin ferric $\mathrm{C}_{4 \mathrm{v}}$ case, the $4 p$ orbitals transform as $e$ and $a_{1}$ and if the main distortion is along the $z$ axis, the dominant effect will be that of the $4 p_{z}$ orbital (of $a_{1}$ symmetry) mixing with the $d_{z}{ }^{2}$ orbital of $a_{1}$ symmetry. In this scenario, the transition to the ${ }^{5} A_{1}$ state that contains the 
$e^{2} b_{2} b_{1}$ component (Scheme 6 ) should be the most intense. Due to the increased ligand repulsion along the $z$-axis, the $d_{x z}, d_{y z}$ and the $d_{z} 2$ orbitals will increase in energy with respect to the $d_{x y}$ and $d_{x}{ }^{2}-y^{2}$ orbitals. This effect increases the energy of the ${ }^{5} A_{1}$ state so that it is the state highest in energy. Thus, the higher energy pre-edge feature should be the most intense since $4 \mathrm{p}_{\mathrm{z}}$ mixing into the $3 \mathrm{~d}_{\mathrm{z}}{ }^{2}$ orbital will increase the intensity of the ${ }^{5} \mathrm{~A}_{1}$ state. This explains the distinctive shape of all the $\mu$-oxo bridged complexes (Figure 4.16 and Table 4.2).

The amount of $4 \mathrm{p}_{z}$ mixing can be quantitated. The total pre-edge peak intensities for all the octahedral dimer complexes with $\mu$-oxo bridges ranges from 10.5 to 16.6 (Table 4.2) with the average total peak intensity being 14.2. A dipole intensity of 9.5 is calculated by subtracting the total quadrupole intensity of 4.7 (the value obtained from the octahedral ferric complexes). The amount of $4 p_{z}$ mixing into the $d_{z}{ }^{2}$ orbital is $9.5 / 1.43$, or $6.6 \%$. As in the high spin ferric square pyramidal case, there is only one state that gains intensity from the $4 p_{2}$ mixing into the $d_{z}^{2}$ orbital; thus, the axial interaction will be reflected in the intensity and position of the most intense pre-edge feature.

\subsubsection{Low Spin Iron Complexes}

4.3.4.1. Ferrous Complexes. Fe K-edge XAS data were obtained for the low spin ferrous complexes $\mathrm{Fe}\left(\mathrm{HB}(\mathrm{pz})_{3}\right)_{2}, \mathrm{Fe}$ (prpep $)_{2}$ and $\mathrm{K}_{4} \mathrm{Fe}(\mathrm{CN})_{6}$. XAS spectra for these three complexes are shown in Figure 4.17 with the inset showing an expanded view of the $1 \mathrm{~s} \longrightarrow 3 \mathrm{~d}$ pre-edge region. All three complexes have a weak, single pre-edge feature with the feature of $\mathrm{Fe}\left(\mathrm{HB}(\mathrm{pz})_{3}\right)_{2}$ being the lowest in energy and that of $\mathrm{K}_{4} \mathrm{Fe}(\mathrm{CN})_{6}$ being highest in energy (Table 4.3).

All three of these low spin ferrous complexes have an octahedral iron site and, therefore, the only intensity mechanism for the $1 \mathrm{~s} \rightarrow 3 \mathrm{~d}$ pre-edge feature is the allowed quadrupole transition. The ground state of these low spin ferrous complexes has an electronic hole configuration of $e^{4}$ with $e^{3}$ being the only excited state configuration (Scheme 7). Coupling of the three $e$ holes produces a ${ }^{2} \mathrm{E}$ excited many-electron state.

\section{ground state \\ configuration}

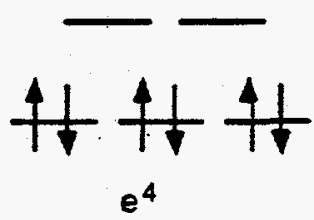

promotion of an 15

electron into the 3o manifold excited state configuration

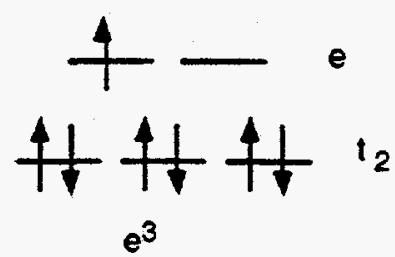

Scheme 7 
Table 4.3. XAS Pre-Edge Energies and Areas for Low Spin Iron Model Complexes.

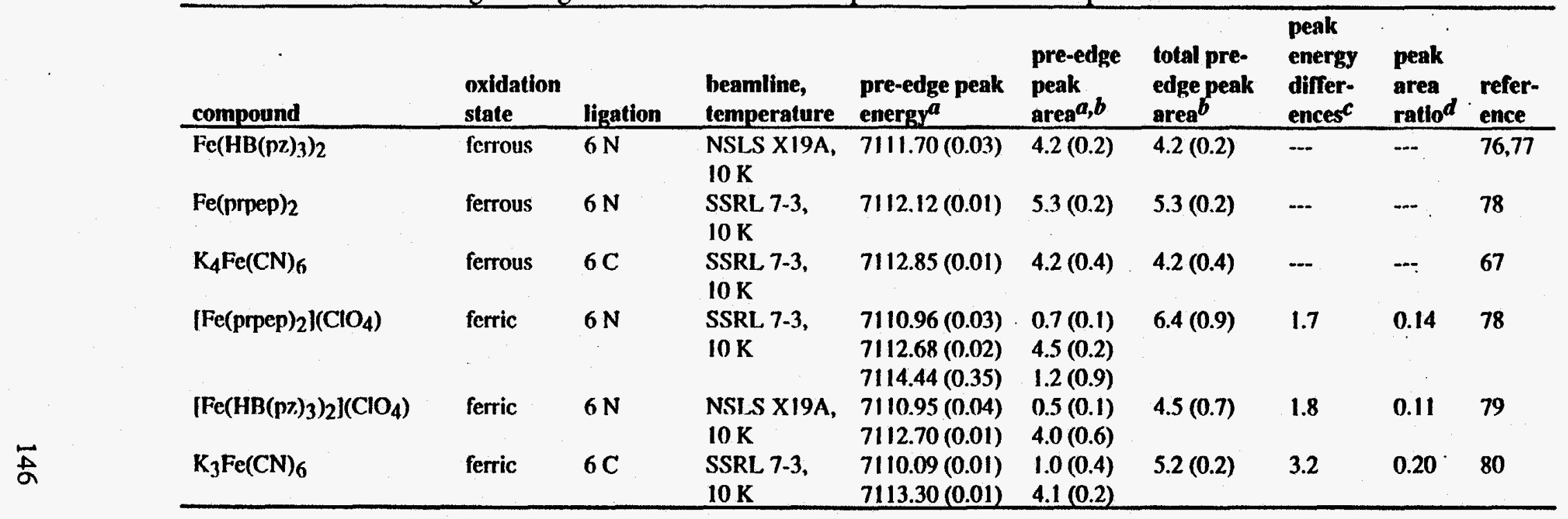

a Pre-edge energies and intensities were determined by fits to the data (see the Experimental Section for details on the fitting procedure). $b$ The values reported for the pre-edge areas were multiplied by 100 . $c$ Peak energy differences are the energy differences between the first and second pre-edge feature. ${ }^{d}$ Peak area ratios are the area of the first pre-edge feature divided by the total pre-edge area. 


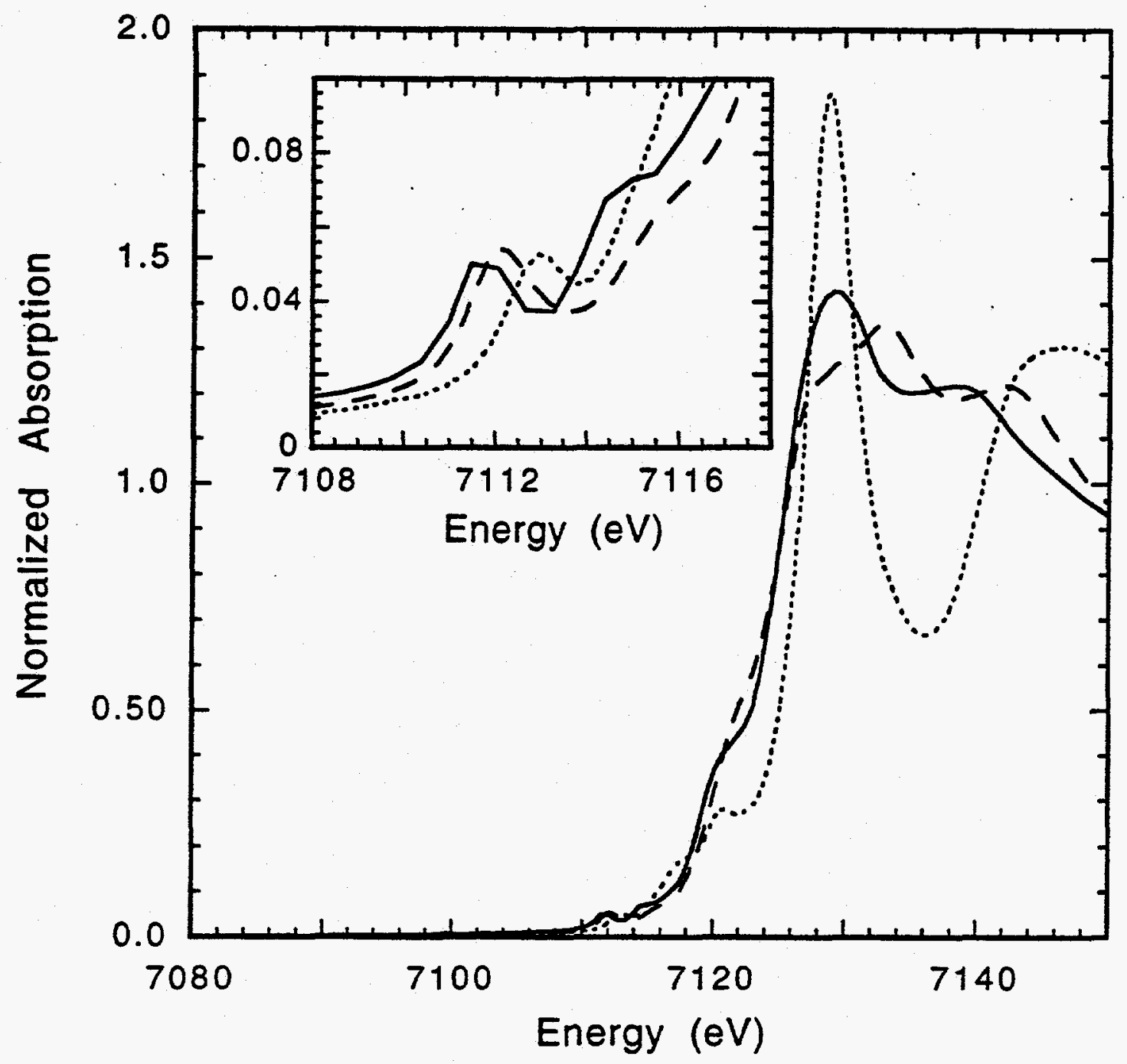

Figure 4.17. Fe K-edge XAS spectra of $\mathrm{Fe}\left(\mathrm{HB}(\mathrm{pz})_{3}\right)_{2}(-), \mathrm{Fe}(\mathrm{prpep})_{2}(--)$, and $\mathrm{K}_{4} \mathrm{Fe}(\mathrm{CN})_{6}(\cdots \cdots)$, where the inset is an expansion of the $1 \mathrm{~s} \longrightarrow 3 \mathrm{~d}$ pre-edge region. 
A transition into the ${ }^{2} \mathrm{E}$ state gives rise to a single pre-edge feature (Figure 4.17 and Table 4.3). The average total pre-edge areas for these low spin complexes is 4.6 , which is similar to that of the high spin ferrous complexes.

4.3.4.2. Ferric Complexes. Fe K-edge XAS data were obtained for the low spin ferric complexes $\left[\mathrm{Fe}\left(\mathrm{HB}(\mathrm{pz})_{3}\right)_{2}\right]\left(\mathrm{ClO}_{4}\right)$, $\left[\mathrm{Fe}(\text { prpep })_{2}\right]\left(\mathrm{ClO}_{4}\right)$, and $\mathrm{K}_{3} \mathrm{Fe}(\mathrm{CN})_{6}$. XAS spectra for these three complexes are shown in Figure 4.18 with an expanded view of the $1 \mathrm{~s} \rightarrow 3 \mathrm{~d}$ pre-edge region shown in the inset. All three of these complexes have a weak pre-edge feature with a lower energy shoulder. The splitting between the lower energy shoulder and the higher energy feature is the greatest in $\mathrm{K}_{3} \mathrm{Fe}(\mathrm{CN})_{6}$ (Table 4.3).

These low spin ferric complexes all have an octahedral iron site and therefore the $1 \mathrm{~s} \rightarrow 3 \mathrm{~d}$ pre-edge feature only gains intensity through an allowed quadrupole transition. The ground state of an octahedral low spin ferric complex has a $t_{2} e^{4}$ configuration, with two excited state configurations of $\mathrm{e}^{4}$ and $\mathrm{t}_{2} \mathrm{e}^{3}$ (Scheme 8).

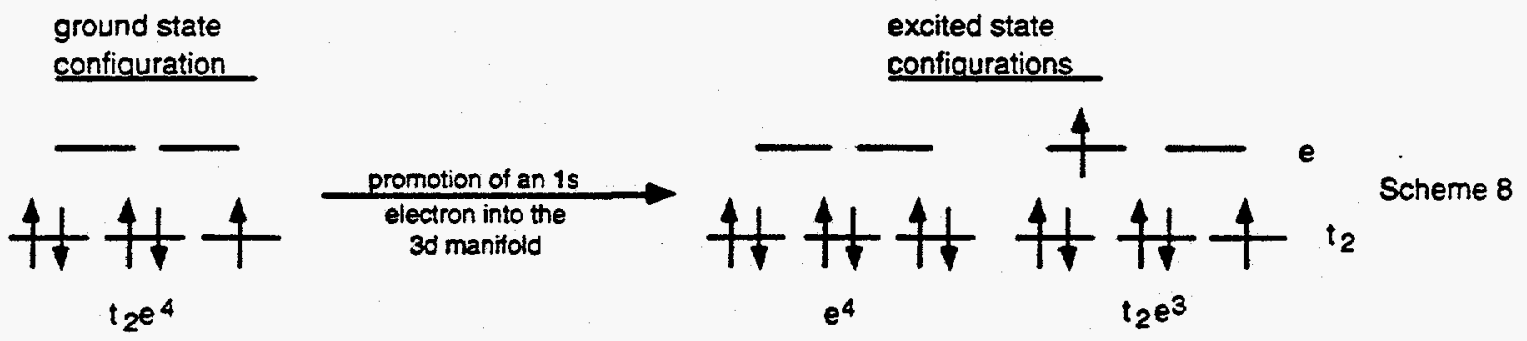

Coupling of the four e holes produces a ${ }^{1} A_{1}$ state, while coupling of the holes in the $t_{2} e^{3}$ configuration produces ${ }^{1} \mathrm{~T}_{1},{ }^{3} \mathrm{~T}_{1},{ }^{1} \mathrm{~T}_{2}$, and ${ }^{3} \mathrm{~T}_{2}$ states. Using a ${ }^{10 \mathrm{Dq}}$ value of $20,000 \mathrm{~cm}^{-1}$, a B value of $1058 \mathrm{~cm}^{-1}$, and a $C$ value of $4500 \mathrm{~cm}^{-1}$, energy splittings of the five states were calculated. The lowest energy state is the ${ }^{1} \mathrm{~A}_{1}$ with the ${ }^{3} \mathrm{~T}_{1}$ state being $0.9 \mathrm{eV}$ higher in energy, the ${ }^{3} \mathrm{~T}_{2}$ state being $0.8 \mathrm{eV}$ above the ${ }^{3} \mathrm{~T}_{1}$ state, the ${ }^{1} \mathrm{~T}_{1}$ state being $0.5 \mathrm{eV}$ above the ${ }^{3} \mathrm{~T}_{2}$ state, and the ${ }^{1} \mathrm{~T}_{2}$ being $1.4 \mathrm{eV}$ above the ${ }^{1} \mathrm{~T}_{1}$ state (Figure 4.19). Comparison of these calculated energies to the fit of the $\left[\mathrm{Fe}(\mathrm{prpep})_{2}\right]\left(\mathrm{ClO}_{4}\right)$ pre-edge region, it appears that the lower energy feature originates from a transition to the ${ }^{1} \mathrm{~A}_{1} \mathrm{~d}^{(\mathrm{n}+1)}$ final state, while the higher energy feature gains intensity from the ${ }^{3} \mathrm{~T}_{1},{ }^{3} \mathrm{~T}_{2}$, and ${ }^{1} \mathrm{~T}_{1}$ states. The ${ }^{1} \mathrm{~T}_{2}$ state is highest in energy and difficult to resolve due to the onset of the edge features (Figure 4.19).

Assuming that the feature at $-7112.7 \mathrm{eV}$ contains the intensity from transitions in the ${ }^{3} \mathrm{~T}_{1},{ }^{3} \mathrm{~T}_{2}$, and ${ }^{1} \mathrm{~T}_{1}$ states, the theoretical intensity ratio of the three pre-edge features should be 1:9:3. The fits to the $\left[\mathrm{Fe}\right.$ (prpep) $\left.{ }_{2}\right]\left(\mathrm{ClO}_{4}\right)$ pre-edge region give an intensity ratio of 1:6.4:1.7. The discrepancy between the theoretical intensity and that which is 


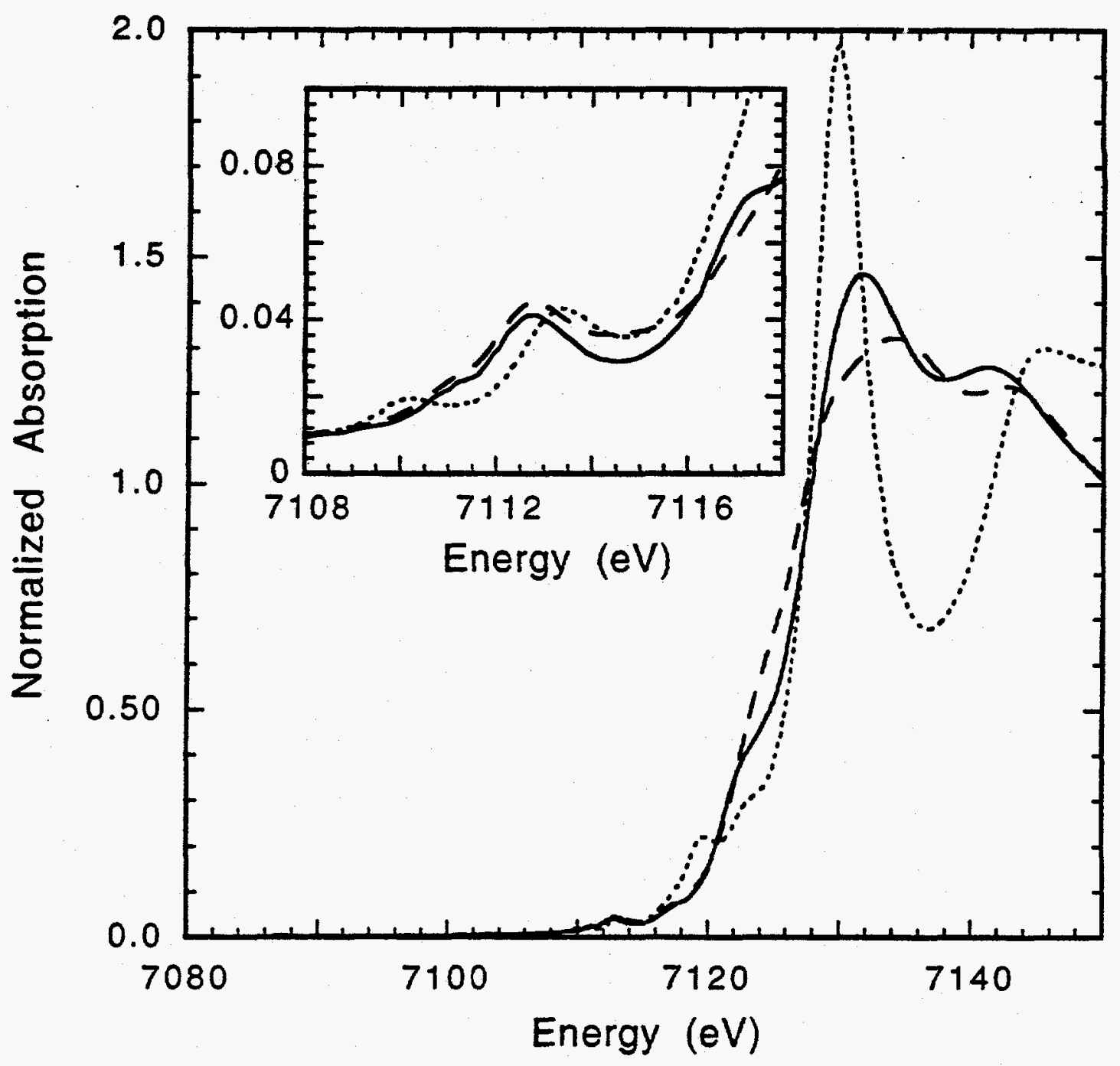

Figure 4.18. Fe K-edge XAS spectra of $\left[\mathrm{Fe}\left(\mathrm{HB}(\mathrm{pz})_{3}\right)_{2}\right]\left(\mathrm{ClO}_{4}\right)(-)$, $\left[\mathrm{Fe}(\mathrm{prpep})_{2}\right]\left(\mathrm{ClO}_{4}\right)$ $(--)$, and $\mathrm{K}_{3} \mathrm{Fe}(\mathrm{CN})_{6}(\cdots \cdot \cdot)$, where the inset is an expansion of the $1 \mathrm{~s} \rightarrow 3 \mathrm{~d}$ pre-edge region. 


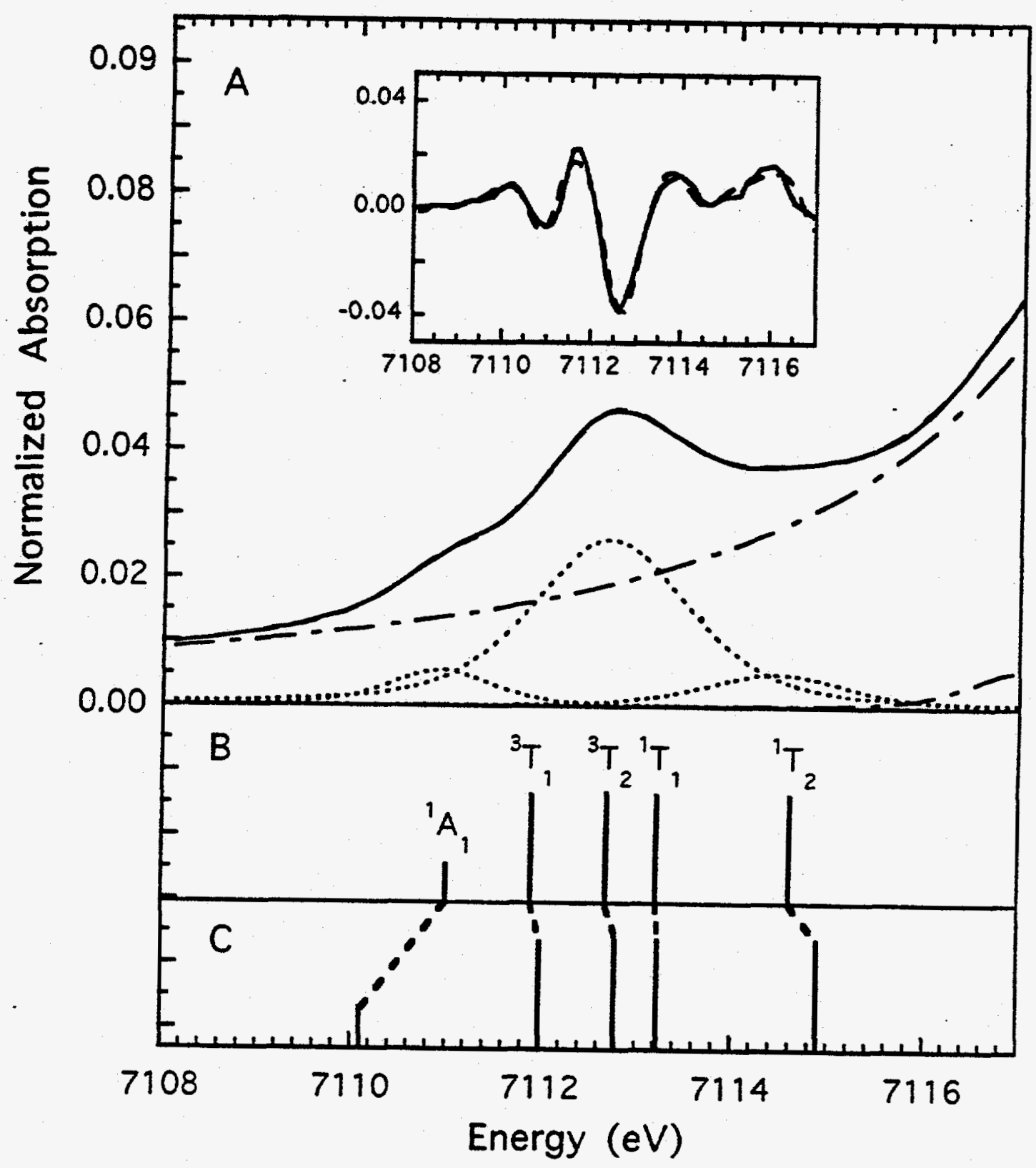

Figure 4.19. A) Fit to the Fe K-edge XAS pre-edge region of $\mathrm{K}_{3} \mathrm{Fe}(\mathrm{CN})_{6}$, where the solid line is the data, the dashed line is the fit to the data, the dashed-dot line is the background function, and the dotted lines are the individual pre-edge peaks. The inset is the second derivative of the data (-) and the second derivative of the fit to the data (--). B) The energy splitting and the quadrupole intensity distribution of the ${ }^{1} \mathrm{~A}_{1},{ }^{3} \mathrm{~T}_{1},{ }^{3} \mathrm{~T}_{2}$, ${ }^{1} T_{1}$, and ${ }^{1} T_{2}$ states. C) The effect of increased $10 \mathrm{Dq}$ on the energy splitting of the ${ }^{1} \mathrm{~A}_{1}$, ${ }^{3} \mathrm{~T}_{1},{ }^{3} \mathrm{~T}_{2},{ }^{1} \mathrm{~T}_{1}$, and ${ }^{1} \mathrm{~T}_{2}$ states. 
observed can be explained by covalency effects. The e set of orbitals is more covalent than the $t_{2}$ set due to $\sigma$ bonding interactions with the ligands; therefore, the transition to the ${ }^{1} \mathrm{~A}_{1}$ state (the state containing the $\mathrm{e}^{4}$ component) should have more relative intensity than transitions to the $T$ states which is seen in the data. The energies of the four states were recalculated using a larger $10 \mathrm{Dq}$ value of $28,000 \mathrm{~cm}^{-1}$ ( $80 \%$ of the ground state $10 \mathrm{Dq}$ for $\left.\mathrm{K}_{3} \mathrm{Fe}(\mathrm{CN})_{6}\right)^{41}$ (see Figure $4.19 \mathrm{C}$ ). Again, the lower energy feature appears to originate from a transition to the ${ }^{1} A_{1}$ state, while the higher energy feature gains intensity from transitions to the ${ }^{3} \mathrm{~T}_{1},{ }^{3} \mathrm{~T}_{2}$, and ${ }^{1} \mathrm{~T}_{1}$ states. The transition to the ${ }^{1} \mathrm{~T}_{2}$ state is difficult to resolve from the edge features. In fact, a third higher energy pre-edge feature at $\sim 7115 \mathrm{eV}$ could be included in the fits to the $\left[\mathrm{Fe}\left(\mathrm{HB}(\mathrm{pz})_{3}\right)_{2}\right]\left(\mathrm{ClO}_{4}\right)$ and $\mathrm{K}_{3} \mathrm{Fe}(\mathrm{CN})_{6}$ data. However, the data could be fit well with only two pre-edge features (Table 4.3), while a third pre-edge feature was necessary to fit the data of $\left[\mathrm{Fe}(\text { prpep })_{2}\right]\left(\mathrm{ClO}_{4}\right)$ (Figure 4.19).

\subsection{Discussion}

The $1 \mathrm{~s} \rightarrow 3 \mathrm{~d}$ pre-edge feature in the iron complexes studied was shown to be sensitive to the electronic and geometric structure of the iron site. For an iron atom in a symmetric six-coordinate environment, the number of features and the energy positions of those features differ with oxidation and spin state of the iron atom due to the differing many-electron excited states. In particular, upon promoting a 1 s electron into the $3 \mathrm{~d}$ manifold, i) a high spin ferrous atom in an $\mathrm{O}_{\mathrm{h}}$ ligand field has three many-electron excited states, ${ }^{4} \mathrm{~T}_{1},{ }^{4} \mathrm{~T}_{2}$, and ${ }^{4} \mathrm{~T}_{1}$. The lowest energy ${ }^{4} \mathrm{~T}_{1}$ state is barely resolvable from the ${ }^{4} \mathrm{~T}_{2}$ state at the Fe K-edge with the higher energy ${ }^{4} \mathrm{~T}_{1}$ state being $\sim 2 \mathrm{eV}$ above the other two states. Hence, a high spin ferrous atom in an octahedral environment has a pre-edge feature at $-7111.5 \mathrm{eV}$ from transitions into the ${ }^{4} \mathrm{~T}_{1}$ and ${ }^{4} \mathrm{~T}_{2}$ states and a feature at $-7113.5 \mathrm{eV}$ from a transition into the higher energy ${ }^{4} \mathrm{~T}_{1}$ state. Splitting of the lower energy feature may be observable if the energy difference between the ${ }^{4} \mathrm{~T}_{1}$ and ${ }^{4} \mathrm{~T}_{2}$ is greater than the experimental energy resolution at the Fe K-edge. ii) A high spin ferric atom in an $\mathrm{O}_{\mathrm{h}}$ ligand field typically has two $1 \mathrm{~s} \longrightarrow 3 \mathrm{~d}$ pre-edge features at approximately 7113.0 and $7114.5 \mathrm{eV}$ split by $10 \mathrm{Dq}$ due to transitions into the ${ }^{5} \mathrm{~T}_{2}$ and ${ }^{5} \mathrm{E}$ excited states. A direct measurement of the excited state $10 \mathrm{Dq}$ value is obtainable from the magnitude of the pre-edge splitting and, thus, trends in ligand field strength are observable. iii) A low spin ferrous atom only has one excited state and, thus, only a single pre-edge feature is observed at $\sim 7112 \mathrm{eV}$ for complexes with nitrogen ligation. iv) A low spin ferric complex in an $\mathrm{O}_{h}$ environment has five allowed excited states with the lowest in energy being a ${ }^{1} \mathrm{~A}_{1}$ state and the ${ }^{3} \mathrm{~T}_{1},{ }^{3} \mathrm{~T}_{2},{ }^{1} \mathrm{~T}_{1}$, and ${ }^{1} \mathrm{~T}_{2}$ states at higher energy. Consequently, 
the $1 \longrightarrow 3 d$ pre-edge feature of a low spin ferric complex will have a low energy feature below $7111.0 \mathrm{eV}$ due to the transition into the ${ }^{1} \mathrm{~A}_{1}$ state with more intense features at higher energy due to transitions into the ${ }^{3} \mathrm{~T}_{1},{ }^{3} \mathrm{~T}_{2},{ }^{1} \mathrm{~T}_{1}$, and ${ }^{1} \mathrm{~T}_{2}$ states. In each of the cases mentioned above where the iron atom is in a symmetric site, the pre-edge features are very weak since the intensity originates from only from quadrupole transitions. The average total pre-edge intensity for the ferrous complexes is close to 4 , while that of the ferric complexes is closer to 5 . The increase in intensity on going from ferrous to ferric sites is due to the fact that a ferrous iron atom has four holes in the ground state $3 \mathrm{~d}$ manifold, while in the ferric oxidation state there are five holes in the $3 \mathrm{~d}$ manifold.

The $1 \mathrm{~s} \rightarrow 3 \mathrm{~d}$ pre-edge feature is also sensitive to the geometry of the iron site as noncentrosymmetric distortions allow for $4 \mathrm{p}-3 \mathrm{~d}$ mixing, thereby, increasing the intensity of the pre-edge feature. Dipole transitions are two orders of magnitude more intense than quadrupole transitions, so even a few percent of $4 p$ mixing into the $3 d$ orbitals will be visible in the intensity of the $1 \mathrm{~s} \rightarrow 3 \mathrm{~d}$ pre-edge feature. The trend of increasing total intensity with decreasing coordination number has been observed previously. ${ }^{1,20}$ However, a more detailed analysis of the intensity distribution over the pre-edge features allows for more accuracy in determining the coordination number of the iron atom. For example, $\left(\mathrm{BF}_{4}\right)[\mathrm{Fe}(\mathrm{TMC}) \mathrm{Cl}]$, a five-coordinate ferrous complex, and $\left(\mathrm{Et}_{4} \mathrm{~N}\right)_{2}\left[\mathrm{FeCl}_{4}\right]$, a four-coordinate ferrous complex, have the same total pre-edge intensity of 12.9. However, the pre-edge features of these complexes look very different. $\left(\mathrm{BF}_{4}\right)[\mathrm{Fe}(\mathrm{TMC}) \mathrm{Cl}]$ has an intense pre-edge feature at $7111.4 \mathrm{eV}$ with an extremely weak feature at $7113.4 \mathrm{eV}$ (Figure 4.7), while $\left(\mathrm{Et}_{4} \mathrm{~N}\right)_{2}\left[\mathrm{FeCl}_{4}\right]$ has two intense peaks at 7111.6 and $7113.1 \mathrm{eV}$ (Figure 4.5). In the $T_{d}$ case, the dipole intensity (from $4 \mathrm{p}$ mixing into the $3 d$ orbitals of $t_{2}$ symmetry) is distributed over both pre-edge features (Figure 4.5), while in the $\mathrm{C}_{4 \mathrm{v}}$ case, where one axial ligand is removed, the dipole intensity from $4 \mathrm{p}_{\mathrm{z}}$ mixing into the $d_{z} 2$ orbital is located only in the lowest energy pre-edge feature (Figure 4.7). The effect is not quite as dramatic for ferric complexes (Figure 4.12), as there is no splitting of the pre-edge feature for ferric complexes in $T_{d}$ ligand fields (10Dq is lower than the experimental energy resolution at the Fe K-edge). There are two pre-edge features in the spectrum of the ferric $\mathrm{C}_{4 v}$ complex $\mathrm{Fe}$ (salen) $\mathrm{Cl}$ with the lower energy feature being much more intense than the higher energy feature (Figure 4.14). The increase in intensity of the lower energy feature is due to $4 \mathrm{p}_{z}$ mixing into $\mathrm{d}_{\mathrm{z}} 2$ where removing an axial ligand decreases the energy of $d_{z} 2$. The amount of $4 p$ mixing into the $3 d$ orbitals can be quantitated since the total pre-edge intensity is a sum of the quadrupole and dipole intensity and the quadrupole intensity can be estimated from the intensity of the pre-edge features of iron in symmetric environments. The values obtained for the amount of $4 p$ 
mixing into the $3 \mathrm{~d}$ orbitals for the iron model complexes in this study agree very well with theoretical estimates of the mixing from density functional calculations.

Analysis of the $1 \mathrm{~s} \rightarrow 3 \mathrm{~d}$ transitions in high spin ferric complexes is simplified by the fact that there is only one many-electron state for each excited state configuration, which is not the true for the high spin ferrous case. Thus, when a ferric atom is in a distorted site, the $1 \mathrm{~s} \rightarrow 3 \mathrm{~d}$ pre-edge feature can be used as a direct probe of the distortion. For example, when a ferric atom is in a $\mathrm{C}_{4 \mathrm{v}}$ site, the pre-edge feature is sensitive to the strength of the axial interaction. Since the $4 p_{z}$ orbital only mixes into the $d_{z} 2$ orbital, only the transition into the $d_{z}{ }^{2}$ orbital (corresponding to a transition into the ${ }^{5} A_{1}$ state). will gain dipole intensity. Hence, the energy of the most intense pre-edge feature reflects the energy of the $\mathrm{d}_{\mathrm{z}^{2}}$ orbital and the intensity of this feature is affected by the strength of the axial interaction. When an axial ligand is removed, as is the case in a five-coordinate $\mathrm{C}_{4 \mathrm{v}}$ complex, the $d_{z^{2}}$ orbital decreases in energy resulting in an intense lower energy $(\sim 7112.9 \mathrm{eV})$ feature in the pre-edge region (Figure 4.13$)$. When there is a strong axial interaction, as is the case in a $\mu$-oxo bridged binuclear complex, the $\mathrm{d}_{\mathrm{z}} 2$ orbital increases in energy resulting in an intense higher energy $(-7114.2 \mathrm{eV})$ feature in the pre-edge region (Figure 4.16). This intense higher energy feature can be used as a diagnostic tool for determining whether or not there is a $\mu$-oxo bridge in binuclear proteins and model complexes.

Using both the edge and EXAFS region of the XAS spectrum, one can obtain a very detailed description of the iron active site in non-heme iron enzymes. An EXAFS analysis provides information on the type of ligating atoms and gives very accurate iron-ligand distances. This study demonstrates that an analysis of the energy splitting and intensity distribution of the $1 \mathrm{~s} \rightarrow 3 \mathrm{~d}$ pre-edge feature can aid in elucidating the coordination number and geometry of the iron active site. In addition, the multiplet structure of the pre-edge feature is specific for a given oxidation and spin state of an iron atom. The results presented in this chapter should further aid in the interpretation of the $1 s \rightarrow 3 d$ pre-edge region for non-heme iron enzymes as the energy splitting and intensity pattern of the pre-edge features are directly related to the oxidation state, spin state and geometry of the iron site.

\subsection{Acknowledgments}

Professors Nobu Kitajima, Stephen Lippard, Richard Holm, Pradip Mascharak, and the Smithsonian Institute should be thanked for providing some of the model complexes in this study. Lawrence Roe should be thanked for measuring the data on the 
$\left(\mathrm{BF}_{4}\right)[\mathrm{Fe}(\mathrm{TMC}) \mathrm{X}]$ series and Jane DeWitt for measuring the data on the majority of the dimer complexes. Pierre Kennepohl performed the density functional calculations alluded to in the text. Susan Shadle and Cecelia Campochiaro should be thanked for their helpful discussions on the intricacies of many-electron wave functions and ligand field theory. This research is supported by grants from the NIH (GM40392, E.I.S.) and NSF (CHE-9121576, K.O.H.). The data were collected at the Stanford Synchrotron Radiation Laboratory and the National Synchrotron Light Source, Brookhaven National Laboratory, which are supported by the U.S. Department of Energy, Office of Basic Energy Science, Divisions of Chemical and Materials Sciences. SSRL is also supported in part by the National Institutes of Health, Biomedical Research Technology Program (RR-01209) and the U.S. Department of Energy, Office of Health and Environmental Research.

\subsection{References and Notes}

(1) Roe, A. L.; Schneider, D. J.; Mayer, R. L.; Pyrz, J. W.; Widom, J.; Que, L., Jr. J. Am. Chem. Soc. 1984, 106, 1676.

(2) Kauzlarich, S. M.; Teo, B. K.; Zirino, T.; Burman, S.; Davis, J. C.; Averill, B. A. Inorg. Chem. 1986, 25, 2781.

(3) Hedman, B.; Co, M. S.; Armstrong, W. H.; Hodgson, K. O.; Lippard, S. J. Inorg. Chem. 1986, 25, 3708.

(4) Bunker, G.; Petersson, L.; Sjöberg, B.-M.; Sahlin, M.; Chance, M.; Chance, B.; Ehrenberg, A. Biochemistry 1987, 26, 4708.

(5) Scarrow, R. C.; Maroney, M. J.; Palmer, S. M.; Que, L., Jr.; Roe, A. L.; Salowe, S. P.; Stubbe, J. J. Am. Chem. Soc. 1987, 109, 7857.

(6) Zhang, K.; Stern, E. A.; Ellis, F.; Sanders-Loehr, J.; Shiemke, A. K. Biochemistry 1988, 27, 7470.

(7) Tsang, H.-T.; Batie, C. J.; Ballou, D. P.; Penner-Hahn, J. E. Biochemistry 1989, $28,7233$.

(8) True, A. E.; Orville, A. M.; Pearce, L. L.; Lipscomb, J. D.; Que, L., Jr. Biochemistry 1990, 29, 10847.

(9) DeWitt, J. G.; Bentsen, J. G.; Rosenzweig, A. C.; Hedman, B.; Green, J.; Pilkington, S.; Papaefthymiou, C. G.; Dalton, H.; Hodgson, K. O.; Lippard, S. J. J. Am. Chem. Soc. 1991, 113, 9219.

(10) Van der Heijdt, L. M.; Feiters, M. C.; Navaratnam, S.; Nolting, H.-F.; Hermes, C.; Veldink, G. A.; Vliegenthart, J. F. G. Eur. J. Biochem. 1992, $207,793$. 
(11) True, A. E.; Scarrow, R. C.; Randall, C. R.; Holz, R. C.; Que, L., Jr. J. Am. Chem. Soc. 1993, 115, 4246.

(12) Randall, C. R.; Zang, Y.; True, A. E.; Que, L., Jr.; Charnock, J. M.; Garner, C. D. Biochemistry 1993, 32, 6664.

(13) Scarrow, R. C.; Trimitsis, M. G.; Buck, C. P.; Grove, G. N.; Cowling, R. A.; Nelson, M. J. Biochemistry 1994, 33, 15023.

(14) Bertini, I.; Briganti, F.; Mangani, S.; Nolting, H. F.; Scozzafava, A. Biochemistry 1994, 33, 10777.

(15) Loeb, K. E.; Zaleski, J. M.; Westre, T. E.; Guajardo, R. J.; Mascharak, P. K.; Hedman, B.; Hodgson, K. O.; Solomon, E. I. J. Am. Chem. Soc. 1995, 117, 4545.

(16) Pavlosky, M. A.; Zhang, Y.; Westre, T. E.; Gan, Q.-F.; Pavel, E. G.; Campochiaro, C.; Hedman, B.; Hodgson, K. O.; Solomon, E. I. J. Am. Chem. Soc. $1995,117,4316$.

(17) Westre, T. E.; Loeb, K. E.; Zaleski, J. M.; Hedman, B.; Hodgson, K. O.; Solomon, E. I. J. Am. Chem. Soc. 1995, 117, 1309.

(18) DeWitt, J. G.; Rosenzweig, A. C.; Salifoglou, A.; Hedman, B.; Lippard, S. J.; Hodgson, K. O. Inorg. Chem. 1995, 34, 2505.

(19) Shulman, R. G.; Yafet, Y.; Eisenberger, P.; Blumberg, W. E. Proc. Natl. Acad. Sci. USA 1976, 73, 1384.

(20) Randall, C. R.; Shu, L.; Chiou, Y.-M.; Hagen, K. S.; Ito, M.; Kitajima, N.; Lachicotte, R. J.; Zang, Y.; Que, L., Jr. Inorg. Chem. 1995, 34, 1036.

(21) DeWitt, J. G., Ph.D. Thesis, Stanford University, 1994.

(22) Blair, R. A.; Goddard, W. A. Phys. Rev. B 1980, 22, 2767.

(23) Brouder, C. J. Phys.: Condens. Matter 1990, 2, 701.

(24) Hahn, J. E.; Scott, R. A.; Hodgson, K. O.; Doniach, S.; Desjardins, S. R.; Solomon, E. I. Chem. Phys. Let. 1982, 88, 595.

(25) Wong, J.; Lytle, F. W.; Messmer, R. P.; Maylotte, D. H. Phys. Rev. B 1984, 30, 5596.

(26) Kau, L.-S.; Spira-Solomon, D. J.; Penner-Hahn, J. E.; Hodgson, K. O.; Solomon, E. I. J. Am. Chem. Soc. 1987, 109, 6433.

(27) Colpas, G. J.; Maroney, M. J.; Bagyinka, C.; Kumar, M.; Willis, W. S.; Suib, S. L.; Baidya, N.; Mascharak, P. K. Inorg. Chem. 1991, 30, 920.

(28) Sano, M.; Komorita, S.; Yamatera, H. Inorg. Chem. 1992, 31, 459.

(29) Abbreviations used: acac $=$ acetylacetonate; $\mathrm{BIPhMe}_{2}=2,2$ '-bis $(1$ methylimidazolyl)phenylmethoxymethane; $\mathrm{Bz}_{\mathrm{PhMe}} \mathrm{N}=$ benzyldimethylphenylammonium; Chel $=4$-hydroxo-2,6-pyridinedicarboxylate; Dipic $=2,6$ - 
pyridinedicarboxylate; EDTA $=$ ethylenediaminetetraacetic acid; $\mathrm{HB}(\mathrm{pz})_{3}=[$ tri1-pyrazolylborate ${ }^{1-} ; \mathrm{HB}\left(3,5-\mathrm{PP}_{2} \mathrm{pz}\right)_{3}=$ hydrotris(3,5-diisopropyl-1pyrazolyl)borate; HPTB $=\left[N, N, N^{\prime}, N^{\prime}\right.$-tetrakis(2-benzimidazoylmethyl)-2hydroxy-1,3-diaminopropane $]^{-}$; PrpepH $=N$-(2-(4-imidazole)ethyl)pyrimidine-4carboxamide; salmp $=[2 \text {-bis(salicylideneamino)methylphenolate }]^{3-} ; \mathrm{TMC}=$ 1,4,8,11-tetramethyl-1,4,8,11-tetraazacyclotetradecane; $T P A=\operatorname{tris}(2-$ pyridylmethyl)amine; $O A C=$ acetate bridge; $O P h=$ phenolate bridge; $O P h t=$ phthalate bridge; $O B z=$ benzoate bridge.

(30) Scott, R. A.; Hahn, J. E.; Doniach, S.; Freeman, H. C.; Hodgson, K. O. J. Am. Chem. Soc. 1982, 104, 5364.

(31) Lytle, F. W. In Applications of Synchrotron Radiation; Winick, H.; Xiam, D.; Ye, M.-H.; Huang, T., Eds.; Gordon and Breach Science Publishers: New York, 1989; p 135.

(32) Argonne National Laboratory; B.S. Garbow, K.E. Hillstrom, J.J. More.

(33) Agarwal, B. K. X-ray Spectroscopy; Springer-Verlag: Berlin, 1979, p 276.

(34) Lytle, F. W.; Greegor, R. B.; Sandstrom, D. R.; Marques, E. C.; Wong, J.; Spiro, C. L.; Huffman, G. P.; Huggins, F. E. Nucl. Instrum. Methods 1984, $226,542$.

(35) Tyson, T. A.; Roe, A. L.; Frank, P.; Hodgson, K. O.; Hedman, B. Phy. Rev. B 1989, 39, 6305.

(36) Griffith, J. S. The Theory of Transition Metal Ions; Cambridge University Press: Cambridge, 1961.

(37) de Groot, F. M. F. J. Electron Spectrosc. Relat. Phenom. 1994, 67, 529.

(38) Sugano, S.; Tanabe, Y.; Kamimura, H. Multiplets of Transition-Metal Ions in Crystals; Academic Press: New York, 1970.

(39) Stout, J. W.; Reed, S. A. J. Am. Chem. Soc. 1954, 76, 5279.

(40) Karshipin, T. B.; Gebhard, M. S.; Solomon, E. I.; Raymond, K. N. J. Am. Chem. Soc. 1991, 113, 2977.

(41) Lever, A. B. P. Inorganic Electronic Spectroscopy; 2 ed.; Elsevier: Amsterdam, 1984.

(42) Gewirth, A. A.; Cohen, S. L.; Schugar, H. J.; Solomon, E. I. Inorg. Chem. 1987, $26,1133$.

(43) Shadle, S. E.; Penner-Hahn, J. E.; Schugar, H. J.; Hedman, B.; Hodgson, K. O.; Solomon, E. I. J. Am. Chem. Soc. 1993, 115, 767.

(44) Karplus, M.; Porter, R. N. Atoms and Molecules; W. A. Benjamin, Inc.: London, 1970, p 228.

(45) Furlane, E.; Cervone, E.; Valent, V. J. Nucl. Chem. 1963, 25, 159. 
(46) Cotton, F. A. Chemical Applications of Group Theory; Interscience Publishers: New York, 1963.

(47) Westre, T. E.; Kennepohl, P.; DeWitt, J. G.; Hedman, B.; Hodgson, K. O.; Solomon, E. I. to be published.

(48) . Deaton, J. C.; Gebhard, M. S.; Solomon, E. I. 1989, 88, 877.

(49) Bertrand, J. A.; Breece, J. L.; Eller, P. G. Inorg. Chem. 1974, 13, 125.

(50) Dong, Y.; Menage, S.; Brennan, B. A.; Elgren, T. E.; Jang, H. G.; Pearce, L. L.; Que, L., Jr. J. Am. Chem. Soc. 1993, 115, 1851.

(51) Armstrong, W. H.; Lippard, S. J. J. Am. Chem. Soc. 1984, 106, 4632.

(52) Neuse, E. W.; Kahn, F. B. D.; Berhalter, K.; Thewalt, U. J. Cryst. Spectr. Res. 1986, 16, 483.

(53) Lauher, J. W.; Ibers, J. A. Inorg. Chem. 1975, 14, 348.

(54) Briat, B.; Canit, J. C. Molec. Phys. 1983, 48, 33.

(55) Kitajima, N. 1994, private communication.

(56) Pabst, A. Am. Mineralogist 1943, 28, 372.

(57) Pabst, A. Acta Crystallogr. 1959, 12, 733.

(58) Hodges, K. D.; Wollmann, R. G.; Barfield, E. K.; Hendrickson, D. N. Inorg. Chem. 1977, 16, 2746.

(59) Ferrari, A.; Braibanti, A.; Bigliardi, G. Acta Crystallogr. 1963, 16, 846.

(60) Gregory, N. W. J. Phys. Chem 1963, 67, 927.

(61) Hatfield, W. E.; Fay, R. C.; Pfluger, C. E.; Piper, T. S. J. Am. Chem. Soc. 1963, $85,265$.

(62) Beattie, J. K.; Moore, C. J. Inorg. Chem. 1982, 21, 1291.

(63) Iball, J.; Morgan, C. H. Acta Crystallogr. 1967, 23, 339.

(64) Holt, S.; Dingle, R. Acta Chem. Scand. 1968, 22, 1091.

(65) Jaeger, F. M.; Mees, R. T. A. Proc. Acad. Sci. Amsterdam 1918, $20,283$.

(66) Clark, H. C.; Curtis, N. F.; Odell, A. L. J. Chem. Soc. 1954, 63.

(67) Wyckoff, R. W. G. Crystal Structures; 2 ed.; Robert E. Krieger Publishing Company: Malabar, Florida, 1982; Vol. 3, p 875,898.

(68) Feng, X.; Bott, S. G.; Lippard, S. J. J. Am. Chem. Soc. 1989, 111, 8046.

(69) Tolman, W. B.; Bino, A.; Lippard, S. J. J. Am. Chem. Soc. 1989, 111, 8522.

(70) Armstrong, W. H.; Spool, A.; Papaefthymiou, G. C.; Frankel, R. B.; Lippard, S. J. J. Am. Chem. Soc. 1984, 106, 3653.

(71) Thich, J. A.; Ou, C. C.; Powers, D.; Vasiliou, B.; Mastropaolo, D.; Potenza, J. A.; Schugar, H. J. J. Am. Chem. Soc. 1976, 98, 1425. 
(72) Norman, R. E.; Yan, S.; Que, L., Jr.; Backes, G.; Ling, J.; Sanders-Loehr, J.; Zhang, J. H.; O'Connor, C. J. J. Am. Chem. Soc. 1990, 112, 1554.

(73) Norman, R. E.; Hoiz, R. C.; Ménage, S.; O'Connor, C. J.; Zhang, J. H.; Que, L., Jr. Inorg. Chem. 1990, 29, 4629.

(74) Gaines, A.; Hammett, L. P.; Walden, G. H. J. Am. Chem. Soc. 1963, 58, 1668 :

(75) Lippard, S. J.; Schugar, H.; Walling, C. Inorg. Chem. 1967, 6, 1825.

(76) Trofimenko, S. J. Am. Chem. Soc. 1967, 89, 3170.

(77) Oliver, J. D.; Mullica, D. F.; Hutchinson, B. B.; Milligan, W. O. Inorg. Chem. 1980, 19, 165.

(78) Brown, S. J.; Olmstead, M. M.; Mascharak, P. K. Inorg. Chem. 1990, 29, 3229.

(79) Rosenzweig, A. C.; Lippard, S. J. 1992, private communication.

(80) Figgis, B. N.; Skelton, B. W.; White, A. H. 1978, 31, 1195. 
Chapter 5

Characterization of the Electronic and Geometric Structure of Non-Heme Iron Active Sites Using Fe K-Edge XAS 


\subsection{Mononuclear Non-Heme Iron Enzymes}

\subsubsection{Mechanistic and Structural Background .}

Mononuclear non-heme iron active sites are present in a variety of enzymes involved in a wide range of important biological functions requiring dioxygen. These non-heme iron enzymes include superoxide dismutases, oxidases, extra- and intradiol dioxygenases, cis-hydroxylases, pterin- and $\alpha$-ketoglutarate-dependent hydroxylases, lipoxygenases, and bleomycin. Several recent reviews of non-beme iron enzymes have appeared in the literature. ${ }^{1-4}$ Both the ferric and ferrous oxidation states have been determined to be involved in catalysis for different enzymes in this class with the iron usually coordinated to four, five, or six oxygen or nitrogen ligands. For several enzymes, substrate and oxygen bound intermediates are reasonably stable. Futhermore, nitric oxide derivatives of the ferrous sites bave been reported for many of these enzymes which serve as reversible analogues of possible dioxygen intermediates. Representative enzymes and the reaction they catalyze are given in Table 5.1. More detailed mechanistic and structural information for these enzymes is presented below.

Iron superoxide dismutase (FeSOD) catalyzes the dismutation of superoxide anions to peroxide and molecular oxygen in procaryotes. ${ }^{5,6}$ The determination of the three-dimensional crystal structure of FeSOD from $E$. coli has given the first insight into the coordination chemistry of this class of enzymes and has underlined its similarity to the bacterial manganese-dependent enzymes catalyzing the same reaction. $5,7,8$ $\mathrm{Fe}$ (III)SOD has a distorted trigonal bipyramidal structure with four endogenous protein ligands (His-26, His-73, Asn-156, and His-160). His-26 coordinates axially with the other three ligands forming the trigonal plane. ${ }^{5,7,8}$ There is some controversy regarding the possible coordination of solvent in the axial fifth position. Stoddard et al. ${ }^{7}$ propose that the fifth site is devoid of a bound water molecule or an anion and place the metal at $0.5 \AA$ out of the plane of the trigonal ligands towards the axial histidine. Stallings et al. ${ }^{5}$ position the metal ion in the plane of the trigonal ligands and find that the solvent (hydroxide) occupies the fifth, axial coordination position on the $\mathrm{Fe}^{3+}$. There is little structural change upon reduction to the ferrous state; however, the uptake of one proton per electron is observed from titration experiments which is accounted for in the Stallings et al. structure by the bound hydroxide going to water. ${ }^{8}$ Magnetic circular dichroism (MCD) and Mössbauer studies indicate that a five-coordinate structure is present in the reduced state, ${ }^{5}$ with a more square pyramidal electronic symmetry indicated from the MCD analysis. ${ }^{9}$ Azide is an inhibitor of dismutase activity and binds to the ferric site 
Table 5.1. Mononuclear Non-Heme Iron Enzymes.

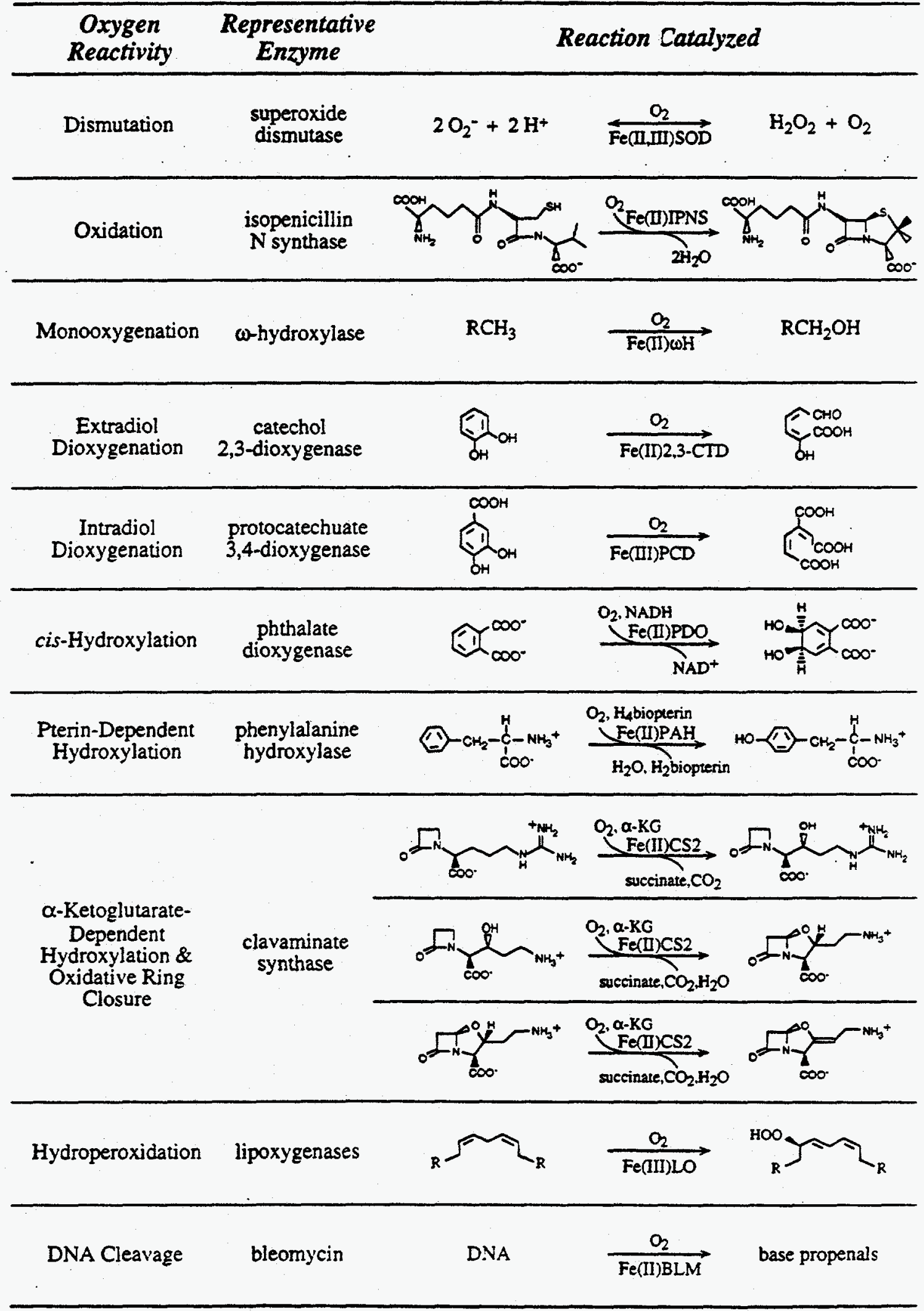

reproduced in part from reference 4 
with an increase in the coordination number. ${ }^{5}$ Superoxide binding to the ferric center is proposed to parallel azide binding, and catalysis involves a cyclic oxidation/reduction mechanism ${ }^{5,6}$ which ultimately results in the dismutation process.

Isopenicillin $N$ synthase (IPNS) is a non-heme, $\mathrm{Fe}^{2+}$ - and oxygen-dependent enzyme found in $\beta$-lactam antibiotic-producing microorganisms. ${ }^{10}$ This enzyme catalyzes the stepwise, ${ }^{11}$ desaturative cyclization of $\delta$-(L- $\alpha$-aminoadipoyl)-L-cysteinyl$D$-valine (LLD-ACV) to form isopenicillin $N,{ }^{12}$ the precursor of all penicillins. ${ }^{13}$ This key step in the biosynthesis of penicillins and cephalosporins results in the four-electron reduction of one equivalent of dioxygen to two equivalents of water. ${ }^{14}$ The loss of four hydrogen atoms is stereospecific and the internal C-N and C-S bonds are.formed with complete retention of configuration. ${ }^{15,16}$ Although no crystal structure of this enzyme exists, information on the active site has been gained using a variety of spectroscopic techniques including optical absorption, ${ }^{14}$ electron paramagnetic resonance (EPR), ${ }^{17}$ electron spin echo envelope modulation (ESEEM), ${ }^{13}$ nuclear magnetic resonance (NMR), ${ }^{18}$ Mössbauer, ${ }^{17}$ and X-ray absorption spectroscopy (XAS). ${ }^{19-21}$ The native enzyme is believed to be coordinated by four endogenous residues, three histidines and one monodentate aspartate, with water or hydroxide occupying the remaining sites in a distorted octahedral geometry. There are two conserved cysteine residues which have been shown by site directed mutagenisis studies ${ }^{22}$ to affect substrate and small molecule binding, but are not directly ligated to the iron. Three independent observations support the notion that substrate binds to the metal center through a thiolate sulfur. First, the Mössbauer isomer shift of the iron center in Fe(II)IPNS is decreased from 1.3 to $1.1 \mathrm{~mm} / \mathrm{s}$ upon ACV binding. ${ }^{17}$ Second, Cu(II)IPNS develops an intense band at $390 \mathrm{~nm}$ upon addition of ACV, which is assigned as a thiolate-to-metal charge transfer transition. ${ }^{14}$ Lastly, EXAFS analysis of the Fe(II)IPNS-ACV and Fe(II)IPNS-ACV-NO complexes require a sulfur scatterer at about $2.3 \AA .^{19-21}$ Nitric oxide also binds to the native metal center to form a complex with a characteristic $S=3 / 2$ EPR signal. At this point little is known about the catalytic mechanism of this enzyme, although a high-valent iron-oxo intermediate has been proposed. $15,23,24$

The hydroxylation of fatty acids and alkanes and the epoxidation of alkenes using molecular oxygen are catalyzed by $\omega$-Hydroxylase $(\omega \mathrm{H}){ }^{25} \omega \mathrm{H}$ is one of the three components, including a flavoprotein reductase and rubredoxin, of the non-heme iron monooxygenase system from Pseudomonas oleovarans. This system has proven difficult to purify, therefore limiting the available mechanistic and structural information. ${ }^{26,27}$ Phospholipids and a stoichiometry of one atom of iron per polypeptide chain are required for full activity. ${ }^{25}$ NMR has shown that the epoxidation of terminal olefins to the 
corresponding 1,2-oxides proceeds with a high degree of stereoselectivity and specificity, resulting primarily in inversion of the original olefin geometry. ${ }^{28}$ The stepwise mechanism does not involve complex hydrogen atom abstraction but rather initial attack of "activated" oxygen and final ring closure to the epoxide product. ${ }^{26}$ At most, one cysteine sulfur may be ligated to the iron of $\omega \mathrm{H}$. However, iron contributes only slightly to the visible absorption spectrum which displays no charge transfer transitions. Thus $\omega \mathrm{H}$ is proposed to have principally nitrogen and/or oxygen coordination. ${ }^{25}$

The catechol dioxygenases serve as part of nature's strategy for degrading aromatic molecules in the environment. They are found in soil bacteria such as pseudomonads and bacilli and act in the last step of transforming aromatic precursors into aliphatic products. The catechol substrates can be oxidatively cleaved by non-heme iron dioxygenases in two distinct modes: intra- and extradiol cleavage. The intradiol cleaving enzymes have a ferric active site and act on catechol and protocatechuate, while the extradiol cleaving enzymes have a ferrous active site and act on a larger number of substrates including the two already mentioned. ${ }^{2,29}$ The ferrous site of extradiol cleaving enzymes is stable in the presence of dioxygen, binds substrate in a bidentate fashion, and has a third coordination position available for small molecule binding and oxygen reactivity. ${ }^{30}$ The intradiol cleaving enzymes involve a ferric site with two open coordination positions for substrate binding ${ }^{2}$ and are the best studied with high resolution crystal structures available for protocatechuate 3,4-dioxygenase (PCD) from Pseudomonas aeruginosa ${ }^{31}$ and Brevibacterium fuscum. ${ }^{32}$ Both active sites have a trigonal bipyramidal metal environment with Tyr-147 and His-162 coordinated axially and Tyr-118, His-160, and a hydroxide bound in the equatorial plane. The substrate is thought to bind as a chelate replacing the hydroxide and axial tyrosine. ${ }^{33}$ Binding to the ferric site is believed to activate this substrate either by inducing semiquinone- $\mathrm{Fe}^{2+}$ character through high covalency, ${ }^{34}$ with oxygen then reacting with either the substrate or the iron, ${ }^{35}$ or by a weakening of the binding of one of the catecholate oxygens with $\mathrm{Fe}^{3+}$ to induce ketonization of the substrate followed by oxygen attack on the coordinated hydroxyl group. ${ }^{29}$ For either model of substrate activation, the enzyme-substrate- $\mathrm{O}_{2}$ intermediate observed in the initial phase of this reaction is thought to involve a peroxy adduct of the substrate coordinated to the iron. This converts to enzyme-substrate- $\mathrm{O}_{2}{ }^{*}$ which is a product complex. For the extradiol dioxygenase catechol 2,3-dioxygenase (2,3-CTD), substrate binding activates the ferrous site for small molecule binding. ${ }^{36} \mathrm{~A}$ crystal structure of an extradiol dioxygenase 2,3-dihydroxybiphenyl 1,2-dioxygenase (1,2-DBD) has just been completed ${ }^{37}$ and shows that this site is square pyramidal as predicted by MCD spectroscopy on the analogous enzyme 2,3-CTD. The ligation 
consists of two bound water molecules, a nitrogen from His-210, and an oxygen from a carboxylate group which form the equatorial plane, and a nitrogen from His-146 coordinated axially.

Phthalate dioxygenase (PDO) catalyzes the cis-hydroxylation of phthalate to 1,2-dihydroxy-4,5-dicarboxy-3,5-cyclohexadiene in the presence of phthalate dioxygenase reductase (PDR), NADH as an electron source, substrate, and oxygen. PDO is substrate specific, activating only phthalate or aromatics with vicinal anionic groups. Although PDO can oxygenate phthalate in the presence of oxygen, PDR is reguired for efficient catalysis. ${ }^{38}$ PDO is a member of a broad class of environmentally significant bacterial dioxygenases which activate aromatic substrates to their cis-dihydrodiols for further degradation and catabolism. Other enzymes in this family include benzene dioxygenase, ${ }^{39}$ benzoate dioxygenase, ${ }^{40}$ naphthalene dioxygenase, ${ }^{41}$ pyrazon dioxygenase, ${ }^{42}$ and toluene dioxygenase, ${ }^{43}$ all of which contain a [2Fe-2S] Rieske site and a mononuclear non-heme iron center. In addition to the bacterial dioxygenases, there are other enzymes which also contain a Rieske cluster in addition to a mononuclear non-heme iron site but do not yield the dihydrodiol products: ${ }^{44}$ 4-chlorophenylacetate 3,4 dioxygenase converts substrate to catechol with chloride elimination, ${ }^{45}$ vanillate demethylase participates in the biodegradation of lignin, ${ }^{46}$ and 4 -methoxybenzoate $O$-demethylase (putidamonooxin) catalyzes the conversion of 4-methoxybenzoic acid to 4-hydroxybenzoic acid and formaldehyde. ${ }^{47}$ In all of these systems, the mononuclear non-heme iron is believed to be the active site for catalysis and presumably binds and activates molecular oxygen. PDO is the best studied enzyme in its class because it is available in high yield and is extremely stable even at room temperature. However, spectroscopic studies that have been performed on PDO, including resonance Raman $(\mathrm{rR}),{ }^{48,49}$ electron-nuclear double resonance (ENDOR), ${ }^{50,51}$ and XAS ${ }^{52,53}$ have focused primarily on investigation of the Rieske site due to its dominant spectroscopic features. The mononuclear non-heme active site has been probed indirectly through XAS studies of $\mathrm{Co}^{2+}$ and $\mathrm{Zn}^{2+-}$-substituted protein. ${ }^{52,53}$ From XAS Co- and $\mathrm{Zn}$-edge and EXAFS studies, ${ }^{53}$ the mononuclear site has been shown to have predominantly oxygen ligation with a coordination number of five or six. Oxidation of the Rieske cluster and/or binding of phthalate seem to favor a five-coordinate form of the site, while reduction of the Rieske and/or removal of phthalate appear to favor a six-coordinate form of the mononuclear site. The mononuclear iron site has also been probed directly by low temperature $\mathrm{MCD}$ since the Rieske site can be rendered diamagnetic (i.e. no temperature dependent $M C D$ ) by oxidation to the antiferromagnetically-coupled [Fe ${ }^{\mathrm{III}} \mathrm{Fe}{ }^{\mathrm{II}}$ ] state..$^{54}$ This data shows that the resting enzyme has a six-coordinate ferrous site, while addition 
of substrate causes the active site to be five-coordinate. Azide binds to the ferrous site in the enzyme-substrate form to produce a new six-coordinate form, this indicates that substrate binding causes the ferrous site to convert from six-to five-coordinate, opening a coordination position on the iron for $\mathrm{O}_{2}$ reactivity.

The hydroxylation of phenylalanine, tyrosine and tryptophan to tyrosine, 3,4-dihydroxyphenylalanine, and 5-hydroxytryptophan, respectively, are catalyzed by hydroxylases that depend on $\mathrm{Fe}^{2+}$ and tetrahydropterins. ${ }^{55}$ Phenylalanine hydroxylase (PAH) is the most well-studied of these enzymes. PAH is found in the liver and kidney and its deficiency is responsible for the genetic disorder phenylketonuria that is associated with severe mental retardation. ${ }^{56}$ It has been thought that the role of the metal ion is to generate a 4a-hydroperoxy intermediate and activate it through forming a $\mu$-peroxy pterin-iron containing complex which could further generate a non-heme oxo-ferryl intermediate and the experimentally observed 4a-hydroxypterin species. ${ }^{57}$ In support of this, PAH from Chromobacterium violaceum (CVPAH), thought to be a copper enzyme, showed an ESEEM spectrum of a pterin directly coordinated to the metal ion ${ }^{58}$ and crystallographic studies on $\mathrm{Cu}$-pterin model complexes indicate that the pterin cofactor binds to the metal center through positions 04 and N5. ${ }^{59}$ Further, paramagnetic NMR studies on $\mathrm{Co}^{2+}$-substituted tyrosine hydroxylase show that phenylalanine, which is also a substrate for this enzyme, binds such that the aromatic ring is in the "second coordination sphere" of the metal ion. ${ }^{60}$ Recent studies of $\mathrm{CVPAH}^{61}$ have questioned whether the copper ion is in fact required for activity and thus whether the iron in mammalian PAHs (which is strictly required for activity) plays a direct oxygen activation role or an indirect structural role in catalysis. As reduction of the $\mathrm{Fe}^{2+}$ state is required for catalysis, it is critically important to probe the interaction of pterin cofactor with this reduced iron site. However, all studies thus far reported have been on the EPR-active resting ferric site. These studies ${ }^{62,63}$ have shown that both active (axial) and inactive (rhombic) iron sites are present which do not interconvert and have a relative ratio dependent on the preparative conditions. ${ }^{64}$

Bacterial resistance to penicillin antibiotics is largely due to the hydrolytic activity of the $\beta$-lactamase enzymes. Clavaminate synthase (CS) catalyzes the key biosynthetic ring closure step in the formation of clavulanic acid, a potent $\beta$-lactamase inhibitor. ${ }^{65} \mathrm{CS}$ is a member of a large class of non-heme iron enzymes dependent on $\alpha$-ketoglutarate $(\alpha-K G)$ as a cofactor. This class also includes prolyl and lysyl hydroxylases (involved in collagen synthesis), ${ }^{66}$ thymine hydroxylase, ${ }^{67}$ and 4-hydroxyphenylpyruvate dioxygenase, ${ }^{68}$ all of which catalyze the hydroxylation of substrate. In contrast, CS catalyzes oxidative ring closure similar to the reaction catalyzed by IPNS, ${ }^{23}$ which is not 
an $\alpha$-KG-dependent enzyme. CS is a particularly interesting enzyme in that, depending on substrate, this enzyme can also catalyze hydroxylation ${ }^{69}$ as well as ring closure and desaturation chemistry. ${ }^{65,70}$ The mechanism of $\alpha$-KG-dependent enzymes generally involves the reaction of an $\alpha-K G$-bound ferrous active site with dioxygen. Model studies ${ }^{71}$ have shown that an $\alpha-\mathrm{KG}$ analog binds to the ferrous site in a bidentate fashion through one carboxylate oxygen and one carbonyl oxygen. The enzymatic reaction generates succinate, $\mathrm{CO}_{2}$, and an activated iron-oxygen species, thought to be an oxo-ferryl unit ${ }^{72}$ which goes on to hydroxylate substrate or catalyze oxidative ring closure through the heteroatom.

The lipoxygenases (LOS) catalyze the hydroperoxidation of cis, cis-1,4-pentadiene-containing fatty acids. Mammalian LOs catalyze the conversion of arachidonic acid to leukotrienes (5-LOs), which mediate hypersensitivity and inflammation, and lipoxins (15-LOs), which inhibit cellular immunity. ${ }^{73} 15-\mathrm{LO}$ are also implicated in the oxidation of low density lipoprotein to the athrogenic form which leads to the growth of atherosclerotic lesions. ${ }^{74}$. Most mechanistic studies have been performed on the plant enzyme from soybeans (SLO-1) which has linoleic acid as a substrate. The resting enzyme is air-stable in its ferrous oxidation state, but is activated by reaction with the hydroperoxide product to produce the ferric site. ${ }^{75}$ The fatty acid substrate reacts with this ferric site to produce an intermediate, which then oxygenates to generate the hydroperoxide. The substrate-active site intermediate has been proposed to be either an organometallic complex formed by proton abstraction and coordination of the carbanion substrate to the ferric center ${ }^{76}$ or a ferrous center with a fatty acid radical, which may not be directly coordinated to the iron. ${ }^{77,78}$ It has been of significant pharmacological importance to develop inhibitors of the lipoxygenases which are mostly found to be reversible and function by reducing the active ferric center to the ferrous state. ${ }^{79}$ Spectroscopic studies have generally indicated that the ferrous site is six-coordinate with at least three histidine ligands. ${ }^{9,80,81}$ Recently, two crystal structures have appeared ${ }^{82,83}$ on the resting enzyme which disagree with respect to the active site ligation. Four amino acid ligands are common to both structures: His-499(NE), His-504(NE), His-609 (NE), and Ile-389 (OT2). The Boyington et al. crystallographic description ${ }^{82}$ of the iron site is four coordinate with a ligand arrangement described as "distorted octahedral with two adjacent unoccupied positions". Alternatively, the Minor et al. structure ${ }^{83}$ has an additional residue as a ligand, Asn-694.(O81), and further possibility of a sixth water based ligand as has been proposed from EPR line-broadening studies on the ferric enzyme. ${ }^{84}$ 
Bleomycin (BLM), a histidine-containing glycopeptide antibiotic produced by strains of Streptomyces verticillus, is used in treatment against a variety of carcinomas and lymphomas. ${ }^{85}$ The anticancer activity of the drug Blenoxane involves selective DNA cleavage at certain GT and GC sites in the presence of metal ions and dioxygen. ${ }^{86-88}$ The structure of BLM contains three domains: the bithiazole tail which is involved in BLM binding to DNA, the sugar moiety which is believed to be responsible for uptake by cancer cells, and the $\beta$-aminoalanine-pyrimidine-histidine region which binds metal ions. BLM is generally thought to be most reactive as a ferrous complex with histidine, deprotonated amide, pyrimidine, and secondary amine nitrogens as equatorial ligands. ${ }^{86,87}$ No crystal structure exists of the ferrous complex and present descriptions are based on correlations to a $\mathrm{Cu}$ (II) complex of the related biosynthetic precursor P-3A, which has a square-pyramidal geometry. ${ }^{89}$ However, $\mathrm{Cu}^{2+}$ has strong structural preferences due to the Jahn-Teller effect which Fe ${ }^{2+}$ does not, and P-3A lacks the disaccharide and bithiazole moieties of BLM, the former being a potential ligand. Thus, there is some controversy with respect to axial ligation where both the carbamoyl group of the mannose sugar ${ }^{90}$ and the primary amine from the $\beta$-aminoalanine ${ }^{91}$ have been implicated, with the sixth position being the site of exogenous ligand binding. BLM binds to DNA via intercalation and/or ionic interactions with the minor groove, with both the bithiazole tail and the metal chelating regions playing a significant role in the binding and specificity. ${ }^{92-94}$ Kinetic and spectral studies ${ }^{95,96}$ have demonstrated that the mechanism of activation involves a high-spin $\mathrm{Fe}(\mathrm{II}) \mathrm{BLM}$ species reacting with dioxygen to form oxygenated BLM, described as a ferric-superoxide complex. This in turn acquires an additional electron either from a second Fe(II)BLM molecule or another reductant to generate activated BLM. Activated BLM reacts with DNA by hydrogen atom abstraction from the C-4' position of the deoxyribose sugar, leading to DNA degradation concomitant with base propenal formation. ${ }^{86}$

XAS edge and EXAFS analysis can be used to obtain information on the electronic and geometric structure of the iron active sites in the above mentioned enzymes. A detailed analysis of the intensity and splitting of the pre-edge features allows one to obtain the oxidation state, spin state, and coordination geometry of the iron active site (as described in Chapter 4). EXAFS analysis provides accurate first shell distances with information on the number and type of coordinating atoms. Not only can these types of analyses be performed on the resting enzymes, but stable substrate and oxygen bound intermediates can also be studied including the nitric oxide derivatives of the ferrous active sites which serve as reversible analogues of possible dioxygen intermediates. Mechanistic information can be gained by studying the various forms of 
the enzymes and comparing them to the resting enzyme. In the following sections XAS has been used to characterize the iron site in specific enzymes. In section 5.2, edge structure analysis has been used to determine the oxidation state of the iron in $\{\mathrm{FeNO}\}^{7}$ systems. The ferrous active sites of BLM and a related model complex were characterized by both Fe K-edge and EXAFS analyses as described in section 5.3. Then, XAS was used to determine the oxidation, spin state and coordination geometry of activated BLM as shown in section 5.4. The iron active sites of both mammalian and soybean lipoxygenases were characterized utilizing XAS and the results are presented in section 5.5. An Fe K-edge and EXAFS study of phenylalanine hydroxylase is described in section 5.6. Finally, the ferrous, ferric, substrate-bound and NO-bound forms of protocatechuate 3,4-dioxygenase were studied and the results are presented in section 5.7.

\subsubsection{References}

(1) Solomon, E. I.; Zhang, Y. Acc. Chem. Res. 1992, 25, 343.

(2) Que, L., Jr. In Bioinorganic Catalysis; Reedijk, J., Ed.; Marcel Dekker, Inc.: New York, 1993; p 347.

(3) Feig, A. L.; Lippard, S. J. Chem. Rev. 1994, 94, 759.

(4) Solomon, E. I.; Pavel, E. G.; Loeb, K. E.; Campochiaro, C. Coord. Chem. Rev. 1995 , in press.

(5) Stallings, W. C.; Bull, C.; Fee, J. A.; Lah, M. S.; Ludwig, M. L. In Molecular Biology of Free Radical Scavenging Systems; Scandolios, J. G., Ed.; Cold Spring Harbor Laboratory Press: New York, 1992; p 193.

(6) Stalling, W.; Bull, C.; Pattridge, K. A.; Powers, T. B.; Fee, J. A.; Ludwig, M. L.; Ringe, D.; Petsko, G. A. In Oxygen Radicals in Chemistry and Biology; Bors, W.; Saran, M.; Tait, D., Eds.; Walter de Gruyter \& Co.: Berlin, 1984; p 779.

(7) Stoddard, B. L.; Howell, P. L.; Ringe, D.; Petsko, G. A. Biochemistry 1990, 29, 8885.

(8) Stallings, W. C.; Metzger, A. L.; Pattridge, K. A.; Fee, J. A.; Ludwig, M. L. Free Rad. Res. Comms. 1991, 12-13, 259.

(9) Whittaker, J. W.; Solomon, E. I. J. Am. Chem. Soc. 1988, 110, 5329.

(10) Baldwin, J. E.; Blackburn, J. M.; Sutherland, J. D.; Wright, M. C. Tetrahedron 1991, 47, 5991.

(11) Baldwin, J. E.; Lynch, G. P.; Schofield, C. J. J. Chem. Soc., Chem. Commun. $1991,736$. 
(12) Baldwin, J. E.; Bradley, M.; Adlington, R. M.; Norris, W. J.; Tumer, N. J. Tetrahedron 1991, 47, 457.

(13) J.ang, F.; Peisach, J.; Ming, L.-J.; Que, L., Jr.; Chen, V. J. Biochemistry 1991, 30, 11437.

(14) Ming, L.-J.; Que, L., Jr.; Kriauciunas, A.; Frolik, C. A.; Chen, V. J. Inorg. Chem. $1990,29,1111$.

(15) Baldwin, J. E.; Adlington, R. M.; Bradley, M.; Norris, W. J.; Turner, N. J.; Yoshida, A. J. Chem. Soc., Chem. Commun. 1988, 1125.

(16) Baldwin, J. E.; Adlington, R. M.; Marquess, D. G.; Pitt, A. R.; Russell, A. T. J. Chem. Soc., Chem. Commun. 1991, 856.

(17) Chen, V. J.; Orville, A. M.; Harpel, M. R.; Frolik, C. A.; Surerus, K. K.; Münck, E.; Lipscomb, J. D. J. Biol. Chem. 1989, 264, 21677.

(18) Ming, L.-J.; Que, L., Jr.; Kriauciunas, A.; Frolik, C. A.; Chen, V. J. Biochemistry 1991, 30, 11653.

(19) Fujishima, Y.; Schofield, C. J.; Baldwin, J. E.; Charnock, J. M.; Garner, C. D. J. Inorg. Biochem. 1991, 43, 564.

(20) Scott, R. A.; Wang, S.; Eidsness, M. K.; Kriauciunas, A.; Frolik, C. A.; Chen, V. J. Biochemistry 1992, 31, 4596.

(21) Randall, C. R.; Zang, Y.; True, A. E.; Que, L., Jr.; Charnock, J. M.; Garner, C. D.; Fujishima, Y.; Schofield, C. J.; Baldwin, J. E. Biochemistry 1993, 32, 6664.

(22) Orville, A. M.; Chen, V. J.; Kriauciunas, A.; Harpel, M. R.; Fox, B. G.; Münck, E.; Lipscomb, J. D. Biochemistry 1992, 31, 4602.

(23) Baldwin, J. E.; Bradley, M. Chem. Rev. 1990, 90, 1079.

(24) Baldwin, J. E.; Lynch, G. P.; Schofield, C. J. Tetrahedron 1992, 48, 9085.

(25) Ruettinger, R. T.; Griffith, G. R.; Coon, M. J. Arch. Biochem. Biophys. 1977, 183, 528.

(26) Katopodis, A. G.; Wimalasena, K.; Lee, J.; May, S. W. J. Am. Chem. Soc. 1984, 106, 7928.

(27) McKenna, E. J.; Coon, M. J. J. Biol. Chem. 1970, 245, 3882.

(28) May, S. W.; Gordon, S. L.; Steltenkamp, M. S. J. Am. Chem. Soc. 1977, 99, 2017.

(29) Lipscomb, J. D.; Orville, A. M. In Metals in Biological Systems; Sigel, H.; Sigel, A., Eds.; Marcel Dekker Inc.: New York, 1992; Vol. 28; p 243.

(30) Arciero, D. M.; Orville, A. M.; Lipscomb, J. D. J. Biol. Chem. 1985, 260, 14035.

(31) Ohlendorf, D. H.; Lipscomb, J. D.; Weber, P. C. Nature 1988, 336, 403.

(32) Earhart, C. A.; Radhakrishnan, R.; Orville, A. M.; Lipscomb, J. D.; Ohlendorf, D. H. J. Mol. Biol. 1994, 236, 374. 
(33) Lipscomb, J. D. personal communication, 1994.

(34) Jang, H. G.; Cox, D. D.; Que, L., Jr. J. Am. Chem. Soc. 1991, 113, 9200.

(35) Funabiki, T.; Konishi, T.; Kobayashi, S.; Mizoguchi, A.; Takano, M.; Yoshida, S. Chem. Lett. 1987, 719.

(36) Mabrouk, P. A.; Orville, A. M.; Lipscomb, J. D.; Solomon, E. I. J. Am. Chem. Soc. 1991, 113, 4053.

(37) Han, S.; Eltis, L.; Bolin, J. personal communication, 1994.

(38) Shiman, R. In Folates and Pterins: Chemistry and Biochemistry of Pterins; Blakley, R. L.; Benkovic, S. J., Eds.; John Wiley and Sons: New York, 1985; Vol. 2; 179.

(39) Crutcher, S. E.; Geary, P. J. Biochem. J. 1979, 177, 393.

(40) Yamaguchi, M.; Fujisawa, H. J. Biol. Chem. 1980, 255, 5058.

(41) Ensley, B. D.; Gibson, D. T. J. Bacteriol. 1983, 155, 505.

(42) Sauber, K.; Fröhner, C.; Rosenberg, G.; Eberspächer, J.; Lingens, F. Eur. J. Biochem. 1977, 74, 89.

(43) Gibson, D. T.; Yeh, W.-K.; Liu, T.-N.; Subramanian, V. In Oxygenases and Oxygen Metabolism; Nozaki, M.; Yamamoto, S.; Ishimura, Y.; Coon, M. J.; Ernster, L.; Estabrook, R. W., Eds.; Academic Press: New York, 1982; p 51.

(44) Batie, C. J.; Ballou, D. P.; Correll, C. C. Chem. Biochem. Flavoenzymes 1992, 3, 543.

(45) Markus, A.; Krekel, D.; Lingens, F. J. Biol. Chem. 1986, 261, 12883.

(46) Brunel, F.; Davison, J. J. Bacteriol. 1988, 170, 4924.

(47) Bernhardt, F.-H.; Heymann, E.; Traylor, P. S. Eur. J. Biochem. 1978, 92, 209.

(48) Kuila, D.; Fee, J. A.; Schoonover, J. R.; Woodruff, W. H.; Batie, C. J.; Ballou, D. P. J. Am. Chem. Soc. 1987, 109, 1559.

(49) Kuila, D.; Schoonover, J. R.; Dyer, R. B.; Batie, C. J.; Ballou, D. P.; Fee, J. A.; Woodruff, W. H. Biochim. Biophys. Acta 1992, 1140, 175.

(50) Gurbiel, R. J.; Batie, C. J.; Sivaraja, M.; True, A. E.; Fee, J. A.; Hoffman, B. M.; Ballou, D. P. Biochemistry 1989, 28, 4861.

(51) Cline, J. F.; Hoffman, B. M.; Mims, W. B.; LaHaie, E.; Ballou, D. P.; Fee, J. A. J. Biol. Chem. 1985, 260, 3251.

(52) .Tsang, H.-T.; Batie, C. J.; Ballou, D. P.; Penner-Hahn, J. E. Biochemistry 1989, $28,7233$.

(53) Penner-Hahn, J. E. Basic Life Sci., 1988, 51 (Synchr. Radiat. in Struct. Biology), 177. 
(54) Pavel, E. G.; Martins, L. J.; Ellis, W. R., Jr.; Solomon, E. I. Chemistry \& Biology $1994,1,173$.

(55) Dix, T. A.; Benkovic, S. J. Acc. Chem. Res. 1988, 21, 101.

(56) Kaufman, S. In Advances in Neurochemistry; Agranoff, B. W.; Aprison, M. H., Eds.; Plenum Press: New York, 1976; Vol. 2; p 1.

(57) Dix, T. A.; Bollag, G. E.; Domanico, P. L.; Benkovic, S. J. Biochemistry 1985, 24, 2955.

(58) McCracken, J.; Pember, S.; Benkovic, S. J.; Villafranca, J. J.; Miller, R. J.; Peisach, J. J. Am. Chem. Soc. 1988, 110, 1069.

(59) Perkinson, J.; Brodie, S.; Yoon, K.; Mosny, K.; Carroll, P. J.; Morgan, T. V.; Burgmayer, S. J. N. Inorg. Chem. 1991, 30, 719.

(60) Martínez, A.; Abeygunawardana, C.; Haavik, J.; Flatmark, T.; Mildvan, A. S. Biochemistry 1993, 32, 6381.

(61) Carr, R. T.; Benkovic, S. J. Biochemistry 1993, 32, 14132.

(62) Bloom, L. M.; Benkovic, S. J.; Gaffney, B. J. Biochemistry 1986, 25, 4204.

(63) Wallick, D. E.; Bloom, L. M.; Gaffney, B. J.; Benkovic, S. J. Biochemistry 1984, $23,1295$.

(64) Caradonna, J. P. personal communication, 1994.

(65) Salowe, S. P.; Marsh, E. N.; Townsend, C. A. Biochemistry 1990, 29, 6499.

(66) Kivirikko, K. I.; Myllylä, R. In The Enzymology of Post-Translational Modifications of Proteins; Freedman, R. B.; Hawkins, H. C., Eds.; Academic Press: London, 1980; Vol. 1; p 53.

(67) Thomburg, L. D.; Lai, M.-T.; Wishnok, J. S.; Stubbe, J. Biochemistry 1993, 32, 14023.

(68) Lindstedt, S.; Odelhög, B.; Rundgren, M. Biochemistry 1977, 16, 3369.

(69) Baldwin, J. E.; Lloyd, M. D.; Wha-Son, B.; Schofield, C. J.; Elson, S. W.; Baggaley, K. H.; Nicholson, N. H. J. Chem. Soc., Chem. Commun. 1993, 500.

(70) Salowe, S. P.; Krol, W. J.; Iwata-Reuyl, D.; Townsend, C. A. Biochemistry 1991, $30,2281$.

(71) Chiou, Y.-M.; Que, L., Jr. J. Am. Chem. Soc. 1992, 114, 7567.

(72) Siegel, B. Bioorg. Chem. 1979, 8, 219.

(73) Samuelsson, B.; Dahlén, S.-E.; Lindgren, J. Å; Rouzer, C. A.; Serhan, C. N. Science 1987, 237, 1171.

(74) Kühn, H.; Barnett, J.; Grunberger, D.; Baeker, P.; Chow, J.; Nguyen, B.; Bursztyn-Pettegrew, H.; Chan, H.; Sigal, E. Biochim. Biophys. Acta 1993, 1169 , 80 . 
(75) Feiters, M. C.; Aasa, R.; Malmström, B. G.; Slappendel, S.; Veldink, G. A.; Vliegenthart, J. F. G. Biochim. Biophys. Acta 1985, 831, 302.

(76) Corey, J.; Nagata, R. J. Am. Chem. Soc. 1987, 109, 8107.

(77) Veldink, G. A.; Vliegenthart, J. F. G. Adv. Inorg. Biochem. 1984, 6, 139.

(78) Nelson, M. J.; Cowling, R. A.; Seitz, S. P. Biochemistry 1994, 33, 4966.

(79) Nelson, M. J.; Batt, D. G.; Thompson, J. S.; Wright, S. W. J. Biol. Chem. 1991, 266,8225 .

(80) Dunham, W. R.; Carroll, R. T.; Thompson, J. F.; Sands, R. H.; Funk, M. O., Jr. Eur. J. Biochem. 1990, 190, 611.

(81) Van der Heijdt, L. M.; Feiters, M. C.; Navaratnam, S.; Nolting, H.-F.; Hermes, C.; Veldink, G. A.; Vliegenthart, J. F. G. Eur. J. Biochem. 1992, 207, 793.

(82) Boyington, J. C.; Gaffney, B. J.; Amzel, L. M. Science 1993, $260,1482$.

(83) Minor, W.; Steczko, J.; Bolin, J. T.; Otwinowski, Z.; Axelrod, B. Biochemistry $1993,32,6320$.

(84) Nelson, M. J. J. Am. Chem. Soc. 1988, 110, 2985.

(85) Bleomycins: Current Status and New Developments; Carter, S. K.; Crooke, S. T.; Umezawa, H., Eds.; Academic Press, Inc.: New York, 1978.

(86) Stubbe, J.; Kozarich, J. W. Chem. Rev. 1987, 87, 1107.

(87) Petering, D. H.; Bymes, R. W.; Antholine, W. E. Chem.-Biol. Interactions 1990 , $73,133$.

(88) Dabrowiak, J. C. In Metal Ions in Biological Systems; Sigel, H., Ed.; Marcel Dekker, Inc.: New York, 1980; Vol. 11; p 305.

(89) Iitaka, Y.; Nakamura, H.; Nakatani, T.; Muraoka, Y.; Fujii, A.; Takita, T.; Umezawa, H. J. Antibiot. 1978, 31, 1070.

(90) Akkerman, M. A. J.; Neijman, E. W. J. F.; Wijmenga, S. S.; Hilbers, C. W.; Bermel, W. J. Am. Chem. Soc. 1990, 112, 7462.

(91) Sugiura, Y. J. Am. Chem. Soc. 1980, 102, 5208.

(92) Hamamichi, N.; Natrajan, A.; Hecht, S. M. J. Am. Chem. Soc. 1992, 114, 6278.

(93) Sakai, T. T.; Riordan, J. M.; Glickson, J. D. Biochim. Biophys. Acta 1983, 758, 176.

(94) Povirk, L. F.; Hogan, M.; Dattagupta, N. Biochemistry 1979, $18,96$.

(95) Burger, R. M.; Peisach, J.; Horwitz, S. B. J. Biol. Chem. 1981, 256, 11636.

(96) Burger, R. M.; Horwitz, S. B.; Pẹisach, J.; Wittenberg, J. B. J. Biol. Chem. 1979, $254,12299$. 


\section{2. $\{\text { FeNO }\}^{7}$ Complexes}

\subsubsection{Introduction}

As described in the previous section, mononuclear non-heme iron centers are present in the catalytic cycle of a number of proteins involved in oxygen activation. ${ }^{1,2}$ In order to understand the reactivity of these systems it is necessary to have a detailed description of the electronic as well as geometric structure of the non-heme iron active site. $^{3}$ Since most of these systems are involved in oxygen activation, identification of oxygen intermediates during the catalytic cycle is necessary to obtain insight into reactivity at the molecular level. These intermediates, however, are often very short-lived and difficult to study experimentally.

As a probe of non-heme iron active sites, nitric oxide has been shown to react with the ferrous state of many mononuclear non-heme iron enzymes, e.g. soybean lipoxygenase, ${ }^{4,5}$ protocatechuate 4,5 -dioxygenase, ${ }^{6}$ metapyrocatechase, ${ }^{7}$ putidamonooxin, ${ }^{8}$ and isopenicillin $\mathrm{N}$ synthase, ${ }^{9}$ to form stable nitrosyl complexes which can serve as reversible analogs of the possible dioxygen intermediates involved in catalysis. These non-heme iron enzyme nitrosyl derivatives have a distinctive $S=3 / 2$ ground state. This distinctive $S=3 / 2$ electron paramagnetic resonance (EPR) signal allows one to probe non-heme ferrous complexes which are normally EPR silent. For intra- and extradiol dioxygenases Fe-NO EPR has been used as an analytical probe of substrate and water coordination through superhyperfine effects on the spectrum., ${ }^{6,7}$ Mössbauer spectroscopy has also proved to be a very useful analytical probe, ${ }^{10,11}$ but conflicting descriptions of the electronic origin of the $S=3 / 2$ ground state have been suggested. The Mössbauer isomer shift for the NO complex of protocatechuate 4,5-dioxygenase has been used to argue against a ferric configuration, ${ }^{12}$ while the equivalent isomer shift for the substrate-NO ternary complex of putidamonooxin has been used to support its description as an intermediate spin ferric site. ${ }^{8}$

The description of metal nitrosyl complexes is a problem of both theoretical and experimental interest. An early classic review by Enemark and Feltham gives a basic description of metal-nitrosyl complexes and their bonding. ${ }^{13}$ In the Enemark/Feltham notation, the metal nitrosyl unit is described as a discrete unit, i.e. (MNO) n, where $n$ represents the sum of the metal $d$ and NO $\pi^{*}$ electrons. ${ }^{13}$ The Fe-NO species generated in non-heme ferrous enzymes is thus designated $\{\mathrm{FeNO}\}^{7}$. A variety of descriptions of the electronic structure of these complexes have been put forth in the literature. These

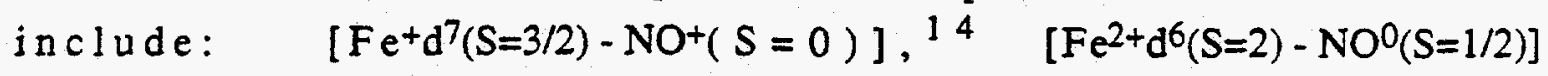


antiferromagnetically coupled, ${ }^{15} \quad\left[\mathrm{Fe}^{3}+\mathrm{d}^{5}(\mathrm{~S}=1 / 2)-\mathrm{NO}-(\mathrm{S}=1)\right]$ ferromagnetically coupled, ${ }^{16}$ and $\left[\mathrm{Fe}^{3+} \mathrm{d}^{5}(\mathrm{~S}=3 / 2)-\mathrm{NO}-(\mathrm{S}=0)\right] .{ }^{11}$ None of these descriptions, however, are consistent with all of the experimental and theoretical data that exist.

In this study, the XAS edge spectra of three $S=3 / 2$ \{FeNO $\}^{7}$ model complexes, FeEDTA-NO, Fe(salen)NO and $[\mathrm{Fe}(\mathrm{TMC}) \mathrm{NO}]\left(\mathrm{BF}_{4}\right)_{2},{ }^{17}$ were compared to spectra of ferrous and ferric complexes with similar ligation to elucidate the oxidation state of the iron in the $[\mathrm{FeNO}]^{7}$ systems. In addition, an analysis of the intensity of the pre-edge features provided information on the distortion of the iron site. The results of this XAS study were combined with results from absorption (abs), variable-temperature, variable-field magnetic circular dichroism (MCD), resonance Raman ( $r R), E P R$, and magnetism measurements (performed by Yan Zhang and Mark Pavlosky) and self consistent field-X $\alpha$-scattered wave (SCF-X $\alpha-S W$ ) theoretical calculations (performed by Carl Brown) to develop a detailed description of the distinctive $S=3 / 2\{\mathrm{FeNO}\}^{7}$ site. $^{18,19}$ These studies indicate that the iron is best described as high spin ferric $(S=5 / 2)$ and the NO as NO- $(S=1)$ which are spin coupled antiferromagnetically to produce the correct total spin state of $S=3 / 2$.

\subsubsection{Experimental Section}

The X-ray absorption spectra were recorded at the Stanford Synchrotron Radiation Laboratory on unfocused beamline 7-3 during dedicated conditions ( $3 \mathrm{GeV}$, $25-95 \mathrm{~mA}$ ). The radiation was monochromatized using a Si(220) double crystal monochromator detuned $50 \%$ at $7474 \mathrm{eV}$ to minimize harmonic contamination. An Oxford Instruments continuous-flow liquid helium CF1208 cryostat was used to maintain a constant temperature. Data were measured to $k=9.5 \AA^{-1}$ with $1 \mathrm{~mm}$ high pre-monochromator beam defining slits. Energies were calibrated using an internal $\mathrm{Fe}$ foil standard, assigning the first inflection point to $7111.2 \mathrm{eV} .{ }^{20}$ The spectrometer energy resolution was approximately $1.4 \mathrm{eV}^{21}$ with reproducibility of edge position determination of $<0.2 \mathrm{eV}$.

$\left.[\mathrm{Fe}(\mathrm{TMC}) \mathrm{NO}]\left(\mathrm{BF}_{4}\right)_{2},{ }^{22} \mathrm{Fe}(\mathrm{TMC}) \mathrm{N}_{3}\right]\left(\mathrm{BF}_{4}\right),{ }^{23} \mathrm{Fe}$ (salen) $\mathrm{NO}^{24}$ and $\mathrm{Fe}$ (salen)Cl ${ }^{25}$ were prepared as described in the literature. With the exception of $\mathrm{Fe}($ salen $) \mathrm{Cl}$, all samples were prepared in an inert atmosphere, i.e. in an anaerobic wet or dry box or on a Schlenk line and handled in a nitrogen-filled inert atmosphere dry glove box during the following sample preparation. [Fe(TMC)NO](BF $)_{4}$, $\left[\mathrm{Fe}(\mathrm{TMC}) \mathrm{N}_{3}\right]\left(\mathrm{BF}_{4}\right), \mathrm{Fe}$ (salen)NO and $\mathrm{Fe}$ (salen) $\mathrm{Cl}$ crystalline samples were mixed with $\mathrm{BN}$ and ground into a fine powder. The $\mathrm{BN} / \mathrm{sample}$ mixture was pressed into a $1 \mathrm{~mm}$ thick $\mathrm{Al}$ spacer that was sealed with 
63.5 $\mu \mathrm{m}$ Mylar tape windows and frozen in liquid nitrogen. Data were measured in transmission mode with $\mathrm{N}_{2}$-filled ionization chambers. Data were measured at $10 \mathrm{~K}$ for $\mathrm{Fe}$ (salen) $\mathrm{Cl}$, at room temperature for $\left[\mathrm{Fe}(\mathrm{TMC}) \mathrm{N}_{3}\right]\left(\mathrm{BF}_{4}\right)$ and at $220 \mathrm{~K}$ for the $\mathrm{S}=3 / 2$ forms of $\mathrm{Fe}($ salen $) \mathrm{NO}$ and $[\mathrm{Fe}(\mathrm{TMC}) \mathrm{NO}]\left(\mathrm{BF}_{4}\right)_{2}$.

[Fe $\left(\mathrm{OH}_{2}\right)$ EDTA] 2 - solution, $50 \mathrm{mM}$ in $\mathrm{Fe}$, was prepared by anaerobic addition of ferrous ammonium sulfate to a $60 \mathrm{mM}$ solution of $\mathrm{Na}_{2} \mathrm{EDTA}$ in $\mathrm{pH}=6.5,0.1 \mathrm{M}$ deoxygenated phosphate buffer. Oxidizing this solution produced [Fe( $\left.\mathrm{OH}_{2}\right)$ EDTA]- An FeEDTA-NO solution was prepared by purging an [ $\left.\mathrm{Fe}\left(\mathrm{OH}_{2}\right) \mathrm{EDTA}\right]^{2-}$ solution with NO gas under anaerobic conditions for approximately 20 minutes, or until the solution stopped changing color, indicating completion of the reaction. To form an ice crystal-free glass, solution samples were prepared by adding 50\% (by volume) glycerol resulting in samples which were $25 \mathrm{mM}$ in $\mathrm{Fe}$. [Fe( $\left(\mathrm{OH}_{2}\right)$ EDTA] ${ }^{2-},\left[\mathrm{Fe}\left(\mathrm{OH}_{2}\right)\right.$ EDTA] and FeEDTA-NO solution samples were loaded into $140 \mu \mathrm{L}$ Lucite EXAFS cells $(23 \times 2 \times 3 \mathrm{~mm})$ with $37 \mu \mathrm{m}$ Kapton windows and frozen in liquid nitrogen. Data were collected in fluorescence mode at $10 \mathrm{~K}$. The fluorescence signal was collected by an Ar-filled ionization chamber, ${ }^{26,27}$ equipped with Soller slits and a Mn filter.

Five to nine scans were averaged for each EDTA solution while two scans were averaged for each powder sample. A smooth pre-edge background was removed from the averaged spectra by fitting a first or second orcier polynomial to the pre-edge region and subtracting this polynomial from the entire spectrum. A one-segment spline of order one was fit to the EXAFS region and the data normalized to an edge jump of one at $7130 \mathrm{eV}$. The intensities and energies of pre-edge features were quantitated by fits to the data. The fitting program EDG_FIT, which utilizes the double precision version of the public domain MINPAK fitting library ${ }^{28}$ was used. EDG_FIT was written by Dr. Graham N. George of the Stanford Synchrotron Radiation Laboratory. All spectra were fit over the range 7108-7118 eV. Pre-edge features were modeled by pseudo-Voigt line shapes (simple sums of Lorentzian and Gaussian functions). ${ }^{21,27,29,30}$ A fixed 50:50 ratio of Lorentzian to Gaussian contribution for the pre-edge feature successfully reproduced these spectral features. The value reported for the area of a fitted feature was calculated by multiplying the height of the feature by the full-width-at-half-maximum (FWHM).

\subsubsection{Results and Analysis}

To determine the oxidation state of the iron in $\{\mathrm{FeNO}\}^{7}$ systems the $\mathrm{Fe} \mathrm{K}$ edge $\mathrm{X}$-ray absorption spectra of $\mathrm{S}=3 / 2$ \{FeNO $\}^{7}$ complexes were compared to the spectra of ferrous and ferric complexes with similar ligation. XAS edge data were collected on 
three $S=3 / 2 \quad\{\mathrm{FeNO}\}^{7}$ complexes: FeEDTA-NO, [Fe(TMC)NO] $\left(\mathrm{BF}_{4}\right)_{2}$, and Fe(salen)NO. The FeEDTA-NO spectrum was compared with spectra of ferrous and ferric complexes of EDTA, [Fe $\left(\mathrm{OH}_{2}\right)$ EDTA] ${ }^{2-}$ and $\left[\mathrm{Fe}\left(\mathrm{OH}_{2}\right) \mathrm{EDTA}\right]$. [Fe(TMC)NO] $\left(\mathrm{BF}_{4}\right)_{2}$ data were compared to those for the ferrous complex $\left[\mathrm{Fe}(\mathrm{TMC}) \mathrm{N}_{3}\right]\left(\mathrm{BF}_{4}\right)$, and $\mathrm{Fe}$ (salen)NO data to those for the ferric complex $\mathrm{Fe}$ (salen)Cl. The XAS edge spectra are shown in Figure 5.1 for $\left[\mathrm{Fe}\left(\mathrm{OH}_{2}\right) \mathrm{EDTA}\right]^{2-}$, $\left[\mathrm{Fe}\left(\mathrm{OH}_{2}\right)\right.$ EDTA]and FeEDTA-NO, in Figure 5.2 for [Fe(TMC)NO](BF $\left.)_{4}\right)_{2}$ and $\left[\mathrm{Fe}(\mathrm{TMC}) \mathrm{N}_{3}\right]\left(\mathrm{BF}_{4}\right)$, and in Figure 5.3 for $\mathrm{Fe}$ (salen)NO and $\mathrm{Fe}$ (salen)Cl. The lowest energy transitions are the weak $1 \mathrm{~s} \longrightarrow 3 \mathrm{~d}$ pre-edge peaks at approximately $7112 \mathrm{eV}$ followed by the $1 \mathrm{~s} \rightarrow 4 \mathrm{p}$ transition at approximately $7123 \mathrm{eV}$. The $1 \mathrm{~s} \longrightarrow 3 \mathrm{~d}$ pre-edge region has been expanded and is shown as insets in Figures 5.1, 5.2 and 5.3. The energies and areas of the pre-edge features were determined by fits to the data and are presented in Table 5.2. The spectrum of $\left[\mathrm{Fe}\left(\mathrm{OH}_{2}\right) \mathrm{EDTA}\right]^{2-}$ has two low intensity pre-edge peaks that are split by $\sim 2 \mathrm{eV}$ (solid line in Figure 5.1 inset). The $\left[\mathrm{Fe}(\mathrm{TMC}) \mathrm{N}_{3}\right]\left(\mathrm{BF}_{4}\right)$ data contain an intense feature at $7111.5 \mathrm{eV}$ with the second derivative of the data (not shown) clearly showing another feature at $7113.3 \mathrm{eV}$ (solid line in Figure 5.2 inset). [Fe( $\left(\mathrm{OH}_{2}\right) \mathrm{EDTA}$-, FeEDTA-NO, $[\mathrm{Fe}(\mathrm{TMC}) \mathrm{NO}]\left(\mathrm{BF}_{4}\right)_{2}, \mathrm{Fe}(\mathrm{salen}) \mathrm{Cl}$ and $\mathrm{Fe}($ salen) $\mathrm{NO}$ all have a features whose maximum lie between 7112.6 and $7113.0 \mathrm{eV}$ (Figure 5.1, 5.2, and 5.3 insets). The pre-edge features of FeEDTA-NO and Fe(salen)NO are more intense than the pre-edge features of $\left[\mathrm{Fe}\left(\mathrm{OH}_{2}\right) \mathrm{EDTA}\right]^{-}$and $\mathrm{Fe}$ (salen)Cl (Figure 5.1 and 5.3 insets), respectively.

The $1 \mathrm{~s} \rightarrow 3 \mathrm{~d}$ pre-edge feature can be used to probe the iron oxidation state in $\{\mathrm{FeNO}\}^{7}$ systems. The $1 \mathrm{~s} \rightarrow 3 \mathrm{~d}$ transition is formally electric dipole forbidden, but gains intensity through an allowed quadrupole transition and more dominantly by $4 p$ mixing into the $3 \mathrm{~d}$ states as a result of the noncentrosymmetric environment of the metal site. Because the final state $\left(1 \mathrm{~s}^{1} 3 \mathrm{~d}^{\mathrm{n}+1}\right)$ has a different electronic configuration for high spin ferric and high spin ferrous ions, the $1 \mathrm{~s} \rightarrow 3 \mathrm{~d}$ feature is sensitive to the oxidation state of the iron site. In the weak field limit, two final states of maximum spin multiplicity can be reached in the $\mathrm{d}^{7}$ high spin ferrous case, ${ }^{4} \mathrm{~F}$ and ${ }^{4} \mathrm{P}$, while only one state of maximum spin multiplicity can be reached in the $d^{6}$ high spin ferric case, ${ }^{5 D} .^{31}$ The free ion splitting of the ${ }^{4} \mathrm{~F}$ and ${ }^{4} \mathrm{P}$ is $2 \mathrm{eV}^{32}$ and is readily resolvable at the $\mathrm{Fe}$ $\mathrm{K}$-edge. However, when the iron atom is placed in an octahedral ligand field the splitting of the $d$ orbitals into the $t_{2}$ and $e$ set causes the free ion terms to split into many-electron states. In the ferrous case, the ${ }^{4} \mathrm{~F}$ term splits into ${ }^{4} \mathrm{~T}_{1},{ }^{4} \mathrm{~T}_{2}$, and ${ }^{4} \mathrm{~A}_{2}$ many-electron states, while the ${ }^{4} \mathrm{P}$ term turns into a ${ }^{4} \mathrm{~T}_{1}$ state. ${ }^{33}$ The ${ }^{4} \mathrm{~A}_{2}$ many-electron state will not be allowed as it would require a two-electron transition (see Chapter 4). In a relatively weak field, the splitting of the three states is determined mainly by electron repulsion ( $v s$. 
ligand field) and the pre-edge feature that arises from transitions into the ${ }^{4} \mathrm{~T}_{1}$ and ${ }^{4} \mathrm{~T}_{2}$ states from the ${ }^{4} \mathrm{~F}$ term will lie $-2 \mathrm{eV}$ lower in energy than that from a transition into the ${ }^{4} \mathrm{~T}_{1}$ state from the ${ }^{4} \mathrm{P}$ term. In the hi gh spin ferric case, in an octahedral ligand field the ${ }^{5 \mathrm{D}}$ term splits into the ${ }^{5} \mathrm{~T}_{2}$ and ${ }^{5 \mathrm{E}}$ states. ${ }^{33}$ Thus, an octahedral high spin ferric complex has two pre-edge features from transitions into the ${ }^{5} \mathrm{~T}_{2}$ and ${ }^{5} \mathrm{E}$ states split by $10 \mathrm{Dq}$.

The splitting of the pre-edge in $\left[\mathrm{Fe}\left(\mathrm{OH}_{2}\right) \text { EDTA] }\right]^{2-}$ is attributed to the splitting of the ${ }^{4} \mathrm{~T}_{1}$ and ${ }^{4} \mathrm{~T}_{2}$ states from the ${ }^{4} \mathrm{~F}$ term and the ${ }^{4} \mathrm{~T}_{1}$ state from the ${ }^{4} \mathrm{P}$ term (Figure 5.1 and Table 5.2). The two pre-edge features in the spectrum of $\left[\mathrm{Fe}\left(\mathrm{OH}_{2}\right) \mathrm{EDTA}\right]^{2-}$ have an intensity ratio of $\sim 2: 1$ (Table 5.2) as would be expected since the lower energy feature arises from transitions into two triplet states, while the higher energy feature arises from a transition into one triplet state. The total area of the $\left[\mathrm{Fe}\left(\mathrm{OH}_{2}\right) \mathrm{EDTA}\right]^{2-}$ pre-edge feature is higher than that observed for octahedral high spin ferrous complexes (Table 4.1, Chapter 4) due to distortions of the iron site from variation in ligation and bond distances. The pre-edge region of $\left[\mathrm{Fe}\left(\mathrm{OH}_{2}\right) \text { EDTA] }\right]^{-}$was fit with two features at 7112.8 and $7113.8 \mathrm{eV}$ (Figure 5.1 and Table 5.2). As in the ferrous case, the total area of the $\left[\mathrm{Fe}\left(\mathrm{OH}_{2}\right)\right.$ EDTA] pre-edge feature is higher than that observed for octahedral high spin ferric complexes (Table 4.1, Chapter 4) due distortions of the iron site from variation in ligation and bond distances. The pre-edge feature of FeEDTA-NO was fit with a single feature at $7112.8 \mathrm{eV}$ (Figure 5.1 and Table 5.2). This feature is positioned at approximately the same energy as that for [Fe( $\left.\left.\mathrm{OH}_{2}\right) \mathrm{EDTA}\right]^{-}$, however, the pre-edge feature of FeEDTA-NO is more intense than that of [Fe( $\left(\mathrm{OH}_{2}\right)$ EDTA]- One does not observe the two features at -7111.5 and $7113.5 \mathrm{eV}$ in the spectrum of FeEDTA-NO that are characteristic of high spin ferrous complexes (Table 4.1, Chapter 4).

The pre-edge region of the spectrum [Fe(TMC) $\left.\mathrm{N}_{3}\right]\left(\mathrm{BF}_{4}\right)$ can be fit with an intense feature at $7111.5 \mathrm{eV}$ and a very weak feature at $7113.3 \mathrm{eV}$ (Figure 5.2 and Table 5.2). Since the high spin ferrous atom in $\left[\mathrm{Fe}(\mathrm{TMC}) \mathrm{N}_{3}\right]\left(\mathrm{BF}_{4}\right)$ is in a noncentrosymmetric site, $4 \mathrm{p}$ mixing into the $3 \mathrm{~d}$ orbitals is allowed. As explained in detail in Chapter 4 , the pre-edge feature for a high spin ferrous complex in a square pyramidal geometry has a lower energy, intense peak due to $4 \mathrm{p}_{\mathrm{z}}$ character mixing into the $3 \mathrm{~d}_{\mathrm{z}} 2$ orbital with a weak peak $\sim 2 \mathrm{eV}$ higher in energy (see Figure 4.7 in Chapter 4). The pre-edge region for [Fe(TMC)NO] $\left(\mathrm{BF}_{4}\right)_{2}$ can also be fit with two features, however, the most intense feature is the higher energy feature which is $-1 \mathrm{eV}$ higher in energy than that for $\left[\mathrm{Fe}\left(\mathrm{TMC}_{\mathrm{N}} \mathrm{N}_{3}\right]\left(\mathrm{BF}_{4}\right)\right.$. The energy of the intense pre-edge feature for $[\mathrm{Fe}(\mathrm{TMC}) \mathrm{NO}]\left(\mathrm{BF}_{4}\right)_{2}$ is much more similar to that of high spin ferric complexes (Table 4.1, Chapter 4). In addition to the intense feature at $7112.6 \mathrm{eV}$, the pre-edge region for $[\mathrm{Fe}(\mathrm{TMC}) \mathrm{NO}]\left(\mathrm{BF}_{4}\right)_{2}$ was fit with a weak feature at $7111.5 \mathrm{eV}$. This feature can be explained if the iron is in a 


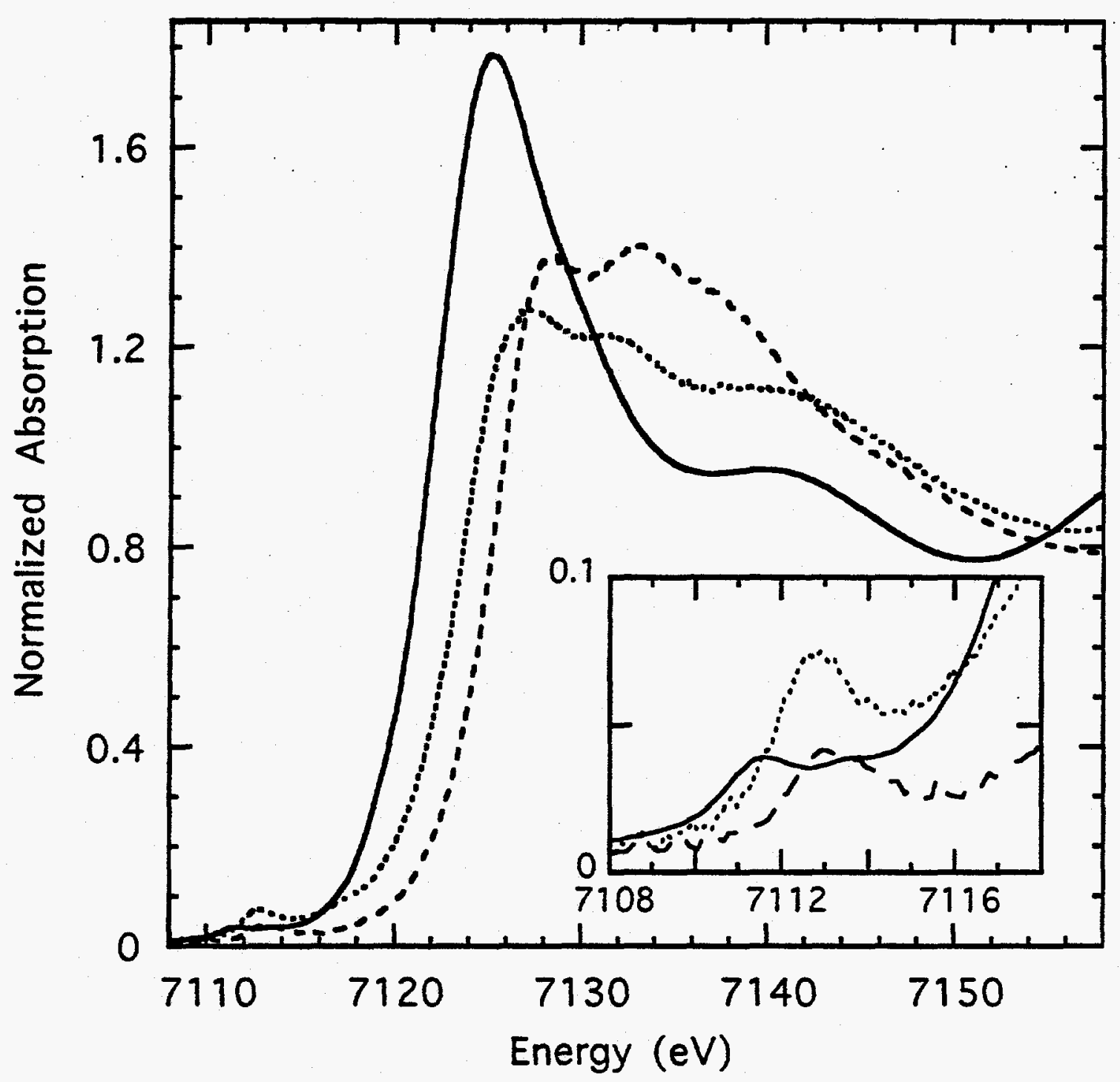

Figure 5.1. XAS Fe K-edge spectra of $\left[\mathrm{Fe}\left(\mathrm{OH}_{2}\right) \mathrm{EDTA}\right]^{2-}(-),\left[\mathrm{Fe}\left(\mathrm{OH}_{2}\right) \mathrm{EDTA}\right]^{-}(---)$, and FeEDTA-NO (....). The inset shows an expansion of the $1 \mathrm{~s} \rightarrow 3 \mathrm{~d}$ pre-edge region. 


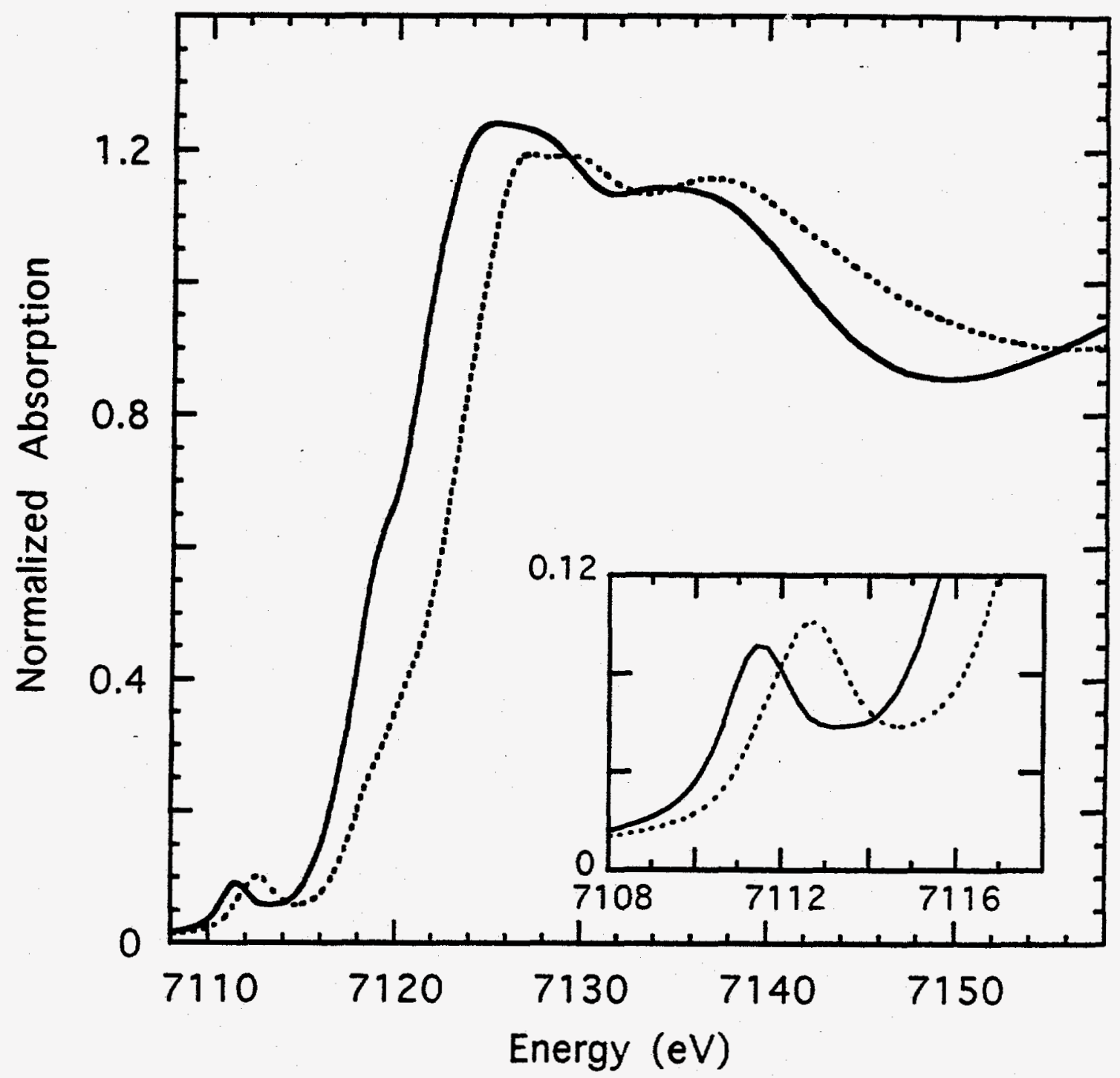

Figure 5.2. XAS Fe K-edge spectra of $\left[\mathrm{Fe}\left(\mathrm{TMC}^{\mathrm{N}} \mathrm{N}_{3}\right]\left(\mathrm{BF}_{4}\right)(-)\right.$ and $[\mathrm{Fe}(\mathrm{TMC}) \mathrm{NO}]\left(\mathrm{BF}_{4}\right)_{2}(\cdots \cdot \cdots)$. The inset shows an expansion of the $1 \mathrm{~s} \rightarrow 3 \mathrm{~d}$ pre-edge region. 


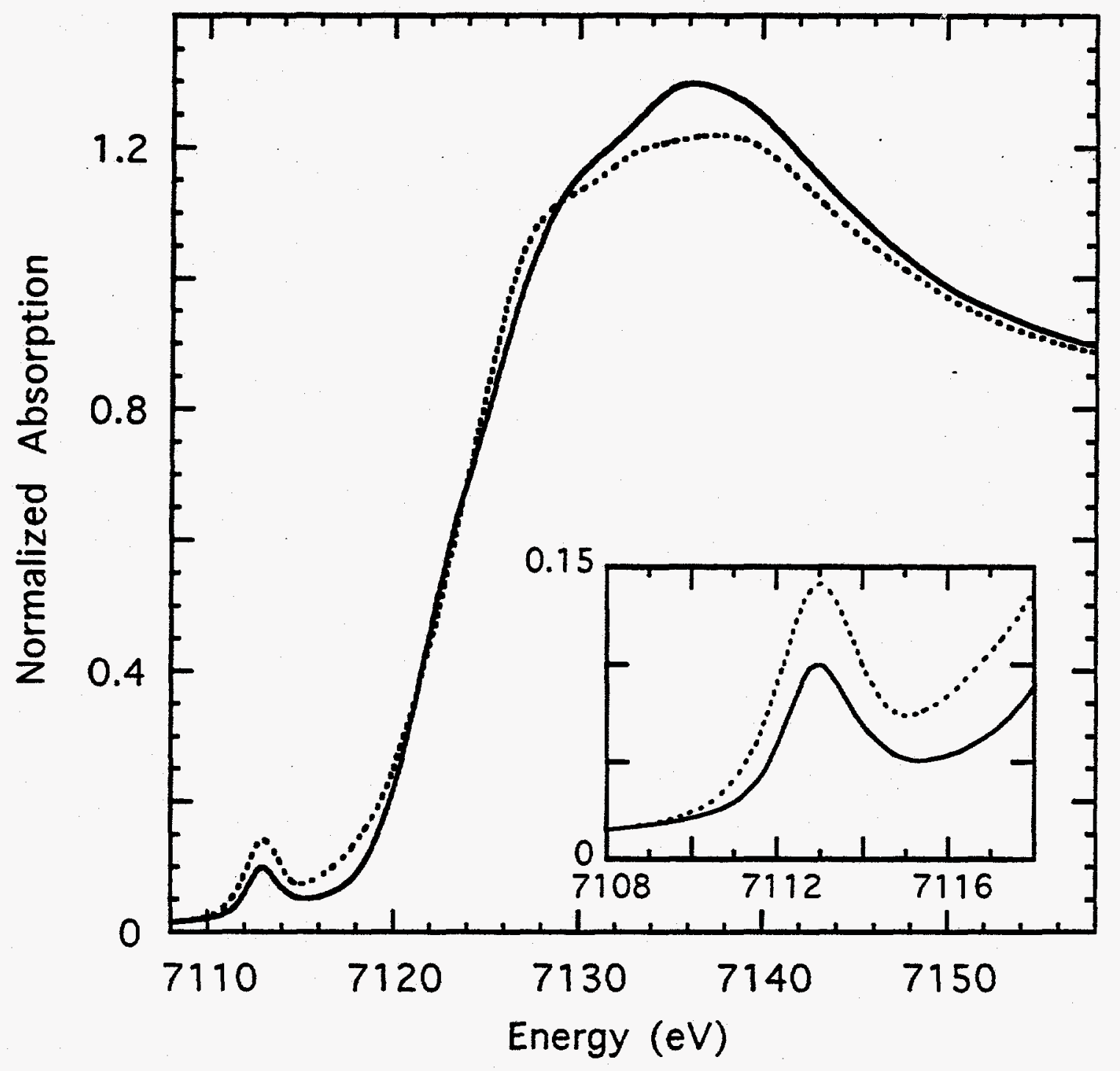

Figure 5.3. XAS Fe K-edge spectra of $\mathrm{Fe}$ (salen) $\mathrm{Cl}(\longrightarrow$ ) and $\mathrm{Fe}$ (salen)NO (.....). The inset shows an expansion of the $1 \mathrm{~s} \longrightarrow 3 \mathrm{~d}$ pre-edge region. 
Table 5.2. XAS Pre-Edge Energies and Areas for $[\mathrm{FeNO}]^{7}$ and Related Complexes.

\begin{tabular}{|c|c|c|c|c|c|}
\hline compound & $\begin{array}{l}\text { Fe oxida- } \\
\text { tion state }\end{array}$ & $\begin{array}{l}\mathrm{Fe}-\mathrm{N}-\mathrm{O} \\
\text { angle }\end{array}$ & $\begin{array}{l}\text { pre-edge } \\
\text { energy }(\mathrm{eV})^{a}\end{array}$ & $\begin{array}{l}\text { pre-edge } \\
\text { area } a, b\end{array}$ & $\begin{array}{l}\text { total } \\
\text { pre-edge } \\
\text { area } b\end{array}$ \\
\hline$\left[\mathrm{Fe}\left(\mathrm{H}_{2} \mathrm{O}\right) \mathrm{EDTA}\right]^{2-}$ & +2 & $\cdots$ & $\begin{array}{l}7111.48(0.02) \\
7113.45(0.04)\end{array}$ & $\begin{array}{l}3.9(0.2) \\
1.8(0.05)\end{array}$ & $5.7(0.5)$ \\
\hline$\left[\mathrm{Fe}\left(\mathrm{H}_{2} \mathrm{O}\right)\right.$ EDTA] & +3 & -- & $\begin{array}{l}7112.82(0.03) \\
7113.81(0.07)\end{array}$ & $\begin{array}{l}2.9(0.4) \\
4.1(0.5)\end{array}$ & $7.0 .(0.2)$ \\
\hline $\begin{array}{l}\text { FeEDTA-NO } \\
{\left[\mathrm{Fe}(\mathrm{TMC}) \mathrm{N}_{3}\right]\left(\mathrm{BF}_{4}\right)}\end{array}$ & $\overline{+-}$ & $156^{\circ} \mathrm{C}$ & $\begin{array}{l}7112.75(0.02) \\
7111.47(0.01) \\
7113.30(0.05)\end{array}$ & $\begin{array}{l}10.4(0.4) \\
12.4(0.5) \\
1.0(0.2)\end{array}$ & $\begin{array}{l}10.4(0.4) \\
13.4(0.3)\end{array}$ \\
\hline$[\mathrm{Fe}(\mathrm{TMC}) \mathrm{NO}]\left(\mathrm{BF}_{4}\right)_{2}$ & - & $178^{\circ}$ & $\begin{array}{l}7111.52(0.05) \\
7112.64(0.02)\end{array}$ & $\begin{array}{l}1.1(0.4) \\
14.3(1.4)\end{array}$ & $15.5(1.1)$ \\
\hline $\mathrm{Fe}($ salen $) \mathrm{Cl}$ & +3 & $\cdots$ & $\begin{array}{l}7112.91(0.01) \\
7114.25(0.07)\end{array}$ & $\begin{array}{l}12.9(0.3) \\
1.5(0.4)\end{array}$ & $14.4(0.6)$ \\
\hline $\mathrm{Fe}($ salen)NO & $\ldots$ & $149^{\circ}$ & $7112.96(0.01)$ & $24.2(0.6)$ & $24.2(0.6)$ \\
\hline
\end{tabular}


ferric oxidation state and the increased electronic repulsion from the short $\mathrm{Fe}-\mathrm{N}(\mathrm{O})$ bond increases the energy of the $d_{z} 2$ orbital so that it is no longer the lowest $3 d$ orbital in energy. Hence, the weak feature at $7111.5 \mathrm{eV}$ could arise from transitions into $d_{x y}$ or $d_{x z}, d_{x z}$ (see Scheme 6 in Chapter 4). The pre-edge feature of Fe(salen)Cl can be fit with an intense feature at 7112.91 and a weaker feature at $7114.25 \mathrm{eV}$ (see Figure 4.13), while that of $\mathrm{Fe}(\mathrm{salen}) \mathrm{NO}$ can be fit with at single more intense feature at $7112.96 \mathrm{eV}$ (Figure 5.3 and Table 5.2). From the energies of the pre-edge features, the iron in $\{\mathrm{FeNO}\}^{7}$ appears to be in the ferric oxidation state as the pre-edge feature of FeEDTA-NO is much more similar in shape and in energy to that of $\left[\mathrm{Fe}\left(\mathrm{OH}_{2}\right) E D T A\right]^{-}$than of $\left[\mathrm{Fe}\left(\mathrm{OH}_{2}\right) \mathrm{EDTA}\right]^{2-}$. In addition, the pre-edge feature of $[\mathrm{Fe}(\mathrm{TMC}) \mathrm{NO}]\left(\mathrm{BF}_{4}\right)_{2}$ and of $\mathrm{Fe}$ (salen)NO are much more similar to that of the ferric square pyramidal complex, $\mathrm{Fe}$ (salen)NO, than to that of the ferrous square pyramidal complex, $\left[\mathrm{Fe}(\mathrm{TMC}) \mathrm{N}_{3}\right]\left(\mathrm{BF}_{4}\right)$.

The energy of the edge position ( $1 s \longrightarrow 4 p$ transition) is dependent upon the effective nuclear charge of the absorbing metal atom. The effective nuclear charge of the metal ion is governed by a combination of effects including the formal oxidation state of the metal, the number and type of ligating atoms and the coordination geometry. ${ }^{32,34-38}$ Therefore, the oxidation state of a metal atom cannot be determined by the edge position without careful consideration of ligation effects on the effective nuclear charge of the metal atom. In the three cases in this study, differences between the types of ligating atoms and the coordination spheres are minimized so that changes in the edge energy can be considered to be strongly related to the oxidation state of the iron (Figure 5.1, 5.2, and 5.3). The energy of the FeEDTA-NO edge lies between that of the reduced and oxidized iron-EDTA edges (Figure 5.1). However, the FeEDTA-NO spectrum is similar in intensity and shape to the $\left[\mathrm{Fe}\left(\mathrm{OH}_{2}\right) \mathrm{EDTA}\right]-$ spectrum. The edge of $[\mathrm{Fe}(\mathrm{TMC}) \mathrm{NO}]\left(\mathrm{BF}_{4}\right)_{2}$ is shifted to higher energy than the edge of $\left[\mathrm{Fe}\left(\mathrm{TMC}^{\mathrm{T}} \mathrm{N}_{3}\right]\left(\mathrm{BF}_{4}\right)\right.$ by $\sim 2 \mathrm{eV}$ (Figure 5.2), which is consistent with the difference in oxidation state between ferrous and ferric. ${ }^{32}$ The edges of $\mathrm{Fe}$ (salen) $\mathrm{Cl}$ and $\mathrm{Fe}$ (salen)NO (Figure 5.3) occur at the same energy indicating that the iron in each site has the same +3 oxidation state.

In a previous study (presented in Chapter 3 ), ${ }^{39}$ iron-ligand bond distances were obtained by extended X-ray absorption fine structure (EXAFS) for [ $\mathrm{Fe}\left(\mathrm{OH}_{2}\right)$ EDTA] ${ }^{2-}$, [Fe $\left(\mathrm{OH}_{2}\right)$ EDTA] $]^{-}$and FeEDTA-NO. The first shell bond distances obtained from the [Fe $\left(\mathrm{OH}_{2}\right)$ EDTA] data were 2.04 and $2.34 \AA$ for the $\mathrm{Fe}-\mathrm{O}$ and $\mathrm{Fe}-\mathrm{N}$ bonds, respectively. The analogous distances obtained from the $\left[\mathrm{Fe}\left(\mathrm{OH}_{2}\right) \mathrm{EDTA}\right]^{2-}$ data were 2.17 and $2.34 \AA$. The first shell bond distance obtained from the FeEDTA-NO data were $1.76 \AA$ for the $\mathrm{Fe}-\mathrm{N}(\mathrm{O})$ bond and 2.05 and $2.27 \AA$ for the $\mathrm{Fe}-\mathrm{O}$ and $\mathrm{Fe}-\mathrm{N}$ bond distances of the EDTA ligand. The Fe-EDTA bond distances in FeEDTA-NO are more closely matched to the 
ferric-EDTA bond distances, in further experimental support of the ferric oxidation state description.

The XAS edge and pre-edge features as well as the iron-ligand distances obtained from EXAFS data are all consistent and indicate that the iron atom in these $\{\mathrm{FeNO}\}^{7}$ complexes is in the ferric oxidation state. However, there are quantitative differences in the edge spectra between the ferric reference complexes and the $\{\mathrm{FeNO}\}^{7}$ complexes, giving insight into the geometric and electronic structure of the these $\{\mathrm{FeNO}\}^{7}$ complexes. First, the pre-edge feature of the iron-nitrosyl complexes is more.intense than that of their ferric counterparts. Empirically, the strength of the pre-edge transition is found to be dependent on the amount of $3 \mathrm{~d}-4 \mathrm{p}$ mixing. ${ }^{36}$ A distortion of the metal site allows for more $3 d-4 p$ mixing resulting in an increase in the pre-edge intensity since the $1 \mathrm{~s} \rightarrow 4 \mathrm{p}$ transition is electric dipole allowed. This effect is the origin of the increase in the pre-edge intensity of $\mathrm{Fe}$ (salen) $\mathrm{Cl}$ (solid line in Figure 5.3 inset), which has a square pyramidal geometry relative to $\left[\mathrm{Fe}\left(\mathrm{OH}_{2}\right) \mathrm{EDTA}\right]^{-}$(dashed line Figure 5.1 inset), which has a distorted octahedral geometry. The effect can also be seen in a comparison of [Fe $\left(\mathrm{OH}_{2}\right)$ EDTA] ${ }^{2-}$ (solid line in Figure 5.1 inset) and $\left[\mathrm{Fe}(\mathrm{TMC}) \mathrm{N}_{3}\right]\left(\mathrm{BF}_{4}\right)$ (solid line in Figure 5.2 inset) where the first pre-edge feature in $\left[\mathrm{Fe}(\mathrm{TMC}) \mathrm{N}_{3}\right]\left(\mathrm{BF}_{4}\right)$ increased in intensity due to the lowering of symmetry from distorted octahedral to square pyramidal. In the case of FeEDTA-NO, [Fe(TMC)NO] $\left(\mathrm{BF}_{4}\right)_{2}$, and $\mathrm{Fe}($ salen)NO complexes, the short $\mathrm{Fe}-\mathrm{N}(\mathrm{O})$ bond lowers the symmetry around the iron site resulting in an increase in the intensity of the $1 \mathrm{~s} \rightarrow 3 \mathrm{~d}$ pre-edge feature (dotted lines in Figure 5.1, 5.2, and 5.3 insets).

The intensity of the $1 \mathrm{~s} \rightarrow 3 \mathrm{~d}$ pre-edge feature can be used to gain information on the distortion around the iron site. As described in detail in Chapter 4 , the intensity of the $1 \mathrm{~s} \rightarrow 3 \mathrm{~d}$ pre-edge feature can be used to quantitate the amount of $4 \mathrm{p}$ mixing into the $3 \mathrm{~d}$ orbitals. For example, FeEDTA-NO has a total pre-edge area of 10.4. Assuming that 5 units originate from quadrupole transition intensity (the value for centrosymmetric high spin ferric complexes, Table 4.1 in Chapter 4), 5.4 units originate from dipole transition intensity (i.e. $4 \mathrm{p}$ mixing into the $3 \mathrm{~d}$ orbitals). If the quadrupole intensity is $1 \%$ of the dipole intensity, then there is $5.4 \% 4 \mathrm{p}$ mixing into the $3 \mathrm{~d}$ orbitals for FeEDTA-NO. This percentage of $4 \mathrm{p}$ mixing is very similar to the $5.5 \% 4 \mathrm{p}$ mixing into the $3 \mathrm{~d}$ orbitals obtained for $\left.\left(\mathrm{enH}_{2}\right)\left[\mathrm{Fe}_{2} \mathrm{O} \text { (HEDTA }\right)_{2}\right] \cdot 6 \mathrm{H}_{2} \mathrm{O}$ (Chapter 4 ), where instead of a short $1.8 \AA$ $\mathrm{Fe}-\mathrm{N}(\mathrm{O})$ bond as in FeEDTA-NO there is a short $1.8 \AA \mathrm{Fe}-\mathrm{O}$ bond from a $\mu$-oxo bridge. Distorting the iron site further would increase the amount of $4 p$ mixing into the $3 d$ orbitals and, thus, increase the pre-edge intensity. Such is the case upon removing an axial ligand and creating a square pyramidal iron site. [ $\mathrm{Fe}(\mathrm{TMC}) \mathrm{NO}]\left(\mathrm{BF}_{4}\right)_{2}$, which has a 
linear Fe-N-O unit, has a total pre-edge area of 15.5 which corresponds to $10.5 \% 4 \mathrm{p}$ mixing into the $3 \mathrm{~d}$ orbitals. The amount of $4 \mathrm{p}$ mixing into the $3 \mathrm{~d}$ orbitals is much greater for [Fe(TMC)NO] $\left(\mathrm{BF}_{4}\right)_{2}$, a square-pyramidal complex, than for FeEDTA-NO, a purported seven-coordinate complex (see Chapter 3). The amount of $4 \mathrm{p}$ character in the $3 \mathrm{~d}$ orbitals of [Fe(TMC)NO](BF 4$)_{2}$ is slightly more than the value of $9.4 \%$ obtained for $\mathrm{Fe}($ salen $) \mathrm{Cl}$, also a square pyramidal complex, due to the shorter iron-axially ligand bond in $[\mathrm{Fe}(\mathrm{TMC}) \mathrm{NO}]\left(\mathrm{BF}_{4}\right)_{2}$. $\mathrm{Fe}(\mathrm{salen}) \mathrm{NO}$, which has a bent $\mathrm{Fe}-\mathrm{N}-\mathrm{O}$ unit, has a total pre-edge area of 24.2 , which corresponds to $19.2 \% 4 \mathrm{p}$ mixing into the $3 \mathrm{~d}$ orbitals. The main difference in the iron site symmetry between [Fe(TMC)NO] $\left(\mathrm{BF}_{4}\right)_{2}$ and $\mathrm{Fe}($ salen)NO is a linear $v s$. bent $\mathrm{Fe}-\mathrm{N}-\mathrm{O}$ unit. Thus, the differences in the pre-edge area and the related $4 p$ mixing into the $3 d$ orbitals must be affected by the bending of the Fe-N-O unit. The increase in $4 \mathrm{p}$ mixing into the $3 \mathrm{~d}$ orbitals upon bending the $\mathrm{Fe}-\mathrm{N}-\mathrm{O}$ unit has also been observed in SCF-X $\alpha$-SW calculations of an $\{\mathrm{FeNO}\}^{7}$ model complexes where the amount of $4 \mathrm{p}_{2}$ mixing into the $3 \mathrm{~d}_{\mathrm{z}} 2$ orbital doubled upon bending the Fe-N-O unit from $180^{\circ}$ to $150^{\circ} .{ }^{19}$

The second quantitative difference involves the energy of the rising edge, with that of FeEDTA-NO being lower than that of [Fe( $\left(\mathrm{OH}_{2}\right)$ EDTA]- (Figure 5.1). As has been stated previously, the $1 \mathrm{~s} \rightarrow 4 \mathrm{p}$ edge energy is affected by changes in the effective nuclear charge of the metal atom as well as ligand field effects. In this case, the $\mathrm{H}_{2} \mathrm{O}$ of the ferric EDTA complex is replaced by NO- in FeEDTA-NO. The resulting short Fe-N $(O)$ bond causes a strong axial distortion that splits the $4 p$ levels with the $4 p_{z}$ orbital shifting to higher energy. Thus, one would expect the $1 \mathrm{~s} \longrightarrow 4 \mathrm{p}$ transition to split with the transition to the $4 p_{x, y}$ orbital occurring at approximately the same energy and the transition to the $4 \mathrm{p}_{z}$ orbital occurring at higher energy. However, experimentally the FeEDTA-NO edge is at lower energy than the $\left[\mathrm{Fe}\left(\mathrm{OH}_{2}\right)\right.$ EDTA] edge (Figure 5.1). This shift to lower energy can be explained by charge donation from the NO- to the ferric center which reduces the effective nuclear charge of the iron and causes the FeEDTA-NO edge to be at a lower energy. Alternatively, in the $\mathrm{Fe}($ salen $) \mathrm{Cl}$ and $\mathrm{Fe}(\mathrm{salen}) \mathrm{NO}$ comparison (Figure 5.3), the edges lie at the same energy. Since $\mathrm{Fe}($ salen) $\mathrm{Cl}$ is square pyramidal, the $4 \mathrm{p}$ orbitals are split with the $4 \mathrm{p}_{\mathrm{z}}$ orbital lying lower in energy. Thus, when the NO- replaces the $\mathrm{Cl}^{-}$with a much shorter bond length (1.78 vs. $2.24 \AA$ ) the $1 \mathrm{~s} \longrightarrow>4 \mathrm{pz}_{2}$ should increase in energy resulting in an edge shift to higher energy. Experimentally the edges are at the same energy, thus there must also be charge donation from the NO- to the ferric center of the salen complex reducing the effective nuclear charge of the iron. 


\subsubsection{Discussion}

In summary, both the energy of the rising edge and the shape of the edge of the $\{\mathrm{FeNO}\}^{7}$ complexes are similar to the ferric complexes with corresponding ligation (i.e. [Fe( $\left(\mathrm{OH}_{2}\right)$ EDTA] vs. FeEDTA-NO and $\mathrm{Fe}$ (salen)Cl vs. Fe(salen)NO, Figure 5.1 and 5.3, respectively). All three of the $\{\mathrm{FeNO}\}^{7}$ complexes exhibit an intense pre-edge feature at $-7113 \mathrm{eV}$ which is consistent with a ferric complex that has a distorted iron site. In the $\{\mathrm{FeNO}\}^{7}$ complexes, the short $\mathrm{Fe}-\mathrm{N}(\mathrm{O})$ bond of $\sim 1.7 \AA$ provides a strong axial distortion of the iron active site. The bond distances obtained from EXAFS data of [Fe $\left(\mathrm{OH}_{2}\right)$ EDTA] ${ }^{2-},\left[\mathrm{Fe}\left(\mathrm{OH}_{2}\right)\right.$ EDTA] and FeEDTA-NO show that the Fe-EDTA distances of FeEDTA-NO are much more similar to the distances in [ $\mathrm{Fe}\left(\mathrm{OH}_{2}\right)$ EDTA] than in $\left[\mathrm{Fe}\left(\mathrm{OH}_{2}\right) \mathrm{EDTA}\right]^{2-{ }^{39}}$ On a quantitative level the rising edge of FeEDTA-NO is shifted by $2 \mathrm{eV}$ to lower energy relative to [Fe( $\left(\mathrm{OH}_{2}\right)$ EDTA] $]^{-}$consistent with significant charge donation from the $\mathrm{NO}^{-}$ligand to the $\mathrm{Fe}^{3+}$ center.

The results obtained from the XAS experiments on $\{\mathrm{FeNO}\}^{7}$ systems can be used in conjunction with experimental (absorption, variable-temperature, variable-field $M C D$, resonance Raman, EPR, and magnetism) and theoretical (SCF-X $\alpha-S W$ ) results to obtain a detailed electronic description of $S=3 / 2 \quad \mathrm{FeNO}^{7}$ systems. The theoretical and experimental electronic structure description we have developed for the $[\mathrm{FeNO}\}^{7} \mathrm{~S}=3 / 2$ non-heme iron complex involves a high spin $\mathrm{Fe}^{3+} \mathrm{S}=5 / 2$ ion antiferromagnetically coupled to an NO- ligand with $S=1 .{ }^{18,19}$ From the SCF-X $\alpha$-SW calculations, the high spin ferric description derives from the large exchange stabilization of the $d^{5}$ configuration. ${ }^{19}$ In the spin unrestricted calculations, which allow spin up and spin down electrons to occupy different orbitals, the occupied spin up $d$ orbitals are stabilized below the unoccupied spin down counterparts by $6 \mathrm{eV}$ due to this exchange interaction. The occupied spin down NO $\pi^{*}$ orbitals are stabilized due to their direct overlap with the spin up iron $d_{x z}$ and $d_{y z}$ orbitals. ${ }^{19}$ Thus, this orbital overlap leads to the $S=1$ on the NO- unit and its antiferromagnetic coupling to the high spin ferric center.

Experimental support for this description comes from XAS, $r R$ and abs/MCD/rR profile excited state spectroscopies. ${ }^{19}$ From the XAS, the pre-edge peak at $\sim 7113 \mathrm{eV}$, rather than two peaks split by $1.9 \mathrm{eV}$ as in the ferrous complexes, and the shape and energy of the rising edge both indicate that the iron is in the high spin ferric oxidation state. Furthermore, the first shell EXAFS distances of the NO complex are $\sim 0.1 \AA$ shorter than the ferrous complex with corresponding ligation but very close to those of the ferric analog. From $\mathrm{rR}$, the intra-ligand frequency of $\sim 1750 \mathrm{~cm}^{-1}$ combined with metal ligand vibrational data on the Fe-NO unit via a normal coordinate analysis give an 
NO stretching force constant which, compared to molecular NO species, is significantly reduced, indicating electron transfer into the NO $\pi^{*}$ orbital resulting in an NO- species. ${ }^{19}$ From excited state spectroscopies, the ligand field and charge transfer (CT) transitions have been identified using $\mathrm{t} R$ profiles. The ligand field transition energies are consistent with those observed for high spin ferric complexes and the CT transitions are in an energy range and ordering consistent with predictions from the SCF-X $\alpha$-SW electronic structure calculations for $\mathrm{NO}-\pi^{*} \longrightarrow \mathrm{Fe}^{3+3 \mathrm{~d}} \mathrm{CT}$ transitions.

The data further show evidence for a highly covalent $\mathrm{Fe}^{3+}-\mathrm{NO}^{-}$ligand donor bond. ${ }^{19}$ First, from magnetic susceptibility the electrons of the NO- are strongly antiferromagnetically coupled to the iron to give the $S=3 / 2$ spin state which requires significant orbital overlap. Secondly, the rising edges of the XAS spectra of the $\mathrm{Fe}^{3+}-\mathrm{NO}^{-}$complexes are at lower energy than the respective ferric complexes, even when ligand field effects on the $4 p$ orbitals are taken into account, indicating charge donation from the NO- to the ferric center. Finally, the intra-ligand frequency and calculated force constant are significantly higher than those found for molecular NO- again indicating charge donation from an antibonding orbital on the NO- of the iron-nitrosyl complex. The spin unrestricted SCF-X $\alpha$-SW calculations ${ }^{19}$ provide insight into the nature of the strong $\mathrm{Fe}^{3+-N O^{-}}$bond. There are two strong donor interactions which are, in fact, $\sigma$ in character. These involve the NO $4 \sigma^{+}$and $5 \sigma^{+}$orbitals. The former is $\sigma$ antibonding with respect to the $\mathrm{NO}^{-}$and dominates the charge donation to the $\mathrm{Fe}^{3+}$ due to better overlap (i.e. increased $\mathrm{N}$ character in the antibonding NO molecular orbital). This charge donation thus removes electron density from a strongly antibonding orbital, which significantly increases the strength of the intramolecular $\mathrm{NO}^{-}$bond, leading to the large increase in the bound NO force constant observed experimentally. It is important to emphasize that reduction of the NO by the ferrous complex involves electron transfer into the NO $\pi^{*}$ orbital while charge donation of the bound $\mathrm{NO}^{-}$to the $\mathrm{Fe}^{3+}$ dominantly involves electron transfer from an NO $\sigma^{*}$ orbital. Thus charge donation from the bound $\mathrm{NO}^{-}$to the $\mathrm{Fe}^{3+}$ can significantly increase the strength of the NO bond.

Thus an experimentally and theoretically consistent electronic structure has been generated for the $\{\mathrm{FeNO}\}^{7} \mathrm{~S}=3 / 2$ systems, which can be described as high spin ferric $(S=5 / 2)$ antiferromagnetically coupled to NO- $(S=1)$ to produce the $S=3 / 2$ ground state. ${ }^{19}$ This new bonding description provides a general understanding of the interaction of NO with the high-spin ferrous site, and the spectral features can be quantitated to provide insight into differences in bonding and electron distribution which can be related to dioxygen activation by non-heme iron enzymes. 


\subsubsection{Acknowledgments}

The EDTA solution samples were prepared by Mark Pavlosky and Yan Zhang. Mark Pavlosky and Yan Zhang performed the absorption, variable-temperature, variable-field $M C D$, resonance Raman, EPR, and magnetism experiments alluded to in the discussion. Carl Brown performed the SCF-X $\alpha$-SW theoretical calculations. This research was supported by grants from the NIH (GM40392, E.I.S.) and NSF (CHE-9121576, K.O.H.). The Stanford Synchrotron Radiation Laboratory is supported by the Department of Energy, Office of Basic Energy Sciences, Divisions of Chemical Science and Materials Science, and in part by the National Institutes of Health, National Center of Research Resources, Biomedical Research Technology Program (RR-01209, K.O.H.) and the DOE's Office of Health and Environmental Research.

\subsubsection{References and Notes}

(1) Feig, A. L.; Lippard, S. J. Chem. Rev. 1994, 94, 759.

(2) Howard, J. B.; Rees, D. C. Advances in Protein Chemistry; Academic Press: New York, 1991; Vol. 42, p 199.

(3) Solomon, E. I.; Zhang, Y. Acc. Chem. Res. 1992, 25, 343.

(4) Nelson, M. J. J. Biol. Chem. 1987, 262, 12137.

(5) Galpin, J. R.; Veldink, G. A.; Vliegenthart, J. F. G.; Boldingh, J. Biochim. Biophys. Acta 1978, 536, 356.

(6) Arciero, D. M.; Lipscomb, J. D. J. Biol. Chem. 1986, 261, 2170.

(7) - Arciero, D. M.; Orville, A. M.; Lipscomb, J. D. J. Biol. Chem. 1985, 260, 14035.

(8) Twilfer, H.; Bernhardt, F.-H.; Gersonde, K. Eur. J. Biochem. 1985, 147, 171.

(9) Chen, V. J.; Orville, A. M.; Harpel, M. R.; Frolik, C. A.; Surerus, K. K.; Münck, E.; Lipscomb, J. D. J. Biol. Chem. 1989, 264, 21677.

(10) Ming, L.-J.; Que, L., Jr.; Kriauciunas, A.; Frolik, C. A.; Chen, V. J. Biochemistry 1991, 30, 11653.

(11) Bill, E.; Bernhardt, F.-H.; Trautwein, A. X.; Winkler, H. Eur. J. Biochem. 1985, 147, 177.

(12) Arciero, D. M.; Lipscomb, J. D.; Huynh, B. H.; Kent, T. A.; Münck, E. J. Biol. Chem. 1983, 258, 14981.

(13) Enemark, J. H.; Feltham, R. D. Coord. Chem. Rev. 1974, 13, 339.

(14) Wells, F. V.; McCann, S. W.; Wickman, H. H.; Kessel, S. L.; Hendrickson, D. N.; Feltham, R. D. Inorg: Chem. 1982, 21, 2306. 
(15) Salemo, J. C.; Siedown, J. N. Biochim. Biophys. Acta 1979, 579, 246.

(16) Pohl, K.; Wieghardt, K.; Nuber, B.; Weiss, J. J. Chem. Soc. Dalton Trans. 1987, 187.

(17) Abbreviations used: EDTA $=$ ethylenediaminetetraacetic acid; TMC = 1,4,8,11-tetramethyl-1,4,8,11-tetraazacyclotetradecane; salen = $N, N$-ethylenebis(salicylideneiminato).

(18) Zhang, Y.; Pavlosky, M. A.; Brown, C. A.; Westre, T. E.; Hedman, B.; Hodgson, K. O.; Solomon, E. I. J. Am. Chem. Soc. 1992, 114, 9189.

(19) Brown, C. A.; Pavlosky, M. A.; Westre, T. E.; Zhang, Y.; Hedman, B.; Hodgson, K. O.; Solomon, E. I. J. Am. Chem. Soc. 1995, 117, 715.

(20) Scott, R. A.; Hahn, J. E.; Doniach, S.; Freeman, H. C.; Hodgson, K. O. J. Am. Chem. Soc. 1982, 104, 5364.

(21) Lytle, F. W. In Applications of Synchrotron Radiation; Winick, H.; Xian, D.; Ye, M.-h.; Huang, T., Eds.; Gordon \& Breach: New York, 1989; p 135.

(22) Hodges, K. D.; Wollmann, R. G.; Kessel, S. L.; Hendrickson, D. N.; Van Derveer, D. G.; Barefield, E. K. J. Am. Chem. Soc. 1979, 101, 906.

(23) Hodges, K. D.; Wollmann, R. G.; Barefield, E. K.; Hendrickson, D. N. Inorg. Chem. 1977, 16, 2746.

(24) Haller, K. J.; Johnson, P. L.; Feltham, R. D.; Enemark, J. H.; Ferraro, J. R.; Basile, L. J. Inorg. Chim. Acta 1979, 33, 119.

(25) Gerloch, M.; Mabbs, F. E. J. Chem. Soc. (A) 1967, 1598.

(26) Stern, E. A.; Heald, S. M. Rev. Sci. Instrum. 1979, 50, 1579.

(27) Lytle, F. W.; Greegor, R. B.; Sandstrom, D. R.; Marques, E. C.; Wong, J.; Spiro, C. L.; Huffman, G. P.; Huggins, F. E. Nucl. Instrum. Meth. Phys. Res. 1984, 226, 542.

(28) Argonne National Laboratory; B. S. Garbow, K. E. Hillstrom, J. J. More.

(29) Agarwal, B. K. X-ray Spectroscopy; Springer-Verlag: New York, 1979, p 276.

(30) Tyson, T. A.; Roe, A. L.; Frank, P.; Hodgson, K. O.; Hedman, B. Phys. Rev. B 1989, 39, 6305.

(31) Sugano, S.; Tanabe, Y.; Kamimura, H. Multiplets of Transition-Metal lons in Crystals; Academic Press: New York, 1970.

(32) - Shulman, R. G.; Yafet, Y.; Eisenberger, P.; Blumberg, W. E. Proc. Natl. Acad. Sci. USA 1976, 73, 1384.

(33) Griffith, J. S. The Theory of Transition Metal lons; Cambridge University Press: Cambridge, 1961.

(34) Srivastava, U. C.; Nigam, H. L. Coord. Chem. Rev. 1973, 9, 275. 
(35) Cramer, S. P.; Eccles, T. K.; Kutzler, F. W.; Hodgson, K. O. J. Am. Chem. Soc. 1976, 98, 1287.

(36) Roe, A. L.; Schneider, D. J.; Mayer, R. J.; Pyrz, J. W.; Widom, J.; Que, L., Jr. J. Am. Chem. Soc. 1984, 106, 1676.

(37) Wong, J.; Lytle, F. W.; Messmer, R. P.; Maylotte, D. H. Phys. Rev. B. 1984, 30 , 5596.

(38) Kau, L.-S.; Spira-Solomon, D. J.; Penner-Hahn, J. E.; Hodgson, K. O.; Solomon, E. I. J. Am. Chem. Soc. 1987, 109, 6433.

(39) Westre, T. E.; Di Cicco, A.; Filipponi, A.; Natoli, C. R.; Hedman, B.; Solomon, E. I.; Hodgson, K. O. J. Am. Chem. Soc. 1994, 116, 6757. 


\subsection{Iron(II) Bleomycin}

\subsubsection{Introduction}

Bleomycin (BLM) is a histidine-containing glycopeptide antibiotic isolated as a $\mathrm{Cu}$ (II) complex from the fungus Streptomyces verticillus. ${ }^{1-3}$ The drug Blenoxane, composed mainly of $B L M A_{2}$, is used in treatment against a variety of carcinomas because of its ability to degrade DNA with sequence specificity. ${ }^{4}$ The structure of BLM A $\mathrm{A}_{2}$ (Figure 5.4a) includes three domains: the bithiazole tail is believed to be involved in BLM binding to DNA, ${ }^{5-7}$ the sugar moiety is thought to control uptake of BLM by cancer cells, ${ }^{8,9}$ and the $\beta$-aminoalanine-pyrimidine-histidine region is responsible for metal chelation. ${ }^{10,11}$ DNA degradation by BLM is oxygen and metal ion dependent ${ }^{12-15}$ with the greatest enhancement observed for iron. ${ }^{12,16-21}$ Oxygen binds to $\mathrm{Fe}$ (II)BLM producing a ternary complex (oxygenated BLM) that is stable in the presence of excess DNA. ${ }^{22-24}$ Transfer of an additional electron from a second $\mathrm{Fe}(\mathrm{II}) \mathrm{BLM}$ molecule (or other reductant) to oxygenated BLM produces activated BLM, the species responsible for DNA strand scission. ${ }^{25}$ The similarity in mechanism to cytochrome $\mathrm{P}-450$ has led researchers to postulate a ferryl $(\mathrm{Fe}(\mathrm{TV})=0) \mathrm{BLM}$ intermediate, ${ }^{10,19}$ as has generally been considered to be present in heme chemistry. ${ }^{26}$ Alternatively, electron paramagnetic resonance (EPR), ${ }^{19}$ Mössbauer, ${ }^{24}$ mass spectrometry, ${ }^{27}$ and X-ray absorption spectroscopy (XAS) ${ }^{28}$ of activated BLM are most consistent with a low spin Fe(III)-peroxide site (see section 5.4. Activated BLM).

No crystal structure exists for the ferrous complex of BLM and there is some controversy concerning the ligation of $\mathrm{BLM}$ to $\mathrm{Fe}^{2+}$. Present descriptions are based on correlation to $\mathrm{a} \mathrm{Cu}(\mathrm{II})$ complex of the related biosynthetic precursor P-3A, which has been crystallographically defined and shown to have a five-coordinate, square pyramidal geometry with pyrimidine, imidazole, deprotonated amide, and secondary amine functions forming the equatorial plane, and a primary amine function coordinating axially. ${ }^{29}$ However, $\mathrm{Cu}^{2+}$ has strong structural preferences due to the Jahn-Teller effect which $\mathrm{Fe}^{2+}$ does not and P-3A lacks the disaccharide and bithiazole moieties of BLM, the former being a potential ligand. Axial coordination in Fe(II)BLM of the carbamoyl group of the mannose sugar has been implicated by ${ }^{1} \mathrm{H}$ nuclear magnetic resonance (NMR) studies ${ }^{30-32}$ and two-dimensional NMR studies of the carbon monoxide bound form. ${ }^{33}$ In contrast, earlier studies of CO-bound $\mathrm{Fe}$ (II)BLM maintained axial coordination of the primary amine and proposed that the sugar portion played a steric role in governing $\mathrm{CO}$ binding constants via hydrogen bonding to the $\beta$-amino-propionamide group. ${ }^{34}$ EPR spectroscopy on the 
(a) Bleomycin $A_{2}$<smiles>Cc1c(N)nc([C@H](CC(N)=O)NC[C@H](N)C(N)=O)nc1C(=O)N[C@H](C(=O)NC(C)C(O)C(C)C(=O)NC(C(=O)NCCc1nc(-c2nc(C(=O)NCCC[S+](C)C)cs2)cs1)C(C)O)C(OC1OC2OC(C(O)C2O)C(O)C1O)c1c[nH]cn1</smiles>

(b) $\mathrm{PMAH}$<smiles>NCCNCc1ncc(Br)c(C(=O)NCCc2c[nH]cn2)n1</smiles>

Figure 5.4. Structural ligand framework of (a) Bleomycin $A_{2}$ and (b) the analogous model complex PMAH. Coordinating nitrogens are denoted with asterisks $\left({ }^{*}\right)$ for each structure. 
nitrosyl complex of $\mathrm{Fe}$ (II)BLM has been used to further support the model that the axial donor trans to the bound NO is the primary nitrogen of the $\alpha$-amino group of the $\beta$-aminoalanine portion. ${ }^{35-37}$ Additional evidence for axial coordination of the primary amine derives from the variation in EPR parameters for Fe(III)BLM and the ferric complexes of depyruvamide BLM and deamido BLM. ${ }^{38}$

There is little direct spectroscopic evidence available on $\mathrm{Fe}(\mathrm{II}) \mathrm{BLM}$ as the Mössbauer spectra exhibit broad, unresolved magnetic features and high spin $\mathrm{Fe}^{2+}$ non-Kramers ions generally do not exhibit EPR signals. ${ }^{24}$ As a result, most experiments have focused on the spectroscopically accessible ferric forms of the drug. In this study $\mathrm{Fe}$ $\mathrm{K}$-edge XAS was used to characterize the electronic and geometric structure of $\mathrm{Fe}(\mathrm{II}) \mathrm{BLM}$. Examination of the pre-edge feature can give insight into the coordination number and geometry of the iron site, while the position of the edge is dependent upon the effective nuclear charge of the iron. In addition, an EXAFS analysis can be used to accurately determine the distances to the ligands. Due to the complexity of the glycopeptide and the range of possible metal coordinating functional groups in BLM, synthetic models have been prepared in an effort to obtain insight into the structure. ${ }^{39-45}$ The synthetic analog $\mathrm{PMAH}^{46}$ which has a similar metal binding domain but lacks the sugar moiety and the bithiazole tail (Figure 5.4b) has also been investigated. The ferrous complex serves as a good model of Fe(II)BLM in that it reacts with dioxygen to generate an analog of activated BLM which has a nearly identical EPR spectrum and causes DNA cleavage with similar specificity. ${ }^{39}$ XAS data was collected on [Fe(II)PMA]+ both in the solid and solution form. The results from this study were combined with results from optical absorption (Abs), magnetic circular dichroism (MCD), and resonance Raman (rR) spectroscopies to develop a detailed description of the geometric and electronic structure of the $\mathrm{Fe}(\mathrm{I}) \mathrm{BLM}$ active site. $^{47}$

\subsubsection{Experimental Section}

Bleomycin sulfate (Blenoxane), a mixture of $60 \%$ BLM A2, $30 \%$ BLM B2, and $10 \%$ other BLM fractions, was obtained as a gift from Bristol-Meyers Squibb and used without further purification. The individual components differ only in the terminal amine functionality which has no influence on the spectroscopic characteristics of the metal binding domain. ${ }^{19}$ All concentrations were calculated with a molecular weight of -1510 Daltons. The Fe(II)BLM sample was prepared as a glass in 50\% (v/v) glycerol- $\mathrm{d}_{3}$ (98 atom \% D; Aldrich), HEPES buffer (Sigma) dissolved in $\mathrm{D}_{2} \mathrm{O}$ (99.9 atom \% D; Aldrich) and adjusted to a $\mathrm{pD}$ in the range 6.0 to 9.0 with $40 \mathrm{wt} \% \mathrm{NaOD}$ (Aldrich) in $\mathrm{D}_{2} \mathrm{O}$. 
Septum stoppered reaction flasks were not sufficient to prevent $\mathrm{O}_{2}$ reaction, therefore, all solutions were rigorously degassed with 10-15 freeze, pump, thaw cycles at $10^{-3}$ Torr in Teflon or ground glass stoppered flasks and immediately placed in a $\mathrm{N}_{2}$ atmosphere $\left(<10 \mathrm{ppm} \mathrm{O}\right.$ ). Solid forms of metal free BLM and $\mathrm{Fe}\left(\mathrm{NH}_{4}\right)_{2}\left(\mathrm{SO}_{4}\right)_{2} \cdot 6 \mathrm{H}_{2} \mathrm{O}$ (FeAS; MCB Manufacturing Chemists, Inc.) were solvated just prior to use in an inert atmosphere with buffered solution and $\mathrm{D}_{2} \mathrm{O}$, respectively. $\mathrm{Fe}(\mathrm{II}) \mathrm{BLM}$ was prepared in the presence of $20 \%$ excess ligand by addition of $5 \mu \mathrm{L}$ of the FeAS stock solution to $5 \mathrm{mM}$ metal free BLM to give a final sample concentration of $\sim 4 \mathrm{mM}$. Sample integrity was confirmed by optical absorbtion ( $\left.\varepsilon_{476}=380 \mathrm{M}^{-1} \mathrm{~cm}^{-1}\right)^{18}$ and EPR. The use of reducing agents to inhibit $\mathrm{Fe}^{2+}$ oxidation was strictly avoided as it was determined to significantly alter both the $C D$ and MCD spectra of Fe(II)BLM.

The ligand $\mathrm{PMAH}^{48}$ was synthesized according to published procedures, followed by anaerobic complexation with $\mathrm{Fe}^{2+}$ to form [Fe(II)(PMA)]Cl-MeOH. A [Fe(II)PMA] ${ }^{+}$ solution was prepared in a $\mathrm{N}_{2}$ atmosphere with degassed methanol- $\mathrm{d}_{4}$ (99.9 atom \% D; Aldrich) and ethanol- $d_{6}(99+$ atom $\% \mathrm{D}$; Aldrich) in a ratio of 1 to 4 to facilitate glassing. Sample concentration was determined by optical absorption $\left(\varepsilon_{670}\left([\mathrm{Fe}(\mathrm{II}) \mathrm{PMA}]^{+}\right)=\right.$ $1850 \mathrm{M}^{-1} \mathrm{~cm}^{-1}$ ) to be $\sim 4 \mathrm{mM}$.

$\mathrm{X}$-ray absorption spectra were recorded at the Stanford Synchrotron Radiation Laboratory on unfocused beamline 7-3 during dedicated conditions ( $3 \mathrm{GeV}, 50-100 \mathrm{~mA}$ ). The radiation was monochromatized using a $\mathrm{Si}(220)$ double-crystal monochromator. Unless specified otherwise, data were measured to $k=15 \AA^{-1}$ with $1 \mathrm{~mm}$ high pre-monochromator beam defining slits and the monochromator was detuned by $50 \%$ at $7998 \mathrm{eV}$ to minimize harmonic contamination. An Oxford Instruments continuous-flow liquid helium CF 1208 cryostat was used to maintain a constant temperature of $10 \mathrm{~K}$. Energies were calibrated using an internal Fe foil standard, assigning the first inflection point to $7111.2 \mathrm{eV} .{ }^{49}$ The spectrometer energy resolution was $\sim 1.4 \mathrm{eV}$ with reproducibility in edge position determination of $\angle 0.2 \mathrm{eV}$.

The $[\mathrm{Fe}(\mathrm{II}) \mathrm{PMA}]^{+}$solution and $\mathrm{Fe}(\mathrm{II}) \mathrm{BLM}$ XAS samples were prepared as described above. The solution samples were syringed into Lucite EXAFS cells ( $23 \mathrm{x} 1 \mathrm{x}$ $3 \mathrm{~mm}$ ) with $37 \mu \mathrm{m}$ Kapton windows under a $\mathrm{N}_{2}$ atmosphere and subsequently frozen in liquid nitrogen prior to exposure to air. The fluorescence signal was monitored by using a 13-element $\mathrm{Ge}$ solid-state array detector ${ }^{50}$ windowed on the $\mathrm{Fe} \mathrm{K} \alpha$ signal. During the experiment, count rates of approximately $30,000 \mathrm{~s}^{-1}$ total per element were not exceeded. Approximately thirty scans were averaged for $\mathrm{Fe}(\mathrm{I}) \mathrm{BLM}$ and eleven scans were averaged for the [Fe(II)PMA]+ solution. The powder [Fe(II)PMA] ${ }^{+}$sample was mixed with $B N$ and ground into a fine powder in a $\mathrm{N}_{2}$-filled glove box. This BN/sample mixture was 
pressed into a $1 \mathrm{~mm}$ thick $\mathrm{A}$ spacer that was sealed with $63.5 \mu \mathrm{m}$ Mylar tape windows and frozen in liquid nitrogen. Data were measured in transmission mode with $\mathrm{N}_{2}$-filled ionization chambers. The final spectrum represents a six scan average.

EXAFS data reduction was performed on the averaged spectra according to established methods. ${ }^{51-53}$ A smooth pre-edge background was removed from the averaged spectra by fitting a second order polynomial to the pre-edge region and subtracting this polynomial from the entire spectrum. A three-segment spline approximately even in $k$-space was fit to the EXAFS region and the data normalized to an edge jump of one at $7130 \mathrm{eV}$. The spline was chosen so that it minimized residual low-frequency background but did not reduce the EXAFS amplitude as checked by monitoring the Fourier transform of the EXAFS during the background subtraction process. The normalized data were converted to $k$-space. The photoelectron wave vector, $k$, is defined by $\left[2 \mathrm{~m}_{e}\left(\mathrm{E}-\mathrm{E}_{0}\right) / \hbar^{2}\right]^{1 / 2}$ where $m_{e}$ is the electron mass, $E$ is the photon energy, $\hbar$ is Planck's constant divided by $2 \pi$, and $E_{0}$ is the threshold energy of the absorption edge, which was defined to be $7130 \mathrm{eV}$ for the $\mathrm{Fe} \mathrm{K}$ absorption edge. The empirical EXAFS data analyses were performed with nonlinear least-square curve-fitting ${ }^{49,51-53}$ techniques using empirical phase and amplitude parameters. The following models were used to obtain the empirical $\mathrm{Fe}-\mathrm{X}$ backscattering parameters of interest: $\mathrm{Fe}-\mathrm{O}$ from $\left[\mathrm{Fe} \text { (acetylacetonate) }{ }_{3}\right]^{54,55}$ and Fe-N from [ $\left.\mathrm{Fe}(1,10 \text {-phenanthroline) })_{3}\right]\left(\mathrm{ClO}_{4}\right)_{3}{ }^{56,57}$ Fourier transforms (from $k$ to $\mathrm{R}$ space) were performed for the data range 3.5-12.5 $\AA^{-1}$ with a Gaussian window of $0.1 \AA^{-1}$. The window widths used in the backtransforms (from $\mathrm{R}$ to $k$ space) are given in the Results and Analysis section. The window widths were kept as similar as possible to those used to extract amplitude and phase parameters from the model compounds to minimize artifacts introduced by the Fourier filtering technique. All curve-fitting was based on $k^{3}$-weighted data and applied to the individual filtered shell of interest. Only the structure-dependent parameters, the distance and coordination number, were varied unless stated otherwise. A "goodness of fit" parameter, $F$, was calculated as $F=\left\{\left[k^{6}\right.\right.$ (data fit) $\left.{ }^{2}\right] /$ (no. of points) $\}^{1 / 2}$ for each fit.

Fe K-edge spectra were also collected on a five- and a six-coordinate ferrous model complex. $\left[\mathrm{Fe}(\mathrm{TMC}) \mathrm{N}_{3}\right] \mathrm{BF}_{4}{ }^{58}$ (where TMC refers to tetramethylcylcam) and [Fe(imidazole $\left.)_{6}\right] \mathrm{Cl}_{2}{ }^{59}$ were prepared as described in the literature. The XAS samples were prepared in the same manner as the [Fe(II)PMA] ${ }^{+}$solid sample. Data were measured in transmission mode with $\mathrm{N}_{2}$-filled ionization chambers to $k=9.5 \AA^{-1}$ detuning the monochromator $50 \%$ at $7474 \mathrm{eV}$ to minimize harmonic contamination. Two to three scans were averaged for each sample. A smooth pre-edge background was removed from the averaged spectra by fitting a first order polynomial to the pre-edge region and subtracting 
this polynomial from the entire spectrum. A two segment spline of order two was fit to the EXAFS region and the data normalized to an edge jump of one at $7130 \mathrm{eV}$.

\subsubsection{Results and Analysis}

5.3.3.1. Fe K-Edge XAS. Fe $\mathrm{K}$ edge spectra of $[\mathrm{Fe}(\mathrm{II}) \mathrm{PMA}]^{+}$solid, [Fe(II)PMA] solution and Fe(II)BLM are shown in Figure 5.5A. The lowest energy peaks arise from the weak $1 \mathrm{~s} \rightarrow 3 \mathrm{~d}$ pre-edge transition which is at $-7113 \mathrm{eV}$ followed by the $1 \mathrm{~s} \longrightarrow 4 \mathrm{p}$ transition at $\sim 7127 \mathrm{eV}$. An expanded view of the $1 \mathrm{~s} \rightarrow 3 \mathrm{~d}$ pre-edge region is shown for the same samples in Figure 5.5B. Figure 5.5C contains the $1 \mathrm{~s} \longrightarrow 3 \mathrm{~d}$ pre-edge region of representative five-and six-coordinate high spin ferrous model complexes, $\left[\mathrm{Fe}(\mathrm{TMC}) \mathrm{N}_{3}\right] \mathrm{BF}_{4}{ }^{58}$ and $\left[\mathrm{Fe}\right.$ (imidazole) $\left.{ }_{6}\right] \mathrm{Cl}_{2},{ }^{59}$ respectively. The [Fe(imidazole) $6 \mathrm{Cl}_{2}$ spectrum (solid line) has two very weak pre-edge features at -7111.5 and $-7113.5 \mathrm{eV}$, while the $\left[\mathrm{Fe}(\mathrm{TMC}) \mathrm{N}_{3}\right] \mathrm{BF}_{4}$ spectrum (dashed line) has a much more intense feature at $7111.5 \mathrm{eV}$ (Figure 5.5C). The spectra of [Fe(II)PMA] $]^{+}$solid (solid line) and [Fe(II)PMA] ${ }^{+}$solution (dashed line) show two features at -7112 and $-7113.5 \mathrm{eV}$ with the first transition at $7112 \mathrm{eV}$ for [Fe(II)PMA]+ solid being more intense (Figure 5.5B). The spectrum of $\mathrm{Fe}(\mathrm{II}) \mathrm{BLM}$ (dotted line) has two weak features at approximately 7111.5 and $7113.5 \mathrm{eV}$ (Figure 5.5B). The feature at $\sim 7127 \mathrm{eV}$, attributed to the $1 \mathrm{~s} \longrightarrow 4 \mathrm{p}$ transition, is more intense and at slightly lower energy for $\mathrm{Fe}$ (I)BLM relative to that of [Fe(II)PMA] ${ }^{+}$ samples. The [Fe(II)PMA] ${ }^{+}$solid feature lies $\sim 1 \mathrm{eV}$ above that of [Fe(II)PMA] ${ }^{+}$in solution (Figure 5.5A).

The energy of the $1 s \rightarrow 4 p$ transition is dependent upon the effective nuclear charge of the absorbing metal atom. This charge is governed by a combination of effects including the formal oxidation state of the metal, the number and type of ligating atoms, and the coordination geometry. ${ }^{60-63}$ Differences in the edge energy position of the ferrous BLM and PMA complexes in Figure 5.5A thus reflect variation in the coordination number and the ligand-iron bonding interactions. One would expect the edge of a five-coordinate complex to be at lower energy than that of a six-coordinate complex ${ }^{63}$ due to reduced ligand repulsion, however the edge of [Fe(II)PMA]+ solid occurs at slightly higher energy than $[\mathrm{Fe}(\mathrm{II}) \mathrm{PMA}]^{+}$solution indicating increased charge donation to the ligand set. This likely reflects the pyrimidine backbonding (vide infra), which from the edge energy positions, follows the trend: [Fe(II)PMA] ${ }^{+}$solid $>$[Fe(II)PMA] $]^{+}$solution $>\mathrm{Fe}$ (II)BLM.

As discussed in detail in Chapter 4 , the $1 \mathrm{~s} \longrightarrow 3 \mathrm{~d}$ pre-edge feature can be used to probe the coordination number of an iron active site. The $1 \mathrm{~s} \rightarrow 3 \mathrm{~d}$ transition is formally electric dipole forbidden, but gains intensity through an allowed quadrupole transition and 


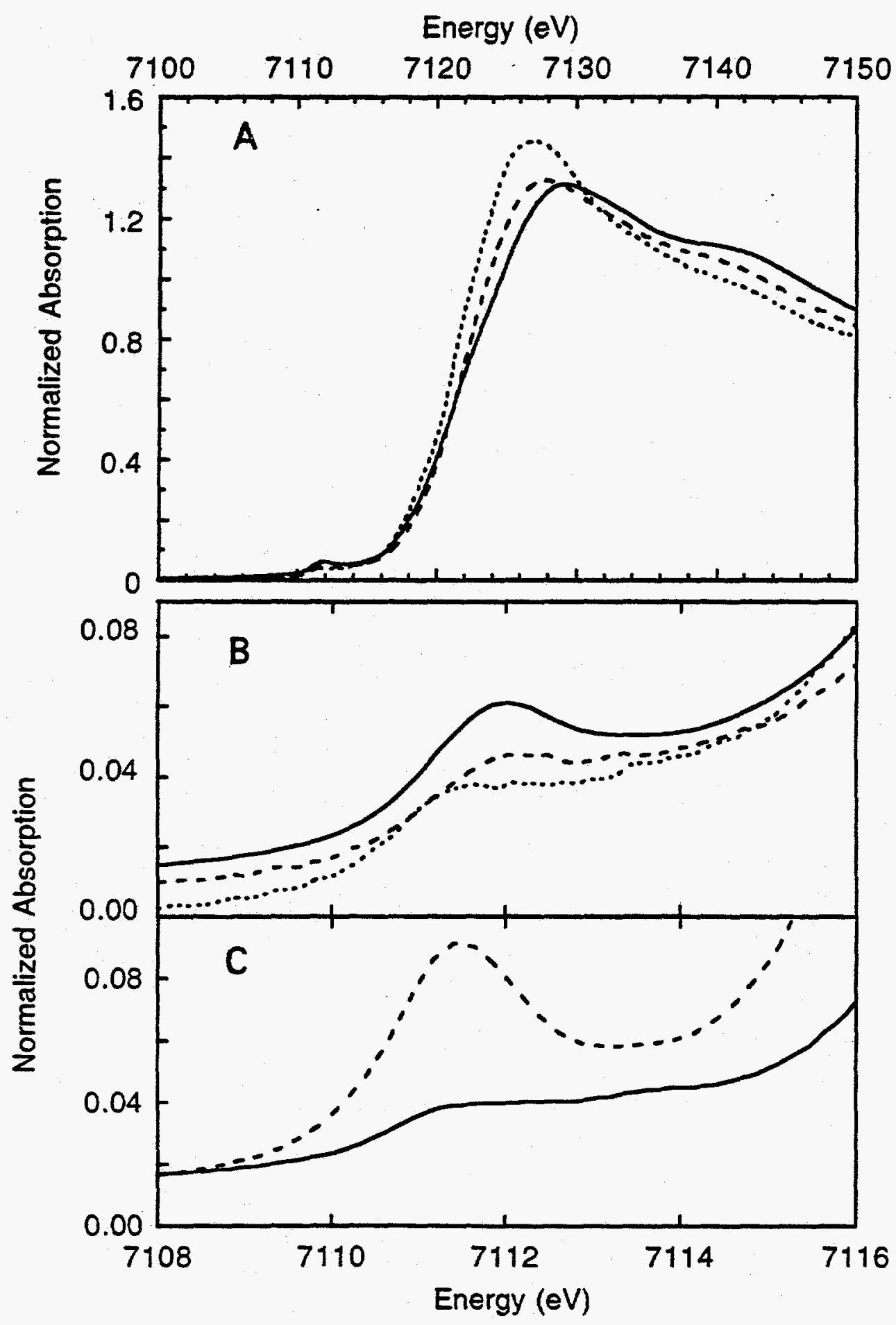

Figure 5.5. A) Fe-K XAS edge spectra of [Fe(II)PMA]+ solid (-), [Fe(II)PMA]+ solution (-.--), and Fe(II)BLM (......). B) Expansion of the 1s $\rightarrow$ 3d pre-edge region of $A$. C) The 1s $\rightarrow 3 d$ pre-edge region of a six-coordinate high spin ferrous model complex [Fe(imidazole) $6 \mathrm{Cl}_{2}\left(-\right.$ ) and a five-coordinate complex $\left[\mathrm{Fe}(\mathrm{TMC}) \mathrm{N}_{3}\right] \mathrm{BF}_{4}(-----)$. 
more dominantly by $4 \mathrm{p}$ mixing into the $3 \mathrm{~d}$ states as a result of the non-centrosymmetric environment of the metal site. It has been shown for ferric and ferrous complexes that when the symmetry of the iron site is lowered, the pre-edge intensity increases due to an increase in the 3d-4p mixing. ${ }^{63.64}$ When a high spin ferrous atom is in an octahedral site, three transitions are observed into ${ }^{4} \mathrm{~T}_{1},{ }^{4} \mathrm{~T}_{2}$, and ${ }^{4} \mathrm{~T}_{1}$ many-electron excited states. (see Chapter 4). The transitions into these states are only quadrupole allowed as the iron is in a centrosymmetric site and therefore there is no $4 \mathrm{p}-3 \mathrm{~d}$ mixing. Therefore, one observes three weak peaks (solid line in Figure 5.5C) positioned at approximately 7111.2, 7112.3, and $7113.67 \mathrm{eV}$ for octahedral high spin ferrous complexes with nitrogen ligation. The pre-edge area of five-coordinate high spin ferrous model complexes is greater than that of six-coordinate complexes (Figure 5.5C). This increase in intensity is due to $4 \mathrm{p}$ mixing into the $3 \mathrm{~d}$ orbitals as a result of the loss of the inversion center at the iron site. When an axially ligand is removed and the high spin ferrous atom is in a square pyramidal site, the intensity of the lower energy feature increases dramatically since there is now $4 \mathrm{pz}_{z}$ mixing into the $3 \mathrm{~d}^{2}$ orbital due to the loss of a center of inversion, as can be seen in the $\left(\mathrm{BF}_{4}\right)\left[\mathrm{Fe}(\mathrm{TMC}) \mathrm{N}_{3}\right]$ spectrum (dashed line in Figure 5.5C). Empirically the pre-edge of the $[\mathrm{Fe} \text { (II)PMA] }]^{+}$solution and $\mathrm{Fe}(\mathrm{II}) \mathrm{BLM}$ look much more similar in shape and intensity to the six-coordinate ferrous complex (Figure 5.5B and 5.5C). The pre-edge of [Fe(II)PMA]+ solid has more intensity in the lowest energy feature, as does [Fe(TMC) $\left.\mathrm{N}_{3}\right] \mathrm{BF}_{4}$, which is consistent with the $\mathrm{MCD}$ results of [Fe(II)PMA] $]^{+}$solid being five-coordinate. ${ }^{47}$ However, the first pre-edge transition of $[\mathrm{Fe}(\mathrm{II}) \mathrm{PMA}]^{+}$solid is not as intense as that of $\left[\mathrm{Fe}(\mathrm{TMC}) \mathrm{N}_{3}\right] \mathrm{BF}_{4}$. This could be due to the $\mathrm{Fe} 4 \mathrm{p}_{2}$ orbital of [Fe(II)PMA]+ solid mixing with the $\pi^{*}$ orbital of the pyrimidine. This would diminish the intensity of the pre-edge feature since for square pyramidal geometry the $4 \mathrm{p}_{z}$ orbital should be the dominant iron p-orbital mixing into the d-orbitals based on its lower energy ${ }^{62}$ and the lack of inversion along the $\mathrm{z}$-axis. This $4 \mathrm{p}_{\mathrm{z}}$-pyrimidine $\pi^{*}$ mixing would shift electric dipole intensity from the pre-edge into a higher energy region of the XAS spectrum.

5.3.3.2. EXAFS. EXAFS studies of [Fe(II)PMA]+ solid, [Fe(II)PMA]+ solution and $\mathrm{Fe}(\mathrm{II}) \mathrm{BLM}$ were also pursued to obtain metrical information on the iron active site. The EXAFS spectra of [Fe(II)PMA]+ solid, [Fe(II)PMA]+ solution and Fe(II)BLM are shown in Figure 5.6A and the Fourier transforms (FTs), taken over the $k$ range of 3.5 12.5 $\AA^{-1}$, are shown Figure 5.6B. Curve-fitting was performed on filtered first coordination shell contributions with FT backtransform windows given in Table 5.3 over the $k$ range $4-12 \AA^{-1}$ varying bond distances and coordination numbers. The results from the curve-fitting analysis are presented in Table 5.3. It should be noted that EXAFS analysis cannot readily distinguish between $\mathrm{O}$ and $\mathrm{N}$ coordination. However, given the 


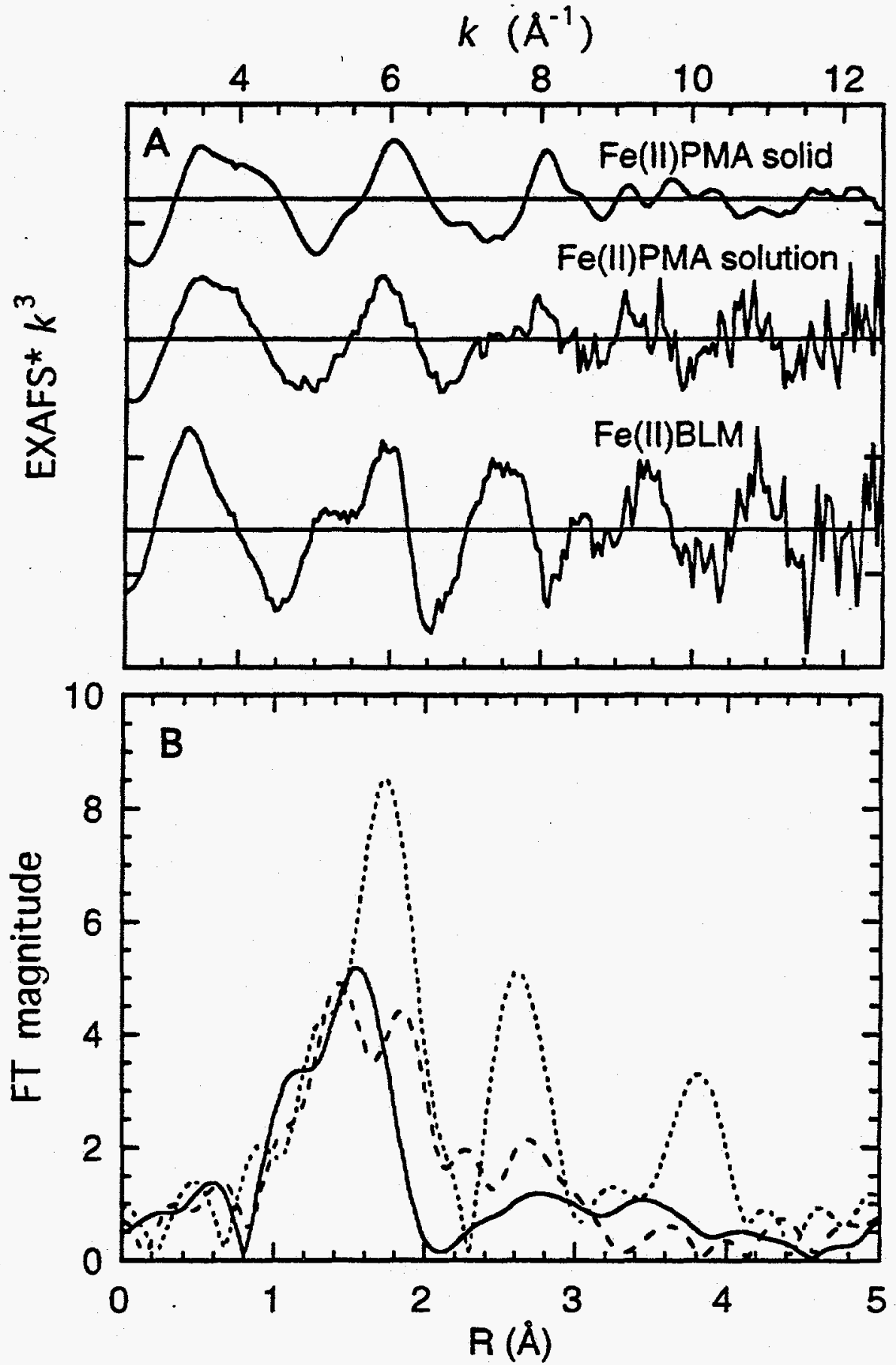

Figure 5.6. A) EXAFS data $\left({ }^{*} k^{3}\right)$ for [Fe(II)PMA] ${ }^{+}$solid, [Fe(II)PMA] ${ }^{+}$solution, and $\mathrm{Fe}$ (II)BLM (the ordinate scale is 5 between major tick marks on an absolute scale from the abscissa with solid horizontal lines indicating the zero point of each plot). B) The Fourier transforms (non-phase shift corrected) over the $k$-range $3.5-12.5 \AA^{-1}$ of the EXAFS data for [Fe(II)PMA] $]^{+}$solid (-), [Fe(II)PMA]+ solution (---.-), and $\mathrm{Fe}(\mathrm{II}) \mathrm{BLM}$ $(\cdots \cdots)$. 
Table 5.3. Summary of EXAFS Curve-Fitting Results.

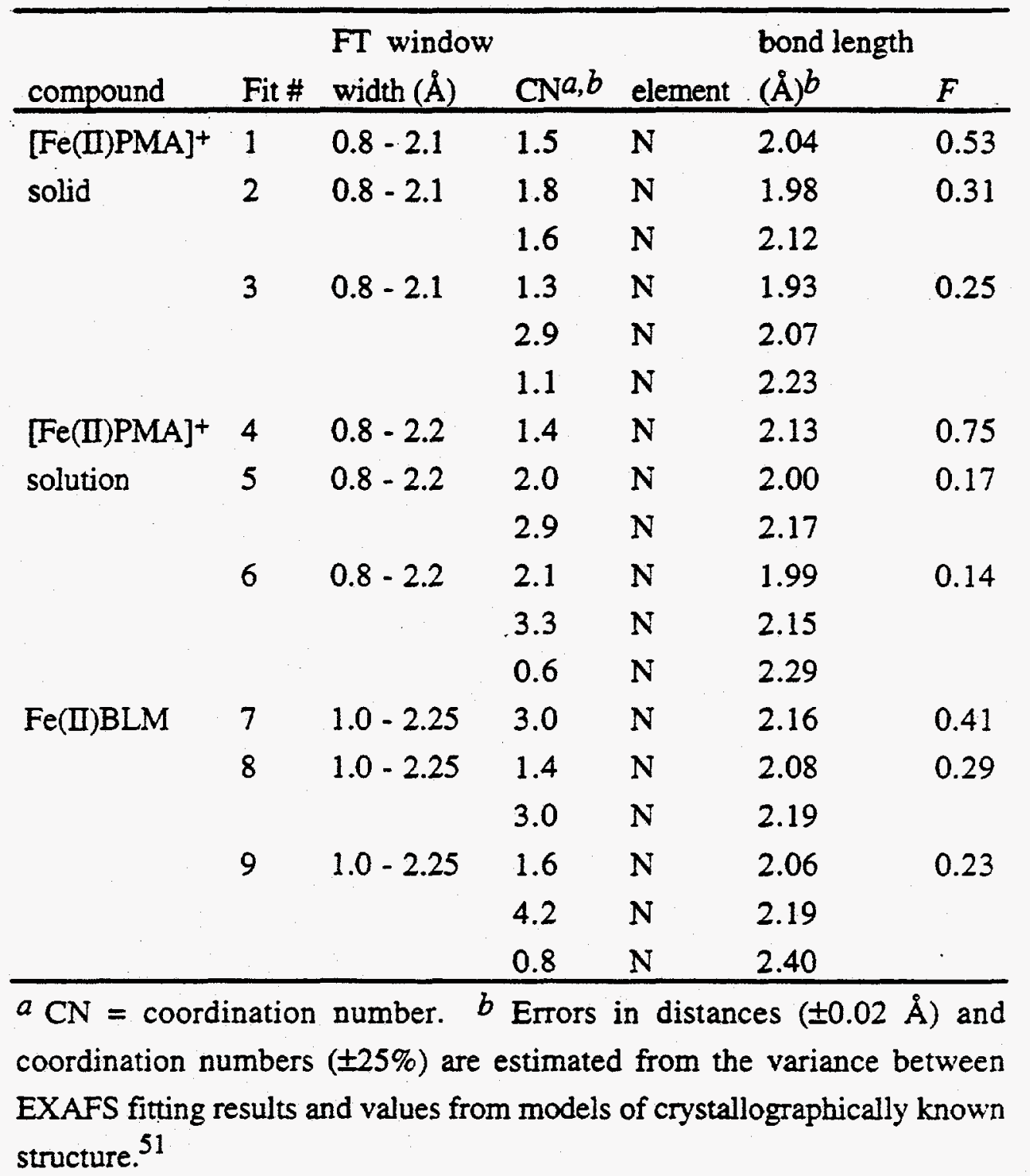



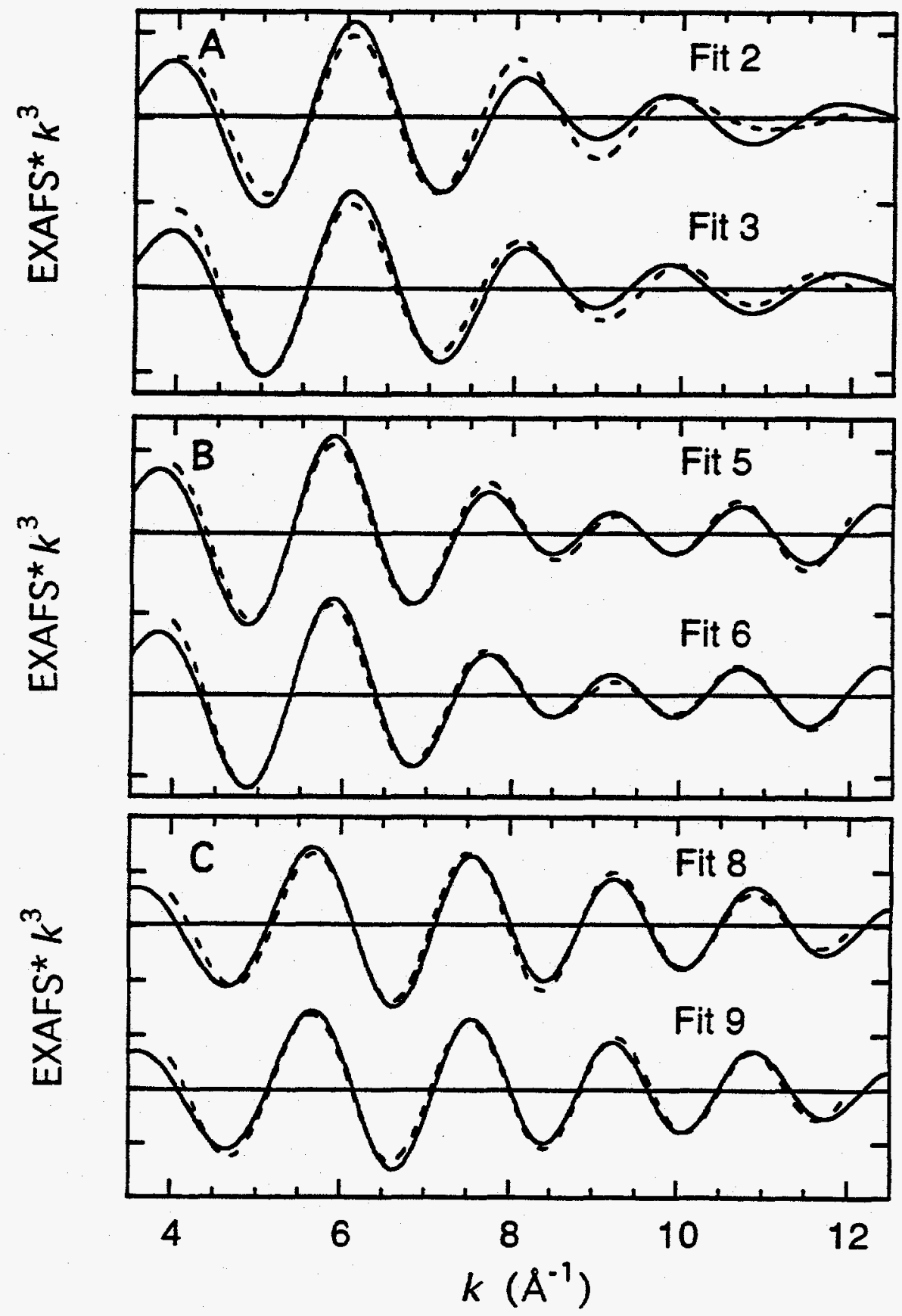

Figure 5.7. Empirical first shell fits to the Fourier-filtered EXAFS data with the solid lines representing the experimental data and the dashed lines representing the fits to the data. A) Fits 2 and 3 to the $[\mathrm{Fe}(\mathrm{II}) \mathrm{PMA}]^{+}$solid data (Table 5.3). B) Fits 5 and 6 to the [Fe(II)PMA]+ solution data. C) Fits 8 and 9 to the Fe(II)BLM data. (The ordinate scale is 2 between consecutive tick marks with solid horizontal lines going through the zero point of each plot.) 
likely ligation, only $\mathrm{N}$ parameters were used in the fits to the data. The EXAFS data of [Fe(II)PMA] ${ }^{+}$solid could not be adequately fit with one shell of $\mathrm{N}$ atoms (Fit 1). The "goodness of fit parameter", $F$, was dramatically reduced when a second shell of $\mathrm{N}$ was added to the fit (Fit 2) giving $N$ distances of 1.98 and $2.12 \AA$. However, the coordination number of 3.4 was still low and there was a mismatch in the fit to the data in the higher $k$ region. Thus, a third shell of $\mathrm{N}$ was added to the fit (Fit 3 ). The $F$ value was somewhat reduced and the fit matched the data more closely in the higher $k$ region, (Figure 5.7A) giving the best fit to the data with $1 \mathrm{~N}$ at $1.93 \AA, 3 \mathrm{~N}$ at $2.07 \AA$, and $1 \mathrm{~N}$ at $2.23 \AA$. The [Fe(II)PMA] ${ }^{+}$solution data also could not be fit adequately with a single shell of $\mathrm{N}$ (Fit 4). The $F$ value dropped by a factor of 4 when a second shell of $\mathrm{N}$ was added (Fit 5), giving 2 $\mathrm{N}$ at $2.00 \AA$ and $3 \mathrm{~N}$ at $2.17 \AA$. Inclusion of a third shell of $\mathrm{N}$ (Fit 6 ) did not substantially improve the fit (Figure 5.7B) and this contribution bad a relatively low coordination number of 0.6. Thus, the physical significance of the contribution at $2.29 \AA$ is more questionable. The Fe(I)BLM EXAFS data could be fit reasonably well with one shell of $\mathrm{N}$ at $2.16 \AA$ (Fit 7). A two shell fit (Fit 8 ) improved the $F$ value and increased the coordination numbers. A three shell fit was also tried (Fit 9), however the fit to the data did not improve substantially (Figure 5.7C). The $F$ value improved slightly and the total coordination numbers increased to 6.6 ( $v$ s. 4.4 for Fit 8).

The average first shell distance of [Fe(II)PMA] ${ }^{+}$in solution is $\sim 0.1 \AA$ longer than that of the [Fe(II)PMA]+ solid, consistent with the Fe K-edge data which indicates that [Fe(II)PMA $]^{+}$solid is five-coordinate while [Fe(II)PMA] ${ }^{+}$solution is six-coordinate. Both [Fe(II)PMA]+ solid and [Fe(II)PMA]+ solution have a first coordination sphere that has several contributions, as is evident from the curve-fitting results and the multiple peaks in the FTs (Figure 5.6B). Fe(I)BLM has a more regular first shell with an average first shell distance of $2.16 \AA$ which is consistent with that of other six-coordinate high spin ferrous complexes $(2.16 \pm 0.03 \AA) .{ }^{65-68}$ Importantly, a short $\mathrm{Fe}(\mathrm{I})-\mathrm{N}$ contribution is present in all three complexes, which increases in length along the series [Fe(II)PMA] ${ }^{+}$solid (1.93 $\AA$ ) $<$ [Fe(II)PMA $]^{+}$solution $(2.00 \AA)<\mathrm{Fe}(\mathrm{II}) \mathrm{BLM}(2.06-2.08 \AA)$. This short $\mathrm{N}$ can reasonably be assigned to the pyrimidine based upon the high degree of covalency derived from Abs, MCD, and rR results. ${ }^{47}$ The trend in $\mathrm{Fe}-\mathrm{N}_{\mathrm{Pm}}$ distances correlates with the $\mathrm{Fe}$ K-edge energy positions and with differences in $\pi$-backbonding as discussed below.

\subsubsection{Discussion}

The results obtained from these XAS experiments on $\mathrm{Fe}(\mathrm{II}) \mathrm{BLM}$ and $[\mathrm{Fe}(\mathrm{II}) \mathrm{PMA}]^{+}$ can be used in conjunction with results from $A b s, M C D$, and $\mathrm{rR}$ spectroscopies to develop 
a detailed description of the geometric and electronic of the $\mathrm{Fe}(\mathrm{II}) \mathrm{BLM}$ active site. ${ }^{47}$ With the array of data in the literature regarding the solution structure of $F e(I I) B L M$, some controversy still remains as to the def nitive metal-chelating mode of this ligand. This problem has been addressed by comparison to [Fe(II)PMA] ${ }^{+}$, which has been found by molecular modeling to be most stable with the pyrimidine, imidazole, deprotonated amide and secondary amine coordinated equatorially and the primary amine bound axially, and is thought to have a solvent molecule coordinated at the open site in solution. ${ }^{69}$ Binding of solvent to the sixth site is confirmed from the ligand field $M C D$ spectra of solid and solution [Fe(II)PMA] $]^{+}{ }^{47}$ Solid [Fe(II)PMA] ${ }^{+}$has a ${ }^{5} \mathrm{E}_{\mathrm{g}}$ splitting of $6100 \mathrm{~cm}^{-1}$ indicating a five-coordinate, square pyramidal structure. In solution the ${ }^{5} \mathrm{E}_{\mathrm{g}}$ splitting for [Fe(II)PMA] decreases to $2110 \mathrm{~cm}^{-1}$ indicating addition of a solvent molecule to the vacant axial position. The increase in coordination number is consistent with a decrease in the XAS pre-edge intensity as shown in Figure 5.5 and an increase in $\mathrm{Fe}-\mathrm{N}$ bond lengths proceeding from five- to six-coordination as determined from the EXAFS analysis in Table 5.3. The similarities in the $M C D$ ligand field spectrum and $\Delta^{5} E_{g}$ splitting of $F e(I I) B L M$ to that of the $[\mathrm{Fe}(\mathrm{I}) \mathrm{PMA}]^{+}$in solution, ${ }^{47}$ as well as the comparable pre-edge shapes and intensities suggest that the coordination spheres of $\mathrm{Fe}$ (II)BLM and [Fe(II)PMA] ${ }^{+}$in solution are not markedly different. The EXAFS analysis does indicate an overall increase in the $\mathrm{Fe}-\mathrm{N}$ bond lengths of $\mathrm{Fe}$ (II)BLM relative to those of [Fe(II)PMA] ${ }^{+}$which is consistent with the increased steric bulk of the BLM ligand compared with the more compact PMAH framework. Thus, a proposed solution structure of Fe(II)BLM is given in Figure 16 of reference 47 which is based on the PMAH ligand set and the experimental evidence that solvent coordinates at the sixth position.

The steric constraints of the BLM ligand framework coupled with the labile coordination site occupied by the solvent molecule suggest that oxygen binding to the $\mathrm{Fe}^{2+}$ center occurs via a dissociative mechanism. This is supported by the fact that in solution the $\Delta^{5} E_{g}$ splitting in the room temperature $C D$ spectrum of $F e(I I) B L M$ increases to $-3600 \mathrm{~cm}^{-1}$ indicating that an axial ligand, likely $\mathrm{H}_{2} \mathrm{O}$ or $\mathrm{OH}^{-}$, in $\mathrm{Fe}(\mathrm{II}) \mathrm{BLM}$ is weakly coordinated to the iron. Our spectral studies ${ }^{47}$ of solid [Fe(II)PMA] ${ }^{+}$have defined the five-coordinate complex as being square pyramidal which produces an open coordination position available for $\mathrm{O}_{2}$ reaction. On the basis of the similarity in spectral features and activation chemistry of [Fe(II)PMA] $]^{+}$and $\mathrm{Fe}(\mathrm{II}) \mathrm{BLM}$ in solution, this square pyramidal structure is reasonably correlated with the reactive intermediate in the mechanism of $\mathrm{O}_{2}$ binding to $\mathrm{Fe}(\mathrm{II}) \mathrm{BLM}$.

In addition to the geometric structure obtained for $\mathrm{Fe}(\mathrm{II}) \mathrm{BLM}$ through the MCD excited state ligand field and XAS analyses, the electronic structures of $\mathrm{Fe}(\mathrm{I}) \mathrm{BLM}$ and 
[Fe(II)PMA]+ have been elucidated through MCD ligand field ground state, charge transfer excited state, and $\mathrm{rR}$ spectroscopies. ${ }^{47}$ From the ligand field analysis, [Fe(II)PMA]+ solid exhibits nested saturation magnetization curves consistent with the five-coordinate, square pyramidal geometry. The value of $\mathrm{V} / 2 \Delta(0.33)$ derived from this analysis indicates that the structure is highly rhombic due to the short Fe-N bond distance in the equatorial plane determined from the EXAFS results which supports the presence of strong metal-ligand $\pi$ backbonding. In solution [Fe(II)PMA] ${ }^{+}$and $\mathrm{Fe}(\mathrm{II}) \mathrm{BLM}$ also exhibit nested saturation magnetization curves that result in unusually small values of the spin Hamiltonian parameter $(\delta)$, not generally observed for six-coordinate non-heme ferrous active sites. This corresponds to a large splitting of the $t_{2 g}$ set of $d$-orbitals $(-\Delta)$ which requires strong $\pi$-bonding interactions between the metal and pyrimidine $\pi^{*}$ orbitals. The values of V/2 $\Delta$ of 0.24 for [ $\mathrm{Fe}(\mathrm{II}) \mathrm{PMA}]^{+}$and 0.18 for $\mathrm{Fe}$ (II)BLM indicate a more moderate degree of rhombic distortion which decreases with increasing $\mathrm{Fe}-\mathrm{N}$ bond length. Thus the magnitudes of $\delta$ (and $-\Delta$ ) are different from typical six-coordinate model complexes indicating that strong $\pi$-bonding effects are present, consistent with the EXAFS results exhibiting a short $\mathrm{Fe}-\mathrm{N}$ distance.

An important deviation from non-heme ferrous behavior demonstrated by $\mathrm{Fe}(\mathrm{II}) \mathrm{BLM}$ and [Fe(II)PMA]+ is the existence of low energy CT transitions analogous to $\mathrm{d}_{\pi} \longrightarrow \pi^{*}$ MLCT transitions in heme systems. ${ }^{70}$ The presence of moderately intense, low energy MLCT transitions in the low temperature $A b s$ and MCD spectra of [Fe(II)PMA] ${ }^{+}$ and $\mathrm{Fe}(\mathrm{I}) \mathrm{BLM}^{47}$ represents an important deviation from other non-heme iron centers and reflects high covalency. The MLCT transition energies and intensities determine the degree of metal-ligand $\pi$-backbonding which decreases along the series solid [Fe(II)PMA] ${ }^{+}>$ solution $[\mathrm{Fe}(\mathrm{II}) \mathrm{PMA}]^{+}>\mathrm{Fe}(\mathrm{II}) \mathrm{BLM}$. Assignment of these bands as $\mathrm{Fe}(\mathrm{II}) \longrightarrow$ pyrimidine MLCT transitions is derived from the strong resonance enhancement of the pyrimidine normal modes at $680,744,1519$, and $1542 \mathrm{~cm}^{-1}$ in the [Fe(II)PMA] ${ }^{+}$Raman spectrum, thereby implicating pyrimidine as the specific ligand associated with the large $\mathrm{d}_{\pi}$ splitting and short $\mathrm{Fe}-\mathrm{N}$ bond. ${ }^{47}$

These MLCT transitions in Fe(II)BLM and [Fe(II)PMA]+ indicate the presence of significant $\pi$-backbonding not observed for other non-heme iron centers. This results from the presence of five strong field nitrogen ligands (with solvent occupying the sixth site) complexed to the $\mathrm{Fe}^{2+}$ which raises the energy of the d-manifold, reduces the energy gap between the $d_{\pi}$ and ligand $\pi^{*}$ orbitals, and increases the propensity for metal-ligand backbonding at the site. This $\pi$-backbonding contributes to the unique chemistry and reactivity of $\mathrm{Fe}$ (II)BLM. Unlike other non-heme iron active sites, $\mathrm{Fe}$ (II)BLM reversibly binds $\mathrm{CO}$ and reacts with $\mathrm{NO}$ and other strong field ligands to form low spin complexes. 
Addition of this sixth strong field ligand induces the low spin conversion through an increase in the already sizable splitting (i.e., all nitrogen ligands) of the d-orbitals (10Dq) which overcomes the electron-spin pairing energy. The unusual binding of $\mathrm{CO}$ derives from the tendency of the Fe(II)BLM site to backbond. Similar "symbiotic" behavior is common in inorganic coordination complexes and is rationalized by the relative softness (i.e., polarizability) of the metal center induced by the coordination sphere. ${ }^{71}$ Formation of the $\mathrm{Fe}(\mathrm{II}) \mathrm{BLM}-\mathrm{CO}$ complex is further promoted by the increased ligand field stabilization energy of the resultant low spin $d^{6}$ configuration. The presence of backbonding also contributes to the unusual $\mathrm{O}_{2}$ reactivity of this site relative to other non-heme $\mathrm{Fe}$ systems. Backbonding to the pyrimidine ligand decreases the extent of charge transfer to the $\mathrm{O}_{2}$ which shifts the equilibrium in eq. (1) to the left, making FeBLM less likely to dissociate $\mathrm{O}_{2}^{-}$, and contributing to the reversible binding of $\mathrm{O}_{2}$ by the site.

$$
\mathrm{Fe}^{2+}+\mathrm{O}_{2} \rightleftharpoons \mathrm{Fe}^{3+}-\mathrm{O}_{2}^{-} \rightleftharpoons \mathrm{Fe}^{3+}+\mathrm{O}_{2}^{-}
$$

This reduced charge transfer to the bound superoxide also enhances its propensity for further reduction to form the $\mathrm{Fe}(\mathrm{III})-\mathrm{O}_{2}{ }^{2-}$ site of activated $\mathrm{BLM}$. This is in contrast to a pathway observed in other non-heme iron systems where strong $\pi$-backbonding is absent. In these cases, a diffusable superoxide radical may be formed by direct autoxidation of the $\mathrm{Fe}^{2+}$ site (eq. (1), right). ${ }^{72,73}$ In the presence of DNA, a superoxide radical would lead to non-selective cleavage products which are not observed in BLM chemistry. 10

The low energy MLCT transitions, the reversible binding of $\mathrm{O}_{2}$, and the formation of low spin complexes with $\mathrm{CO}$ and NO suggest that $\mathrm{Fe}(\mathrm{II}) \mathrm{BLM}$ more closely parallels heme rather than non-heme iron chemistry. Yet the differences in these ferrous centers are significant as heme systems exhibit more intense $\left(\varepsilon=10,000 \mathrm{M}^{-1} \mathrm{~cm}^{-1}\right)$, lower energy $\left(\sim 10,000 \mathrm{~cm}^{-1}\right)$ MLCT transitions than $\mathrm{Fe}(\mathrm{II}) \mathrm{BLM}$ indicating much greater $\mathrm{Fe}^{2+}$ backbonding into the tetrapyrrole macrocycle. ${ }^{70}$ This difference in backbonding correlates with differences in the $\mathrm{O}_{2}$ reactivity of BLM relative to heme iron centers. In particular, the oxygen intermediate of cytochrome $\mathrm{P}-450$ is thought to be a ferryl species ${ }^{26,74}$ which is structurally distinct from the peroxide-level intermediate of activated BLM. ${ }^{27,28}$ Our results suggest that other non-heme $\mathrm{Fe}$ enzymes which exhibit little or no low energy MLCT transitions relative to $\mathrm{Fe}$ (II)BLM may be expected to form intermediates which more closely resemble the ferric-peroxide species of activated BLM than the ferryl species of heme systems.

In summary, these studies have provided significant insight into the oxygen reactivity of $\mathrm{Fe}$ (II)BLM. The lability of high spin iron combined with steric constraints of 
the BLM ligand framework and its weaker axial interaction with solvent support a dissociative mechanism for $\mathrm{O}_{2}$ reactivity. These spectroscopic studies of solid [Fe(II)PMA $]^{+}$have defined the nature of such a five-coordinate intermediate as square pyramidal which provides an open coordination position for reaction with $\mathrm{O}_{2}$. A major electronic structure difference between $\mathrm{Fe}(\mathrm{II}) \mathrm{BLM}$ (and [Fe(II)PMA] ${ }^{+}$) and other non-heme ferrous sites is the presence of low energy CT transitions which reflect strong $\mathrm{Fe}$ (II)->pyrimidine backbonding. Relative to non-heme iron complexes this backbonding results in reduced charge donation to the $\mathrm{O}_{2}$ which 1) contributes to the reversible binding of $\mathrm{O}_{2}$ exhibited by $\mathrm{Fe}(\mathrm{II}) \mathrm{BLM}, 2$ ) stabilizes the initial $\mathrm{Fe}(\mathrm{III})-\mathrm{O}_{2}$ - intermediate from loss of $\mathrm{O}_{2}^{-}$and reaction with substrate, and 3) promotes the further reduction of $\mathrm{Fe}(\mathrm{III}) \mathrm{BLM}-\mathrm{O}_{2}^{-}$ to form activated BLM. Despite generally being considered a non-heme iron system due to the absence of an extensive delocalized $\pi$ network, the existence of low energy MLCT transitions with reasonable intensity, hence the presence of some backbonding, identify BLM as an important link bridging the chemistry of non-heme and heme active sites.

\subsubsection{Acknowledgments}

Pradip Mascharak and Richard Guarjardo of UC Santa Cruz provided the $[\mathrm{Fe}(\mathrm{II})(\mathrm{PMA})] \mathrm{Cl} \cdot \mathrm{MeOH}$ sample. Kelly Loeb and Jeff Zaleski prepared the Fe(II)BLM sample and performed the Abs, $r R$, and MCD experiments alluded to in the Discussion. This work was supported by grants from the National Institutes of Health (GM40392, E.I.S.) and National Science Foundation (CHE-9121576, K.O.H.). SSRL is supported by the U.S. Department of Energy, Office of Basic Energy Science, Divisions of Chemical and Materials Sciences, and in part by the National Institutes of Health, Biomedical Research Technology Program (RR-01209), and the US Department of Energy, Office of Health and Environmental Research.

\subsubsection{References and Notes}

(1) Umezawa, H.; Maeda, K.; Takeuchi, T.; Oakami, Y. J. Antibiot. 1966, 19, 200.

(2) Umezawa, H.; Surhara, Y.; Takita, T.; Maeda, K. J. Antibiot. 1966, 19, 210.

(3) Umezawa, H. Biomed. 1973, 18, 459.

(4) Hutchinson, F.; Povirk, L. F. In Bleomycin: Chemical, Biochemical, and Biological Aspects; Hecht, S. M., Ed.; Springer-Verlag: New York, 1979; p 255.

(5) Chien, M.; Grollman, A. P.; Horwitz, S. B. Biochemistry 1977, 16, 3641. 
(6) Kross, J.; Henner, W. D.; Haseltine, W. A.; Rodriquez, L.; Levin, M. D.; Hecht, S. M. Biochemistry 1982, 21, 3711.

(7) Fisher, L. M.; Kuroda, R.; Sakai, T. T. Biochemistry 1985, 24, 3199.

(8) Umezawa, H. Pure Appl. Chem. 1971, 28, 665.

(9) DeReimer, L. H.; Meares, C. F.; Goodwin, D. A.; Diamanti, C. I. J. Med. Chem. $1979,22,1019$.

(10) Stubbe, J.; Kozarich, J. W. Chem. Rev. 1987, 87, 1107.

(11) Petering, D. H.; Bymes, R. W.; Antholine, W. E. Chem.-Biol. Interactions 1990, $73,133$.

(12) Sausville, E. A.; Peisach, J.; Horwitz, S. B. Biochemistry 1978, 17, 2740.

(13) Ishida, R.; Takahashi, T. Biochem. Biophys. Res. Commun. 1975, 66, 1432.

(14) Oppenheimer, N. J.; Chang, C.; Rodriguez, L. O.; Hecht, S. M. J. Biol. Chem. 1981, 256, 1514.

(15) Ehrenfeld, G. M.; Murugesan, N.; Hecht, S. M. Inorg. Chem. 1984, 23, 1496.

(16) Lown, J. W.; Sim, S.-K. Biochem. Biophys. Res. Commun. 1977, 77, 1150.

(17) Sausville, E. A.; Peisach, J.; Horwitz, S. B. Biochem. Biophys. Res. Commun. $1976,73,814$.

(18) Sausville, E. A.; Stein, R. W.; Peisach, J.; Horwitz, S. B. Biochemistry 1978, $17,2746$.

(19) Burger, R. M.; Peisach, J.; Horwitz, S. B. J. Biol. Chem. 1981, 256, 11636.

(20) Giloni, L.; Takeshita, M.; Johnson, F.; Iden, C.; Grollman, A. P. J. Biol. Chem. $1981,256,8608$.

(21) Burger, R. M.; Berkowitz, A. R.; Peisach, J.; Horwitz, S. B. J. Biol. Chem. $1980,255,11832$.

(22) Fulmer, P.; Petering, D. H. Biochemistry 1994, 33, 5319.

(23) Albertini, J.-P.; Garnier-Suillerot, A.; Tosi, L. Biochem. Biophys. Res. Commun. 1982, 104, 557.

(24) Burger, R. M.; Kent, T. A.; Horwitz, S. B.; Münck, E.; Peisach, J. J. Biol. Chem. 1983, 258, 1559.

(25) Hecht, S. M. Acc. Chem. Res. 1986, 19, 383.

(26) Ortiz de Montellano, P. R. Cytochrome P-450: Structure, Mechanism, and Biochemistry; Plenum Press: New York, 1986, p 429.

(27) Sam, J. W.; Tang, X.-J.; Peisach, J. J. Am. Chem. Soc. 1994, 116, 5250.

(28). Westre, T. E.; Loeb, K. E.; Zaleski, J. M.; Hedman, B.; Hodgson, K. O.; Solomon, E. I. J. Am. Chem. Soc. 1995, 117, 1309. 
(29) Iitaka, Y.; Nakamura, H.; Nakatani, T.; Muraoka, Y.; Fujii, A.; Takita, T.; Umezawa, H. J. Antibiot. 1978, 31, 1070.

(30) Sugiura, Y.; Suzuki, T.; Otsuka, M.; Kobayashi, S.; Ohno, M.; Takita, T.; Umezawa, H. J. Biol. Chem. 1983, 258, 1328.

(31) Oppenheimer, N. J.; Chang, C.; Chang, L.-H.; Ehrenfeld, G.; Rodriguez, L. O.; Hecht, S. M. J. Biol. Chem. 1982, 257, 1606.

(32) Oppenheimer, N. J.; Rodriguez, L. O.; Hecht, S. M. Proc. Natl. Acad. Sci. USA 1979, 76, 5616.

(33) Akkerman, M. A. J.; Neijman, E. W. J. F.; Wijmenga, S. S.; Hilbers, C. W.; Bermel, W. J. Am. Chem. Soc. 1990, 112, 7462.

(34) Sugiura, Y.; Kuwahara, J.; Suzuki, T. FEBS Letters 1985, 182, 39.

(35) Sugiura, Y.; Ishizu, K. J. Inorg. Biochem. 1979, 11, 171.

(36) Sugiura, Y. J. Antibiot. 1978, 31, 1206.

(37) Sugiura, Y.; Ishizu, K.; Miyoshi, K. J. Antibiot. 1979, $32,453$.

(38) Sugiura, Y. J. Am. Chem. Soc. 1980, 102, 5208.

(39) Guajardo, R. J.; Hudson, S. E.; Brown, S. J.; Mascharak, P. K. J. Am. Chem. Soc. 1993, 115, 7971.

(40) Henichart, J.-P.; Bernier, J.-L.; Helbecque, N.; Houssin, R. Nucl. Acid Res. $1985,13,6703$.

(41) Kenani, A.; Bailly, C.; Helbecque, N.; Houssin, R.; Bernier, J.-L.; Henichart, J.P. Eur. J. Med. Chem. 1989, 24, 371.

(42) Sugiyama, T.; Ohno, M.; Shibasaki, M.; Otsuka, M.; Sugiura, Y.; Kobayashi, S.; Maeda, K. Heterocycles 1994, 37, 275.

(43) Shepherd, R. E.; Lomis, T. J.; Koepsel, R. R.; Hedge, R.; Mistry, J. S. Inorg. Chim. Acta 1990, 171, 139.

(44) Brown, S. J.; Tao, X.; Wark, T. A.; Stephan, D. W.; Mascharak, P. K. Inorg. Chem. 1988, 27, 1581.

(45) Muetterties, M.; Mascharak, P. K.; Cox, M. B.; Arora, S. K. Inorg. Chim. Acta $1989,160,123$.

(46) The chemical formula for PMAH is 2-((N-(aminoethyl)amino)methyl)-4-(N-(2-(4imidazolyl)ethyl)carbomoyl)-5-bromopyrimidine where $\mathrm{H}$ is the dissociable amide proton. PMAH forms a 1:1 iron complex with pyrimidine, imidazole, amide, secondary and primary amine functionalities.

(47) Loeb, K. E.; Zaleski, J. M.; Westre, T. E.; Guajardo, R. J.; Mascharak, P. K.; Hedman, B.; Hodgson, K. O.; Solomon, E. I. J. Am. Chem. Soc. 1995, 117, 4545 . 
(48) Brown, S. J.; Mascharak, P. K.; Stephan, D. W. J. Am. Chem. Soc. 1988, 110, 1996.

(49) Scott, R. A.; Hahn, J. E.; Doniach, S.; Freeman, H. C.; Hodgson, K. O. J. Am. Chem. Soc. 1982, 104, 5364.

(50) Cramer, S. P.; Tench, O.; Yocum, M.; George, G. N. Nucl. Instrum. Methods Phys. Res. 1988, A266, 586.

(51) Cramer, S. P.; Hodgson, K. O.; Stiefel, E. I.; Newton, W. E. J. Am. Chem. Soc. 1978, 100, 2748.

(52) Cramer, S. P.; Hodgson, K. O. Prog. Inorg. Chem. 1979, $25,1$.

(53) Scott, R. A. Methods Enzymol. 1985, 117, 414.

(54) Iball, J.; Morgan, C. H. Acta Crystallogr. 1967, 23, 239.

(55) Roof, R. B., Jr. Acta Crystallogr. 1956, 9, 781.

(56) Johansson, L.; Molund, M.; Oskarsson, A. Inorg. Chim. Acta. 1978, $31,117$.

(57) Johansson, L. Chemica Scripta. 1976, 9, 30.

(58) Hodges, K. D.; Wollman, R. G.; Barefield, E. K.; Hendrickson, D. N. Inorg. Chem. 1977, 16, 2746.

(59) Burbridge, C. D.; Goodgame, D. M. L. Inorg. Chim. Acta 1970, 4, 231.

(60) Shulman, R. G.; Yafet, Y.; Eisenberger, P.; Blumberg, W. E. Proc. Natl. Acad. Sci. USA 1976, 73, 1384.

(61) Cramer, S. P.; Eccles, T. K.; Kutzler, F. W.; Hodgson, K. O.; Mortenson, L. E. J. Am. Chem. Soc. 1976, 98, 1287.

(62) Kau, L.-S.; Spira-Solomon, D. J.; Penner-Hahn, J. E.; Hodgson, K. O.; Solomon, E. I. J. Am. Chem. Soc. 1987, 109, 6433.

(63) Roe, A. L.; Schneider, D. J.; Mayer, R. J.; Pyrz, J. W.; Widom, J.; Que, L., Jr. J. Am. Chem. Soc. 1984, 106, 1676.

(64) Randall, C. R.; Shu, L.; Chiou, Y.-M.; Hagen, K. S.; Ito, M.; Kitajima, N.; Lachicotte, R. J.; Zang, Y.; Que, L., Jr. Inorg. Chem. 1995, 34, 1036.

(65) Montgomery, H.; Chastain, R. V.; Natt, J. J.; Witkowska, A. M.; Lingafelter, E. C. Acta Cryst. 1967, 22, 775.

(66) Price, D. C. Can. J. Phys. 1987, 65, 1280.

(67) Miller, L. L.; Jacobsen, R. A.; Chen, Y.-S.; Kurtz, D. M., Jr. Acta Cryst. 1989, C45, 527.

(68) Kitajima, N.; Fukui, H.; Moro-oka, Y. J. Am. Chem. Soc. 1990, 112, 6402.

(69) Wu, Y.-D.; Houk, K. N.; Valentine, J. S.; Nam, W. Inorg. Chem. 1992, 31, 718. 
(70) Lever, A. B. P.; Gray, H. B. Iron Porphyrins: Part One; Addison-Wesley Publishing Co., Inc.: Reading, 1983.

(71) Jørgensen, C. K. Inorg. Chem. 1964, 3, 1201.

(72) Feig, A. L.; Lippard, S. J. Chem. Rev. 1994, 94, 759.

(73) Brown, E. R.; Mazzarella, J. D. J. Electroanal. Chem. 1987, 222, 173.

(74) Dawson, J. H. Science 1988, 240, 433.

(75) Solomon, E. I.; Pavel, E. G.; Loeb, K. E.; Campochiaro, C. Coord. Chem. Rev. in press. 


\subsection{Activated Bleomycin}

\subsubsection{Introduction}

Bleomycin (BLM), a glycopeptide antibiotic produced by strains of Streptomyces verticillus, is currently used in treatment against a variety of carcinomas and lymphomas. ${ }^{1}$ Its therapeutic activity involves selective DNA cleavage at certain GT and GC sites in the presence of metal ions (in particular $\mathrm{Fe}^{2+}$ ) and dioxygen. ${ }^{2,3}$ The coordination environment of the iron is believed to be square pyramidal with the iron ligated to five nitrogens from histidine, pyrimidine, a deprotonated peptide function, and primary and secondary amine groups (see section 5.3). ${ }^{4}$ Kinetic and spectral studies have demonstrated that the activation mechanism involves high spin Fe(II)BLM reacting with dioxygen and an electron to form activated BLM which is formally at the peroxo-ferric level. ${ }^{5}$ Activated BLM (and the activated form of the analogous PMA model complex) ${ }^{6}$ is the first mononuclear non-heme iron oxygen intermediate stable enough for detailed spectroscopic study. DNA degradation by activated BLM involves $\mathrm{C}-\mathrm{H}$ bond cleavage at the $\mathrm{C}^{\prime}$ 'position of deoxyribose moieties and results in the production of base propenals. ${ }^{3,7}$ This mechanism is similar to the monooxygenation mechanism of cytochrome P-450 and has led researchers to postulate an oxo-ferryl BLM intermediate, ${ }^{3}$ as has been generally considered to be present in P-450 chemistry. Alternatively, spectroscopic model studies ${ }^{6,8}$ and mass spectrometric studies ${ }^{9}$ have indicated activated BLM to have an iron (III)-peroxide site. In this study, X-ray absorption spectroscopy (XAS) has been used to directly probe the oxidation and spin state of the iron in activated BLM and to determine if a short iron-oxo bond is present, which would be characteristic of the oxo-ferryl species of heme iron. Both the pre-edge and edge regions of the $\mathrm{Fe}$ $\mathrm{K}$-edge spectra indicate that activated BLM is a low spin ferric complex. Bond distances obtained from extended X-ray absorption fine structure (EXAFS) are similar to those in low spin $\mathrm{Fe}(\mathrm{III}) \mathrm{BLM}$ and show no evidence for a short iron-oxo bond. These data indicate that the Fe(III)-peroxide formalism is an appropriate description of the iron center in activated BLM and suggest that such an intermediate may play an important role in activating $\mathrm{O}_{2}$ for further chemistry in the catalytic cycles of mononuclear non-heme iron enzymes. 


\subsubsection{Experimental Section}

Blenoxane (a mixture of $60 \%$ BLM A2, $30 \% \mathrm{BLM} \mathrm{B}_{2}$, and $10 \%$ other BLMs) was obtained as a gift from Bristol-Meyers Squibb and used without further purification. For all XAS samples a $5 \mathrm{mM}$ apo-BLM solution consisting of equal volumes of $300 \mathrm{mM}, \mathrm{pH}$ 7.0, HEPES buffer (Sigma) and ethylene glycol (Mallinckrodt) was prepared. Subsequent anaerobic and aerobic additions of $5 \mu \mathrm{L}$ of a $\mathrm{Fe}\left(\mathrm{NH}_{4}\right)_{2}\left(\mathrm{SO}_{4}\right)_{2} \cdot 6 \mathrm{H}_{2} \mathrm{O}(\mathrm{FeAS}$; MCB Manufacturing Chemists, Inc.) stock solution yielded $\sim 4 \mathrm{mM}$ Fe(II)BLM and Fe(III)BLM, respectively. Sample integrity (< 5\% ferric impurity) was confirmed by optical absorption with an HP 8452A diode array spectrophotometer and by electron paramagnetic resonance (EPR) using a Bruker ER 220D-SRC spectrometer interfaced to an IBM XT computer and a Bruker ER 042MRH microwave bridge ( $X$-band). The $\mathrm{Fe}$ (II)BLM sample was syringed into the XAS sample cell (Lucite cell $23 \times 1 \times 3 \mathrm{~mm}$ with $37 \mu \mathrm{m}$ Kapton windows) under a $\mathrm{N}_{2}$ atmosphere and frozen in liquid $\mathrm{N}_{2}$ before exposure to air. Activated BLM was formed from the reaction of low spin Fe(III)BLM with $\mathrm{H}_{2} \mathrm{O}_{2}$ (Mallinckrodt), ${ }^{5}$ because the reaction of $\mathrm{Fe}(\mathrm{II}) \mathrm{BLM}$ with $\mathrm{O}_{2}$ yields a 50:50 mixture of activated BLM and low spin Fe(III)BLM. The intermediate was prepared at $4{ }^{\circ} \mathrm{C}$ by rapid addition of $3.8 \mu \mathrm{L}$ of $\mathrm{H}_{2} \mathrm{O}_{2}$ (100-fold excess) to $80 \mu \mathrm{L} \mathrm{Fe}$ (III)BLM followed by simultaneous freezing in the XAS sample cell and a $3 \mathrm{~mm}$ EPR tube, after a $25 \mathrm{sec}$ incubation This method resulted in samples with superimposable EPR spectra. The concentrations of the individual components of the activated BLM sample were determined by spin quantitation of the sample in the EPR tube using a $1 \mathrm{mM}$ $\mathrm{CuSO}_{4} \cdot 5 \mathrm{H}_{2} \mathrm{O}$ (Mallinckrodt) solution, in $2 \mathrm{mM} \mathrm{HCl}$ and $2 \mathrm{M} \mathrm{NaClO} 4$ (Fisher), and a $3 \mathrm{mM}$ Fe(III)EDTA solution in 50/50 glycerol (Baker)/water (prepared by stirring $\mathrm{Fe}$ (III) $\mathrm{Cl}_{3} \cdot 6 \mathrm{H}_{2} \mathrm{O}$ (Baker) with excess $\mathrm{Na}_{2} \mathrm{EDTA} \cdot 2 \mathrm{H}_{2} \mathrm{O}$ (Aldrich) for 12 hours) as standards in the $g=2.0$ and $g=4.3$ regions, respectively. The proportionality of the high field feature in the Fe(III)BLM EPR spectrum $(g=1.89)$ to the total low spin ferric signal was used to account for this contribution to the overlapping features in the EPR spectrum of the intermediate and quantitate the remaining low spin signal attributed to activated BLM. Quantitation of the EPR silent degradation product was achieved by subtracting the percentages of the EPR detectable components from the total Fe(III)BLM concentration determined by quantitation of the sample prior to activation and accounting for the $3.8 \mu \mathrm{L} \mathrm{H} \mathrm{H}_{2}$ dilution. This revealed a sample composed of 81(4)\% activated BLM, 7(1)\% low spin Fe(III)BLM, 4(1)\% high spin ferric, and 8(1)\% Fe degradation product undetectable by EPR at $77 \mathrm{~K}$. A separate sample with the degradation product of 
the $\mathrm{Fe}$ (III)BLM- $\mathrm{H}_{2} \mathrm{O}_{2}$ reaction was generated by allowing activated BLM to decay for 12 hours producing $8 \%$ high spin ferric with the remaining $92 \% \mathrm{Fe}$ EPR silent at $77 \mathrm{~K}$.

$\mathrm{X}$ ray absorption spectra were recorded at the Stanford Synchrotron Radiation Laboratory (SSRL) on unfocused wiggler beamline 7-3 during dedicated conditions ( $3 \mathrm{GeV}, 50-100 \mathrm{~mA}$ ). The radiation was monochromatized using a $\mathrm{Si}(220)$ double-crystal monochromator. An Oxford Instruments continuous-flow liquid helium CF1208 cryostat was used to maintain a constant temperature of $10 \mathrm{~K}$. Energies were calibrated using an internal $\mathrm{Fe}$ foil standard, assigning the first inflection point to $7111.2 \mathrm{eV}^{10}$ The spectrometer energy resolution was approximately $1.4 \mathrm{eV}^{11}$ with reproducibility in edge position determination of $<0.2 \mathrm{eV}$.

Data were collected on Fe(II)BLM, Fe(III)BLM, 81(4)\% activated BLM, and the degradation product of the $\mathrm{Fe}(\mathrm{III}) \mathrm{BLM}-\mathrm{H}_{2} \mathrm{O}_{2}$ reaction . Data were measured to $k=15 \AA^{-1}$ with $1 \mathrm{~mm}$ high pre-monochromator beam defining slits, detuning the monochromator $50 \%$ at $7998 \mathrm{eV}$ to minimize harmonic contamination. The fluorescence signal was monitored by using a 13 -element $\mathrm{Ge}$ solid-state array detector ${ }^{12}$ windowed on the Fe K $\alpha$ signal. During the experiment, count rates of approximately $30,000 \mathrm{~s}^{-1}$ total per element were not exceeded. Approximately thirty scans were averaged for each sample. A smooth pre-edge background was removed from the averaged spectra by fitting a second order polynomial to the pre-edge region and subtracting this polynomial from the entire spectrum. A three-segment spline approximately even in $k$-space was fit to the EXAFS region and the data normalized to an edge jump of one at $7130 \mathrm{eV}$. The spline was chosen so that it minimized residual low-frequency background but did not reduce the EXAFS amplitude as checked by monitoring the Fourier transform of the EXAFS during the background subtraction process.

$\mathrm{Fe} \mathrm{K}$-edge spectra were also collected on several iron model complexes. [ $\mathrm{Fe}$ (Prpep) $)_{2} \cdot 2 \mathrm{CH}_{3} \mathrm{OH}$ and [ $\mathrm{Fe}$ (Prpep) $\left.)_{2}\right] \mathrm{ClO}_{4} \cdot 2 \mathrm{CH}_{3} \mathrm{OH} \cdot \mathrm{CH}_{3} \mathrm{CN}$ were obtained as gifts from Pradip Mascharak. ${ }^{13}$ [Fe(imidazole) $6 \mathrm{Cl}_{2}{ }^{14}$ and $\mathrm{Fe}$ (acac) ${ }_{3}{ }^{15,16}$ were prepared as described in the literature. [ $\left.\mathrm{Fe}(\mathrm{Prpep})_{2}\right] \cdot 2 \mathrm{CH}_{3} \mathrm{OH}$, [ $\left.\mathrm{Fe}(\mathrm{Prpep})_{2}\right] \mathrm{ClO}_{4} \cdot 2 \mathrm{CH}_{3} \mathrm{OH} \cdot \mathrm{CH}_{3} \mathrm{CN}$, and $\left[\mathrm{Fe}\right.$ (imidazole) $\left.{ }_{6}\right] \mathrm{Cl}_{2}$ are air-sensitive and thus the following procedure was carried out in a nitrogen-filled glove box. The crystalline samples were each mixed with boron nitride (BN) and ground into a fine powder. The BN/sample mixture was pressed into a $1 \mathrm{~mm}$ thick Al spacer that was sealed with $63.5 \mu \mathrm{m}$ Mylar tape windows and frozen in liquid nitrogen. Data were measured in transmission mode with $\mathrm{N}_{2}$-filled ionization chambers to $k=9.5 \AA^{-1}$ detuning the monochromator $50 \%$ at $7474 \mathrm{eV}$ to minimize harmonic contamination. Two to three scans were averaged for each sample. A smooth pre-edge background was removed from the averaged spectra by fitting a first order 
polynomial to the pre-edge region and subtracting this polynomial from the entire spectrum. A two segment spline of order two was fit to the EXAFS region and the data normalized to an edge jump of one at $7130 \mathrm{eV}$.

EXAFS data reduction was performed on the normalized BLM spectra according to established methods. ${ }^{17-19}$ The normalized data were converted to $k$-space. The photoelectron wave vector, $k$, is defined by $\left[2 \mathrm{~m}_{e}\left(E-E_{0}\right) / \hbar^{2}\right]^{1 / 2}$ where $\mathrm{m}_{\mathrm{e}}$ is the electron mass, $E$ is the photon energy, $\hbar$ is Planck's constant divided by $2 \pi$, and $E_{0}$ is the threshold energy of the absorption edge, which was defined to be $7130 \mathrm{eV}$ for the $\mathrm{Fe} \mathrm{K}$ absorption edge. The empirical EXAFS data analyses were performed with nonlinear least-square curve-fitting ${ }^{10,17-19}$ techniques using empirical phase and amplitude parameters. The following models were used to obtain the empirical $\mathrm{Fe}-\mathrm{X}$ backscattering parameters of interest: $\mathrm{Fe}-\mathrm{O}$ from $\mathrm{Fe}(\mathrm{acac})_{3}{ }^{15,16}$ and $\mathrm{Fe}-\mathrm{N}$ from $\left[\mathrm{Fe}(1,10 \text {-phenanthroline })_{3}\right]\left(\mathrm{ClO}_{4}\right)_{3}{ }^{20,21}$. Fourier transforms (from $k$ to $\mathrm{R}$ space) were performed for the data range 3.5-12.5 $\AA^{-1}$ with a Gaussian window of $0.1 \AA^{-1}$. The window widths used in the backtransforms (from R to $k$ space) for the BLM samples are given in the Results and Analysis section. The window widths were kept as similar as possible to those used to extract amplitude and phase parameters from the model compounds to minimize artifacts introduced by the Fourier filtering technique. All curve-fitting was based on $k^{3}$-weighted data and applied to the individual filtered shell of interest. Only the structure-dependent parameters, the distance and coordination number, were varied unless stated otherwise. A "goodness of fit" parameter, $F$, was calculated as $F=\left\{\left[k^{6}(\text { data }-\mathrm{fit})^{2}\right] /(\text { no. of points })\right\}^{1 / 2}$ for each fit.

Contributions to the XAS spectrum due to low spin Fe(III)BLM and degradation product were subtracted from the normalized data of the $81 \%$ activated BLM spectrum to obtain a $96 \%$ activated BLM spectrum. As stated above, all the averaged spectra were normalized to an edge jump of one. Fe(III)BLM and degradation product data were used to subtract off $7 \%$ and $8 \%$, respectively, from the normalized $81 \%$ activated BLM spectrum, giving a $96 \%$ pure activated BLM spectrum which was then re-normalized to an edge jump of one. The remaining $4 \%$ corresponds to a high spin ferric impurity for which no reference is available. The $96 \%$ activated BLM edge spectrum was identical in shape to that of the $81 \%$ pure sample, however, the edge shifted $0.7 \mathrm{eV}$ to higher energy. The $96 \%$ activated BLM spectrum was used in the edge analysis, whereas the original $81 \%$ activated BLM spectrum was used in the EXAFS analysis. 


\subsubsection{Results and Analysis}

5.4.3.1. Fe K-Edge XAS. The Fe-K edge spectra of Fe(II)BLM, Fe(III)BLM, and activated BLM are shown in Figure 5.8, whereas the inset shows an expanded view of the $1 \mathrm{~s} \rightarrow 3 \mathrm{~d}$ pre-edge region. The lowest energy peaks arise from the weak $1 \mathrm{~s} \rightarrow 3 \mathrm{~d}$ transition at $-7113 \mathrm{eV}$ followed by the $1 \mathrm{~s} \rightarrow 4 \mathrm{p}$ transition at $-7125 \mathrm{eV}$. The spectrum of $\mathrm{Fe}$ (II)BLM (solid line) has two low-intensity pre-edge peaks at 7111.4 and $7113.6 \mathrm{eV}$ (Figure 5.8 inset). The data for both Fe(III)BLM (dashed line) and activated BLM (dotted line) have similar pre-edge features, each with a maximum at $7112.5 \mathrm{eV}$ with similar intensity and a barely resolvable low energy shoulder for Fe(III)BLM at $\sim 7111 \mathrm{eV}$ and a sloping tail in this lower energy region in activated BLM. The rising edge inflection points occur at $7121.3 \mathrm{eV}$ for $\mathrm{Fe}$ (II) $\mathrm{BLM}, 7127.0 \mathrm{eV}$ for $\mathrm{Fe}$ (III)BLM, and $7127.3 \mathrm{eV}$ for activated BLM. Figure 5.9 displays the $1 \mathrm{~s} \longrightarrow 3 \mathrm{~d}$ pre-edge region for representative iron model complexes: a) a low spin ferrous complex, $\left[\mathrm{Fe}\right.$ (Prpep) $\left.{ }_{2}\right] \cdot 2 \mathrm{CH}_{3} \mathrm{OH}^{22}$; b) a high spin ferrous complex, [Fe(imidazole) 6$] \mathrm{Cl}_{2}{ }^{14}$; c) a low spin ferric complex, [ $\mathrm{Fe}\left(\mathrm{Prpep}_{2}\right] \mathrm{ClO}_{4} \cdot 2 \mathrm{CH}_{3} \mathrm{OH} \cdot \mathrm{CH}_{3} \mathrm{CN}^{22}$; and d) a high spin ferric complex, $\mathrm{Fe}(\mathrm{acac})_{3} \cdot{ }^{15,16}$ The [Fe(Prpep) 2$] \cdot 2 \mathrm{CH}_{3} \mathrm{OH}$ spectrum has a single peak in the pre-edge region located at $7112.1 \mathrm{eV}$, while the [Fe(imidazole) $6 \mathrm{Cl}_{2}$ spectrum has three features positioned at approximately $7111.2,7112.3$, and $7113.67 \mathrm{eV}$. The data for [Fe(Prpep) $\left.{ }_{2}\right] \mathrm{ClO}_{4} \cdot 2 \mathrm{CH}_{3} \mathrm{OH} \cdot \mathrm{CH}_{3} \mathrm{CN}$ shows three pre-edge features: a low-energy shoulder at $7111.0 \mathrm{eV}$, a more intense peak at $7112.7 \mathrm{eV}$, and a weaker feature at $7114.4 \mathrm{eV}$. The pre-edge region in the spectrum for $\mathrm{Fe}(\mathrm{acac})_{3}$ has two features positioned at 7112.8 and $7114.3 \mathrm{eV}$.

The energy of the edge position (dominated by the $1 s \rightarrow 4 p$ transition) is dependent upon the effective nuclear charge of the absorbing metal atom. This charge is governed by a combination of effects, including the formal metal oxidation state, the number and type of ligating atoms, and the coordination geometry. ${ }^{23-28}$ In this case, the types of ligating atoms and the coordination sphere are similar, thus changes in the edge energy can be correlated to the iron oxidation state. From Figure 5.8, the edge spectrum of activated BLM is very close in energy and similar in shape to that of $F e(I I) B L M$, in contrast to the $\mathrm{Fe}(\mathrm{II}) \mathrm{BLM}$ spectrum which is $\sim 4 \mathrm{eV}$ lower in energy and has a more intense $1 \mathrm{~s} \rightarrow 4 \mathrm{p}$ feature, indicating that activated BLM contains a ferric metal center. It should be noted that the edge inflection point of horseradish peroxidase compound I, which has a purported $\mathrm{Fe}(\mathrm{IV})=\mathrm{O}$ metal site, was observed to be at $-2 \mathrm{eV}$ higher in energy than that of the resting ferric horseradish peroxidase. ${ }^{29}$ 


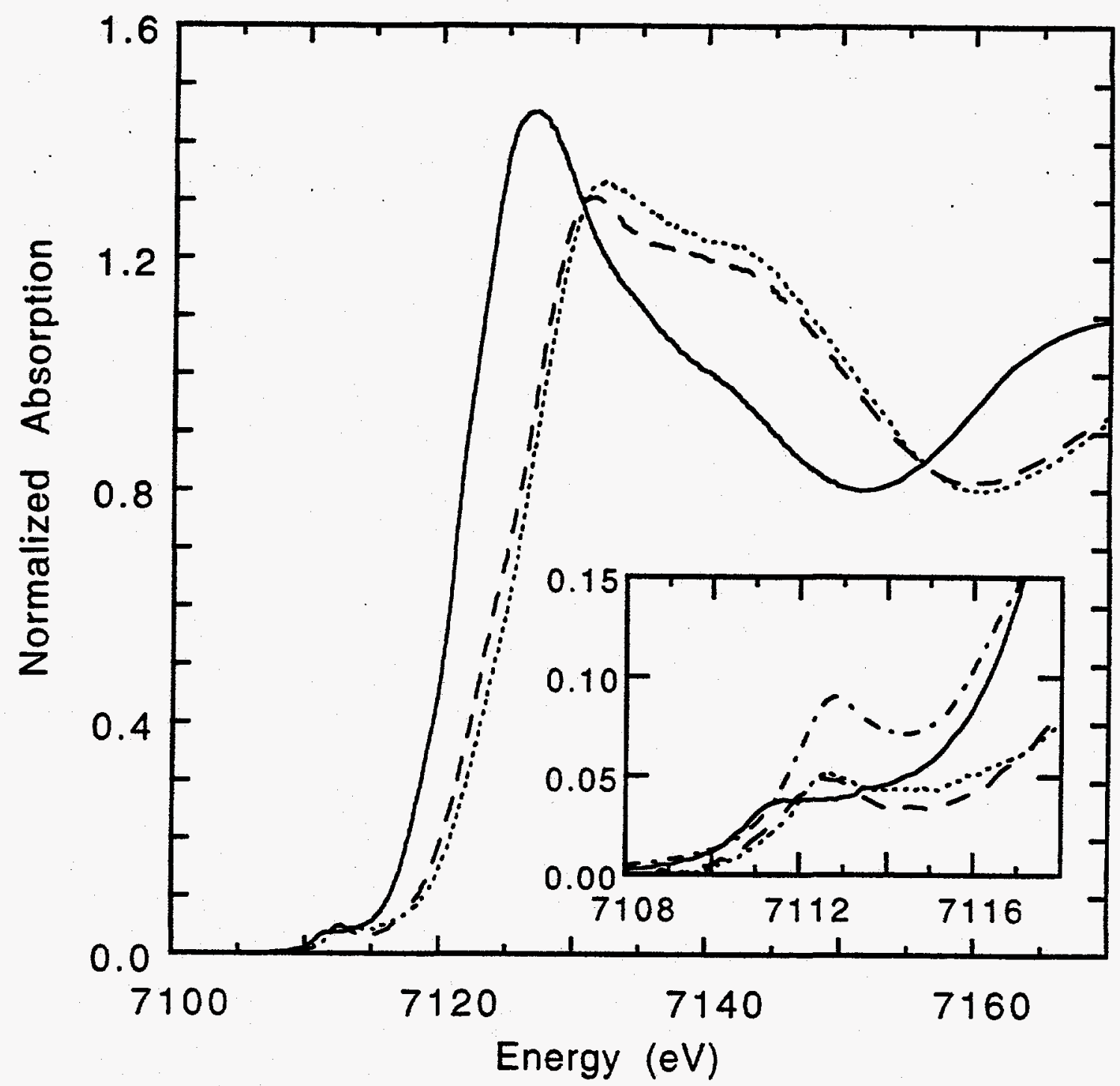

Figure 5.8. Fe-K XAS edge spectra of Fe(II)BLM (-), Fe(III)BLM (---), and activated BLM (.....). The inset shows an expansion of the $1 \mathrm{~s} \rightarrow 3 \mathrm{~d}$ pre-edge region and includes pre-edge data of $\mathrm{Fe}\left(\mathrm{Me}_{3} \mathrm{TACN}\right)(\mathrm{NO})\left(\mathrm{N}_{3}\right)_{2}(--\cdot)$, which has a $\mathrm{Fe}-\mathrm{N}(\mathrm{O})$ bond of $1.74 \AA$. 


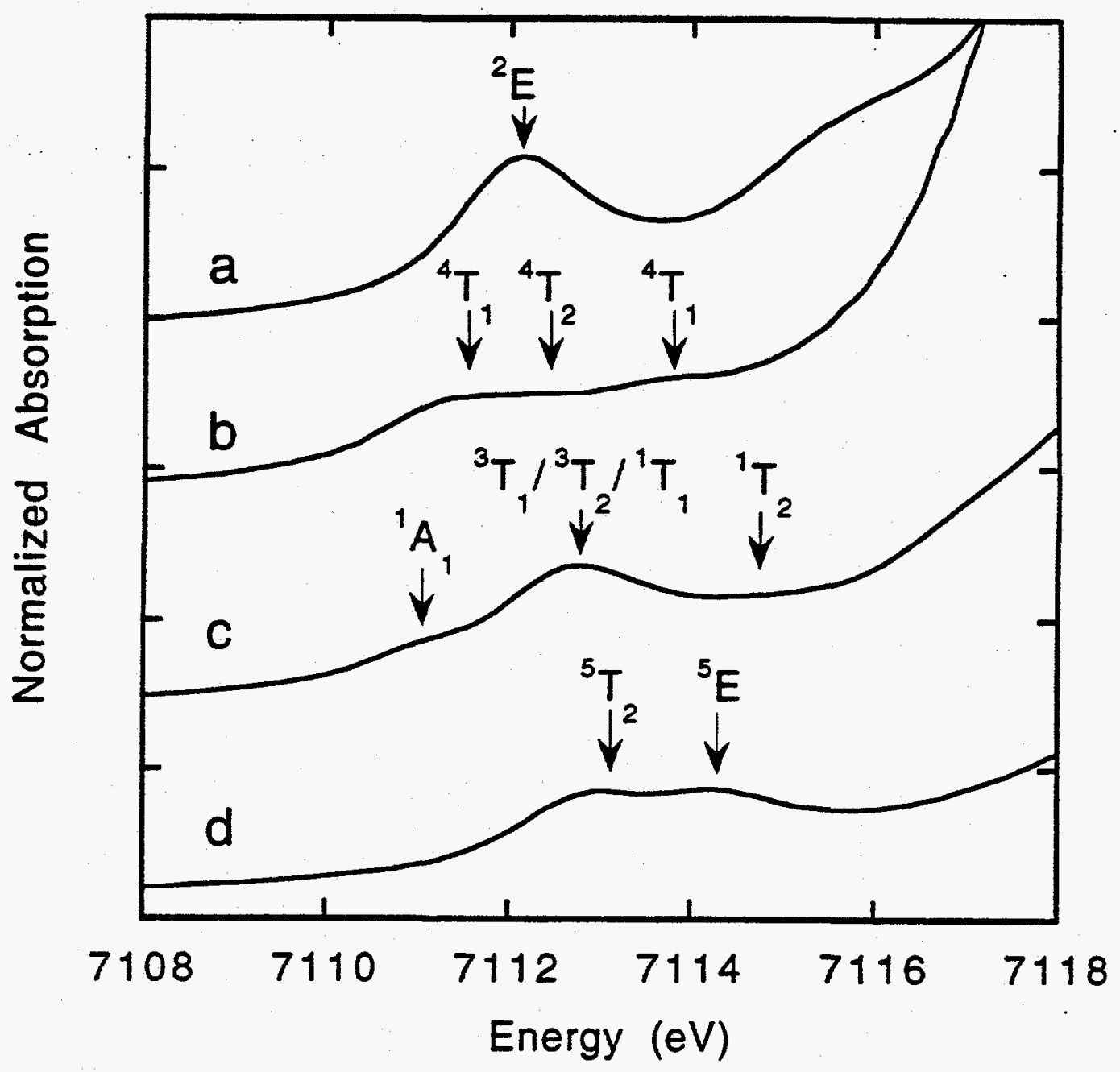

Figure 5.9. The $1 \mathrm{~s} \rightarrow 3 \mathrm{~d}$ pre-edge region of representative iron model complexes. a) a low spin ferrous complex, [ $\left.\mathrm{Fe}(\mathrm{Prpep})_{2}\right] \cdot 2 \mathrm{CH}_{3} \mathrm{OH}$; b) a high spin ferrous complex, [Fe(imidazole) 6$] \mathrm{Cl}_{2} ; \mathrm{c}$ ) a low spin ferric complex, [Fe(Prpep) $\left.)_{2}\right] \mathrm{ClO}_{4} \cdot 2 \mathrm{CH}_{3} \mathrm{OH} \cdot \mathrm{CH}_{3} \mathrm{CN}$; and d) a high spin ferric complex, $\mathrm{Fe}(\mathrm{acac})_{3}$. The individual spectra are offset vertically' by increments of 0.05 on the $y$-scale. 
The $1 \mathrm{~s} \rightarrow 3 \mathrm{~d}$ pre-edge feature can be used to probe the spin and oxidation states of the iron site in activated BLM, since the $3 \mathrm{~d}^{\mathrm{n}+1}$ final state has a different multiplet splitting for high spin ferrous $\left({ }^{4} \mathrm{~T}_{1},{ }^{4} \mathrm{~T}_{2},{ }^{4} \mathrm{~T}_{1}\right)$, high spin ferric $\left({ }^{5} \mathrm{~T}_{2},{ }^{5} \mathrm{E}\right)$, low spin ferrous ( $\left.{ }^{2} \mathrm{E}\right)$, and low spin ferric $\left({ }^{1} \mathrm{~A}_{1}, \mathrm{~T}_{1},{ }^{3} \mathrm{~T}_{2},{ }^{1} \mathrm{~T}_{1}, \mathrm{~T}_{2}\right)$ cases (as described in detail in Chapter 4). The number, intensity ratio, and energies of the pre-edge multiplet features are indicative of the specific oxidation and spin states of the iron. As can be seen in Figure 5.9, the low spin ferrous complex has a single pre-edge feature which has a maximum at $-7112 \mathrm{eV}$ (Figure 5.9a), while high spin ferrous complexes have three features positioned at approximately $7111.2,7112.3$, and $7113.7 \mathrm{eV}$ (Figure 5.9b). This correlates with the fact that there is a single allowed final state for low spin ferrous complexes $\left({ }^{2} E\right)$ while there are three final states of maximum spin multiplicity for the high spin ferrous complexes $\left({ }^{4} \mathrm{~T}_{1},{ }^{4} \mathrm{~T}_{2},{ }^{4} \mathrm{~T}_{1}\right)$. A typical pre-edge for a low spin ferric complex has a low energy feature at $-7111 \mathrm{eV}$ with a more intense feature at $\sim 7112.5 \mathrm{eV}$ (Figure $5.9 \mathrm{c}$ ), corresponding to transitions into the ${ }^{1} \mathrm{~A}_{1}$ and ${ }^{3} \mathrm{~T}_{1},{ }^{3} \mathrm{~T}_{2}$, and ${ }^{1} \mathrm{~T}_{1}$ many-electron states, respectively (features from transitions into the higher energy ${ }^{1} \mathrm{~T}_{2}$ state are much harder to resolve due to the onset of the rising edge). There are two features in pre-edge region of $\mathrm{Fe}(\mathrm{acac})_{3}$ (Figure 5.9d) corresponding to transitions into the two final states of maximum spin multiplicity allowed for high spin ferric complexes, the ${ }^{5} \mathrm{~T}_{2}$ and ${ }^{5} \mathrm{E}$ states. The pre-edge region for Fe(II)BLM (inset Figure 5.8 solid line) exhibits a weak broad feature that is similar in shape and energy to that observed for octahedral high spin ferrous complexes. Fe(III)BLM and activated BLM both have a pre-edge feature with maximum at $7112.5 \mathrm{eV}$ with a lower energy shoulder being barely resolved in the Fe(III)BLM data. The energy position of the pre-edge features and the shape of both the Fe(III)BLM and the activated BLM indicate that both complexes have a low spin ferric active site.

The pre-edge features can also provide information on the geometric structure of the active site. The $1 \mathrm{~s} \rightarrow 3 \mathrm{~d}$ pre-edge feature is formally electric dipole forbidden, but gains intensity through an allowed quadrupole transition and through $4 \mathrm{p}$ mixing into the $3 d$ states as a result of the non-centrosymmetric environment of the metal site. When the symmetry of the iron site is lowered, the pre-edge intensity increases due to an increase in the $3 d-4 p$ mixing (see Chapter 4). ${ }^{27,30}$ Iron-oxo ${ }^{31}$ and iron-nitrosyl ${ }^{32}$ complexes typically have pre-edge features that are a factor of two more intense than their centrosymmetric counterparts, due to their short $\sim 1.8 \AA \mathrm{Fe}-\mathrm{O} / \mathrm{N}$ bond. See, for example, the factor of two increase for $\mathrm{Fe}\left(\mathrm{Me}_{3} \mathrm{TACN}\right)(\mathrm{NO})\left(\mathrm{N}_{3}\right)_{2}{ }^{33}$ (where $\mathrm{TACN}=N, N^{\prime}, N^{\prime \prime}-$ trimethyl-1,4,7-triazacylcononane) which has been included in inset in Figure 5.8 (dot dashed line) as a reference. This iron-nitrosyl complex has an Fe-N(O) bond length of $1.74 \AA$. The pre-edge intensity of a five-coordinate oxo-ferryl porphyrin complex with an 
$\mathrm{Fe}-\mathrm{O}$ bond of $1.65 \AA$ is even greater than that of $\mathrm{Fe}\left(\mathrm{Me}_{3} \mathrm{TACN}\right)(\mathrm{NO})\left(\mathrm{N}_{3}\right)_{2}$, due to the shorter Fe-O bond length. ${ }^{34}$ In contrast, the spectrum of activated BLM (inset Figure 5.8 dotted line) has a pre-edge intensity which is typical of a six coordinate low spin ferric complex with no severe distortion around the iron site, eliminating the possibility of a short Fe-O bond.

5.4.3.2. EXAFS. The EXAFS spectra of Fe(II)BLM, Fe(III)BLM, and activated BLM are shown in Figure 5.10A and the Fourier transforms (FTs), calculated over the $k$ range of 3.5-12.5 $\AA^{-1}$, are shown in Figure 5.10B. Curve-fitting was performed on filtered first shell contributions over the $k$ range 4-12 $\AA^{-1}$ varying bond distances and coordination numbers. The results of the curve-fitting are presented in Table 5.4 with the best fits shown in Figure 5.11. The first shell of each sample could not be adequately fit with a single low- $Z$ wave (Fits $1,3,5$ in Table 5.4). The Fe(II)BLM data were well fit by 2 shells of $\mathrm{N}$ atoms (Fit 2 in Table 5.4 and Figure 5.11) with an average Fe-N distance of $2.16 \AA$. The Fe(II)BLM and activated BLM data were also fit with two shells of low $Z$ atoms (Fits 4,6 in Table 5.4 and Figure 5.11), but with an average first shell distance $0.2 \AA$ shorter than in Fe(II)BLM (see FTs in Figure 5.10B). The two $\mathrm{N}$ shells from the fit to the $\mathrm{Fe}(\mathrm{III}) \mathrm{BLM}$ and activated BLM data have very similar distances with slight changes in the coordination numbers consistent with both being low spin ferric complexes. In contrast, an oxo-ferryl species would have a bond length of $\sim 1.65 \AA \AA^{29}$ The presence of a short $\mathrm{Fe}-\mathrm{O}$ was further examined by fixing an $\mathrm{Fe}-\mathrm{O}$ distance of 1.65 and $1.75 \AA$ with a coordination number of 1 , while varying the coordination numbers and distances of the two longer shells of $\mathrm{N}$. These fits to the data do not support a shorter $\mathrm{Fe}-\mathrm{O}$ distance, in that there was a much worse fit to the data when a 1.65 or $1.75 \AA$ contribution was added (Fit 7, Figure 5.11) and the coordination numbers of the other $\mathrm{N}$ shells became unreasonable (total $\mathrm{CN}$ of $\sim 11$ ). When the third shell distance was allowed to vary it refined to a value of $1.83 \AA$ with the fit having a similar $F$ value as Fit 6 (Table 5.4). These studies indicate that an oxo-ferryl species is clearly not present from the analysis of the EXAFS data.

\subsubsection{Discussion}

Both the energy position and shape of the rising edge of the activated BLM XAS spectrum are very similar to those for Fe(III)BLM, indicating that the iron in activated BLM is ferric. Both Fe(III)BLM and activated BLM exhibit a weak pre-edge feature at $7112.5 \mathrm{eV}$ with a lower energy shoulder, indicative of a low spin ferric active site with no severe distortion (i.e. no short iron-oxo bond). The first shell distances obtained from the 

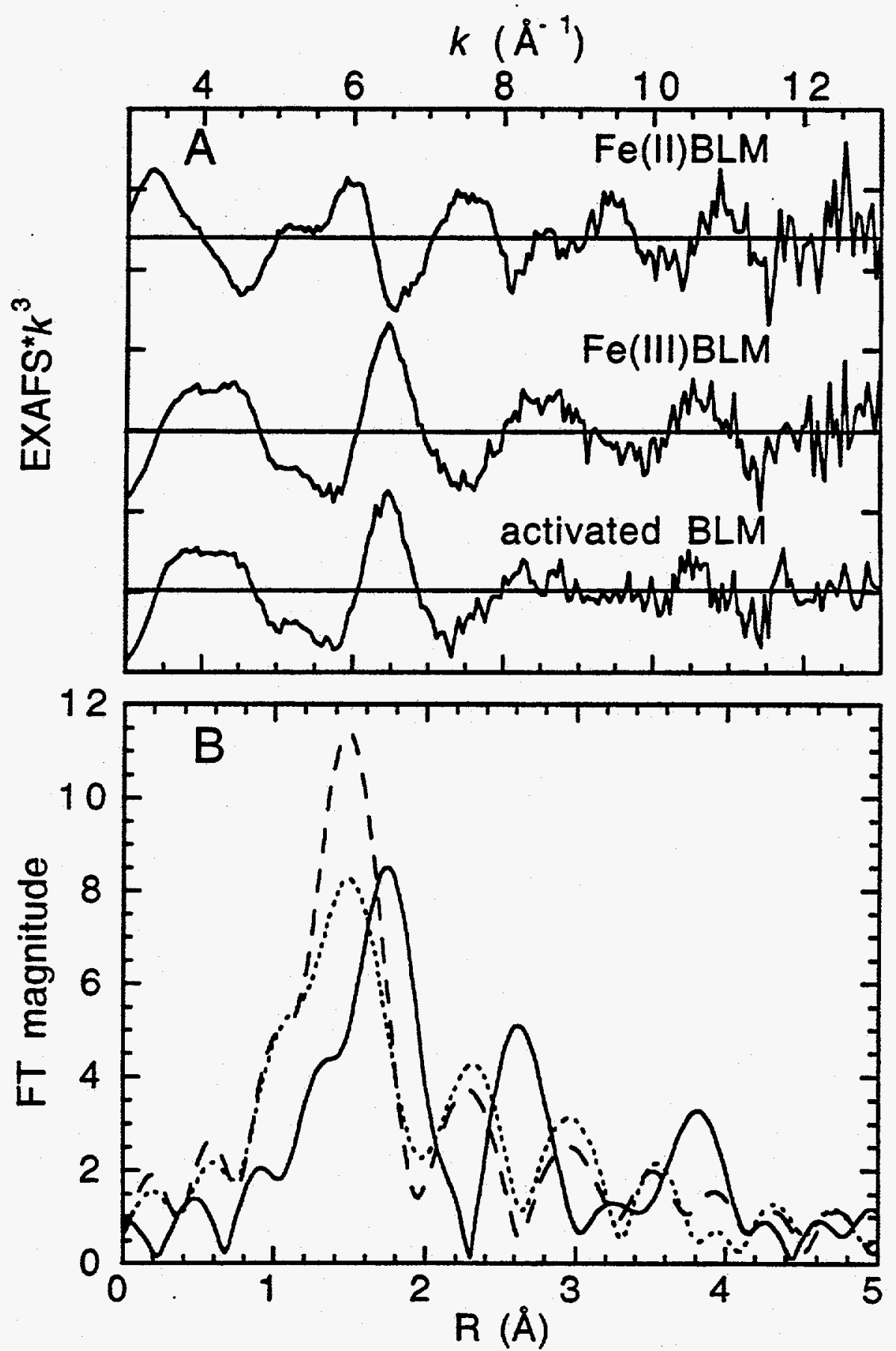

Figure 5.10. A) EXAFS data $\left({ }^{*} k^{3}\right)$ for $\mathrm{Fe}(\mathrm{II}) \mathrm{BLM}, \mathrm{Fe}(\mathrm{III}) \mathrm{BLM}$, and activated BLM (the ordinate scale is 5 between major tick marks on an absolute scale from the abscissa with solid horizontal lines indicating the zero point of each plot). B) The Fourier transforms (non-phase shift corrected) over the $k$-range $3.5-12.5 \AA^{-1}$ of the EXAFS data for $\mathrm{Fe}(\mathrm{II}) \mathrm{BLM}(-), \mathrm{Fe}(\mathrm{III}) \mathrm{BLM}(---)$, and activated BLM (.....). 
Table 5.4. Summary of EXAFS Curve-Fitting Results.

\begin{tabular}{|c|c|c|c|c|c|c|}
\hline \multirow[b]{2}{*}{ sample } & \multirow[b]{2}{*}{$F_{t \#}$} & \multicolumn{3}{|l|}{ FT window } & \multicolumn{2}{|c|}{ bond length } \\
\hline & & width $(\AA)$ & element & $\mathrm{CN}^{a} a, b$ & $(\AA)^{b}$ & $F$ \\
\hline \multirow[t]{3}{*}{$\mathrm{Fe}$ (II)BLM } & 1 & {$[1.0-2.25]$} & $\mathrm{N}$ & 3.0 & 2.16 & 0.41 \\
\hline & 2 & [1.0-2.25] & $\mathrm{N}$ & 1.4 & 2.08 & 0.29 \\
\hline & & & $\mathrm{N}$ & 3.0 & 2.19 & \\
\hline \multirow[t]{3}{*}{$\mathrm{Fe}(\mathrm{III}) \mathrm{BLM}$} & 3 & {$[0.75-2.0]$} & $\mathrm{N}$ & 3.3 & 1.94 & 0.73 \\
\hline & 4 & {$[0.75-2.0]$} & $\mathrm{N}$ & 3.4 & 1.90 & 0.31 \\
\hline & & & $\mathrm{N}$ & 2.5 & 2.03 & \\
\hline \multirow[t]{6}{*}{ activated $B L M$} & 5 & {$[0.7-2.0]$} & $\mathbf{N}$ & 2.5 & 1.96 & 0.84 \\
\hline & 6 & {$[0.7-2.0]$} & $N$ & 2.5 & 1.89 & 0.36 \\
\hline & & & $\mathbf{N}$ & 3.0 & 2.03 & \\
\hline & 7 & {$[0.7-2.0]$} & 0 & $1^{*}$ & $1.65 *$ & 0.75 \\
\hline & & & $\mathrm{N}$ & 7.0 & 1.91 & \\
\hline & & & $\mathrm{N}$ & 2.9 & 2.07 & \\
\hline
\end{tabular}




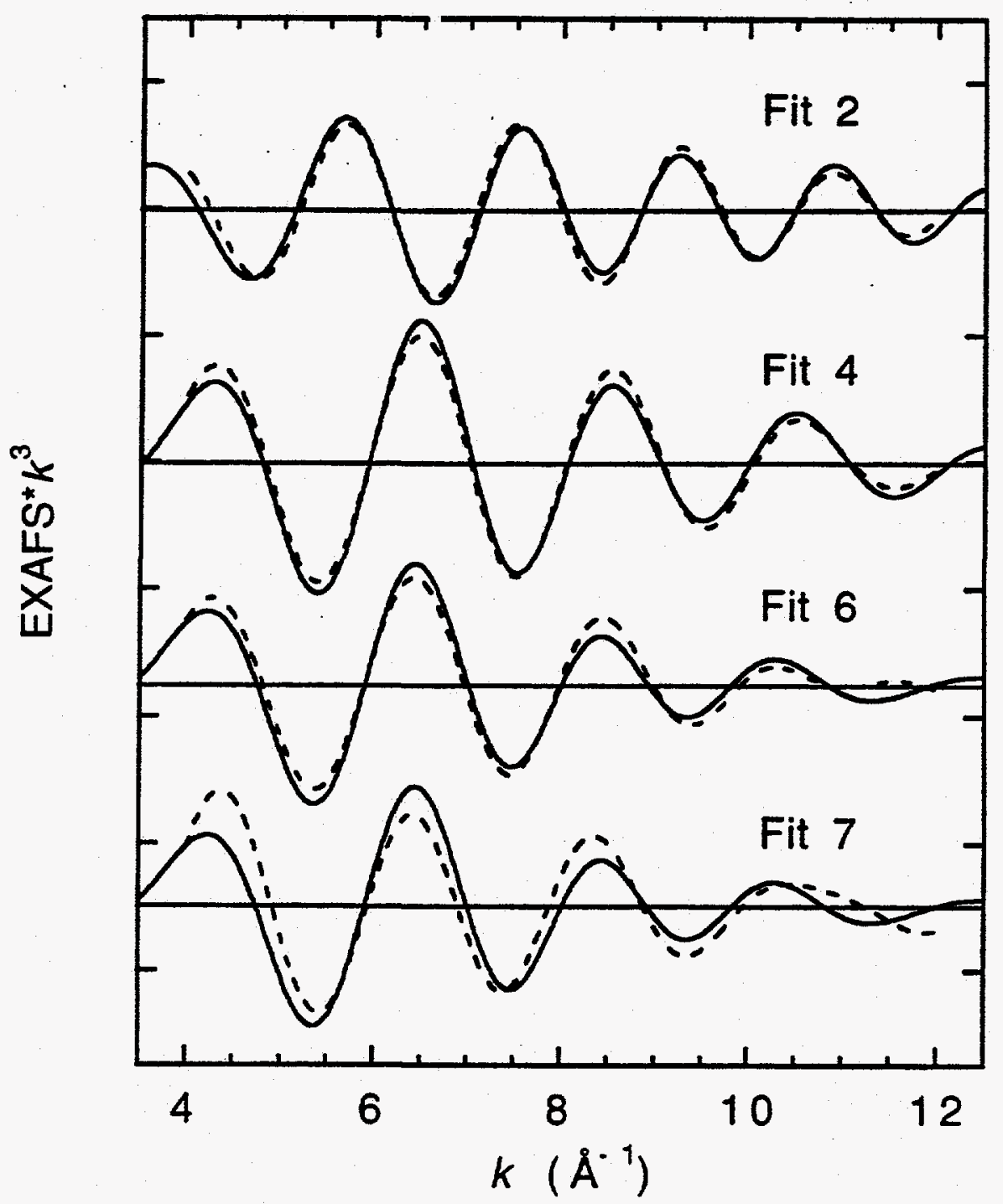

Figure 5.11. Empirical first shell fits to the Fourier-filtered EXAFS data with the solid lines representing the experimental data and the dashed line representing the fit to the data. Fits 2, 4, and 6 are the best empirical fits to the Fe(II)BLM, Fe(III)BLM and activated BLM data, respectively. Fit 7 is a fit to the activated BLM data where a short $1.65 \AA \mathrm{Fe}-\mathrm{O}$ bond distance was included. (The ordinate scale is 4 between major tick marks on an absolute scale from the abscissa with solid horizontal lines indicating the zero point of each plot.) 
EXAFS data of activated BLM are within $0.01 \AA$ of the Fe(III)BLM distances with slight changes in the coordination numbers. Thus, the XAS data of activated BLM are not consistent with a $\mathrm{Fe}(\mathrm{IV})=0$ species, but rather wit a low spin ferric-peroxide description of the active site. These results confirm the description of activated BLM being a peroxy-Fe(II)BLM species presented in a recent mass spectrometry study. ${ }^{9}$ The XAS results provide a direct determination of both the iron oxidation and spin states and the fact that there is no short iron-oxo bond. Activated BLM is the first mononuclear non-heme iron oxygen intermediate to be characterized and its description suggests that such a peroxy-ferric complex may play an important role in $\mathrm{O}_{2}$ activation by this class of enzymes. Experiments are currently underway to define the peroxide binding mode and to generate a detailed electronic structure description of activated BLM in order to understand the nature of the oxygen activation.

\subsubsection{Acknowledgments}

The BLM samples were prepared by Kelly Loeb and Jeffrey Zaleski. This research is supported by grants from the NIH (GM40392, E.I.S.) and NSF (CHE-9121576, K.O.H.). SSRL is supported by the U.S. Department of Energy, Office of Basic Energy Science, Divisions of Chemical and Materials Sciences, and in part by the National Institutes of Health, Biomedical Research Technology Program (RR-01209) and the US Department of Energy, Office of Health and Environmental Research.

\subsubsection{References and Notes}

(1) Carter, S. K. Bleomycin Chemotherapy; Academic Press: New York, 1985, p 3.

(2) Hecht, S. M. Acc. Chem. Res. 1986, 19, 383.

(3) Stubbe, J.; Kozarich, J. W. Chem. Rev. 1987, 87, 1107.

(4) Sugiura, Y. Biochem. Biophys. Res. Commun. 1979, 87, 643.

(5) Burger, R. M.; Peisach, J.; Horwitz, S. B. J. Biol. Chem. 1981, 256, 11636.

(6) Guajardo, R. J.; Hudson, S. E.; Brown, S. J.; Mascharak, P. K. J. Am. Chem. Soc. 1993, 115, 7971.

(7) McGall, G. H.; Rabow; L. E.; Ashley, G. W.; Wu, S. H.; Kozarich, J. W.; Stubbe, J. J. Am. Chem. Soc. 1992, 114, 4958.

(8) Burger, R. M.; Kent, T. A.; Horwitz, S. B.; Münck, E.; Peisach, J. J. Biol. Chem. 1983, 258, 1559.

(9) Sam, J. W.; Tang, X.-J.; Peisach, J. J. Am. Chem. Soc. 1994, 116, 5250. 
(10) Scott, R. A.; Hahn, J. E.; Doniach, S.; Freeman, H. C.; Hodgson, K. O. J. Am. Chem. Soc. 1982, 104, 5364.

(11) Lytle, F. W. In Applications of Synchrotron Radiation; Winick, H.; Xian, D.; Ye, M.-h.; Huang, T., Eds.; Gordon and Breach Science Publishers: New York, 1989; p 135.

(12) Cramer, S. P.; Tench, O.; Yocum, M.; George, G. N. Nucl. Instrum. Methods Phys. Res. 1988, A266, 586.

(13) Abbreviations used: PrpepH = N-(2-(4-imidazoyl)ethyl)pyrimidine-4carboxamide; acac $=$ acetylacetonate.

(14) Burbridge, C. D.; Goodgame, D. M. L. Inorg. Chim. Acta 1970, 4, 231.

(15) Roof, R. B., Jr. Acto Crystallogr 1956, 9, 781.

(16) Iball, J.; Morgan, C. H. Acta Crystallogr. 1967, 23, 239.

(17) Cramer, S. P.; Hodgson, K. O.; Stiefel, E. I.; Newton, W. E. J. Am. Chem. Soc. 1978, 100, 2748.

(18) Cramer, S. P.; Hodgson, K. O. Prog. Inorg. Chem. 1979, $25,1$.

(19) Scott, R. A. Methods Enzymol. 1985, 117, 414.

(20) Johansson, L. Chem. Scr. 1976, 9, 30.

(21) Johansson, L.; Molund, M.; Oskarsson, Å. Inorg. Chim. Acta 1978, 31, 117.

(22) Brown, S. J.; Olmstead, M. M.; Mascharak, P. K. Inorg. Chem. 1990, $29,3229$.

(23) Srivastava, U. C.; Nigam, H. L. Coord. Chem. Rev. 1973, 9, 275.

(24) Shulman, R. G.; Yafet, Y.; Eisenberger, P.; Blumberg, W. E. Proc. Natl. Acad. Sci. USA 1976, 73, 1384.

(25) Cramer, S. P.; Eccles, T. K.; Kutzler, F. W.; Hodgson, K. O. J. Am. Chem. Soc. 1976, 98, 1287.

(26) Wong, J.; Lytle, F. W.; Messmer, R. P.; Maylotte, D. H. Phys. Rev. B. 1984, 30, 5596.

(27) Roe, A. L.; Schneider, D. J.; Mayer, R. J.; Pyrz, J. W.; Widom, J.; Que, L., Jr. J. Am. Chem. Soc. 1984, 106, 1676.

(28) Kau, L.-S.; Spira-Solomon, D. J.; Penner-Hahn, J. E.; Hodgson, K. O.; Solomon, E. I. J. Am. Chem. Soc. 1987, 109, 6433.

(29) Penner-Hahn, J. E.; McMurry, T. J.; Renner, M.; Latos-Grazynsky, L.; Eble, K. S.; Davis, I. M.; Balch, A. L.; Groves, J. T.; Dawson, J. H.; Hodgson, K. O. J. Biol. Chem. 1983, 258, 12761.

(30). Randall, C. R.; Shu, L.; Chiou, Y.-M.; Hagen, K. S.; Ito, M.; Kitajima, N.; Lachicotte, R. J.; Zang, Y.; Que, L., Jr. Inorg. Chem. 1995, 34, 1036.

(31) DeWitt, J. G., Ph.D. Thesis, Stanford University, 1993. 
(32) Brown, C. A.; Pavlosky, M. A.; Westre, T. E.; Zhang, Y.; Hedman, B.; Hodgson, K. O.; Solomon, E. I. J. Am. Chem. Soc. 1995, 117, 715.

(33) Pohl, K.; Wieghardt, K.; Nuber, B.; Weiss, J. J. Chem. Soc., Daltori Trans. 1987, 187.

(34) Liu, H. I.; Gold, A.; Dawson, J. H.; Hedman, B.; Hodgson, K. O. unpublished data. 


\subsection{Lipoxygenases}

\subsubsection{Introduction}

Lipoxygenases (LOs) are non-heme iron enzymes which catalyze the reaction of dioxygen with cis, cis-1,4-pentadiene-containing fatty acids, e.g., linoleic and arachidonic acids, to form hydroperoxide products (see Table 5.1). Certain LOs are positionally specific and are classified by the carbon position at which the hydroperoxidation of arachidonic acid occurs. Mammalian 5-, 12-, and 15-LOs catalyze the formation of the direct precursors to leukotrienes and lipoxins, ${ }^{1}$ compounds which mediate inflammation, hypersensitivity, and cellular immunity. LOs have also been implicated in the oxidation of low-density lipoprotein to its atherogenic form. ${ }^{2,3}$ Thus, the development of $\mathrm{LO}$ inhibitors has been the target of many pharmaceutical companies. In plants, LOs are involved in immunity and growth regulation. ${ }^{4}$ Soybean lipoxygenase-1 (SLO-1) and mammalian 5and 15-LOs have regions of high amino acid sequence identity. ${ }^{5}$ Maximal alignment of the LOs shows that the middle and $\mathrm{COOH}$ terminal portions are very similar. The non-homology of the first 117 residues of SLO-1 (MW -95,000) with the mammalian LOs (MW $\sim 75,000)$ is thought to be due to an additional region present only in plant LOs. ${ }^{5,6}$

SLO-1, a 15-LO, catalyzes the reaction of $\mathrm{O}_{2}$ with linoleic acid to give primarily 13(S)-hydroperoxy-9,11-(E,Z)-octadecadienoic acid (13-HPOD) as product. The native ferrous enzyme is stable in $\mathrm{air}^{7}$ and must be oxidized to the active ferric form for reaction with substrate. There are two classes of proposed mechanisms for LO catalysis. The first is a radical-based mechanism in which the fatty acid is activated through oxidation by the ferric center to form a fatty acid radical. ${ }^{8,9}$ The ferrous site generated may then activate $\mathrm{O}_{2}$ for reaction with the radical, or $\mathrm{O}_{2}$ may react with the radical to generate product. The second mechanism involves a direct reaction of the ferric center and the substrate, forming an organo-iron complex ${ }^{10}$ to which $\mathrm{O}_{2}$ inserts, forming a ferric peroxo complex and subsequently product.

Two recent X-ray crystal structures ${ }^{6,11}$ of ferrous SLO-1 have identified four common amino acid active site ligands: histidine ${ }_{499} \mathrm{~N} \varepsilon$, histidine ${ }_{504} \mathrm{~N} \varepsilon$, histidine $690 \mathrm{~N} \varepsilon$, and isoleucine ${ }_{839} \mathrm{OT} 2$, the terminal carboxylate. Each of these residues is conserved in all sequenced lipoxygenases, with the exception of a valine replacement of the isoleucine in rat leukocyte 5-LO.12 One crystallographic ąctive site description is four-coordinate distorted octahedral with two adjacent unoccupied ligand positions. ${ }^{6}$ The other crystal structure ${ }^{11}$ has an additional ligand, asparagine $6940 \delta 1$, with the further possibility of a sixth water-based ligand; such a ligand has been proposed from electron paramagnetic resonance 
(EPR) line broadening studies on the ferric enzyme. ${ }^{13}$ This asparagine residue is conserved in all sequenced LOs with the exception of rabbit reticulocyte and human 15-LOs, in which a histidine is in the analogous position. ${ }^{5}$

There have been a limited number of spectroscopic studies aimed at defining the geometric and electronic structure of the ferrous site of SLO-1. Magnetic susceptibility measurements ${ }^{7}$ show that the ferrous active site ground state is high-spin with $S=2$. $\mathrm{X}$-ray absorption (XAS), ${ }^{14}$ Mössbauer, ${ }^{15}$ and circular and magnetic circular dichroism ${ }^{16}$ (CD/MCD) studies predicted a distorted octahedral geometry. However, a more recent XAS study on a frozen solution of ferrous SLO-1 found the $1 \mathrm{~s} \rightarrow 3 \mathrm{~d}$ pre-edge intensity greater than what was found for six-coordinate high-spin iron(II) model complexes, but comparable to that of a five-coordinate model. ${ }^{17}$ These studies now appear to have been complicated by solvent effects as a recent $C D / M C D$ study ${ }^{18}$ in the near-infrared (NIR) region on native SLO-1 in solution using sucrose as a non-perturbing low-temperature glassing agent showed that native ferrous SLO-1 exists as a mixture of two forms, one five-coordinate and one six-coordinate, and that the addition of glycerol converts the mixture to the pure six-coordinate form. ${ }^{19}$

Considering the discrepancies between the two crystals structures ${ }^{6,11}$ (which predict a four- and five-coordinate active site, respectively) and the spectroscopic studies (which predict a five $-{ }^{17}$ and six-coordinate ${ }^{14-16}$ active site), both Fe K-edge and extended $\mathrm{X}$-ray absorption fine structure (EXAFS) measurements were performed on SLO-1 in glycerol to obtain information on the coordination number and geometry of the iron active site in a pure form. ${ }^{18}$ In addition Fe K-edge XAS data were obtained for two mammalian LOs, rabbit reticulocyte 15-LO (15-RLO) and recombinant buman 15-LO (15-HLO), to compare to SLO-1. The XAS results have been combined with the results from a NIR CD/MCD study of SLO-1, 15-RLO and 15-HLO in solution and glycerol to obtain an understanding of the geometric and electronic structure of these three LOS.

\subsubsection{Experimental Section}

SLO-1 was purified from soybeans (Williams Variety, Tabor Seed Division, Decatur, II) following published procedures. ${ }^{20}$ Enzyme activity was determined by monitoring the absorption of 13-HPOD product formed during the enzymatic reaction at $234 \mathrm{~nm}\left(\varepsilon_{234}=2.5 \times 10^{4} \mathrm{M}^{-1} \mathrm{~cm}^{-1}\right)$. The specific activity of the final enzyme was -200 units/mg. Native SLO-1 samples were buffered in $0.1 \mathrm{M}$ sulfonic acid solutions: MĖS, 5.6<pD<7; HEPES, 7.5<pD<8.2; CHES, 8.5<pD<9.5; CAPS, $10<\mathrm{pD}<11$. Human 15-lipoxygenase was purified from an baculovirus/insect cell expression system by 
anion-exchange chromatography on a Mono Q column. ${ }^{21}$ Rabbit 15-lipoxygenase was purified from a $55 \%$ ammonium sulfate precipitate of rabbit reticulocyte lysate to greater than $98 \%$ purity according to the published method with the following modifications: desalting the protein with a PD-10 column instead of dialysis and replacement of the Mono $S$ with a Mono $Q$ column. ${ }^{22}$ The Bradford method was used for determining protein concentration..$^{21}$ All purification steps were carried out at $4^{\circ} \mathrm{C}$ or on ice.

$\mathrm{X}$-ray absorption spectra were recorded at the Stanford Synchrotron Radiation Laboratory on unfocused beamline 7-3 during dedicated conditions ( $3 \mathrm{GeV}, 50-100 \mathrm{~mA}$ ). The radiation was monochromatized using a Si(220) double-crystal monochromator. An Oxford Instruments continuous-flow liquid helium CF1208 cryostat was used to maintain a constant temperature of $10 \mathrm{~K}$. Energies were calibrated using an internal $\mathrm{Fe}$ foil standard, assigning the first inflection point to $7111.2 \mathrm{eV} .^{23}$ The spectrometer energy resolution was approximately $1.4 \mathrm{eV}$ with reproducibility in edge position determination of $<0.2 \mathrm{eV}$.

The SLO-1, 15-RLO, and 15-HLO XAS samples were prepared as described above with $50 \%$ glycerol. The samples were loaded into Lucite EXAFS cells $(23 \times 1 \times 3 \mathrm{~mm})$ with $37 \mu \mathrm{m}$ Kapton windows and frozen in liquid nitrogen. Data were measured to $k=$ $13 \AA^{-1}$ with $1 \mathrm{~mm}$ high pre-monochromator beam defining slits, detuning the monochromator $50 \%$ at $7824 \mathrm{eV}$ to minimize harmonic contamination. The fluorescence signal was monitored by using a 13-element Ge solid-state array detector ${ }^{24}$ windowed on the Fe $\mathrm{K} \alpha$ signal. During the experiment, count rates of approximately $30,000 \mathrm{~s}^{-1}$ total per element were not exceeded. Approximately 30 scans were averaged for each protein sample. A pre-edge subtraction was performed by fitting the tail of a Gaussian to the pre-edge region and subtracting this polynomial from the averaged spectra. ${ }^{25} \mathrm{~A}$ three-segment spline approximately even in $k$-space was fit to the EXAFS region and the data normalized to an edge jump of one at $7130 \mathrm{eV}$. The spline was chosen so that it minimized residual low-frequency background but did not reduce the EXAFS amplitude as checked by monitoring the Fourier transform of the EXAFS during the background subtraction process.

Fe K-edge spectra were also collected on four-, five-, and six-coordinate ferrous model complexes. $\mathrm{Fe}\left(\mathrm{HB}(3,5-\mathrm{Pr} 2 \mathrm{pz})_{3}\right) \mathrm{Cl}^{26}$ was obtained as a gift from N. Kitajima. ${ }^{28}$ [Fe(imidazole) $6 \mathrm{Cl}_{2}{ }^{29}$ and $\left(\mathrm{BF}_{4}\right)\left[\mathrm{Fe}(\mathrm{TMC}) \mathrm{Cl}^{27}\right.$ was prepared as described in the literature. These three samples are air-sensitive and thus the following procedure was carried out in a nitrogen-filled glove box. The crystalline samples were each mixed with $\mathrm{BN}$ and ground into a fine powder. The BN/sample mixture was pressed into a $1 \mathrm{~mm}$ thick $\mathrm{Al}$ spacer that was sealed with $63.5 \mu \mathrm{m}$ Mylar tape windows and frozen in liquid nitrogen. Data were measured in transmission mode with $\mathrm{N}_{2}$-filled ionization chambers to $k=$ 
$9.5 \AA^{-1}$, detuning the monochromator $50 \%$ at $7474 \mathrm{eV}$ to minimize harmonic contamination. Two to three scans were averaged for each sample. A smooth pre-edge background was removed from the averaged spectra by fitting a first order polynomial to the pre-edge region and subtracting this polynomial from the entire spectrum. A two-segment spline of order two was fit to the EXAFS region and the data normalized to an edge jump of one at $7130 \mathrm{eV}$.

EXAFS data reduction was performed on the normalized protein spectra according to established methods. ${ }^{30-32}$ The normalized data were converted to $k$-space. The photoelectron wave vector, $k$, is defined by $\left[2 \mathrm{~m}_{e}\left(\mathrm{E}-\mathrm{E}_{0}\right) / \hbar^{2}\right]^{1 / 2}$ where $\mathrm{me}_{\mathrm{e}}$ is the electron mass, $E$ is the photon energy, $h$ is Planck's constant divided by $2 \pi$, and $E_{0}$ is the threshold energy of the absorption edge, which was defined to be $7130 \mathrm{eV}$ for the $\mathrm{Fe} \mathrm{K}$ absorption edge. The empirical EXAFS data analyses were performed with nonlinear least-squares curve-fitting ${ }^{23,30-32}$ techniques using empirical phase and amplitude parameters. The following models were used to obtain the empirical $\mathrm{Fe}-\mathrm{X}$ backscattering parameters of interest: $\mathrm{Fe}-\mathrm{O}$ from $\left[\mathrm{Fe}(\text { acetylacetonate) }]^{33,34}\right.$ and $\mathrm{Fe}-\mathrm{N}$ from [Fe(1,10-phenanthroline $\left.)_{3}\right]\left(\mathrm{ClO}_{4}\right)_{3} \cdot{ }^{35,36}$ Fourier transforms (from $k$ to $\mathrm{R}$ space) were performed for the data range $3.5-12.5 \AA^{-1}$ with a Gaussian window of $0.1 \AA^{-1}$. The window widths used in the backtransforms (from $\mathrm{R}$ to $k$ space) for the proteins are given in the Results and Analysis. The window widths were kept as similar as possible to those used to extract amplitude and phase parameters from the model compounds to minimize artifacts introduced by the Fourier filtering technique. All curve-fitting was based on $k^{3}$-weighted data and applied to the individual filtered shell of interest. Only the structure-dependent parameters, the distance and coordination number, were varied unless stated otherwise. A "goodness of fit" parameter, $F$, was calculated as $F=\left\{\left[k^{6}\right.\right.$ (data fit $\left.)^{2}\right] /$ (no. of points) $\}^{1 / 2}$ for each fit.

The intensities and energies of pre-edge features of the model complex and protein data were quantitated by fits to the data. The fitting program EDG_FIT, which utilizes the double precision version of the public domain MINPAK fitting library ${ }^{37}$ was used. EDG_FIT was written by Dr. Graham N. George of the Stanford Synchrotron Radiation Laboratory. All spectra were fit over the range $7108-7118 \mathrm{eV}$. Pre-edge features were modeled by pseudo-Voigt line shapes (simple sums of Lorentzian and Gaussian functions). ${ }^{38-41}$ A fixed 50:50 ratio of Lorentzian to Gaussian contribution for the pre-edge feature successfully reproduced these spectral features. Functions modeling the background underneath the pre-edge features were chosen empirically to give the best fit. The second derivative of the data was compared to the second derivative of the fit. In all cases, a number of fits were obtained which reproduced the data and the second derivative. 
The value reported for the area of a fitted feature (where the peak area was approximated by the height $x$ full width at half-maximum (FWHM)) is the average of all the pseudo-Voigts which successfully fit the feature. For each sample, the standard deviation of the average of the areas was calculated to quantitate the error.

\subsubsection{Results and Analysis}

5.5.3.1. Fe K-Edge XAS. Fe K-edge X-ray absorption spectra of ferrous SLO-1, 15-RLO, and 15-HLO have been compared to spectra of four-, five-, and six-coordinate ferrous model complexes to obtain information on the coordination number of the iron active site in the LOs. All three protein samples contained glycerol to ensure that a pure form was being studied. ${ }^{18,19}$ The XAS edge spectra for SLO-1, 15-RLO, and 15-HLO are shown in the upper portion of Figure 5.12, while the lower portion contains spectra of representative four-, five-, and six-coordinate ferrous model complexes: $\mathrm{Fe}\left(\mathrm{HB}\left(3,5-i \mathrm{Pr}_{2} \mathrm{pz}\right)_{3}\right) \mathrm{Cl}_{,}{ }^{26}\left(\mathrm{BF}_{4}\right)[\mathrm{Fe}(\mathrm{TMC}) \mathrm{Cl}],{ }^{27}$ and $\left[\mathrm{Fe}\right.$ (imidazole) $\left.{ }_{6}\right] \mathrm{Cl}_{2},{ }^{29}$ respectively. The lowest energy peaks arise from the weak $1 \mathrm{~s} \rightarrow 3 \mathrm{~d}$ transition which is at $-7112 \mathrm{eV}$ followed by the $1 \mathrm{~s} \rightarrow-4 \mathrm{p}$ transition at $\sim 7125 \mathrm{eV}$. An expanded view of the $1 \mathrm{~s} \longrightarrow 3 \mathrm{~d}$ pre-edge region is shown for the three LOs as well as for the model complexes in Figure 5.12 insets $A$ and $B$, respectively. The energies and areas of the pre-edge features were determined by fits to the data and are presented in Table 5.5. The SLO-1, 15-RLO, and 15-HLO spectra all have a very broad low-intensity pre-edge feature which can be fit with two features that have a total pre-edge area of -8 . The three ferrous model complexes, $\mathrm{Fe}\left(\mathrm{HB}\left(3,5-i \mathrm{Pr}_{2} \mathrm{pz}\right)_{3}\right) \mathrm{Cl},\left(\mathrm{BF}_{4}\right)[\mathrm{Fe}(\mathrm{TMC}) \mathrm{Cl}]$, and $\left.[\mathrm{Fe} \text { (imidazole })_{6}\right] \mathrm{Cl}_{2}$, all have what appear to be two pre-edge features split by $\sim 2 \mathrm{eV}$ with varying intensities (Figure 5.12 inset $B$ ). The four-coordinate complex has the most intense pre-edge feature with a total area of 19.8, while the six-coordinate complex has the least intense pre-edge feature with a total pre-edge area of 3.8 . The "white line", attributed to the $1 \mathrm{~s} \rightarrow 4 \mathrm{p}$ transition at $-7125 \mathrm{eV}$, is very similar in shape and intensity in all three LO samples, while this feature differs drastically in shape between the four-, five-, and six-coordinate ferrous complexes (Figure 5.12).

The $1 \mathrm{~s} \rightarrow 3 \mathrm{~d}$ pre-edge feature can be used to probe the coordination number of the iron in the active site of the LOs. The $1 s \rightarrow 3 d$ transition is formally electric dipole forbidden, but gains intensity through an allowed quadrupole transition and more dominantly by $4 p$ mixing into the $3 d$ states as a result of the noncentrosymmetric environment of the metal site. It has been shown for ferric and ferrous complexes that when the symmetry of the iron site is lowered, the pre-edge intensity increases due to an 


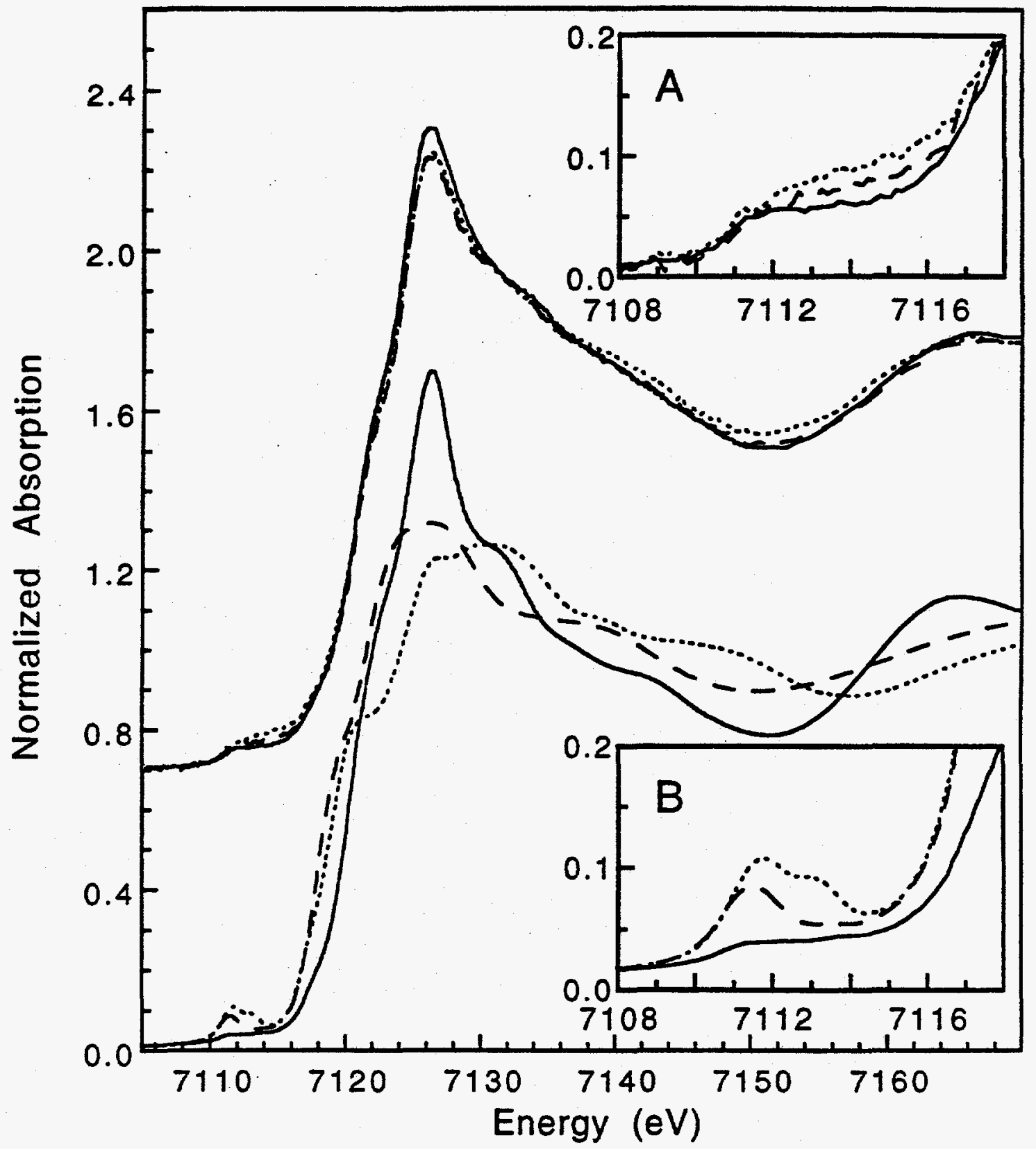

Figure 5.12. Fe K-edge XAS spectra (offset by +0.7) of SLO-1 (-), 15-RLO (---), and 15-HLO (.....) are shown in the upper portion, where inset $A$ shows an expansion of the $1 \mathrm{~s} \rightarrow 3 \mathrm{~d}$ pre-edge region. The lower portion shows the Fe K-edge XAS spectra of four-, five- and six-coordinate ferrous model complexes: $\mathrm{Fe}\left(\mathrm{HB}(3,5-i \mathrm{Pr} 2 \mathrm{pz})_{3}\right) \mathrm{Cl}(\cdots \cdot \cdots)$, $\left(\mathrm{BF}_{4}\right)[\mathrm{Fe}(\mathrm{TMC}) \mathrm{Cl}](--)$, and $\left[\mathrm{Fe}(\text { imidazole })_{6}\right] \mathrm{Cl}_{2}(-)$. Inset $\mathrm{B}$ is an expansion of the $1 \mathrm{~s} \rightarrow 3 \mathrm{~d}$ pre-edge region for the model complexes. 
Table 5.5. XAS Pre-Edge Energies and Areas for LO's and Ferrous Model Complexes.

\begin{tabular}{|c|c|c|c|c|}
\hline sample & $\mathrm{CN}^{a}$ & $\begin{array}{l}\text { pre-edge } \\
\text { energy }(\mathrm{eV})^{b}\end{array}$ & $\begin{array}{l}\text { pre-edge } \\
\text { area } b, c\end{array}$ & $\begin{array}{l}\text { total } \\
\text { pre-edge } \\
\text { areac }\end{array}$ \\
\hline \multirow[t]{2}{*}{$\mathrm{Fe}\left(\mathrm{HB}(3,5-\mathrm{iP} 2 \mathrm{pz})_{3}\right) \mathrm{Cl}$} & 4 & $7111.64(0.02)$ & $14.2(0.5)$ & $19.8(0.9)$ \\
\hline & & $7113.17(0.02)$ & $5.6(0.9)$ & \\
\hline \multirow[t]{2}{*}{$\left(\mathrm{BF}_{4}\right)[\mathrm{Fe}(\mathrm{TMC}) \mathrm{Cl}]$} & 5 & $7111.41(0.01)$ & $10.9(0.1)$ & $12.9(0.2)$ \\
\hline & & $7113.43(0.02)$ & $2.0(0.3)$ & \\
\hline \multirow[t]{3}{*}[\mathrm{Fe}\text{(imidazole)}6]{$\mathrm{Cl}_{2}$} & 6 & $7111.24(0.03)$ & $1.6(0.5)$ & $3.8(0.3)$ \\
\hline & & $7112.35(0.16)$ & $1.6(0.8)$ & - \\
\hline & & $7113.66(0.02)$ & $0.6(0.2)$ & \\
\hline \multirow[t]{2}{*}{ SLO-1 in glycerol } & - & $7111.64(0.07)$ & $4.4(0.9)$ & $8.7(1.1)$ \\
\hline & & $7113.34(0.15)$ & $4.3(1.2)$ & \\
\hline \multirow[t]{2}{*}{ 15-RLO } & -- & $7111.85(0.08)$ & $4.1(1.4)$ & $8.1(2.6)$ \\
\hline & & $7113.35(0.32)$ & $4.0(1.7)$ & \\
\hline \multirow[t]{2}{*}{ 15-HLO } & -. & $7111.69(0.08)$ & $4.0(0.9)$ & $7.9(1.4)$ \\
\hline & & $7113.35(0.19)$ & $3.9(0.7)$ & \\
\hline
\end{tabular}

$a \mathrm{CN}=$ coordination number. $b$ Pre-edge energies and areas were determined by fits to the data as described in the Experimental Section. $c$ Pre-edge areas were calculated by multiplying the height of the fitted feature by the FWHM (the values reported were multiplied by 100 ). 
increase in the $3 \mathrm{~d}-4 \mathrm{p}$ mixing. 42,43 As described in more detail in Chapter 4, the pre-edge splitting and intensity distribution is indicative of the site symmetry of the iron atom. When a high spin ferrous atom is in an octahedral site, three transitions are observed into ${ }^{4} T_{1}$, ${ }^{4} \mathrm{~T}_{2}$, and ${ }^{4} \mathrm{~T}_{1}$ excited states (a transition into the ${ }^{4} \mathrm{~A}_{2}$ state is not observed as it would involve a two electron transition). The transitions into these states are only quadrupole allowed as the iron is in a centrosymmetric site and therefore there is no 4p-3d mixing. Thus, one observes three weak peaks as can be seen in the [Fe(imidazole) $\left.{ }_{6}\right] \mathrm{Cl}_{2}$ spectrum (Figure 5.12 inset $B$ ) with the first two features being barely resolvable and centered at 7111.2 and $7112.4 \mathrm{eV}$ and the third feature positioned at $7113.7 \mathrm{eV}$. Since the difference in energy between the lowest energy ${ }^{4} \mathrm{~T}_{1}$ state and the ${ }^{4} \mathrm{~T}_{2}$ state is about the same as the energy resolution at the Fe K-edge, occasionally only two pre-edge features are observed for high spin ferrous complexes with the first feature attributed to transitions into both the ${ }^{4} \mathrm{~T}_{1}$ and the ${ }^{4} \mathrm{~T}_{2}$ states and the second feature $-2 \mathrm{eV}$ higher in energy attributed to a transition into the higher energy ${ }^{4} \mathrm{~T}_{1}$ state. When a high spin ferrous atom is in a square pyramidal site, the intensity of the lower energy feature increases dramatically since there is now $4 \mathrm{p}_{\mathrm{z}}$ mixing into the $3 \mathrm{~d}_{\mathrm{z}} 2$ orbital due to the loss of a center of inversion, as can be seen in the $\left(\mathrm{BF}_{4}\right)[\mathrm{Fe}(\mathrm{TMC}) \mathrm{Cl}]$ spectrum (dashed line in Figure 5.12 inset $\mathrm{B}$ ). When a high spin ferrous atom is in a tetrahedral site, there are two intense pre-edge features due to $4 \mathrm{p}$ mixing into the $d_{x y}, d_{x z}, d_{y z}$ orbitals, with the lower energy fearure being more intense than the higher energy feature, as can be seen in the $\left(\mathrm{Fe}\left(\mathrm{HB}\left(3,5-i \mathrm{Pr}_{2} \mathrm{pz}\right) 3\right) \mathrm{Cl}\right.$ spectrum (dotted line in Figure 5.12 inset B). See Chapter 4 for a more detailed analysis of the distribution of the dipole intensity in the $1 \mathrm{~s} \longrightarrow 3 \mathrm{~d}$ pre-edge features of high spin ferrous complexes.

Empirically the pre-edge features of SLO-1, 15-RLO, and 15-HLO look much more similar in shape and intensity to that of the six-coordinate ferrous model and quite different from those of the four- and five-coordinate model complexes. The total fitted area of the pre-edge features of the LOs at $\sim 8$ is higher than that of $\mathrm{Fe}$ (imidazole) $6 \mathrm{Cl}_{2}$ at 4 . The larger pre-edge in the LOs can be attributed to an overall less symmetric iron site caused by mixed ligation and variation in bond lengths. However, the shape of the LOs' pre-edge feature is very similar to that of the $[\mathrm{Fe}$ (imidazole) 6$] \mathrm{Cl}_{2}$ and other six-coordinate ferrous complexes (see Chapter 4). In addition, the shapes of the "white line" in the SLO-1, 15-RLO, and 15-HLO spectra at $\sim 7127 \mathrm{eV}$ are very much alike and similar to that in the six-coordinate [Fe(imidazole) $\left.{ }_{6}\right] \mathrm{Cl}_{2}$ spectrum. These results indicate that all three $\mathrm{LOs}$ in glycerol have a six-coordinate iron active site.

5.5.3.2. EXAFS. EXAFS studies of SLO-1 in glycerol were also pursued to obtain metrical information on the iron active site of the pure six-coordinate form. ${ }^{18,19}$ High-quality, high- $k$ EXAFS data could not be obtained on the 15-HLO or 15-RLO 
samples due to the low protein concentration of $-1 \mathrm{mM}$. The EXAFS spectrum of SLO-1 in glycerol is shown in Figure 5.13A, and the Fourier transform (FT), taken over the $k$ range of $3.5-12.5 \AA^{-1}$, is shown in Figure 5.13C. Curve-fitting was performed on filtered first shell contributions over the $k$ range $4-12 \AA^{-1}$. Fits were performed by stepping through fixed coordination numbers while allowing the bond distance and $c 2$ to vary. $c 2$ is the amplitude parameter which has the functional form of the Debye-Waller factor, i.e., $\exp \left(\mathrm{x} k^{2}\right)$, where $\mathrm{x}=\mathrm{c} 2$ or $\mathrm{x}=-2 \sigma_{a s}^{2}{ }^{23,30-32}$ As determined in these fits, the value of $c 2$ is correlated to $\sigma_{a s}^{2}$ only in a relative manner. Since $\Delta c 2=-2 \Delta \sigma_{a s}^{2}$, a more negative value of $c 2$ denotes a larger value of $\sigma_{\omega s}^{2}$ and thus a weaker bond or a greater distribution of distances for scatterers. The values of $\mathrm{c} 2$ for $\mathrm{Fe}-\mathrm{O}$ from $\mathrm{Fe}$ (acetylacetonate $)_{3}$ and $\mathrm{Fe}-\mathrm{N}$ from $\left[\mathrm{Fe}(1,10 \text {-phenanthroline })_{3}\right]\left(\mathrm{ClO}_{4}\right)_{3}$ were -0.02391 and -0.02083 , respectively. These values correspond to a well-ordered shell of nearest neighbors.

The FT peak centered at $\sim 1.8 \AA$ (non-phase shift corrected) could be adequately fit with either one shell of O's or N's (Fits 1-6 in Table 5.6; Figure 5.13B) with an average first shell distance of 2.15 and $2.18 \AA$, respectively. Fits $1-3$ with all $O$ ligation matched the data better in the lower $k$ region, while Fits 4-6 with all $N$ ligation matched the data better in the higher $k$ region. The coordination number and $c 2$ are highly correlated, as can be seen in Fits 1-3 and 4-6 in Table 5.6, with the $c 2$ values becoming more negative as the coordination number is increased. The $F$ value is lowest with a coordination number of 4 using the Fe-O parameters (Fit 1) and a coordination number of 5 or 6 using the $\mathrm{Fe}-\mathrm{N}$ parameters (Fit 6, Figure 5.13B). Two shell fits with both an $\mathrm{O}$ and a $\mathrm{N}$ contribution were also attempted (Fits 7-9). These fits gave no indication that the first shell has two components since the distances remained the same (and thus not separable), the $c 2$ parameters were highly correlated, and the $c 2$ parameter of $O$ increased to such a level where its resulting contribution was negligible. Previous results on SLO-1 in frozen buffered solution ${ }^{14}$ indicated that there were $2 O$ at a distance of $1.94 \AA$. Therefore, the possibility of a shorter low $\mathbf{Z}$ contribution was further examined by fixing an Fe-O distance at $1.95,2.00,2.05$ and $2.10 \AA$ (Fits 10-15) and varying both the $c 2$ value and the second shell distance. When both distances of these two shell fits were allowed to vary, the same results as in Fits 7-9 were obtained. These fits to the data on SLO-1 in glycerol thus do not support a shorter Fe-O distance. The fit became significantly worse with the addition of a second shell in that the $\mathrm{c} 2$ value for the shorter $\mathrm{Fe}-\mathrm{O}$ contribution is more than an order a magnitude more negative than is chemically reasonable.

Fits were also performed where $c 2$ was fixed to -0.02391 and -0.02083 for the $\mathrm{Fe}-\mathrm{O}$ and $\mathrm{Fe}-\mathrm{N}$ contributions, respectively, letting both the distance and coordination 

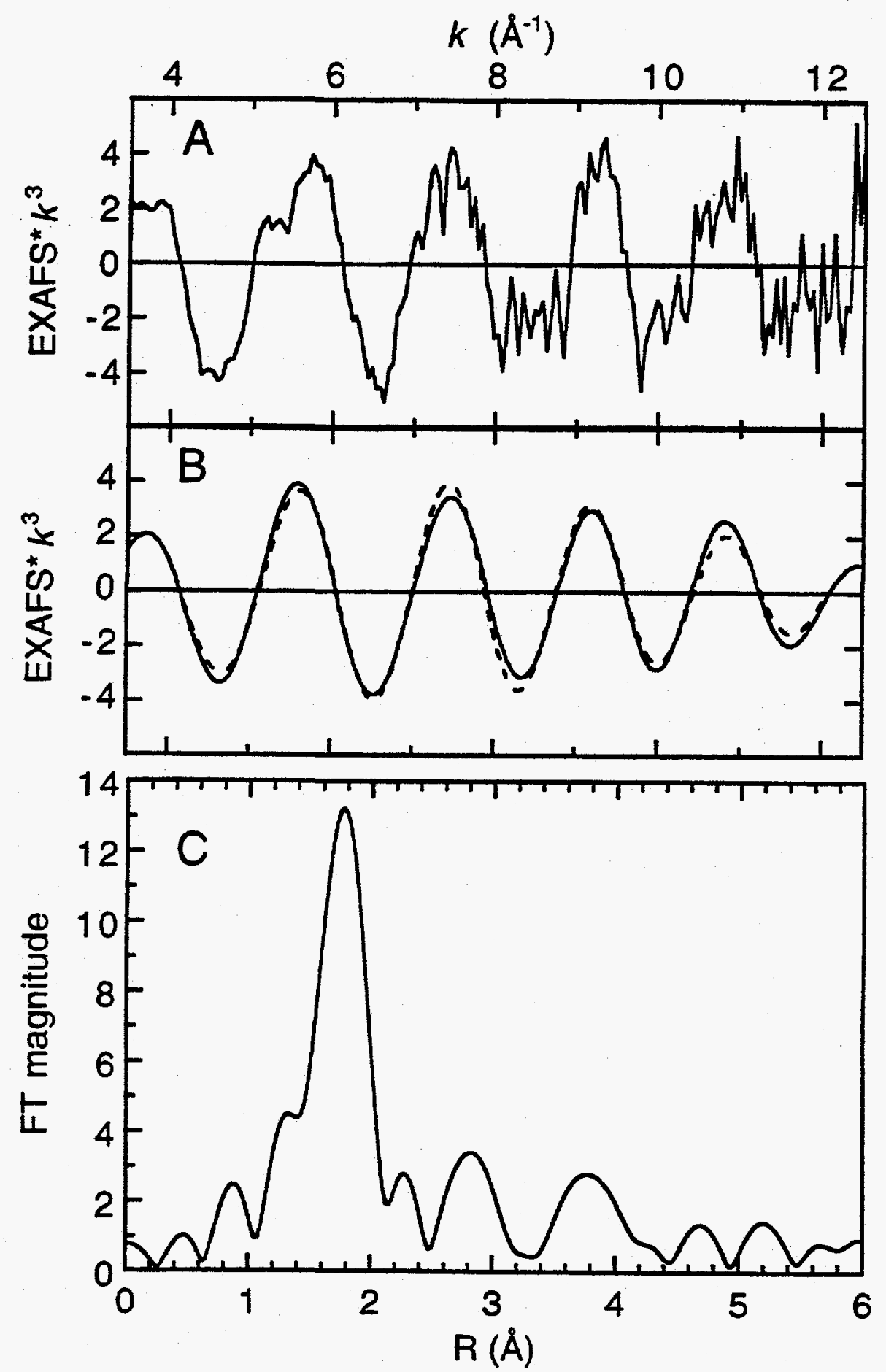

Figure 5.13. A) EXAFS data $\left({ }^{*} k^{3}\right)$ for SLO-1 in glycerol. B) - Fit to the Fourier filtered data. The solid line represents the data (FT backtransform range $1.0-2.25 \AA$ ) while the dashed line is the fit to the data with one shell of $N$ (Table 5.6, Fit 6). C) Fourier transform (non-phase shift corrected) over the $k$ range $3.5-12.5 \AA^{-1}$ of the EXAFS data of SLO-1. 
Table 5.6. Summary of EXAFS Curve-Fitting Results for SLO-1 in Glycerol.

\begin{tabular}{cccccc}
\hline Fit & element & $\begin{array}{c}\text { CN } \\
\text { (fixed) }\end{array}$ & distance & c2d & $F$ \\
\hline 1 & $\mathrm{O}$ & 4 & 2.15 & -0.02449 & 0.32 \\
2 & $\mathrm{O}$ & 5 & 2.15 & -0.02814 & 0.47 \\
3 & $\mathrm{O}$ & 6 & 2.15 & -0.03158 & 0.63 \\
4 & $\mathrm{~N}$ & 4 & 2.18 & -0.01973 & 0.45 \\
5 & $\mathrm{~N}$ & 5 & 2.18 & -0.02250 & 0.34 \\
6 & $\mathrm{~N}$ & 6 & 2.18 & -0.02500 & 0.32 \\
7 & $\mathrm{O}$ & 1 & 2.15 & -0.05671 & 0.28 \\
& $\mathrm{~N}$ & 5 & 2.18 & -0.02299 & \\
8 & $\mathrm{O}$ & 2 & 2.15 & -0.05148 & 0.25 \\
& $\mathrm{~N}$ & 4 & 2.18 & -0.02086 & \\
9 & $\mathrm{O}$ & 3 & 2.15 & -0.04811 & 0.22 \\
& $\mathrm{~N}$ & 3 & 2.18 & -0.01830 & \\
10 & $\mathrm{O}$ & 1 & $1.95^{*}$ & -0.69547 & 0.34 \\
& $\mathrm{~N}$ & 5 & 2.18 & -0.02250 & \\
11 & $\mathrm{O}$ & 2 & $1.95^{*}$ & -0.64971 & 0.45 \\
& $\mathrm{~N}$ & 4 & 2.18 & -0.01973 & \\
12 & $\mathrm{O}$ & 3 & $1.95^{*}$ & -0.65984 & 0.63 \\
& $\mathrm{~N}$ & 3 & 2.18 & -0.01650 & \\
13 & $\mathrm{O}$ & 2 & $2.00^{*}$ & -0.68207 & 0.45 \\
14 & $\mathrm{~N}$ & 4 & 2.18 & -0.01973 & \\
& $\mathrm{O}$ & 2 & $2.05^{*}$ & -0.07232 & 0.42 \\
& $\mathrm{~N}$ & 4 & 2.18 & -0.01978 & \\
& $\mathrm{O}$ & 2 & $2.10^{*}$ & -0.04990 & 0.30 \\
& $\mathrm{~N}$ & 4 & 2.18 & -0.02053 & \\
\hline
\end{tabular}

a FT range $3.5-12.5 \AA^{-1}$; FT back-transform range $1.0-2.5 \AA . b \mathrm{CN}=$ coordination number. $c$ Errors in distances $( \pm 0.02 \AA)$ are estimated from the variance between EXAFS fitting results and values from models of crystallographically known structure. ${ }^{30} d$ c2 values for $\mathrm{Fe}-\mathrm{O}$ from $\mathrm{Fe}$ (acetylacetonate) ${ }_{3}$ and $\mathrm{Fe}-\mathrm{N}$ from $\left[\mathrm{Fe}(1,10 \text {-phenanthroline })_{3}\right]\left(\mathrm{ClO}_{4}\right)_{3}$ were -0.02391 and -0.02083 , respectively. $*$ Values fixed. 
number vary. As expected, this gave the same results. The coordination numbers obtained from these fits were low, 3.8 for a fit with one $O$ shell and 4.6 for a fit with one $N$ shell. The low coordination numbers from these fits and the fact that the $c 2$ values (when $\mathrm{CN}=$ 6) are more negative than the respective values for $\mathrm{Fe}$ (acetylacetonate) 3 and [Fe(1,10-phenanthroline $\left.)_{3}\right]\left(\mathrm{ClO}_{4}\right)_{3}$ indicate that SLO-1 has a greater distribution of scatterers than the model complexes, as would be expected for the active site of a protein. Experimentally for a spread in first shell distances of $0.15 \AA$ for a six-coordinate complex with mixed $O$ and $N$ ligation, it has been observed that the $c 2$ values were -0.03408 for a fit with one shell of $O$ and -0.02816 for a fit with one shell of $N$. The fact that Fits 3 and Fit 6 have less negative $c 2$ values, respectively, suggests that the spread of first shell distances in SLO-1 is less than $0.15 \AA$. This is also supported by the fact that a two shell fit (Fits 7-9) does not match the data significantly better than a one shell fit (Fits 1-6) and that two shells should be readily discemible over the $k$ range $4-12 \AA^{-1}$ if the spread of first-shell distances is greater than $\sim 0.1 \AA$.

\subsubsection{Discussion}

In summary, the $\mathrm{Fe} \mathrm{K}$-edge shape and pre-edge intensity for all three 15-LO active sites are very similar to each other and all consistent with six-coordination. The EXAFS data of SLO-1 show that the iron active site has $5 \pm 1$ O/N ligands at $-2.16 \AA$. The average bond length also supports a six-coordinate site: crystallographic information on ferrous model complexes with oxygen and nitrogen ligation reports average bond lengths of $2.16 \pm 0.03 \AA$ for six-coordinate sites, ${ }^{44-48} 2.12 \pm 0.01 \AA$ for five-coordinate sites, ${ }^{26,27}$ and $2.00 \pm 0.01 \AA$ for four-coordinate sites. ${ }^{26}$ There is no indication of the previously reported shorter $\mathrm{Fe}-\mathrm{O}$ distance of Van der Heijdt et. al. ${ }^{14}$ Furthermore, these results show an average first shell distance that is $\sim 0.1 \AA$ longer than the previous result of $2.05 \AA .{ }^{14}$ However, the EXAFS data collected by Van der Heijdt et. al. was of a pH 9 sodium borate buffered frozen solution and lyophilized native SLO-1 samples. There is now evidence that native SLO-1 consists of more than one form. ${ }^{18}$ The fact that native SLO-1 contains a five-coordinate and a six-coordinate form could also explain the higher pre-edge intensity observed by Scarrow et. al. in the active site of native SLO-1. ${ }^{17}$ The edge and EXAFS analysis of SLO-1 in 50\% glycerol from this work and that of SLO-1 ${ }^{\mathrm{N}} \cdot \mathrm{MeOH}$ of Scarrow et. al. give very similar first-shell coordination and distances, ${ }^{17}$ indicating that iron active site in SLO-1 in glycerol and methanol is six-coordinate. 
The XAS results can be combined with NIR CD/MCD and VTVH MCD to better understand the coordination geometries of the iron active site in plant and mammalian LOs. ${ }^{19}$ NIR CD and MCD spectroscopies allow the direct determination of the splitting of the ferrous ${ }^{5} \mathrm{E}_{\mathrm{g}}$ excited state which through ligand field (LF) theory is dependent on the coordination geometry. ${ }^{49,50}$ Through analysis of the NIR CD and MCD spectra, it has been determined that native SLO-1 in solution exists as a nearly equal mixture of five- and six-coordinate forms. ${ }^{18}$ Addition of linoleate substrate or alcohols shifts the mixture to the purely six-coordinate form, which is consistent with the XAS pre-edge results and the EXAFS results of SLO-1 in glycerol which show the iron active site to have $5 \pm 1 \mathrm{~N} / \mathrm{O}$ at $-2.16 \AA$.

The two crystal structures ${ }^{6,11}$ of SLO-1 have four common ligands, three histidines and the carboxylate terminus isoleucine. The NIR CD/MCD and XAS spectroscopic results indicate that one and two additional ligands are present in the five- and six-coordinate forms of SLO-1, respectively. One ligand is likely a water or hydroxide, as Nelson ${ }^{13}$ has performed a EPR line broadening experiment on $\mathrm{Fe}^{+3} \mathrm{SLO}-1$ in $\mathrm{H}_{2}{ }^{17} \mathrm{O}$ which showed evidence that in the ferric enzyme a water or hydroxide is present. Minor $e t$ al. leave a water-based ligand open as a possibility in their structure, while Boyington et al. find no evidence for a water-based ligand. A significant difference in the crystal structures is in the binding of Asn694. Minor et al. show this residue bound through the carbonyl oxygen of the amide side chain, while the side chain is $3.3 \AA$ from the $\mathrm{Fe}^{+2}$ in the Boyington et al. structure. An amide carbonyl oxygen would be a relatively weak ligand which could be perturbed by hydrogen bonding or steric effects. Boyington et al. have the asparagine side chain hydrogen bonded to a neighboring glycine. The asparagine is also conserved in all sequenced LOs, except for rabbit reticulocyte and human 15-LOs. It is reasonable for the sixth ligand in the native six-coordinate SLO-1 site to be this asparagine.

Since the ligands perturb the d-orbital energy levels in a way dependent on their arrangement around the ferrous center, ${ }^{51,52} \mathrm{LF}$ calculations ${ }^{51}$ were performed on two spectroscopically effective active site models ${ }^{19}$ (one five-coordinate without Asn 694 bound and one six- coordinate with Asn 694 bound) of SLO-1 built upon the crystallography ${ }^{6,11}$ as well as on the four-coordinate structure of Boyington et al. (described as a distorted octahedron missing two cis ligands). Comparison of the calculated ${ }^{5} \mathrm{E}_{\mathrm{g}}$ energy levels to those of the experimental ${ }^{5} \mathrm{E}_{\mathrm{g}}$ splittings shows that the five- and six-coordinate LF calculations accurately model the experimentally observed splittings of the two components in the native SLO-1 spectrum. The four-coordinate SLO-1 calculation show the presence of two transitions in the $5000-13,000 \mathrm{~cm}^{-1}$ region and an additional high-energy transition at $>13,000 \mathrm{~cm}^{-1}$. Since the experimental NIR CD/MCD data on SLO-1 do not 
exhibit such high-energy transitions, the four-coordinate crystal structure does not accurately describe the ferrous site in solution. The observed $C D / M C D$ transitions for native ferrous SLO-1 are consistent with the five- and six-coordinate sites.

The effect of alcohols on the NIR CD/MCD data for the native ferrous SLO-1 site is also dramatic, shifting the mixture of forms to a purely six-coordinate site which has very similar spectral features to the six-coordinate component of the native enzyme. The ferric enzyme has been shown by EPR studies 53,54 to be a mixture of two fairly axial forms with $g=6.2$ and 7.4. Addition of alcohols causes the mixture of EPR signals to shift to the clean $g=6.2$ peak. ${ }^{53}$ The coordination number of the two ferric forms is not known, but $\mathrm{MCD}$ studies ${ }^{55}$ indicate that a six-coordinate site may also be appropriate for the $\mathrm{g}=6.2$ species, as it is the only form present when alcohols and glycerol are added to the ferric enzyme. NMR studies ${ }^{56}$ of the ferric enzyme showed that alcohols bind near the active site but not directly to the iron, likely in the hydrophobic fatty acid substrate binding site. This may also relate to the alcohol effects on the native ferrous site, which have the same effect as substrate binding to native ferrous SLO-1, and thus the alcohols are likely to bind at the substrate binding site. Asn 694 is at the end of a cavity which starts at the surface of the enzyme ${ }^{6}$ and which may relate to the fatty acid binding site. It appears that when linoleate (or alcohols) are present at the fatty acid binding site, a change in the active site environment occurs allowing the $O \delta 1$ of $\mathrm{Asn}_{694}$ to bind to the iron, converting the active site to the purely six-coordinate form. Thus the ferrous SLO-1 plus linoleate substrate form may be the most relevant with respect to catalysis.

The NIR CD and MCD studies on the two native mammalian 15-LO active sites show that in contrast to SLO-1, they exist as purely six-coordinate structures rather than a mixture of five- and six-coordinate sites. ${ }^{19}$ XAS pre-edge intensities also indicate that the mammalian 15-LOs in glycerol are six-coordinate. The edge shapes are also very similar to those of six-coordinate model complexes. The corresponding Asn694 amino acid position in SLO-1 is substituted with histidine in rabbit reticulocyte and human 15-LOs. ${ }^{5}$ The differences in the NIR CD/MCD spectra of the native plant vs. these mammalian 15-LOs is reasonably associated with this substitution, as histidine would be a stronger ligand than the carbonyl oxygen of asparagine and lead to a stronger bond. A LF calculation was also performed on a model structure, in which the asparagine $O$ has been replaced with a histidine N. ${ }^{19}$ Comparison of the resulting calculated d-orbital energy levels to the experimentally observed transitions for 15-RLO and 15-HLO shows general agreement for the ${ }^{5} \mathrm{E}_{\mathrm{g}}$ splittings. The calculated ${ }^{5} \mathrm{~T}_{2 \mathrm{~g}}$ splittings for both the soybean and mammalian enzymes are also qualitatively similar to the ground-state splittings obtained though the 
VTVH MCD analysis, showing a larger ${ }^{5} \mathrm{~T}_{2 g}$ splitting for the five-coordinate SLO-1 site than for the six-coordinate LOs.

The saturation magnetization data for the mammalian 15-LOs are qualitatively different from those of the six-coordinate SLO-1, exhibiting tighter nesting compared to the plant enzyme data. The VTVH MCD analysis of these data shows that both the five- and six-coordinate sites of SLO-1 have a positive zero field splitting (+D), while the mammalian LOs have a negative zero field splitting ( $-D)$. Thus replacing one ligand going from the plant to the mammalian enzymes causes large perturbations in the electronic structure of the ferrous site which are clearly exhibited in the behavior of the VTVH MCD data. LF calculations which differ only by the parameters associated with the Asn $\rightarrow$ His substitution along the molecular $\mathrm{z}$-axis (with the asparagine $\mathrm{O}$ weaker than the histidine $\mathrm{N}$ ) reproduce the $+D$ ground state $d$-orbital pattern for six-coordinate SLO-1 and the $-D$ pattern for the mammalian LOs. Therefore, although the spectra of six-coordinate SLO-1 and 15-R/HLO are qualitatively similar, the ground-state VTVH MCD and LF analyses demonstrate significant differences in the electronic structure of the ferrous site between the soybean and mammalian enzymes reasonably ascribed to the Asn $\rightarrow$ His ligand substitution.

\subsubsection{Acknowledgments}

Mark Pavlosky prepared the SLO-1 sample. The 15-RLO and 15-HLO samples were prepared by Yan Zhang and Qing-Fen Gan of Syntex. Dr. Elliott Sigal, Executive Director of Cell Biology of Syntex Discovery Research, provided the human recombinant 15-lipoxygenase and rabbit reticulocyte 15-lipoxygenase protein samples. Mark Pavlosky, Yan Zhang, Elizabeth Pavel, and Cecelia Campochiaro performed the NIR CD/MCD experiments and the LF calculations alluded to in the Discussion. This research was supported by grants from the NIH (GM40392, E. I. Solomon) and NSF (CHE-9121576, K. O. Hodgson). The Stanford Synchrotron Radiation Laboratory is supported by the Department of Energy, Office of Basic Energy Sciences, Divisions of Chemical Science and Materials Science, and in part by the National Institutes of Health, National Center of Research Resources, Biomedical Research Technology Program (RR-01209, K. O. Hodgson) and the DOE's Office of Health and Environmental Research.

\subsubsection{References and Notes}

(1) Samuelsson, B.; Dahlén, S.-E.; Lindgren, J. Å.; Rouzer, C. A.; Serhan, C. N. Science 1987, 237, 1171. 
(2) Sigal, E.; Laughton, C. W.; Mulkins, M. A. Ann. New York Acad. Sci. 1994, $714,211$.

(3) Steinberg, D.; Parthasarathy, S.; Carew, 1. E.; Khoo, J. C.; Witztum, J. L. N. Engl. J. Med. 1989, 320, 915.

(4) Gardner, H. W. Biochim. Biophys. Acta 1991, 1084, 221.

(5) Sigal, E. Am. J. Physiol. 1991, 260, L13.

(6) Boyington, J. C.; Gaffney, B. J.; Amzel, L. M. Science 1993, 260, 1482.

(7) Petersson, L.; Slappendel, S.; Vliegenthart, J. F. G. Biochim. Biophys. Acta $1985,828,81$.

(8) Veldink, G. A.; Vliegenthart, J. F. G. Adv. Inorg. Biochem. 1984, 6, 139.

(9) Nelson, M. J.; Cowling, R. A.; Seitz, S. P. Biochemistry 1994, 33, 4966.

(10) Corey, E. J.; Nagata, R. J. Am. Chem. Soc. 1987, 109, 8107.

(11) Minor, W.; Steczko, J.; Bolin, J. T.; Otwinowski, Z.; Axelrod, B. Biochemistry 1993, 32, 6320.

(12) Sigal, E.; Craik, C. S.; Highland, E.; Grunberger, D.; Costello, L. L.; Dixon, R. A. F.; Nadel, J. A. Biochem. Biophys. Res. Comm. 1988, 157, 457.

(13) Nelson, M. J. J. Am. Chem. Soc. 1988, 110, 2985.

(14) Van der Heijdt, L. M.; Feiters, M. C.; Navaratnam, S.; Nolting, H.-F.; Hermes, C.; Veldink, G. A.; Vliegenthart, J. F. G. Eur. J. Biochem. 1992, $207,793$.

(15) Dunham, W. R.; Carroll, R. T.; Thompson, J. F.; Sands, R. H.; Funk, M. O., Jr. Eur. J. Biochem. 1990, 190, 611.

(16) Whittaker, J. W.; Solomon, E. I. J. Am. Chem. Soc. 1988, 110, 5329.

(17) Scarrow, R. C.; Trimitsis, M. G.; Buck, C. P.; Grove, G. N.; Cowling, R. A.; Nelson, M. J. Biochemistry 1994, 33, 15023.

(18) Pavlosky, M. A.; Solomon, E. I. J. Am. Chem. Soc. 1994, 116, 11610.

(19) Pavlosky, M. A.; Zhang, Y.; Westre, T. E.; Gan, Q.-F.; Pavel, E. G.; Campochiaro, C.; Hedman, B.; Hodgson, K. O.; Solomon, E. I. J. Am. Chem. Soc. 1995, 117, 4316.

(20) Nelson, M. J. Biochemistry 1988, 27, 4273.

(21) Kühn, H.; Barnett, J.; Grunberger, D.; Baeker, P.; Chow, J.; Nguyen, B.; Bursztyn-Pettegrew, H.; Chan, H.; Sigal, E. Biochim. Biophys. Acta 1993, $1169,80$.

(22) Sloane, D. L.; Browner, M. F.; Dauter, Z.; Wilson, K.; Fletterick, R. J.; Sigal, E. Biochem. Biophys. Res. Comm. 1990, 173, 507.

(23) Scott, R. A.; Hahn, J. E.; Doniach, S.; Freeman, H. C.; Hodgson, K. O. J. Am. Chem. Soc. 1982, 104, 5364. 
(24) Cramer, S. P.; Tench, O.; Yocum, M.; George, G. N. Nucl. Instrum. Methods Phys. Res. 1988, A266, 586.

(25) George, G. N.; Pickering, I., to be published.

(26) Kitajima, N., private communication.

(27) Hodges, K.D.; Wollmann, R.G.; Barfield, E.K.; Hendrickson, D.N. Inorg. Chem. 1977, 16, 2746.

(28) Abbreviations used: $\mathrm{HB}\left(3,5-i \mathrm{Pr}_{2} \mathrm{pz}\right)_{3}=$ hydrotris(3,5-diisopropyl-1pyrazolyl)borate; TMC = 1,4,8,11-tetramethyl-1,4,8,11-tetraazacyclotetradecane.

(29) Burbridge, C. D.; Goodgame, D. M. L. Inorg. Chim. Acta 1970, 4, 231.

(30) Cramer, S. P.; Hodgson, K. O.; Stiefel, E. I.; Newton, W. E. J. Am. Chem. Soc. 1978, 100, 2748.

(31) Cramer, S. P.; Hodgson, K. O. Prog. Inorg. Chem. 1979, $25,1$.

(32) Scott, R. A. Methods Enzymol. 1985, 117, 414.

(33) Iball, J.; Morgan, C. H. Acta Crystallogr. 1967, 23, 239.

(34) Roof, R. B., Jr. Acta Crystallogr. 1956, 9, 781.

(35) Johansson, L. Chem. Scripta 1976, 9, 30.

(36) Johansson, L.; Molund, M.; Oskarsson, Å. Inorg. Chim. Acta. 1978, 31, 117.

(37) Argonne National Laboratory; Garbow, B.S.; Hillstrom, K.E.; More, J.J.

(38) Agarwal, B. K. X-ray Spectroscopy; Springer-Verlag: New York, 1979; p 276.

(39) Lytle, F. W.; Greegor, R. B.; Sandstrom, D. R.; Marques, E. C.; Wong, J.; Spiro, C. L.; Huffman, G. P.; Huggins, F. E. Nucl. Instrum. Methods Phys. Res. 1984, 226, 542.

(40) Lytle, F. W. In Applications of Synchrotron Radiation; Winick, H.; Xian, D.; Ye, M.-h.; Huang, T., Eds.; Gordon and Breach Science Publishers: New York, 1989; p 135.

(41) Tyson, T. A.; Roe, A. L.; Frank, P.; Hodgson, K. O.; Hedman, B. Phys. Rev. B 1989, 39, 6305.

(42) Roe, A. L.; Schneider, D. J.; Mayer, R. J.; Pyrz, J. W.; Widom, J.; Que, L., Jr. J. Am. Chem. Soc. 1984, 106, 1676.

(43) Randall, C. R.; Shu, L.; Chiou, Y.-M; Hagen, K.S.; Ito, M.; Kitajima, N.; Lachicotte, R.J.; Zang, Y.; Que, L., Jr. Inorg. Chem. 1995, 34, 1036.

(44) Miller, L. L.; Jacobson, R. A.; Chen, Y.-S.; Kurtz, D. M., Jr. Acta Crystallogr. 1989, C45, 527.

(45) Oliver, J. D.; Mullica, D. F.; Hutchinson, B. B.; Milligan, W. O. Inorg. Chem. $1980,19,165$. 
(46) Kitajima, N.; Fukui, H.; Moro-oka, Y.; Mizutani, Y.; Kitagawa, T. J. Am. Chem. Soc. 1990, 112, 6402 .

(47) Montgomery, H.; Chastain, R. V.; Natt, J. J.; Witkowska, A. M.; Lingafelter, E. C. Acta Crystallogr. 1967, 22, 775.

(48) Price, D. C. Can. J. Phys. 1987, 65, 1280.

(49) Solomon, E. I.; Zhang, Y. Acc. Chem. Res. 1992, 25, 343.

(50) Solomon, E. I.; Pavel, E. G.; Loeb, K. E.; Campochiaro, C. Coord. Chem. Rev. in press.

(51) Companion, A. L.; Komarynsky, M. A. J. Chem. Ed. 1964, 41, 257.

(52) Pulver, S.; Froland, W. A.; Fox, B. G.; Lipscomb, J. D.; Solomon, E. I. J. Am. Chem. Soc. 1993, 115, 12409.

(53) Slappendel, S.; Aasa, R.; Malmström, B. G.; Verhagen, J.; Veldink, G. A.; Vliegenthart, J. F. G. Biochim. Biophys. Acta 1982, 708, 259.

(54) Gaffney, B. J.; Mavrophilipos, D. V.; Doctor, K. S. Biophys. J. 1993, 64, 773.

(55) Zhang, Y.; Gebhard, M. S.; Solomon, E. I. J. Am. Chem. Soc. 1991, 113, 5162.

(56) Slappendel, S.; Aasa, R.; Falk, K.-E.; Malmström, B. G.; Vänngård, T.; Veldink, G. A.; Vliegenthart, J. F. G. Biochim. Biophys. Acta 1982, 708, 266. 


\subsection{Phenylalanine Hydroxylase}

\subsubsection{Introduction}

Phenylalanine hydroxylase (PAH), tyrosine hydroxylase (TH), and tryptophan hydroxylase (TPH) are mammalian aromatic amino acid hydroxylases that uniquely use tetrahydropterins as obligatory cofactors. The reactions catalyzed by these enzymes are all used in amino acid metabolism: PAH initiates the detoxification of high levels of phenylalanine ${ }^{1,2}$ while TH and TPH catalyze the committed steps in the biosynthesis of the neurotransmitters dihydroxyphenylalanine $e^{3,4}$ and serotonin, 5,6 respectively. A role for the non-heme iron in oxygen activation is strongly suggested, since all of the mammalian hydroxylases contain one non-heme iron per subunit and each enzyme requires the presence of its metal center for reactivity. ${ }^{7-9}$ It appears certain that $\mathrm{Fe}(\mathrm{II})$ is the catalytically important oxidation state, ${ }^{10}$ but the $\mathrm{Fe}$ (III) state may play a role in feedback regulation. ${ }^{11}$ Due to the relatively large quantity of PAH available from rat liver as well as its soluble nature and ease of purification by substrate-induced hydrophobic binding, much more work has been done on this enzyme than the other hydroxylases. Thus, PAH serves as the prototype aromatic amino acid hydroxylase. ${ }^{12}$

PAH catalyzes the rate-limiting step in the removal of phenylalanine from blood. Dysfunction of this enzyme leads to a condition called phenylketonuria, which is characterized by irreversible, progressive brain damage that results from the buildup of phenylalanine and its neurotoxic metabolites. ${ }^{13}$ PAH is a tetramer of $-50 \mathrm{kDa}$ subunits, each of which contains a non-heme iron that is absolutely required for reactivity. ${ }^{7-9}$ During the hydroxylation of phenylalanine, the enzyme uses molecular oxygen and one tetrahydrobiopterin $\left(\mathrm{H}_{4} \mathrm{BP}\right)$ to generate tyrosine and a molecule of oxidized pterin cofactor:

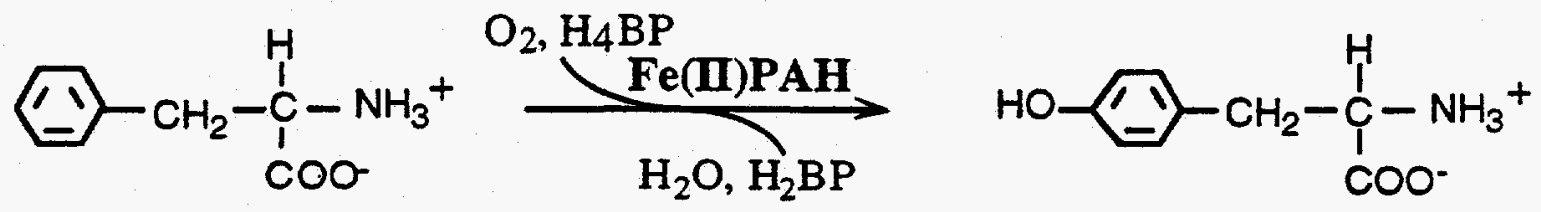

Dioxygen is partitioned heterolytically between the phenolic hydroxy group of tyrosine and the initial C4a-carbinolamine form of the oxidized pterin, which subsequently dehydrates to yield the oxidized, quinonoid dihydropterin. In order to avoid depletion of phenylalanine from the liver, the enzyme is only active in the presence of elevated levels of its amino acid substrate. In studies of the protein, it has been observed that phenylalanine binds to an allosteric effector site (as distinguished from the active phenylalanine binding site) concomitant with an increase in the specific activity of the protein. This "activation" 
process has a large energetic barrier ( $30-40 \mathrm{kcal} / \mathrm{mol}$ by Arthenius analysis) and is accompanied by a large structural rearrangement of the PAH protein. ${ }^{14}$ The transition between the resting low affinity state (the " $T$ " state) and the activated high affinity state (the " $R$ " state) is required for the enzyme to achieve catalytic competence. ${ }^{14}$ Enzyme activation can also be accomplished using lysolecithin, $\alpha$-chymotrypsin, or $N$-ethylmaleimide. ${ }^{2}$ For the reaction of the resting enzyme with $\mathrm{H}_{4} \mathrm{BP}$ an ordered sequential mechanism is observed, ${ }^{15}$ phenylalanine $+\mathrm{H}_{4} \mathrm{BP}+\mathrm{O}_{2}$, because tight binding of the pterin to the resting enzyme inhibits its conversion to the activated state.

By analogy to flavin chemistry, it was originally proposed that dioxygen reacts directly with the pterin cofactor to generate 4a-hydroperoxy-tetrahydrobiopterin (4a-OOH), which decays to form the active oxidant. Substantial experimental evidence supports this hypothesis. ${ }^{12,16,17}$ According to this mechanism, Fe(II) would react with the peroxidated cofactor, rather than with dioxygen itself, forming an $\mathrm{Fe}$ (II)peroxypterine (Fe-OO-4a) adduct. Heterolytic decomposition of this intermediate is proposed to yield $4 \mathrm{a}-\mathrm{OH}$ (which has been observed experimentally ${ }^{12}$ ) and a ferryl species that is the active oxidant. This theory appeared to be supported by the existence of a "copper-free" form of PAH from Chromobacterium violaceum, ${ }^{18}$ since the heterolytic cleavage of hydroperoxides is known to occur under certain metal free conditions. Conversely, a reaction between the reduced metal and dioxygen may precede formation of the $4 \mathrm{a}-\mathrm{OOH}$ intermediate. The strongest evidence for such a role for iron in PAH is the diminished oxidation rate of the cofactor in absence of the metal ion. ${ }^{12}$ On the basis of chemistry observed in other enzymes and model systems and the clear requirement for reduced iron, it may be that a ferricsuperoxide/ferrous-dioxygen adduct forms initially which then peroxidizes the $\mathrm{H}_{4} \mathrm{BP} .{ }^{19}$ The reaction would yield the same $\mathrm{Fe}$ (II)-OO-4a cofactor adduct discussed above and result in possibly the same heterolytic decomposition pathway. The current experimental data unfortunately cannot differentiate these two pathways.

As isolated from either rat liver or an $E$. coli-based overexpression system, ${ }^{14} \mathrm{PAH}$ is oxidized and in the " $\mathrm{T}$ " state (Fe(III)PAH $)$. Either reduction or activation may occur next, to give $\mathrm{Fe}$ (II)PAH $\mathrm{PA}^{\mathrm{T}}$ or $\mathrm{Fe}$ (III)PAHR, respectively. Finally, the generation of active enzyme follows activation or reduction to give $\mathrm{Fe}(\mathrm{II}) \mathrm{PAH}^{\mathrm{R}}$. Note that $\mathrm{Fe}(\mathrm{II}) \mathrm{PAH}^{\mathrm{R}}$ has phenylalanine in both the allosteric and the active site. It is this activated form of the ferrous enzyme that reacts with $\mathrm{H}_{4} \mathrm{BP}$ and $\mathrm{O}_{2}$ to generate tyrosine and an oxidized form of the pterin cofactor (see previous page).

$\mathrm{X}$-ray absorption spectroscopy (XAS) can be used to characterize the essential non-heme iron site, both in the absence and presence of substrates and competitive inhibitors of substrates. An understanding of the structure of this iron site will aid in 
elucidating the complex mechanism of PAH catalysis. In the study presented here, XAS was used to define the geometric and electronic structure of the non-heme iron active site of $\mathrm{Fe}$ (II)PAHR $, \mathrm{Fe}(\mathrm{II}) \mathrm{PAH}^{\mathrm{T}}, \mathrm{Fe}$ (III)PAHR, and $\mathrm{Fe}(\mathrm{III}) \mathrm{PAH}^{\mathrm{T}}$. Fe K-edge data were collected for these four forms of PAH and compared to data of four-, five-, and six-coordinate ferrous and ferric model complexes to determine the coordination number of the non-heme active site. In addition, an extended X-ray absorption fine structure (EXAFS) analysis was performed on the data of $\mathrm{Fe}$ (II)PAH ${ }^{\mathrm{R}}, \mathrm{Fe}$ (II)PAH $\mathrm{PA}^{\mathrm{T}}, \mathrm{Fe}$ (III)PAHR, and $\mathrm{Fe}$ (III) $\mathrm{PAHT}$ to provide information on the types of ligands surrounding the iron site and the distances to those ligands.

\subsubsection{Experimental Section}

The PAH samples were prepared in Prof. John Caradonna's laboratory at Yale University using an $E$. coli-based overexpression system. ${ }^{14}$ As-isolated PAH is in the $\mathrm{Fe}$ (III)PAH $\mathrm{PA}^{\mathrm{T}}$ form. Each of the four samples (Fe(II)PAHR, $\mathrm{Fe}(\mathrm{II}) \mathrm{PAH}^{\mathrm{T}}, \mathrm{Fe}$ (III)PAH ${ }^{\mathrm{R}}$, and $\left.\mathrm{Fe}(\mathrm{III}) \mathrm{PAH}^{\mathrm{T}}\right)$ were exchanged into low-salt $50 \mathrm{mM} 3-(N$-morpholino)propanesulfonic acid (MOPS) buffer and concentrated over a YM30 ultrafiltration membrane to $2 \mathrm{ml}$. At this point, 2 volumes of $50 \mathrm{mM}$ MOPS $/ 75 \%$ glycerol were added and then the samples were concentrated back down to $\sim 0.2 \mathrm{~mL}$. The Fe(II)PAH ${ }^{\mathrm{T}}$ sample was obtained by reducing the $\mathrm{Fe}$ (III)PAH ${ }^{\mathrm{T}}$ sample with one equivalent of dithionite. The $\mathrm{R}$ state samples were generated by dissolving the protein in $50 \mathrm{mM}$ MOPS buffer $\left(\mathrm{pH} 7.3\right.$ at $25^{\circ} \mathrm{C}$ ) that contained $1 \mathrm{mM}$ phenylalanine and activated by heating the sample to $25^{\circ} \mathrm{C}$ for 10 minutes. The samples were then concentrated as described above. Two volumes of $50 \mathrm{mM}$ MOPS $/ 75 \%$ glycerol $/ 10 \mathrm{mM}$ phenylalanine were added and then the $\mathrm{R}$ state samples were concentrated back down to $\sim 0.2 \mathrm{~mL}$. The iron concentrations of all four samples were between $2.0-2.7 \mathrm{mM}$ in Fe. The starting specific activity for the Fe(III)PAH $\mathrm{PA}^{\mathrm{T}}$ sample was $7.9 \mathrm{U} / \mathrm{mg}$ with about $0.75 \mathrm{Fe} /$ subunit. The starting specific activity for the $\mathrm{Fe}(\mathrm{III}) \mathrm{PAH}^{\mathrm{R}}$ sample was $6.0 \mathrm{U} / \mathrm{mg}$ with about $0.75 \mathrm{Fe} /$ subunit. The samples were loaded into Lucite EXAFS cells $(23 \times 1 \times 3 \mathrm{~mm}$ ) with $37 \mu \mathrm{m}$ Kapton windows and frozen in liquid nitrogen. The samples were characterized before and after exposure to the $\mathrm{X}$-ray beam using electron paramagnetic resonance (EPR).

$\mathrm{X}$-ray absorption spectra were recorded at the Stanford Synchrotron Radiation Laboratory (SSRL) on unfocused beamline 7-3 during dedicated conditions ( $3 \mathrm{GeV}$, 50-100 mA). The radiation was energy resolved using a Si(220) double-crystal monochromator with $1 \mathrm{~mm}$ high pre-monochromator beam defining slits. An Oxford Instruments continuous-flow liquid helium CF1208 cryostat was used to maintain a 
constant temperature of $10 \mathrm{~K}$. Energies were calibrated using an internal Fe foil standard, assigning the first inflection point to $7111.2 \mathrm{eV} .^{20}$ The spectrometer energy resolution was approximately $1.4 \mathrm{eV}$ with reproducibility in edge position determination of $<0.2 \mathrm{eV} .40$ Data were measured to $k=15 \AA^{-1}$, detuning the monochromator $50 \%$ at $7998 \mathrm{eV}$ to minimize harmonic contamination. The fluorescence signal was monitored using a 13 -element $\mathrm{Ge}$ solid-state array detector ${ }^{21}$ windowed on the $\mathrm{Fe} \mathrm{K} \alpha$ signal. During the experiment, count rates of approximately $30,000 \mathrm{~s}^{-1}$ total per element were not exceeded. Thirty scans were collected on the $\mathrm{Fe}(\mathrm{II}) \mathrm{PAH}^{\mathrm{T}}$ sample and twenty-two scans were collected on the $\mathrm{Fe}$ (II)PAHR sample. Both the $\mathrm{Fe}$ (III)PAHR and $\mathrm{Fe}(\mathrm{III}) \mathrm{PAH}^{\mathrm{T}}$ samples photo-reduced in the beam, as was evident by changes in the edge shape and position. Therefore, the Fe(III)PAH ${ }^{\mathrm{T}}$ sample was exposed to the beam in two different spots and thus two 'first' scans and a total of eleven scans were collected. Since the photo-reduction of $\mathrm{Fe}$ (III)PAH $\mathrm{PA}^{\mathrm{R}}$ was anticipated, the sample was exposed in four different spots giving four 'first' scans and a total of twenty scans. The edge position of the data in the 'fifth' scans was at $\sim 1 \mathrm{eV}$ lower in energy than that in the 'first' scans for both ferric protein samples. From comparison of 'first' scan data to 'second' scan data to 'third' scan data, etc., no discernible changes in the EXAFS could be detected. Therefore, the edge spectra of $\mathrm{Fe}$ (III) $\mathrm{PAH}^{\mathrm{T}}$ and $\mathrm{Fe}$ (III)PAHR presented in this section are an average of the 'first' scans only, while the EXAFS data shown are an average of all the scans collected. A pre-edge subtraction was performed by fitting the tail of a Gaussian to the pre-edge region and subtracting this polynomial from the averaged spectra. ${ }^{22}$ A three-segment spline approximately even in $k$-space was fit to the EXAFS region and the data normalized to an edge jump of one at $7130 \mathrm{eV}$. The spline was chosen so that it minimized residual low-frequency background but did not reduce the EXAFS amplitude as checked by monitoring the Fourier transform of the EXAFS during the background subtraction process.

Fe K-edge spectra were also collected on four-, five-, and six-coordinate ferrous and ferric model complexes. $\mathrm{Fe}(\mathrm{acac})_{3}$ was purchased from Aldrich. ${ }^{23} \mathrm{Fe}(\mathrm{HB}(3,5-$ $\left.i \mathrm{Pr}_{2} \mathrm{Pz}_{3}\right) \mathrm{Cl}^{24}$ was obtained as a gift from N. Kitajima. $\left(\mathrm{BF}_{4}\right)[\mathrm{Fe}(\mathrm{TMC}) \mathrm{Cl}],{ }^{25}$ [Fe(imidazole) 6$] \mathrm{Cl}_{2},{ }^{26} \mathrm{Fe}$ (salen) $\mathrm{Cl}_{1}{ }^{27}$ and $\left(\mathrm{Et}_{4} \mathrm{~N}\right)\left[\mathrm{FeCl}_{4}\right]^{28}$ were prepared as described in the literature. The model complex XAS samples were prepared in the following manner. [Fe(imidazole) $\left.)_{6}\right] \mathrm{Cl}_{2}, \mathrm{Fe}\left(\mathrm{HB}\left(3,5-\mathrm{Pr}_{2} \mathrm{pz}\right)_{3}\right) \mathrm{Cl}$, and $\left(\mathrm{BF}_{4}\right)[\mathrm{Fe}(\mathrm{TMC}) \mathrm{Cl}]$ are air-sensitive and thus the following procedure was carried out in a nitrogen-filled glove box for these three samples. The crystalline samples were each mixed with $B N$ and ground into a fine powder. The BN/sample mixture was pressed into a $1 \mathrm{~mm}$ thick $\mathrm{Al}$ spacer that was sealed with $63.5 \mu \mathrm{m}$ Mylar tape windows and frozen in liquid nitrogen. Data were measured in 
(44) Montgomery, H.; Chastain, R. V.; Natt, J. J.; Witkowska, A. M.; Lingafelter, E. C. Acta Crystallogr. 1967, 22, 775.

(45) Cingi, M. B.; Lanfredi, A. M. M.; iripicchio, A.; Cornelissen, J. P.; Haasnoot, J. G.; Reedijk, J. Acta Crystallogr. 1986, C42, 1296.

(46) Kitajima, N.; Fukui, H.; Moro-oka, Y.; Mizutani, Y.; Kitagawa, T. J. Am. Chem. Soc. 1990,6402 .

(47) Ainscough, E. W.; Brodie, A. M.; Plowman, J. E.; Brown, K. L.; Addison, A. W.; Gainsford, A. R. Inorg. Chem. 1980, 19, 3655.

(48) Cox, D. D.; Que, L., Jr. J. Am. Chem. Soc. 1988, 110, 8085.

(49) Lauffer, R. B.; Heistand, R. H., II; Que, L., Jr. Inorg. Chem. 1983, 22, 50.

(50) Malfant, I.; Morgenstern-Badarau, I.; Philoche-Levisalles, M.; Lloret, F. J. Chem. Soc., Chem. Commun. 1990, 1338.

(51) McDevitt, M. R.; Addison, A. W.; Sinn, E.; Thompson, L. K. Inorg. Chem. $1990,29,3425$.

(52) Okamoto, K.-I.; Kanamori, K.; Hidaka, J. Acta Crystallogr. 1990, C46, 1640.

(53) Raymond, K. N.; Isied, S. S.; Brown, L. D.; Fronczek, F. R.; Nibert, J. H. J. Am. Chem.Soc. 1976, 98, 1767.

(54) Clarke, E. T.; Martell, A. E.; Reibenspies, J. Inorg. Chim. Acta 1992, 196, 177.

(55) Miller, L. L.; Jacobson, R. A.; Chen, Y.-S.; Kurtz, D. M., Jr. Acta Crystallogr. 1989, C45, 527.

(56) Chiou, Y.-M.; Que, L., Jr. J. Am. Chem. Soc. 1992, 114, 7567.

(57) Zang, Y.; Elgren, T. E.; Dong, Y.; Que, L., Jr. J. Am. Chem. Soc. 1993, 115, 811.

(58) Kitajima, N.; Tamura, N.; Amagai, H.; Fukui, H.; Moro-oka, Y.; Mizutani, Y.; Kitagawa, T.; Mathur, R.; Heerwegh, K.; Reed, C. A.; Randall, C. R.; Que, L., Jr.; Tatsumi, K. J. Am. Chem. Soc. 1994, 116, 9071.

(59) Hagen, K. private communication.

(60) Heistand, R. H., II; Roe, A. L.; Que, L., Jr. Inorg. Chem. 1982, 21, 676.

(61) Koch, S. A.; Millar, M. J. Am. Chem. Soc. 1982, 104, 5255.

(62) Loeb, K.; Solomon, E. I. private communication. 


\subsection{Protocatechuate 3,4-Dioxygenase}

\subsubsection{Introduction}

The catechol dioxygenases serve as part of nature's strategy for degrading aromatic molecules in the environment. ${ }^{1}$ A critical point of this degradation is the opening of the chemically stable aromatic ring. In typical aerobic pathways, the ring is first activated by hydroxylation on adjacent carbons to form a catecholate-like complex. Ring cleavage, catalyzed by dioxygenase, then proceeds between the hydroxylated carbon atoms (intradiol- or ortho-cleavage) or adjacent to one of these carbon atoms (extradiolor meta-cleavage). ${ }^{2}$ The intradiol cleaving enzymes utilize a ferric active site and act on catechol and protocatechuate resulting in muconic acids, while the extradiol cleaving enzymes utilize a ferrous active site and act on a larger number of substrates producing muconic semialdehydes (Table 5.1). ${ }^{3,4}$

The most well-characterized intradiol dioxygenase is protocatechuate 3,4-dioxygenase (PCD). Crystal structures of $\mathrm{PCD}$ from Psuedomonas aeruginosa ${ }^{5,6}$ and Brevibacterium fuscum ${ }^{7}$ both have trigonal bipyramidal iron sites with a tyrosine (Tyr) and histidine (His) coordinated axially and a Tyr, His, and water-based molecule bound in the equatorial plane. The iron coordination environment defined by the crystal structure corresponds remarkably well to the active site proposed on the basis of spectroscopic studies. ${ }^{8}$ The enzyme exhibits a distinct burgundy red color $\left(\lambda_{\max } \sim\right.$ $460 \mathrm{~nm}$ ) that is associated with a tyrosinate $\rightarrow \mathrm{Fe}$ (III) charge transfer (CT) transition with the presence of two distinct tyrosine ligands indicated by the appearance of two $v_{\mathrm{CO}}$ bands in the resonance Raman ( $\mathrm{rR}$ ) spectrum. ${ }^{9,10}$ Histidine ligation was also suggested by $\mathrm{r} R$ studies with the observation of a low-energy $V_{F e-N(I m)}$ feature at $276.5 \mathrm{~cm}^{-1}$ and by the presence of second and third shell features in the Fourier transform (FT) of the extended X-ray absorption fine structure (EXAFS) data ascribable to the imidazole ring. ${ }^{11} \mathrm{~A}$ bound water ligand was indicated by the line broadening found in the electron paramagnetic resonance (EPR) spectrum of the native enzyme from Brevibacterium fuscum when dissolved in $\mathrm{H}_{2}{ }^{17} \mathrm{O} .^{12}$ A subsequent EXAFS study suggested that the bound water-based molecule is a hydroxide based on the $\mathrm{Fe}-\mathrm{O}$ distance. ${ }^{13}$

The extradiol dioxygenases are less understood in comparison to their intradiol counterparts, since the active iron site is in the ferrous oxidation state and thus more difficult to study spectroscopically. However, a recent crystal structure of extradiol dioxygenase 2,3 dihydroxybiphenyl 1,2-dioxygenase (1,2-DBD) showed a squarepyramidal iron site with ligands from two histidine and one glutamate residues and two 
water molecules. ${ }^{14}$ A square pyramidal geometry was predicted by magnetic circular dichroism (MCD) spectroscopy on an analogous enzyme, catechol 2,3-dioxygenase $(2,3-\mathrm{CTD}) .^{15}$

Knowing the structure of the iron active site of the native enzymes is only the first step in the broader objective of understanding the iron's role in the enzyme mechanism. It is clear that the native intradiol dioxygenases have a high spin ferric active site $^{16}$ and that the enzyme mechanism involves initial substrate binding followed by $\mathrm{O}_{2}$ attack. ${ }^{17}$ An attractive mechanism postulated early on suggested reduction of the ferric site by substrate, followed by dioxygen binding to the ferrous center. However, Mössbauer and EPR studies have shown that the iron retains its ferric oxidation state even after the substrate is bound. ${ }^{18,19}$ Stopped-flow kinetic studies have revealed the involvement of three intermediates subsequent to $\mathrm{O}_{2}$ attack on the enzyme-substrate complex, all of which retain their visible chromophores, indicating that the metal center remains high spin ferric. ${ }^{20,21}$ These results led to the postulation of a substrate activation mechanism in which the coordination of catechol to the ferric center activates the catechol for direct attack by the $\mathrm{O}_{2}$. The mechanism of the extradiol dioxygenases is less understood. The active form of the extradiol dioxygenases have a high spin ferrous site, as has been ascertained from Mössbauer spectroscopy. ${ }^{22,23}$ It is also known from MCD studies on 2,3-CTD that substrate binding activates the ferrous site for small molecule binding. ${ }^{15}$

The changes that occur to the iron active site due to substrate binding and $\mathrm{O}_{2}$ activation in catechol dioxygenases can be further probed using $\mathrm{NO}$ as an $\mathrm{O}_{2}$ analog. Intradiol dioxygenases do not bind $\mathrm{NO}$ in their native ferric state, however, the ferrous form of the enzyme binds NO to form an $S=3 / 2$ \{FeNO ${ }^{7}$ species. ${ }^{24}$ A different $S=3 / 2$ complex is formed when NO binds to a preformed enzyme-substrate complex. ${ }^{24}$ Additionally, substrate does not bind to the enzyme-NO complex indicating that initial NO binding blocks the single site for exogenous ligand binding to the iron, thereby inhibiting substrate association. In contrast, substrate binding before NO appears to evoke an enzyme conformational change that allows simultaneous NO binding in another site. ${ }^{24}$ On the other hand, native extradiol dioxygenases bind NO to form a $\left.\{\mathrm{FeNO}\}\right\}^{7}$ $\mathrm{S}=3 / 2$ species, as does the enzyme-substrate form. Particularly interesting is the fact that the NO binding constant increases by two orders of magnitude upon substrate binding. ${ }^{25,26}$ From EPR studies on the NO forms of protocatechuate 4,5-dioxygenase and gentisate 1,2-dioxygenase, it has been shown that substrate binds in a bidentate manner in the NO complex and thus there are three available sites on the iron for exogenous ligand binding, two for substrate and one for $\mathrm{NO}$ (and presumably $\mathrm{O}_{2}$ ). ${ }^{25,26}$ 
This fact is supported by the MCD studies on $2,3-\mathrm{CTD}^{15}$ and the crystal structure of $1,2-\mathrm{DBD} .^{14}$

Since X-ray absorption spectroscopy (XAS) is not dependent on the oxidation or spin state of the iron, comparable data can be obtained on the different forms of the intraand extradiol dioxygenases to obtain more detailed information on the electronic and geometric structures of the iron active site. The ferrous and ferric forms of these enzymes can be studied, as well as the enzyme-NO, enzyme-substrate, and enzyme-substrate-NO forms. The changes observed in the edge and EXAFS region of the XAS spectra can be used to obtain a detailed description of the iron active site in stages of the mechanism for which forms can be isolated or stabilized. In this study, both Fe K edge and EXAFS data were collected on $\mathrm{Fe}$ (III)PCD, $\mathrm{Fe}$ (II)PCD, $\mathrm{Fe}$ (III)PCD + 3,4 dihydroxybenzoic acid (Fe(III)PCA), and FePCD-NO. The intensities and energy splitting of the $1 \mathrm{~s} \rightarrow 3 \mathrm{~d}$ pre-edge features and the energy and shape of the rising edge indicate that $\mathrm{Fe}(\mathrm{II}) \mathrm{PCD}$ has a six-coordinate iron site, while Fe(III)PCD, Fe(III)PCA, and FePCD-NO have five-coordinate active sites. The edge shape and intensity of FePCD-NO indicates that the iron is in the ferric oxidation state. An EXAFS analysis provided iron-ligand distances for each of the four forms of the protein. Additionally, the GNXAS approach was used to fit the experimental EXAFS data of Fe(III)PCD and FePCD-NO in order to investigate the Fe-N-O angle in FePCD-NO.

\subsubsection{Experimental Section}

5.7.2.1. Sample Preparation. PCD was isolated from Brevibacterium fuscum as previously described. ${ }^{12,19,27}$ All XAS samples had a concentration of $-3 \mathrm{mM}$ in $\mathrm{Fe}$ in $50 \mathrm{mM}$ 3-( $N$-morpholino)propanesulfonic acid (MOPS) buffer at $\mathrm{pH} 7.0$ and contained $50 \%$ glycerol by volume. As isolated, the protein is in the ferric oxidation state and thus the Fe(III)PCD sample was obtained by concentrating isolated PCD in $50 \mathrm{mM}$ MOPS to $-6 \mathrm{mM}$ in $\mathrm{Fe}$ and then adding $50 \%$ glycerol by volume. The sample was then syringed into a Lucite XAS cell $(23 \times 1 \times 3 \mathrm{~mm})$ with $37 \mu \mathrm{m}$ Kapton windows and frozen in liquid nitrogen. The Fe(II)PCD XAS sample was characterized using liquid He EPR and showed the typical high spin ferric signal at 4.3. ${ }^{19} \mathrm{Fe}(\mathrm{II}) \mathrm{PCD}$ was obtained by reducing degassed $\mathrm{Fe}(\mathrm{III}) \mathrm{PCD}(\sim 6 \mathrm{mM}$ in $\mathrm{Fe}$ in $50 \mathrm{mM}$ MOPS) with $5 \mu \mathrm{l}$ of $-1 \mathrm{M}$ degassed dithionite solution ( $\sim 15$-fold reducing equivalents of $\mathrm{Fe}$ ) anaerobically. The protein sample turned from burgundy to clear indicating a complete reduction of the iron site. Degassed glycerol was then added to the sample under anaerobic conditions. The Fe(II)PCD sample was loaded into the XAS cell in a nitrogen-filled glove box and 
immediately frozen in liquid nitrogen. FePCD-NO was prepared by bubbling NO gas over the headspace of Fe(II)PCD ( $\sim 3 \mathrm{mM}$ in Fe in $50 \mathrm{mM}$ MOPS with $50 \%$ glycerol by volume) while tipping/mixing for $\mathbf{- 2 0} \mathrm{min}$. under anaerobic conditions until the color of the sample changed to a yellowish green and stopped changing color. The FePCD-NO sample was then loaded into an XAS cell in a nitrogen-filled glove box and immediately frozen in liquid nitrogen. The quality of the FePCD-NO XAS sample was characterized by the $S=3 / 2$ He EPR signal. EPR spectra taken before and after the sample was exposed to the $\mathrm{X}$-ray beam showed the characteristic $\{\mathrm{FeNO}\}^{7} \mathrm{~S}=3 / 2 \mathrm{EPR}$ signal, ${ }^{24}$ thus showing that NO was bound. In addition, there is a significant change in the edge feature between $\mathrm{Fe}$ (II)PCD and FePCD-NO (vide infra), indicative of the NO being bound to the iron. The edge shape and position of FePCD-NO was closely monitored during data collection to ensure that the NO was not photo-dissociating with there being no change in the edge feature for the duration of the experiment. Fe(III)PCA was prepared by anaerobically mixing $\mathrm{Fe}$ (III)PCD ( $\sim \mathrm{mM}$ in $\mathrm{Fe}$ in $50 \mathrm{mM}$ MOPS) with 3,4-dihydroxybenzoic acid (30 mM in MOPS) and glycerol (all three solutions were thoroughly degassed prior to mixing). The sample was then loaded into an XAS cell in a nitrogen-filled glove box and immediately frozen in liquid nitrogen. The liquid He EPR signal of Fe(III)PCA was identical to that previously reported. ${ }^{19}$

5.7.2.2. Data Collection and Reduction. X-ray absorption spectra were recorded at the Stanford Synchrotron Radiation Laboratory (SSRL) on unfocused beamline 7-3 during dedicated conditions $(3 \mathrm{GeV}, 50-100 \mathrm{~mA})$. The radiation was energy resolved using a $\mathrm{Si}(220)$ double-crystal monochromator with $1 \mathrm{~mm}$ vertical pre-monochromator beam defining slits. An Oxford Instruments continuous-flow liquid helium CF 1208 cryostat was used to maintain a constant temperature of $10 \mathrm{~K}$. Energies were calibrated using an internal Fe foil standard, assigning the first inflection point to $7111.2 \mathrm{eV} .^{28}$ The spectrometer energy resolution was approximately $1.4 \mathrm{eV}^{29}$ with reproducibility in edge position determination of $<0.2 \mathrm{eV}$. Data were measured to $k=$ $15 \AA^{-1}$, detuning the monochromator $50 \%$ at $7998 \mathrm{eV}$ to minimize harmonic contamination. The fluorescence signal was monitored using a 13 -element $\mathrm{Ge}$ solid-state array detector ${ }^{30}$ windowed on the $\mathrm{Fe} \mathrm{K} \alpha$ signal. During the experiment, count rates of approximately $30,000 \mathrm{~s}^{-1}$ total per element were not exceeded. Thirty-five scans were collected on the Fe(II)PCD sample. Thirty-two scans were collected on the Fe(III)PCD and $\mathrm{Fe}$ (III)PCA samples. Both the Fe(III)PCD and Fe(III)PCA samples photo-reduced in the beam, as was evident by changes in the edge shape and position. Therefore, these samples were exposed to the beam in eight different spots (four spots on two separate cells) producing eight 'first' scans and a total of thirty-two scans. There was less than a 
$0.5 \mathrm{eV}$ energy shift between the 'first' and 'fourth' scans. There was no discemible change in the EXAFS data from comparison of the average of the 'first' scan data to the average of the 'fourth' scan data. Therefore, the spectra of Fe(III)PCD and Fe(III)PCA presented in this section are an average all thirty-two scans. Two data sets were collected on FePCD-NO (on separate protein samples). The first data set contained a thirty-four scan average and the second data set contained a thirty scan average. The data presented in this section is an average of the two data sets (i.e. a sixty-four scan average), since both the edge and EXAFS features of the two data sets were identical within the noise level. A pre-edge subtraction was performed by fitting the tail of a Gaussian to the pre-edge region and subtracting this polynomial from the averaged spectra. ${ }^{31}$ A three-segment spline approximately even in $k$-space was fit to the EXAFS region and the data normalized to an edge jump of one at $7130 \mathrm{eV}$. The spline was chosen so that it minimized residual low-frequency background but did not reduce the EXAFS amplitude as checked by monitoring the FT of the EXAFS during the background subtraction process.

Fe K-edge spectra were also collected on four-, five-, and six-coordinate ferrous and ferric model complexes. $\mathrm{Fe}(\mathrm{acac})_{3}$ was purchased from Aldrich. ${ }^{32}$ $\mathrm{Fe}\left(\mathrm{HB}(3,5-i \mathrm{Pr} 2 \mathrm{pz})_{3}\right) \mathrm{Cl}^{33}$ was obtained as a gift from N. Kitajima. (BF $)[\mathrm{Fe}(\mathrm{TMC}) \mathrm{Cl}]{ }^{34}$ $\left[\mathrm{Fe}\right.$ (imidazole)6]Cl $2,{ }^{35} \mathrm{Fe}($ salen$) \mathrm{Cl},{ }^{36}\left(\mathrm{AsPh}_{4}\right)\left[\mathrm{Fe}\left(\mathrm{N}_{3}\right)_{5}\right],{ }^{37}$ and $\left(\mathrm{Et}_{4} \mathrm{~N}\right)[\mathrm{FeCl} 4]^{38}$ were prepared as previously described. The model complex XAS samples were prepared in the following manner. The crystalline samples were each mixed with BN and ground into a fine powder. The BN/sample mixture was pressed into a $1 \mathrm{~mm}$ thick $\mathrm{Al}$ spacer that was sealed with $63.5 \mu \mathrm{m}$ Mylar tape windows and frozen in liquid nitrogen. [Fe(imidazole $\left.)_{6}\right] \mathrm{Cl}_{2}, \mathrm{Fe}\left(\mathrm{HB}\left(3,5-\mathrm{Pr}_{2} \mathrm{pz}\right)_{3}\right) \mathrm{Cl}$, and $\left(\mathrm{BF}_{4}\right)[\mathrm{Fe}(\mathrm{TMC}) \mathrm{Cl}]$ are air-sensitive and thus the preparation was carried out in a nitrogen-filled glove box for these three samples. Data were measured in transmission mode with $\mathrm{N}_{2}$-filled ionization chambers to $k=$ $9.5 \AA^{-1}$ detuning the monochromator $50 \%$ at $7474 \mathrm{eV}$ to minimize harmonic contamination. Two to three scans were averaged for each sample. A smooth pre-edge background was removed from the averaged spectra by fitting a first order polynomial to the pre-edge region and subtracting this polynomial from the entire spectrum. A two segment spline of order two was fit to the EXAFS region and the data normalized to an edge jump of one at $7130 \mathrm{eV}$. The intensities and energies of the pre-edge features were determined as described below.

5.7.2.3. Empirical EXAFS Analysis. EXAFS data analysis was performed on the normalized protein spectra according to established methods. ${ }^{39-41}$ The normalized data were converted to $k$-space. The photoelectron wave vector, $k$, is defined by 
[2 $\left.m_{e}\left(E-E_{0}\right) / \hbar^{2}\right]^{1 / 2}$ where $m_{e}$ is the electron mass, $E$ is the photon energy, $\hbar$ is Planck's constant divided by $2 \pi$, and $E_{0}$ is the threshold energy of the absorption edge, which was defined to be $7130 \mathrm{eV}$ for the Fe $\mathrm{K}$ absorption edge. The empirical EXAFS data analyses were performed with nonlinear least-square curve-fitting techniques ${ }^{28,39-41}$ using empirical phase and amplitude parameters. The following models were used to obtain the empirical $\mathrm{Fe}-\mathrm{X}$ backscattering parameters of interest: $\mathrm{Fe}-\mathrm{O}$ from $\left[\mathrm{Fe}(\mathrm{acac})_{3}\right]^{42,43}$ and $\mathrm{Fe}-\mathrm{N}$ from $\left[\mathrm{Fe}(1,10 \text {-phenanthroline })_{3}\right]\left(\mathrm{ClO}_{4}\right)_{3} .{ }^{44,45}$ Fourier transforms (from $k$ to $\mathrm{R}$ space) were performed for the data range $3.5-14.5 \AA^{-1}$ with a Gaussian window of $0.1 \AA^{-1}$. The window widths used in the backtransforms (from $R$ to $k$ space) for the proteins are given in the results section. The window widths were kept as similar as possible to those used to extract amplitude and phase parameters from the model compounds to minimize artifacts introduced by the Fourier filtering technique. All curve-fitting was based on $k^{3}$-weighted data and applied to the individual filtered shell of interest. Only the structure-dependent parameters, the distance and coordination number, were varied unless stated otherwise. A "goodness of fit" parameter, $F$, was calculated as $F=\left\{\left[k^{6}(\text { data }- \text { fit })^{2}\right] / \text { (no. of points) }\right\}^{1 / 2}$ for each fit.

5.7.2.4. GNXAS Data Analysis. As described in detail in Chapter 2 and elsewhere, ${ }^{46-48}$ the GNXAS programs generate model EXAFS signals for each shell around the photoabsorber based on an initial structural model. Both single-scattering and multiple-scattering contributions are summed to generate a theoretical spectrum for the model which is then fit to the non-Fourier-filtered experimental data. ${ }^{46-48}$

The crystallographic coordinates were used as input for Fe(III)PCD. ${ }^{6}$ Phase shifts were calculated using the standard muffin-tin approximation with all the atoms associated with each compound and up to an energy limit of $70 \mathrm{Ry}(950 \mathrm{eV})$ above the $\mathrm{Fe} \mathrm{K}$ edge. The Mattheiss prescription ${ }^{49}$ of overlapping, self-consistent atomic charge densities of the atoms of the cluster was used to construct the Coulomb portion of the effective one-electron potential. Proper account of the charge relaxation around the core hole was taken. The Hedin-Lundqvist plasmon-pole approximation was used to model its exchange and correlation part. ${ }^{50}$ The imaginary part of the latter takes into account inelastic scattering processes of the photoelectron propagating out of the system and models a priori its mean-free path. The muffin-tin radii were chosen by scaling Norman radii of the cluster atoms by a factor of about 0.8 as to match the nearest neighbor distance.

The theoretical EXAFS spectrum was calculated to include contributions from two-atom and three-atom configurations. Within each $n$-atom configuration, all the MS contributions were taken into account. ${ }^{46-48}$ The two-atom and three-atom configurations 
were identified in each cluster up to $4.5 \AA$ and averaged with a frequency tolerance of $0.1 \AA$. The resultant information was used to calculate the various EXAFS $\gamma^{(2)}$ and $\gamma^{(3)}$ signals associated with each two-atom and three-atom contribution using the crystallographic bond lengths and distances.

The GNXAS fitting program constructs the theoretical absorption spectrum by summing all the $\gamma(2)$ and $\gamma^{(3)}$ signals and compares this theoretical spectrum with the experimental absorption spectrum with the residual function $R$ being a measure of the quality of the fit. ${ }^{48}$ Least-squares fits are performed on the averaged, energy-calibrated, raw absorption data without prior background subtraction or Fourier filtering. Raw data are compared directly with a model absorption coefficient composed of an appropriate background plus the oscillatory structural contribution from the theoretically calculated EXAFS. ${ }^{48}$ A spline of orders $4,4,4$ with defining energy points of $7147,7269,7577$, and $7998 \mathrm{eV}$ was used for $\mathrm{Fe}(\mathrm{III}) \mathrm{PCD}$. The first spline point used for FePCD-NO was $7149 \mathrm{eV}$ to reduce low-frequency noise in the FT. Least-squares fits were done with $k^{3}$-weighting where the first and the last spline points determined the range of the fit.

The structural parameters varied in the refinements were the distance $(R)$ and the bond variance $\left(\sigma_{R}^{2}\right)$, the mean square variation in the bond distance, for each two-atom configuration and the distances, the angle and the covariance matrix elements ${ }^{46-48}$ for the three-atom configurations. Distances and angles were allowed to vary within a preset range, typically $\pm 0.05 \AA$ and $\pm 5^{\circ}$. Bond and angle variances were also allowed to vary in restricted ranges: $\pm 0.005 \AA^{2}$ and \pm 50 (degrees) ${ }^{2}$, respectively. The off-diagonal covariance matrix elements were kept fixed at zero. The coordination numbers were kept fixed. The nonstructural parameters in the fits were $E_{0}$ (a parameter that aligns the experimental energy scale to the theoretical energy scale), $S_{0}{ }^{2}$ (many-body amplitude reduction factor), $\Gamma_{\mathrm{c}}$ (core-hole lifetime), and $E_{\mathrm{r}}$ (experimental resolution). These parameters were refined within narrow limits around expected values. ${ }^{51,52}$

5.7.2.5. 1s $\longrightarrow 3 d$ Pre-Edge Analysis. The intensities and energies of pre-edge features of the model complex and protein data were quantitated by fits to the data. The fitting program EDG_FIT, which utilizes the double precision version of the public domain MINPAK fitting library was used. EDG_FIT was written by Dr. Graham N. George of the Stanford Synchrotron Radiation Laboratory. All spectra were fit over the range $7108-7118 \mathrm{eV}$. Pre-edge features were modeled by pseudo-Voigt line shapes (simple sums of Lorentzian and Gaussian functions). ${ }^{29,53-55}$ A fixed 50:50 ratio of Lorentzian to Gaussian contribution for the pre-edge feature successfully reproduced these spectral features. Functions modeling the background underneath the pre-edge features were chosen empirically to give the best fit. The second derivative of the data 
was compared to the second derivative of the fit. In all cases, a number of fits were obtained which reproduced the data and the second derivative. The value reported for the area of a fitted feature (where peak area was approximated by the height $\mathrm{x}$ full-width-athalf-maximum (FWHM)) is the average of all the pseudo-Voigts which successfully fit the feature. For each sample, the standard deviation of the average of the areas was calculated to quantitate the error.

\subsubsection{Results and Analysis}

5.7.3.1. Fe K-Edge XAS. Fe K-edge XAS data for $F e(I I) P C D, F e(I I) P C D$, $\mathrm{Fe}$ (III)PCA, and FePCD-NO were measured to obtain information on the electronic and geometric structure of the iron sites of these forms of the protein. The protein data were compared to data of four-, five-, and six-coordinate ferrous and ferric model complexes to obtain information on the coordination number and the geometry of the non-heme iron site. Information on the oxidation state and the site symmetry of the iron in FePCD-NO was also obtained from the edge spectra. The XAS edge spectra for $\mathrm{Fe}(\mathrm{II}) \mathrm{PCD}$, $\mathrm{Fe}$ (III)PCD, $\mathrm{Fe}$ (III)PCA, and FePCD-NO are shown in Figure 5.19. Figure 5.20 displays spectra of representative four-, five-, and six-coordinate ferrous model complexes, $\mathrm{Fe}\left(\mathrm{HB}\left(3,5-\mathrm{IPr}_{2} \mathrm{pz}\right)_{3}\right) \mathrm{Cl}^{33},{ }^{33}\left(\mathrm{BF}_{4}\right)[\mathrm{Fe}(\mathrm{TMC}) \mathrm{Cl}],{ }^{34}$ and $\left.[\mathrm{Fe} \text { (imidazole) })_{6}\right] \mathrm{Cl}_{2},{ }^{35}$ in part $\mathrm{A}$ and ferric model complexes, (Et4N)[FeCl4], ${ }^{38} \mathrm{Fe}($ salen $) \mathrm{Cl}^{36},{ }^{36}\left(\mathrm{AsPh}_{4}\right)\left[\mathrm{Fe}\left(\mathrm{N}_{3}\right)_{5}\right],{ }^{37}$ and $\mathrm{Fe}(\mathrm{acac})_{3},{ }^{42,43}$ in part $\mathrm{B}$. The lowest energy peaks arise from the weak $1 \mathrm{~s} \longrightarrow 3 \mathrm{~d}$ transition which is at $-7112 \mathrm{eV}$ followed by the $1 \mathrm{~s} \rightarrow 4 \mathrm{p}$ transition at $-7125 \mathrm{eV}$. An expanded view of the $1 \mathrm{~s} \rightarrow 3 \mathrm{~d}$ pre-edge region is shown for the proteins as well as for the model complexes as insets in Figures 5.19 and 5.20, respectively. The energies and areas of the pre-edge features were determined by fits to the data and are presented in Table 5.9 .

$\mathrm{Fe}$ (II)PCD has a broad, low-intensity pre-edge feature which can be fit with two peaks. Fe(III)PCD has an intense pre-edge feature centered at $\sim 7113.4 \mathrm{eV}$, while $\mathrm{Fe}$ (III)PCA has a broader feature centered $\sim 0.1 \mathrm{eV}$ lower in energy. FePCD-NO has a relatively intense, broad pre-edge feature that can be fit with two pre-edge peaks. The three ferrous model complexes, $\mathrm{Fe}\left(\mathrm{HB}\left(3,5-i \mathrm{Pr}_{2} \mathrm{pz}\right)_{3}\right) \mathrm{Cl},\left(\mathrm{BF}_{4}\right)[\mathrm{Fe}(\mathrm{TMC}) \mathrm{Cl}]$, and [Fe(imidazole) $\left.{ }_{6}\right] \mathrm{Cl}_{2}$, all have what appear to be two pre-edge features split by $-2 \mathrm{eV}$ with varying intensities (Figure 5.20A). The four-coordinate complex has the most intense pre-edge feature with a total area of 19.8 , while the six-coordinate complex has the least intense pre-edge feature with a total pre-edge area of 3.8. The ferric four-coordinate complex, $\left(\mathrm{Et4N}_{4}\right)\left[\mathrm{FeCl}_{4}\right]$, has a single intense pre-edge feature with a total fitted pre-edge 


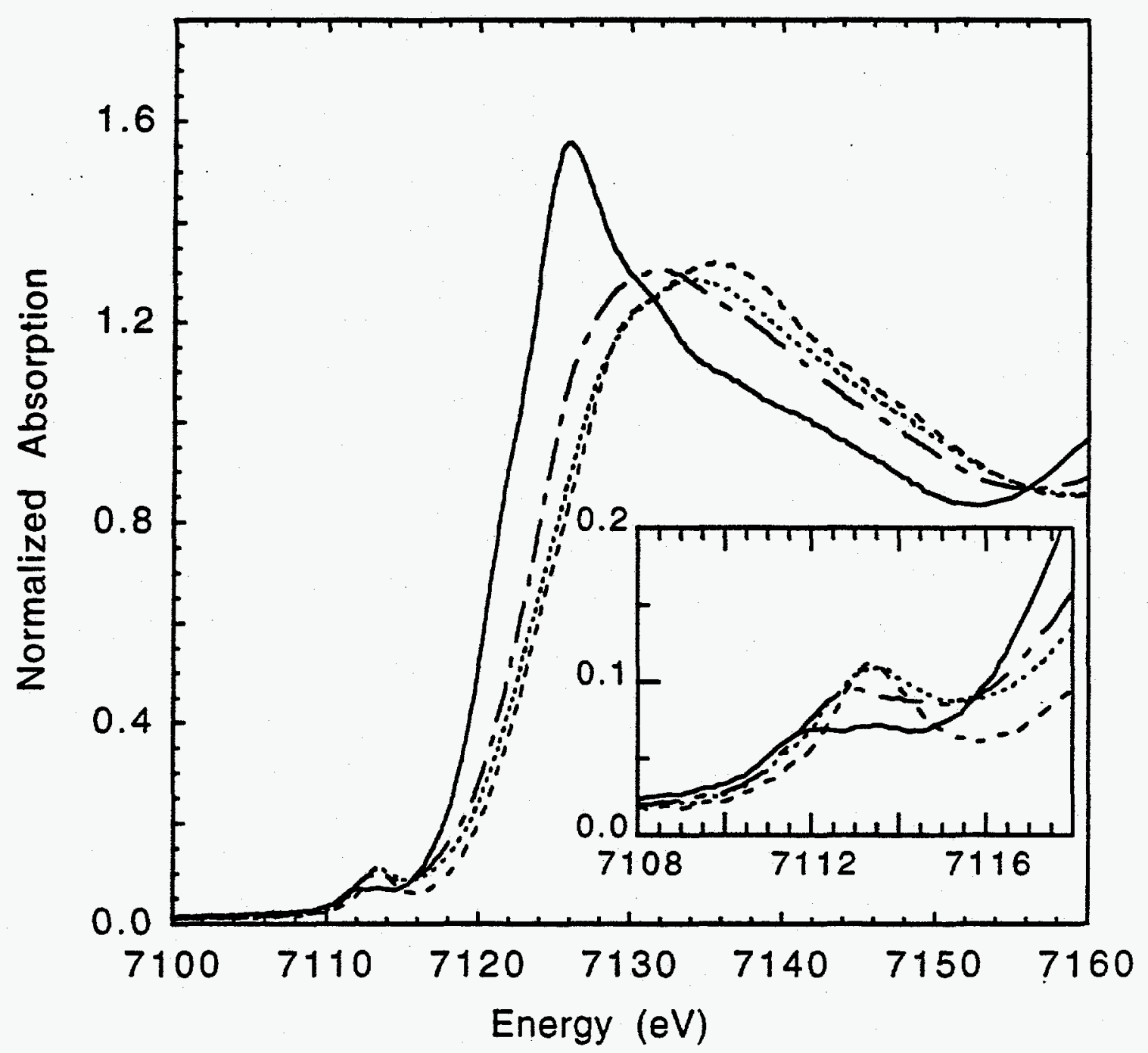

Figure 5.19. Fe K-edge XAS spectra of Fe(II)PCD (-), Fe(III)PCD (---), $\mathrm{Fe}$ (III)PCA $(\cdots)$, and FePCD-NO (- -). The inset is an expansion of the $1 \mathrm{~s} \rightarrow 3 \mathrm{~d}$ pre-edge region. 


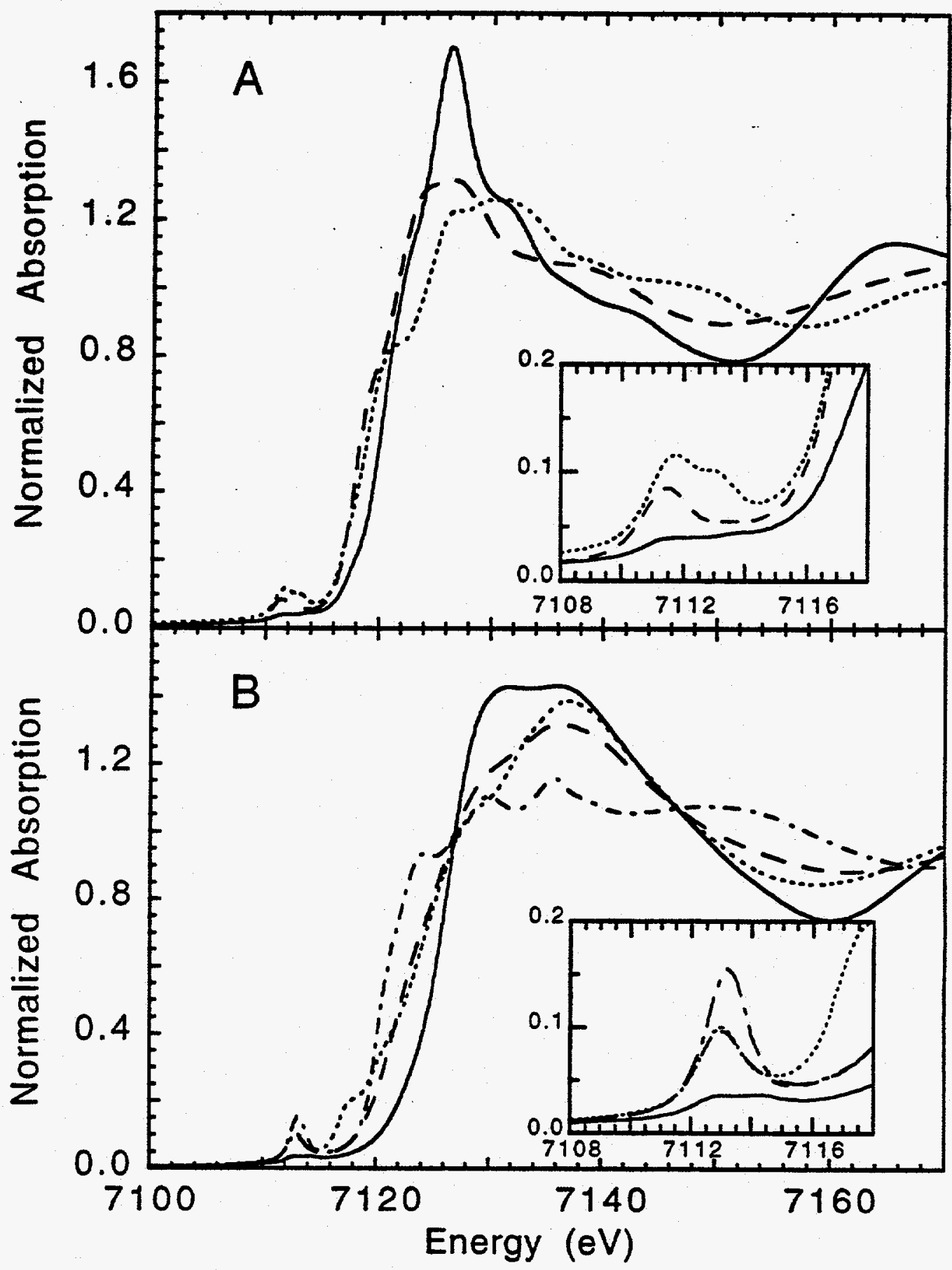

Figure 5.20. Fe K-edge XAS spectra of four-, five-, and six-coordinate ferrous model complexes are shown in part $\mathrm{A}: \mathrm{Fe}\left(\mathrm{HB}\left(3,5-i \mathrm{Pr}_{2} \mathrm{pz}\right)_{3}\right) \mathrm{Cl}(\cdots \cdot),\left(\mathrm{BF}_{4}\right)[\mathrm{Fe}(\mathrm{TMC}) \mathrm{Cl}](---)$, and $\left[\mathrm{Fe}\right.$ (imidazole) $\left.{ }_{6}\right] \mathrm{Cl}_{2}(-)$. XAS spectra of four-, five-, and six-coordinate ferric model complexes are shown in part B: $\left(\mathrm{Et}_{4} \mathrm{~N}\right)\left[\mathrm{FeCl}_{4}\right](-),[\mathrm{Fe}($ salen $) \mathrm{Cl}](--)$, $(\mathrm{AsPh} 4)\left[\mathrm{Fe}\left(\mathrm{N}_{3}\right)_{5}\right](\cdots \cdot)$, and $\mathrm{Fe}(\mathrm{acac})_{3}(-)$. The insets are expansions of the $1 \mathrm{~s} \rightarrow 3 \mathrm{~d}$ pre-edge region. 
Table 5.9. XAS Pre-Edge Energies and Areas for PCD and Model Complexes.

\begin{tabular}{|c|c|c|c|c|c|}
\hline sample & $\begin{array}{l}\mathrm{Fe} \\
\text { oxid. } \\
\text { state } \\
\end{array}$ & $\mathrm{CN}^{a}$ & $\begin{array}{l}\text { pre-edge } \\
\text { energy }(\mathrm{eV})^{b}\end{array}$ & $\begin{array}{l}\text { pre-edge } \\
\text { area } b, c\end{array}$ & $\begin{array}{l}\text { total } \\
\text { pre-edge } \\
\text { area } c\end{array}$ \\
\hline \multirow[t]{2}{*}{$\mathrm{Fe}\left(\mathrm{HB}(3,5-\mathrm{IPr} 2 \mathrm{pz})_{3}\right) \mathrm{Cl}$} & $2+$ & 4 & $7111.64(0.02)$ & $14.2(0.5)$ & $19.8(0.9)$ \\
\hline & & & $7113.17(0.02)$ & $5.6(0.9)$ & \\
\hline \multirow{2}{*}{$\left(\mathrm{BF}_{4}\right)[\mathrm{Fe}(\mathrm{TMC}) \mathrm{Cl}]$} & $2+$ & 5 & $7111.41(0.01)$ & $10.9(0.1)$ & $12.9(0.2)$ \\
\hline & & & $7113.43(0.02)$ & $2.0(0.3)$ & \\
\hline \multirow[t]{3}{*}[\mathrm{Fe}(\text{imidazole})_{6}]{$\mathrm{Cl}_{2}$} & $2+$ & 6 & $7111.24(0.03)$ & $1.6(0.5)$ & $3.8(0.3)$ \\
\hline & & & $7112.35(0.16)$ & $1.6(0.8)$ & 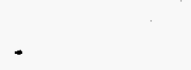 \\
\hline & & & $7113.66(0.02)$ & $0.6(0.2)$ & \\
\hline \multirow{2}{*}{$\mathrm{Fe}($ II)PCD } & $2+$ & -- & $7111.81(0.02)$ & $5.7(0.8)$ & $8.8(0.9)$ \\
\hline & & & $7113.43(0.03)$ & $3.0(0.4)$ & \\
\hline$\left(\mathrm{Et}_{4} \mathrm{~N}\right)\left[\mathrm{FeCl}_{4}\right]$ & $3+$ & 4 & $7113.16(0.00)$ & $20.7(0.8)$ & $20.7(0.8)$ \\
\hline \multirow[t]{2}{*}{$\mathrm{Fe}($ salen $) \mathrm{Cl}$} & $3+$ & 5 & $7112.91(0.01)$ & $12.9(0.3)$ & $14.4(0.6)$ \\
\hline & & & $7114.25(0.07)$ & $1.5(0.4)$ & \\
\hline$\left(\mathrm{AsPh}_{4}\right)\left[\mathrm{Fe}\left(\mathrm{N}_{3}\right)_{5}\right]$ & $3+$ & 5 & $7112.94(0.01)$ & $11.7(0.6)$ & $11.7(0.6)$ \\
\hline \multirow[t]{2}{*}{$\mathrm{Fe}(\mathrm{acac})_{3}$} & $3+$ & 6 & $7112.79(0.02)$ & $2.7(0.3)$ & $4.6(0.3)$ \\
\hline & & & $7114.31(0.04)$ & $1.9(0.1)$ & \\
\hline $\mathrm{Fe}$ (III) $\mathrm{PCD}$ & $3+$ & - & $7113.35(0.02)$ & $16.4(1.7)$ & $16.4(1.7)$ \\
\hline $\mathrm{Fe}$ (II)PCA & $3+$ & -- & $7113.27(0.01)$ & $20.3(0.7)$ & $20.3(0.7)$ \\
\hline \multirow[t]{2}{*}{ FePCD-NO } & -- & -- & $7112.71(0.04)$ & $14.2(2.0)$ & $18.3(2.8)$ \\
\hline & & & $7114.47(0.08)$ & $4.1(1.6)$ & \\
\hline
\end{tabular}

$a \mathrm{CN}=$ coordination number. $b$ Pre-edge energies and areas were determined by fits to the data as described in the Experimental Section. $c$ Pre-edge areas were calculated by multiplying the height of the fitted feature by the FWHM (the values reported were multiplied by 100 ). 
area of 20.7 at $7113.2 \mathrm{eV}$. The two five-coordinate models, $\mathrm{Fe}($ salen)Cl and $\left(\mathrm{AsPh}_{4}\right)\left[\mathrm{Fe}\left(\mathrm{N}_{3}\right)_{5}\right]$, have pre-edge features that are less intense with fitted areas of 14.4 and 11.7, respectively (Figure 5.20B and Table 5.9). The ferric six-coordinate complex, $\mathrm{Fe}(\mathrm{acac})_{3}$, has two relatively weak features with a total fitted area of 4.6 and an intensity ratio of 3:2 (Figure 5.20B and Table 5.9). The main edge feature, attributed to the 1s- $>4 \mathrm{p}$ transition at $\sim 7125 \mathrm{eV}$, is very narrow and intense for the Fe(II)PCD data (Figure 5.19). The main edge feature of Fe(II)PCD, Fe(III)PCA, and FePCD-NO all look similar with the onset of the edge at approximately $4 \mathrm{eV}$ higher in energy than that of the respective ferrous protein with that of FePCD-NO being slightly lower in energy (Figure 5.19). The edge features of the ferrous and ferric model complexes differ significantly in shape (Figure 5.20) with the onset of the edge for the ferric complexes being $\sim 4 \mathrm{eV}$ higher in energy than that of the ferrous complexes.

The $1 \mathrm{~s} \rightarrow 3 \mathrm{~d}$ pre-edge feature can be used to probe the coordination number of the non-heme iron site in these various forms of PCD. The $1 s \rightarrow 3 d$ transition is formally electric dipole forbidden, but gains intensity through an allowed quadrupole transition and also by $4 p$ mixing into the $3 d$ states as a result of the noncentrosymmetric environment of the metal site. It has been shown for ferric and ferrous complexes that when the symmetry of the iron site is lowered, the pre-edge intensity increases due to an increase in the $3 \mathrm{~d}-4 \mathrm{p}$ mixing. 56,57

As described in more detail in Chapter 4, the pre-edge splitting and intensity distribution is indicative of the site symmetry of the iron atom. When a high spin ferrous atom is in an octahedral site, three transitions are observed into ${ }^{4} T_{1},{ }^{4} T_{2}$, and ${ }^{4} T_{1} d^{(n+1)}$ excited states (a transition into the ${ }^{4} \mathrm{~A}_{2}$ state is not observed as it would involve a two electron transition). The transitions into these states are only quadrupole allowed as the iron is in a centrosymmetric site and therefore there is no $4 \mathrm{p}-3 \mathrm{~d}$ mixing. Thus, one observes three weak peaks as can be seen in the $[\mathrm{Fe}$ (imidazole) 6$] \mathrm{Cl}_{2}$ spectrum (Figure 5.20A) with the first two features being barely resolvable and centered at 7111.2 and $7112.4 \mathrm{eV}$ and the third feature positioned at $7113.7 \mathrm{eV}$. Since the difference in energy between the lowest energy ${ }^{4} \mathrm{~T}_{1}$ state and the ${ }^{4} \mathrm{~T}_{2}$ state is about the same as the energy resolution at the Fe $\mathrm{K}$-edge, occasionally only two pre-edge features are observed for high spin ferrous complexes with the first feature attributed to transitions into both the ${ }^{4} \mathrm{~T}_{1}$ and the ${ }^{4} \mathrm{~T}_{2}$ states and the second feature $\sim 2 \mathrm{eV}$ higher in energy attributed to a transition into the higher energy ${ }^{4} \mathrm{~T}_{1}$ state.

When a high spin ferrous atom is in a square pyramidal site, the intensity of the lower energy feature increases dramatically since there is now $4 \mathrm{p}_{z}$ mixing into the $3 \mathrm{~d}_{z} 2$ orbital due to the loss of a center of inversion, as can be seen in the $\left(\mathrm{BF}_{4}\right)[\mathrm{Fe}(\mathrm{TMC}) \mathrm{Cl}]$ 
spectrum (dashed line in Figure 5.20A). When a high spin ferrous atom is in a tetrahedral site, there are two intense pre-edge features due to $4 p$ mixing into the $d_{x y}, d_{x z}, d_{y z}$ orbitals, with the lower energy feature being more intense than the higher energy feature, as can be seen in the $\left(\mathrm{Fe}\left(\mathrm{HB}\left(3,5-i \mathrm{Pr}_{2} \mathrm{Pz}\right)_{3}\right) \mathrm{Cl}\right.$ spectrum (dotted line in Figure 5.20A). See Chapter 4 for a more detailed analysis of the distribution of the dipole intensity in the $1 \mathrm{~s} \rightarrow 3 \mathrm{~d}$ pre-edge features of high spin ferrous complexes.

Empirically the pre-edge feature of $\mathrm{Fe}$ (II)PCD looks much more similar in shape and intensity to that of the six-coordinate ferrous complex and quite different from that of the four- and five-coordinate complexes (Figure 5.19 and 5.20A). The broad pre-edge feature of $\mathrm{Fe}(\mathrm{II}) \mathrm{PCD}$ can be fit with two peaks, with the lower energy peak having a wider FWHM than the higher energy peak (solid line in Figure 5.19). In addition, the area of the pre-edge feature centered at $7111.8 \mathrm{eV}$ is twice as large as that of the feature centered at $7113.4 \mathrm{eV}$ (Table 5.9), indicating that the first feature contains transitions into both the ${ }^{4} \mathrm{~T}_{1}$ and the ${ }^{4} \mathrm{~T}_{2}$ states while the second feature contains the transition into the higher energy ${ }^{4} \mathrm{~T}_{1}$ state. The energies of the pre-edge peaks of $\mathrm{Fe}(\mathrm{II}) \mathrm{PCD}$ are consistent with those seen in octahedral high spin ferrous complexes (Table 4.1 in Chapter 4). The total fitted area of the pre-edge features is higher for Fe(II)PCD than for octahedral ferrous model complexes (Table 4.1 in Chapter 4), which can be attributed to an overall less symmetric iron site caused by mixed ligation and variation in bond lengths. Additional support of a six-coordinate iron site for $F e(\Pi) P C D$ comes from the shape of the edge feature. The shape of the main edge feature in the Fe(II)PCD spectrum (solid line in Figure 5.19) is very similar to that in the six-coordinate [ $\mathrm{Fe}$ (imidazole) ${ }_{6} \mathrm{Cl}_{2}$ spectrum (solid line in Figure 5.20A) and other octahedral high spin ferrous model complexes with oxygen and nitrogen ligation (Figure 4.2 in Chapter 4). The results from the pre-edge and edge analysis are consistent in indicating that Fe(II)PCD has a six-coordinate iron active site.

The intensity pattern and energy splitting of the pre-edge feature of high spin ferric iron are also indicative of the iron's site symmetry. When a high spin ferric atom is in an octahedral site, two pre-edge features are observed from transitions to ${ }^{5} \mathrm{~T}_{2}$ and ${ }^{5} \mathrm{E}$ $d^{(n+1)}$ many-electron excited states. The transitions into these states are only quadrupole allowed as the iron is in a centrosymmetric site and therefore there is no $4 \mathrm{p}-3 \mathrm{~d}$ mixing. Thus, one observes two weak features with an intensity ratio of 3:2 as can be seen in the $\mathrm{Fe}(\mathrm{acac})_{3}$ spectrum (solid line in Figure 5.20B). When a high spin ferric atom is in a five-coordinate site (either square pyramidal or trigonal bipyramidal), the intensity of the pre-edge feature increases dramatically due to $4 p$ mixing into the $3 d$ orbital due to the loss of a center of inversion, as can be seen in the $\mathrm{Fe}($ salen $) \mathrm{Cl}$ and $(\mathrm{AsPh})\left[\mathrm{Fe}\left(\mathrm{N}_{3}\right)_{5}\right]$ 
spectra (dashed and dotted lines in Figure 5.20B). When a high spin ferric atom is in a tetrahedral site, there is a single very intense pre-edge feature due to $4 \mathrm{p}$ mixing into the $d_{x y}, d_{x z}, d_{y z}$ orbitals, as can be seen in the $\left(\mathrm{Et}_{4} \mathrm{~N}\right)\left[\mathrm{FeCl}_{4}\right] \mathrm{s}_{\mathrm{y}}$ jectrum shown in Figure 5.20B.

The pre-edge feature of Fe(III)PCD and Fe(III)PCA (dashed and dotted line, respectively, in Figure 5.19) can be fit with a single broad peak at $\sim 7113.3 \mathrm{eV}$ (Table 5.9). The fitted area of this feature, which is 16.4 for Fe(III)PCD and 20.3 for $\mathrm{Fe}$ (III)PCA, is more similar to that of the five-coordinate models, where $\mathrm{Fe}($ salen)Cl and $\left(A s \mathrm{Ph}_{4}\right)\left[\mathrm{Fe}\left(\mathrm{N}_{3}\right)_{5}\right]$ have areas of 14.4 and 11.7 , respectively, than to that of the six-coordinate model, $\mathrm{Fe}(\mathrm{acac})_{3}$, which has an intensity of 4.6. The total pre-edge area of $\mathrm{Fe}$ (III)PCA is more similar to that of the four-coordinate model $\left(\mathrm{Et}_{4} \mathrm{~N}\right)\left[\mathrm{FeCl}_{4}\right]$ (Table 5.9), however, the shape of the pre-edge feature more closely resembles that of the five-coordinate models. Also, the fitted area of the pre-edge features for protein complexes is typically higher than that of model complexes, since the iron sites of the proteins are often more distorted than the iron sites in model complexes due to mixed ligation and varying bond distances. A further indication of the coordination number of the iron is the shape of the main edge feature. Fe(III)PCD and Fe(III)PCA have a main edge feature that is very similar to the five-coordinate ferric model complexes with a feature at $\sim 7128 \mathrm{eV}$ that is slightly lower in intensity than a feature at $\sim 7135 \mathrm{eV}$ (Figure 5.19 and $5.20 \mathrm{~B}$ ). In contrast, the features at 7128 and $7135 \mathrm{eV}$ are of equal intensity in the six-coordinate octahedral complex (solid line in Figure 20B) and the four-coordinate complex has three features at approximately 7124,7130 , and $7136 \mathrm{eV}$ (dashed-dotted line in Figure 20B). Thus, the pre-edge and edge features of both Fe(II)PCD and Fe(III)PCA indicate that the iron is in a five-coordinate site.

The pre-edge and edge features were used to probe the oxidation state of the iron in FePCD-NO. The pre-edge region of FePCD-NO was fit with an intense feature at $7112.7 \mathrm{eV}$ and a weaker feature at $7114.5 \mathrm{eV}$. The energies of these peaks are much more similar to those of ferric complexes than ferrous complexes (Table 5.9), with the lower energy feature of ferrous complexes typically being below $7111.8 \mathrm{eV}$ in energy (see Table 4.1 in Chapter 4). The pre-edge peak energies and areas for FePCD-NO is very similar to that of the ferric five-coordinate model complex, $\mathrm{Fe}$ (salen) $\mathrm{Cl}$ (Table 5.9). The rising edge of FePCD-NO lies between that of Fe(II)PCD and Fe(III)PCD in energy with the shape and intensity of the edge being much more similar to that of $\mathrm{Fe}(\mathrm{III}) \mathrm{PCD}$ (Figure 5.19). The differences in the edge spectra of Fe(II)PCD, Fe(III)PCD, and FePCD-NO are very similar to the differences observed in the spectra of Fe(II)EDTA, Fe(III)EDTA, and FeEDTA-NO (Figure 5.1). Previous studies on FeEDTA-NO and 
other $\mathrm{S}=3 / 2$ \{FeNO $\}^{7}$ complexes indicate that the iron in these systems is in the high spin ferric form and that there is significant charge donation from the NO- to the ferric atom. ${ }^{58}$ The pre-edge and edge features of FePCD-NO also indicate that the iron atom in

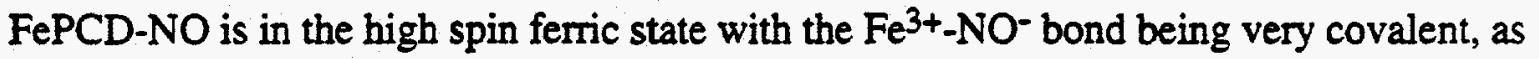
is evident by the fact that the FePCD-NO rising edge is at lower energy than the $\mathrm{Fe}$ (III) $\mathrm{PCD}$ rising edge.

The intensity of the $1 \mathrm{~s} \longrightarrow 3 \mathrm{~d}$ pre-edge feature of FePCD-NO was used to determine the coordination environment of the iron site. As has been previously discussed the intensity of the $1 \mathrm{~s} \rightarrow 3 \mathrm{~d}$ pre-edge feature is sensitive to the coordination environment and geometry of the iron site. $\{\mathrm{FeNO}\}^{7}$ complexes have been shown to have intense pre-edge features due to the distortion caused by the short Fe-N(O) bond. ${ }^{57,58}$ Additionally, the pre-edge feature has been shown to also be sensitive to the $\mathrm{Fe}-\mathrm{N}-\mathrm{O}$ angle in $\{\mathrm{FeNO}\}^{7}$ complexes (section 5.2). Bending of the Fe-N-O unit increases the distortion around the iron site, thus, increasing the amount of $4 \mathrm{p}$ mixing into the $3 \mathrm{~d}$ orbitals. The increase of $1 \mathrm{~s} \rightarrow 3 \mathrm{~d}$ pre-edge intensity upon bending of the Fe-N-O angle has been observed for [Fe(TMC)NO] $\left(\mathrm{BF}_{4}\right)_{2}$ which has a total pre-edge intensity of 15.5 and $\mathrm{Fe}$ (salen)NO which has a total pre-edge intensity of 24.2 (Table 5.2) where the main difference in the iron site symmetry between [Fe(TMC) NO] $\left(\mathrm{BF}_{4}\right)_{2}$ and $\mathrm{Fe}$ (salen)NO is a linear vs. bent $\mathrm{Fe}-\mathrm{N}-\mathrm{O}$ unit, respectively. ${ }^{59,60}$ The increase of $4 \mathrm{p}$ mixing into the 3d orbitals upon bending of the Fe-N-O unit has also been predicted by self consistent field-X $\alpha$-scattered wave calculations. ${ }^{58}$ Hence, an analysis of the intensity of the pre-edge feature for FePCD-NO allows for determination of the coordination number of the iron site as well as the mode of NO binding (linear vs. bent).

The fitted pre-edge area for FePCD-NO of 18.3 is much higher than that of FeEDTA-NO, a seven-coordinate $\{\text { FeNO }\}^{7}$ complex, which has a pre-edge area of 10.4 and $\mathrm{Fe}(\mathrm{Me} 3 \mathrm{TACN})(\mathrm{NO})\left(\mathrm{N}_{3}\right)_{2},{ }^{61}$ a six-coordinate $\{\mathrm{FeNO}\}^{7}$ complex, which has a pre-edge area of 10.8 (Table 5.2). In fact, the pre-edge area of FePCD-NO is in the range of the five-coordinate $\{\mathrm{FeNO}\}^{7}$ complexes as $[\mathrm{Fe}(\mathrm{TMC}) \mathrm{NO}]\left(\mathrm{BF}_{4}\right)_{2}$ has a total pre-edge intensity of 15.5 and $\mathrm{Fe}$ (salen)NO has a total pre-edge intensity of 24.2 (Table 5.2). From a first-shell empirical EXAFS analysis of FePCD-NO (vide infra), the Fe-N(O) bond length in FePCD-NO is $-1.9 \AA$, substantially longer than the 1.74 and $1.78 \AA$ $\mathrm{Fe}-\mathrm{N}(\mathrm{O})$ bond lengths for $[\mathrm{Fe}(\mathrm{TMC}) \mathrm{NO}]\left(\mathrm{BF}_{4}\right){ }_{2}{ }^{60}$ and $\mathrm{Fe}($ salen $) \mathrm{NO}^{59}$, respectively. The increase in the Fe-N(O) bond length in FePCD-NO should decrease the distortion around the iron site and thus the pre-edge intensity should be lower than that of a $\{\mathrm{FeNO}\}^{\text {? }}$ model complexes of similar geometry and shorter $\mathrm{Fe}-\mathrm{N}(\mathrm{O})$ bond length. Thus, it appears 
that the FePCD-NO has a bent Fe-N-O unit where the pre-edge area of 18.3 is lower the value of 24.2 for $\mathrm{Fe}$ (salen)NO.

5.7.3.2. First-Shell Empirical EXAFS Analysis. Analysis of the EXAFS data for $\mathrm{Fe}$ (II)PCD, $\mathrm{Fe}$ (III)PCD, Fe(III)PCA, and FePCD-NO was pursued to obtain metrical information on the iron active site of each of these forms of the protein. The EXAFS spectra are shown in Figure 5.21. The EXAFS oscillations for Fe(II)PCD have a higher frequency than those for Fe(III)PCD (Figure 5.21), indicative of longer first-shell bond distances in the ferrous protein. The EXAFS data for Fe(III)PCD and Fe(III)PCA are similar in the low- $k$ region, but differ in the higher $k$ region. The EXAFS data for FePCD-NO are unique with respect to the other three forms of the protein.

The FTs of the EXAFS data taken over the $k$ range of $3.5-14.5 \AA^{-1}$ are shown in Figure 5.22. The FTs of the EXAFS data for Fe(II)PCD and FePCD-NO are fairly similar, however, $\mathrm{Fe}$ (II)PCD has a feature at $\sim 2.8 \AA$ that is nearly absent in the data for FePCD-NO (Figure 5.22). The FT of the EXAFS data for Fe(III)PCD has a very intense feature at $\sim 1.5 \AA$, while that for $\mathrm{Fe}(\mathrm{III}) \mathrm{PCA}$ has a split feature at this distance and has additional features at -2.1 and $4.1 \AA$. Curve-fitting was performed on filtered first-shell contributions over the $k$ range $4-14 \AA^{-1}$ varying bond distances and coordination numbers (the FT backtransform windows are given in Table 5.10). Note that curvefitting was also performed over the $k$ range of $4-12 \AA^{-1}$ for all four of the protein samples. The results over the shorter $k$ range were all very similar (all within the error of the technique) to the results over the $k$ range of $4-14 \AA^{-1}$ that are presented in Table 5.10. In each of the four cases, the FT peak centered at $\sim 1.8 \AA$ (non-phase shift corrected) could not be adequately fit with waves representing a single shell of oxygens or nitrogens (Fits $1,4,5,6$, and 9). These fits did not match the frequency of the data at higher $k$ and gave unreasonably low coordination numbers. The $F$ values were significantly reduced when a wave from a second oxygen or nitrogen contribution was included in the fit to the data. Since most non-heme iron active sites contain both oxygen and nitrogen ligation, fits were performed with two oxygen waves, an oxygen (shorter) and a nitrogen wave, an oxygen and a nitrogen (shorter) wave, and two nitrogen waves. All four types of fits gave similar results, since distinguishing between an oxygen and nitrogen wave from EXAFS data on proteins is inherently extremely difficult due to their strong similarity in backscattering properties and further exacerbated by the relatively low quality of the data. Only the fits with nitrogen signals are therefore reported in Table 5.10 .

The EXAFS data of $\mathrm{Fe}(\mathrm{II}) \mathrm{PCD}$ were reasonably well fit with two nitrogen waves (Fit 2) having $1 \mathrm{~N}$ at $1.99 \AA$ and $3 \mathrm{~N}$ at $2.15 \AA$ with an $F$ value of 0.37 (Table 5.10 and 


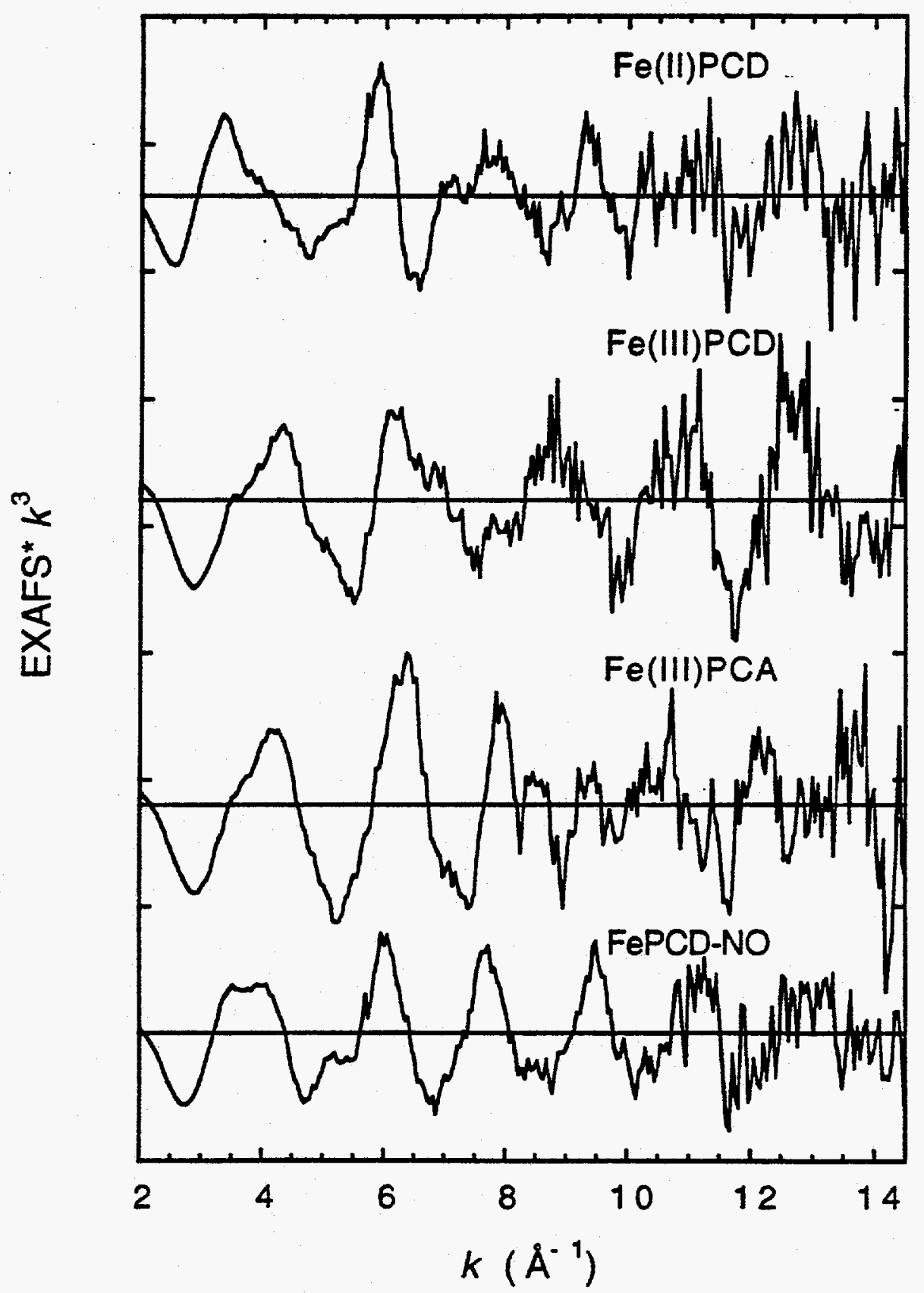

Figure 5.21. EXAFS data $\left({ }^{*} k^{3}\right)$ for $\mathrm{Fe}($ II)PCD, Fe(III)PCD, Fe(II)PCA, and FePCD-NO (the ordinate scale is 5 between tick marks with solid horizontal lines indicating the zero point of each plot). 


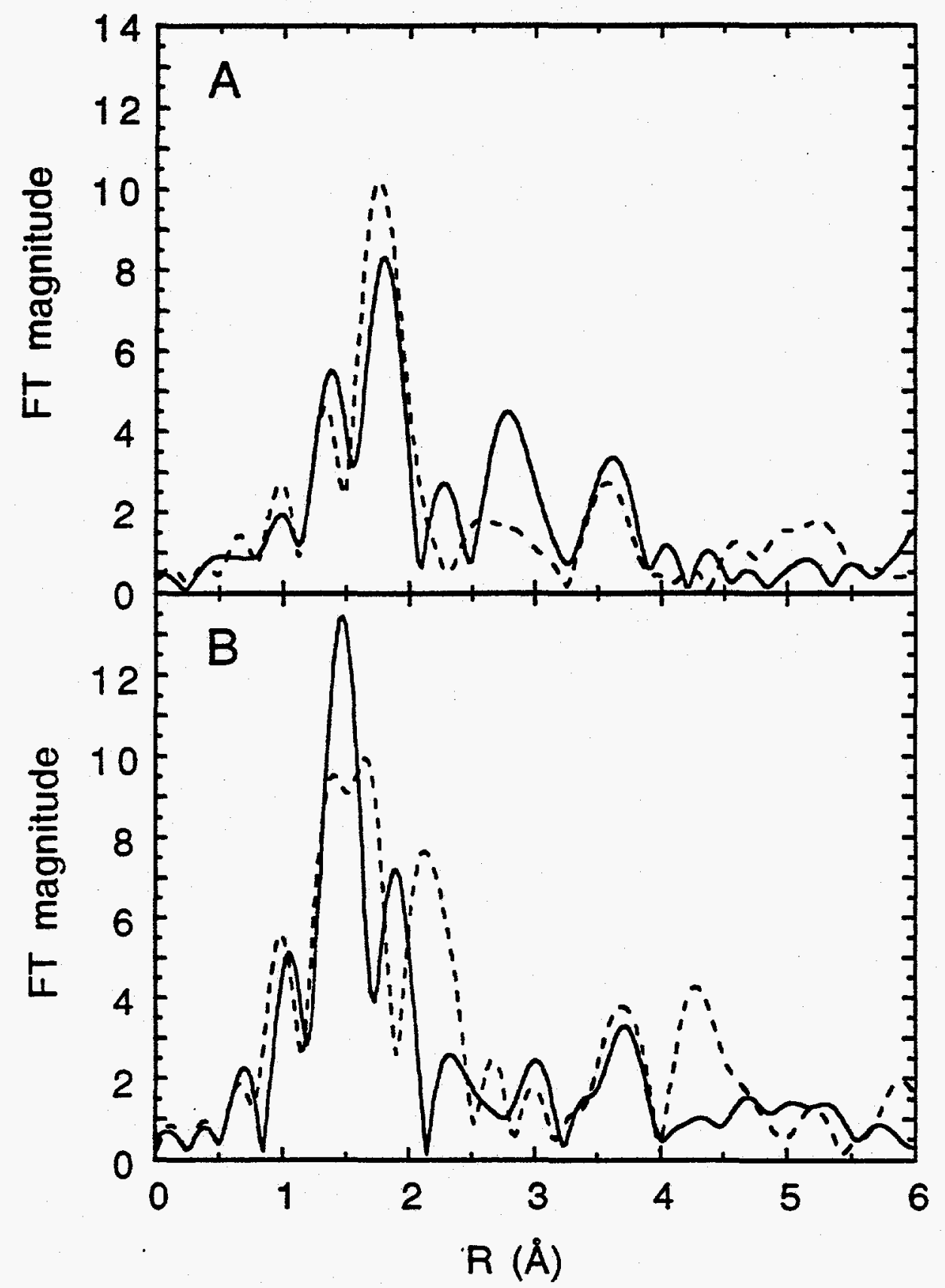

Figure 5.22. Fourier transforms over the $k$-range $3.5-14.5 \AA^{-1}$ (non-phase shift corrected) for the EXAFS data of Fe(II)PCD (-) and FePCD-NO (---) (shown in part A) and $\mathrm{Fe}$ (III)PCD (-) and Fe(III)PCA (--) (shown in part B). 
Table 5.10. Summary of EXAFS Curve-Fitting Results.*

\begin{tabular}{|c|c|c|c|c|c|c|}
\hline sample & Fit \# & $\begin{array}{l}\text { FT window } \\
\text { width }(\AA)\end{array}$ & element & $\mathrm{CN}^{a, b}$ & $\begin{array}{l}\text { ond length } \\
(\AA)^{b}\end{array}$ & $F$ \\
\hline \multirow{6}{*}{$\mathrm{Fe}($ II)PCD } & $T$ & {$[1.1-2.1]$} & $\overline{\mathrm{N}}$ & 2.3 & 2.14 & 0.56 \\
\hline & 2 & {$[1.1-2.1]$} & $\mathrm{N}$ & 1.0 & 1.99 & 0.37 \\
\hline & & & $\mathrm{N}$ & 3.1 & 2.15 & \\
\hline & 3 & {$[1.1-2.1]$} & $\mathrm{N}$ & 1.5 & 1.98 & 0.31 \\
\hline & & & $\mathbf{N}$ & 4.1 & 2.15 & \\
\hline & & & $\mathrm{N}$ & 1.0 & 2.31 & \\
\hline \multirow[t]{3}{*}{$\mathrm{Fe}(\mathrm{III}) \mathrm{PCD}$} & 4 & {$[0.88-2.15]$} & $\mathrm{N}$ & 2.6 & 1.91 & 1.2 \\
\hline & 5 & {$[0.88-2.15]$} & $N$ & 4.3 & 1.92 & 0.38 \\
\hline & & & $\mathrm{N}$ & 3.1 & 2.12 & \\
\hline \multirow[t]{6}{*}{$\mathrm{Fe}$ (III)PCA } & 6 & {$[0.75-2.55]$} & $\mathrm{N}$ & 2.7 & 2.00 & 1.4 \\
\hline & 7 & {$[0.75-2.55]$} & $\mathrm{N}$ & 3.7 & 1.97 & 1.2 \\
\hline & & & $\mathrm{N}$ & 2.5 & 2.11 & \\
\hline & 8 & {$[0.75-2.55]$} & $N$ & 3.0 & 1.97 & 0.80 \\
\hline & & & $\mathrm{N}$ & 1.8 & 2.10 & \\
\hline & & & 0 & 2.2 & 2.47 & \\
\hline \multirow{9}{*}{ FePCD-NO } & 9 & {$[0.8-2.3]$} & $\mathrm{N}$ & 2.8 & 2.12 & 0.58 \\
\hline & 10 & {$[0.8-2.3]$} & $\mathrm{N}$ & 1.1 & 1.94 & 0.33 \\
\hline & & & $N$ & 3.7 & 2.12 & \\
\hline & 11 & {$[0.8-2.3]$} & N & 1.1 & 1.91 & 0.31 \\
\hline & & & $\mathrm{N}$ & 1.9 & 2.07 & \\
\hline & & & $\mathrm{N}$ & 2.6 & 2.14 & \\
\hline & 12 & [0.8-2.3] & $\mathrm{N}$ & 1.0 & 1.94 & 0.27 \\
\hline & & & $\mathrm{N}$ & 3.6 & 2.12 & \\
\hline & & & $\mathrm{N}$ & 0.5 & 2.50 & \\
\hline
\end{tabular}

* EXAFS data were fit over the $k$-range $4-14 \AA^{-1}$. a $\mathrm{CN}=$ coordination number. $b$ Errors in distances $( \pm 0.02 \AA)$ and coordination numbers $( \pm 25 \%)$ are estimated from the variance between EXAFS fitting results and values from models of crystallographically known structure. 39 
Figure 5.23). However, this fit gave a total coordination number of four which is much lower than the value of six predicted by the edge analysis. The $F$ value decreased to 0.31 when a third contribution of $1 \mathrm{~N}$ at $2.31 \AA$ was added to the fit (Fit 3). This fit matches the data more accurately at higher $k$ (Figure 5.23) and has a total coordination number of 6.6, which is more consistent with the edge data. Also, the average first-shell distance for Fit 3 of $2.14 \AA$ is more consistent with six-coordination than the average first-shell distance for Fit 2 of $2.11 \AA$. Crystallographic determination of ferrous model complexes with oxygen and nitrogen ligation reports average bond lengths of $2.17 \pm 0.03 \AA$ for six-coordinate sites, ${ }^{62-68} 2.11 \pm 0.02 \AA$ for five-coordinate sites, ${ }^{69,70}$ and $2.00 \pm 0.01 \AA$ for four-coordinate sites. ${ }^{33}$

The EXAFS data of Fe(III)PCD could not be simulated with a wave from a single shell of nitrogens (Fit 4, Table 5.10). Inclusion of a second nitrogen signal (Fit 5) decreased the $F$ value by a factor of three (Table 5.10) and gave a fit that matched the EXAFS data extremely well (Fit 5, Figure 5.23). Fit 5 has $4.3 \mathrm{~N}$ at $1.92 \AA$ and $3.1 \mathrm{~N}$ at $2.12 \AA$. The total coordination number of Fit 5 is 7.4 which is much higher than the coordination number of five predicted by the edge analysis. However, the two EXAFS waves from nitrogens at $1.92 \AA$ and $2.12 \AA$ are $-180^{\circ}$ out-of-phase with one another in the $k$ region where the signals are the strongest, $6-10 \AA^{-1}$. This causes high correlation and thus high uncertainty in the coordination numbers. The distances and the relative strengths of the two O/N waves obtained from these fits to the EXAFS data are consistent with a previous study ${ }^{13}$ which reported a best fit to the EXAFS data for Fe(III)PCD of 3 O N at $1.90 \AA$ and $2 \mathrm{O} / \mathrm{N}$ at $2.08 \AA$.

The EXAFS data of Fe(III)PCA could not be reproduced accurately with fits that contained only one (Fit 6 ) or two Fe-N waves (Fit 7). Fits 6 and 7 have $F$ values of 1.4 and 1.2, respectively (Table 5.10). A fit that included signals from nitrogens at 1.97 and $2.10 \AA$ and oxygens at $2.47 \AA$ was necessary to simulate the EXAFS data in the higher $k$ region (Fit 8 , Figure 5.23). The addition of the longer oxygen distance in the fit is also justified by the fact that there is a feature in the FT at $-2.2 \AA$ (non-phase shift corrected). Fit 8 has a total coordination number of seven, which is inconsistent with the pre-edge and edge analysis that indicates that the iron site is five-coordinate.

The EXAFS data of FePCD-NO could be simulated accurately with fits that contained either two or three-Fe-N signals (Fit 10,11, and 12 in Table 5.10 and Figure 5.23). The $F$ values of Fits 10 and 11 are nearly identical ( 0.33 and 0.31 , respectively). Both fits have a contribution from a single nitrogen at $-1.9 \AA$ with another contribution of approximately four nitrogens at $2.1 \AA$. The only difference between Fits 10 and 11 is that the contribution at $2.1 \AA$ is split into two contributions in Fit 11 . Fit 12 contains an 


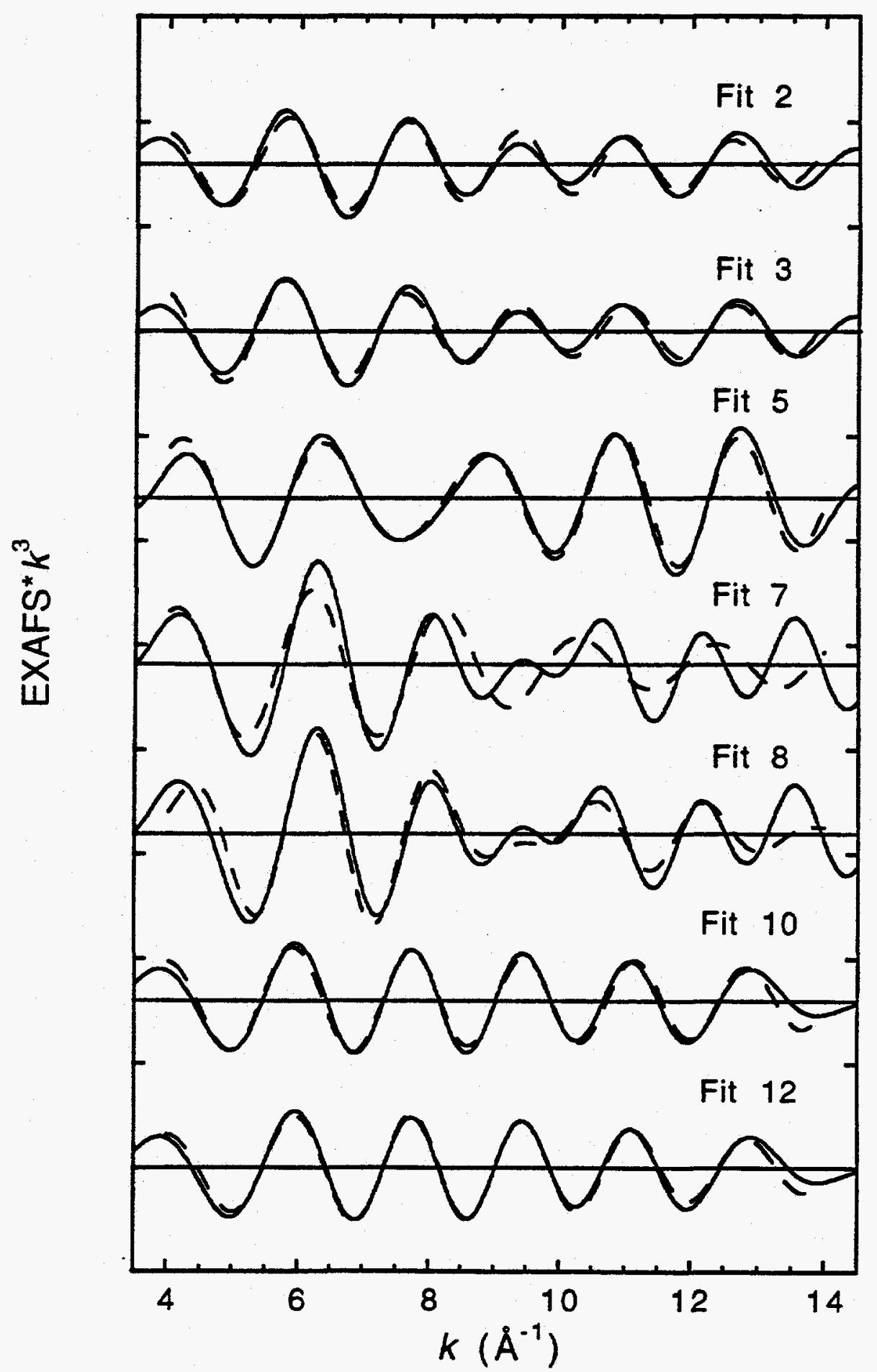

Figure 5.23. Fits to the Fourier filtered data of Fe(II)PCD (Fit 2 and 3), Fe(III)PCD (Fit 5), Fe(II)PCA (Fit 7 and 8), and FePCD-NO (Fit 10 and 12), where the solid line represents the backtransformed data while the dashed line is the fit to the data. (The ordinate scale is 5 between tick marks with solid horizontal lines indicating the zero point of each plot). 
additional contribution from a N at $-2.5 \AA$. The contribution from an $\mathrm{O} N$ at $2.5 \AA$ is questionable as the $F$ value only decreases slightly to 0.27 when this contribution is added and the match to the experimental data is only slightiy better (Figure 5.23). The short $1.9 \AA$ distance can be assigned as the $\mathrm{Fe}-\mathrm{N}(\mathrm{O})$ bond with this distance being longer than the typical $\mathrm{Fe}-\mathrm{N}(\mathrm{O})$ distance of $\sim 1.75 \AA$ reported for $\{\mathrm{FeNO}\}^{7}$ model complexes $^{59-61}$ and isopenicillin $\mathrm{N}$ synthase-substrate-NO. ${ }^{71}$ The presence of a shorter $\mathrm{Fe}-\mathrm{N}$ interaction was further examined by fixing a $\mathrm{Fe}-\mathrm{N}$ distance at $1.75 \AA$ with a coordination number of 1 , while varying the distances and coordination numbers of the other two $\mathrm{N}$ contributions. Including the shorter $\mathrm{Fe}-\mathrm{N}$ distance at $1.75 \AA$ increased the $F$ value to 0.6 indicating that there is no contribution from a shorter Fe-N distance.

5.7.3.3. Fe-N-O Angle Determination Using GNXAS. The GNXAS approach was used to fit the experimental EXAFS data of Fe(III)PCD and FePCD-NO in order to investigate the Fe-N-O angle in FePCD-NO. GNXAS requires an initial structural model. Since the crystal structure of Fe(III)PCD is known, the EXAFS data of Fe(III)PCD were fit using the GNXAS approach to determine the accuracy of the technique for studying the active site of this protein. The GNXAS approach was then used to fit the EXAFS data of FePCD-NO to gain information on the Fe-N-O angle.

Crystal structures of Fe(III)PCD from Psuedomonas aeruginosa $a^{5,6}$ and Brevibacterium fuscum ${ }^{7}$ both show that the iron atom is coordinate axially by a Tyr and His with a Tyr, His, and water-based molecule bound in the equatorial plane. Crystallographic coordinates from the Psuedomonas aeruginosa structure at $2.15 \AA^{6}$ were used as input to build the initial structural model. EXAFS contributions for each two-atom and three-atom configuration were calculated using the crystallographic bond distances and angles. The individual contributions were then summed to generate a theoretical EXAFS spectrum which was then fit to the non-Fourier-filtered experimental EXAFS data without prior background subtraction. In the fits the crystallographic bond distances and angles were allowed to vary to fit the experimental EXAFS data unless stated otherwise.

Due to the complexity of the structure, contributions to the fits of the Fe(II)PCD EXAFS data were systematically introduced. The first-shell fit contained waves from the following two-atom configurations: $\mathrm{Fe}-\mathrm{O}(\mathrm{H})$ [1], $\mathrm{Fe}-\mathrm{O}(\mathrm{Tyr}$ ) [2], and $\mathrm{Fe}-\mathrm{N}(\mathrm{His})$ [2], where the number in brackets indicates the coordination number. This first-shell fit (not shown) gave an $R$ value of $0.286 \times 10^{-5}$ with good agreement between the FT of the experimental data and the theoretical signal up to $2.0 \AA$ (corresponding to $-2.4 \AA$ in the cluster when the phase shift is taken into account). The major contributions in the EXAFS signal were accounted for using the three first-shell distances with especially 
good agreement at higher $k$. The next fit included signals from three-body configurations: $\mathrm{Fe}-\mathrm{O} 2-\mathrm{C} 4$ [2], $\mathrm{Fe}-\mathrm{N} 1-\mathrm{C} 7$ [2], and Fe-N1-C6 [2] (with the atom designations shown in Figure 5.24A and $\mathrm{B}$ ). The $R$ value decreased only slightly to $0.222 \times 10^{-5}$ when these three signals from the second-shell carbon atoms were included. The four Fe-N1-C6/C7 contributions could not be summed together as the His ligand is bound in such a way that the Fe-N1-C6 angle is $-140^{\circ}$ while the Fe-N1-C7 angle is $-110^{\circ}$ in the crystal structure. Fits that included signals from third and fourth shells were then performed to investigate the dominant multiple-scattering pathways. There were approximately 35 unique three-atom configurations which ranged in distance from 3.0 to 4.5 $\AA$. The seven main contributions were from Fe-O2-C4, Fe-N1-C7, Fe-N1-C6, $\mathrm{Fe}-\mathrm{O} 2-\mathrm{C} 3, \mathrm{Fe}-\mathrm{C} 7-\mathrm{N} 2, \mathrm{Fe}-\mathrm{N} 1-\mathrm{N} 2$, and Fe-N1-C8 (with the atom designations shown in Figure 5.24A and $B$ ).

The best fit to the data was obtained when the last seven contributions were included with the three $\mathrm{Fe}-\mathrm{O} / \mathrm{N}$ first-shell contributions. This fit had an $R$ value of $0.178 \times 10^{-5}$. A comparison of the theoretical EXAFS spectrum to the experimental data (along with the individual EXAFS signal from each contribution) is presented in Figure 5.24C for this fit to the Fe(III)PCD data with the FTs shown in Figure 5.25. A comparison of the bond distances and angles obtained from the GNXAS fit to the crystallographic values is given in Table 5.11. The low-frequency EXAFS is dominated by the three waves from the first-shell contributions. The Fe-O distances obtained from the GNXAS fit show excellent agreement with the crystallographic values for $\mathrm{Fe}(\mathrm{III}) \mathrm{PCD}^{6}$ with the $\mathrm{Fe}-\mathrm{O} 1 \mathrm{(H}$ ) distance being $1.92 \AA$ (vs. the crystallographic value of $1.91 \AA$ ) and the average of the two Fe-O2 (Tyr) distances being $1.88 \AA$, which is within the crystallographic range of $1.80-1.90 \AA$. The Fe-N1 (His) distance obtained from the GNXAS fit to the data is $2.14 \AA$ which is $-0.15 \AA$ shorter than the crystallographic value. The experimental EXAFS data could not be reproduced with an Fe-N1 (His) distance of $-2.29 \AA$. The shorter Fe-Nl (His) distance obtained from the GNXAS fit is consistent with the value obtained from the first-shell empirical fits to the EXAFS data of $\mathrm{Fe}$ (III)PCD in this study (see Fit 5 in Table 5.10 and Figure 5.23) and in the study by True et. al. ${ }^{13}$

The EXAFS data of Fe(III)PCD was fit reasonably well including signals from the three two-atom configurations and the seven main three-body configurations (Figure 5.24C and 5.25). The bond distances and angles obtained from this fit were all within $10 \%$ of the crystallographic values (Table 5.11). However, the frequency distribution of the signals from the three-body configurations does not match the data particularly well in the $2-4 \AA$ region of the FT (Figure 5.25A). The match to the data was significantly 


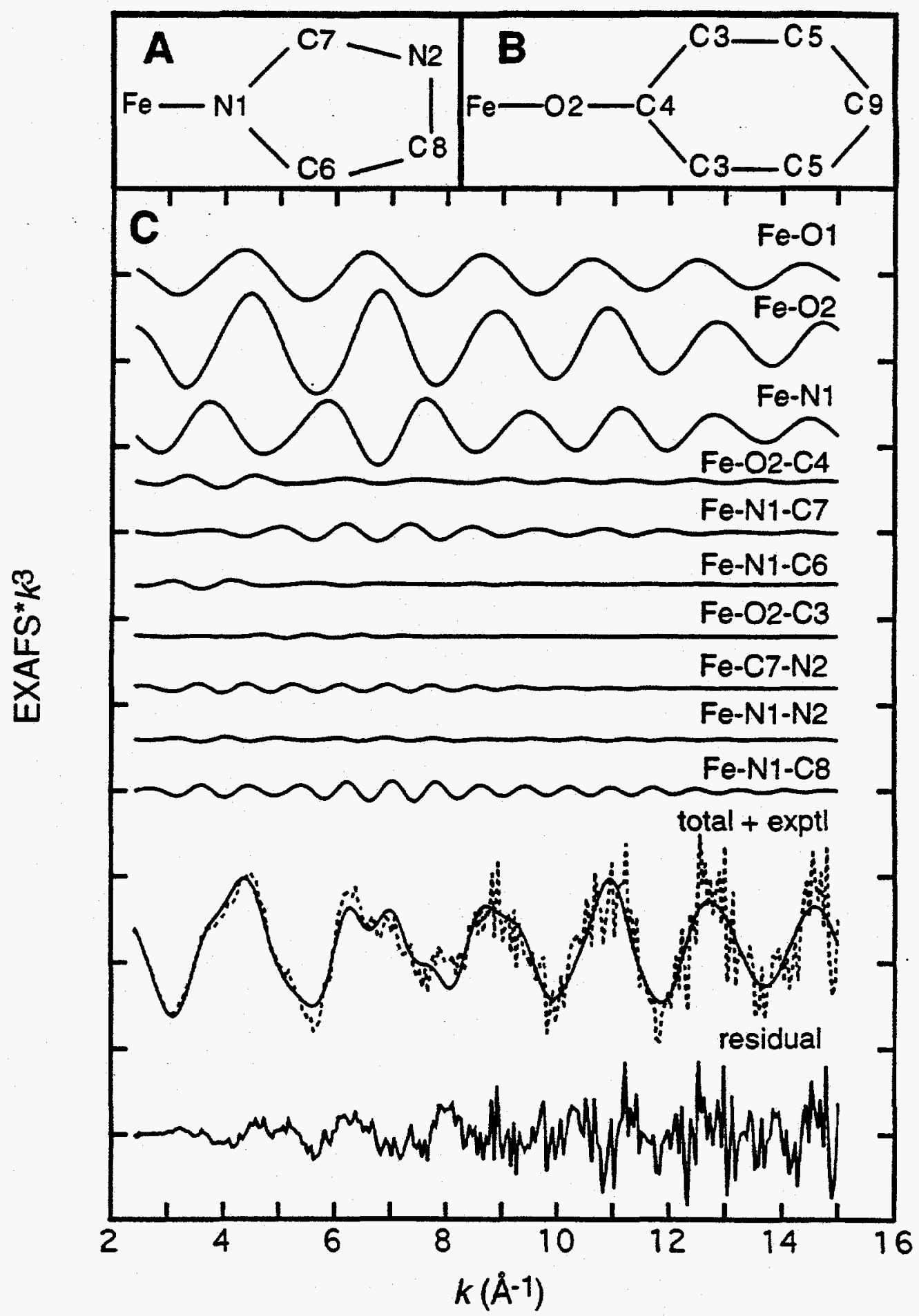

Figure 5.24. Atom designations for the A) Fe-His and the B) Fe-Tyr subunits. C) EXAFS signals for individual contributions in the best fit for the Fe(III)PCD data. The total signal (-) is also shown and compared with the experimental data ( (...) with the residual being the difference between the experimental EXAFS and the theoretical EXAFS. (The ordinate scale is 5 between two consecutive tick marks.) 


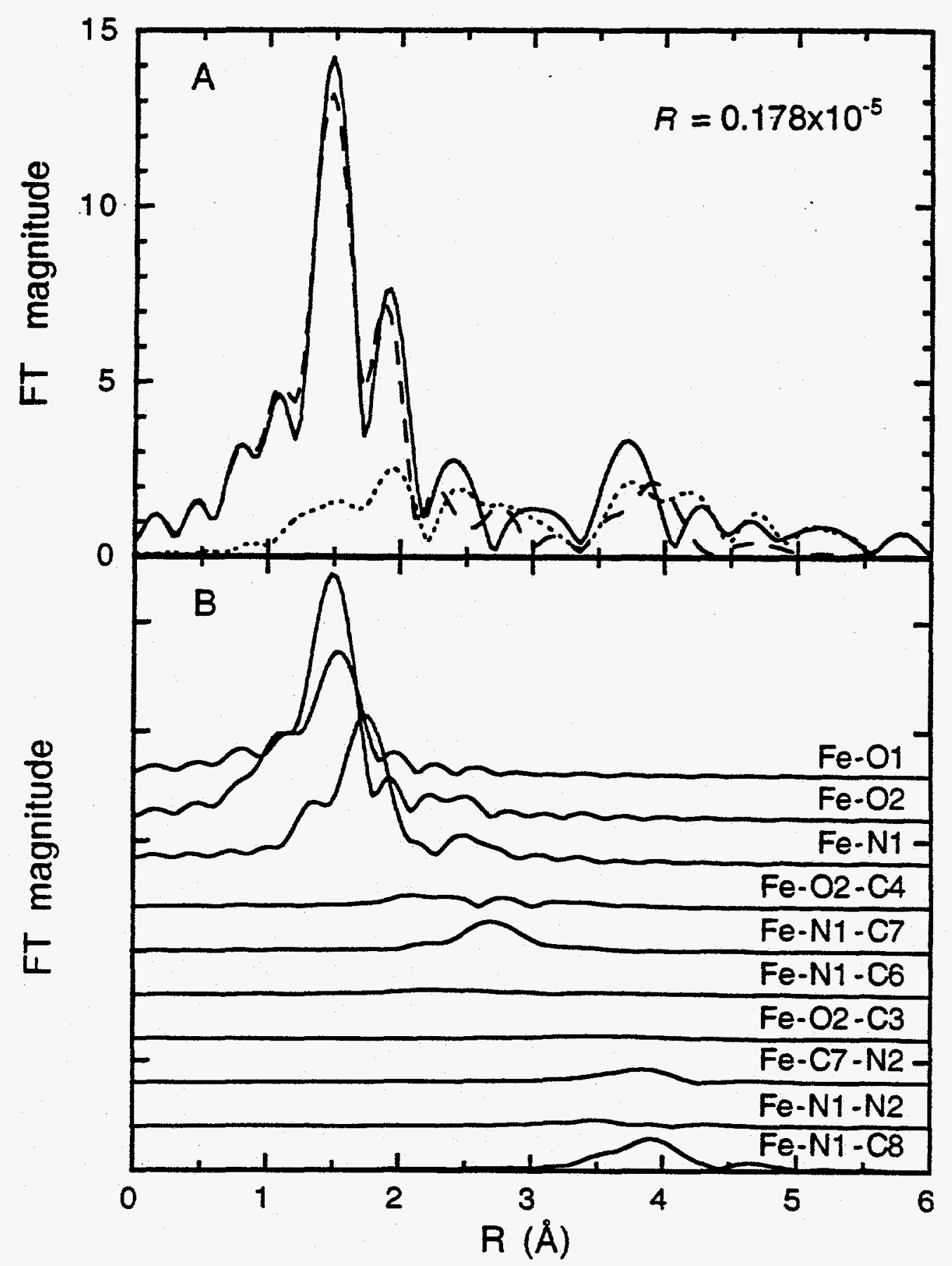

Figure 5.25. A) A comparison of the theoretical (---) and experimental (-) non-phase shift corrected FT of Fe(III)PCD EXAFS data, along with the FT of the EXAFS residual $(\cdots)$. The $R$ value is an indication of the goodness of the fit. B) FT of the EXAFS signals of $\mathrm{Fe}(\mathrm{II}) \mathrm{PCD}$ for the individual contributions shown in Figure 5.24C. This display is a useful way to determine which signals contribute in which regions. (The ordinate scale is 5 between two consecutive tick marks.) 
Table 5.11. Crystallographic Bond Distances and Angles Compared to GNXAS Results for Fe(III)PCD.

\section{GNXAS}

bond variance

\begin{tabular}{|c|c|c|c|}
\hline $\begin{array}{l}\text { structural feature } a \\
\left(\mathrm{CN}^{b}\right)\end{array}$ & $\begin{array}{l}\text { GNXAS } \\
\text { distancel } \\
\text { angle }\end{array}$ & $\begin{array}{l}\left(\sigma_{R}^{2}\right) \prime \\
\text { angle variance } \\
\left(\sigma_{\theta}^{2}\right)^{c}\end{array}$ & $\begin{array}{l}\text { crystallographic } \\
\text { values } \\
\text { [range] }\end{array}$ \\
\hline $\mathrm{Fe}-\mathrm{O} 1(\mathrm{H})(1)$ & $1.92 \AA$ & 0.002 & $1.91 \AA$ \\
\hline $\mathrm{Fe}-\mathrm{O} 2(\mathrm{Tyr})(2)$ & $1.88 \AA$ & 0.002 & $1.85 \AA[1.80-1.90]$ \\
\hline $\mathrm{Fe}-\mathrm{N}$ (His) (2) & $2.14 \AA$ & 0.002 & $2.29 \AA[2.26-2.33]$ \\
\hline $\mathrm{O} 2-\mathrm{C} 4(2)$ & $1.30 \AA$ & 0.003 & $1.37 \AA[1.36-1.38]$ \\
\hline $\mathrm{N} 1-\mathrm{C} 7(2)$ & $1.37 \AA$ & 0.004 & $1.32 \AA[1.31-1.32]$ \\
\hline N1-C6 (2) & $1.32 \AA$ & 0.006 & $1.37 \AA[1.36-1.38]$ \\
\hline $\mathrm{O} 2-\mathrm{C} 3(4)$ & $2.45 \AA$ & 0.007 & $2.4 \AA[2.35-2.42]$ \\
\hline C7-N2 (2) & $1.32 \AA$ & 0.004 & $1.31 \AA[1.30-1.31]$ \\
\hline $\mathrm{Fe}-\mathrm{C} 7(2)$ & $3.14 \AA$ & 0.007 & $3.01 \AA[2.97-3.05]$ \\
\hline N1-N2 (2) & $2.11 \AA$ & 0.004 & $2.13 \AA[2.11-2.14]$ \\
\hline $\mathrm{N} 1-\mathrm{C} 8(2)$ & $2.25 \AA$ & 0.004 & $2.18 \AA[2.16-2.20]$ \\
\hline $\mathrm{Fe}-\mathrm{O} 2-\mathrm{C} 4(1)$ & $139^{\circ}$ & $3 \times 10^{1}$ & $138^{\circ}[130-145]$ \\
\hline $\mathrm{Fe}-\mathrm{N} 1-\mathrm{C} 7(2)$ & $119^{\circ}$ & $5 \times 10^{0}$ & $110^{\circ}[1.09-110]$ \\
\hline $\mathrm{Fe}-\mathrm{N} 1-\mathrm{C} 6(2)$ & $136^{\circ}$ & $6 \times 10^{1}$ & $141^{\circ}[140-142]$ \\
\hline $\mathrm{Fe}-\mathrm{O} 2-\mathrm{C} 3(2)$ & $130^{\circ}$ & $9 \times 10^{1}$ & $132^{\circ}[111-166]$ \\
\hline $\mathrm{Fe}-\mathrm{C} 7-\mathrm{N} 2(2)$ & $154^{\circ}$ & $5 \times 10^{0}$ & $154^{\circ}[153-155]$ \\
\hline Fe-N1-N2 (2) & $146^{\circ}$ & $3 \times 10^{0}$ & $146^{\circ}[145-146]$ \\
\hline $\mathrm{Fe}-\mathrm{N} 1-\mathrm{C} 8(2)$ & $176^{\circ}$ & $5 \times 10^{0}$ & $177^{\circ}[176-178]$ \\
\hline
\end{tabular}

$a$ Atom designation are shown in Figure 5.24. $b \mathrm{CN}=$ number of configurations in the complex. $c$ Bond and angle variances are reported in $\AA^{2}$ and degrees ${ }^{2}$, respectively. 
worse when the bond angles and distances were fixed to crystallographic values. Numerous fits were tried including signals from other three-body configurations with none of the fits having a good match to the data in the FT range of $2-4 \AA$. The inability to match the signals from second-, third-, and fourth-shell atoms is most likely due to the number of and the complexity of the multiple-scattering pathways. As mentioned earlier there were 35 unique three-body configurations in the range of 3.0 to $4.5 \AA$. Since all the atoms surrounding the iron site are low $Z(O, N$, and $C)$, the signals are weak and there is no dominant signal. In addition, this fit is slightly underdetermined with 40 fitting parameters compared with 38 independent points, where the number of independent points was calculated by the expression $N_{l}=(2 \delta k \delta R \pi)+2 .{ }^{72}$ It is difficult to say whether the mismatch in the FT region between the experimental data and the theoretical signal is due to an inaccuracy in the bond distances and angles of the initial structural model as it was derived from the crystal structure determined at a resolution of $2.15 \AA$ or from the fact that there are so many unique multiple-scattering pathways all contributing very weak signals, or both.

The GNXAS approach was then applied to the EXAFS data of FePCD-NO using the theoretical signals that were generated from the Fe(III)PCD crystallographic bond distances and angles. Since the structure of FePCD-NO is unknown, the EXAFS data was fit with signals from differing first-shell two-body configurations to develop an understanding of the first coordination sphere of the iron atom. The data could not be reproduced well with a single contribution of oxygen or nitrogen ligands. A fit (not shown) with 50 at $2.06 \AA$ had an $R$ value of $0.826 \times 10^{-5}$. The match to the experimental data improved significantly when a second $\mathrm{Fe}-\mathrm{O}$ wave was included with the $R$ value decreasing by almost a factor of four to $0.246 \times 10^{-5}$. This fit included signals from a single $O$ at $1.85 \AA$ and four $O$ at $2.05 \AA$. These distances are similar to those observed in the first-shell empirical fit to the FePCD-NO EXAFS data (see Fit 10 in Table 5.10 and Figure 5.23). Yet, there was still a mismatch between the data and the theoretical signal in the FT at $2 \AA$ (corresponding to $-2.4 \AA$ in the cluster). Thus, a third first-shell contribution was included. The best first-shell fit to the data had an $R$ value of $0.134 \times 10^{-5}$ and included signals from one $\mathrm{O}$ at $1.89 \AA$, four $\mathrm{N}$ at $2.11 \AA$, and one $\mathrm{N}$ at $2.45 \AA$ (see Figure 5.26 and Table 5.12). Fits to the data were also tried where the distance of the short Fe-O contribution was fixed to $1.85,1.80$ and $1.75 \AA$. In each of these cases the $R$ values increased with the greatest increase for the shortest distance and there was not a good match between the experimental and theoretical signal. The distance of the longer Fe- $\mathrm{N}$ contribution was also fixed to shorter values. Again the $R$ values increased with the greatest increase for the shortest distance and there was not a 

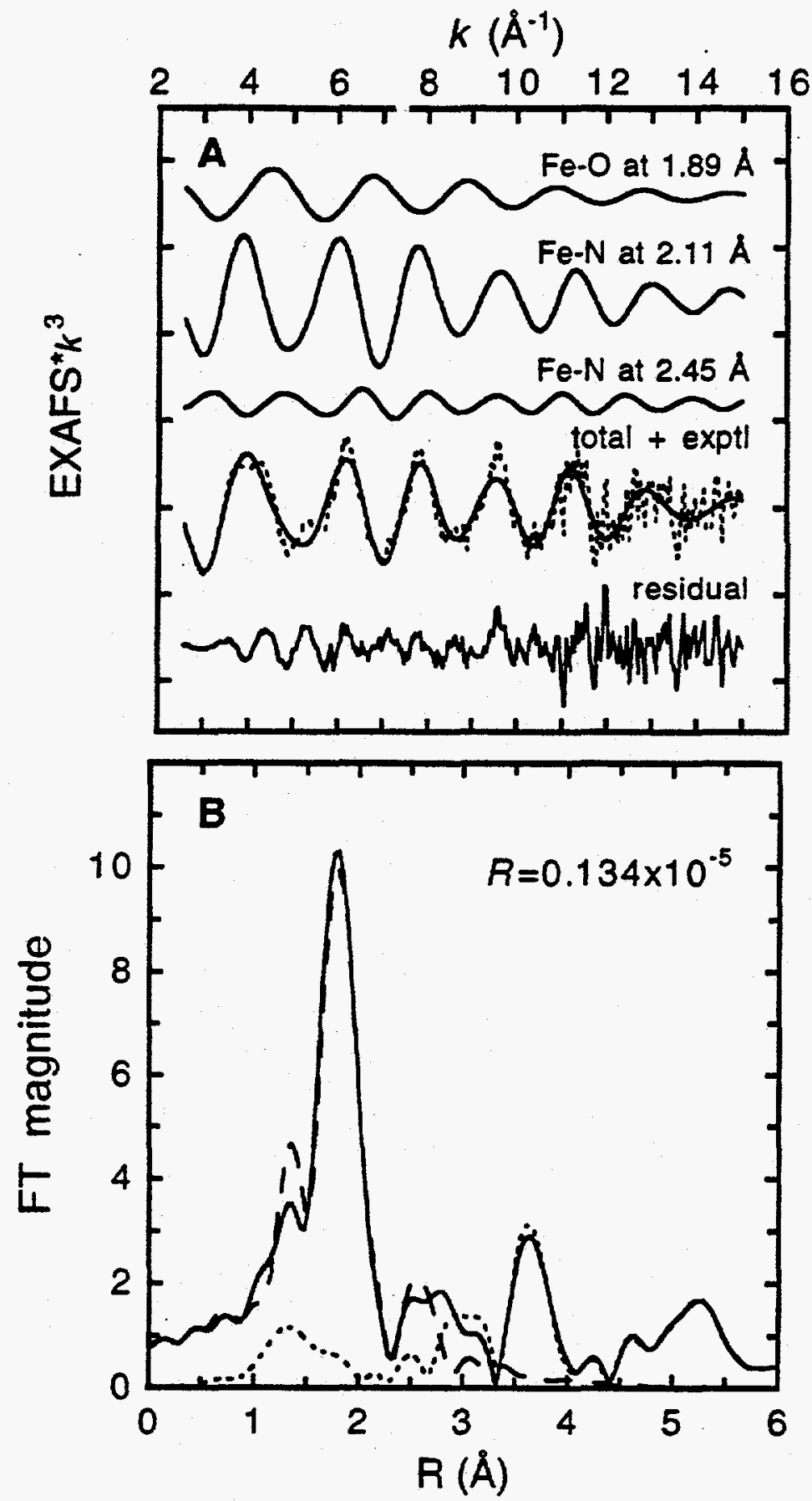

Figure 5.26. Comparison of the theoretical and experimental signals of the $k^{3}$-weighted EXAFS data and the FT of the best two-body fit for FePCD-NO. Part A contains the individual EXAFS contributions where the total signal (-) is also shown and compared with the experimental data (---). The residual is the difference between the experimental and theoretical EXAFS. (The ordinate scale is 5 between consecutive tick marks.) B) The non-phase-shift-corrected FT of the EXAFS data of the experimental data (-) and that of the total theoretical signal (---). Also shown is the FT of the residual (....). 
Table 5.12. GNXAS Results for FePCD-NO.

\begin{tabular}{|c|c|c|c|c|}
\hline Fit & $\begin{array}{l}\text { structural feature } \\
\left(\mathrm{CN}^{a}\right)\end{array}$ & $\begin{array}{l}\text { GNXAS } \\
\text { distance/ } \\
\text { angle }\end{array}$ & $\begin{array}{l}\text { GNXAS } \\
\text { bond variance } \\
\left(\sigma_{R}^{2}\right) / \\
\text { angle variance } \\
\left(\sigma_{\theta}^{2}\right)^{b}\end{array}$ & $R$ vạlue \\
\hline best fit with only & $\mathrm{Fe}-\mathrm{O}(1)$ & $1.89 \AA$ & 0.005 & $0.134 \times 10^{-5}$ \\
\hline \multirow{2}{*}{ first-shell signals } & $\mathrm{Fe}-\mathrm{N} s(4)$ & $2.11 \AA$ & 0.005 & \\
\hline & $\mathrm{Fe}-\mathrm{N} l(1)$ & $2.45 \AA$ & 0.002 & \\
\hline best fit with three- & $\mathrm{Fe}-\mathrm{O}(1)$ & $1.89 \AA$ & 0.005 & $0.120 \times 10^{-5}$ \\
\hline body signals & $\mathrm{Fe}-\mathrm{Ns}(4)$ & $2.11 \AA$ & 0.005 & \\
\hline \multirow[t]{5}{*}{ included } & $\mathrm{Fe}-\mathrm{N} l(1)$ & $2.45 \AA$ & 0.002 & \\
\hline & $\mathrm{Ns}-\mathrm{C}(4)$ & $1.32 \AA$ & 0.004 & \\
\hline & $\mathrm{N} l-\mathrm{C}(1)$ & $1.37 \AA$ & 0.003 & \\
\hline & $\mathrm{Fe}-\mathrm{Ns}-\mathrm{C}(4)$ & $125^{\circ}$ & $1 \times 10^{2}$ & \\
\hline & $\mathrm{Fe}-\mathrm{Nl}-\mathrm{C}(1)$ & $145^{\circ}$ & $6 \times 10^{1}$ & \\
\hline
\end{tabular}


good match between the experimental and theoretical signal. Note that an $\mathrm{Fe}-\mathrm{O}$ signal was used to mimic the $\mathrm{Fe}-\mathrm{N}(\mathrm{O})$ signal as the shortest Fe-ligand distance in the $\mathrm{Fe}$ (III)PCD structure was an $\mathrm{Fe}-\mathrm{O}$ distance.

To investigate the Fe- $\mathrm{N}-\mathrm{O}$ bond angle, the distances and $\sigma^{2}$ values of the three first-shell contributions were kept fixed to those values obtained from the best first-shell fit (Table 5.12 and Figure 5.26) while the Fe-N-O angle was varied. The sensitivity of the fit to the Fe-N-O angle was tested by calculating a theoretical EXAFS spectrum with $\mathrm{Fe}-\mathrm{N}-\mathrm{O}$ angles ranging from $90^{\circ}$ to $180^{\circ}$. The signal for the $\mathrm{Fe}-\mathrm{N}-\mathrm{O}$ unit was generated from a $\{\text { FeNO }\}^{7}$ model complex, $\mathrm{Fe}(\mathrm{TACN})\left(\mathrm{N}_{3}\right)_{2} \mathrm{NO}$ (where $\mathrm{TACN}=N, N^{\prime} N^{\prime \prime}$-trimethyl1,4,7-triazacyclononane). ${ }^{73}$ A plot of the $\log (R$ value) $v s$. Fe-N-O angle is shown in Figure 5.27A where a minimum in this type of plot is indicative of a better fit to the experimental EXAFS data. The $R$ value drops drastically as the Fe-N-O angle is decreased from $180^{\circ}$ to $155^{\circ}$ and then increases slightly as the angle is lowered to $135^{\circ}$. The $R$ value is fairly constant between $140^{\circ}$ and $100^{\circ}$ and then increases as the Fe-N-O angle is lowered to $90^{\circ}$. A comparison of the FTs of the experimental data and calculated spectra at $180^{\circ}, 155^{\circ}$, and $140^{\circ}$ are presented in Figure 5.28 where the $R$ value was largest at $180^{\circ}$, lowest at $155^{\circ}$, and had leveled off by $140^{\circ}$. The FT of the calculated spectrum with the Fe-N-O angle equal to $180^{\circ}$ does not match the FT of the experimental EXAFS data at $\sim 2.5 \AA$ (Figure 5.28A) which is the region where the signal from the Fe-N-O multiple-scattering appears. ${ }^{73}$ The Fe-N-O signal is very strong when the angle is $180^{\circ}$ due to the intervening atom focusing effect. ${ }^{73}$ The Fe-N-O signal has decreased significantly when the angle is at $155^{\circ}$ matching the experimental data more accurately (Figure 5.28B). When the Fe-N-O angle is below $\sim 150^{\circ}$, the signal from the $\mathrm{Fe}-\mathrm{N}-\mathrm{O}$ unit is relatively weak. ${ }^{73}$ Thus, there is not much variation in the match to the experimental data for Fe-N-O angles between $100^{\circ}$ and $145^{\circ}$ as is indicated by the similar $R$ values (Figure 5.27A). The $R$ value increases when the $\mathrm{Fe}-\mathrm{N}-\mathrm{O}$ angle is below $100^{\circ}$ as the single-scattering signal from the $\mathrm{Fe}-\mathrm{O}$ of the $\mathrm{Fe}-\mathrm{N}-\mathrm{O}$ unit increases (Figure 5.27A).

The signal from the Fe- $\mathrm{N}-\mathrm{O}$ configuration was the only three-body contribution that was taken into account in the calculated spectra above. In reality there will also be signals from second-shell carbon atoms in the FT range of $\sim 2.3-3 \AA$. Accordingly, the FePCD-NO data was fit with the three first-shell contributions, Fe-O at $1.89 \AA$, Fe-N at $2.11 \AA$ (Fe-Ns), and Fe-N at $2.45 \AA$ ( $\mathrm{Fe}-\mathrm{N} l$ ), and two second-shell contributions, Fe-Ns-C and $\mathrm{Fe}-\mathrm{Nl}$-C. The best fit to the FePCD-NO data that included the Fe-N-C contributions had an $R$ value of $0.120 \times 10^{-5}$ with the resulting bond distances and angles shown in Table 5.12. The signals (not shown) from the Fe-N-C configurations were very weak as was also observed for the Fe-O-C and Fe-N-C signals for Fe(III)PCD (Figure 5.24C). 


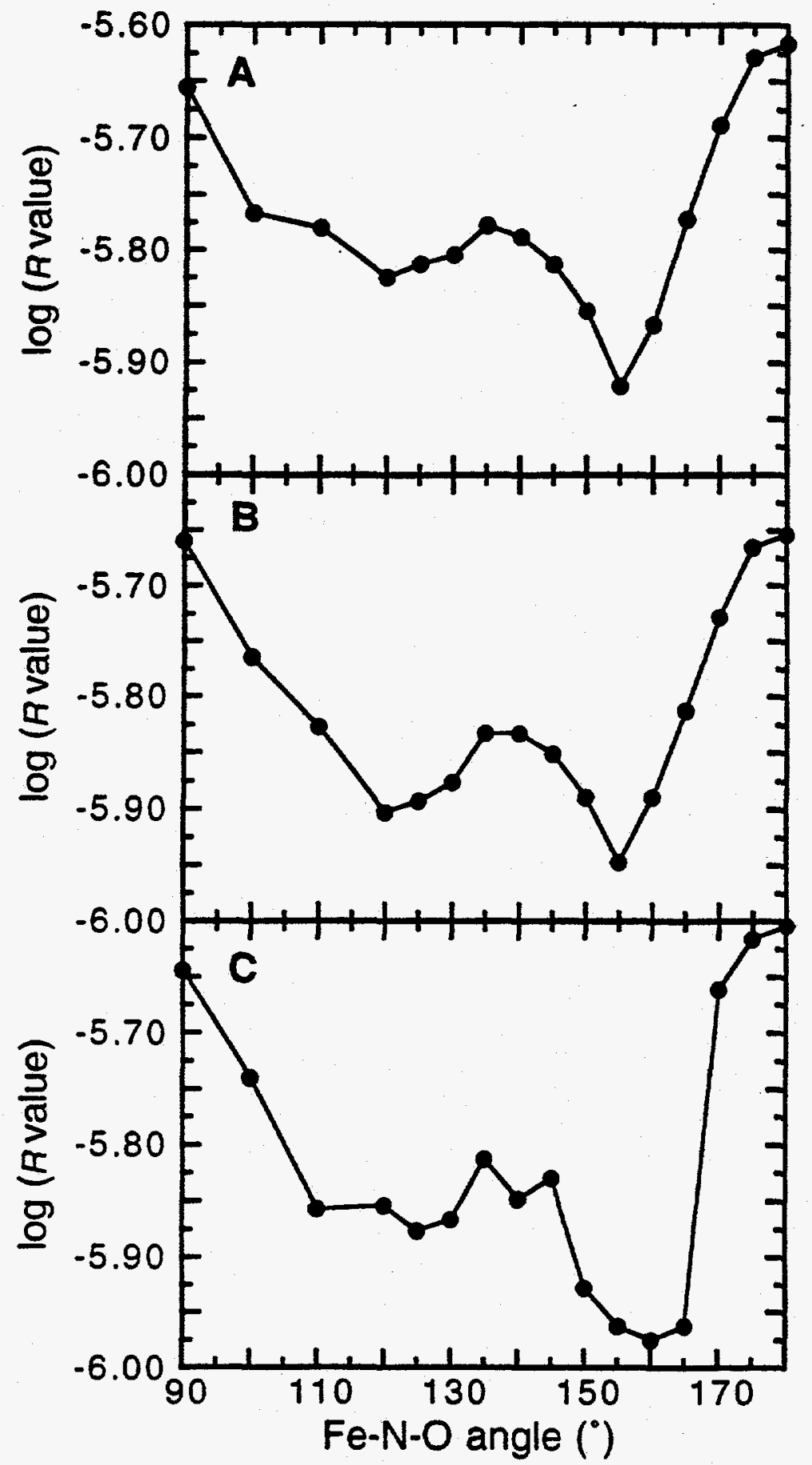

Figure 5.27. Plots of the $\log (R$ value $)$ vs Fe-N-O angle of FePCD-NO where each point represents a match of the calculated EXAFS spectrum to the data. The lower the $R$ value the better the fit. Plot A contains calculated spectra that included only first-shell contributions and an $\mathrm{Fe}-\mathrm{N}-\mathrm{O}$ contribution while $\mathrm{Fe}-\mathrm{N}-\mathrm{C}$ contributions were included in the spectra of plots $B$ and $C$ with all the Fe-N-C parameters fixed for the calculated spectra in plot $B$ and varied for those in plot $C$. 


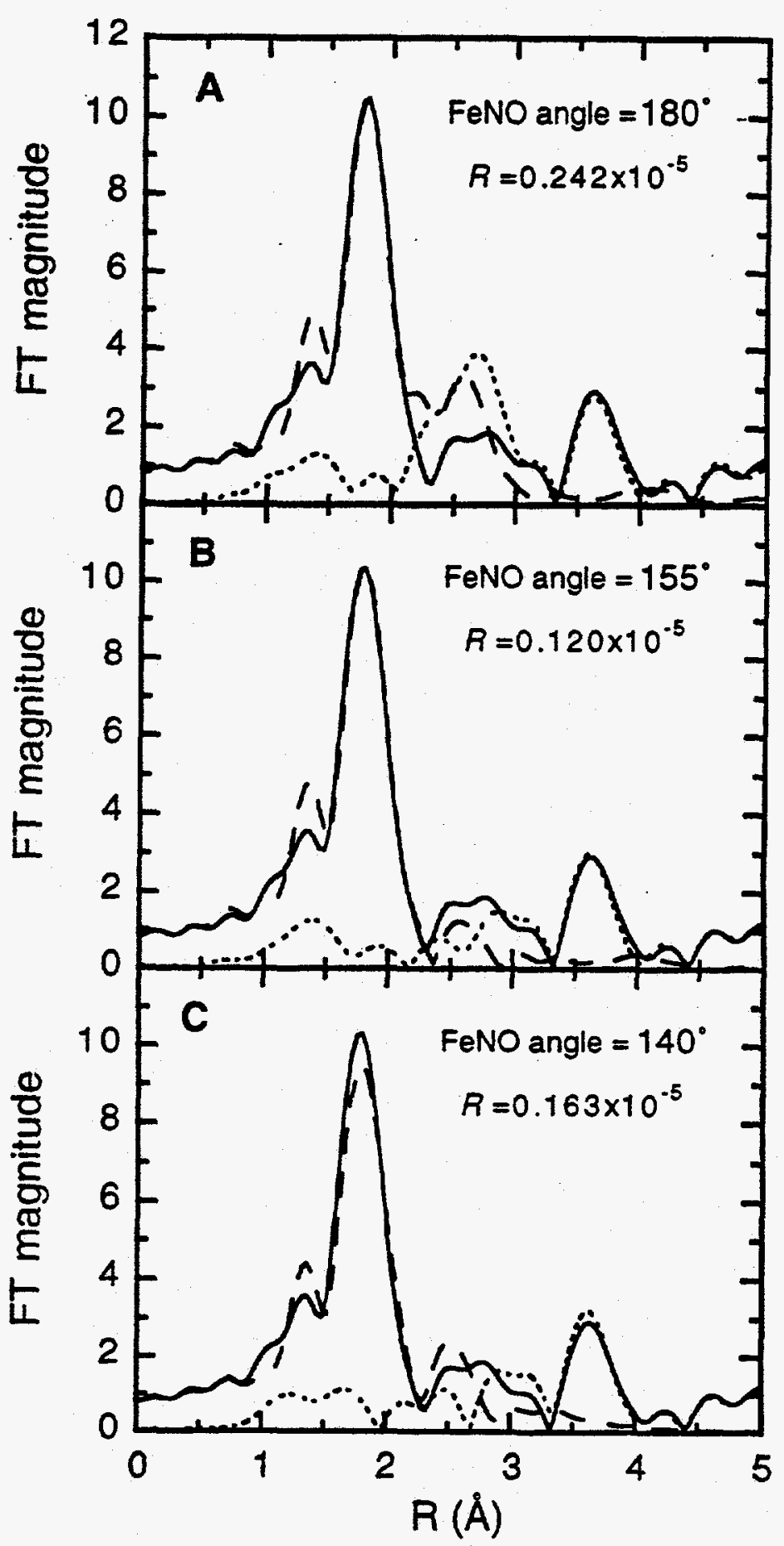

Figure 5.28. A comparison of the theoretical (--) and experimental $(-)$ non-phase shift corrected FT of FePCD-NO EXAFS data, along with the FT of the EXAFS residual $(\cdots .$.$) . The R$ value is an indication of the goodness of the fit. Calculated spectra for several different Fe-N-O bond angles are shown: A) $180^{\circ}$, B) $155^{\circ}$, and C) $140^{\circ}$. The calculated spectra included the three first-shell contributions and an $\mathrm{Fe}-\mathrm{N}-\mathrm{O}$ contribution. 
The sensitivity of this fit to the Fe-N-O angle was tested by fixing all the distances, angles, and variances to the values shown in Table 5.12 and calculating a theoretical EXAFS spectrum with Fe-N-O angles ranging from $90^{\circ}$ to $180^{\circ}$. A plot of the $\log (R$ value) vs. Fe-N-O angle is shown in Figure 5.27B. The $R$ value decreases substantially in lowering the $\mathrm{Fe}-\mathrm{N}-\mathrm{O}$ angle from $180^{\circ}$ to $155^{\circ}$, rises slightly upon lowering the angle further to $140^{\circ}$, decreases slightly upon lowering the angle further to $120^{\circ}$, and then increases upon lowering the angle to $90^{\circ}$. A comparison of the FTs for the calculated spectra and experimental data are shown for Fe-N-O angles of $180^{\circ}, 155^{\circ}$, and $140^{\circ}$ in Figure 5.29. Again, the FT of the calculated spectrum with the Fe-N-O angle equal to $180^{\circ}$ does not match the FT of the experimental EXAFS data at $\sim 2.5 \AA$ due to the strength of the signal from a linear $\mathrm{Fe}-\mathrm{N}-\mathrm{O}$ unit. Once the Fe-N-O angle decreases below $\sim 160^{\circ}$, the signal weakens and the calculated spectra match the experimental data more accurately. The plot of the $\log (R$ value $) v s$. Fe-N-O angle shows a double minimum. This double minimum has been observed before for $\{\mathrm{FeNO}\}^{7}$ model complexes ${ }^{73}$ where the minimum at lower angles is due to the single-scattering ( $\mathrm{Fe}-\mathrm{O}$ ) signal having a phase and amplitude that matches the experimental data and the minimum at higher angles is due to the multiple-scattering ( $\mathrm{Fe}-\mathrm{N}-\mathrm{O}$ ) signal having a phase and amplitude that matches the data.

Since FePCD-NO is not structurally defined, one may be biasing the results by fixing the distances, angles, and variances as was done in the previous set of calculated spectra with varying Fe-N-O angle. Thus, spectra were also calculated for a range of $\mathrm{Fe}-\mathrm{N}-\mathrm{O}$ angles over $90^{\circ}$ to $180^{\circ}$ allowing the distances, angles, and variances of the $\mathrm{Fe}-\mathrm{N} s-\mathrm{C}$ and $\mathrm{Fe}-\mathrm{N} l-\mathrm{C}$ configurations to vary. A plot of the $\log (R$ value $)$ vs. Fe-N-O angle for the calculated spectra is shown in Figure 5.27C. The $R$ value drops dramatically on going from an Fe-N-O angle of $180^{\circ}$ to $165^{\circ}$ with there being a minimum between $165^{\circ}$ and $150^{\circ}$. The $R$ value increases slightly when the Fe-N-O angle is decreased from $150^{\circ}$ to $145^{\circ}$, is approximately constant for $\mathrm{Fe}-\mathrm{N}-\mathrm{O}$ angles between $145^{\circ}$ and $110^{\circ}$, and then increases upon decreasing the Fe- $\mathrm{N}-\mathrm{O}$ angle from $110^{\circ}$ to $90^{\circ}$. A comparison of the FTs for the calculated spectra and experimental data are shown for $\mathrm{Fe}-\mathrm{N}-\mathrm{O}$ angles of $180^{\circ}$, $155^{\circ}$, and $140^{\circ}$ in Figure 5.30. Again, the FT of the calculated spectrum with the Fe-N-O angle equal to $180^{\circ}$ does not match the FT of the experimental EXAFS data at $-2.5 \AA$ due to the strength of the signal from a linear Fe-N-O unit. In general, the plot of $\log (R$ value) vs. Fe-N-O angle between the calculated spectra where the Fe-N-C parameters were kept fixed $v s$. allowed to vary are similar. The one striking difference is the precipitous drop in the $R$ value when the Fe-N-O angle is decreased from $170^{\circ}$ to $165^{\circ}$ in the set of fits where the Fe-N-C parameters were allowed to vary. Comparison of the 


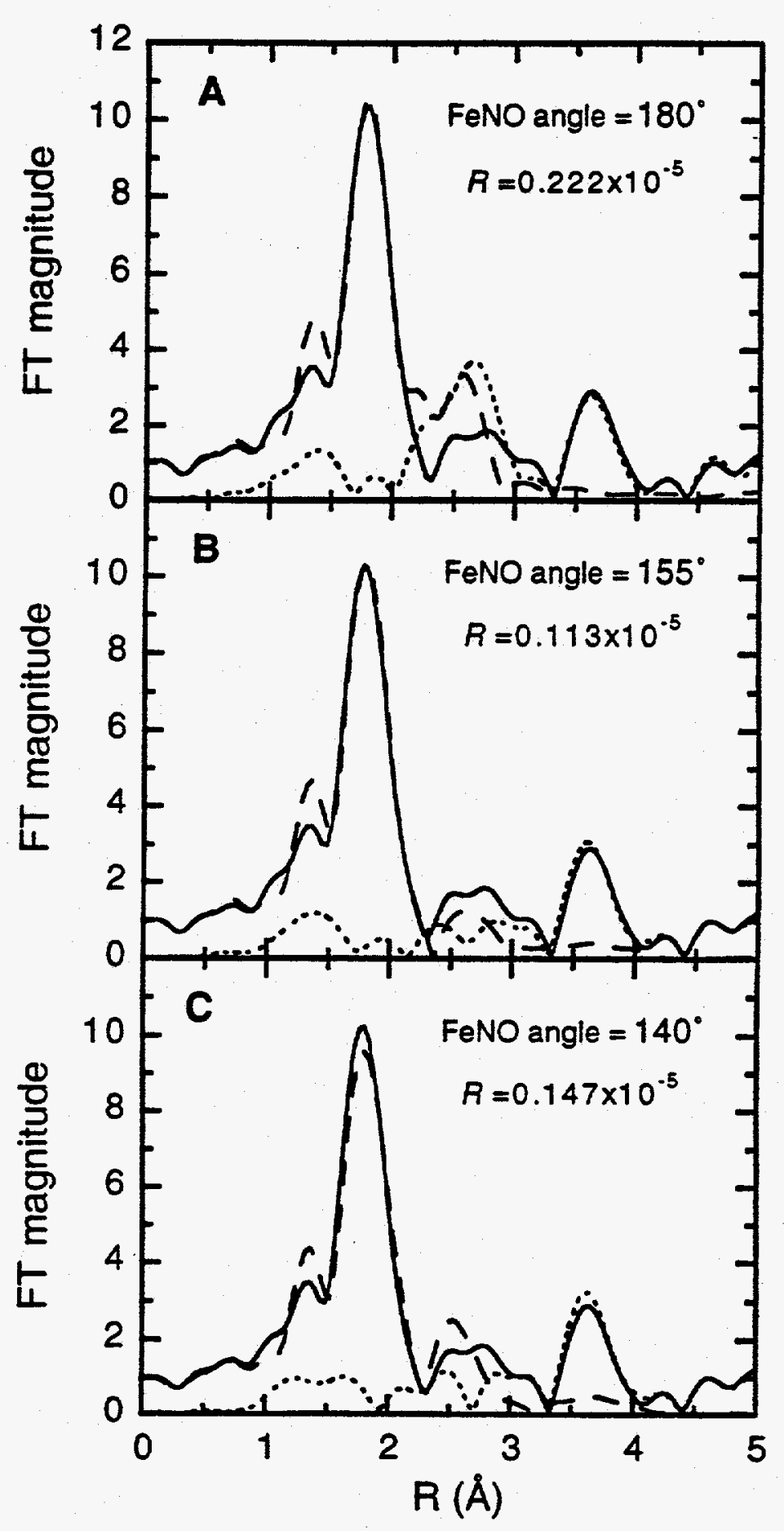

Figure 5.29. Comparison of the theoretical (--) and experimental $(-)$ non-phase shift corrected FT of FePCD-NO EXAFS data, along with the FT of the EXAFS residual (…). The $R$ value is an indication of the goodness of the fit. Calculated spectra for several different Fe-N-O bond angles are shown: A) $180^{\circ}$, B) $155^{\circ}$, and C) $140^{\circ}$. The calculated spectra included the three first-shell, two Fe-N-C (fixed), and an Fe-N-O contributions. 


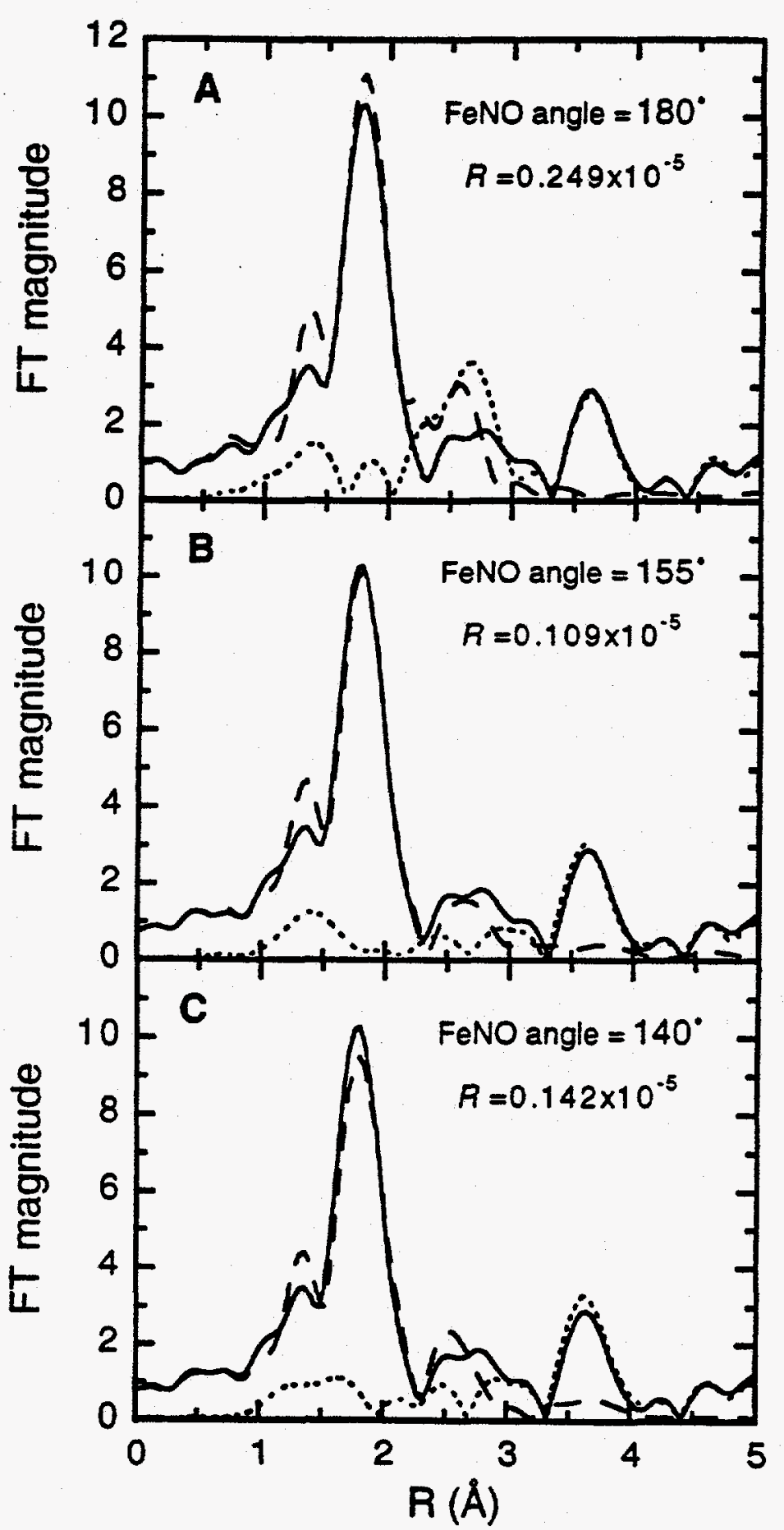

Figure 5.30. Comparison of the theoretical (--) and experimental (-) non-phase shift corrected FT of FePCD-NO EXAFS data, along with the FT of the EXAFS residual (....). The $R$ value is an indication of the goodness of the fit. Calculated spectra for several different Fe-N-O bond angles are shown: A) $180^{\circ}$, B) $155^{\circ}$, and C) $140^{\circ}$. The calculated spectra included the three first-shell, two Fe-N-C (varied), and an Fe-N-O contributions. 
individual EXAFS signals for the calculated spectra that had Fe-N-O angles of $165^{\circ}$ shows that in the latter set of fits the signal from the Fe-Ns-C configuration has increased dramatically where the strongest portion of the signal is exactly out-of-phase with the strongest portion of the $\mathrm{Fe}-\mathrm{N}-\mathrm{O}$ signal (Figure 5.31). The angle variance of the $\mathrm{Fe}-\mathrm{Ns}-\mathrm{C}$ configuration dropped from 100 (see Table 5.12) down to 1 when the Fe-N-O was fixed to $165^{\circ}$ and the $\mathrm{Fe}-\mathrm{N}-\mathrm{C}$ parameters were allowed to vary. An angle variance of 1 for four different $\mathrm{Fe}-\mathrm{Ns}-\mathrm{C}$ angles is physically unreasonable as all four of the $\mathrm{Fe}-\mathrm{Ns}-\mathrm{C}$ angles would have to be identical.

In summary, the signal in the FT of the FePCD-NO EXAFS data at $-2.5 \AA$ is very weak. The lack of signal in this region either implies that the Fe-N-O unit is bent or that there is a strong interference effect between the Fe-N-O signal and other $\mathrm{Fe}-\mathrm{O}-\mathrm{C}$ and Fe-N-C signals. Since all the Fe-O-C and Fe-N-C signals in Fe(III)PCD EXAFS data were relatively weak, it is not expected that they would be strong in the data of FePCD-NO. Thus, the FePCD-NO unit appears to be bent. An examination of the minimum in the $\log (R$ value $) v s$. Fe-N-O angle plots for the three types of fits studied indicates that the $\mathrm{Fe}-\mathrm{N}-\mathrm{O}$ angle is between $110^{\circ}$ and $160^{\circ}$. These plots look very similar to those of $\{\mathrm{FeNO}\}^{7}$ model complexes with bent $\mathrm{Fe}-\mathrm{N}-\mathrm{O}$ units and unlike that of a complex with a linear Fe-N-O unit. ${ }^{73}$ The $\log (R$ value $)$ vs. $\mathrm{Fe}-\mathrm{N}-\mathrm{O}$ angle plot for a complex with a linear Fe-N-O unit shows a minimum at $180^{\circ}$ with the $R$ value increasing dramatically as the Fe-N-O angle is lowered from $180^{\circ}$ to $150^{\circ}$.

\subsubsection{Discussion}

The analysis of the XAS pre-edge and edge features of Fe(II)PCD indicate that the iron site is six-coordinate. In addition, the fits to the EXAFS data of Fe(II)PCD show that the iron active site has $5 \pm 1$ O/N ligation with an average first-shell distance of $2.14 \AA$ which is consistent with a six-coordinate iron site. MCD results for $F e(\Pi) P C D$ also indicate that the iron site is six-coordinate. ${ }^{74}$ The iron active site of Fe(III)PCD and Fe(III)PCA appear to be five-coordinate as the pre-edge and edge features are similar to those of high spin ferric five-coordinate model complexes. The results are consistent with the crystal structure of Fe(III)PCD from Psuedomonas aeruginosa show that the iron active site to be trigonal bipyramidal with tyrosine and histidine residues bound axially and a tyrosine, histidine and water-based molecule bound in the equatorial plane. 5 An analysis of the EXAFS data for Fe(III)PCD shows O/N ligands at $1.92 \AA$ and $2.12 \AA$. These results agree well with a previous study which reported a best fit to the EXAFS data for Fe(III)PCD of $3 \mathrm{O} / \mathrm{N}$ at $1.90 \AA$ and $2 \mathrm{O} / \mathrm{N}$ at $2.08 \AA$ and attributed 


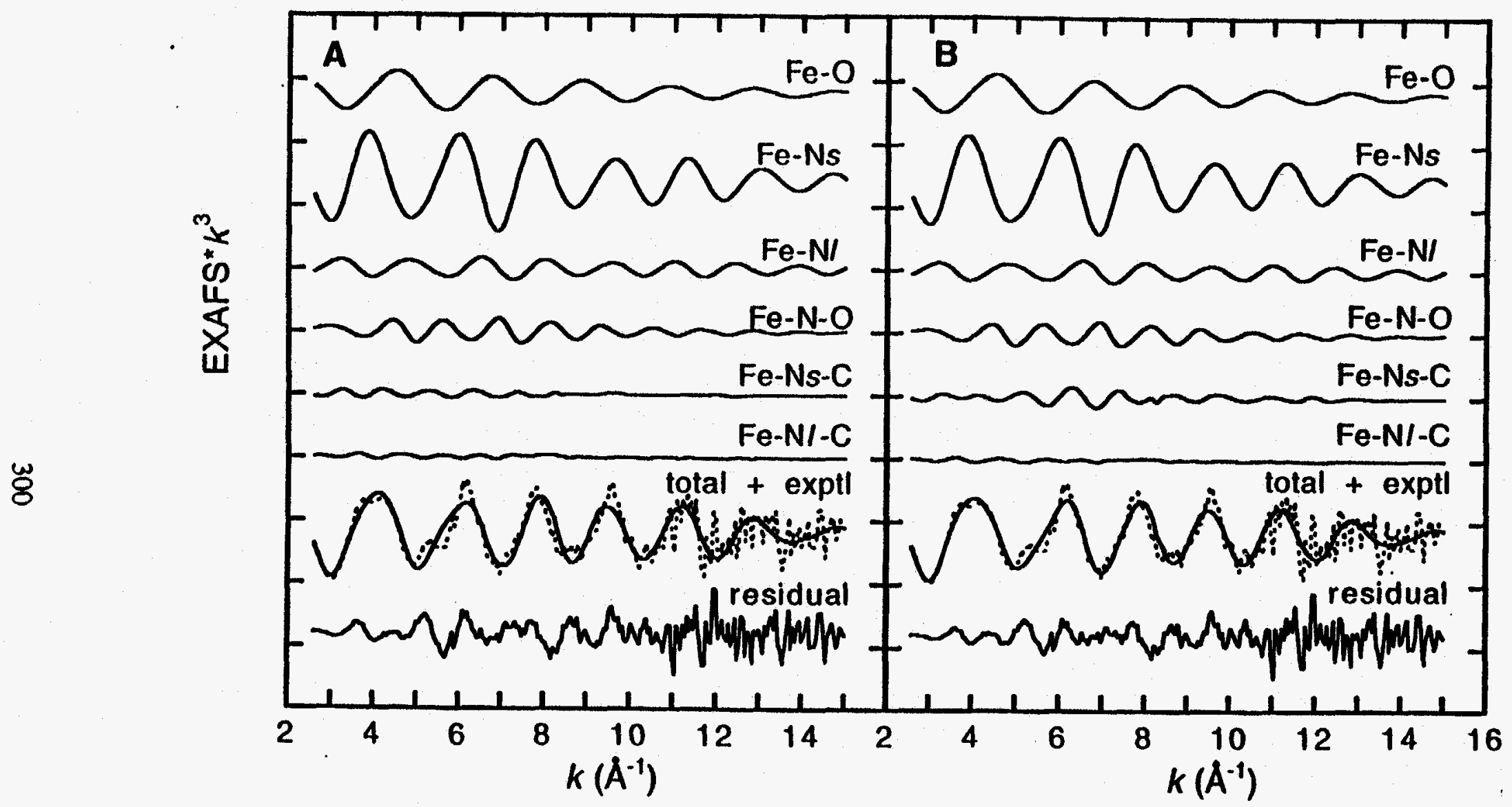

Figure 5.31. EXAFS signals for individual contributions in the GNXAS fits to the FePCD-NO data where the Fe-N-O angle was fixed to $165^{\circ}$. The total signal $(-)$ is also shown and compared with the experimental data (‥) with the residual being the difference between the experimental EXAFS and the theoretical EXAFS. (The ordinate scale is 5 between two consecutive tick marks.) Part A contains a fit where the distances, angles, and $\sigma^{2}$ values of both $\mathrm{Fe}-\mathrm{N}-\mathrm{C}$ configurations were kept fixed. Part B contains a fit where those values were allowed to vary. Note the difference in the strength of the Fe-Ns-C contribution in part A vs. B. 
the shorter distance to the bound tyrosine and hydroxide ligands and the longer distance to the twr histidine ligands. ${ }^{13}$ Thus, the coordination number increases from 5 to 6 upon reduction of the iron site. Addition of an electron may decrease the ability of the $\mathrm{Tyr} \rightarrow$ Fe charge transfer which lengthens the Fe-Tyr bond as is evident by the longer first shell distances observed for Fe(II)PCD. This relaxtion the ligand sphere allows for an additional ligand.

The EXAFS data of $\mathrm{Fe}(\mathrm{III}) \mathrm{PCA}$ could not be fit well without a longer $\mathrm{ON}$ contribution at $2.47 \AA$ in addition to contributions from $O / N$ at 1.97 and $2.10 \AA$. A fit to the EXAFS data of $\mathrm{Fe}$ (II)PCA that includes all three contributions gives a first-shell coordination number of seven which is much higher than value of five indicated by the pre-edge and analysis. The discrepancy in the coordination number between the edge and EXAFS analysis can either be explained by the fact that there are three Fe-O/N EXAFS waves beating against one another causing high correlation and thus high uncertainty in the total coordination number or that the $\mathrm{O} / \mathrm{N}$ contribution at $-2.5 \AA$ does not effect the local site symmetry of the iron which is probed in the edge and pre-edge region.

A previous EXAFS study examined the binding of terephthalate, a competitive inhibitor, and homoprotocatechuate, a slow substrate, to Fe(III)PCD. ${ }^{13}$ The study showed that when the inhibitor, terephthalate, was bound to Fe(III)PCD the pre-edge feature indicated that the iron site is six-coordinate and the EXAFS data showed a beat pattern which could only be simulated with a long Fe-O/N interaction at $2.46 \AA$. True et. al. suggested that the oxygens of the carboxylate group in terephthalate displaced the hydroxide ligand and chelated to the ferric site, but in an asymmetric fashion. In contrast, a pre-edge analysis of homoprotocatechuate bound to Fe(III)PCD showed the iron site to remain five-coordinate. ${ }^{13}$ There is strong evidence that substrate binds to Fe(III)PCD in a bidentate manner. ${ }^{8,75,76}$ If the coordination number of Fe(III)PCD bound to homoprotocatechuate is five, then the homoprotocatechuate must displace not only the hydroxide but also one of the endogenous protein ligands. True et. al. favor substrate displacing the axially histidine ligand, but do not rule out the possibility that the axially tyrosine may be displaced instead. In the present study, the pre-edge feature of $\mathrm{Fe}(\mathrm{III}) \mathrm{PCA}$ also indicates that the iron is five-coordinate. In addition, a long $\mathrm{Fe}-\mathrm{O} / \mathrm{N}$ interaction at $2.46 \AA$ was needed to simulate the EXAFS data (this contribution is readily visible in the FT of $\mathrm{Fe}$ (III)PCA at $-2.2 \AA$ (dashed line in Figure 5.22B)). If substrate binds in a bidentate fashion, either it is bound asymmetrically, in which case the long $\mathrm{Fe}-\mathrm{O} / \mathrm{N}$ contribution could be attributed to an oxygen from the substrate, or symmetrically, in which case the long Fe-O/N contribution could be attributed to an oxygen or a nitrogen from the displaced tyrosine or histidine, respectively. A detailed 
study of the multiple-scattering pathways in the various binding modes of the substrate may aid in the interpretation of the EXAFS data; however, the signal-to-noise level is too low in the higher $k$ region of the EXAFS data for Fe(III)PCA for such an analysis to be meaningful. Further study is warranted to distinguish between the various binding modes of the substrate.

The differences observed in the Fe(II)PCD, Fe(III)PCD, and FePCD-NO edge spectra are very similar to those in the Fe(II)EDTA, Fe(III)EDTA, and FeEDTA-NO edge spectra. ${ }^{58}$ FePCD-NO has a rising edge position that lies in between that of $\mathrm{Fe}$ (II)PCD and $\mathrm{Fe}$ (III)PCD with the shape and intensity of the FePCD-NO edge being much more similar to that of $\mathrm{Fe}$ (III)PCD indicating that the iron in Fe(III)PCD is in the ferric oxidation state. The rising edge of FePCD-NO is at lower energy than that of $\mathrm{Fe}$ (III)PCD due to the highly covalent nature of the Fe(II)-NO- bond. ${ }^{58}$ An analysis of the pre-edge intensity for FePCD-NO predicts that the iron site is five-coordinate with a bent Fe-N-O unit. FePCD-NO having a bent Fe-N-O unit is also supported by the GNXAS analysis of the EXAFS data for FePCD-NO. There is not a strong signal in the FT of the EXAFS data at $2.5 \AA$ which would be evident if the Fe-N-O unit were linear and the multiple-scattering of the Fe-N-O unit was enhanced due to the focusing effect. ${ }^{73}$ The plot of the $\log (R$ value) vs. Fe-N-O angle of the EXAFS data for FePCD-NO is extremely similar to that for the EXAFS data of $\mathrm{Fe}(\mathrm{TACN})\left(\mathrm{N}_{3}\right)_{2} \mathrm{NO}$, where $\mathrm{Fe}(\mathrm{TACN})\left(\mathrm{N}_{3}\right)_{2} \mathrm{NO}$ has an $\mathrm{Fe}-\mathrm{N}-\mathrm{O}$ angle of $156^{\circ} .{ }^{73}$ Resonance Raman data and self-consistent field-X $\alpha$-scattered wave calculations have shown that a bent $\mathrm{Fe}-\mathrm{N}-\mathrm{O}$ unit is stabilized by the in-plane bonding interaction of the NO- $\pi^{*}$ orbital with the iron $d$ orbital. ${ }^{58}$

The EXAFS data of FePCD-NO were fit well with $1 \mathrm{ON}$ at $1.89 \AA, 4 \mathrm{O}$ at $2.11 \AA$, and $1 \mathrm{O} / \mathrm{N}$ at $2.45 \AA$. The shorter distance is attributed to the Fe-N(O) bond distance. An Fe-N(O) distance of $1.89 \AA$ is much longer than previously observed $\mathrm{Fe}-\mathrm{N}(\mathrm{O})$ distances for $(\mathrm{FeNO}\}^{7}$ model complexes ${ }^{59-61}$ and for isopenicillin $\mathrm{N}$ synthase-substrate-NO ${ }^{71}$ which have $\mathrm{Fe}-\mathrm{N}(\mathrm{O})$ distances of $\sim 1.75 \AA$. The nature of the longer $\mathrm{Fe}-\mathrm{N}(\mathrm{O})$ bond in FePCD-NO needs to be further investigated keeping in mind that tyrosinate $\rightarrow \mathrm{Fe}$ (III) charge donation may limit the ability of NO- to donate electron density to the ferric site, thereby weakening the $\mathrm{Fe}-\mathrm{N}(\mathrm{O})$ interaction. Further, the $\mathrm{N}-\mathrm{O}$ bond of FePCD-NO should be weaker than that observed for $[\mathrm{FeNO}]^{7}$ model complexes as the $\mathrm{N}-\mathrm{O}$ bond is strengthened in these complexes due to the $\mathrm{NO} \longrightarrow \mathrm{Fe}(\mathrm{III})$ charge donation through the $4 \sigma^{+}$orbital which is antibonding with respect to the $\mathrm{N}-\mathrm{O}$ bond. ${ }^{58}$

It has been shown that an XAS edge and EXAFS analysis can provide valuable information on the electronic and geometric structure of the iron active site. Further XAS 
studies on FePCA-NO and the ferrous and ferric forms of the extradiol dioxygenases, as well as the enzyme-NO, enzyme-sibstrate, and enzyme-substrate-NO forms can be used to understand the differences of the catalytic mechanisms of the intra- vs. extradiol dioxygenases.

\subsubsection{Acknowledgments}

PCD was provided by Prof. John Lipscomb's laboratory at the University of Minnesota. Jeff Zaleski prepared and characterized the four forms of the protein in this study. This research was supported by grants from the NIH (GM40392, E. I. Solomon) and NSF (CHE-9423181, K. O. Hodgson). The Stanford Synchrotron Radiation Laboratory is supported by the Department of Energy, Office of Basic Energy Sciences, Divisions of Chemical Science and Materials Science, and in part by the National Institutes of Health, National Center of Research Resources, Biomedical Research Technology Program (RR-01209, K. O. Hodgson) and the DOE's Office of Health and Environmental Research.

\subsubsection{References and Notes}

(1) Microbial Degradation of Organic Molecules; Gibson, D. T., Ed.; Marcel Decker: New York, 1984.

(2) Harayama, S.; Kok, M.; Neidle, E. L. Annu. Rev. Microbiol. 1992, 46, 565.

(3) Que, L., Jr. In Iron Carriers and Iron Proteins; Loehr, T. M., Ed.; VCH: New York, 1989; p 467.

(4) Lipscomb, J. D.; Orville, A. M. Metal Ions Biol. Sys. 1992, 28, 243.

(5) Ohlendorf, D. H.; Lipscomb, J. D.; Weber, P. C. Nature 1988, 336, 403.

(6). Ohlendorf, D. H.; Orville, A. M.; Lipscomb, J. D. J. Mol. Biol. 1994, 244, 586.

(7) Earhart, C. A.; Radhakrishnan, R.; Orville, A. M.; Lipscomb, J. D.; Ohlendorf, D. H. J. Mol. Biol. 1994, 236, 374.

(8) Pyrz, J. W.; Roe, A. L.; Stern, L. J.; Que, L., Jr. J. Am. Chem. Soc. 1985, 107, 614.

(9) Que, L., Jr.; Heistand, R. H., II; Mayer, R.; Roe, A. L. Biochemistry 1980, 19, 2258.

(10) Que, L., Jr.; Epstein, R. M. Biochemistry 1981, 20, 2545.

(11) Felton, R. H.; Barrow, W. L.; May, S. W.; Sowell, A. L.; Goel, S.; Bunker, G.; Stern, E. A. J. Am. Chem. Soc. 1982, 104, 6132. 
(12) Whittaker, J. W.; Lipscomb, J. D. J. Biol. Chem. 1984, 259, 4487.

(13) True, A. E.; Orville, A. M.; Pearce, L. L.; Lipscomb, J. D.; Que, L., Jr. Biochemistry 1990, 29, 10847.

(14) Han, S.; Eltis, L. D.; Timmis, K. N.; Muchmore, S. W.; Bolin, J. T. Science 1995, in press.

(15) Mabrouk, P. A.; Orville, A. M.; Lipscomb, J. D.; Solomon, E. I. J. Am. Chem. Soc. 1991, 113, 4053.

(16) Fujisawa, H.; Uyeda, M.; Kojima, Y.; Nozaki, M.; Hayaishi, O. J. Biol. Chem. 1972, 247, 4414.

(17) Nozaki, M. In Molecular Mechanisms of Oxygen Activation; Hayaishi, O., Ed.; Academic Press: New York, 1974; p 405.

(18) Que, L., Jr.; Lipscomb, J. D.; Zimmerman, R.; Münck, E.; Orme-Johnson, N. R.; Orme-Johnson, W. H. Biochim. Biophys. Acta 1976, 452, 320.

(19) Whittaker, J. W.; Lipscomb, J. D.; Kent, T. A.; Münck, E. J. Biol. Chem. 1984, $259,4466$.

(20) Bull, C.; Ballou, D. P.; Otsuka, S. J. Biol. Chem. 1981, 256, 12681.

(21) Walsh, T.; Ballou, D. P.; Mayer, R.; Que, L., Jr. J. Biol. Chem. 1983, 258, 14422.

(22) Arciero, D. M.; Lipscomb, J. D.; Huynh, B. H.; Kent, T. A.; Münck, E. J. Biol. Chem. 1983, 258, 14981.

(23) Tatsuno, Y.; Saeki, Y.; Nozaki, M.; Otsuka, S.; Maeda, Y. FEBS Lett. 1980, 112, 83.

(24) Orville, A. M.; Lipscomb, J. D. J. Biol. Chem. 1993, 268, 8596.

(25) Arciero, D. M.; Orville, A. M.; Lipscomb, J. D. J. Biol. Chem. 1985, 260, 14035.

(26) Arciero, D. M.; Lipscomb, J. D. J. Biol. Chem. 1986, 260, 2170.

(27) Whittaker, J. W.; Orville, A. M.; Lipscomb, J. D. Methods Enzymol. 1990, 188, 82.

(28) Scott, R. A.; Hahn, J. E.; Doniach, S.; Freeman, H. C.; Hodgson, K. O. J. Am. Chem. Soc. 1982, 104, 5364.

(29) Lytle, F. W. In Applications of Synchrotron Radiation; Winick, H.; Xiam, D.; Ye, M.-h.; Huang, T., Eds.; Gordon and Breach Science Publishers: New York, 1989; p 135.

(30) Cramer, S. P.; Tench, O.; Yochum, M.; George, G. N. Nucl. Instrum. Methods Phys. Rev. 1988, A266, 586.

(31) George, G. N.; Pickering, I., to be published.

(32) Abbreviations used: $\mathrm{HB}\left(3,5-\mathrm{PP}_{2} \mathrm{Pz}\right)_{3}=$ hydrotris(3,5-diisopropyl-1pyrazolyl)borate; $T M C=$ tetramethylcyclam; salen = 
$N, N^{\prime}$-ethylenebis(salicylideneiminato); acac $=$ acetylacetonate; TACN $=$ $N, N^{\prime}, N^{\prime \prime}$-trimethyl-1,4,7-triazacyclononane.

(33) Kitajima, N. private communication.

(34) Hodges, K. D.; Wollman, R. G.; Barfield, E. K.; Hendrickson, D. N. Inorg. Chem. 1977, 16, 2746.

(35) Burbridge, C. D.; Goodgame, D. M. L. Inorg. Chim. Acta 1970, 4, 231.

(36) Gerloch, M.; Mabbs, F. E. J. Chem. Soc. (A) 1967, 1598.

(37) Drummond, J.; Woods, J. S. Chem. Commun. 1969, 1373.

(38) Kistenmacher, T. J.; Stucky, G.D. Inorg. Chem. 1968, 7, 2150.

(39) Cramer, S. P.; Hodgson, K. O.; Stiefel, E. I.; Newton, W. E. J. Am. Chem. Soc. 1978, 100, 2748.

(40) Cramer, S. P.; Hodgson, K. O. Prog. Inorg. Chem. 1979, $15,1$.

(41) Scott, R. A. Methods Enzymol. 1985, 117, 414.

(42) Tball, J.; Morgan, C. H. Acta Crystallogr. 1967, 23, 239.

(43) Roof, R. B. J. Acta Crystallogr. 1956, 9, 781.

(44) Johansson, L. Chem. Scr. 1976, 9, 30.

(45) Johansson, L.; Molund, M.; Oskarsson, Å. Inorg. Chim. Acta. 1978, 31, 117.

(46) Filipponi, A.; Di Cicco, A.; Tyson, T. A.; Natoli, C. R. Solid State Commun. 1991, 78, 265.

(47) Filipponi, A.; Di Cicco, A. Synchrotron Radiation News 1992, 6, 13.

(48) Westre, T. E.; Di Cicco, A.; Filipponi, A.; Natoli, C. R.; Hedman, B.; Solomon, E. I.; Hodgson, K O. J. Am. Chem. Soc. 1995, 117, 1566.

(49) Mattheiss, L. F. Phys. Rev. 1964, 134, A970.

(50) Hedin, L.; Lundqvist, S. Solid State Phys. 1969, 23, 1.

(51) Krause, M. O.; Oliver, J. H. J. Phys. Chem. Ref. Data 1979, 8, 329.

(52) The principal determining factor for $E_{\mathrm{r}}$ is the monochromator and associated vertical slit opening, with the resolution determined by the relationship $\triangle E / E=$ $\cot (\Theta) \Delta \Theta$, where $\Theta$ is a function of the Darwin width and the vertical angular acceptance of the monochromator. The value at the Fe K-edge for the experimental conditions used for these experiments were $-1.4 \mathrm{eV}$ (see ref 29).

(53) Agarwal, B. K. X-ray Spectroscopy; Springer-Verlag: New York, 1979; p 276.

(54) Lytle, F. W.; Greegor, R. B.; Sandstrom, D. R.; Marques, E. C.; Wong, J.; Spiro, C. L.; Huffman, G. P.; Huggins, F. E. Nucl. Instrum. Methods 1984, 226, 542.

(55) Tyson, T. A.; Roe, A. L.; Frank, P.; Hodgson, K. O.; Hedman, B. Phys. Rev. B 1989, 39A, 6305. 